\title{
Coordinates and Boundary Conditions for the \\ General Relativistic Initial Data Problem
}

\author{
by \\ Jonathan Thornburg \\ B.Sc. Honors 1st class \\ Simon Fraser University, 1983
}

A Thesis Submitted in Partial Fulfillment of

the Requirements for the Degree of

MASTER OF SCIENCE

in

The Faculty of Graduate Studies

Department of Geophysics and Astronomy

We accept this thesis as conforming

to the required,standard

The University of British Columbia

November 1985

(C) Jonathan Thornburg, 1985 
In presenting this thesis in partial fulfilment of the requirements for an advanced degree at the University of British Columbia, I agree that the Library shall make it freely available for reference and study. I further agree that permission for extensive copying of this thesis for scholarly purposes may be granted by the head of my department or by his or her representatives. It is understood that copying or publication of this thesis for financial gain shall not be allowed without my written permission.

Department of Geophysics and Astronomy

The University of British Columbia 1956 Main Mal1 Vancouver, Canada V6T 1 Y3

Date 11 November 1985 


\begin{abstract}
Techniques for numerically constructing initial data in the $3+1$ formalism of general relativity (GR) are studied, using the theoretical framework described in Bowen and York (1980), Physical Review D 21(8), 2047-2056. The two main assumptions made are maximal slicing and 3-conformal flatness of the generated spaces. For ease of numerical solution, axisymmetry is also assumed, but all the results should extend without difficulty to the non-axisymmetric case.
\end{abstract}

The numerical code described in this thesis may be used to construct vacuum spaces containing arbitrary numbers of black holes, each with freely specifyable (subject to the axisymmetry assumption) position, mass, linear momentum, and angular momentum. It should be emphasised that the time evolution of these spaces has not yet been attempted.

There are two significant innovations in this work: the use of a new boundary condition for the surfaces of the black holes, and the use of multiple coordinate patches in the numerical solution.

The new boundary condition studied herein requires the inner boundary of the numerical grid to be a marginally trapped surface. This is in contrast to the approach used in much previous work on this problem area, which requires the constructed spaces to be conformally isometric under a "reflection mapping" which interchanges the interior of a specified black hole with the remainder of the space. The new boundary condition is found to be easy to implement, even for multiple black holes. It may also prove useful in time evolution problems.

The coordinate choice scheme introduced in this thesis uses multiple coordinate 
patches in the numerical solution, each with a coordinate system suited to the local physical symmetries of the region of space it covers. Because each patch need only cover part of the space, the metrics on the individual patches can be kept simple, while the overall patch system still covers a complicated topology. The patches are linked together by interpolation across the interpatch boundaries. Bilinear interpolation suffices to give accuracy comparable with that of common second order difference schemes used in numerical $\mathrm{GR}$.

This use of multiple coordinate patches is found to work very well in both one and two black hole models, and should generalise to a wide variety of other numerical GR problems. Patches are also found to be a useful (if somewhat over-general) way of introducing spatially varying grid sizes into the numerical code. However, problems may arise when trying to use multiple patches in time evolution problems, in that the interpatch boundaries must not become spurious generators or reflectors of gravitational radiation, due to the interpolation errors. These problems have not yet been studied.

The code described in this thesis is tested against Schwarzschild models and against previously published work using the Bowen and York formalism, reproducing the latter within the limits of error of the codes involved. A number of new spaces containing one and two black holes with linear or angular momentum are also constructed to demonstrate the code, although little analysis of these spaces has yet been done. 
Table of Contents

Abstract ........................ . . . . . . .

Table of Contents ................. iv

List of Tables . . . . . . . . . . . . . . . . . v

List of Figures . . . . . . . . . . . . . vii

Acknowledgements ....................

Dedication .........................

Introduction . . . . . . . . . . . . . . . . 1

Theoretical Background .................... 4

Boundary Conditions . . . . . . . . . . . . 15

Choice of coordinates . . . . . . . . . . . . . . 22

Finite Differencing the Equations . . . . . . . . . . . 26

Coordinate Patches -1 . . . . . . . . . . . 36

Coordinate Patches - II . . . . . . . . . . . . . . 42

The Extringic Curvature . . . . . . . . . . . . . 44

Choice of Conformal Fonction . . . . . . . . . . . . . 48

Solving the Algebraic Equations . . . . . . . . . . . . 52

Measuring the Masses of the Models . . . . . . . . . . 56

Testing the Code . . . . . . . . . . . . . . . . 59

Demonstrating the Code . . . . . . . . . . . . 67

Conclusions and Directions for Farther Research . . . . . . . . 77

References ..................... 81

Appendix 1 .....................84

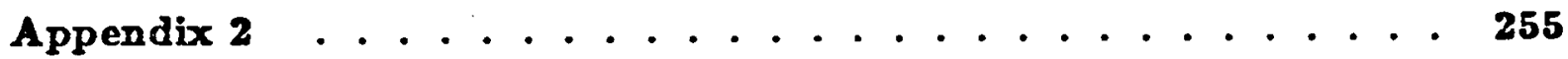




\section{List of Trbles}

1 Comparison of Different Grid Sizes . . . . . . . . . . . . 60

2 Comparison of Different Patch Types ............... 61

3 Comparison of Different Conformal Functions . . . . . . . . . 61

4 Comparison of $\delta \Psi$ with Choptuik's Results . . . . . . . . . . 65

5 Model index for the Schwarzschild test series . . . . . . . . . 85

6 Model index for the Choptuik comparison test series . . . . . . . 86

7 Model index for the $\mathrm{J}$ test series . . . . . . . . . . . . 87

8 Model index for the RSP test series . . . . . . . . . . . . 87

9 Model index for the RSM test series . . . . . . . . . . . 88

10 Model index for the NTO test series . . . . . . . . . . . . 88

11 Model index for the PP test series . . . . . . . . . . . . 89

12 Schwarzschild test series, 10plo100 selected $\Psi$ values . . . . . . . 93

13 Schwarzschild test series, 20plo100 selected $\Psi$ values . . . . . . . 97

14 Schwarzschild test series, 40 plo100 selected $\Psi$ values . . . . . . . 101

15 Schwarzschild test series, c2 selected $\Psi$ values . . . . . . . . . 105

16 Schwarzschild test series, c01 selected $\Psi$ values . . . . . . . . . . 109

17 Schwarzschild test series, c2i selected $\Psi$ values . . . . . . . . . . . 113

18 Schwarzschild test series, poo selected $\Psi$ values . . . . . . . . . . 117

19 Schwarzschild test series, opo selected $\Psi$ values . . . . . . . . . . 121

20 Schwarzschild test series, oop selected $\Psi$ values . . . . . . . . . . 125

$21 \mathrm{~J}$ test series, $\mathrm{j} 0$ selected $\Psi$ values . . . . . . . . . . . 171

22 RSP test series, p0 selected $\Psi$ values $\ldots \ldots$. . . . . . . . . 186

23 RSN test series, p0 selected $\Psi$ values . . . . . . . . . . . . 201

24 KTO test series, po selected $\Psi$ values $\ldots . . . . . . . . . . .216$ 
25 PP test series, m100 selected $\Psi$ values . . . . . . . . . . . 231

26 PP test series, m10 selected $\Psi$ values _. . . . . . . . . . . 233

27 PP test series, $\mathrm{ml}$ selected $\Psi$ values $\ldots \ldots 235$

28 PP test series, $\operatorname{m\times 1}$ selected $\Psi$ values $\ldots \ldots 237$

29 PP test series, mx01 selected $\Psi$ values . . . . . . . . . . . . 239

30 PP test series, 0 selected $\Psi$ values . . . . . . . . . . . 241

31 PP test series, pr01 selected $\Psi$ values . . . . . . . . . . . 243

32 PP test series, px1 selected $\Psi$ values _. . . . . . . . . . . . 245

33 PP test series, p1 selected $\Psi$ values . . . . . . . . . . . . 247

34 PP test series, p10 selected $\Psi$ values f . . . . . . . . . . 249

35 Pp test series, p100 selected $\Psi$ values . . . . . . . . . 251

36 Directories where the code resides . . . . . . . . . . . . 256

37 File index for the main directory . . . . . . . . . . . 257

38 File index for the fortran directory ............. 258

39 File index for the patchutil directory . . . . . . . . . . . 259

40 File index for the util directory ... . . . . . . . . 260 


\section{List of Figares}

1 "Square" vs. "Triangle" Interpolation . . . . . . . . . . . . . 39

2 Bilinear Interpolation Formulae . . . . . . . . . . 40

3 Baseline patch system for table 2 . . . . . . . . . . . 62

4 Augmented patch system for table 2 . . . . . . . . . . 63

$5 \mathrm{~J}$ Test Series Solutions . . . . . . . . . . . . . . . . 69

6 RSP Test Series Solutions . . . . . . . . . . . . . 70

7 RSN Test Series Solutions . . . . . . . . . . . . . 71

8 NTO Test Series Solutions . . . . . . . . . . . . . 72

9 Patch System for the PP Test Series _. . . . . . . . . . 74

10 PP Test Series Solutions $(P \geq 0) \quad \ldots . . . \ldots 75$

11 PP Test Series Solutions $(P \leq 0) \quad \ldots . . . \ldots 76$

12 Choptuik comparison test series, ppro01 quantitative results . . . . 127

13 Choptuik comparison test series, ppx01 quantitative results . . . . . 129

14 Choptuik comparison test series, ppx1 quantitative results . . . . . 131

15 Choptuik comparison test series, pp1 quantitative results . . . . 133

16 Choptuik comparison test series, pp2x5 quantitative results . . . . 135

17 Choptuik comparison test series, pp7×5 quantitative results . . . . 137

18 Choptuik comparison test series, pp12×5 quantitative results . . . . . 139

19 Choptuik comparison test series, mpx001 quantitative results . . . . . 141

20 Choptuilk comparison test series, mpx01 quantitative results . . . . 143

21 Choptuik comparison test series, mpr1 quantitative results . . . . . . 145

22 Choptuik comparison test series, mp1 quantitative results . . . . . 147

23 Choptuik comparison test series, mp2x5 quantitative results . . . . . 149

24 Choptuik comparison test series, mp7×5 quantitative results . . . . 151 
25 Choptuik comparison test series, mp12x5 quantitative results . . . . . . 153

26 Choptuik comparison test series, jz001 quantitative results . . . . . 155

27 Choptuik comparison test series, jx01 quantitative results . . . . . . 157

28 Choptuik comparison test series, $j \times 1$ quantitative results $\ldots . . . . . .159$

29 Choptuik comparison test series, $\mathrm{J} 1$ quantitative results . . . . . . 161

30 Choptuik comparison test series, j10 quantitative results . . . . . . 163

31 Choptuik comparison test series, j100 quantitative results . . . . . . 165

32 Choptuik comparison test series, j1000 quantitative results . . . . . 167

$33 \mathrm{~J}$ test series, $\mathrm{j} \times 01$ quantitative results . . . . . . . . . . . . . 173

$34 \mathrm{~J}$ test series, $\mathrm{j} \times 1$ quantitative results . . . . . . . . . . . . 175

$35 \mathrm{~J}$ test series, $\mathrm{j} 1$ quantitative results ................. 177

$36 \mathrm{~J}$ test series, $\mathrm{j} 10$ quantitative results . . . . . . . . . . . . 179

$37 \mathrm{~J}$ test series, $\mathrm{j} 100$ quantitative results . . . . . . . . . . . 181

38 RSP test series, pr01 quantitative results _. . . . . . . . . . 188

39 RSP test series, pr1 quantitative results . . . . . . . . . . . . . 190

40 RSP test series, p1 quantitative results . . . . . . . . . . . . . . 192

41 RSP test series, p10 quantitative results . . . . . . . . . . . . 194

42 RSP test series, p100 quantitative results . . . . . . . . . . . . 196

43 RSN test series, pr01 quantitative results . . . . . . . . . . . . 203

44 RSM test series, px1 quantitative results . . . . . . . . . . . . . 205

45 RSN test series, p1 quantitative results . . . . . . . . . . . . 207

46 RSM test series, p10 quantitative results . . . . . . . . . . . . . 209

47 RSN test series, p100 quantitative results _. . . . . . . . . . . 211

48 NTO test series, pr01 quantitative results . . . . . . . . . . . . 218

49 NTO test series, pr1 quantitative results . . . . . . . . . . . . . 220 
50 NT0 test series, p1 quantitative results . . . . . . . . . . 222

51 NT0 test series, p10 quantitative results . . . . . . . . . . 224

52 MTO test series, p100 quantitative results . . . . . . . . . 226 


\section{Acknowledgements}

First and foremost, I must acknowledge the many contributions of W. G. Unruh to this thesis. In addition to many stimulating conversations, he provided one of the two key ideas of this thesis (the marginally trapped surface boundary condition), as well as many other helpful suggestions. The exposition of this thesis has also benefited greatly from his comments.

F. Chen gave invaluable help with the $\operatorname{TEX}_{\mathrm{X}}$ typesetting system used to prepare this thesis. M. Choptuik kindly reexamined the results from his own GR code and did a new perturbation analysis of them to provide me with comparison values to test out my code.

My choice of topic for this thesis was helped by early discussions with M. Choptuik, T. Piran and J. York. U. Ascher, M. Choptuik, and M. Yedlin provided valuable "physics" suggestions, and L. Brewin, G. Grieve, and T. Nicol helped me with a variety of computer problems.

I thank The University of British Columbia for financial support. The computer facility used to do the calculations of this thesis was funded by the Natural Sciences and Engineering Research Council. 


\section{Dedication}

This thesis is dedicated to all of my professors, who together have shaped my mind and inspired me to learn. In particular, Hans Thom first interested me in physics, Ken Lyall nurtured that interest, Leigh Hunt Palmer first interested me in general relativity by giving me a copy of Price (1982), and Michael Ovenden gently guided me in the direction of Bill Unruh.

There are certain common Privileges of a Writer, the Benefit whereof, I hope, there will be no Reason to doubt; Particularly, that where I am not understood, it shall be concluded, that something very useful and profound is coucht underneath. - Jonathan Swift, "Tale of a Tub", Preface (1704) 


\section{Introduction}

Due to their complexity, the Einstein equations of general relativity (hereinafter "GR") are very. hard to solve analytically. Indeed, such solutions have been found only for systems with either a high degree of symmetry, or for systems in which the non-linearity of the equations can be taken to be "small". Such restrictions exclude many interesting systems, for example the collision and subsequent merger of two black holes.

One way of examining systems not meeting these restrictions, is to numerically solve the Einstein equations, using the methods developed for other nonlinear PDE problems such as the Navier-Stokes equations. This approach, known as "numerical $\mathrm{GR}^{n}$, has seen substantial use in the past few decades. Most commonly, the "finite difference" technique is used to discretise the equations prior to solving them on a digital computer, although the alternative "finite elements" method has seen some limited use.

This thesis studies the problem of constructing spaces containing multiple moving and/or spinning black holes, and involves the construction of a numerical GR code to do this. This problem was selected for three main reasons. First, the time evolution of such spaces (which has not yet been performed) would be of great interest. Second, previous work has established a general theoretical framework for the solution of this problem. Third, and perhaps most important, it seems to provide a good "test-bed" problem for trying out various coordinates and boundary conditions.

The main results of this thesis are the introduction of a new and more general scheme for choosing coordinate systems, and of a new and physically well-motivated 
boundary condition for black hole surfaces. Together, these should allow the abovementioned theoretical framework to be more easily applied to a wider variety of problems, including the two black hole collision problem mentioned above.

In general, the emphasis in this thesis is more on the techniques used than on the results obtained. However, as well as replicating earlier work involving single black holes, some new spaces are constructed. In particular, the new boundary condition is used in the construction of a series of spaces containing moving single black holes. A series of spaces containing single spinning black holes are also constructed, although here the new boundary condition turns out to be equivalent to that used in previous work. Finally, both the new boundary condition and the new coordinate choice scheme are used in the construction of a series of spaces containing two black holes with equal and opposite momenta. The latter have not been numerically studied before.

The remainder of this thesis will now be outlined. Successive chapters discuss the theoretical backround to the work, the various boundary conditions (both old and new) involved, and the coordinate choice problem. The general technique of finite differencing is then discussed, and applied to the problem of this thesis. The following chapters give more details of the code used in this thesis, including the internal representation of patch systems, the "human-readable" patch description language used to specify them, the detailed means by which the contents (for example " 2 black holes with such-andsuch positions, masses, momenta, and spins ${ }^{\prime}$ ) of the spaces are specified, and some final details of just how the equations are set up and solved. The various "mass functionals" used to measure the mass-energy of the black holes and spaces constructed are then discussed, followed by the means used to test the code. The new spaces used to demonstrate the code are described, followed by some conclusions and directions for 
further research. Two appendices give the detailed input parameters to and quantitative graphs of the results of each model described in the thesis, and the source listings for the code itself.

For the most part, the chapters are organized so that they may be read independently. To this end, all notational conventions are defined near the beginning of the “Theoretical Background" chapter. Also, for the benefit of anyone wishing to replicate the results of this thesis, the main equations used in the code are all (with one exception described in the text) given in the body of the thesis as well, even if their derivations are merely tedious excercises in algebra. This should spare the reader the task of trying to "unprogram" the equations from the code listings. 


\section{Theoretical Background}

As usually written, the Einstein equations refer to spacetime as a whole, without reference to any one coordinate being singled out as a "global time". In contrast, many other physical theories (for example classical electromagnetism as described by the Maxwell equations) can be phrased in terms of the time evolution of various quantities. By analogy, we suspect that such a formulation of GR (as a dynamical theory) would be useful. Such a formulation does indeed exist, under suitable technical assumptions to guarantee the "non-pathological" nature of spacetime. However, the details of how this is set up are beyond the scope of this thesis, and will not be discussed here. The interested reader is referred to Choptuik (1982), Misner, Thorne, and Wheeler (1973 (herinafter cited as "MTW"), chapter 21), Wald (1984, chapter 10 and appendix E), York (1979), York (1983), and York and Piran (1982) for further discussions of this topic. In particular, the cited works by York et al contain excellent discussions of how GR can be formulated as a dynamical theory in which the notion of "time evolution" is well defined. These works all build on the basic theoretical framework of the classic paper of Arnowitt, Deser, and Misner (1962).

We now discuss the theoretical foundations of this approach to GR, as well as the particular theoretical framework used in this thesis. Our discussion gives only a brief overview of the results used in this thesis, and will be kept to the least technical level possible. Tensor calculus will be used, but only in its coordinate form, i.e. the modern "coordinate-free" language and techniques of differential geometry will not be used. More advanced and comprehensive discussions can be found in the cited references.

The basic idea of the "ADM" framework is to view 4-dimensional spacetime as being composed of a 1-dimensionally infinite "stack" of 3-dimensional spacelike hyper- 
surfaces (hereinafter "spaces" or "slices"), each labeled with a "time" coordinate. This decomposition of spacetime will often be referred to as "slicing" it. To give a dynamical interpretation to GR, we choose "initial data" defined on a single slice, and then try to "time evolve" it with the Einstein equations. This " $3+1$ " approach to the dynamics of GR is often referred to as (solving) the "Cauchy problem". However, there are two interrelated difficulties which arise when we try to do this.

First, not all the initial data can be freely specified on the initial slice. This is because of the Bianchi identities, which reduce by four the number of independent metric components. A useful analogy here is the specification in electromagnetism of $E$ and $B$ at a single moment in time-Gauss's law and its magnetic analog prevent these from being completely freely specifyable. Such initial data as can be freely specified on the initial slice, presumably represent the true degrees of freedom of our problem. Recent work by York and others on the problem of defining freely specifyable GR initial data has yielded a general theoretical framework for dealing with this problem, which we adopt for this thesis.

Second, how do we remove the coordinate freedom inherent in the Einstein equations? The problem here is that a "solution" to a GR dynamical problem will presumably consist of a specification of all components of the metric over some time interval. This (together with the ranges of the coordinates) implicitly specifies the coordinate system in use throughout that interval, which clearly is not a true dynamical feature of the spacetime. Thus there can not and must not be enough Einstein equations to determine the evolution of all the metric components-some additional information must be supplied (thus defining the choice of coordinates). This must be done prior to obtaining the spacetime, so there is clearly a risk of choosing coordinates which are 
inappropriate for the symmetries of the spacetime. Worse still, the chosen coordinates may fail to cover the whole spacetime, or they may cover undesired areas of it, for example the immediate neighborhoods of singularities. This problem still lacks a fully satisfactory solution, although considerable progress has been made. This thesis deals only with the construction of initial data, so this difficulty will not be further discussed.

Corresponding to the slicing of spacetime, the $3+1$ approach to GR splits the 10 Einstein equations into 4 "constraint" equations for the initial data, and 6 "evolution" equations which specify how this data evolves with time. This thesis deals solely with the former, i.e. with the construction of spaces which satisfy the constraint equations. The theoretical background for this construction will now be discussed, beginning with the $3+1$ slicing process itself.

Our notation generally follows that of MTW, with units chosen such that $G=c=1$ and the metric having signature $(-,+,+,+)$. We shall sometimes use " $t$ " as a synonym for the coordinate $x^{0}$. Indices $a, b, c, \ldots$ range from 0 to 3 , while indices $i, j, k, \ldots$ have the restricted range of 1 to 3 . The usual summation convention is assumed for both types of indices. Greek letters will not be used for indices. $\partial_{a}$ denotes the partial derivative operator with respect to the coordinate $x^{a}$, while $\nabla_{a}$ denotes the covariant derivative operator with respect to the same coordinate. Where ambiguity exists, quantities defined with respect to the full 4-dimensional spacetime are prefixed with a $^{(4)}$, as in ${ }^{(4)} R$, while quantities defined with respect to a 3-dimensional space are prefixed with a ${ }^{(3)}$, as in ${ }^{(3)} R$.

Non-italic bold letters denote mathematical objects which do not transform as tensors, such as vectors and matrices (for which the index range conventions given above do not apply). Typewriter font identifies objects (for example names of models 
or individual functions) known to or defined in the computer code itself.

We shall adopt one other important notational convention-the Penrose "abstract index" notation. This involves (almost) always writing tensors with the number of indices appropriate to their rank, even if the tensor as a whole is being referred to. Thus, we refer to the 4-metric (viewed as a single object) by the symbol ${ }^{(4)} g_{a b}$, rather than ${ }^{(4)} g$ as is sometimes done. The latter symbol is used to deonote a (presumably related) scalar (rank zero) object. Another way to look at this notation is to just view all tensor equations as component equations with an implied "for all free (not summed over) indices" appended.

We begin the slicing process by considering the full 4-dimensional metric ${ }^{(4)} g_{a b}$. We view ${ }^{(4)} g_{a b}$ as the partitioned matrix

$$
{ }^{(4)} g_{a b}=\left[\begin{array}{l|l}
{ }^{(4)} g_{00} & { }^{(4)} g_{0 j} \\
\hline{ }^{(4)} g_{i 0} & { }^{(4)} g_{i j}
\end{array}\right]
$$

and define the (covariant) " 3 -metric" (3) $g_{i j}$ to be the spatial components of the 4-metric

$$
{ }^{(3)} g_{i j}={ }^{(4)} g_{i j}
$$

so it denotes the lower right submatrix of (1). It can be shown that the ${ }^{(3)} g_{i j}$ so defined is indeed the (covariant) metric induced on the hypersurface $t=$ constant by the 4 'metric ${ }^{(4)} g_{a b}$. Thus ${ }^{(3)} g_{i j}$ is (as the notation implies) a (symmetric) 3-tensor. Given ${ }^{(3)} g_{i j}$, we define the contravariant 3 -metric ${ }^{(3)} g^{i j}$ in the usual way as the matrix inverse of ${ }^{(3)} g_{i j}$.

We now define a scalar $\alpha$ and a 3-vector $\beta_{i}$, known as the "lapse" and "shift" functions respectively, by

$$
\left.\begin{array}{l}
{ }^{(4)} g_{00}=-\left(\alpha^{2}-\beta_{i} \beta^{i}\right) \\
{ }^{(4)} g_{0 i}={ }^{(4)} g_{i 0}=\beta_{i},
\end{array}\right\}
$$


where the index on $\beta^{i}$ is raised with the 3 -metric. The timelike nature of $t$ can be shown to guarantee that $\alpha$ comes out real and positive.

The lapse and shift functions defined above are the means by which the time evolution of our coordinate system itself is defined-they must be given as inputs to the Einstein evolution equations. They can be given an elegant geometric interpretation as the time and space components of a "time step" 4-vector (see for example, York $(1979)$, p. 89). However, this is beyond the scope of this thesis; their only use herein is as a vehicle in the definition of the variables of our problem.

Finally, we define the "extrinsic curvature" $K_{i j}$ by

$$
\frac{\partial^{(3)} g_{i j}}{\partial t}=-2 \alpha K_{i j}+{ }^{(3)} \nabla_{i} \beta_{j}+{ }^{(3)} \nabla_{j} \beta_{i}
$$

So defined, $K_{i j}$ can be shown to transform as a (symmetric) 3-tensor under 3-coordinate transformations, and should perhaps be written ${ }^{(3)} K_{i j}$, but no confusion will arise from the simpler form $K_{i j}$.

As defined, $K_{i j}$ is essentially a "generalized velocity" conjugate to the "generalized position" ${ }^{(3)} g_{i j}$. Indeed, the natural choice

$$
\left.\begin{array}{l}
\alpha=1 \\
\beta^{i}=0,
\end{array}\right\}
$$

(frequently referred to as "Gaussian Normal" or "harmonic" coordinates) leads to

$$
K_{i j}=-\frac{1}{2} \frac{\partial^{(3)} g_{i j}}{\partial t}
$$

$K_{i j}$ can also be interpreted as specifying just how the hypersurface $t=$ constant is embedded into spacetime-this is why it's called the "extrinsic curvature" tensor. However, both of these interpretations are beyond the scope of this thesis, which uses $K_{i j}$ only in specifying the contents of the spaces. 
Having defined the 3-metric and the (3-)extrinsic curvature, we now consider the “initial data" problem of constructing suitable initial data to be time evolved. (This problem is sometimes referred to as the "initial value" problem, but the author feels that this usage invites confusion with the latter term's well established meaning in a number of other fields, notably the study of numerical solutions of both ODEs and PDEs.) As described in the cited references, this amounts to constructing suitable 3-metrics and extrinsic curvatures at a single moment in time. This is the overall problem addressed by this thesis. By "suitable", we mean both consistent with the Einstein constraint equations, and containing the desired physical objects. In this thesis, the latter are multiple moving and/or spinning black holes. The theoretical framework used in this thesis is that of Bowen and York (1980), which we will now discuss.

We begin by writing the Einstein constraint equations for the initial data as

$$
\nabla^{j}\left(K_{i j}-{ }^{(3)} g_{i j} \operatorname{Tr}(K)\right)=8 \pi j_{i}
$$

and

$$
{ }^{(3)} R-{ }^{(3)} g^{i m(3)} g^{j n} K_{i j} K_{m n}+(\operatorname{Tr}(K))^{2}=16 \pi \rho
$$

where $\rho$ and $j_{i}$ are the time and space components of the energy-momentum 4-vector of sources, ${ }^{(3)} R$ is the Reimann curvature scalar of the 3-metric, $\operatorname{Tr}(K)$ is the trace of $K_{i j}$ defined by

$$
\operatorname{Tr}(K)={ }^{(3)} g^{i j} K_{i j}
$$

and indices are raised and lowered with the 3-metric.

We now make two simplifying assumptions. The first is that our initial slice satisfies

$$
\operatorname{Tr}(K)=0
$$


This condition, which is widely used in numerical GR, can be interpreted (see, for example, York and Piran (1982), pp. 157-158) as requiring our space to be of maximal volume as embedded into spacetime, and is thus often referred to as the "maximal slicing" condition. It is known that many "interesting" spacetimes admit slicings satisfying this condition. York and Piran (1982) give as examples the Schwarzschild, Kerr, and Minkowski (flat) spacetimes as well as those "sufficiently close" (in a suitable norm) to them. They also state that "every stationary, axisymmetric, and asymptotically flat spactime that is topologically $\mathbf{R}^{4}$ has maximal spacelike slices." $\delta$ Murchadha and Seifert (1982) have shown that the requirement of axisymmetry may be removed in this statement. Nonetheless, this condition probably excludes some physically interesting spacetimes. It should be noted that much of the present work relies only on $\operatorname{Tr}(K)$ being a constant, although York and Piran suggest non-zero values as being appropriate for asymptotically null spaces, rather than the asymptotically strictly spacelike ones studied in this thesis.

The second simplifying assumption is that the space is 3-conformally flat,

$$
{ }^{(3)} g_{i j}=\Psi^{4} f_{i j}
$$

where $f_{i j}$ is a flat 3-metric and $\Psi$ is an unknown (positive) "conformal factor". The use of the fourth power here is for later convienence, and is of no fundamental significance. This assumption is perhaps the most serious limitation of this approach to the initial data problem. An arbitrary spacetime, even one known to posess maximal slices, in general does not posess any slicing satisfying this condition.. However, the Schwarzschild solution does satisfy this assumption in any coordinate system which uses the usual Schwarzschild time coordinate, so we know that the existence of black holes isn't ruled out. 
The conformal factor can be interpreted as an isotropic "local stretching" of space, in that the transformation

$$
{ }^{(3)} g_{i j} \rightarrow \Psi^{4}{ }^{(3)} g_{i j}
$$

leaves angles locally invariant, but isotropically multiplies local distances by a factor of $\Psi^{2}$. Wald (1984, appendix D) discusses conformal transformations in more detail.

We now subject the other variables in the Einstein constraint equations (7) and (8) to the transformation

$$
\left.\begin{array}{rl}
\rho & =\Psi^{-8} \hat{\rho} \\
j_{i} & =\Psi^{-6} \hat{\jmath}_{i} \\
K_{i j} & =\Psi^{-2} \hat{K}_{i j}
\end{array}\right\}
$$

where "hatted" quantities refer to the "hat-means-flat" transformed (flat) space with 3-metric $f_{i j}$. This results in (the Einstein constraint equations) (7) and (8) simplifying to

$$
\hat{\nabla}^{j} \hat{K}_{i j}=8 \pi \hat{j}_{i}
$$

and

$$
\nabla^{2} \Psi+\frac{1}{8} \hat{K}_{i j} \hat{K}^{i j} \Psi^{-7}=2 \pi \hat{\rho} \Psi^{-3},
$$

where $\nabla^{2}$ is the standard flat-space Laplacian operator. (Strictly speaking, it should have a "hat", but no confusion should arise from the omission.)

We restrict our attention to vacuum spaces (outside black holes), so we take $\hat{\jmath}_{i}=$ $\hat{\rho}=0$. All of the following discussion takes place in the transformed flat space; "hats" and ${ }^{(3)}$ prefixes are often omitted in the interests of typographical clarity. Indices are raised and lowered with the flat 3-metric $f_{i j}$.

We observe that (14) does not involve $\Psi$ and hence is effectively decoupled from (15). In fact, this would still happen even if the maximal slicing assumption (10) were 
replaced by any other slicing satisfying

$$
\partial_{i} \operatorname{Tr}(K)=0
$$

Failure to satisfy this latter condition would result in our having to solve (14) and (15) simultaneously, rather than sequentially as we do in this thesis.

Bowen and York show that (14) may be solved analytically through the use of a vector potential. Their general solution is not needed herein; we shall use only two of their specific solutions, namely those representing single black holes with either specified (linear) momentum or specified spin (angular momentum).

For a single black hole with linear momentum $P^{i}$ (and no spin), Bowen and York give the extrinsic curvature as (the following solution to (14))

$$
\begin{aligned}
\hat{K}_{i j}= & \frac{3}{2 r^{2}}\left[P_{i} n_{j}+P_{j} n_{i}-\left(f_{i j}-n_{i} n_{j}\right) P^{k} n_{k}\right] \\
& -\kappa \frac{3 a^{2}}{2 r^{4}}\left[P_{i} n_{j}+P_{j} n_{i}+\left(f_{i j}-5 n_{i} n_{j}\right) P^{k} n_{k}\right],
\end{aligned}
$$

where $n^{i}$ is the outward pointing unit normal to the 2 -sphere of radius $r$ centered on the black hole, $a$ is an arbitrary parameter (later to be interpreted as one half the mass of the black hole), and $\kappa= \pm 1$. The significance of $\kappa$, as well as possible reasons for taking it equal to zero instead of \pm 1 (thus dropping the second term of (17) entirely), will be discussed in the next chapter.

For a single black hole with spin $J^{i}$ (and no momentum), they give the solution

$$
\hat{K}_{i j}=\frac{3}{r^{3}} J^{l} n^{k}\left(\epsilon_{k i l} n_{j}+\epsilon_{k j l} n_{i}\right)
$$

where $n^{i}$ and $r$ are as before and

$$
\epsilon_{i j k}=\left\{\begin{array}{cl}
+1 & \text { if }(i j k) \text { is an even permutation of (123) } \\
-1 & \text { if }(i j k) \text { is an odd permutation of (123) } \\
0 & \text { otherwise }
\end{array}\right.
$$


is the usual completely antisymmetric unit alternating Levi-Civita symbol.

The only remaining point to be made here about the solution of (14) is that, since it's a linear equation, we may add solutions together to obtain $\hat{K}_{i j}$ (and hence $K_{i j}$ by (13), once (15) has been solved) for spaces containing an arbitrary number of black holes, each with arbitrary and freely specifyable mass, position (obtained by translating the origin of the solutions given above), momentum, and spin.

Given a suitable solution to (14), we define a "source function" $S$ by

$$
S=\frac{1}{8} \hat{K}_{i j} \hat{K}^{i j}
$$

If we view (15), which we rewrite as

$$
\nabla^{2} \Psi+S \Psi^{-7}=0
$$

as a nonlinear Poisson equation, $S \Psi^{-7}$ plays the role (modulo a factor of $4 \pi$ ) of a charge density. This analogy with classical electrostatics will prove helpful in our discussion of the solution of (21). It is the (numerical) solution of this equation which is the main topic of this thesis.

We now make one additional simplifying assumption, namely that our spaces are axisymmetric. This assumption is in no way implicit in the Bowen and York formalism, but is made purely to simplify our numerical solution of (21). It does this by allowing us to neglect one space dimension (cooresponding to rotation about the symmetry axis) in the numerical solution, so we need only consider 2 space dimensions. Obviously, this greatly reduces the computational difficulty of numerically solving (21).

There is, however, another advantage to considering only 2 space dimensions. This is that we can obtain a global "bird's eye" view of a function (for example $S$ or $\Psi$ ) 
defined on our space, with a single plot of the function vs. (2-dimensional) position, viewed in perspective. No comparable technique is available for spaces of $\mathbf{3}$ or more dimensions-one must resort to the piecemeal examination of "slices" of the space. This lack of an easy means of viewing a function over the whole space is perhaps a more serious impediment to the treatment of fully 3-dimensional spaces than the greater computational difficulties, since the latter are diminishing with time as available computers increase in speed and memory capacity. 


\section{Bonndary Conditions}

We now consider what boundary conditions should be imposed on $\Psi$ to specify a unique and physically meaningful solution to (21), and hence a space containing the desired objects. The following discussion takes place in the "hat-means-flat" space with (3-)metric ${ }^{(3)} g_{i j}=f_{i j}$. As mentioned above, we take this space to be axisymmetric, with the axis of symmetry chosen as the $z$ axis and $\phi$ the angle of rotation about it. We introduce the usual polar spherical coordinates $(r, \theta, \phi)$, with the line element $d s^{2}=d r^{2}+r^{2} d \theta^{2}+r^{2} \sin ^{2} \theta d \phi^{2}$. We also introduce cylindrical coordinates $(\rho, z, \phi)$, with the line element $d s^{2}=d \rho^{2}+d z^{2}+\rho^{2} d \phi^{2}$.

We first consider the "outer" boundary. Since the physical "unhatted" space is asymptotically flat, we have

$$
\lim _{r \rightarrow \infty} \Psi=1
$$

However, our numerical "grid" will terminate at some finite radius $r$, so we must choose a boundary condition there which will best approximate the true boundary condition (22).

We could of course just take $\Psi=1$ on our outer boundary, but this would not give very accurate results. Instead, York and Piran (1982) suggest exploiting more of the physics of the problem by observing that if $E$ is the total energy of the space, the asymptotic behavior of $\Psi$ as $r \rightarrow \infty$ will be

$$
\Psi=1+\frac{E / 2}{r}+O\left(\frac{1}{r^{2}}\right)
$$

Thus

$$
\frac{\partial \Psi}{\partial r}+\frac{\Psi-1}{r}=O\left(\frac{1}{r^{3}}\right)
$$


We now neglect the right hand side of this latter equation to obtain a fairly accurate "Robin" outer boundary condition,

$$
\frac{\partial \Psi}{\partial r}+\frac{\Psi-1}{r}=0
$$

We next consider the problem of choosing an "inner" boundary condition for the "surfaces" of the black holes, assuming that we don't want to extend our numerical grid inside the black holes. We first review the approach taken by Bowen and York (1980), which begins by considering the case of a single black hole only.

They suggest requiring that the space be conformally isometric under the "reflection" mapping

$$
r \rightarrow \frac{a^{2}}{r}
$$

where both $a$ and $r$ are as defined in their choice (17) for the extrinsic curvature (which does indeed satisfy this requirement, as does their choice (18)). They then interpret the resulting space as the union of two distinct asymptotically flat spaces, joined at $r=a$ by an Einstein-Rosen bridge which they take as a model for the black hole. They observe that it suffices to solve (21) on only one of these spaces, the "outside" one defined by $r \geq a$, provided that an inner boundary condition enforcing the conformal isometry of the two spaces under (26) is chosen. They derive

$$
\frac{\partial \Psi}{\partial r}+\frac{\Psi}{2 r}=0 \quad(\text { at } r=a)
$$

as the desired "reflection symmetric" boundary condition for enforcing this.

We now digress to explain the significance of the parameter $\kappa$ in (17), as promised in the previous chapter: the momentum $P^{i}$ enters (21) quadratically (through $S$ ), so the "inner" space may possess either equal (if $\kappa=+1$ in (17)) or equal and opposite 
(if $x=-1$ ) momentum to the outer one. Bowen and York point out that the only reason for the second term in (17) is to satisfy their reflection symmetric boundary condition-taking $\kappa=0$ in (17) (thus dropping the second term entirely) still yields a valid solution to (14). Thus if (as is done later in this thesis) Bowen and York's inner boundary condition is abandoned, there seems no particular reason not to choose the simplest form of (17), namely that with $\kappa=0$.

We now return to our discussion of the Bowen and York reflection symmetric inner boundary condition (27). To this author, it seems to suffer from several flaws. It may place the numerical boundary inside the actual black hole event horizon, which seems rather a waste of grid points (as was pointed out to the author by W. G. Unruh) if we are concerned only with the outside world. More serious difficulties may arise in time evolution problems: the grid points inside the horizon may encounter a singularity, and some care is needed to ensure that the numerical scheme remains well-behaved across the apparent horizon despite the change in the causal structure of spacetime which occurs there.

However, these latter effects should be fairly minor, because the reflection symmetric boundary condition seems (York and Piran (1982)) to result in the apparent horizon being only a small distance outside the inner grid boundary. When applied to the Schwarzschild solution, the two are found to coincide.

A more serious difficulty with the reflection symmetric inner boundary condition is that applying it to spaces containing multiple black holes is rather messy, involving (see, for example, Kulkarni, Shepley, and York (1983), Kulkarni (1984a), York (1984), Bowen, Rauber, and York (1984), and Kulkarni (1984b)) the summation of infinite series of "image charges" needed to enforce the isometry condition on reflection through 
any of the $a_{i} s$.

This author also finds somewhat unnatural the modelling of a black hole by a vacuum region of spacetime, possesing a non-Euclidian topology which is a "mirror image" of the rest of the universe. In particular, why should the way a given black hole is modeled depend on the contents of the outside world?

As an alternative inner boundary condition, W. G. Unruh has suggested requiring that the inner boundary be a marginally trapped surface. This appears to avoid most of the difficulties mentioned above. In particular, the grid is kept outside the (apparent) event horizon and the generalization to multiple black holes is straightforward. The author also finds the physical significance of this boundary condition much clearer. It allows us to exclude the interiors of the trapped surfaces from our space, so we need not deal with such difficulties as how to model the actual (non-vacuum) singularaties therein and how to treat them numerically.

It should be noted however, that there is no guarantee that the marginally trapped surface in question will be an outer trapped surface (apparent horizon). In particular, as Cadez (1974) showed, if we have two black holes close enough together, a third (outer) marginally trapped surface forms, enclosing them both. This latter surface is the desired inner grid boundary. One solution to this difficulty is to use the marginally trapped boundary condition on the "inner" grid boundary surrounding each black hole, then search the resulting space for additional marginally trapped surfaces. However, this has not yet been done for the spaces constructed in the present thesis.

The actual boundary condition on $\Psi$ which results from the marginally trapped surface boundary condition will now be derived. Bowen and York (1980, equation 
(44)) find that the equation for a marginally trapped surface (in the "hat-means-flat" space) is

$$
\nabla_{i} s^{i}+4 s^{i} \nabla_{i}(\ln \Psi)+\frac{\hat{K}_{i j} s^{i} s^{j}}{\Psi^{4}}=0
$$

where $s^{i}$ is the outward pointing unit normal to the surface. The first term, being just the 3-divergence of the vector field $s^{i}$, can be rewritten (see for example, MTW page 222 , equation (8.51c)) as

$$
\nabla_{i} s^{i}=\frac{1}{\sqrt{(3) g}} \partial_{i}\left(\sqrt{(3) g} s^{i}\right)
$$

where ${ }^{(3)} g$ denotes the determinant of the matrix ${ }^{(3)} g_{i j}$. The second term can be rewritten as

$$
\begin{aligned}
4 s^{i} \nabla_{i}(\ln \Psi) & =4 s^{i} \partial_{i}(\ln \Psi) \quad \text { (since } \Psi \text { is a scalar) } \\
& =\frac{4 s^{i} \partial_{i} \Psi}{\Psi}
\end{aligned}
$$

Hence (28) becomes

$$
\frac{1}{\sqrt{(3) g}} \partial_{i}\left(\sqrt{(3) g} s^{i}\right)+\frac{4 s^{i} \partial_{i} \Psi}{\Psi}+\frac{\hat{K}_{i j} s^{i} s^{j}}{\Psi^{4}}=0
$$

We must now find the coordinate version of (31) in whatever coordinate system will be used immediately surrounding black holes. Taking this to be polar spherical coordinates centered on the black hole in question and taking the marginally trapped surface to be the 2 -sphere $r=$ constant, we have $\sqrt{(3) g}=r^{2} \sin \theta$ and $s^{i}=(1,0,0)$. (31) then becomes

$$
\frac{1}{r^{2} \sin \theta} \frac{\partial}{\partial r}\left(r^{2} \sin \theta\right)+\frac{4}{\Psi} \frac{\partial \Psi}{\partial r}+\frac{\hat{K}_{r r}}{\Psi^{4}}=0
$$

or

$$
\frac{\partial \Psi}{\partial r}+\frac{\Psi}{2 r}+\frac{1}{4} \frac{\hat{K}_{r r}}{\Psi^{3}}=0
$$

This is the marginally trapped surface boundary condition used in the present work. 
It is desirable to use the same computer code to handle as many as possible of the boundary conditions, so we merge (25), (27) and (33) into a single "generic" boundary condition

$$
\frac{\partial \Psi}{\partial r}+b_{1} \Psi+b_{2}+\frac{b_{3}}{\Psi^{3}}=0
$$

where the coefficients $b_{1}, b_{2}$, and $b_{3}$ are defined by

Robin

Reflection Symmetric

Marginally Trapped

$$
\left.\begin{array}{ccc}
b_{1} & b_{2} & b_{3} \\
+\frac{1}{r} & -\frac{1}{r} & 0 \\
+\frac{1}{2 r} & 0 & 0 \\
+\frac{1}{2 r} & 0 & \frac{1}{4} \hat{K}_{r r}
\end{array}\right\}
$$

with both $r$ and $\hat{K}_{r r}$ interpreted in a polar spherical coordinate system centered on either a "global origin" (for the "Robin" entries) or on the black hole in question (for the "Reflection Symmetric" and "Marginally Trapped" entries). Note that if multiple black holes are present, some care is needed to compute the correct $\hat{K}_{r r}$ values for the latter case; this will be discussed in detail later.

We shall frequently find that our spaces have a mirror image symmetry about the $z=0$ plane (hereinafter the "equator") which can be exploited by solving our problem only on (say) the $z \geq 0$ half-space and imposing a suitable boundary condition on the equator. To derive this boundary condition, we observe that mirror image symmetry about the equator implies that $\Psi$ must be an even function across it. Hence

$$
\frac{\partial \Psi}{\partial z}=0 \quad \text { (on the equator) }
$$

for cylindrical coordinates, and

$$
\frac{\partial \Psi}{\partial \theta}=0 \quad \text { (on the equator) }
$$

for polar spherical coordinates. 
We have now discussed boundary conditions for all the boundaries of our spaces. However, both polar spherical and cylindrical coordinates have the $z$ axis as a boundary of their coordinate systems. We thus require a boundary condition there, which we now derive.

We assume that $\Psi$ itself remains smooth across the $z$ axis, in particular that all $\Psi$ 's directional derivatives are continuous there. But if $\ell$ denotes a vector perpendicular to this axis, then the directional derivative operator in the $\ell$ direction switches from being (in cylindrical coordinates) a $-\rho$ derivative to a $+\rho$ derivative as we cross the axis in the $\ell$ direction, so we must have

$$
\frac{\partial \Psi}{\partial \rho}=0 \quad \text { (on the } z \text { axis) }
$$

For polar spherical coordinates, the same argument yields

$$
\frac{\partial \Psi}{\partial \theta}=0 \quad \text { (on the } z \text { axis) }
$$




\section{Choice of coordinates}

We now discuss the problem of choosing a suitable set of coordinates for spaces containing multiple (say two) black holes.

The numerical treatment of boundary conditions is much simpler if they occur only at constant coordinate surfaces, so we desire each black hole to have its surface be a constant coordinate surface of our coordinate system. Indeed, we would ideally like our coordinates to approach polar spherical (centered on the black hole's "center") as we approach each black hole, to reflect its approximate spherical symmetry.

As mentioned earlier, our space is asymptotically flat, so we again desire our coordinates to reflect the asymptotic spherical symmetry this implies by approaching polar spherical coordinates at large distances from the strong field region containing the black holes. Of course our actual numerical grid will only be of finite size, but it is still reasonable to require that its outer boundary resemble a sphere as much as possible. In particular, this boundary should be a constant coordinate surface (for the same reasons as the black hole surfaces), and its outward directed normal should be fairly close to a radial one, to minimise errors in computing surface integrals over this outer boundary. Because $\Psi$ depends only weakly on $\theta$ near the outer boundary, this condition helps keep its radial and angular dependence decoupled. This allows anisotropic coordinate grids with (for example) very low angular resolution yet fairly high radial resolution.

Finally, it is certainly a reasonable requirement that our coordinate system be free of singularities, at least exterior to the black holes. This is important for numerical reasons, since a coordinate singularity would probably cause severe rounding errors, or even floating point over/underflow problems. 
Unfortunately, the three conditions given above cannot be satisfied by any single coordinate system. This is because there are by assumption two black hole surfaces, each topologically a sphere, but only one outer grid boundary, also topologically a sphere. Clearly no coordinate system can make a continuous transition from having two spheres as constant coordinate surfaces, to only one. Thus one or more of the conditions given must be sacrificed.

Past work on this and similar problems, notably that of Smarr (1975), Eppley (1975), and Čadez (1971), (who studied the head-on collision of two black holes by time evolving analytically obtained initial data) has usually chosen to sacrifice the reqirement that the coordinate system be free of singularities. Coordinates related to the equipotentials of electric charges placed at the black hole positions have often been used; these have saddle point singularities in between the black holes.

In a prototype of the code described in this thesis, bispherical coordinates were used, which placed a coordinate singularity at spatial infinity. One result of this was that the outer grid boundary was no longer a single constant coordinate surface, but was instead the union of three such surfaces. This proved inconvienent in a number of ways, including difficulty in varying the grid spacings in a local manner and problems with joining the two outer boundary surfaces. Physical insight into the numerical solutions was made more difficult by the same coordinate playing the dual role of "radius" near a black hole, and "angle" near the outer grid boundary. Also, these coordinates fail badly to approach polar spherical at large distances from the black holes.

Most such "global analytic" solutions to the coordinate choice problem yield coordinates which give rise to algebraically "messy" and complicated 3-metrics ${ }^{(3)} g_{i j}$, even for the flat case $f_{i j}$. For non-flat 3-metrics (as would probably be used in a time 
evolution calculation) this difficulty would be much more serious.

This thesis describes an alternative solution to the problem, namely the use of multiple coordinate patches in the numerical solution. Thus each black hole can be surrounded with a polar spherical patch centered on it. A polar spherical patch centered on some convenient origin can be used for the outer region of the numerical grid, and the volume in between can be filled with (for example) a rectangular coordinate patch.

Given that the boundaries between the patches can be dealt with, this scheme offers several advantages: It is not specialized to a fixed number of black holes, so the same computer code can deal with a wider variety of physical systems. Because each patch may have its own coordinate system, there is a great deal of flexibility in locally adapting the coordinates to the physics of the system, while still retaining a simple 3-metric in each patch.

In this thesis, the scheme mentioned above of using polar spherical coordinates both for the neighborhoods of black holes and for the outer regions of the numerical grid is used. For single black hole models, no other coordinate types are needed. For multiple ( 2 in this thesis) black hole models, cylindrical coordinates are used to fill the volume in between the neighborhoods of the black holes and the outer part of the grid. (These are preferred over the rectangular coordinates mentioned above because of the axisymmetry of our spaces.) Other types of coordinate systems have thus far been neither needed nor tried, but it would be easy to incorporate them into the computer code described herein.

Another advantage offered by the use of patches is that any remaining coordinate singularaties (for example the one at the origin of polar spherical coordinates) can be 
easily avoided by placing a nonsingular coordinate patch at the offending location. One place in this work where this might have been needed is the $z$ axis, but the singularaties there are sufficiently mild that it proved easy to deal with them analytically. None of the polar spherical coordinate patches used herein contain their origins, so the singularaties occuring there are not a problem.

Another important issue in coordinate system selection is the need for varying and/or anisotropic resolution in the numerical grid. In the present work, this is dealt with by introducing new patches, while keeping the grid size constant (although possibly anisotropic) within each patch. The full generality of the patch structure used in this thesis is not really needed for this function, but the overhead of introducing new patches is small.

A detailed discussion of just how coordinate patches are used in the actual solution of our problem will be delayed until after the manner of discretizing the equations is discussed in the next chapter. 


\section{Finite Differencing the Equations}

The previous chapters have shown that we must solve the nonlinear elliptic PDE

$$
\nabla^{2} \Psi+S \Psi^{-7}=0
$$

on the region of space exterior to the black holes, using both polar spherical and cylindrical coordinates. We must do so subject to the boundary conditions

$$
\frac{\partial \Psi}{\partial r}+b_{1} \Psi+b_{2}+\frac{b_{3}}{\Psi^{3}}=0
$$

on both the inner boundary of the patch surrounding each black hole and the outer boundary of the outermost patch, and

$$
\left.\begin{array}{ll}
\frac{\partial \Psi}{\partial \rho}=0 & \text { (cylindrical coordinates, on the } z \text { axis) } \\
\frac{\partial \Psi}{\partial z}=0 & \text { (cylindrical coordinates, on the equator) } \\
\frac{\partial \Psi}{\partial \theta}=0 & \text { (polar spherical coordinates, on the } z \text { axis) } \\
\frac{\partial \Psi}{\partial \theta}=0 & \text { (polar spherical coordinates, on the equator) }
\end{array}\right\}
$$

on the given boundaries. The techniques by which these equations are converted into a set of coupled nonlinear algebraic equations will now be discussed. Only those aspects of finite differencing used in this thesis will be discussed; see Mitchell and Griffiths (1980) for a more detailed discussion and further references on the topic.

The basic idea is to consider the functions (eg. $\Psi$ ) to be defined on a finite "grid" of points (typically uniformly spaced), and to approximate the differential operators by suitable differences of grid-function values (thus the name "finite differences" for this technique). The resulting finite difference operators are then substituted for the differential operators to yield an algebraic equation, which must hold at each grid point. The boundary conditions are treated in a similar manner, as will be discussed later. 
To illustrate the technique, consider first a single function $f$ of a single variable $x$, and suppose that $x$ has been discretized to a uniform grid $x_{i}=x_{0}+i \Delta x$. (Within this chapter we will use subscripts mainly to denote evaluation at the corresponding grid point.) Taylor expanding $f(x)$ about $x_{i}$, we find that

$$
f_{i}^{\prime} \approx \frac{f_{i+1}-f_{i-1}}{2 \Delta x}
$$

and that

$$
f_{i}^{\prime \prime} \approx \frac{f_{i+1}-2 f_{i}+f_{i-1}}{(\Delta x)^{2}}
$$

where we have made the approximation of neglecting $O\left((\Delta x)^{2}\right)$ and higher terms in the Taylor series.

These two "centered second order" finite difference formulas are very widely used. The letter $h$ is often used as a "generic" grid spacing $\Delta x$; these formulas are then refered to as being "O(h $\left.h^{2}\right)$ accurate". Note that this "accuracy" (more properly known as "local truncation error ${ }^{n}$ ) refers only to the degree to which the difference equations locally approximate the differential equation. Whether or not the solution of the difference equations globally approximates the solution of the differential equation (assuming both to exist) to the same extent is in general a rather difficult question to answer. Fortunately, this latter property turns out to hold for all difference schemes discussed in this thesis, so this problem will not be further considered.

Continuing with our example, suppose that $f$ satisfies the differential equation

$$
f^{\prime \prime}(x)+x=0
$$

We finite difference this by substituting the approximation (44) for $f^{\prime \prime}$ at a typical grid point $x_{i}$, to give the algebraic equation

$$
\frac{f_{i+1}-2 f_{i}+f_{i-1}}{\Delta x}+x_{i}=0
$$


which we require to be satisfied at each grid point, i.e. for each value of $i$.

We now consider boundary conditions for our example problem (45). Suppose we have that

$$
f(0)=0
$$

and

$$
f^{\prime}(0)-1=0 .
$$

Suppose also that we have a grid starting at $x_{0}=0$ (it is usual in finite differencing to choose the grid so that all boundaries occur at grid points, as we have done here) and extending in the positive $x$ direction (we ignore the right hand boundary of the grid for this example).

The boundary condition (47) is easily dealt with-just set $f_{0}=0$. However, boundary condition (48) requires that we have a finite difference approximation to $f^{\prime}(0)$. Using the difference scheme (43) given above, this requires a value $f_{-1}$ for $f(x)$ at the nonexistant grid point $x_{-1}$, as does the finite difference form (46) of the differential equation when evaluated at the grid point $x_{0}$.

One solution to this difficulty lies in using the boundary condition to extrapolate a value for $f_{-1}$, which can then be substituted in the finite difference form (46) of the differential equation for the grid point $x_{0}$. This substitution can be done either analytically (resulting in a modified finite difference form of the differential equation at the boundary point, which implicitly incorporates the boundary conditions), or numerically, by actually including the extrapolated function value as an extra variable in the algebraic equations. The latter technique is used in this thesis, so our example 
boundary condition (48) becomes

$$
\frac{f_{1}-f_{-1}}{2 \Delta x}-1=0
$$

This completes the finite differencing of our example; we now have one algebraic equation for each unknown function value.

We now return to our main problem, namely the finite differencing of (40) subject to the boundary conditions (41) and (42). We will need to do this for each coordinate system type used, namely cylindrical $(\rho, z, \phi)$ and polar spherical $(r, \theta, \phi)$. Finite differencing in a general curvilinear coordinate system is not quite as trivial as the one-dimensional case considered above, so we now discuss the finite difference approximation to $\nabla^{2} \Psi$ for use in (40) above. The $\phi$ terms in the $\nabla^{2}$ operator can be omitted due to the axisymmetry of our problem.

In cylindrical coordinates,

$$
\begin{aligned}
\nabla^{2} \Psi & =\frac{1}{\rho}\left[\frac{\partial}{\partial \rho}\left(\rho \frac{\partial \Psi}{\partial \rho}\right)\right]+\frac{\partial^{2} \Psi}{\partial z^{2}} \\
& =\frac{\partial^{2} \Psi}{\partial \rho^{2}}+\frac{1}{\rho} \frac{\partial \Psi}{\partial \rho}+\frac{\partial^{2} \Psi}{\partial z^{2}}
\end{aligned}
$$

assuming for the moment that $\rho \neq 0$.

While the two forms (50) and (51) are certainly equal, the straighforward application of the approximations (43) and (44) to them will not necessarily give the same finite difference equations. This is symptomatic of a general problem in finite differencingthe finite difference form of a given (continuum) equation is not unique. The different forms may vary greatly in accuracy. We shall defer consideration of the form (50) until later in this chapter, and proceed now with the finite differencing of (51).

We do this (still assuming that $\rho \neq 0$ ) by substituting the standard $O\left(h^{2}\right)$ centered 
approximations (43) and (44) for the partial derivatives. This gives

$$
\begin{aligned}
\left(\nabla^{2} \Psi\right)_{i, j} \approx & {\left[\frac{\Psi_{i-1, j}-2 \Psi_{i, j}+\Psi_{i+1, j}}{(\Delta \rho)^{2}}\right]+\frac{1}{\rho_{i, j}}\left[\frac{\Psi_{i+1, j}-\Psi_{i-1, j}}{2 \Delta \rho}\right] } \\
& +\left[\frac{\Psi_{i, j-1}-2 \Psi_{i, j}+\Psi_{i, j+1}}{(\Delta z)^{2}}\right] \\
= & c_{1} \Psi_{i-1, j}+c_{2} \Psi_{i+1, j}+c_{3} \Psi_{i, j-1}+c_{4} \Psi_{i, j+1}+c_{5} \Psi_{i, j},
\end{aligned}
$$

where we have defined the coefficients

$$
\left.\begin{array}{l}
c_{1}=\frac{1}{(\Delta \rho)^{2}}-\frac{1}{2 \rho \Delta \rho} \\
c_{2}=\frac{1}{(\Delta \rho)^{2}}+\frac{1}{2 \rho \Delta \rho} \\
c_{3}=c_{4}=\frac{1}{(\Delta z)^{2}} \\
c_{5}=-\left(c_{1}+c_{2}+c_{3}+c_{4}\right)
\end{array}\right\}
$$

at the $(i, j)$ th grid point. It is common to write (52) as the convolution

$$
\left(\nabla^{2} \Psi\right)_{i, j}=\left[\begin{array}{lll} 
& c_{4} & \\
c_{1} & c_{5} & c_{2} \\
& c_{3} &
\end{array}\right] \circ\left(\Psi_{i, j}\right)
$$

of the "molecule" with coefficients $\left\{c_{i}\right\}$ with the matrix of $\Psi$ values centered on the point $(i, j)$. In this notation, the molecule coefficients are always assumed to be evaluated at the central grid point $(i, j)$, so for example " $\rho$ " denotes $\rho_{i, j}$.

This difference scheme must be modified on the $z$ axis, since the second term of (51) becomes singular there. The Neumann boundary conditions (42) allow us to apply L'Hopital's rule in its " $0 / 0$ " form to the offending term of (51), to obtain

$$
\nabla^{2} \Psi=2 \frac{\partial^{2} \Psi}{\partial \rho^{2}}+\frac{\partial^{2} \Psi}{\partial z^{2}} \quad(\rho=0) .
$$

We finite difference this in the same manner as before, to give the molecule with coefficients

$$
\left.\begin{array}{l}
c_{1}=c_{2}=\frac{2}{(\Delta \rho)^{2}} \\
c_{3}=c_{4}=\frac{1}{(\Delta z)^{2}} \\
c_{5}=-\left(c_{1}+c_{2}+c_{3}+c_{4}\right) .
\end{array}\right\}
$$


We now consider the finite differencing of $\nabla^{2} \Psi$ in polar spherical coordinates. We have

$$
\begin{aligned}
\nabla^{2} \Psi & =\frac{1}{r^{2}} \frac{\partial}{\partial r}\left(r^{2} \frac{\partial \Psi}{\partial r}\right)+\frac{1}{r^{2} \sin \theta} \frac{\partial}{\partial \theta}\left(\sin \theta \frac{\partial \Psi}{\partial \theta}\right) \\
& =\frac{\partial^{2} \Psi}{\partial r^{2}}+\frac{2}{r} \frac{\partial \Psi}{\partial r}+\frac{1}{r^{2}} \frac{\partial^{2} \Psi}{\partial \theta^{2}}+\frac{\cos \theta}{r^{2} \sin \theta} \frac{\partial \Psi}{\partial \theta},
\end{aligned}
$$

assuming that $\theta \neq 0$ or $\pi$. We again defer consideration of the first of these forms (57), and proceed to finite difference (58).

Using the same procedure as before, we obtain the molecule with coefficients

$$
\left.\begin{array}{l}
c_{1}=\frac{1}{(\Delta r)^{2}}-\frac{1}{r \Delta r} \\
c_{2}=\frac{1}{(\Delta r)^{2}}+\frac{1}{r \Delta r} \\
c_{3}=\frac{1}{r^{2}(\Delta \theta)^{2}}-\frac{\cos \theta}{2 r^{2} \sin \theta \Delta \theta} \\
c_{4}=\frac{1}{r^{2}(\Delta \theta)^{2}}+\frac{\cos \theta}{2 r^{2} \sin \theta \Delta \theta} \\
c_{5}=-\left(c_{1}+c_{2}+c_{3}+c_{4}\right),
\end{array}\right\}
$$

still assuming that $\theta \neq 0$ or $\pi$. If this latter assumption does not hold, we can again apply L'Hopital's rule to the offending term (the last one) in (58) to obtain

$$
\nabla^{2} \Psi=\frac{\partial^{2} \Psi}{\partial r^{2}}+\frac{2}{r} \frac{\partial \Psi}{\partial r}+\frac{2}{r^{2}} \frac{\partial^{2} \Psi}{\partial \theta^{2}} \quad(\theta=0 \text { or } \pi)
$$

Finite differencing this in the same manner as before gives the molecule with coefficients

$$
\left.\begin{array}{l}
c_{1}=\frac{1}{(\Delta r)^{2}}-\frac{1}{r \Delta r} \\
c_{2}=\frac{1}{(\Delta r)^{2}}+\frac{1}{r \Delta r} \\
c_{3}=c_{4}=\frac{2}{r^{2}(\Delta \theta)^{2}} \\
c_{5}=-\left(c_{1}+c_{2}+c_{3}+c_{4}\right) .
\end{array}\right\}
$$

We now return to consider the finite differencing of (57). If we were to apply the approximation (43) to each of the partial derivatives in (57), we would obtain a molecule 
with nonzero entries farther away from the central point than we have previously seen ( 2 grid points rather than 1). This would greatly complicate the treatement of boundary points. As an alternative to this use of (43), we introduce the concept of a "staggered" grid. We first consider this in the context of our example problem (45).

We define the staggered grid by $x_{i+\frac{1}{2}}=x_{0}+\left(i+\frac{1}{2}\right) \Delta x$. We Taylor expand $f(x)$ about $x_{i+\frac{1}{2}}$ to obtain the approximation

$$
f_{i+\frac{1}{2}}^{\prime} \approx \frac{f_{i+1}-f_{i}}{\Delta x}
$$

where we have again dropped the second order and higher terms in the Taylor expansion. Having defined $f^{\prime}$ on the staggered grid, we can now use it to derive an approximation for $f^{\prime \prime}$ on the original grid by Taylor expanding the function $f^{\prime}(x)$ about $x_{i}$ and dropping the second and higher order terms. This gives

$$
f_{i}^{\prime \prime}=\left(f^{\prime}\right)_{i}^{\prime} \approx \frac{f_{i+\frac{1}{2}}^{\prime}-f_{i-\frac{1}{2}}^{\prime}}{\Delta x}
$$

For our example problem (45), this doesn't get us anywhere, since substituting (62) into (63) just gives the approximation (44) again. However, the technique used here is directly applicable to the finite differencing of (57), to which we now return.

We apply the staggered grid technique to the finite differencing of (57) by first applying the approximation (63) to the "outer" derivatives, then using the approximation (62) to evaluate the "inner" derivatives on the staggered grid points where they are 
needed. We have

$$
\begin{aligned}
& \left(\nabla^{2} \Psi\right)_{i, j} \\
& \approx \frac{1}{r_{i, j}^{2}} \frac{\left[\left(r^{2} \frac{\partial \Psi}{\partial r}\right)_{i+\frac{1}{2}, j}-\left(r^{2} \frac{\partial \Psi}{\partial r}\right)_{i-\frac{1}{2}, j}\right]}{\Delta r} \\
& \quad+\frac{1}{r_{i, j}^{2} \sin \theta_{i, j}} \frac{\left[\left(\sin \theta \frac{\partial \Psi}{\partial \theta}\right)_{i, j+\frac{1}{2}}-\left(\sin \theta \frac{\partial \Psi}{\partial \theta}\right)_{i, j-\frac{1}{2}}\right]}{\Delta \theta} \\
& \approx \\
& \quad \frac{1}{r_{i, j}^{2}} \frac{1}{\Delta r}\left[r_{i+\frac{1}{2}, j}^{2}\left(\frac{\Psi_{i+1, j}-\Psi_{i, j}}{\Delta r}\right)-r_{i-\frac{1}{2}, j}^{2}\left(\frac{\Psi_{i, j}-\Psi_{i-1, j}}{\Delta r}\right)\right] \\
& \quad+\frac{1}{r_{i, j}^{2} \sin \theta_{i, j}} \frac{1}{\Delta \theta}\left[\sin \theta_{i, j+\frac{1}{2}}\left(\frac{\Psi_{i, j+1}-\Psi_{i, j}}{\Delta \theta}\right)-\sin \theta_{i, j-\frac{1}{2}}\left(\frac{\Psi_{i, j}-\Psi_{i, j-1}}{\Delta \theta}\right)\right],
\end{aligned}
$$

which gives the molecule with coefficients

$$
\left.\begin{array}{l}
c_{1}=\frac{1}{(\Delta r)^{2}}\left(1-\frac{\Delta r}{2 r}\right)^{2} \\
c_{2}=\frac{1}{(\Delta r)^{2}}\left(1+\frac{\Delta r}{2 r}\right)^{2} \\
c_{3}=\frac{1}{r^{2}(\Delta \theta)^{2}} \frac{\sin \theta_{i, j-\frac{1}{2}}}{\sin \theta_{i, j}} \\
c_{4}=\frac{1}{r^{2}(\Delta \theta)^{2}} \frac{\sin \theta_{i, j+\frac{1}{2}}}{\sin \theta_{i, j}} \\
c_{5}=-\left(c_{1}+c_{2}+c_{3}+c_{4}\right) .
\end{array}\right\}
$$

Finally, if $\theta=0$ or $\pi$, we can again apply L'Hopital's rule to the offending term in (57) to obtain

$$
\nabla^{2} \Psi=\frac{1}{r^{2}} \frac{\partial}{\partial r}\left(r^{2} \frac{\partial \Psi}{\partial r}\right)+\frac{2}{r^{2}} \frac{\partial^{2} \Psi}{\partial \theta^{2}}, \quad(\theta=0 \text { or } \pi)
$$

which gives the molecule with coefficients

$$
\left.\begin{array}{l}
c_{1}=\frac{1}{(\Delta r)^{2}}\left(1-\frac{\Delta r}{2 r}\right)^{2} \\
c_{2}=\frac{1}{(\Delta r)^{2}}\left(1+\frac{\Delta r}{2 r}\right)^{2} \\
c_{3}=c_{4}=\frac{2}{r^{2}(\Delta \theta)^{2}} \\
c_{5}=-\left(c_{1}+c_{2}+c_{3}+c_{4}\right) .
\end{array}\right\}
$$


The application of the staggered grid differencing technique to the finite differencing of the form (50) turns out to give the same molecule (53) as was obtained by finite differencing (51) in the usual way, so we shall not consider this case further.

It can be shown that finite differencing $\nabla^{2}$ (using the staggered grid differencing technique) in the form (50) or (57), results in the solution to the (linear) PDE $\nabla^{2} \Psi=0$ obeying a local finite difference form of Gauss's law. Such finite difference schemes (as (65)) are thus known as "locally conservative". Their properties (in contrast with the "independent terms" difference schemes such as (53) or (59)) are beyond the scope of this thesis, but they are often more accurate. Eppley (1977, pp. 1610-1612) discusses them in more detail, and gives further examples.

We now consider the boundary conditions (41) and (42). These are treated in the same manner as the example (48) above, i.e. the first derivatives are approximated using the standard $O\left(h^{2}\right)$ form (43), which is used to extrapolate a value for $\Psi$ one grid spacing beyond the boundary. This value is then available for use with the $\nabla^{2}$ molecules derived above; its substitution in the appropriate molecule is done numerically. Thus, if the actual boundary occurs at the "end" grid point $\Psi_{e}$ (we supress the coordinate running parallel to the boundary line), we extrapolate an "exterior" value $\Psi_{e \pm 1}$ one grid spacing beyond the boundary (which may be in the direction either of increasing or decreasing grid coordinate) based on $\Psi_{e}$ and the "interior" value $\Psi_{e \mp 1}$ defined one grid spacing inside the boundary.

For the boundary condition (41), we thus have

$$
\Psi_{e \pm 1} \approx \Psi_{e \mp 1}+d_{1} \Psi_{e}+d_{2}+\frac{d_{3}}{\Psi_{e}^{3}},
$$


where

$$
d_{i}= \begin{cases}+2 \Delta r b_{i} & \text { (for an "inner" boundary) } \\ -2 \Delta r b_{i} & \text { (for the "outer" boundary) }\end{cases}
$$

and the $b_{i}$ are defined by (35). The Neumann boundary conditions (42) are treated the same way, and reduce to the simple expression

$$
\Psi_{e \pm 1} \approx \Psi_{e \mp 1}
$$




\section{Coordinate Patches - I}

Now that we have discussed the finite differencing of our problem, the requirements on a patch system (i.e. the questions that the patch data structures must be able to answer) can be defined, and the means chosen to implement patch systems can be discussed in more detail. The key problem here is how to handle the interpatch boundaries. We must also be able to describe the geometry of the patch system, i.e. how the patches fit together to cover our space. We now discuss the basic principles of the patch system used, and the means by which it adresses these problems.

Each patch uses either polar spherical or cylindrical coordinates. The latter are also used as a "global" coordinate system to refer to positions in our space without reference to any particular patch. The coordinate system of any single patch need not be centered on the global origin, although in practice only polar spherical patches will be centered elsewhere, and these must always be centered somewhere on the $z$ axis to preserve axisymmetry.

In order to keep the discussion applicable to both patch types, we introduce generic (2-dimensional) patch coordinates $(x, y)$, corresponding to $(\rho, z)$ and $(r, \theta)$ in cylindrical and polar spherical patches respectively. Given the axisymmetry of our spaces, we will supress the $\phi$ coordinate in the following discussion.

Each patch is then most simply described by first looking at it in its own $(x, y)$ coordinate system, i.e. we view cylindrical patches in $(\rho, z)$ coordinates and polar spherical patches in $(r, \theta)$ coordinates. We take the grid spacings to be uniform within each patch, so the coordinate grid becomes a uniform rectangular "mesh" of straight (coordinate) lines overlaying the patch. We label these lines with integer coordinates $(i, j)$, with 
unit grid spacing in both directions. For the present work, the origins of the $(x, y)$ and the $(i, j)$ systems are taken to coincide. This is in no way essential to the development, but the extra generality of allowing them to be distinct has not been needed.

We now discuss in more detail just how the "geometry" of the patch is described. It suffices to describe the intersection of each (coordinate grid) line with the patch. A patch is said to be "manhattan convex" if and only if each such intersection is a finite line segment. All patches are taken to satisfy this constraint, which has not in practice proved onerous. (At worst, a region of our space which is logically a single patch but is not manhattan convex, must be split into two or more manhattan convex patches. This occurs, for example, in the patch system shown in figure 9.) Given this constraint, it suffices to describe the two ends of each line. In particular, the shape of a patch is described by giving the value of $i$ or $j$ (as appropriate) at each line end. This information is stored as part of a "line end descriptor" for each separate line end.

There remains the problem of how to describe the way the different patches fit together to cover the whole space. Truly "global" information (eg. "patch \#4 is surrounded by patches $\# 2, \# 5$, and $\# 9^{\prime}$ ) is very hard to describe in a useful way, but fortunately is not really needed. What is needed, though, is a means to describe "locally" how the patches fit together.

In particular, as well as describing where each line in a patch ends, a description is needed of how it ends. There are two basic possibilities for this: it can end either by "running into" an actual boundary of the overall coordinate system (hereinafter a "true" boundary), or it can end by encountering another patch (hereinafter a "patch" boundary). A flag is kept (in the line end descriptor) for each line end, telling which of these cases holds. An indication of which patch was "run into" or what type of 
boundary condition (Neumann, Robin, Marginally Trapped, etc.) is in effect at the boundary is also encoded into the same flag value.

If the line ends in a true boundary, the appropriate choice of boundary condition (70) or of (68), (69), and (35), can then be made. The necessary coefficients $d_{1}, d_{2}$, and $d_{3}$ for the latter case are also stored in the line end descriptor.

If, on the other hand, the line ends by encountering another patch, then we must somehow obtain a $\Psi_{e \pm 1}$ (in the notation of the previous chapter) value for the "exterior" point which lies one grid step beyond the end of this line. We require that the exterior point lie within the other patch. This amounts to requiring that any gaps between patches can't exceed the minimum of the grid sizes of the patches in question, which turns out to be fairly easy to arrange. (It usually shows up when switching between polar spherical and rectangular patches, where the effect is to sometimes require an extra polar spherical patch whose only purpose is to match up the grid sizes before the switch is made. An example of this is patch \#2 of the patch system shown in figure 4.)

Since it's grid point lies within the other patch, we can derive $\Psi_{e \pm 1}$ by interpolating $\Psi$ from its values in the other patch. Since we are only approximating $\nabla^{2} \Psi$ to $O\left(h^{2}\right)$, it suffices to use bilinear interpolation, whose error term is also $O\left(h^{2}\right)$. As shown in figure 1 , there are essentially two cases to this interpolation: either we are interpolating into a square (of points in the other patch), or we are interpolating into a triangle.

The coefficients for the interpolation must be derived in a different manner (from the position of the interpolation point) in each case, with the triangle case further broken down into four subcases depending on the triangle's orientation. However, as shown in figure 2 , the net result in all cases is that the interpolated $\Psi_{e \pm 1}$ value is a 


\section{Figure 1 - "Square" vs. "Triangle" Interpolation}

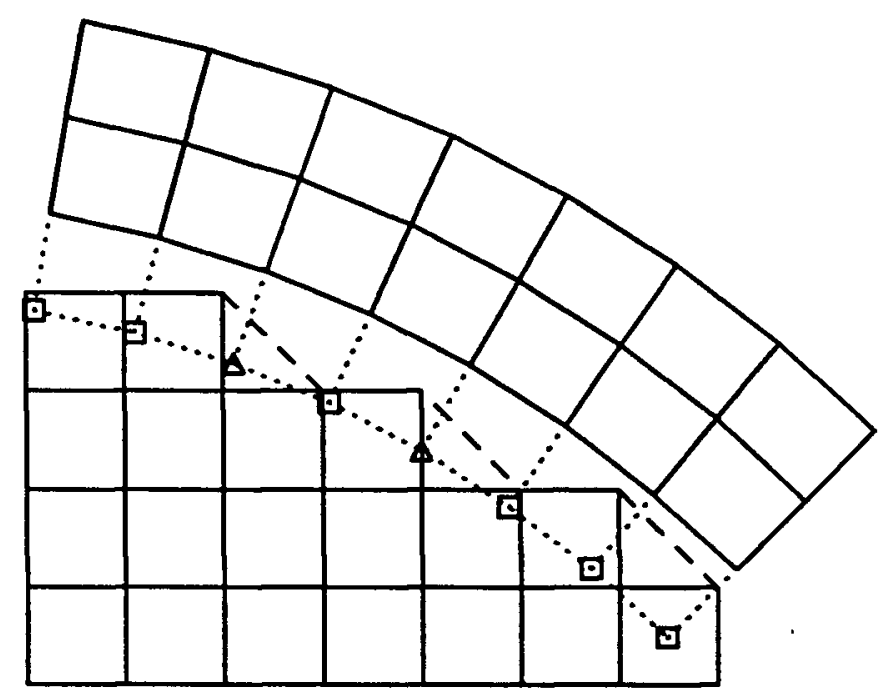

This figure shows interpolation of values from a rectangular coordinate patch into a polar spherical coordinate patch. The dotted lines and the points at the ends of them show the exterior points of the polar spherical coordinate patch, where the interpolation is done. Those points labelled with " $\square$ " lie within a square of the rectangular coordinate patch's grid points; those labelled with " $\Delta$ " lie within a triangle of such points, the "outer" side of which is shown with a dashed line.

linear combination

$$
\Psi_{e \pm 1}=d_{00} \Psi_{00}+d_{01} \Psi_{01}+d_{10} \Psi_{10}+d_{11} \Psi_{11}
$$

of the other patch's $\Psi$ values on the four corners of a square (of side one) in the other patch's $(i, j)$ coordinate system. (The triangle cases all have the "weight" of the "missing corner" set to zero.) The $(i, j)$ coordinates of the lower left corner of that square, and the four interpolation coefficients, are all stored in the line end descriptor.

There is one more detail to be discussed before the description of the patch system is complete. This concerns adjacent patches which have the same coordinate system type. (These can arise either as the result of splitting a "logical" patch into two or 
Figure 2 - Bilinear Interpolation Formulae

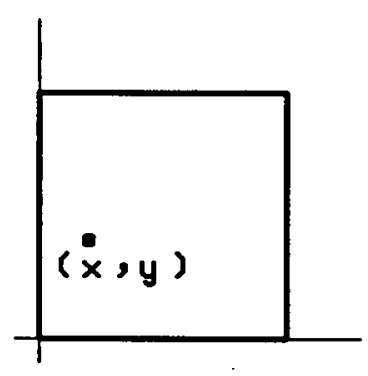

$$
\left.\begin{array}{rl}
\mathbf{d}_{00} & =(1-x)(1-y) \\
\mathbf{d}_{01} & =(1-x) y \\
\mathbf{d}_{10} & =x(1-y) \\
\mathbf{d}_{11} & =x y
\end{array}\right\}
$$

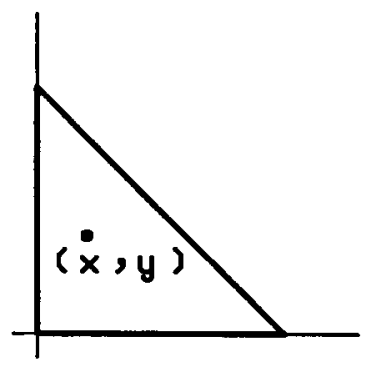

$$
\left.\begin{array}{rl}
\mathrm{d}_{00} & =1-x-y \\
\mathrm{~d}_{01} & =y \\
\mathrm{~d}_{10} & =x \\
\mathrm{~d}_{11} & =0
\end{array}\right\}
$$

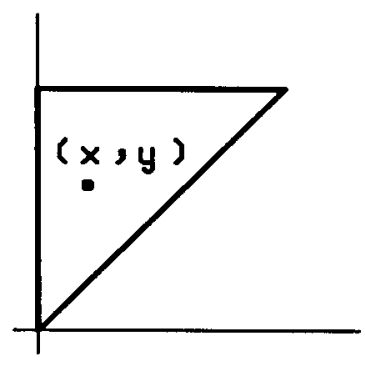

$$
\left.\begin{array}{l}
\mathbf{d}_{00}=1-y \\
d_{01}=y-x \\
d_{10}=0 \\
d_{11}=x
\end{array}\right\}
$$

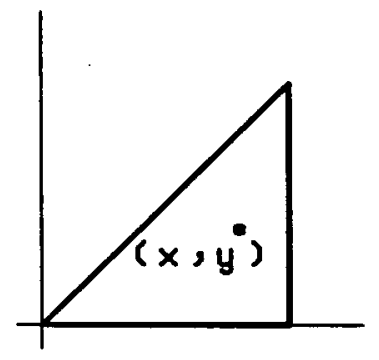

$$
\left.\begin{array}{rl}
d_{00} & =1-x \\
d_{01} & =0 \\
d_{10} & =x-y \\
d_{11} & =y
\end{array}\right\}
$$

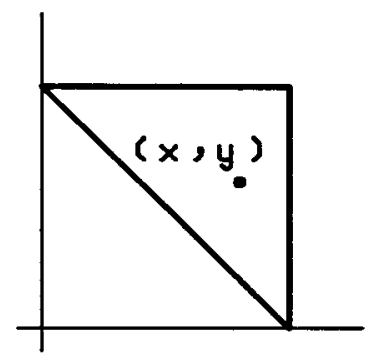

$$
\left.\begin{array}{rl}
d_{00} & =0 \\
d_{01} & =1-x \\
d_{10} & =1-y \\
d_{11} & =x+y-1
\end{array}\right\}
$$

Interpolation coefficients for the different possible cases of bilinear interpolation. For this figure only, $(x, y)$ is the position of the interpolation point in the interpolation region, the latter being taken to run from 0 through 1 in both $x$ and $y$. 
more manhattan convex patches, or they can be the result of using extra patches to allow local variation in the grid spacing.) It would be possible to "butt joint" such patches, since the interpatch interpolation would then reduce to a simple transfer of $\Psi$ values. However, due to finite differencing errors, the $\Psi$ values obtained at the points common to both patches would not exactly match. It is thus simpler to always leave a gap between such patches, of width equal to the minimum of the two grid spacings perpendicular to their common boundary. This has been done in all patch systems used in this thesis. 


\section{Coordinate Patches - II}

The previous chapter has discussed the type of coordinate patch system (also known as "grid") used in this thesis. This system, although well-suited for "internal" computer use, is far too low-level a representation to be used for human input of any nontrivial patch system. Thus, a higher-level "patch description language" is needed, to describe the overall geometry of the patch system. This can then be "compiled" into the appropriate arrays of patch descriptors and line end descriptors. This chapter gives a brief overview of this language. It's detailed syntax and semantics are fully defined only by the programs (listed in appendix 2) which process it, but an overview should be of interest to anyone trying to use coordinate patches in the numerical solution of PDEs. Numerous examples of patch description language input files may be found in appendix 1.

The key idea of the language is the means used to describe the geometry of each patch. This is done by describing all the different ways the patch's grid lines can end, for each of the four "sides" of the patch-minimum $x$, maximum $x$, minimum $y$, and maximum $y$. Each such description takes the form of a series of "boundary definition terms", each of which either says that the grid lines end at a (given) constant value of $x$ or $y$ (as appropriate), or says that they end by encountering such a boundary of some other patch. The actual shape of a patch is determined by the union of all its terms, i.e. a grid line ends as soon as it "hits" any term.

The compilation algorithm has two main phases: the determination of the shapes of the patches, and the determination of the precise boundary conditions in effect at each line end. The first phase is trivial for "regular" patches, defined as those whose definitions contain only "constant" terms. For "irregular" patches (all others), 
we require the specification of rectangular (in the patch's own coordinate systems) "bounding boxes" completely enclosing the patches. These provide starting points for finding patch boundaries by sweeping along grid lines until a term that "hits" is found.

Once the patch shapes have all been determined, each line end of each patch is checked to see which of the available terms it hit. This allows the nature of the boundary conditions in effect at the line end to be determined, so the various flags and coefficients described in previous chapters can be calculated and stored in the appropriate descriptors.

This patch description language has proven adequate for the uses made of it in this thesis, but further enhancements might well be in order. For example, the current language lacks any facilities for parameterizing patch definitions. Defining non-trivial patch systems (such as those used for the two-black hole models of this thesis) is in practice a rather tedious and error-prone task, typically requiring several attempts before the "compilation" is error-free and produces the desired result. Part of this problem may be due to the fairly crude nature of the compilation algorithm and it's present implementation. The basic problem is that almost all "computational geometry" is conceptually simple but complicated in practice. This certainly applies to the compilation code, which runs to about 2000 lines of code, and was very hard to debug. 


\section{The Extrinsic Curvature}

The previous chapters have shown how our problem is discretized to obtain a set of coupled nonlinear algebraic equations. These depend on the particular black hole configuration chosen through the conformally scaled extrinsic curvature tensor $\hat{K}_{i j}$. We now discuss just how this latter object is obtained, starting with the Bowen and York solutions to (14) given by (17) and (18).

These solutions are most sizpply evaluated in polar spherical coordinates centered on a black hole. Choptuik (1982, pp. 56-57) gives the only non-zero components of $\hat{K}_{i j}$ in these coordinates as

$$
\left.\begin{array}{l}
\hat{K}_{r r}=\frac{3 P \cos \theta}{r^{2}}\left(1+\kappa \frac{a^{2}}{r^{2}}\right) \\
\hat{K}_{r \theta}=-\frac{3 P \sin \theta}{2 r}\left(1-\kappa \frac{a^{2}}{r^{2}}\right) \\
\hat{K}_{\theta \theta}=-\frac{3 P \sin \theta}{2}\left(1+\kappa \frac{a^{2}}{r^{2}}\right) \\
\hat{K}_{\phi \phi}=-\frac{3 P \cos \theta \sin ^{2} \theta}{2}\left(1+\kappa \frac{a^{2}}{r^{2}}\right)
\end{array}\right\}
$$

for a black hole with momentum $P$ in the positive $z$ direction, and (correcting 2 typographical errors in the cited source)

$$
\hat{K}_{r \phi}=-\frac{3 J \sin ^{2} \theta}{r^{2}}
$$

for a black hole with spin vector $J$ in the positive $z$ direction.

For our problem, we in general have multiple black holes, each at a different position, and each possibly having both momentum and spin. Thus we need to transform (77) and (78) for each black hole into a common coordinate system, then sum over all black holes. We take cylindrical coordinates for this common system. The necessary partial 
derivatives of $\{r, \theta\}$ with respect to $\{\rho, z\}$ are

$$
\left.\begin{array}{l}
\frac{\partial r}{\partial \rho}=\frac{\rho-\rho_{\mathrm{BH}}}{r} \\
\frac{\partial r}{\partial z}=\frac{z-z_{\mathrm{BH}}}{r} \\
\frac{\partial \theta}{\partial \rho}=\frac{z-z_{\mathrm{BH}}}{r^{2}} \\
\frac{\partial \theta}{\partial z}=-\frac{\rho-\rho_{\mathrm{BH}}}{r^{2}}
\end{array}\right\}
$$

where we take the black hole coordinates to be $\left(\rho_{\mathrm{BH}}, z_{\mathrm{BH}}\right)$. In practice, we must always have $\rho_{\mathrm{BH}}=0$ due to the assumed axisymmetry of our space.

Observing that a black hole with both momentum and spin will in general have all its polar spherical coordinate $\hat{K}_{i j}$ components non-zero except for $\hat{K}_{\theta \phi}$ and recalling the symmetry of $\hat{K}_{i j}$ in any coordinate system, we find the cylindrical coordinate $\hat{K}_{i j}$ components to be

$$
\begin{aligned}
& \hat{K}_{\rho \rho}=\hat{K}_{r r}\left(\frac{\partial r}{\partial \rho}\right)^{2}+2 \hat{K}_{r \theta} \frac{\partial r}{\partial \rho} \frac{\partial \theta}{\partial \rho}+\hat{K}_{\theta \theta}\left(\frac{\partial \theta}{\partial \rho}\right)^{2} \\
& \hat{K}_{\rho z}=\hat{K}_{r r} \frac{\partial r}{\partial \rho} \frac{\partial r}{\partial z}+\hat{K}_{r \theta}\left[\frac{\partial r}{\partial \rho} \frac{\partial \theta}{\partial z}+\frac{\partial \theta}{\partial \rho} \frac{\partial r}{\partial z}\right]+\hat{K}_{\theta \theta} \frac{\partial \theta}{\partial \rho} \frac{\partial \theta}{\partial z} \\
& \hat{K}_{\rho \phi}=\hat{K}_{r \phi} \frac{\partial r}{\partial \rho} \\
& \hat{K}_{z z}=\hat{K}_{r r}\left(\frac{\partial r}{\partial z}\right)^{2}+2 \hat{K}_{r \theta} \frac{\partial r}{\partial z} \frac{\partial \theta}{\partial z}+\hat{K}_{\theta \theta}\left(\frac{\partial \theta}{\partial z}\right)^{2} \\
& \hat{K}_{z \phi}=\hat{K}_{r \phi} \frac{\partial r}{\partial z} \\
& \hat{K}_{\phi \phi}=\hat{K}_{\phi \phi}
\end{aligned}
$$

where the right hand sides are found (in polar spherical coordinates) from (77) and (78).

Having obtained $\hat{K}_{i j}$ in the global cylindrical coordinate system, there are two ways in which we shall use it. First, we must compute the source function

$$
S=\frac{1}{8} \hat{K}_{i j} \hat{K}^{i j}
$$


Since this is a scalar (rank zero) object defined by a tensor equation, it is invariant under coordinate transformation. We thus may choose a coordinate system to compute it in solely on the basis of convienence. Since we have all $\hat{K}_{i j}$ components available in the global cylindrical coordinate system, we choose to compute $S$ also in this system. Since

$$
{ }^{(3)} g^{i j}=\left[\begin{array}{lll}
1 & & \\
& 1 & \\
& & \rho^{-2}
\end{array}\right]
$$

in cylindrical coordinates, we have (again recalling the symmetry of both $\hat{K}_{i j}$ and $\hat{K}^{i j}$ )

$$
\begin{aligned}
S=\frac{1}{8}\left[\left(\hat{K}_{\rho \rho}\right)^{2}\right. & +2\left(\hat{K}_{\rho z}\right)^{2}+\frac{2}{\rho^{2}}\left(\hat{K}_{\rho \phi}\right)^{2} \\
& \left.+\left(\hat{K}_{z z}\right)^{2}+\frac{2}{\rho^{2}}\left(\hat{K}_{z \phi}\right)^{2}+\frac{1}{\rho^{4}}\left(\hat{K}_{\phi \phi}\right)^{2}\right] .
\end{aligned}
$$

This latter expression can be evaluated as it stands, except on the $z$ axis, where $\rho=0$. However, this difficulty is only apparent, since the $\hat{K}_{i \phi}$ components involved all contain cancelling factors of $\rho$. Since $\rho=r \sin \theta$ for polar spherical coordinates centered on (a black hole on) the $z$ axis, the solution is to divide out a factor of $\sin ^{2} \theta$ from $\hat{K}_{\phi \phi}$ in (77) and a factor of $\sin \theta$ from $\hat{K}_{r \phi}$ in (78), then divide out a factor of $r$ from $\hat{K}_{\rho \phi}$ and $\hat{K}_{z \phi}$ and a factor of $r^{2}$ from $\hat{K}_{\phi \phi}$ in (80). We then compute

$$
\begin{aligned}
S=\frac{1}{8}\left[\left(\hat{K}_{\rho \rho}\right)^{2}\right. & +2\left(\hat{K}_{\rho z}\right)^{2}+2\left(\frac{\hat{K}_{\rho \phi}}{\rho}\right)^{2} \\
& \left.+\left(\hat{K}_{z z}\right)^{2}+2\left(\frac{\hat{K}_{z \phi}}{\rho}\right)^{2}+\left(\frac{\hat{K}_{\phi \phi}}{\rho^{2}}\right)^{2}\right] .
\end{aligned}
$$

The second way the global cylindrical coordinate components of $\hat{K}_{i j}$ are used, is in computing $\hat{K}_{r r}$ for use in the marginally trapped surface boundary condition (33). We must thus transform $\hat{K}_{i j}$ from the global cylindrical coordinates back to the local polar spherical coordinates of each black hole that uses the boundary condition (33). We find that

$$
\hat{K}_{r r}=\hat{K}_{\rho \rho}\left(\frac{\partial \rho}{\partial r}\right)^{2}+2 \hat{K}_{\rho z} \frac{\partial \rho}{\partial r} \frac{\partial z}{\partial r}+\hat{K}_{z z}\left(\frac{\partial z}{\partial r}\right)^{2}
$$


where the necessary partial derivatives are given by

$$
\left.\begin{array}{l}
\frac{\partial \rho}{\partial r}=\sin \theta \\
\frac{\partial z}{\partial r}=\cos \theta .
\end{array}\right\}
$$




\section{Choice of Conformal Panction}

As mentioned earlier, our basic equation (21) can be viewed as a nonlinear Poisson equation, with the source function $S$ determining the magnitude of the nonlinearity. To the extent that we can ignore this nonlinearity, our solutions to (21) should thus be superpositions of Schwarzschild solutions. In isotropic coordinates (required by our assumption of 3-conformal flatness), the Schwarzschild solution is

$$
\Psi_{\text {SCHW }}=1+\frac{m / 2}{r}
$$

where $m$ is the black hole mass and $r$ is the usual polar spherical radial coordinate in the "hat-means-flat" space. Thus we expect the "monopole sum"

$$
\Psi=1+\sum_{i} \frac{\lambda_{i}}{r_{i}}
$$

where the sum is over all black holes, $\left\{\lambda_{i}\right\}$ is a set of "monopole strengths" which we set to one half the black hole masses, and $r_{i}$ is the distance from the $i$ th black hole, to be an approximate solution to (21).

Because the boundary conditions play such a crucial role in determining the solution of (21), it is desirable that they be finite differenced as accurately as possible. In particular, this applies to our "generic" boundary condition (34) and its finite difference form (68). When this is applied at an inner (black hole surface) boundary, a single term in the sum of (88) will usually dominate. Likewise, when this is applied at the outer boundary, the asymptotic form (23) is again of the form of (88) with only a single term in the sum.

Thus any mechanism by which we can improve the accuracy of our finite differencing of the boundary condition (34) for solutions of the general form

$$
\Psi=1+\frac{\lambda}{r}
$$


where $\lambda$ is an unknown constant, should also improve our accuracy in more general problems. In particular, we want a good finite difference approximation to $\frac{\partial \Psi}{\partial r}$ for such solutions.

This leads to the idea of working with some function $\Omega(\Psi)$ which linearizes this radial derivative. We adopt the function

$$
\boldsymbol{\Omega}(\Psi)=\frac{1}{\Psi-1}
$$

for this purpose. This gives $\Omega$ as a linear function of $r$ for the solution (89), so our finite difference approximation (68) to the boundary condition (34) is exact for this case.

An alternative way of improving the accuracy to which the boundary conditions (both inner and outer) are approximated was tried by Choptuik (1982), who replaced the $r$ coordinate of polar spherical coordinates with the new coordinate

$$
s=1-\frac{a}{r}
$$

This has the advantage of not putting any constraints on the range of $\Psi$, whereas (90) requires that $\Psi$ be everywhere greater than unity (or everywhere less than it). On a few occasions when very poor initial guesses are used, the author's code produces extrapolated $\Psi$ values which violate this requirement, with generally disastrous results.

Another advantage to Choptuik's $s$ coordinate is that grid points evenly spaced in $s$ are bunched up near the black hole. This is useful since closely spaced grid points are needed to resolve the rapid variation of $\Psi$ in this region. (The author's code uses multiple patches to perform this function.) However, the definition (91) is closely tied to the use of polar spherical coordinates, and does not easily generalise to the case of multiple coordinate patches. A possible solution would be to adopt (91) on polar spherical patches around black holes and in the neighborbood of infinity, while retaining 
more usual coordinates elsewhere. Used in the neighborhood of infinity, (91) allows the direct use of (22) in the form

$$
\Psi(s=1)=1
$$

for an outer boundary condition. Choptuik (1985c) has suggested that this will lead to more reliable values for the total mass-energy of the computed spaces than the Robin outer boundary conditions used in this thesis, but this has not been tried.

We now turn to a more detailed discussion of the use of (90) in the author's code. It was decided to continue to always store and finite difference $\boldsymbol{\Psi}$ (to obtain $\nabla^{2} \Psi$ ), but to allow interpatch interpolation and boundary extrapolation to use either $\Psi$ or $\Omega$, the choice being made independently for each patch. To obtain a value $\Psi_{e \pm 1}$ at an interpatch boundary, we use bilinear interpolation in either $\Psi$ or $\Omega$ based on the other patch's choice of conformal function, since that's the patch $\Psi_{e \pm 1}$ is in. To obtain $\Psi_{e \pm 1}$ at a true boundary of a patch, we extrapolate either $\Psi$ or $\Omega$ based on that patch's choice, since there is no other patch involved in this case.

For the interpolation case, (71) is merely replaced by a bilinear interpolation in $\Omega$,

$$
\Omega_{e \pm 1}=d_{00} \Omega_{00}+d_{01} \Omega_{01}+d_{10} \Omega_{10}+d_{11} \Omega_{11}
$$

where the interpolation coefficients are found as before (figure 2). However, the extrapolation process at true boundaries isn't quite as simple. If the Neumann boundary conditions (42) apply, the extrapolation formula (70) is easily seen to still hold, so we need not consider this case further. However, the generalised boundary condition (41) requires a new treatment, which we now discuss.

From the defining expression (90), we have

$$
\mathbf{\Psi}=1+\frac{1}{\mathbf{\Omega}}
$$


so that

$$
\frac{\partial \Psi}{\partial r} \doteq-\frac{1}{\Omega^{2}} \frac{\partial \Omega}{\partial r}
$$

Our boundary condition (41) thus becomes

$$
-\frac{1}{\Omega^{2}} \frac{\partial \Omega}{\partial r}+b_{1} \Psi+b_{2}+\frac{b_{3}}{\Psi^{3}}=0,
$$

which we finite difference in the same manner as before to obtain

$$
\boldsymbol{\Omega}_{e \pm 1}=\Omega_{e \mp 1}-\left(\Omega_{e}\right)^{2}\left(d_{1} \Psi_{e}+d_{2}+\frac{d_{3}}{\Psi_{e}^{3}}\right)
$$

with the $d_{i}$ being defined by (69) and (35) as before. 


\section{Solving the Algebraic Equations}

The result of finite differencing the PDE under consideration is, as mentioned above, a large set of nonlinear algebraic equations, one for each grid point. In the interior of a patch, each point is "linked" to its four nearest neighbors only; points on interpatch boundarys are linked to the interpolation points in adjacent patches. In either case, the number of points a given point is linked to is far smaller than the total number of grid points in the patch system, i.e. the system of equations is "sparse". We now discuss the methods used herein to solve these equations.

Most past work in numerical GR has used iterative methods to solve such systems of equations, typically without ever explicitly constructing the systems at once. These methods involve iteration even if the equations are linear. This was tried in early versions of the program described in this thesis, but the convergence of these methods (notably the standard "SOR" one) was found to be at best slow, and sometimes nonexistant.

To avoid this, we adopt a global Newton method, where the entire linearized system of equations is solved simultaneously, iteration occuring only due to the nonlinearity. This gives reliable convergence without "tuning parameters", at the expense of very slow execution. Choptuik (1982) found the "multigrid" algorithm of Brandt et al to be very fast in execution. However, he reported that the programming effort to implement it is substantial-at least several person-months of programming over and above that needed to implement SOR. He also found that some tuning of the parameters of the multigrid algorithm was needed to ensure reliable convergence.

We will now discuss the global Newton method used in this thesis. We begin by 
imposing a linear ordering on the grid points, say from 1 to $n$. (For the present work, the natural ordering of the 3-tuples $(p, i, j)$ is used, where $p$ denotes the patch number and $(i, j)$ the integer patch coordinates of the grid point.) The system of equations can then be written as the single vector equation $\mathbf{P}(\mathbf{x})=0$, where $\mathbf{x}$ denotes the vector of $n \Psi$ values, and $\mathbf{P}$ is a suitable vector-valued function.

We now linearize $\mathbf{F}$ about our current solution estimate $\mathbf{x}$,

$$
\mathbf{P}(\mathbf{x}+\delta \mathbf{x}) \approx \mathbf{P}(\mathbf{x})+\mathbf{J} \cdot \delta \mathbf{x}
$$

where $\mathbf{J}$ is the $n$ by $n$ "Jacobian" matrix (in general dependent on $\mathbf{x}$ ) defined by

$$
\mathbf{J}_{k l}(\mathbf{x})=\frac{\partial \mathbf{F}_{k}(\mathbf{x})}{\partial \mathbf{x}_{l}}
$$

to be matrix multiplied by the column vector $\delta x$. Finally, we solve for the "step" $\delta \mathbf{x}$ which would make $\mathbf{F}(\mathbf{x}+\delta \mathbf{x})$ zero if the linear approximation (98) were exact, finding that we must have

$$
\mathbf{J} \cdot \delta \mathbf{x}=-\mathbf{F}(\mathbf{x})
$$

In this case, we interpret $\mathbf{F}$ as the vector of residuals, so $\mathbf{J}_{k l}$ represents the partial derivative of the $k$-th residual with respect to the $l$-th $\Psi$ value. Note that this must take into account the indirect influence of values in other patches through interpatch interpolation at line ends, as well as the effects of boundary conditions. Given that either or both of these may be done using either $\Psi$ or $\Omega$, the actual calculation of the components of the Jacobian is a rather messy task, although conceptually straightforward. The detailed equations used may be found in the listing of the function jacobian.c in appendix 2; they have too many subcases to justify writing them out here.

Like the usual single-variable Newton iteration which it generalizes, this iteration may fail to converge if the initial guess is sufficiently bad, but when it does converge 
it does so quadratically, roughly squaring the relative error (doubling the number of correct digits) each iteration. (This latter property provides a useful check that all components of the Jacobian have been calculated correctly.) Because of these two properties, there is a premium placed on having a fairly good initial guess.

The strategy adopted to provide this is twofold. First, we use the monopole sum (88), which corresponds to a linear superposition of Schwarzschild solutions. As discussed in the previous chapter, this provides a good initial guess when the source function $S$ is small. Second, when computing a series of models differing only in black hole momenta or spins (i.e. in $S$ ), the $\Psi$ values from previously computed models are used as initial guesses for new ones. This latter technique is known as using a "continuation method", and was suggested by M. Yedlin.

Our problem is now reduced to that of solving a large sparse system of linear equations. This is a standard problem in numerical linear algebra, and many algorithms are available for solving it. For the present work, the "ILUCG" algorithm of Kerneilaw (1978) was used, as embodied in a subroutine package written by P. Madderom and supplied by T. Nicol, both of the UBC Computing Center. This was used "as is" except for a minor modification in its internal convergence criteria, as described in the code listing in appendix 2.

However, one further problem arises in the numerical solution. The Jacobian entries are (ignoring boundary conditions) just the "molecule" components defined earlier, and these all depend on the grid spacing as $1 / h^{2}$. Thus, when using a patch system in which different patches have very different grid spacings, the Jacobian will have entries which differ greatly in magnitude. This usually results in the matrix being numerically illconditioned. To try to avoid this, we scale the Jacobian so that the entries on each row 
are approximately the same in size. We also scale the right hand side vector $-\mathbf{F}(\mathbf{x})$ by the same amounts, so the solution $\delta x$ to the system (100) remains unchanged. 


\section{Measuring the Masses of the Models}

It will prove useful to be able to measure the total energy of the spaces we construct. Also, to the extent that any proper subspace can be said to have a mass associated with it, we would like to measure the masses of the individual black holes which populate our spaces. We now discuss the means by which these measurements are made.

The first useful "mass functional" we shall consider is that associated with the area of the event horizon of a black hole. This quantity (summed over all black holes) always increases (second law of black hole dynamics) in any process involving black holes. Unfortunately, the event horizon of a black hole is a global property of spacetime as a whole, and can't be found until the complete time evolution of the spacetime is known. However, every (outer) marginally trapped surface must lie within an event horizon, so we can place a lower bound on the "horizon area mass" of a black hole by calculating the mass associated with a marginally trapped surface surrounding it. We find the latter simply by specifying marginally trapped surface inner boundary conditions, so our inner grid boundary becomes the desired surface.

The mass $M_{h}$ associated with any given horizon area $A_{h}$ is

$$
M_{h}=\sqrt{\frac{A_{h}}{16 \pi}} .
$$

This area is found by integrating over the marginally trapped surface,

$$
\begin{aligned}
A_{\mathrm{MTS}} & =\int_{0}^{2 \pi} \int_{0}^{\pi} \Psi^{4} r^{2} \sin \theta d \theta d \phi \\
& =2 \pi \int_{0}^{\pi} \Psi^{4} r^{2} \sin \theta d \theta,
\end{aligned}
$$

where we have exploited the axisymmetry to do the $\phi$ integral analytically.

We now consider means of computing estimates of the total energy of a model. Defining the total energy of a space is a somewhat difficult matter in GR, and is in any 
case beyond the scope of this thesis. Wald (1984, section 11.2) discusses this topic in more detail. For our purposes it will suffice to consider the mass functional (see Eppley (1977, p. 1610) for a variety of different formulations)

$$
E_{\infty}=-\frac{1}{2 \pi} \oint_{\mathbf{S}} \nabla \Psi d \mathbf{S},
$$

where the integral is taken over the 2-sphere of infinite radius $\mathbf{S}$. We approximate this by taking $\mathbf{S}$ as the outer grid boundary. Since we are integrating over all angles, only the radial derivative of $\Psi$ contributes towards the integral, and the Robin outer boundary condition (25) gives this "for free" as

$$
\frac{\partial \Psi}{\partial r} \approx-\frac{\Psi-1}{r}
$$

Hence (again using the axisymmetry to do the $\phi$ integral analytically)

$$
E_{\infty} \approx \int_{0}^{\pi} \frac{\Psi-1}{r} r^{2} \sin \theta d \theta
$$

Unfortunately, most previous studies have found that such "surface integral" energy functionals are numerically rather inaccurate, due to finite differencing errors. By applying Gauss's theorem to the volume occupied by the model, the bulk of the surface integral (103) can be replaced by a volume integral of $\nabla^{2} \Psi$, which is known analytically by virtue of our basic equation.(21). The remaining surface terms are also available "for free" through our inner boundary condition (34). This procedure, although it would almost certainly lead to more accurate energy values, is not used in the present thesis. This is due to the difficulty of doing a volume integral over the model in the presence of multiple coordinate patches with (small) gaps between them. This difficulty can probably be overcome by using information on the global geometry of the patch system, but this has not yet been tried. 
Both (102) and (105) involve integrating a function which typically varies little with $\theta$, weighted by $r^{2} \sin \theta$. Although this can be done directly, numerical accuracy is considerably improved if a "representative" value of the function is subtracted off the numerical integral and it's (weighted) integral is done analytically and added back. This is particularly useful for (105), since the patch systems used in this thesis tend to have low resolution in $\theta$ near the outer grid boundary.

The actual numerical integrations involved in (102) and (105) are done by fitting a cubic spline (using the Neumann boundary conditions (37) and (39) for the endpoints) and integrating this analytically (via the spline coefficients). We choose this method over the usual choice of Simpson's rule because it does not constrain the number of integration points (angular grid points) to be an odd number. 


\section{Testing the Code}

We use two main types of models to test out the code described in this thesis: Schwarzschild models with a variety of different patch systems, and both moving and spinning single black holes with parameters chosen to match those of Choptuik (1982, 1985a). Recall that detailed information about all models mentioned in this thesis is given in appendix 1.

We need to assess the accuracy of various Schwarzschild models. We will do this by comparing three quantities. The first of these is the mass $M_{\text {Mrs }}$ associated via (101) with the area of the marginally trapped surface at our inner grid boundary, as calculated by (102). The second is the total energy "at infinity" $E_{\infty}$, as calculated by (105). The third (and most reliable) is the maximum deviation $\max \left|\Psi-\Psi_{\text {sCHw }}\right|$ between the calculated $\Psi$ and the exact Schwarzschild solution (87).

The first main result of the Schwarzschild tests is that the independent terms finite difference scheme given by (59) and (61) turns out to be exact (ignoring rounding errors) for the approximate solution (89). In other words, when (89) is substituted into the algebraic equations resulting from this difference scheme, they are satisfied to within the rounding errors, rather than just to $O\left(h^{2}\right)$. Because of this, the independent terms form is used in most of the models run in this thesis. The locally conservative finite difference scheme given by (65) and (67), in contrast, exhibits the expected $O\left(h^{2}\right)$ dependence of errors on grid size. Table 1 gives the results of a test series of models run to verify this dependence; note that each time the grid resolution is doubled, the errors are (approximately) divided by four.

The second main result of the Schwarzschild tests is that good results are (still) 


\section{Table 1 - Comparison of Different Grid Sizes}

$\begin{array}{ccccc}\text { Model } & \text { Resolution } & M_{\text {MTs }} & E_{\infty} & \max \left|\Psi-\Psi_{\text {sCHW }}\right| \\ 10 \text { plo100 } & 10 & 1.00613 & 1.01808 & 6.125 \times 10^{-3} \\ 20 \text { plo100 } & 20 & 1.00147 & 1.00429 & 1.474 \times 10^{-3} \\ 40 \text { plo100 } & 40 & 1.00036 & 1.00105 & 3.621 \times 10^{-4}\end{array}$

Results of running unit mass Schwarzschild models with different grids. "Resolution" is the value of $\Delta r / r$ on the inner boundary of each patch. This is also the approximate number of angular grid points used in the inner patches. Observe that the errors go down by approximately a factor of four each time the resolution is doubled.

obtained when polar spherical and cylindrical patches are intermixed. Table 2 gives the results of two test series of models run to verify this, one each for locally conservative and independent terms finite differencing schemes. Each test series consists of a baseline model using only polar spherical patches, followed by a model in which one of the patches has been augmented with a cylindrical coordinate patch. The same patch systems were used in both test series; the relevant portions of those patch systems are shown in figures 3 and 4 . The table shows that inserting a cylindrical coordinate patch into the (otherwise spherically symmetric) model does not greatly increase the errors. We conclude that the accuracy of our solutions is probably not greatly degraded by the interpatch interpolation process.

The third main result of the Schwarzschild tests is that the use of $\Omega$ as a conformal function does indeed linearize the boundary condition (34), at both the inner and outer boundaries. It also helps to linearize the interpatch interpolation done across patch boundaries. Table 3 gives the results of a test series of models run to demonstrate the effects of using $\Omega$ compared to using $\Psi$ as a conformal function. The series consists of a baseline case using $\boldsymbol{\Omega}$ everywhere, followed by 3 models which succesively substitute $\Psi$ on the innermost patch, all the "middle" patches, and the the outermost patch. All 


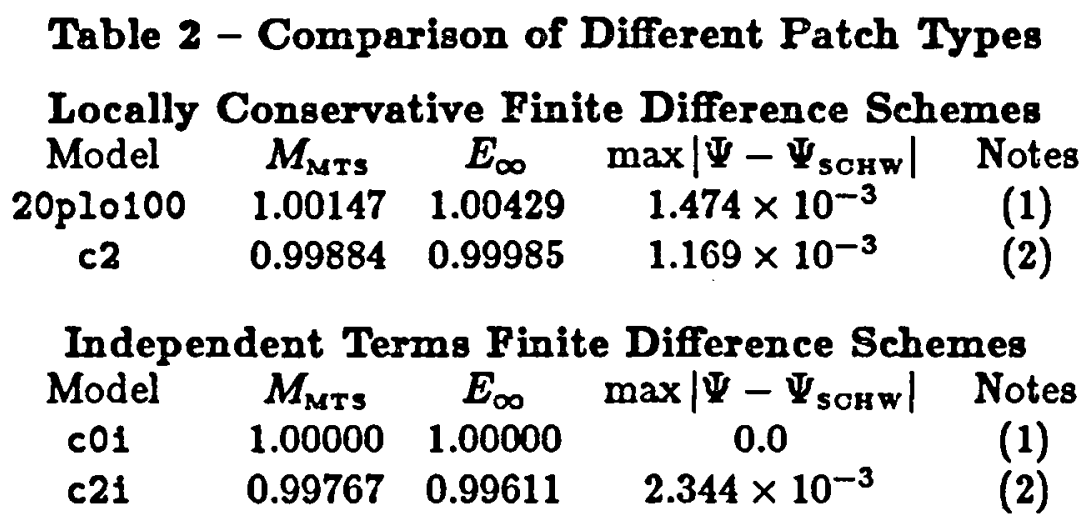

\section{Notes}

(1): Baseline patch system, as shown in figure 3. All patches are polar spherical.

(2): Baseline patch system with patch \#2 augmented with a cylindrical coordinate patch, as shown in figure 4.

Results of running unit mass Schwarzschild models with different grids. Observe that the model results change only slightly when cylindrical coordinate patches are introduced into otherwise spherically symmetric grids. This holds true for both types of finite difference schemes.

of these show larger errors than the baseline model, with the worst being the "inner" case where $\Psi$ is being used to approximate the inner boundary condition. The overall conclusion to be drawn from this test series is that the best accuracy is obtained by using $\Omega$ everywhere.

\section{Table 3 - Comparison of Different Conformal Functions}

\begin{tabular}{|c|c|c|c|c|}
\hline $\begin{array}{c}\text { Model } \\
\text { 20plo100 }\end{array}$ & $\begin{array}{c}M_{\mathrm{MTS}} \\
1.00147\end{array}$ & $\begin{array}{c}E_{\infty} \\
1.00429\end{array}$ & 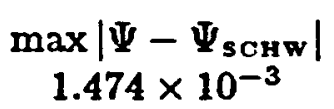 & $\begin{array}{c}\text { Description } \\
\mathbf{\Omega} \text { used for all patches }\end{array}$ \\
\hline poo & 0.99648 & 0.99929 & $3.519 \times 10^{-3}$ & $\Psi$ used for innermost patch \\
\hline opo & 1.00196 & 1.00899 & $1.963 \times 10^{-3}$ & $\Psi$ used for "middlen patches \\
\hline oop & 1.00148 & 1.00493 & $1.480 \times 10^{-3}$ & Tused for outermost patch \\
\hline
\end{tabular}

Results of running unit mass Schwarzschild models with different choices of conformal function. Observe that the smallest errors occur when $\Omega$ is used for all patches. The "middle" patches are those which are neither innermost nor outermost. 
Pigure 3 - Baseline patch gystem for table 2

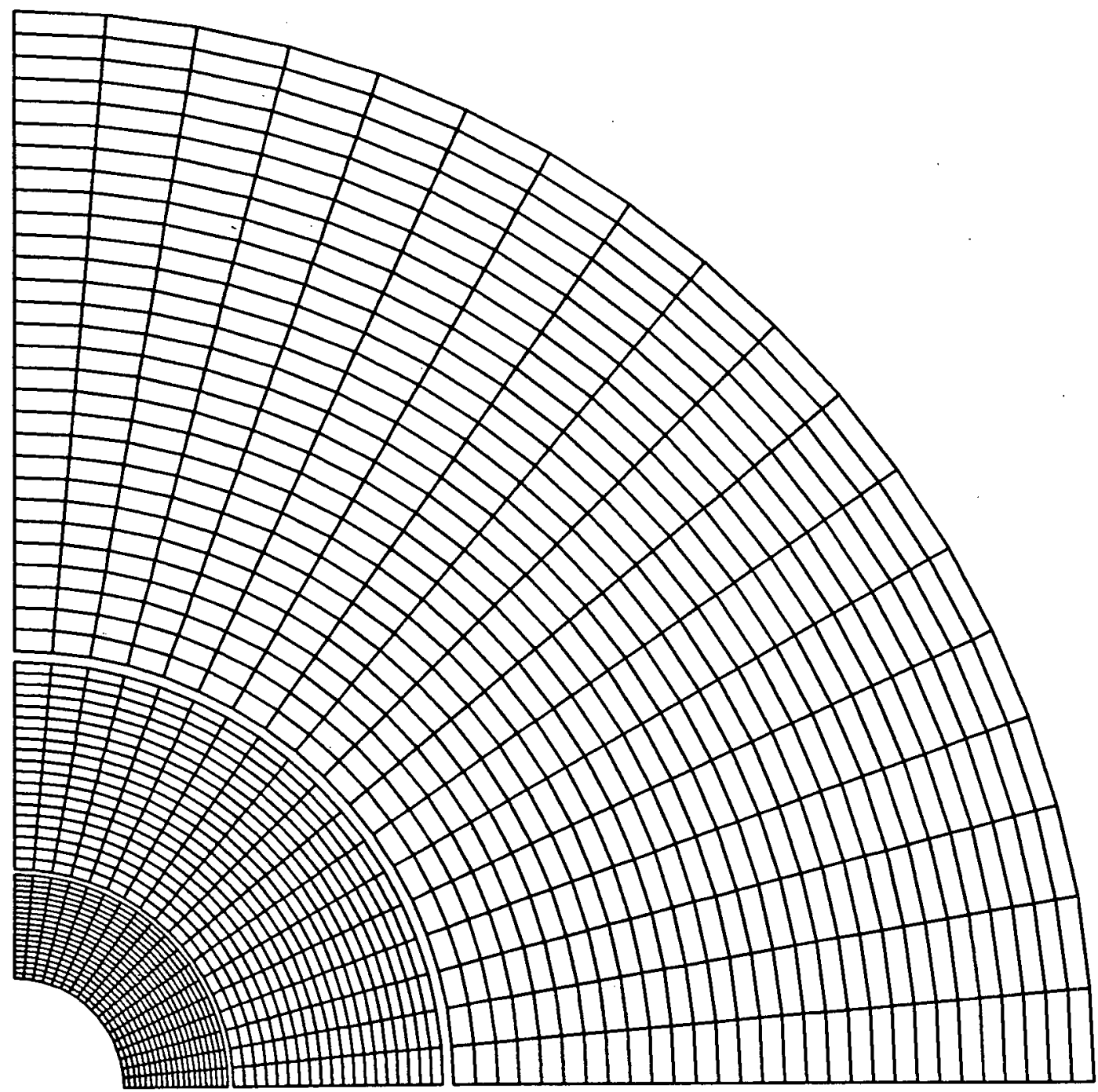

Only the inner section of the patch system is shown (patches \#1, \#2, and \#3, numbering from the inside out). The $\rho$ axis runs horizontally at the bottom, and the $z$ axis runs vertically at the left. The range of $r$ shown is from 0.5 (at the inner boundary of patch \#1) to 4.9 (at the outer boundary of patch \#3). 
Fignre 4 - Angmented patch system for table 2

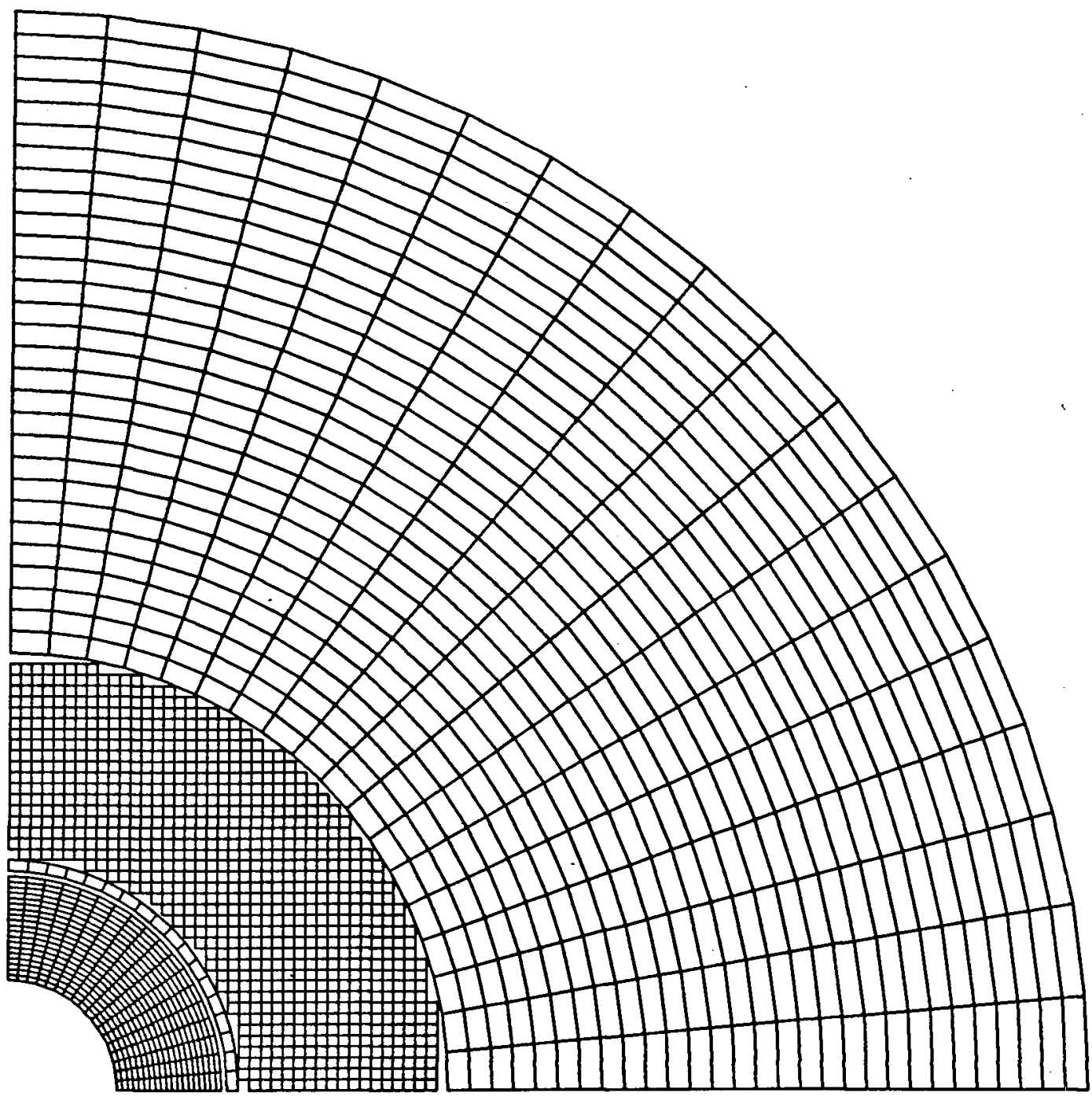

This should be compared to the patch system shown in figure 3 (previous page). The axes and coordinate ranges given there also apply here. The previous patch \#3 is now \#4, and a new cylindrical coordinate patch \#3 has been inserted. Patch \#2 (the "thin" polar spherical one) is used to change to the larger grid size before the polar spherical-cylindrical patch transition. 
The other main way we test the code is by comparing its calculated results with those of Choptuik (1982, 1985a), who studied moving and spinning single black holes using the same Bowen and York formalism as this thesis. It should be noted that Choptuik's results are independent of those of this thesis, in that he used both a different coordinate system and two completely different techniques for solving (21). Choptuik (1982) did a full numerical solution of (21) using a different algorithm to solve the finite differenced equations, while Choptuik (1985a) numerically integrated a perturbation solution to (21) valid for small nonlinearities ( $S$ values). Since he in both cases used the Bowen and York reflection symmetric inner boundary condition (27), we also use it in constructing models meant for comparison with his.

We now discuss the results of these comparisons. There are three main series of models used, with results given in table 4. These series are moving black hole models with a range of momentum (i.e. $P$ in (77)) values and $\kappa=+1$, moving black holes with the same range of momentum values but $\kappa=-1$, and spinning black holes with a range of angular momentum (i.e. $J$ in (78)) values.

For these models, the differences

$$
\delta \Psi=\Psi(\text { north pole })-\Psi(\text { equator })
$$

between the calculated $\Psi$ at the "north pole $(\theta=0)$ and on the equator $(\theta=\pi / 2)$ are compared with Choptuik's values. (A direct check of $\Psi$ itself with his plots was also made, with satisfactory results.) These comparisons are made at the black hole surfaces $(r=a=1)$, where he gave his results. As can be seen from the table, the results agree with his (1985a) perturbation analysis whenever the nonlinearities are small, and generally agree with his (1982) full numerical solution in the nonlinear regime. The moving black hole models with $P=7.5$ and $P=12.5$ for $\kappa=-1$, however, 
Table 4 - Comparison of $\delta \Psi$ with Choptuik's Results

\begin{tabular}{|c|c|c|c|c|c|c|}
\hline Model & $\boldsymbol{P}$ & $\boldsymbol{\kappa}$ & $\boldsymbol{J}$ & $\delta \Psi($ Model) & $\delta \Psi$ (Choptuik) & Notes \\
\hline ppx001 & 0.001 & +1 & 0 & $+7.985 \times 10^{-9}$ & $+8.0 \times 10^{-9^{\circ}}$ & $(1)$ \\
\hline ppx01 & 0.01 & +1 & 0 & $+7.985 \times 10^{-7}$ & $+8.0 \times 10^{-7}$ & (1) \\
\hline$p p \times 1$ & 0.1 & +1 & 0 & $+7.966 \times 10^{-8}$ & $+8.0 \times 10^{-5}$ & (1) \\
\hline pp1 & 1.0 & +1 & 0 & $+6.550 \times 10^{-3}$ & $+8.0 \times 10^{-3}$ & (1) (2) \\
\hline Pp2 $2 \times 5$ & 2.5 & +1 & 0 & $+2.335 \times 10^{-2}$ & - & (3) \\
\hline PP7×5 & 7.5 & +1 & 0 & $+5.586 \times 10^{-2}$ & - & (3) \\
\hline pp12x5 & 12.5 & +1 & $\mathbf{0}$ & $+7.209 \times 10^{-2}$ & - & (3) \\
\hline mpx001 & 0.001 & -1 & $\mathbf{0}$ & $-1.375 \times 10^{-9}$ & $-1.4 \times 10^{-9}$ & (1) \\
\hline mpx01 & 0.01 & -1 & $\mathbf{0}$ & $-1.375 \times 10^{-7}$ & $-1.4 \times 10^{-7}$ & (1) \\
\hline mpx1 & 0.1 & -1 & $\mathbf{0}$ & $-1.372 \times 10^{-3}$ & $-1.4 \times 10^{-5}$ & (1) \\
\hline mp1 & 1.0 & -1 & $\mathbf{0}$ & $-1.152 \times 10^{-3}$ & $-1.4 \times 10^{-3}$ & (1) (2) \\
\hline $\operatorname{mp} 2 \times 5$ & 2.5 & -1 & $\mathbf{0}$ & $-4.240 \times 10^{-3}$ & $-4.2 \times 10^{-3}$ & (4) (5) \\
\hline mp7x5 & 7.5 & -1 & 0 & $-1.030 \times 10^{-2}$ & $-8.0 \times 10^{-3}$ & (4) (5) (6) \\
\hline mp12x5 & 12.5 & -1 & 0 & $-1.328 \times 10^{-2}$ & $-1.1 \times 10^{-2}$ & (4) (5) (6) \\
\hline jx001 & 0 & 0 & 0.001 & $-2.340 \times 10^{-9}$ & $-2.3 \times 10^{-9}$ & (1) (7) \\
\hline jx01 & 0 & 0 & 0.01 & $-2.340 \times 10^{-7}$ & $-2.3 \times 10^{-7}$ & (1) (7) \\
\hline$j \times 1$ & 0 & 0 & 0.1 & $-2.338 \times 10^{-8}$ & $-2.3 \times 10^{-5}$ & (1) (7) \\
\hline j1 & $\mathbf{0}$ & 0 & 1.0 & $-2.210 \times 10^{-3}$ & $-2.3 \times 10^{-3}$ & (1) (2) (7) \\
\hline j10 & 0 & 0 & 10.0 & -0.05451 & -0.05 & (7) (8) \\
\hline j100 & $\mathbf{0}$ & 0 & 100.0 & -0.1799 & -0.18 & (7) (8) \\
\hline j1000 & 0 & $\mathbf{0}$ & 1000.0 & -0.3622 & -0.36 & (7) $(8)$ \\
\hline
\end{tabular}

\section{$\underline{\text { Notes }}$}

(1): Comparison made with Choptuik (1985a) perturbation analysis.

(2): Discrepancy presumably due to nonlinearities invalidating assumptions of Choptuik (1985a) perturbation analysis.

(3): No data available in Choptuik (1982) for this case.

(4): Comparison made with values read graphically from Choptuik (1982, figure 12, page 113) full numerical solution.

(5): Choptuik (1985d) confirms that $\kappa=-1$ is correct here, rather than $\kappa=+1$ as stated in Choptuik (1982).

(6): See text for discussion of discrepancy.

(7): Since $P=0$, the value of $\alpha$ is irrelevant here.

(8): Comparison made with values read graphically from Choptuik (1982, figure 16, page 118) full numerical solution. 
show disagreement at the $20 \%$ level. This corresponds to errors at the $10^{-3}$ level in $\Psi$ itself. Choptuik (1985b) has said that he does not consider such disagreements to be significant, given the uncertainties (mostly truncation errors) of both his (1982) full numerical solution and the present one. Thus the overall conclusion of these test series is that the results of this thesis agree with those of Choptuik (1982, 1985a). 


\section{Demonstrating the Code}

Although the main results of this thesis are the techniques developed, a number of test series of models were run to demonstrate the numerical GR code's capabilities. We now discuss these, beginning with the four single black hole test series. All of the black holes involved have unit nominal mass, in comparison to the unit nominal radia (hence nominal mass of two) used by York and Piran (1982) and Choptuik (1982, 1985a) in similar studies.

The first test series (hereinafter known as the "J" series) consists of models with zero linear momentum $P$ (as defined by (17)) and varying (zero, then from 0.01 to 100 in decade steps) angular momentum $J$ (as defined by (18)). The sign convention is that the angular momentum 3-vector of the black holes is directed along the positive $z$ axis. Since for a single spinning black hole (78) shows $\hat{K}_{r r}=0$, the marginally trapped surface inner boundary condition (33) used in this test series is identical to the Bowen and York reflection symmetric boundary condition (27) used in the previous studies cited above.

The remaining three test series all consist of models with zero angular momentum $J$ and varying (zero; then from 0.01 to 100 in decade steps) linear momentum $P$. The sign convention is that the momentum 3-vector of the black holes is directed along the positive $z$ axis. These test series differ in their choice of inner boundary condition, with one (hereinafter the "RSP" series) using the Bowen and York reflection symmetric condition (27) with $\kappa=+1$, one (hereinafter the "RSN" series) with $\kappa=-1$, and one (hereinafter the "MTO" series) using the marginally trapped surface condition (33) with $\kappa=0$. 
The J, RSP, and RSN models all have a mirror image symmetry about the equator, so $\Psi$ is only numerically solved for on the the half space $z \geq 0$ for these models. The MTO models lack this symmetry due to the nonlinear $\hat{K}_{r r}$ term in (33), so they require that $\Psi$ be numerically solved for on the whole space. All of the single black hole models used the same patch system, with all polar spherical patches. The solutions ( $\Psi$ shown as a function of position in the space) are shown in figures $5,6,7$, and 8 for the J, RSP, RSM, and NTO test series respectively. All of these figures show orthographic (perspective as seen from an "infinite" distance) views of the inner two patches ( $r$ from 0.5 to 1.95) of the numerical solution.

The first thing one notices on viewing these plots is the near spherical symmetry of the J, RSP, and RSN test series solutions. Although a detailed multipole analysis of the solutions has not been done, the perturbation analysis of Choptuik (1985a) found the dipole asymmetry to be dominant for small $S$ (i.e. for small $J$ or $P$ ).

We now consider the extent to which the different boundary conditions used in the three varying momentum test series just described, effect the solutions obtained. It is clear from examining figure 8 that the marginally trapped surface boundary condition gives models which differ noticably from the two reflection symmetric cases. This is perhaps to be expected, since the former lack the mirror image symmetry of the latter, and the extra nonlinear $\hat{K}_{\text {rr }}$ term in (33) changes sign across the equator (see (77)). This seems to be the cause of the "tilt" which shows up in the high $P$ models of this test series.

In contrast to the substantial difference the marginally trapped surface boundary condition makes, the two cases $\kappa= \pm 1$ of the reflection symmetric boundary condition produce very similar results, as can be seen by comparing figures 6 and 7. However, 
Figure 5 - J Test Series Solutions

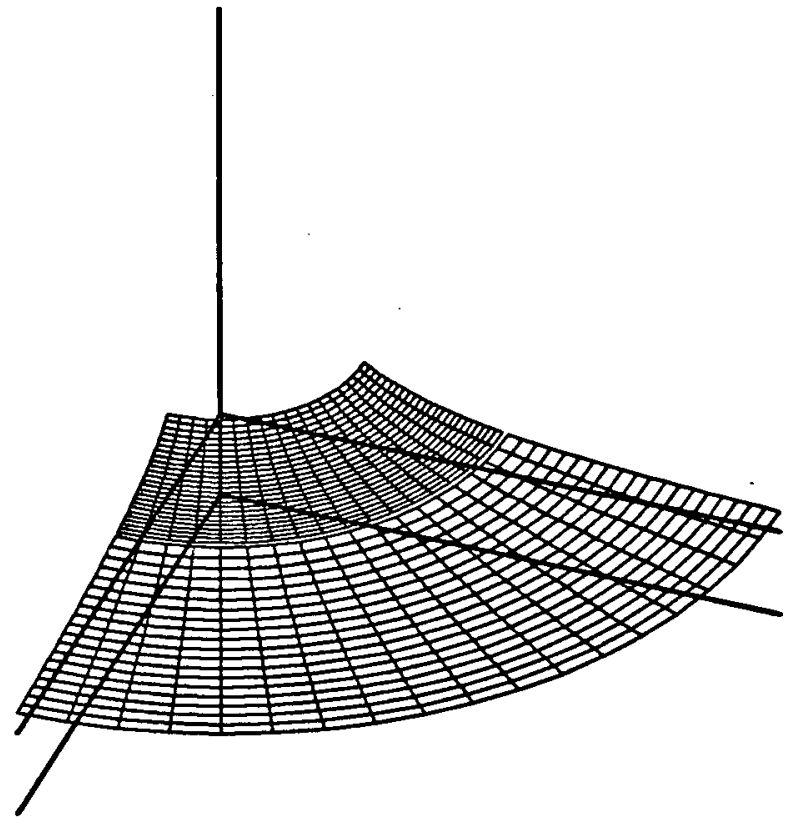

$\mathrm{J}=\mathbf{0}$

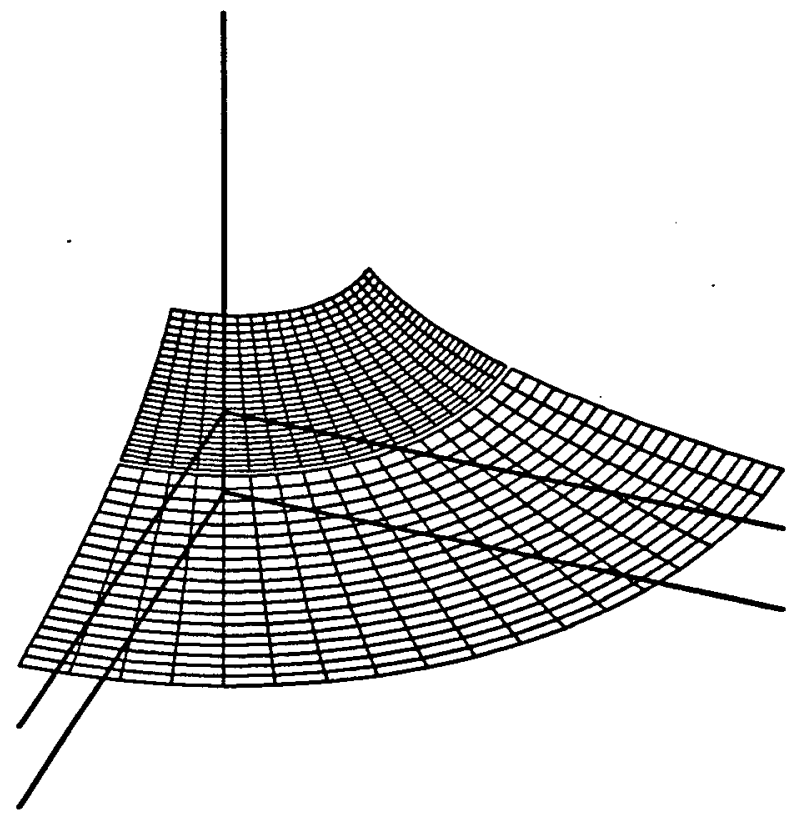

$J=10$

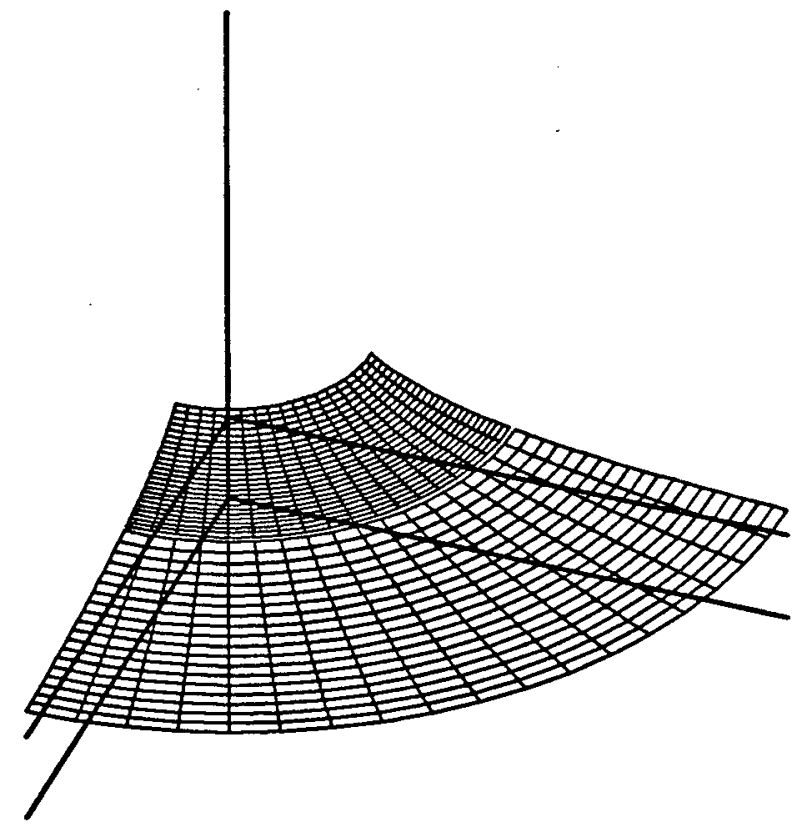

$\mathrm{J}=1$

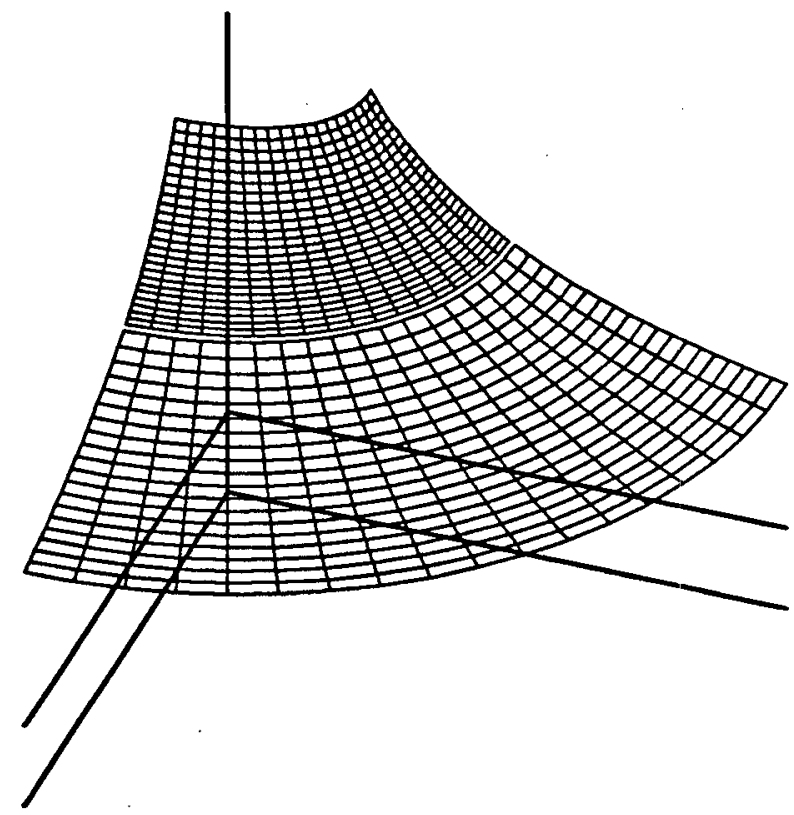

$J=100$

$\Psi$ is plotted vertically from 0 to $6, \rho$ forward from 0 to $1.95, z$ to the right from 0 to 1.95. The lower set of $(\rho, z)$ axes are at $\Psi=0$, the upper set at $\Psi=1$. The $J=0.01$ and $J=0.1$ models are visually indistinguishable from the $J=0$ one at this scale, and are hence not plotted. 
Figure 6 - RSP Test Series Solntions

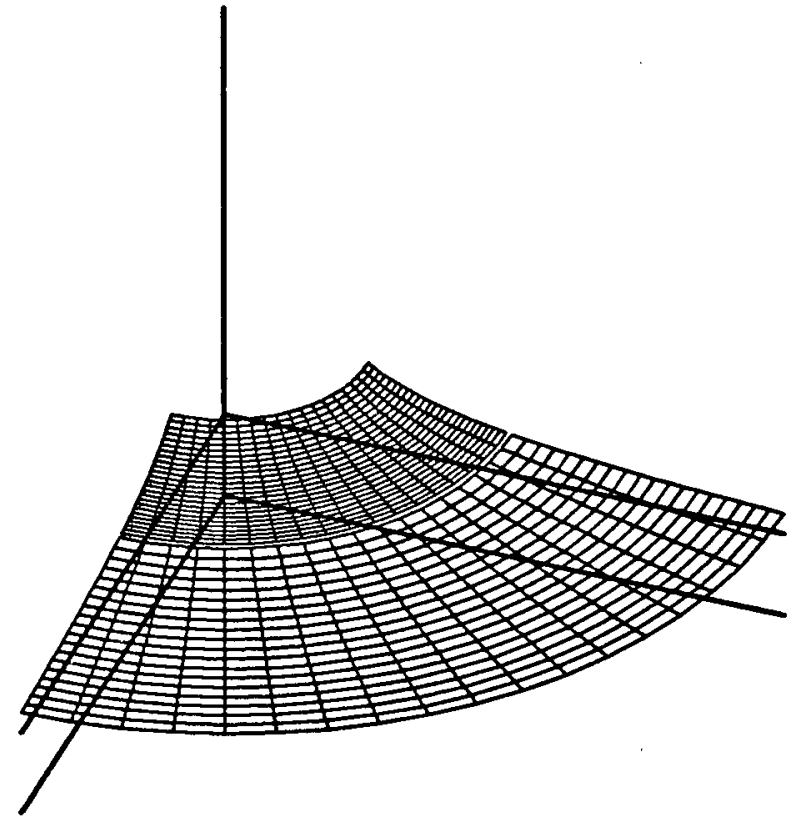

$P=0$

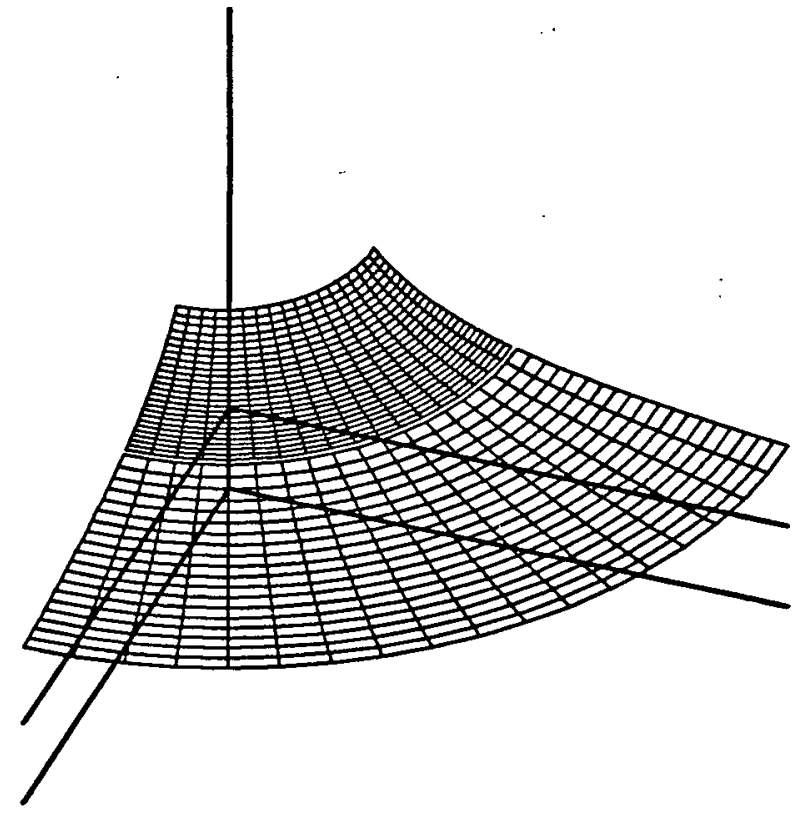

$P=10$

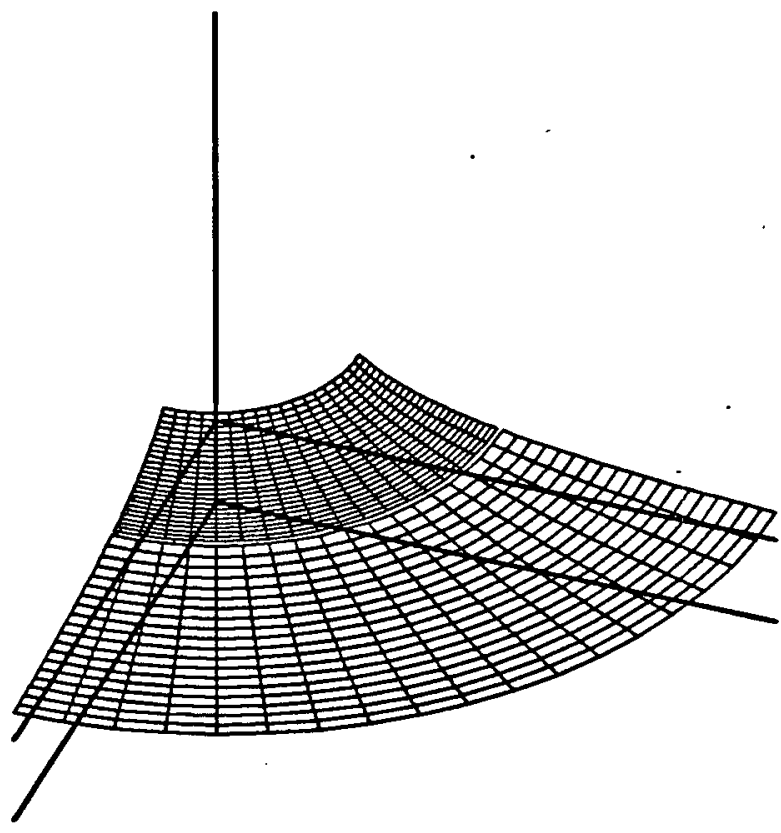

$P=1$

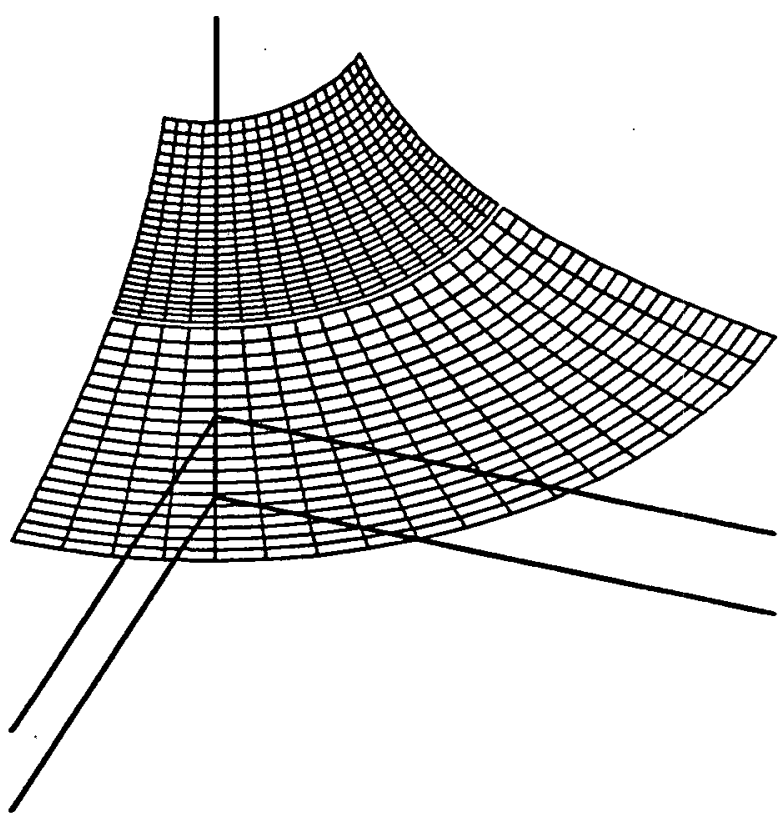

$P=100$

$\Psi$ is plotted vertically from 0 to $6, \rho$ forward from 0 to $1.95, z$ to the right from 0 to 1.95. The lower set of $(\rho, z)$ axes are at $\Psi=0$, the upper set at $\Psi=1$. The $P=0.01$ and $P=0.1$ models are visually indistinguishable from the $P=0$ one at this scale, and are hence not plotted. 
Figure 7 - RSN Test Series Solutions

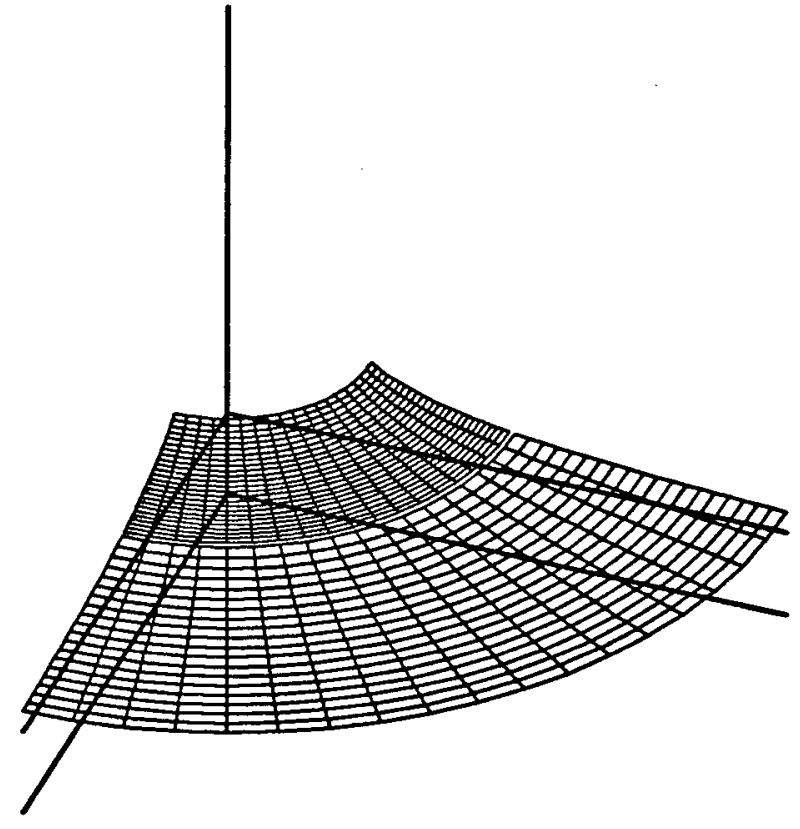

$P=0$

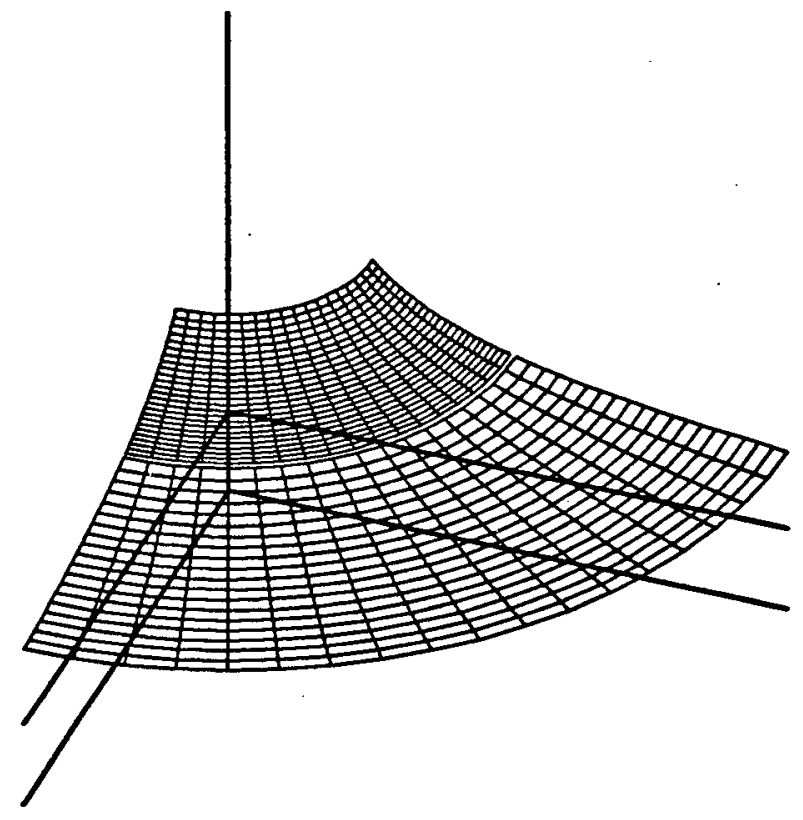

$P=10$

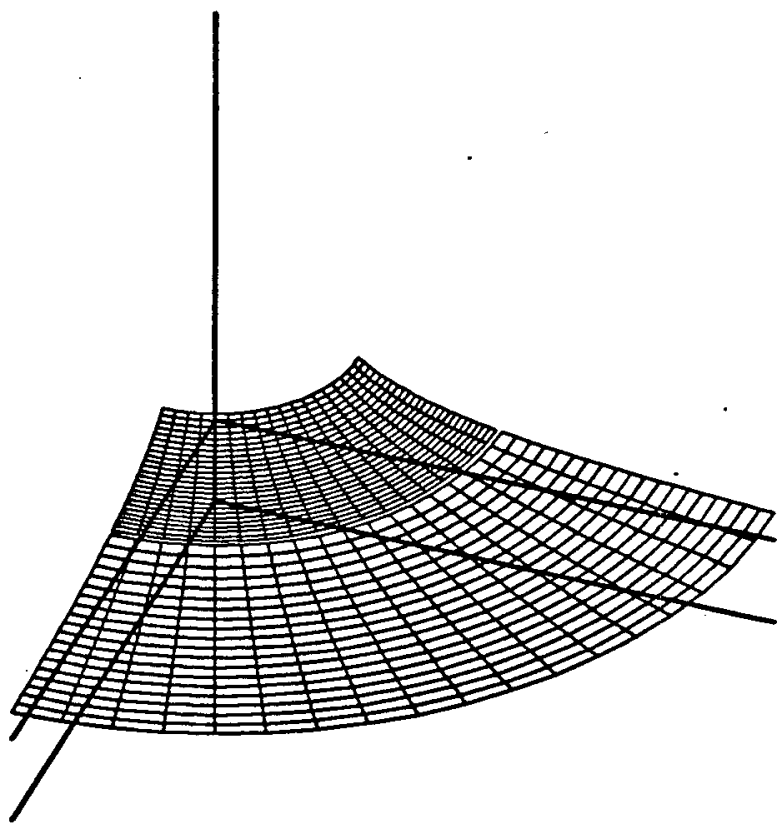

$P=1$

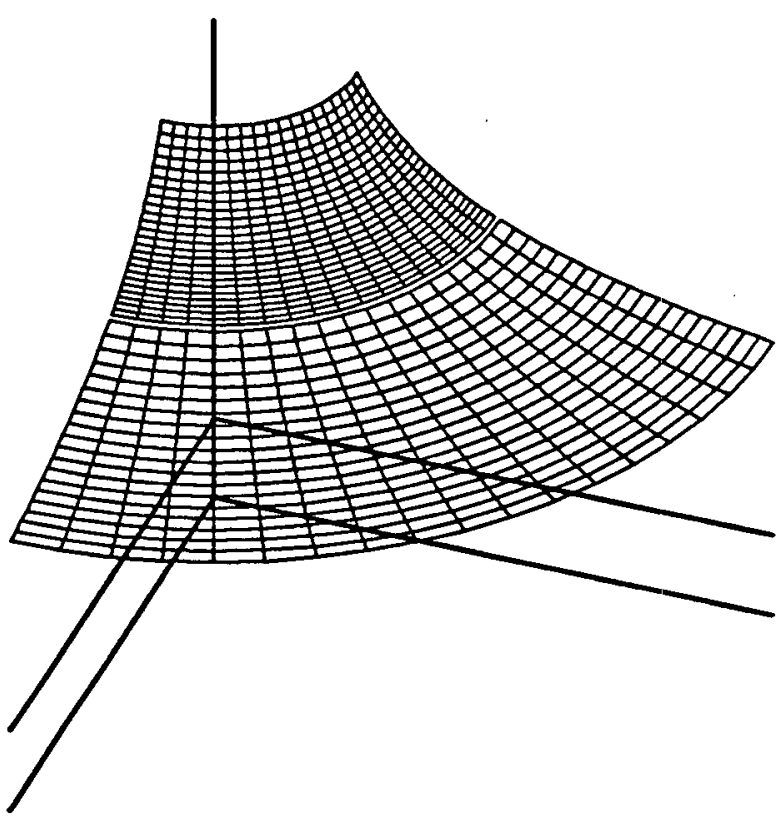

$P=100$

$\Psi$ is plotted vertically from 0 to $6, \rho$ forward from 0 to $1.95, z$ to the right from 0 to 1.95. The lower set of $(\rho, z)$ axes are at $\Psi=0$, the upper set at $\Psi=1$. The $P=0.01$ and $P=0.1$ models are visually indistinguishable from the $P=0$ one at this scale, and are hence not plotted. 
Figure 8 - NTO Test Series Solntions
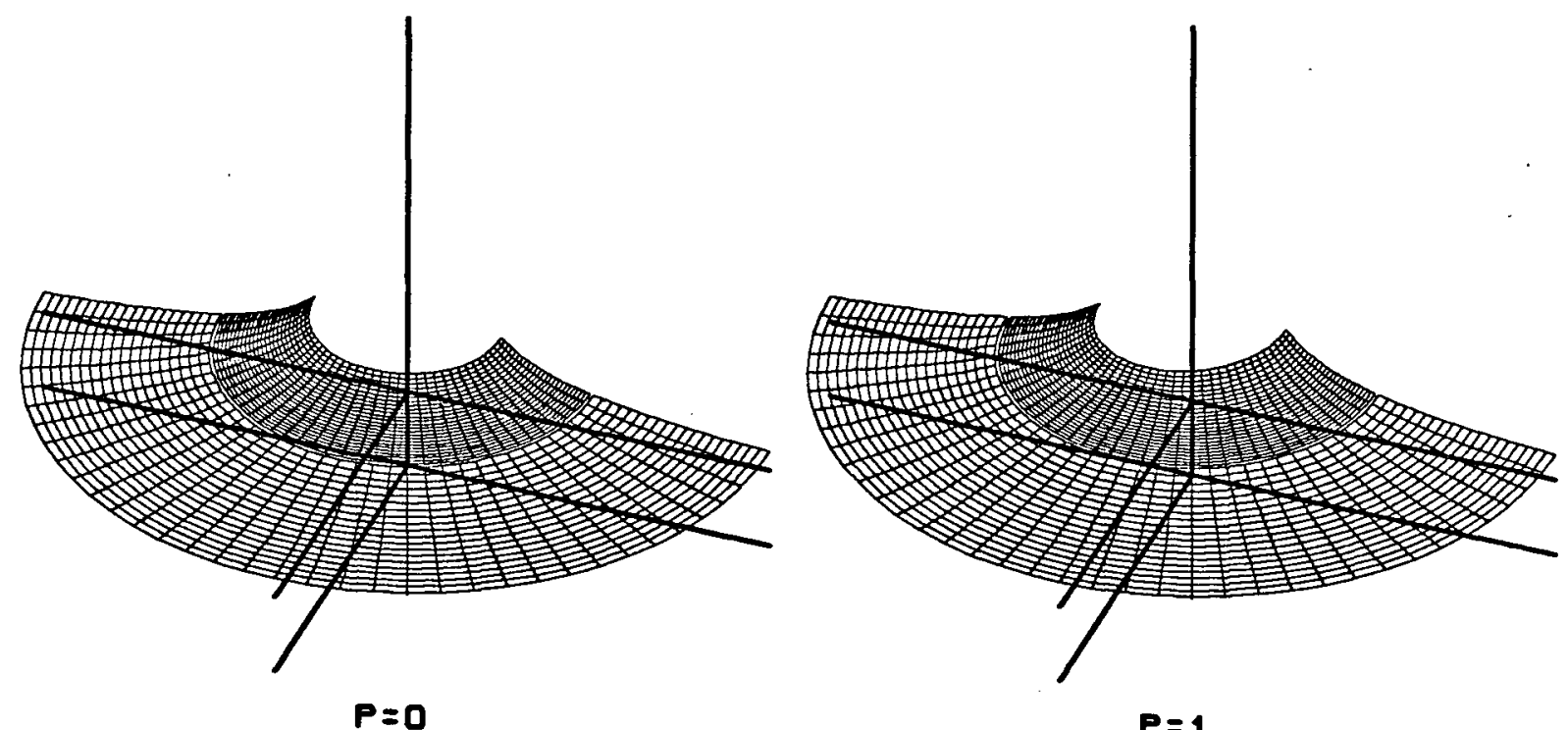

$P=1$

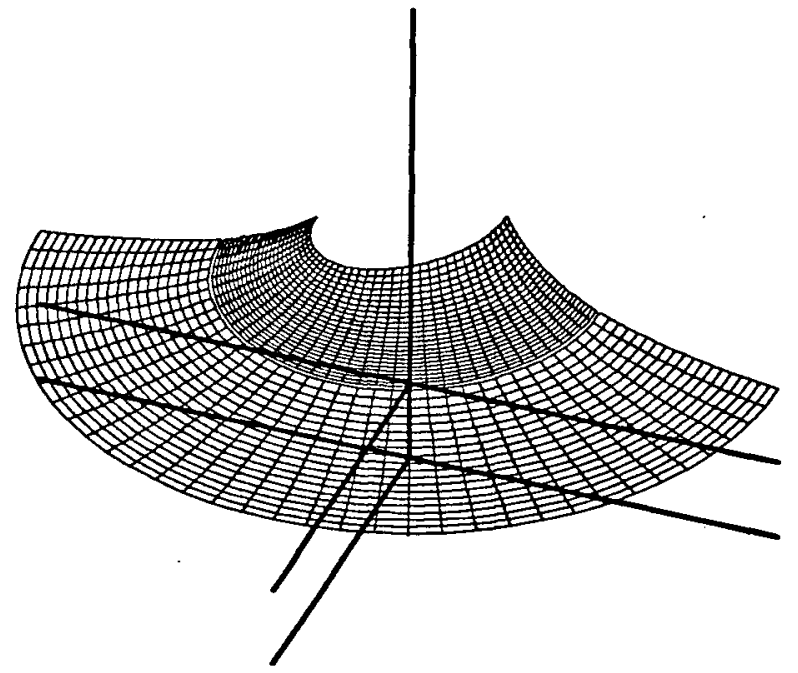

$P=10$

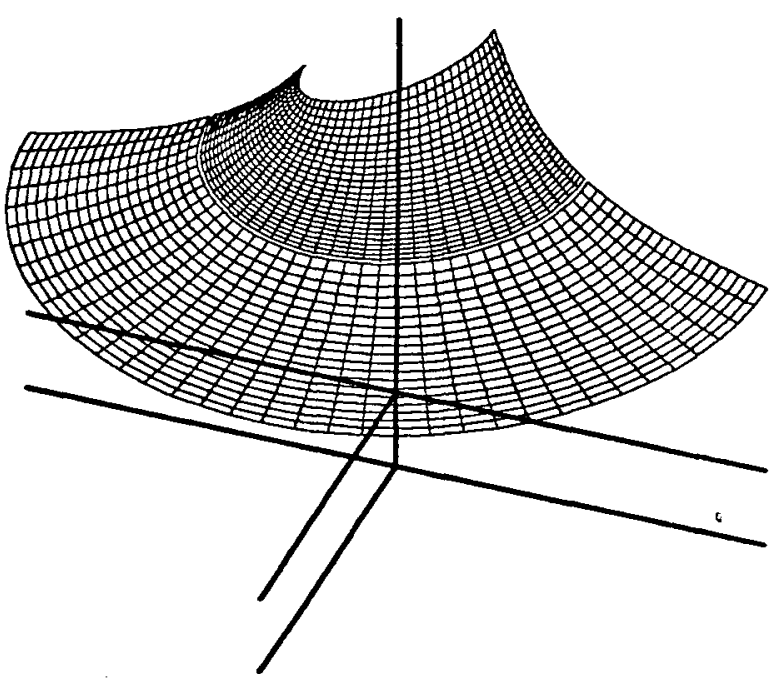

$P=100$

$\Psi$ is plotted vertically from 0 to $6, \rho$ forward from 0 to $1.95, z$ to the right from -1.95 to +1.95 . The lower set of $(\rho, z)$ axes are at $\Psi=0$, the upper set at $\Psi=1$. The black holes are all moving in the positive $z$ direction. The $P=0.01$ and $P=0.1$ models are visually indistinguishable from the $P=0$ one at this scale, and are hence not plotted. 
they do produce rather different radial variations of the (small) nonspherical component of $\Psi$, as shown by the quantitative graphs in appendix 1 .

We now consider models with two black holes. Recall that, due to our assumption of axisymmetry, the black holes must both lie on the $z$ axis, as must their linear and angular momentum vectors. Due to computer memory limitations, only one series of two black hole models has been run so far. This "PP" series has two unit mass black holes with equal and opposite momenta (and no spin). These momenta vary from -100 to -0.01 in decade steps, then zero, then from +0.01 to +100 in decade steps. The sign convention is that positive momenta indicate the black holes are approaching each other. The two black holes lie at positions $z= \pm 1.5$. The patch system used for this test series is shown in figure 9.

The solutions for this test series are shown in figures 10 and 11 , plotted in the same way as the other solutions (although at a different scale, as given in the figure caption). These models again have a mirror image symmetry about the equator, which is treated in the same manner as before. However, note that the contributions of both black holes must be taken into account in determining $\hat{K}_{r r}$ for the marginally trapped surface boundary condition (33) as well as in determining $S$ itself. 
Figure 9 - Patch System for the PP Test Series

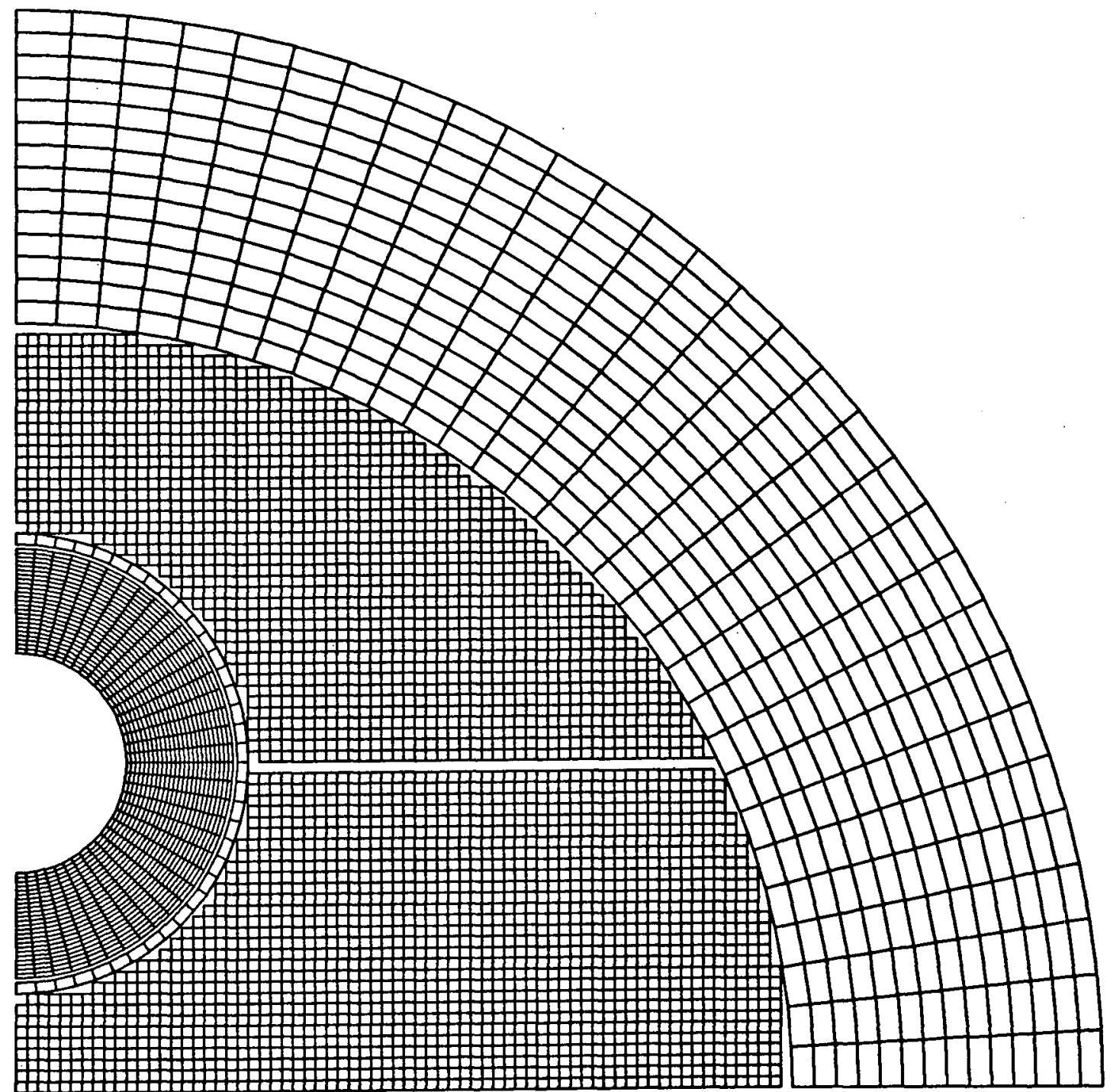

Only the inner 5 patches are shown. The $\rho$ axis runs horizontally at the bottom, and the $z$ axis runs vertically at the left. Both axes range from 0 to 4.9. Note the splitting of the cylindrical coordinate region into two patches 80 that each patch will be manhattan convex, and the use of a "thin" polar spherical patch to change to a larger grid size before the polar spherical-cylindrical patch transition. 
Figure 10 - PP Test Series Solutions $(P \geq 0)$

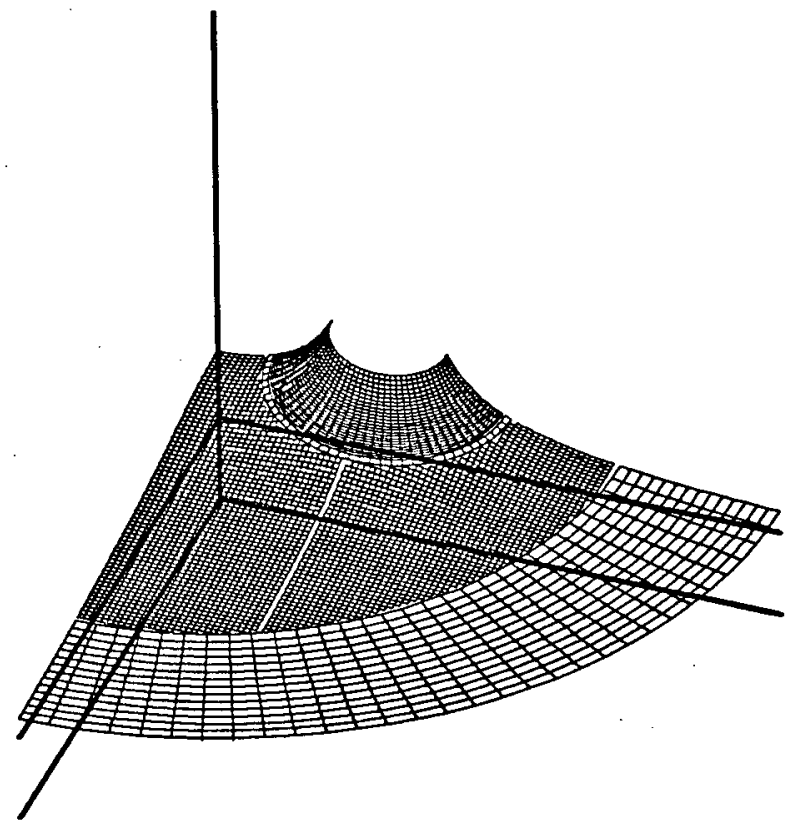

$P=0$

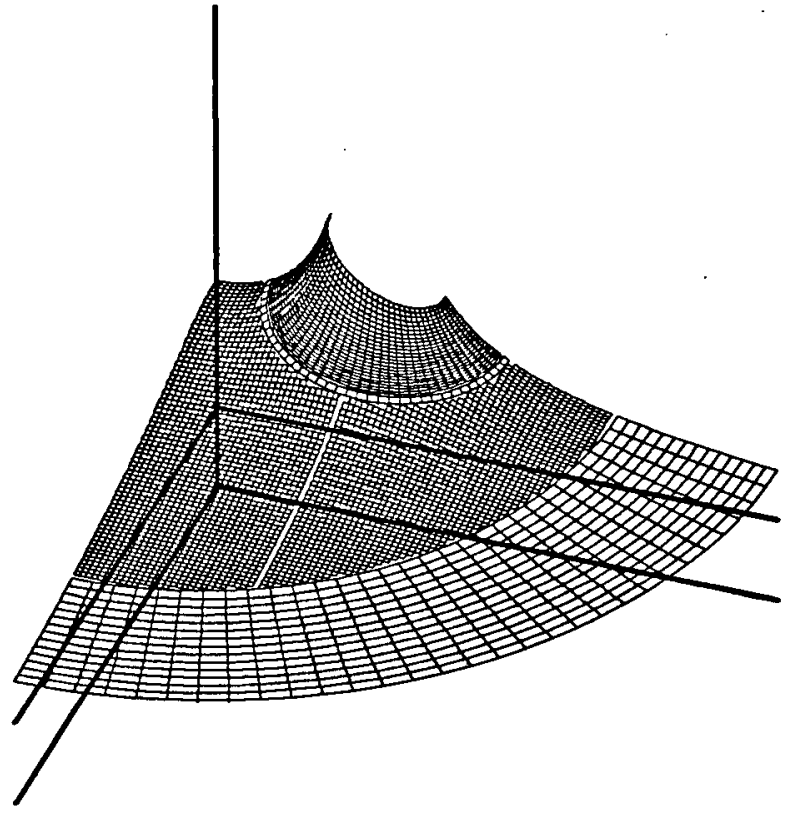

$P=+10$

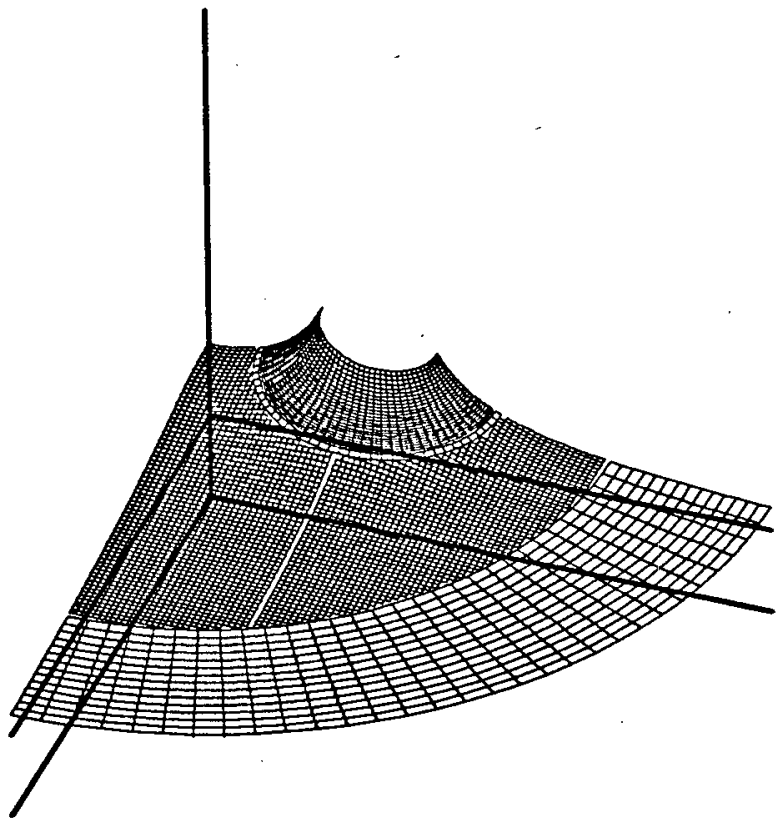

$P=+1$

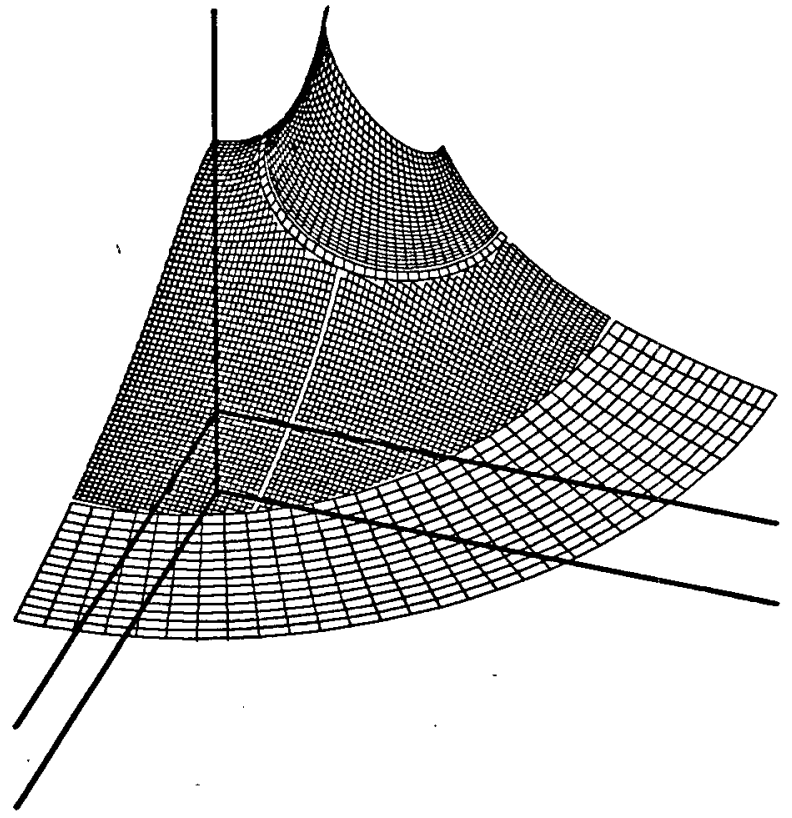

$P=+100$

$\Psi$ is plotted vertically from 0 to $6, \rho$ forward from 0 to $4.9, z$ to the right from 0 to 4.9. The lower set of $(\rho, z)$ axes are at $\Psi=0$, the upper set at $\Psi=1$. The black holes are approaching one another. The $P=+0.01$ and $P=+0.1$ models are visually indistinguishable from the $P=0$ one at this scale, and are hence not plotted. 
Figure 11 - PP Test Series Solntions $(P \leq 0)$

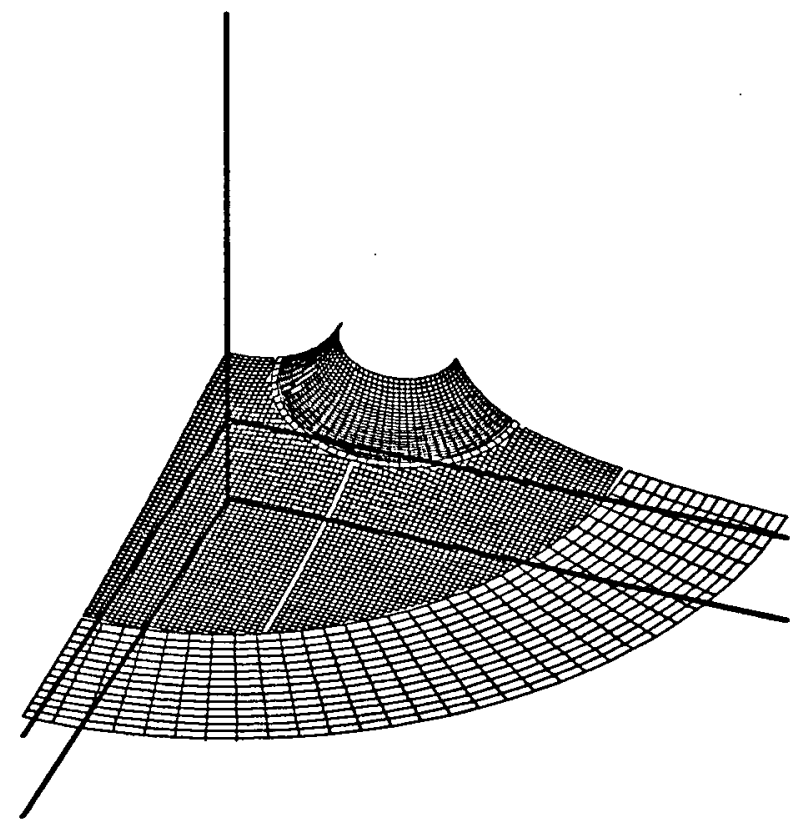

$P=0$

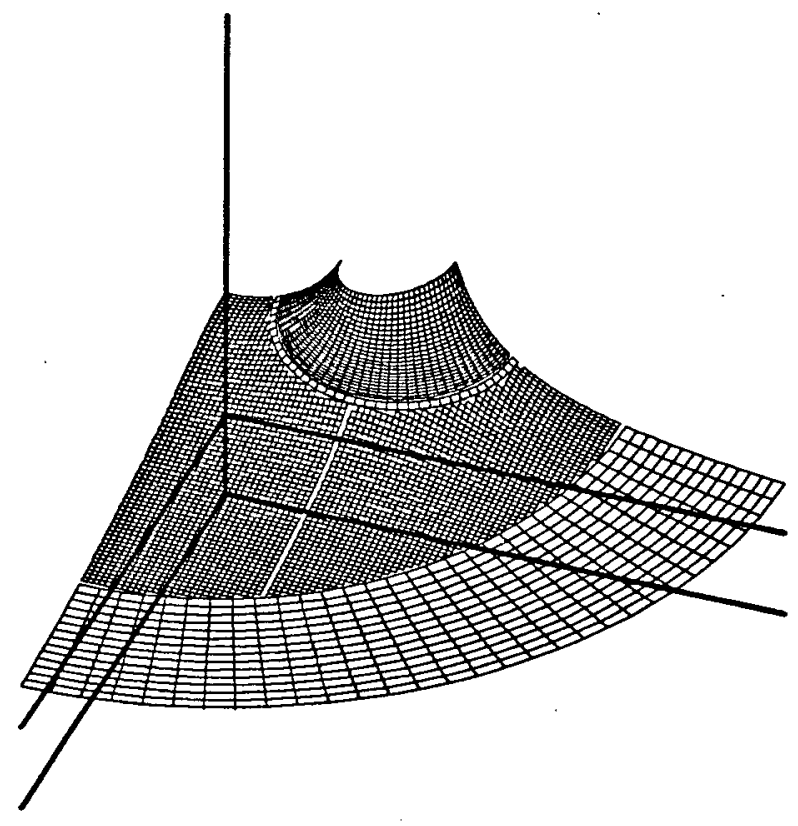

$P=-10$

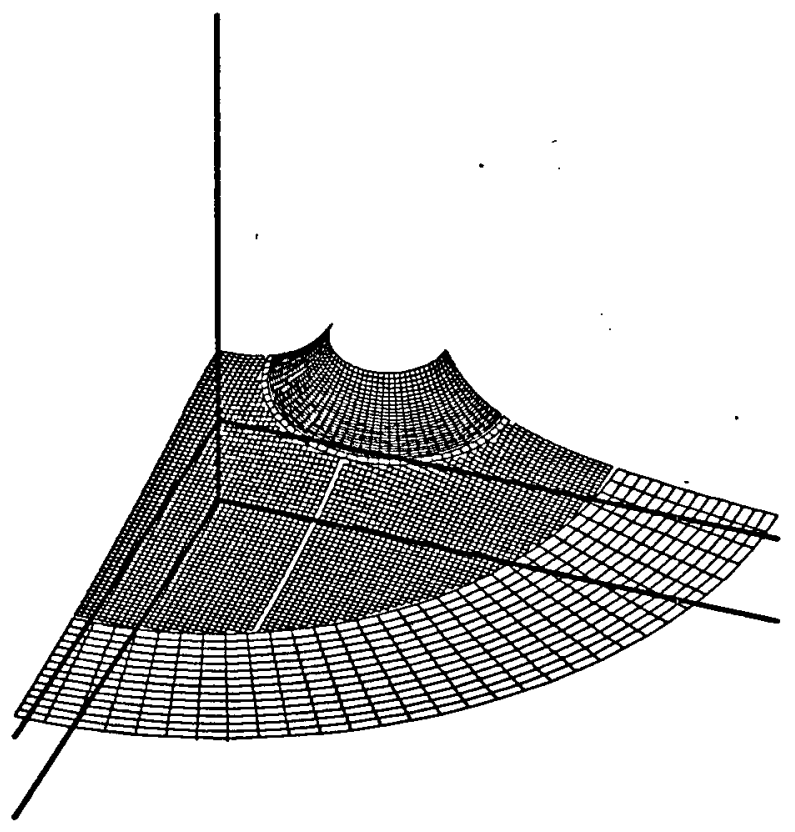

$P=-1$

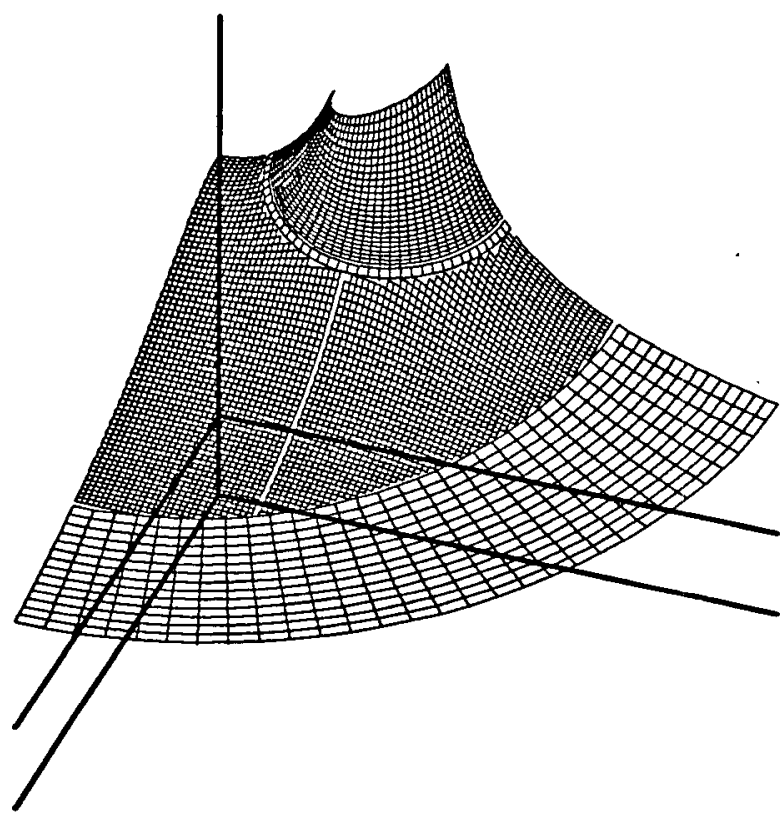

$P=-100$

$\Psi$ is plotted vertically from 0 to $6, \rho$ forward from 0 to $4.9, z$ to the right from 0 to 4.9. The lower set of $(\rho, z)$ axes are at $\Psi=0$, the upper set at $\Psi=1$. The black holes are receding from one another. The $P=-0.01$ and $P=-0.1$ models are visually indistinguishable from the $P=0$ one at this scale, and are hence not plotted. 


\section{Conclusions and Directions for Further Research}

The two main innovations of this thesis (multiple coordinate patches and the marginally trapped surface boundary condition) both proved highly successful. Together, they should allow the Bowen and York formalism for the initial data problem to be used in a wide range of physical situations.

The difficulty of implementing the marginally trapped surface boundary condition seems to be no greater than that of the reflection symmetric one. The former generalizes easily to spaces containing multiple black holes, without the infinite series of "image charges" the latter seems to require. The marginally trapped surface condition also possesses a straightforward physical interpretation. It does, however, place a restriction on one's freedom to choose spatial coordinates, both on the initial slice and on future slices in a time evolution problem.

There is another possible problem with using the marginally trapped surface condition in a time evolution GR code. In order to realise the advantages mentioned above, each marginally trapped surface used as an inner grid boundary should be the outermost such one surrounding its black hole. If the spatial geometry of the slices changes smoothly, this isn't difficult to ensure. However, if a black hole suddenly gains some mass, or (say) two black holes merge, the outer marginally trapped surface will discontinuously move outwards, and the code may well "loose track" of it. A possible solution to this problem is to search the space for further marginally trapped surfaces each time step. This has not yet been done, but should pose no major difficulty. However, the cost of doing this search at each time step may prove prohibitive. Further investigation of this problem would be useful. 
Multiple coordinate patches seem to work very well in providing spatial coordinates for spaces containing multiple black holes. At least for the initial data problem, the required interpolation across the interpatch boundaries is easy to implement and does not significantly degrade the accuracy of the numerical solution. These conclusions should extend to other elliptic PDE problems involving topologies which do not admit simple "analytic" coordinate systems.

However, if multiple coordinate patches are to be used for parabolic or hyperbolic PDE problems (such as time evolving GR initial data), further investigation is needed. The potential problems arise from the errors induced by the interpatch interpolationthey could introduce either instabilities at the interpatch boundaries, or spurious waves which will then propagate forward in time. Since the interpolation errors can be (and are, in this thesis) made of the same order as those incurred in finite differencing the PDE itself, these difficulties should be soluble, but the means needed have yet to be studied.

This thesis also used multiple coordinate patches as a means of spatially varying the grid resolution. This should probably be done automatically by an adaptive mechanism. Choptuik (1982) found such a scheme to be very effective in placing extra grid points just where they were needed, but his code only did adaptive gridding in one space dimension-the grid size in the other dimension was fixed. In multiple black hole systems, a full $n$-dimensional adaptive gridding system is probably needed.

The use of $\Omega$ to linearize the boundary conditions proved rather awkward. Given the desire to do some linearizing transformation, it seems that doing so with the domain (via Choptuik (1982)'s $s$ coordinate or some similar device) is preferable to linearizing in the range (with $\Omega$ ). The former can, if properly chosen, linearize a number of 
different functions at once (useful for vector problems), and places no restrictions on the values of the functions being linearized.

The use of a global Newton method for solving the finite difference equations seems to be useful for "testbed" type codes such as the one constructed in this thesis. It offers reliable convergence, but the cost in run time and memory consumption probably precludes its use in a "production" code.

Like previous studies, this thesis found surface integral total energy formulas somewhat inaccurate. Volume integrals should be better, but means to do volume integrals in the presence of multiple coordinate patches (with gaps in between them) have yet to be developed.

Any means of relaxing either of the two main assumptions (maximal slicing and 3conformal flatness) of this thesis would be of considerable significance. If $\partial_{i} \operatorname{Tr}(K) \neq 0$, the Bowen and York formalism requires the solution of a vector elliptic equation, rather than the scalar one solved herein. An investigation of the difficulty of this would be very useful. Given that the vector equation is soluble, the 3-conformal flatness assumption could probably also be dropped; further investigation of this possibility is certainly warranted.

The new coordinates and boundary, conditions could also be applied to the time evolution of these spaces, as well as to a wide variety of other problems. In particular, the work of Smarr (1975), Eppley (1975), and Cadez (1971) on the head-on collision of two black holes could be replicated and extended. Studies of the collision of spinning black holes would be of special interest, since they should show the effects of the GR spin-spin interaction. It would also be of interest to see if the amount of radiation 
emitted in such collisions could be increased by "throwing" the two black holes together with initial linear momentum.

In the more distant future, the decay and subsequent merger of black hole binary systems would be of great interest, as would be a study of black hole-black hole scattering in general. Such studies (by numerical GR means) are probably some years away, since they require time evolution of 3 (space) dimensional data. Even assuming the required computational capability to be available (which it almost is today, and soon will be), the techniques involved in constructing suitable numerical GR codes are not yet fully understood. 


\section{References}

Arnowitt, R., Deser, S., and Misner, C. W. (1962): "The Dynamics of General Relativity", pp. 227-265 in Witten (1962).

Bowen, J. M., Rauber, J., and York, J. W. (1984): “Two Black Holes with Axisymmetric Parallel Spins: Initial Data", Classical and Quantum Gravity 1, 591-610.

Bowen, J. M., and York, J. W. (1980): “Time-Asymmetric Initial Data for Black Holes and Black Hole Collisions", Physical Review D 21(8), 2047-2056.

Čadež, A (1971): “Colliding Black Holes”, University of North Carolina at Chapel Hill Ph.D. Thesis.

Cadež, A (1974): “Apparent Horizons in the Two-Black-Hole Problem", Annals of Physics 83, 449-457.

Choptuik, M. (1982): "A Study of Numerical Techniques for the Initial Value Problem of General Relativity”, University of British Columbia M.Sc. Thesis.

Choptuik, M. (1985a): Personal communication.

Choptuik, M. (1985b): Personal communication.

Choptuik, M. (1985c): Personal communication.

Choptuik, M. (1985d): Personal communication.

Deruelle, N., and Piran, T., eds. (1983): "Rayonnement Gravitationnel", NorthHolland, Amsterdam.

Eppley, K. R. (1975): “The Numerical Evolution of the Collision of Two Black Holes”, Princeton University Ph.D. Thesis.

Eppley, K. R. (1977): "Evolution of Time-Symmetric Gravitational Waves: Initial Data and Apparent Horizons”, Physical Review D 16(6), 1609-1614.

Kernighan, B. W., and Ritchie, D. M. (1978): “The C Programming Language”, Prentice-Hall, Englewood Cliffs. 
Kernshaw, D. S. (1978): “The Incomplete Cholesky-Conjugate Gradient Method for the Iterative Solution of Systems of Linear Equations", Journal of Computational Physics 26, 43-65.

Kulkarni, A. D. (1984a): "Time-Asymmetric Initial Data for the $N$ Black Hole problem in General Relativity", Journal of Mathematical Physics 25(4), 10281034.

Kulkarni, A. D. (1984b): "Extrinsic Curvature for the Two-Black-Hole Problem", Preprint.

Kulkarni, A. D., Shepley, L. C., and York, J. W. (1983): "Initial Data for $N$ Black Holes", Physics Letters 96A(5), 228-230.

Matzner, R. A., and Shepley, L. C. (1982): "Spacetime and Geometry: The Alfred Schild Lectures", University of Texas Press, Austin.

Misner, C. W., Thorne, K. S., and Wheeler, J. A. (1973): “Gravitation”, W. H. Freeman and Company, San Francisco.

Mitchell, A. R., and Griffiths, D. F. (1980): "The Finite Difference Method In Partial Differential Equations”, Wiley, Chichester.

MTW: Alternate citation for Misner, Thorne, and Wheeler (1973).

Ó Murchadha, N., and Seifert, H. J. (1982): personal communication.

Price, R. H. (1982): "General Relativity Primer", American Journal of Physics 50(4), 300-329; see also Tryon (1984) for errata.

Smarr, L. L. (1975): “The Structure of General Relativity With A Numerical Illustration: The Collision of Two Black Holes", University of Texas at Austin Ph.D. Thesis.

Smarr, L. L., ed. (1979): "Sources of Gravitational Radiation", Cambridge University Press, Cambridge.

Tryon, E. P. (1984): "Comment on 'General Relativity Primer', American Journal of Physics 52(4), 366-367.

Wald, R. M. (1984): “General Relativity”, University of Chicago Press, Chicago. 
Witten, L., ed. (1962): “Gravitation-An Introduction to Current Research", Wiley, New York.

York; J. W. (1979): “Kinematics and Dynamics of General Relativity”, pp. 83-126 in Smarr (1979).

York, J. W. (1983): “The Initial Value Problem and Dynamics", pp. 175-201 in Deruelle and Piran (1983).

York, J. W. (1984): “Initial Data for $N$ Black Holes”, Physica 124A, 629-638.

York, J. W., and Piran, T. (1982): "The Initial Value Problem and Beyond", pp. 147-176 in Matzner and Shepley (1982). 


\section{Appendix 1}

This appendix contains listings of the input parameter files for each model discussed in this thesis. As described in the header file patchut1l:patch.h (listed in appendix 2), the input parameters to a model modelname reside in two files, a modelname.par file describing the physical situation being modeled, and a gridname pat file describing the patch system being used. The latter is typically shared between a number of models.

In addition, this appendix also contains quantitative graphs of both $\Psi$ and $\delta \Psi$ as functions of $s$ for all the non-Schwarzschild single black hole models, and tables of selected values of $\Psi$ for the Schwarzschild and double black hole models. These should prove useful to the reader desiring specific numeric values to check against some other GR code's results.

The modelname.par files typically end with a sequence of comment lines (anything after a semicolon is a comment), which log all programs that modified the modelname.gfn data file.

The next 5 pages give indices to the listings and graphs/tables, giving the page numbers on which each model's information starts. The listings and graphs/tables themselves follow. When all the models of a test series share a common .pat file (as they do for all but the Schwarzschild models), this is listed after all the other information for that series. 
Table 5 - Model index for the Schwarmschild test series

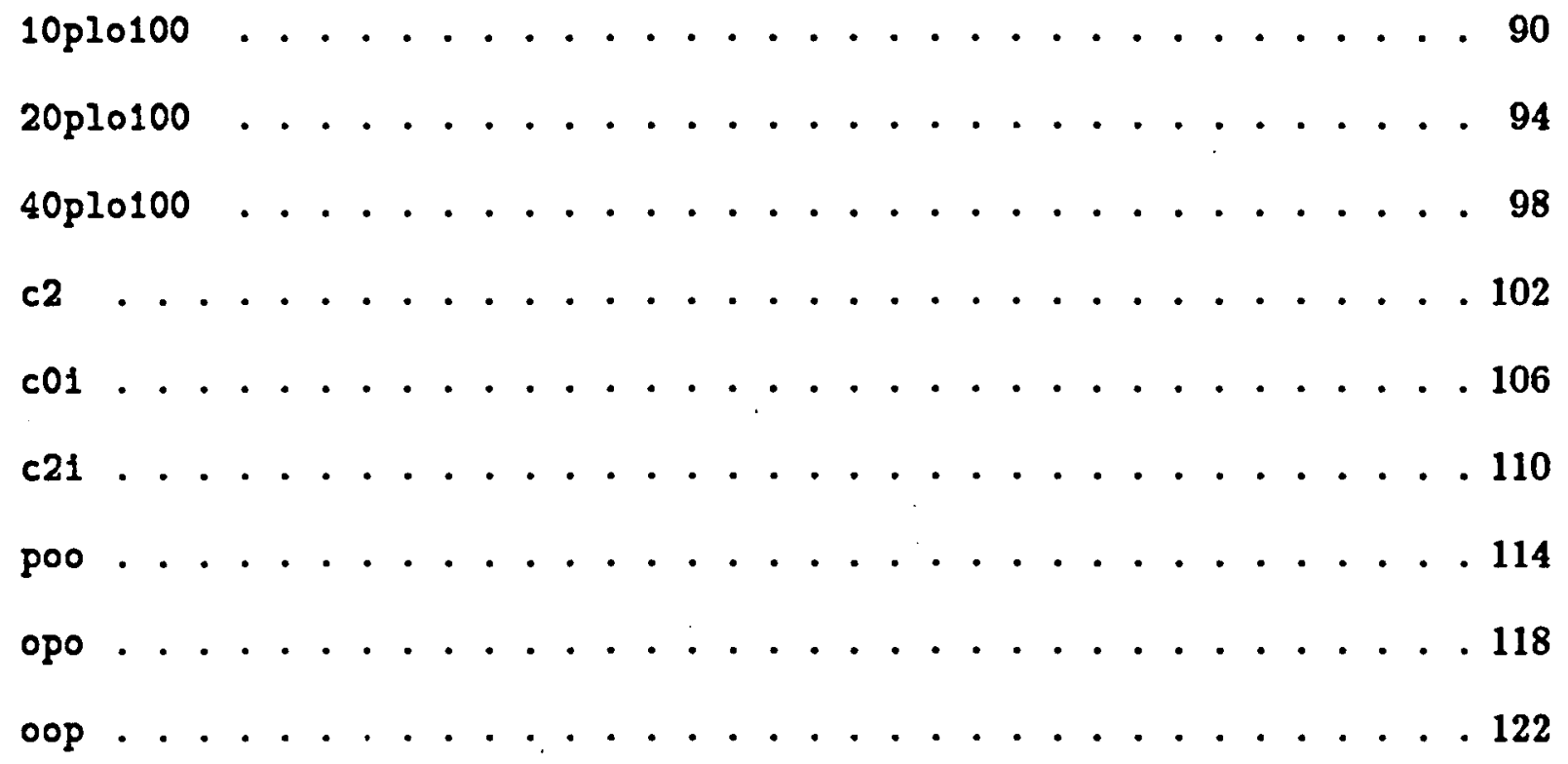


Table 6 - Model index for the Choptuik comparison test geries

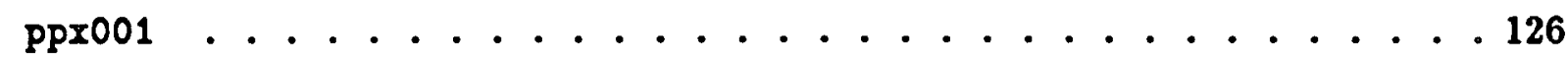

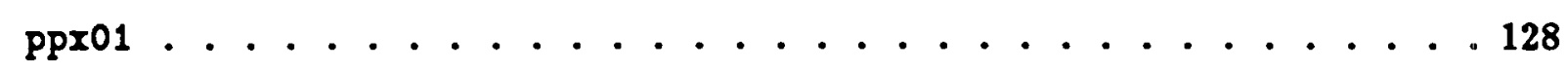
$\operatorname{ppr1} \ldots \ldots . \ldots 130$ $\mathrm{pp1} \ldots \ldots . . \ldots 132$

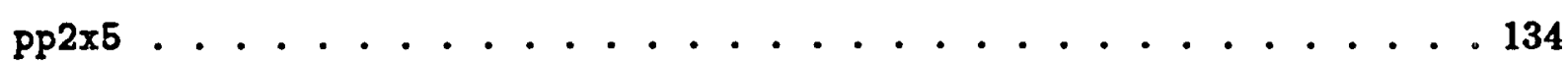
$\mathrm{pp7x5} \ldots . . \ldots 136$

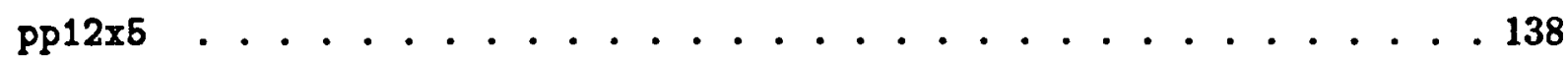

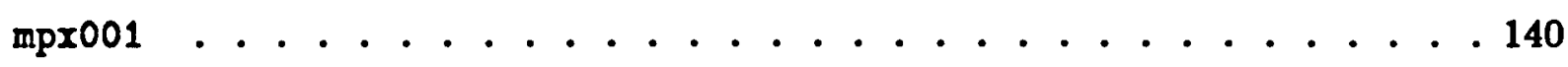
$\operatorname{mpx01} \ldots \ldots 142$ $\operatorname{mpx1} \ldots \ldots 144$ $\operatorname{mp} 1 \ldots \ldots . \ldots 146$ $\operatorname{mp} 2 \times 5 \ldots \ldots 148$ $\operatorname{mp7x5} \ldots \ldots . . \ldots \ldots$

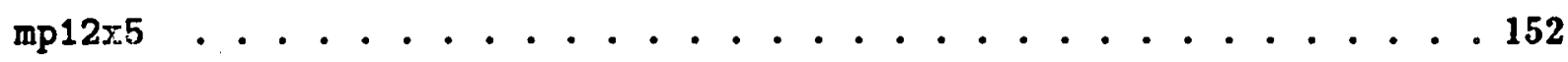

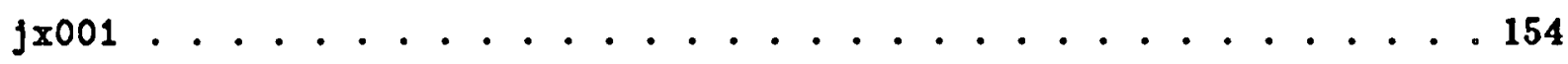

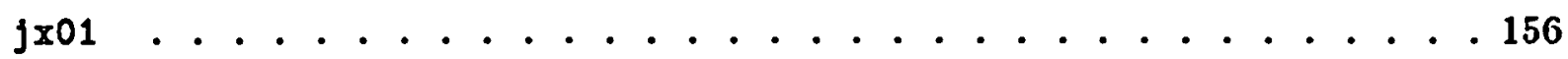

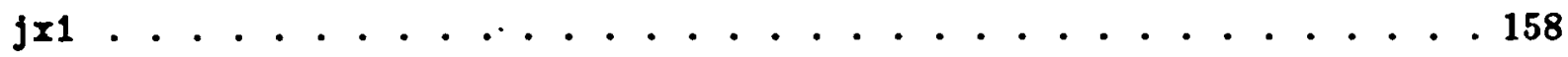

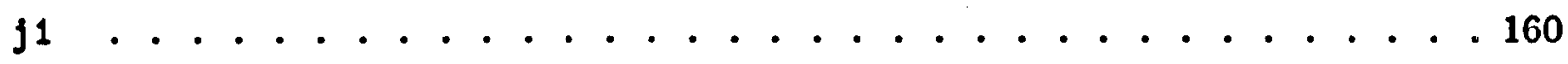

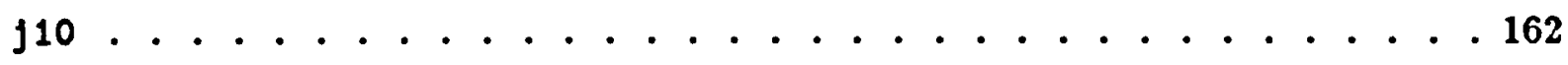

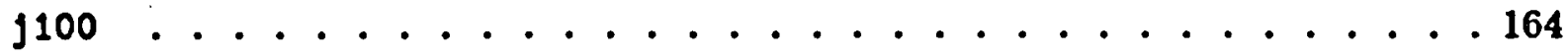

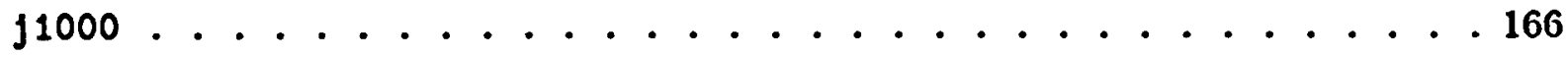

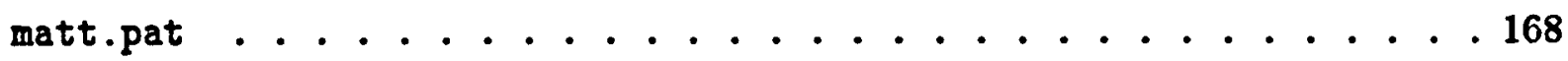


Table 7 - Model index for the J test series

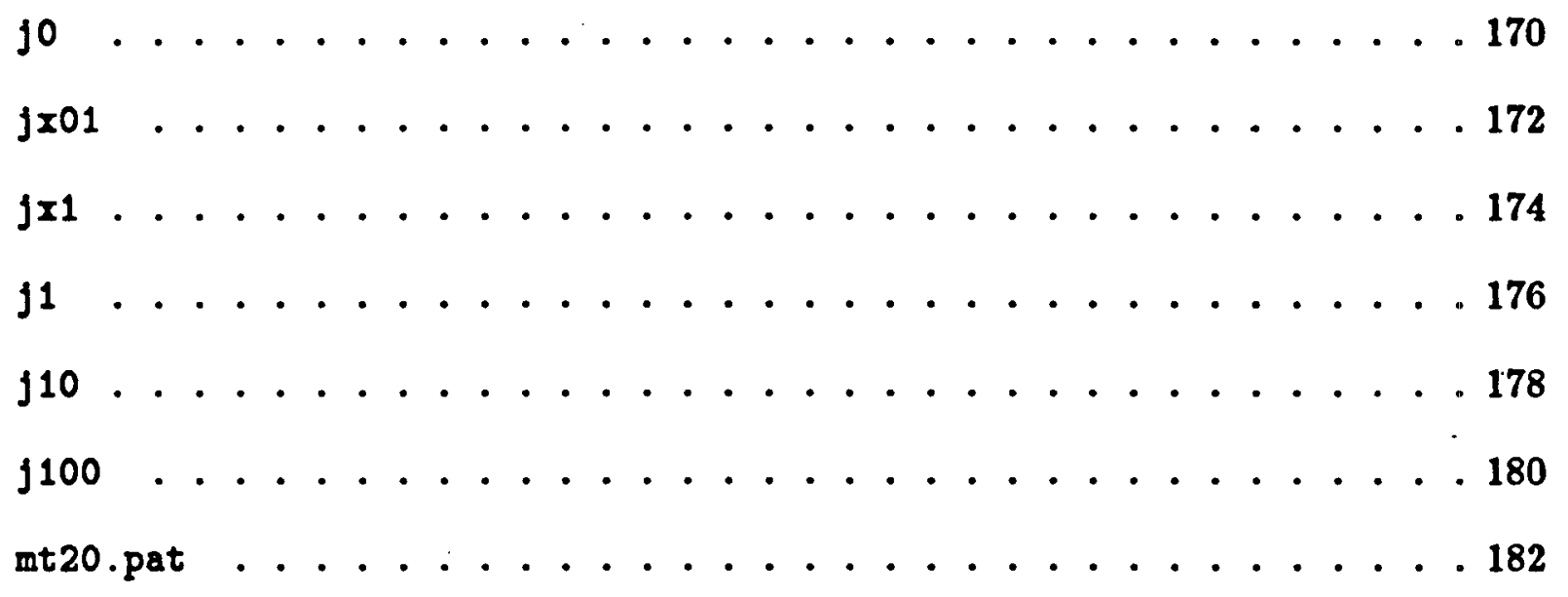

Table 8 - Model index for the RSP test series

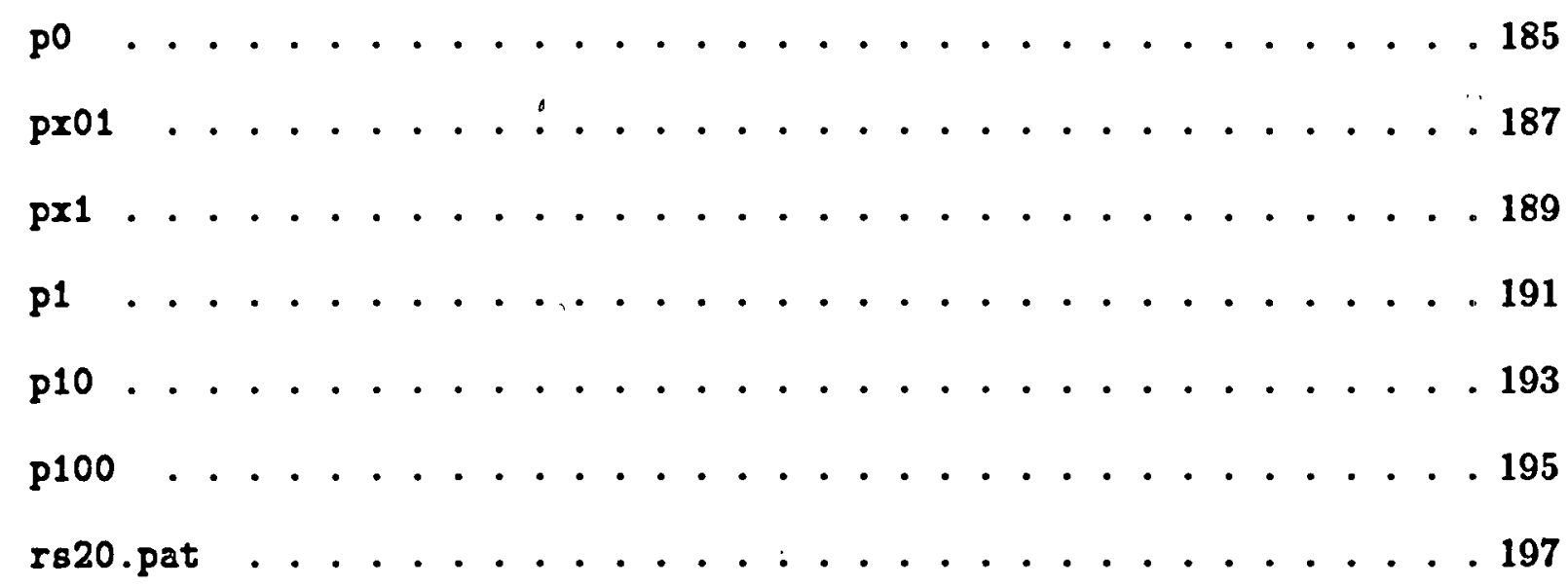


Table 9 - Model index for the RSM test series

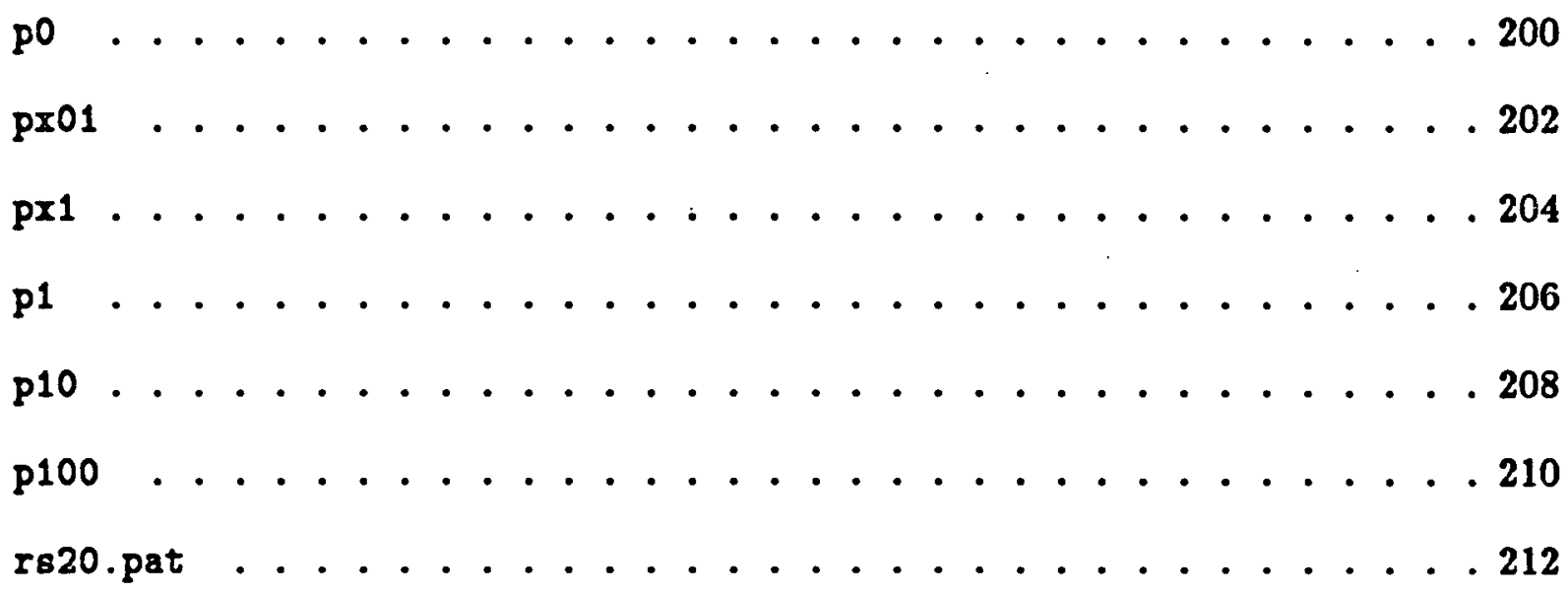

Table 10 - Model index for the MTO test series

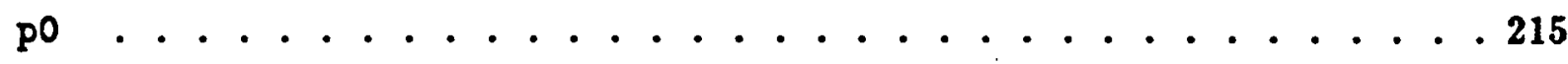

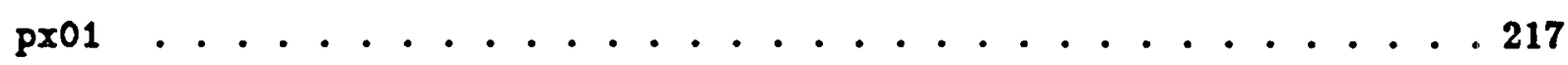
px1 .............................. 219 p1 ................................... 221 p10 . . . . . . . . . . . . . . . . . . . . . . . . . . . 223 p100 . . . . . . . . . . . . . . . . . . . . . . . . 225 mt20big.pat . . . . . . . . . . . . . . . . . . . . 227 
Table 11 - Model index for the PP test series

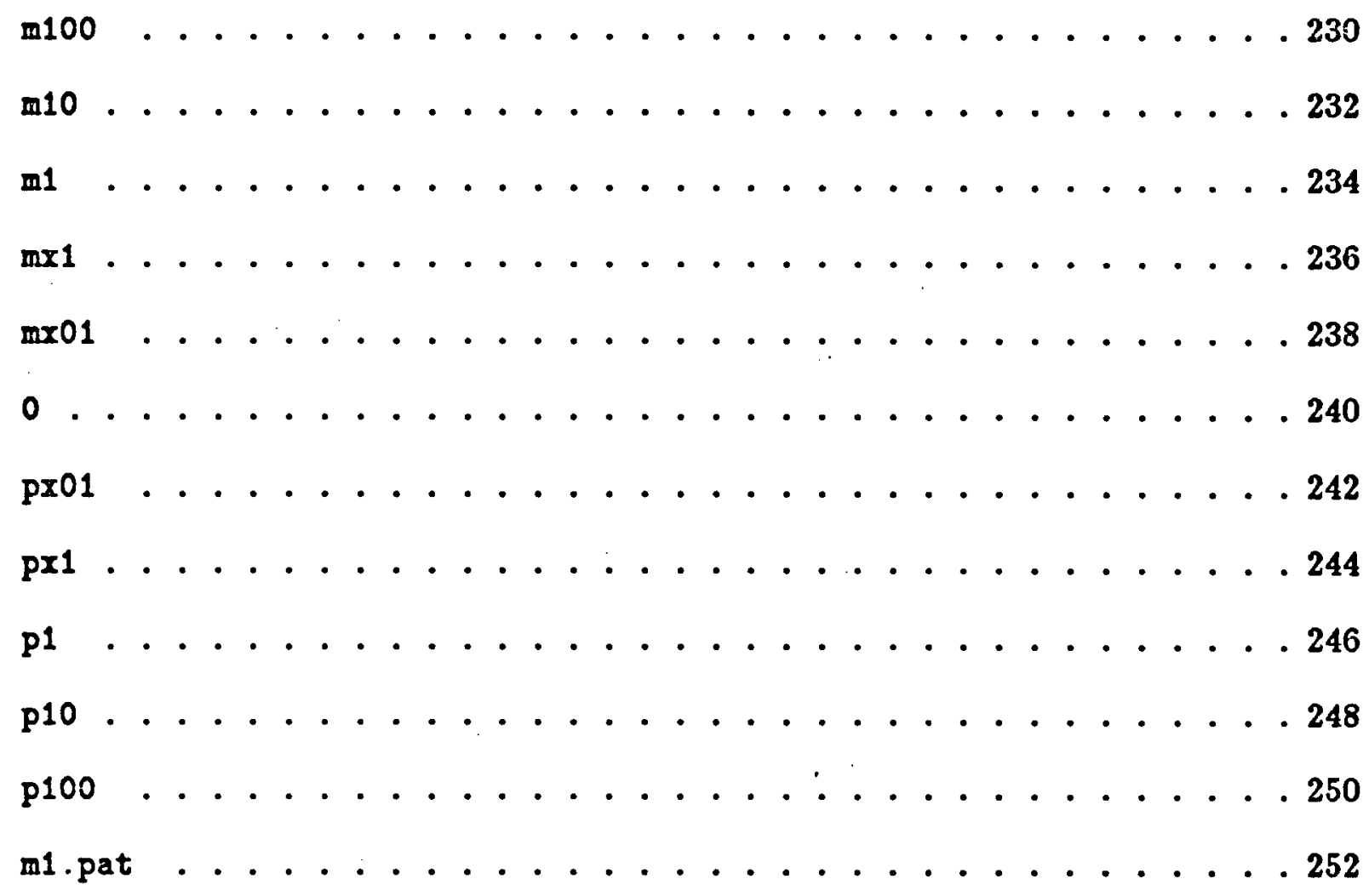


Schwarzschild test geries

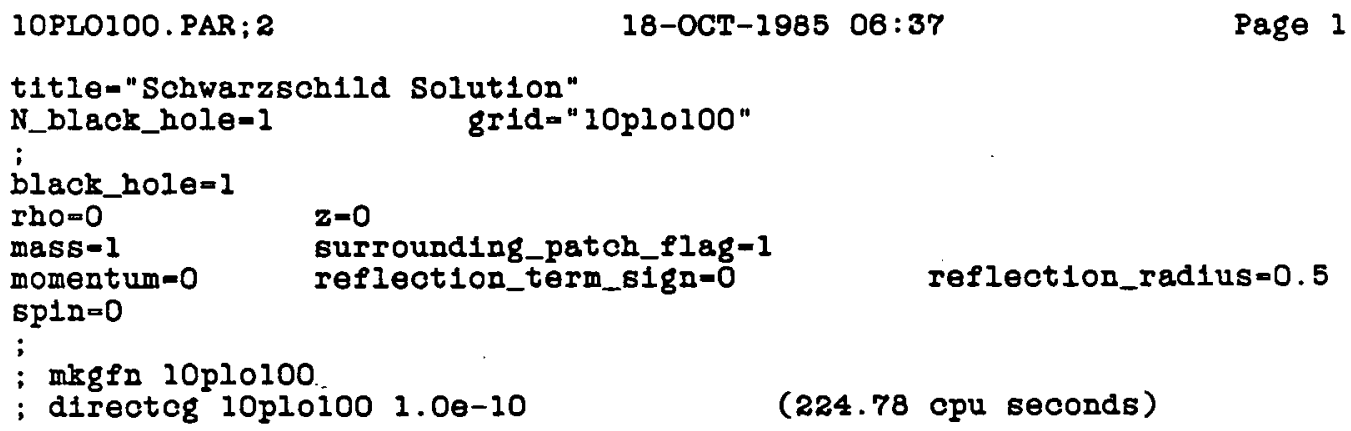




\section{Schwarzschild test series}

\section{PLO100. PAT; 3}

Bach patch approximately $10 \times 10$

All patches polar spherical

All patches using locally conservative differenoing

All patches using omega for conformal funotion

Outer boundary at $r=100$

N_patch=7

; Now describe esch coordinate patch:

patch-1

coord_type="polar spherical"

difference_scheme-"Iocally conservative"

conformal_fn_type-"omega(ps1)"

rho_center $=0$ z_center $=0$

I_m1n="constant $=0.50$ "

$r$ max $=$ "constant $=0.95$ "

theta_m1n="constant $=0 "$

theta_max=" constant $=90$ "

;

patch $=2$

coord_type-"polar spher1cal"

difference_scheme-"10cally conservat1ve"

conformal_fn_type="omega(psi)"

Tho_center $=0$ z_center $=0$

r_min="constant $=1.0$ "

I_max = " constant $=1.9$ "

theta_min="constant $=0$ "

theta_max=" constant $=90$ "

delta_r=0.05 delta_theta $=10$

bndry_cond-"Reflection Symmetr1c"

brdry_cond - "patch=2"

bndry_cond = "Feumann"

bndry_cond= "Neumann"

patch $=3$

coord_type-"polar spherical"

differenoe_scheme-"10cally conservat1ve"

conformal_fn_type-"omega(psi)"

rho_center-0 z_center=0

I_min-" constant $=2.0$ "

I_max=" constant $=4.8$ "

theta_minm" constant $=0$ "

theta_max=" constant $=90$ "

delta_r-0.1 delta_theta=10

bndry_cond=" patch-I"

bndry_cond = "patch=3"

bndry_cond - "Neumann"

bndry_cond= "Neumann"

delta_r=0.2 delta_theta-10

bndry_cond = "patch $=2$ "

bndry_cond-"patch $=4$ "

bndry_cond- "Neumann"

bndry_cond - "Neumann"

:

patch $=4$

coord_type="polar spherical"

difference_scheme-"1ocally conservattve"

conformal_fn_type-"omega(psi)"

rho_center $=0$ z_center-0

$r \_m i n="$ constant $=5.0$ "

I_max=" constant $=9$. B $^{\prime}$

theta_min=" constant $=0$ "

theta_max $=$ "constant $=90$ "

;

patch $=5$

coord_type-"polar spherical"

difference_scheme- "Iocally conservative"

conformal_fn_type="omega(psi)"

rho_center=0 z_center=0 delta_r-1

delta_theta-15

delta_r-0.8 delta_theta-10 bndry_cond= "patch-3" bndry_cond-" patch $=5$ " bndry_cond - "Tieumann" bndry_cond- "Neumann" 


\section{Schwarzschild test series}

10PLOI0O. PAT; 3

I_m1n=" constant -10 "

$r$ max $=$ " constant $=19^{\circ}$

theta_m1n="constant-0"

theta_max="constant=90"

;

patch-6

coord_type="polar spher1cal"

difference_scheme-"locally conservative"

conformal_fn_type="omega(psi)"

rho_center $=0$ z_center $=0$

I_mIn="constant $=20$ "

$I$ max = "constant -48 "

theta_min="constant $=0$ "

theta_max="constant $=90$ "

;

patch-7

coord_type="polar spher10al"

difference_scheme="locally conservative"

conformal_fn_type-"omega(ps1)"

rho_center $=0$ z_center $=0$

r_min="constant $=50 "$

I_max= "constant $=100 "$

theta_m1n $=$ "constant $=0 "$

theta_max-"constant $=90 "$
18-OCT-1985 06:37

Page 2

bndry_cond - "patch $=4$ " bndry_cond = "patch $=6$ "

bndry_cond = "Neumann"

bndry_cond = "Neumann"

delta_r=2

bndry_cond = "patch $=5$ "

bndry_cond=" patch=7"

bndry_cond=" Neumann"

bndry_cond = "Neumann" delta_r-5

bndry_cond = "patch $=6$ "

bndry_cond = "Robin"

bndry_cond= "Neumann"

bndry_cond= "Neumann" 
Table 12 - Schwarzschild test series, 10pl0100 selected $\Psi$ values

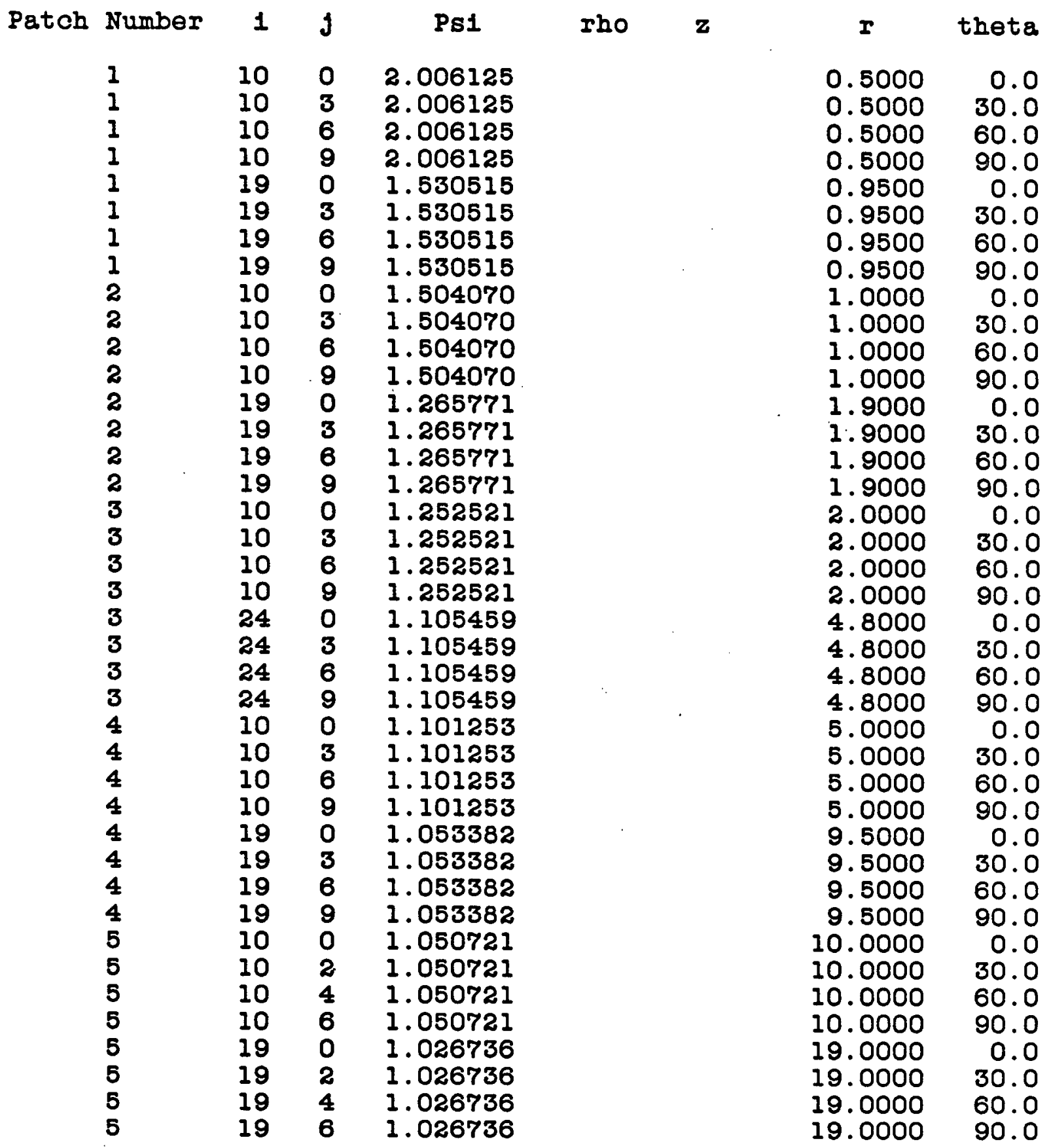

This table gives values of $\Psi$ at selected points in the space. These points are identified by triples (patch number, $i, j$ ), where $(i, j)$ are the generic integer patch coordinates defined in the text. Depending on the patch type, either $(\rho, z)$ or $(r, \theta)$ are also shown. 
Schwarzschild test series

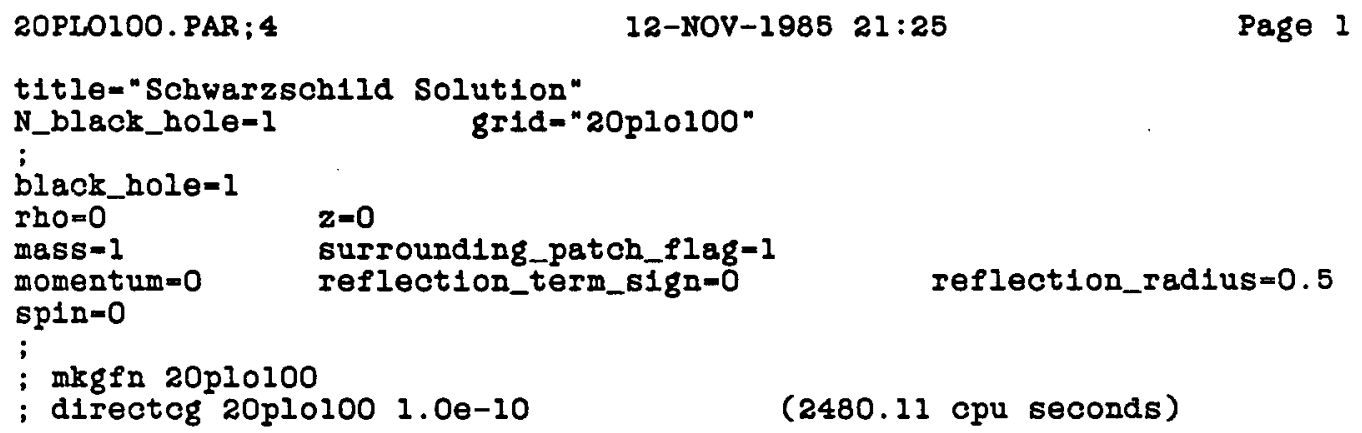




\section{Schwarzschild test series}

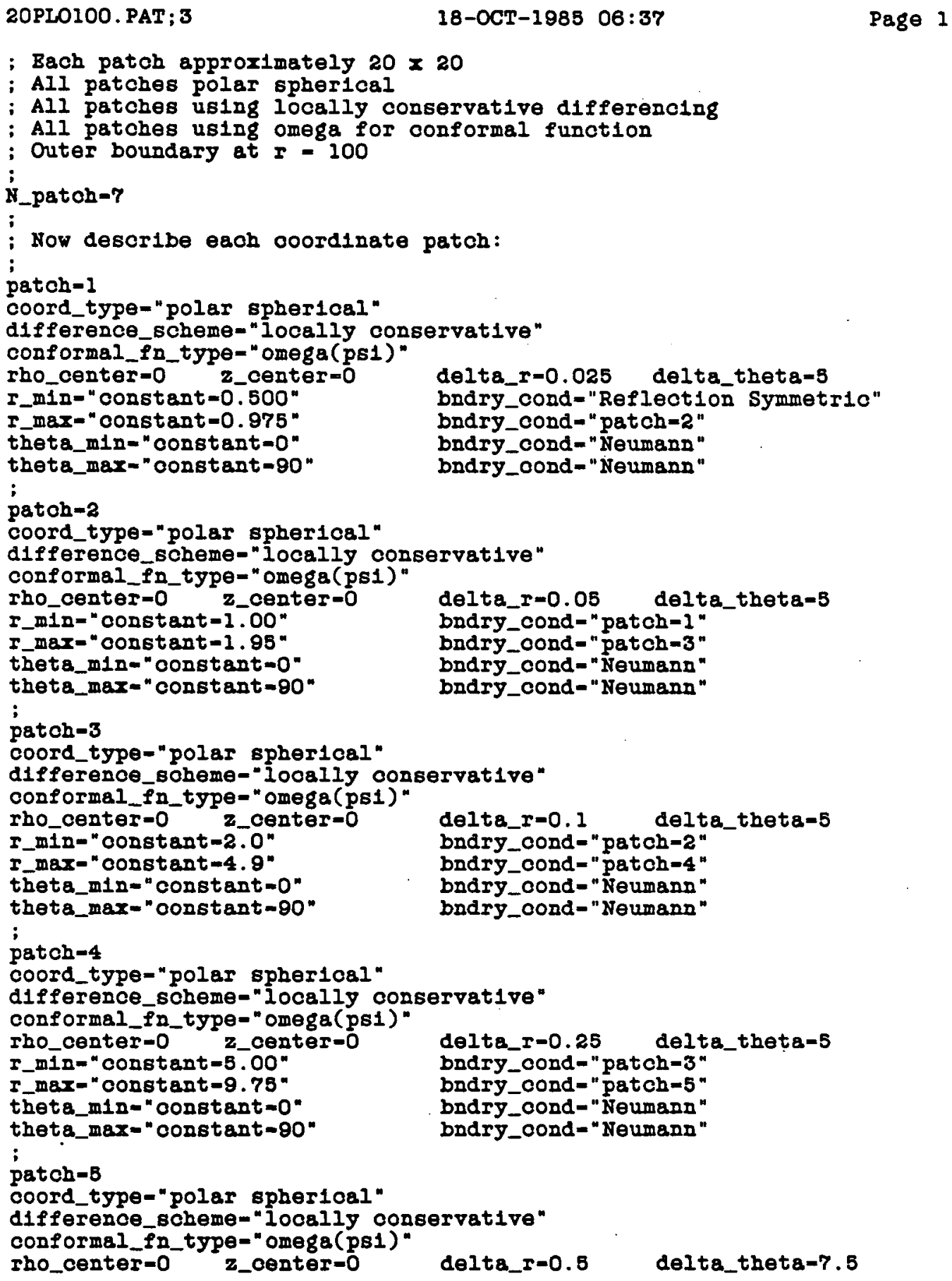




\section{Schwarzschild test series}

2OPLO100. PAT; 3

I_m1n-" constant $=10.0^{n}$

I_max-" constant $=18.5$ "

theta_min="constant $=0$ "

theta_max-"constant $=90$ "

patch $=6$

coord_type="polar spherical"

difference_scheme-" "locally conservat1ve"

conformal_fn_type="omega(psi)"

rho_center $=0$ z_center $=0$

r_m1n-" constant $=20$ "

$r$ max-" constant $=49$ "

theta min=" constant $=0$ "

theta_max="constant $=90$ "

;

patch-7

coord_type-"polar spher1cal"

difference_scheme- "locally conservat1ve"

conformal_fn_type="omega(ps1)"

rho_center $=0$ z_center $=0$

$r$ min="constant $=50$ "

r_max-" constant $=100$ "

theta $m 1 n="$ constant $=0$ "

theta_max $=$ "constant $=90$ "
18-OCT-1985 06:37

Page 2 bary_conde" patch $=4$ "

bndry_cond = "Neumann"

bndry_cond= "Neumenn" delta_r=1

bndry_cond = "patch-5"

bndry_cond = "patch $=7$ "

bndry_cond = "Neumann"

bndry_cond= "Neumann" elta_theta $=7.5$

delta_r=2.5

bndry_cond = "patoh $=6$ "

bndry_cond " Robin"

bndry_conda "Neumann"

bndry_cond = "Neumann" 
Table 13 - Schwarzschild test series, 20plo100 selected $\Psi$ values

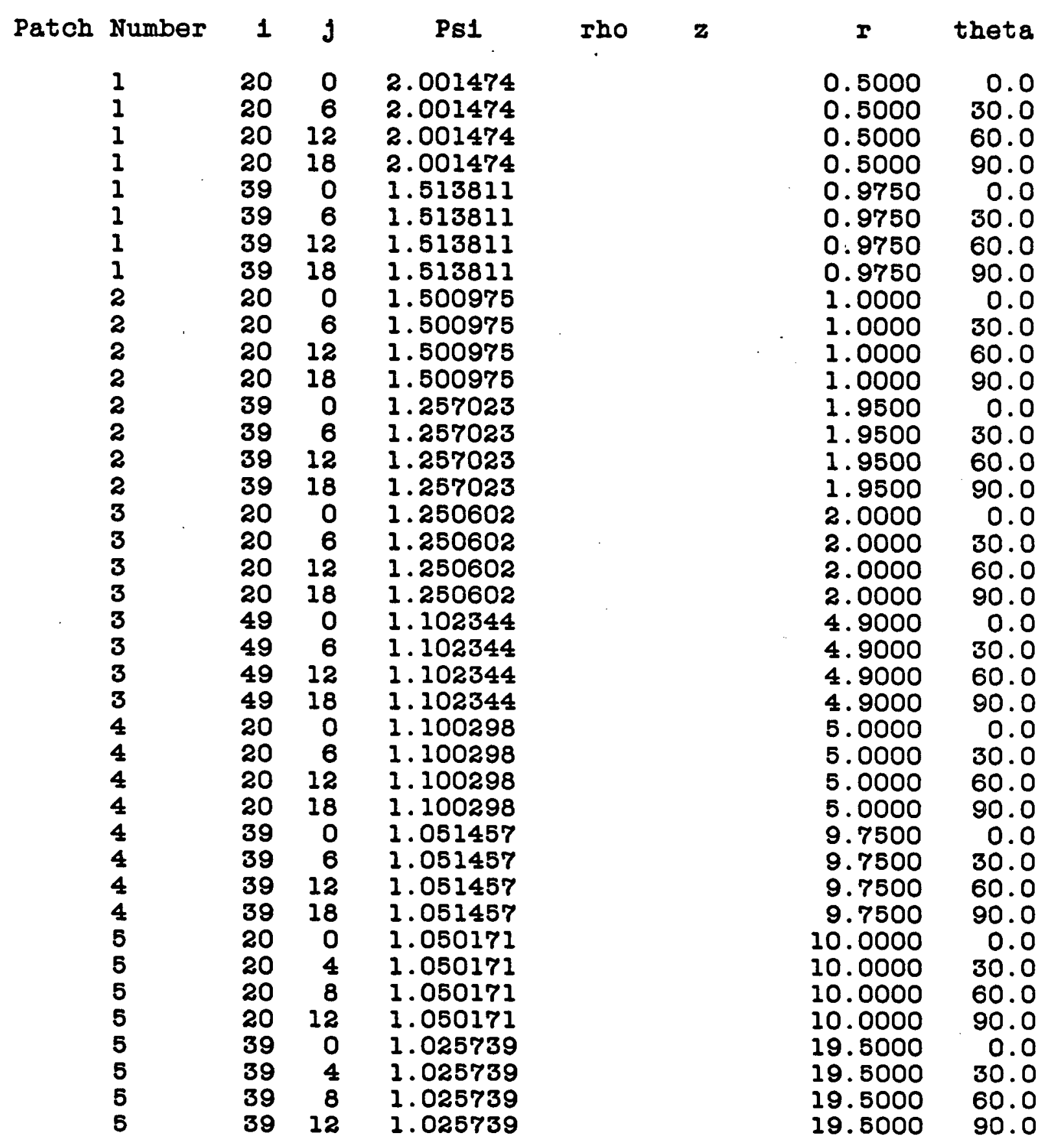

This table gives values of $\Psi$ at selected points in the space. These points are identified by triples (patch number, $i, j$ ), where $(i, j)$ are the generic integer patch coordinates defined in the text. Depending on the patch type, either $(\rho, z)$ or $(r, \theta)$ are also shown. 
Schwarzschild test series

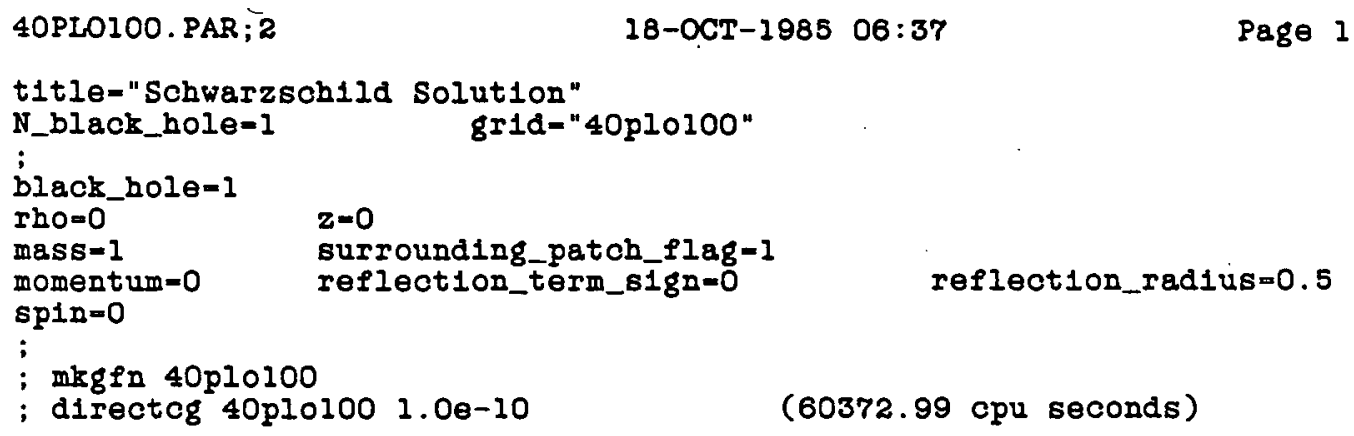




\section{Schwarsschild test series}

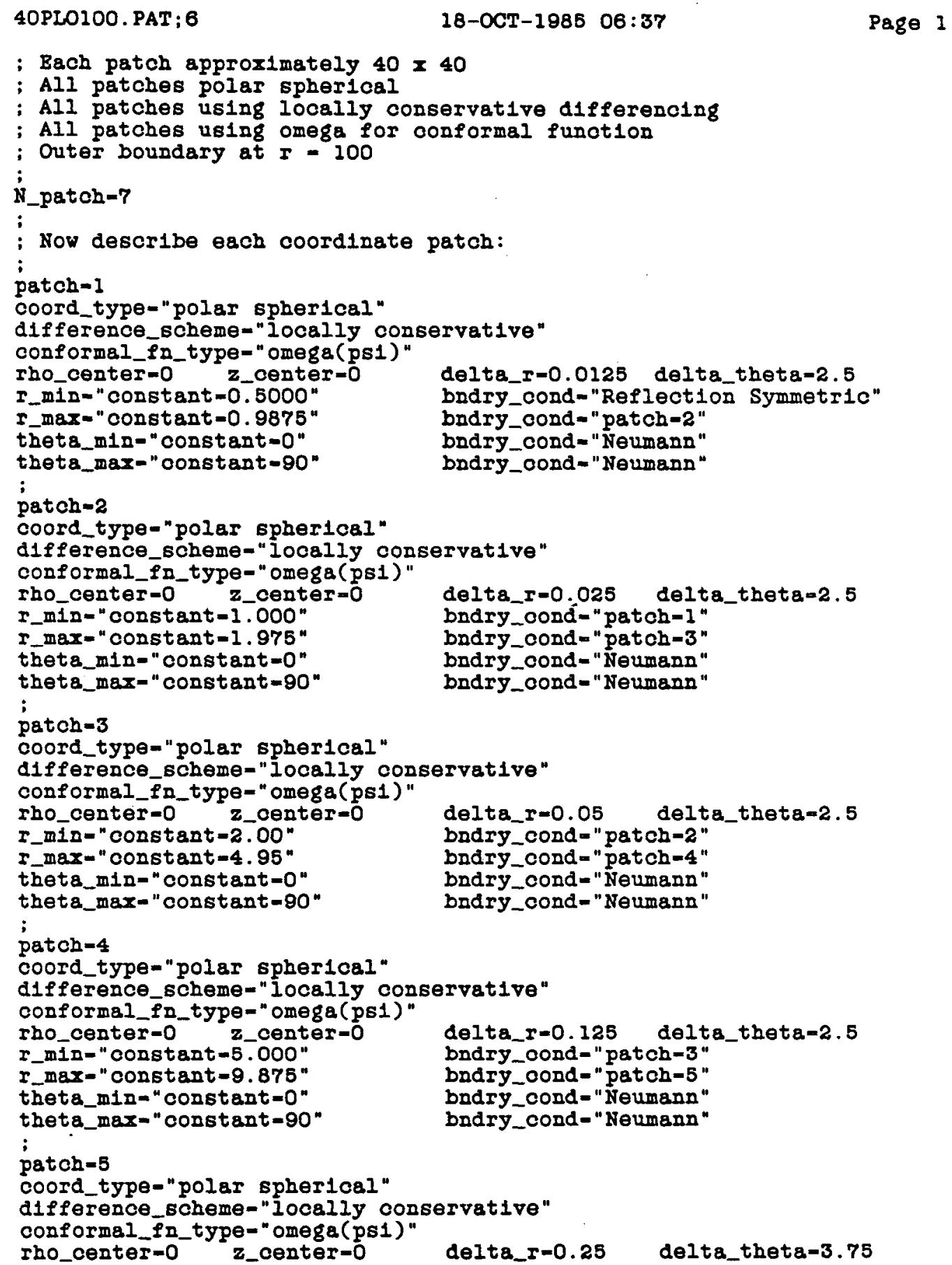


Schwarzschild test series

40PLO100. PAT; 6

I_m1D=" oonstant $=10.00^{\prime \prime}$

$r$ max= "oonstant-19.75"

theta_m1n="constant -0 "

theta_max="constant $=90$ "

;

patch-6

coord_type-"polar spher1cal"

difference_scheme="locally conservat1ve"

conformal_fn_type=" omega(psi)"

rho_center $=0$ z_center $=0$

I_min=" constant $=2 \overline{0}$. O"

I_max $=$ "constant $=49.5$ "

theta_m1n-"constant $=0$ "

theta_max=" constant $=90$ "

;

patch-7

coord_type="polar spher1cal"

difference_scheme="Iocally conservat1ve"

conformal_fn_type-"omega(psi)"

rho_center $=0$ z_center=0

I_mIn=" constant $=50^{\circ}$

$r$ max $=$ " constant $=100$ "

theta_m1n="constant $=0$ "

theta_max-"constant $=90^{\prime \prime}$
18-OCT-1985 06:37

Page 2

bndry_cond = "patch-4"

bndry_cond = "patch $=6$ "

bndry_cond = "Neumann"

bndry_cond " "Neumann" delta_r=0.5 delta_theta $=3.75$

bndry_cond=" patch $=5$ "

bndry_cond = "patch $=7 "$

bndry_cond = "Neumann"

bndry_cond= "Neumann" delta_r=1.25 delta_theta $=3.75$ bndry_cond $=$ "patch $=6$ "

bndry_cond = "Robin"

bndry_cond= "Neumann"

bndry_cond = "Neumann" 
Table 14 - Schwarzschild test series, 40 plo100 selected $\Psi$ values

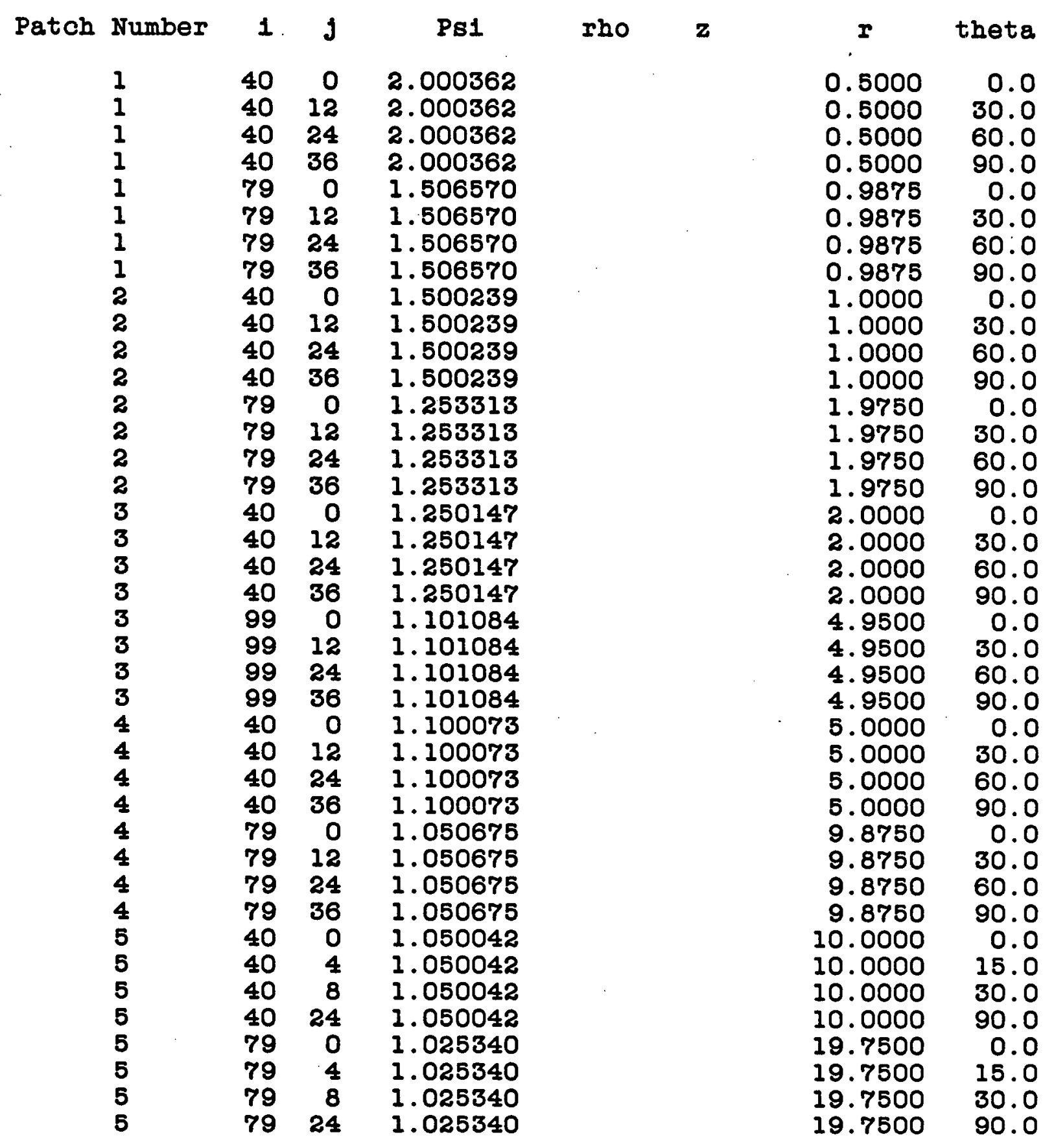

This table gives values of $\Psi$ at selected points in the space. These points are identified by triples (patch number, $i, j$ ), where $(i, j)$ are the generic integer patch coordinates defined in the text. Depending on the patch type, either $(\rho, z)$ or $(r, \theta)$ are also shown. 
Schwarzschild test series

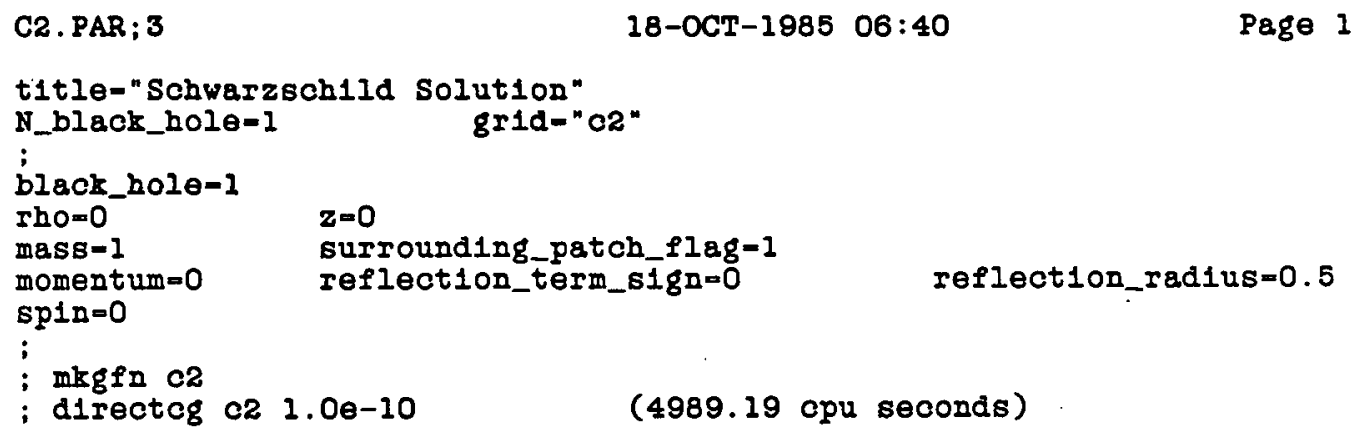




\section{Schwarsschild test series}

; Bach patch approximately $20 \times 20$ All patches polar spherical. except (or1g1n8l) patoh 12 is augmented by - a oylindrical one

All patches using locally conservative differenolng ; All patches using omega for conformal funotion : Outer boundary at $r-100$ : N_patch-8

; Now describe each coordinate patch:

patch-1

coord_type="polar spherical" difference_scheme-" " ocaliy conservat1ve" conformal_fn_type-"omega(ps1)" rho_center $=0$ z_center $=0$ r_min-"oonstant -0.500 " I_max- "constant $=0.975$ " theta_min=" constant $=0 "$ theta_max-" constant $=90$ " delta_I-0.025 delta_theta-5 bndry_cond="Reflect1on Symmetr10" bndry_cond = "patch $=2$ " bndry_cond = "Neumann" ; patch-2 coord_type-"polar spher1cal" difference_scheme- "I0cally conservative" conformal_fn_type="omega(psi)" rho_center $=0 \quad z$ _center $=0$ r_min-" constant $=1.00 "$ r_max= "constant $=1.0$ " $^{\prime}$ theta_min-"constant $=0$ " theta_max=" constant-90" bndry_cond-"Neumann" ;

patch-3

coord_type="cyl1ndr1c8I"

difference_scheme="locally conservat1re" conformal_fn_type="omega(psi)" rho_center $=0$ z_center $=0$ Iho_mIn_bound-0

z_min_bound-0

rho_m1n="patch-2 bndry-r_max"

rho_m1n="constant -0 "

rho_max-"patch-4 bndry-r_min"

z_m1n-"patch=2 bndry-r_max"

z_min-" constant $=0$ "

z_max- "patch=4 budry-r_min"

delta_r-0.05 delta_theta-5

bndry_cond-"patch=1"

bndry_cond = "patch $=3$ "

bndry_cond = "Neumann"

bodry_cond- "Neumann"

patch-4

coord_type-"polar spherical"

difference_scheme-"locally conservat1ve"

conformal_fn_type="omega(psi)"

rho_center $=0$ z_center $=0$

InIn-" constant $=2.0$ "

I_mar-" constant $=4.9$ "

theta_mln-"constant-o"

theta_max="constant $=90$ "

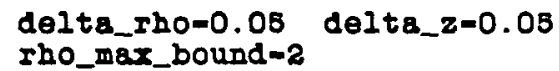

delta_r=0.1 delta_theta-5

bndry_cond = "patch=3"

bodry_cond = "patoh $=8$ "

bndry_cond- "Neumann"

bodry_oond - "Neumen" 


\section{Schwarzschild test series}

patch -5

coord_type="polar spher1cal"

difference_scheme="1ocally conservative"

conformal_fn_type="omega(psi)"

rho_center $=0 \quad$ z_center $=0$

r.min=" constant $=5.00 "$

$r$ max $=$ "constant $=9.75 "$

thets_min="constant $=0$ "

delta_r-0.25 delta_theta $=5$

theta_max-"constant $=90$ "

bndry_cond= "patch $=4$ "

bndry_cond-" "patch-6"

;

badry_cond- "Neumann"

patch $=6$

coord_type-"polar spher1cal"

difference_scheme-"10cally conservative"

conformal_fn_type="omega(psi)"

rho_center $=0$ z_center $=0$

delta_r $=0.5$

badry_cond = "patch $=5$ "

I_m1n=" constant $=10.0$ "

r_max=" constant $=19.5$ "

theta_min="constant $=0$ "

bndry_cond = " patch $=7$ "

theta_mar="constant $=90 "$

bndry_cond = "Neumann"

;

patch $=7$

coord_type="polar spherical"

difference_scheme-"locally conservative"

conformal_fn_type-"omega(psi)"

rho_center $=0$ z_center $=0$

delta_r-1

delta theta $=7.5$

r_min=" constant $=20$ "

$r$ max= "constant $=49$ "

bndry_cond = "patch $=6$ "

theta_min="constant $=0$ "

bndry_cond=" patch=8"

theta_max= " constant $=90$ "

bndry_cond = "Neumann"

;

patch-8

coord_type="polar spher1cal"

difference scheme=" Iocally conservat1ve"

conformal_fn_type="omega(psi)"

rho_center $=0$ z_center $=0$

$r$ mIn="constant $=50$ "

I_max="constant $=100$ "

theta_m1n="constant $=0$ "

theta_max="constant-90"

delta_I-2.5 delta_theta-7.5

bndry_cond= "patch=7"

bndry_cond="Robin"

bndry_cond= "Neumann"

bndry_cond- "Neumann" 
Table 15 - Schwarzschild test series, c2 selected $\Psi$ values

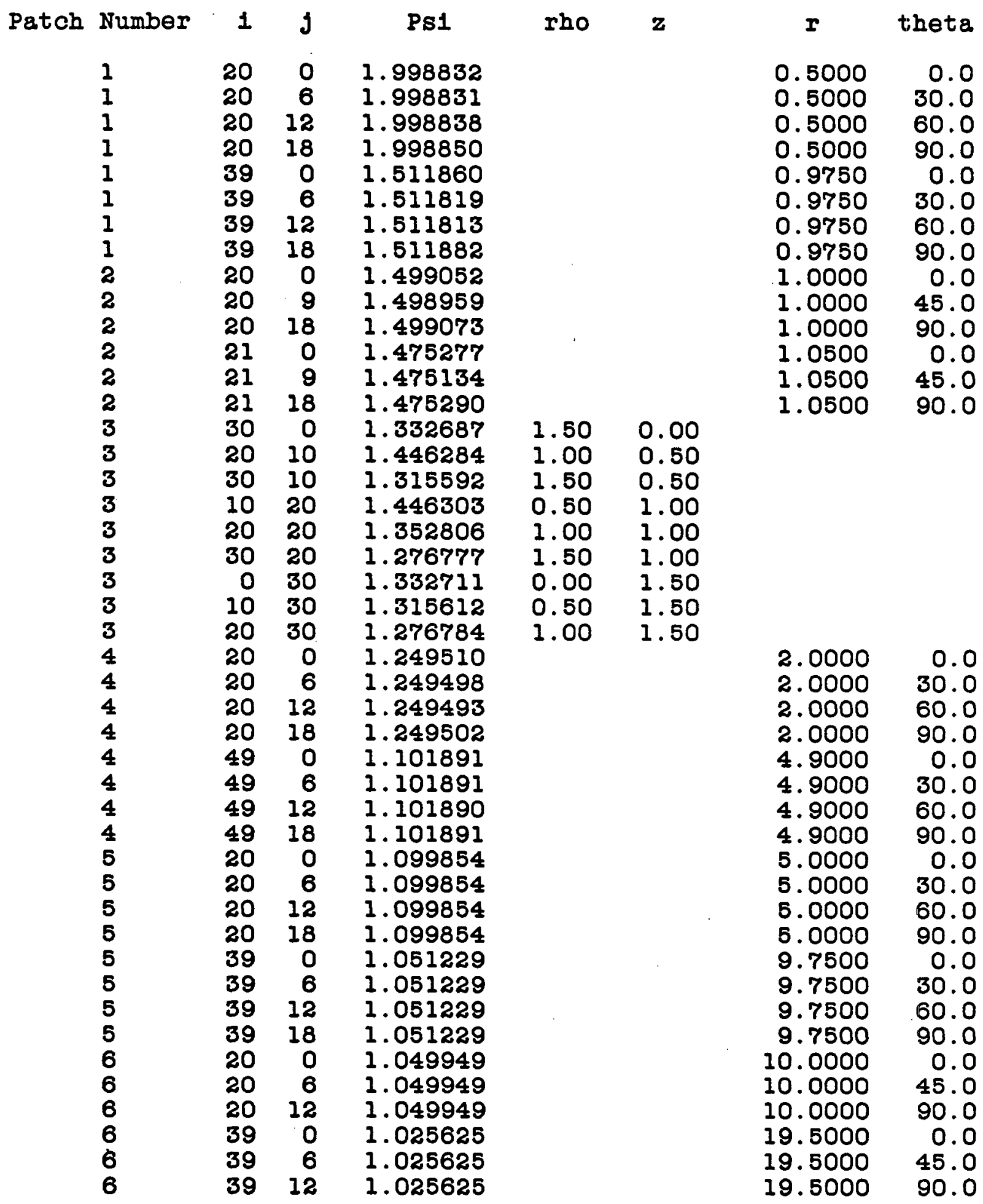

This table gives values of $\Psi$ at selected points in the space. These points are identified by triples (patch number, $i, j$ ), where $(i, j)$ are the generic integer patch coordinates defined in the text. Depending on the patch type, either $(\rho, z)$ or $(r, \theta)$ are also shown. 
Schwarzschild test series

COI. PAR; 2

$18-O C T-1985 \quad 06: 40$

Page 1

t1tle="Sohwarzsohild solution"

N_black_hole=l

grid="co1"

;

black_hole-l

rho $=0$

mass $=1$

$$
\mathbf{z}=0
$$

momentum $=0$

surrounding_patch_flag-1

reflect1on_term_sign=0

reflect10n_radius $=0.5$ spin=0

;

; mkgfn 001

; direotog $0011.00-10$ 


\section{Schwarzschild test series}

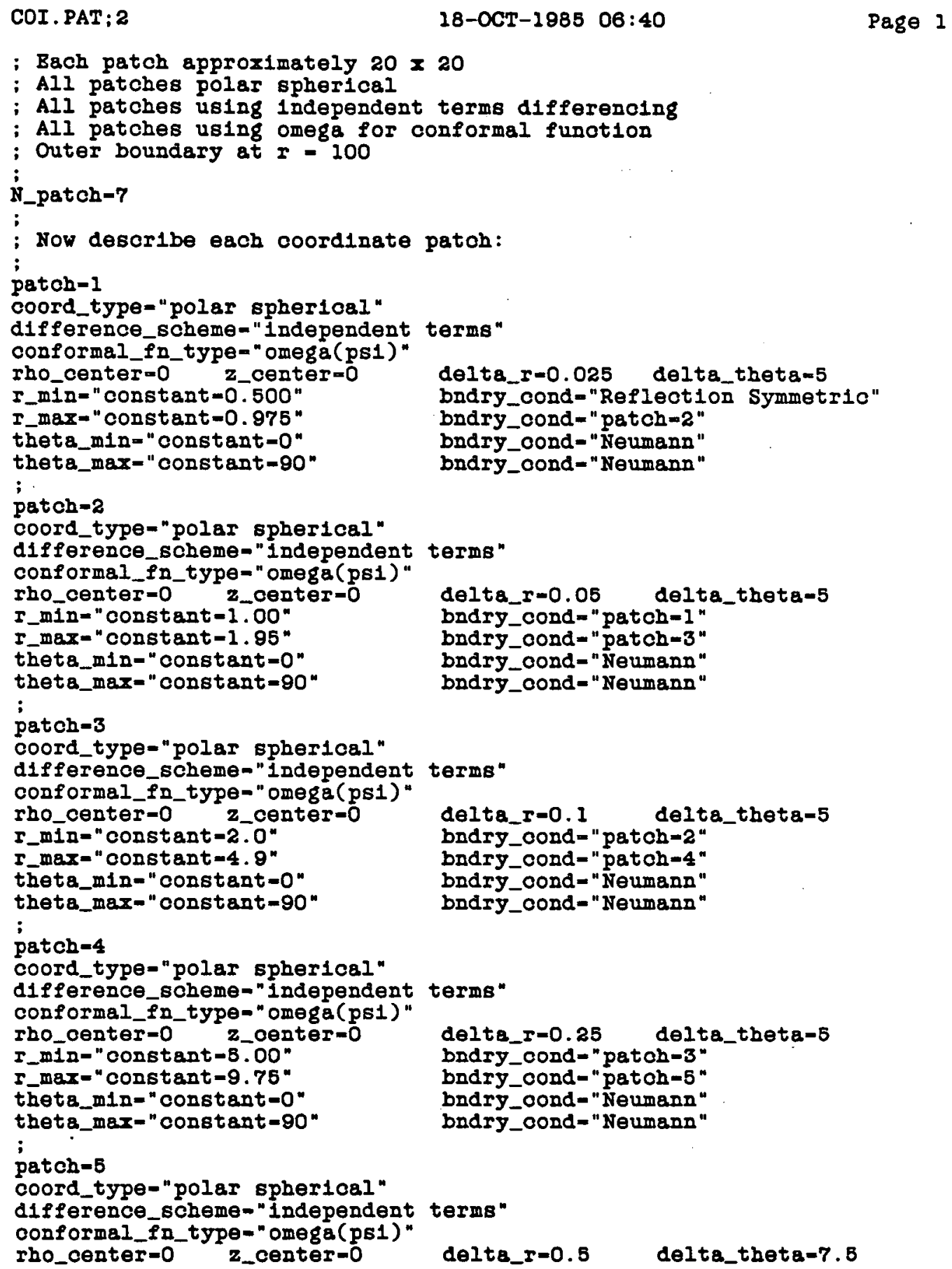

difference_scheme-"independent terms"

delta $r=0.025$ delta theta $=5$

bndry_cond="Reflection Symmetric" bndry_cond = "patch-2" bndry_cond-"Neumann" bndry_cond- "Neumann"

delta $r=0.1$ delta theta-5 bndry_cond = "patch-2" bndry_cond= "patch-4" bndry_cond=" Neumann" bndry_cond = "Neumann"

bndry_cond" "patch=1" bndry_cond=" patch-3" bndry_cond = "Neumann" bndry_cond = "Neumann"

delta_r=0.25 delta_theta-5

bndry_cond="patch-3"

bndry_cond- "patoh-5"

bndry_oond = "Neumann"

bndry_cond = "Neumann" 


\section{Schwarzschild test series}

COI. PAT ; 2

r_min=" constant $=10.0$ "

I_max= "constant $=19.6$ "

theta min="constant-o"

theta_max=" constant-90"

patch $=6$

coord_type="polar spherical"

difference_scheme=" independent terms"

conformal_fn_type="omega(psi)"

rho_center $=0$ z_center $=0$

$r$ min-" constant $=20$ "

I_max=" constant $=49$ "

theta_m1n=" constant $=0$ "

theta_max=" constant $=90$ "

patch-7

coord_type- "polar spher1cal"

conformal_fn_type="omega(ps1)"

rho_center $=0$ z_centermo

r_min-"constant $=5 \overline{0}$ "

$r$ max- " constant $=100$ "

theta_min=" constant $=0$ "

theta_max $=$ " constant $=90$ "
18-OCT-1985 O6:40

Page 2

bndry_cond-" patch -4 "

bndry_cond- "patch 6 "

bndry_cond = "Neumann"

bndry_cond - "Neumsnn"

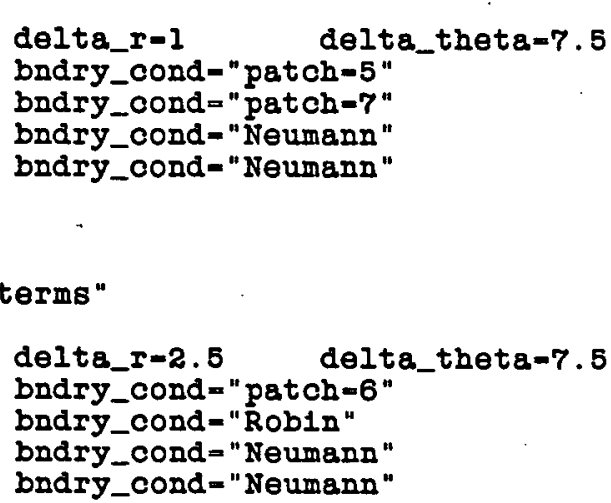


Table 16 - Schwarzschild test series, c0i selected $\Psi$ values

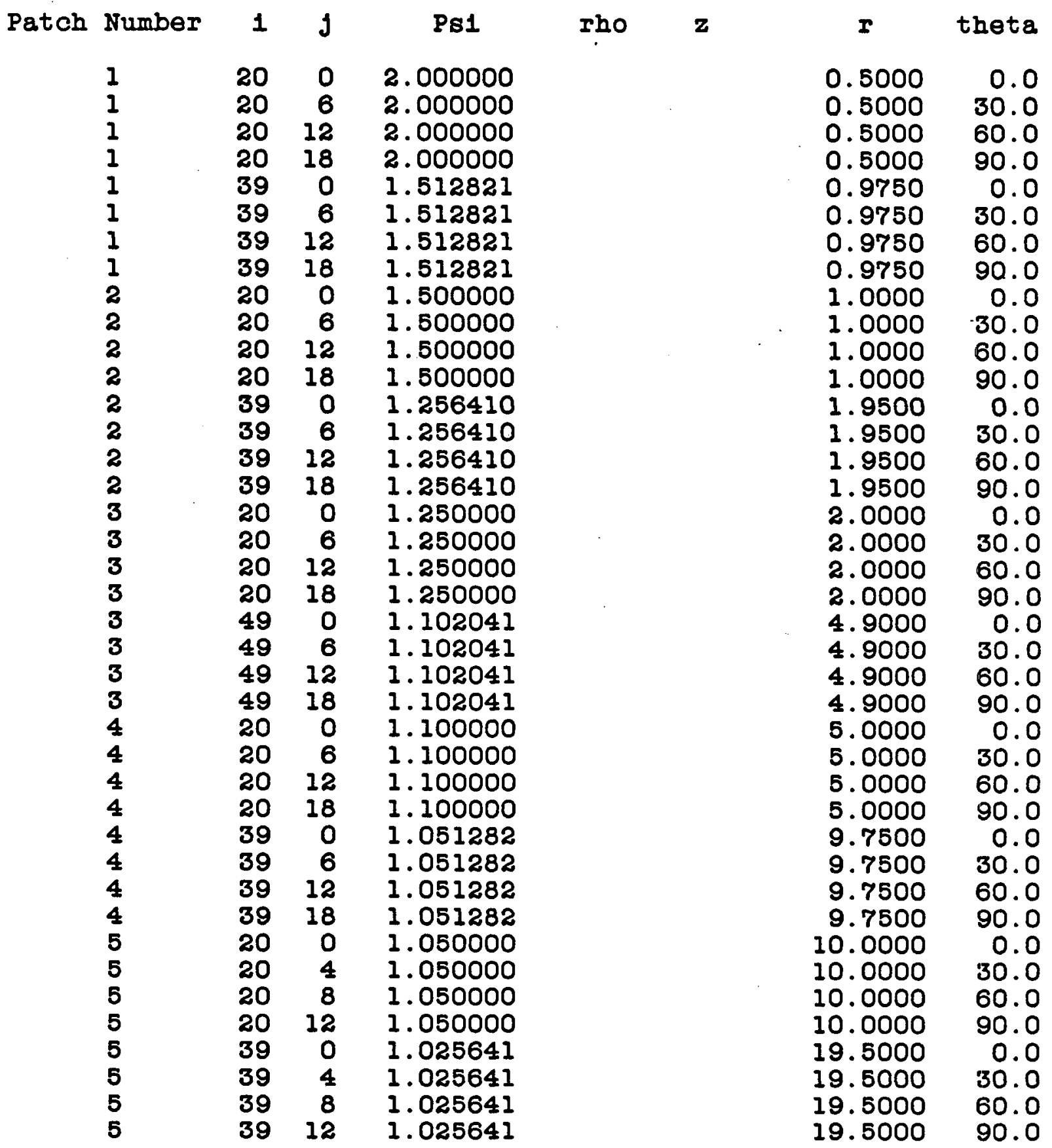

This table gives values of $\Psi$ at selected points in the space. These points are identified by triples (patch number, $i, j$ ), where $(i, j)$ are the generic integer patch coordinates defined in the text. Depending on the patch type, either $(\rho, z)$ or $(r, \theta)$ are also shown. 
Schwarzschild test geries

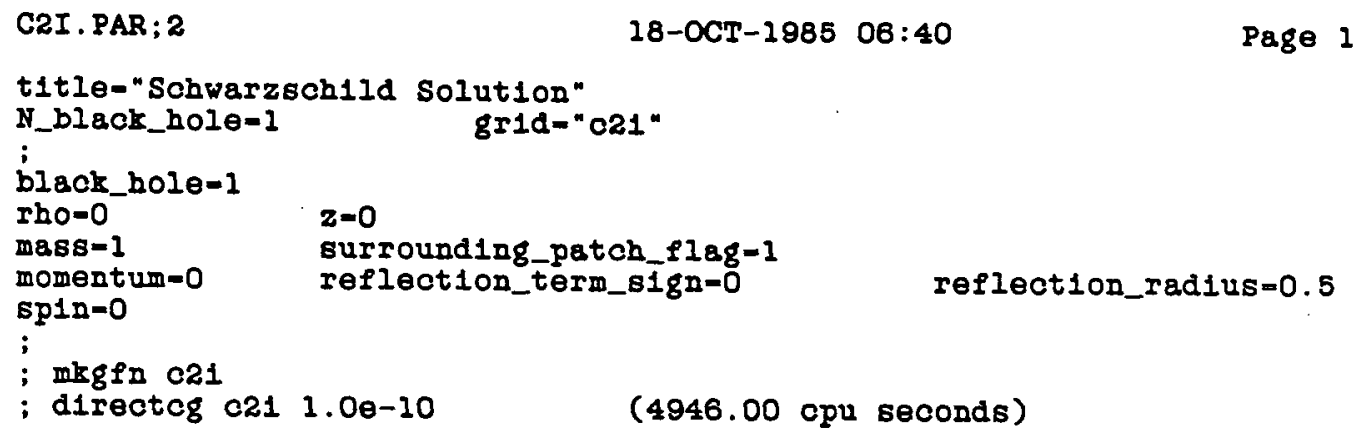




\title{
Schwarzschild test series
}

C2I. PAT; 2

Bach patch approximately 20 × 20

All patches polar spherical, except (or1ginal) patch $\$ 2$ 1s augmented by a cylindrical one

All patches using independent terms differencing

All patches using omega for conformal function

Outer boundary at $r=100$

N_patch-8

Now describe each coordinate patch:

patch $=1$

coord_type-"polar spherical"

difference_scheme-"Independent terms"

conformal_fn_type-"omega(psi)"

rho_center $=0$ z_center $=0$

I_min=" constant $=0.500$ "

I_max= "constant $=0.975 "$

theta_min= "constant $=0$ "

theta_mar $=$ " constant $=90$ "

delta_r=0.025 delta_theta $=5$

bndry_cond="Reflection Symmetr1c"

bndry_cond - "patoh=2"

bndry_cond - "Neumann"

patch $=2$

coord_type-"polar spher1cal"

difference_scheme-"Independent terms"

conformal_fn_type-"omega(ps1)"

rho_center $=0 \quad$ z_center $=0$

r_min=" constant $=1$.00"

I_max=" constant $=1.05$ "

theta_m1n-"constant $=0$ "

theta_max-"constant $=90$ "

bndry_cond=" Neumann"

;

patch-3

coord_type-"oyl1ndr1cal"

difference_scheme-"Independent terms"

conformal_fn_type="omega(ps1)"

rho_center $=0$ z_center $=0$

\author{
delta_r=0.05 delta_theta=5 \\ bndry_cond= "patch-1" \\ bndry_cond="patch-3" \\ bndry_cond= "Neumann" \\ bndry_cond=" Neumann"
}

rho_min_bound $=0$

z_min_bound-0

rho_m1n="patch=2 badry=r_max"

rho_m1n-"constant-0"

rho_max="patch-4 bndry=r_m1n"

z_min="patch-2 bndry=r_mex"

z_min=" constant $=0$ "

z_max="patch=4 bndry=r_min"

;

patch -4

coord_type="polar spher1cal"

difference_scheme-"1ndependent terms"

conformal_fn_type-"omega(ps1)"

rho_center $=0$ z_center $=0$

I_min-"constant -2.0 "

r_max= "constant $=4.9 "$

theta_min="constant-0"

theta_max="constant=90"

delta_rbo-0.05

delta_z=0.05

Tho_max_bound-2

z_max_bound $=2$

bndry_cond- "Teumann"

bndry_cond= "Neumann"

;

delta_r=0.1 delta_theta-5

bndry_cond- "patch=3"

bndry_cond-" "patch-5"

bndry_cond= "Neumann"

bndry_cond- "Neumann" 


\section{Schwarzschild test series}

patch-5

coord_type-"polar spher10al"

difference_scheme-"independent terms"

conformal_fn_type-"omega(psi)"

rho_center $=0$ z_center $=0$

I_min=" constant $=5.00$ "

I_max=" constant $=9.75$ "

theta_m1n=" constant=0"

theta_max-" constant-90"

delta_r=0.25 delta_theta-5
bndry_cond-"patch $=4$ "

bndry_cond-"patoh-6"

bndry_cond- "Neumenn"

;

patch $=6$

coord_type="polar spher1cal"

difference_scheme-" Independent terms"

conformal_fn_type- "omega(ps1)"

rho_center $=0$ z_center $=0$

I_mIn="constant $-1 \overline{0} .0^{\text {" }}$

r_max-"constant $=19$. " $^{\text {" }}$

theta_m1n="constant=0"

theta_max="constant $=90$ "

bndry_cond- "Neumenn"

;

patch -7

coord_type="polar spher1cal"

difference_scheme-"independent terms"

conformal_fn_type="omega(psi)"

rho_center $=0$ z_center-0

r_min=" constant $=20^{\circ}$

r_max-" constant $=49$ "

theta_min="constant $=0$ "

theta_max=" oonstant $=90$ "

delta_r-0.5

delta_theta-7.5

bndry_cond-" patch-5"

bndry_cond = "patch $=7$ "

bndry_cond = "Neumann"

bndry_cond= "Neumann"

;

patch-8

coord_type-"polar spherical"

difference_scheme-"Independent terms"

conformal_fn_type="omega(psi)"

rho_center $=0$ z_center=0

I_mIn="constant $=50^{\circ}$

I_max=" constant $=100$ "

theta_m1n="constant-0"

theta_max = "constant $=90$ "

delta_r=1

bndry_cond= " patch=6"

bndry_cond=" patch=8"

bndry_cond - "Neumann"

bndry_cond= "Neumann" delta_r-2.5 delta_theta-7.5

bndry_cond = "patoh-7"

bndry_cond-"Robin"

bndry_cond- "Neumann"

bndry_cond- "Neumann" 
Table 17 - Schwarzschild test series, c21 selected $\Psi$ values

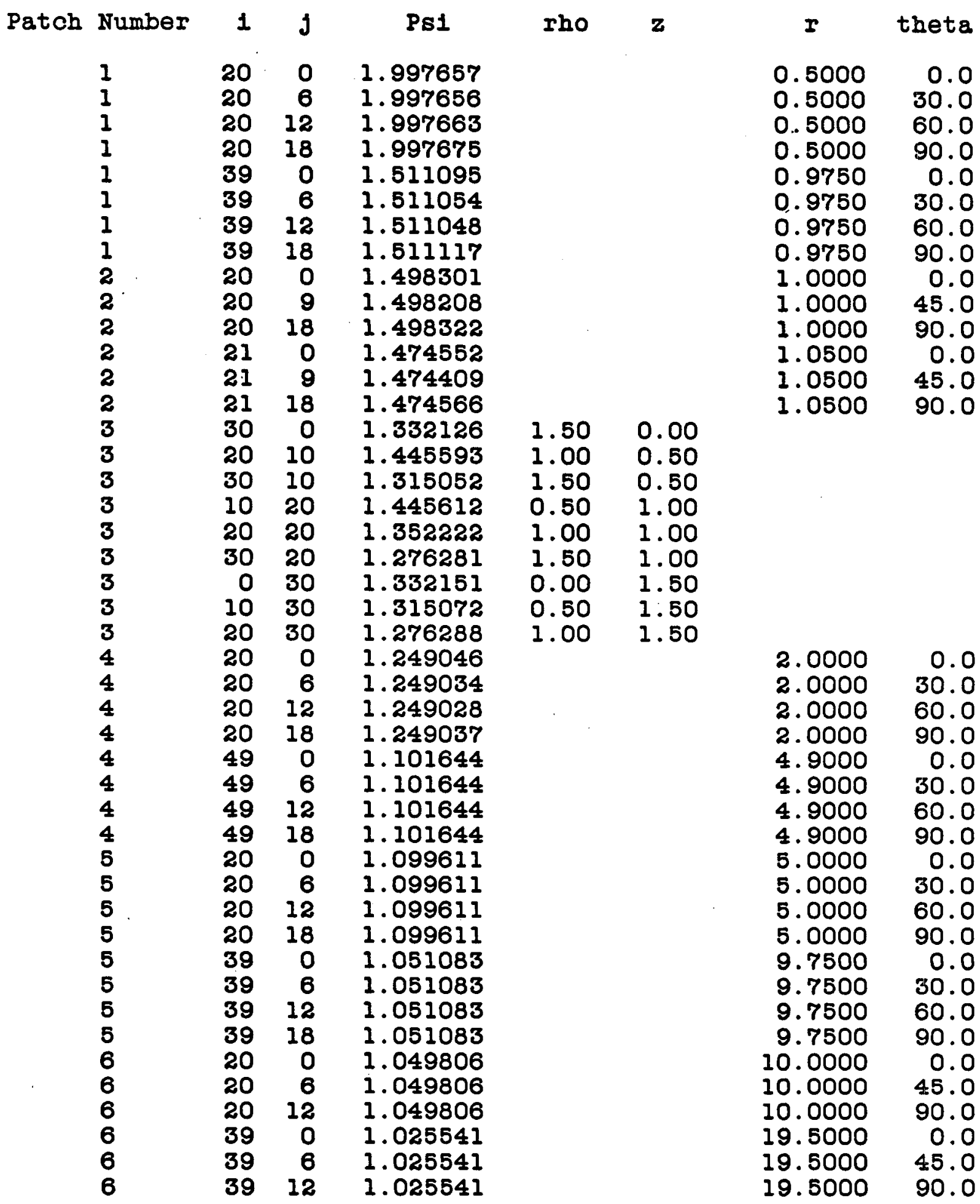

This table gives values of $\Psi$ at selected points in the space. These points are identified by triples (patch number, $i, j$ ), where $(i, j)$ are the generic integer patch coordinates defined in the text. Depending on the patch type, either $(\rho, z)$ or $(r, \theta)$ are also shown. 
Schwarzschild test series

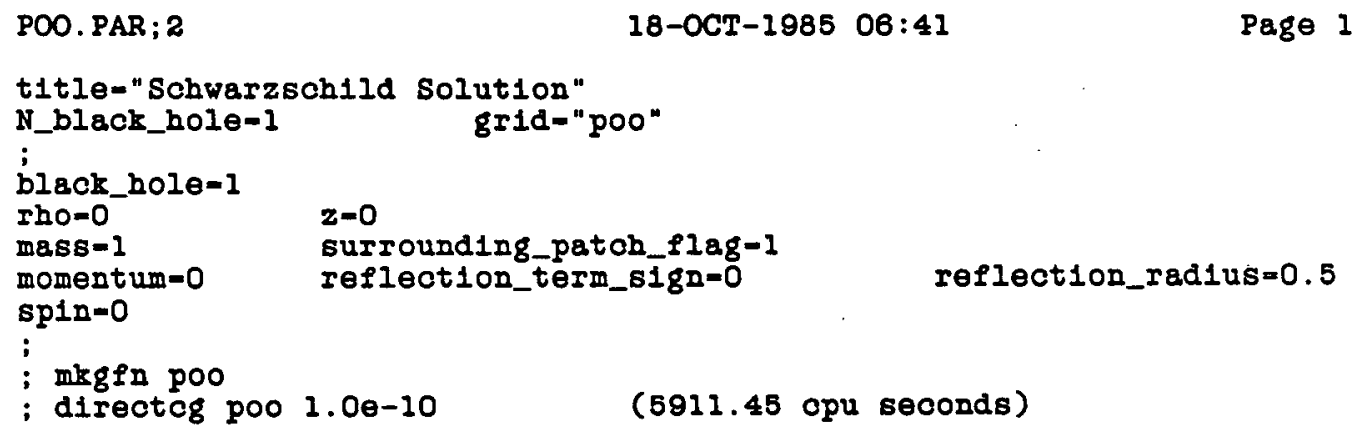


Schwarzschild test geries

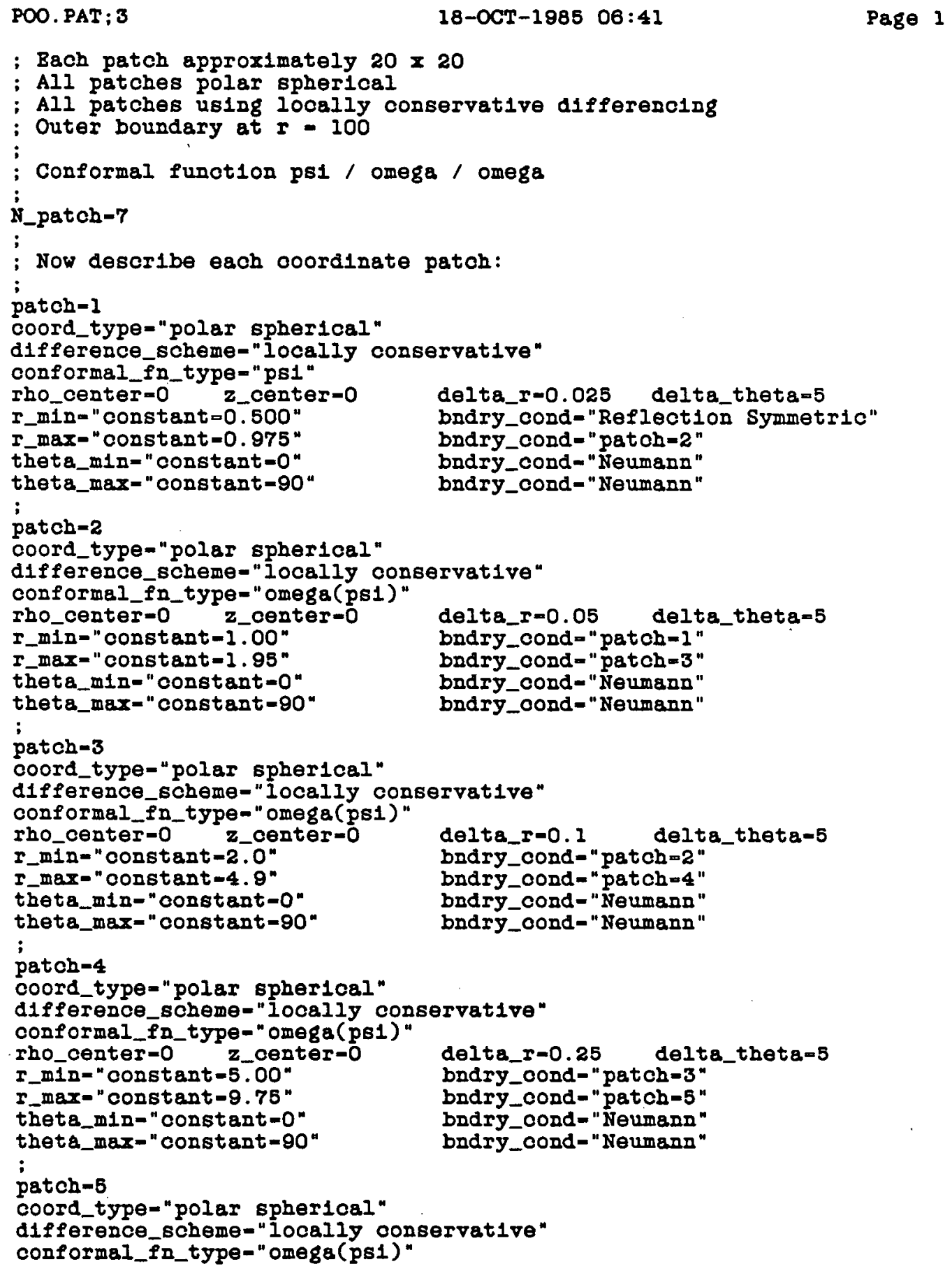




\section{Schwarzschild test series}

POO. PAT; 3

rho_center $=0$ z_center-0

r_min="constant $=10.0 "$

$r$ max $=$ "constant $=19.6$ "

theta_min=" constant $=0$ "

theta_max=" constant $=90$ "

patch $=6$

coord_type-"polar spherical"

difference_scheme-"Iocally conservat1ve"

conformal_fn_type="omega(ps1)"

rho_center $=0$ z_center $=0$

I.m1n- constant $=20$ "

r_max $=$ "constant $=49$ "

theta_min-"constant $=0$ "

theta_max="constant $=90$ "

patch-7

coord_type="polar spherical"

difference_scheme-" "locally conservat1ve"

conformal_fn_type="omega(ps1)"

rho_center $=0 \quad$ z_center $=0$

r_m1n=" constant $=50$ "

$I$ max= "constant $=100$ "

theta_m1n="constant $=0$ "

theta max $=$ "constant $=90$ "

delta_ral
18-OCT-1885 06:41

Page 2

delta_r=0.5 delta_theta-7.5

bndry_cond= "patch=4"

bndry_cond = "patch=6"

bndry_cond = "Neumann"

bndry_cond = "Neumann"

bndry_cond=" patch $=5$ "

bndry_cond- "patch=7"

bndry_cond- "Neumann"

bndry_cond= "Neumann"

delta_theta $=7.5$

delta_r-2.5

delta_theta $=7.5$

bndry_cond= "patch=6"

bndry_cond = "Rob1n"

bndry_cond- " Neumann"

bndry_cond = "Neumann" 
Table 18 - Schwarzschild test series, poo selected $\Psi$ values

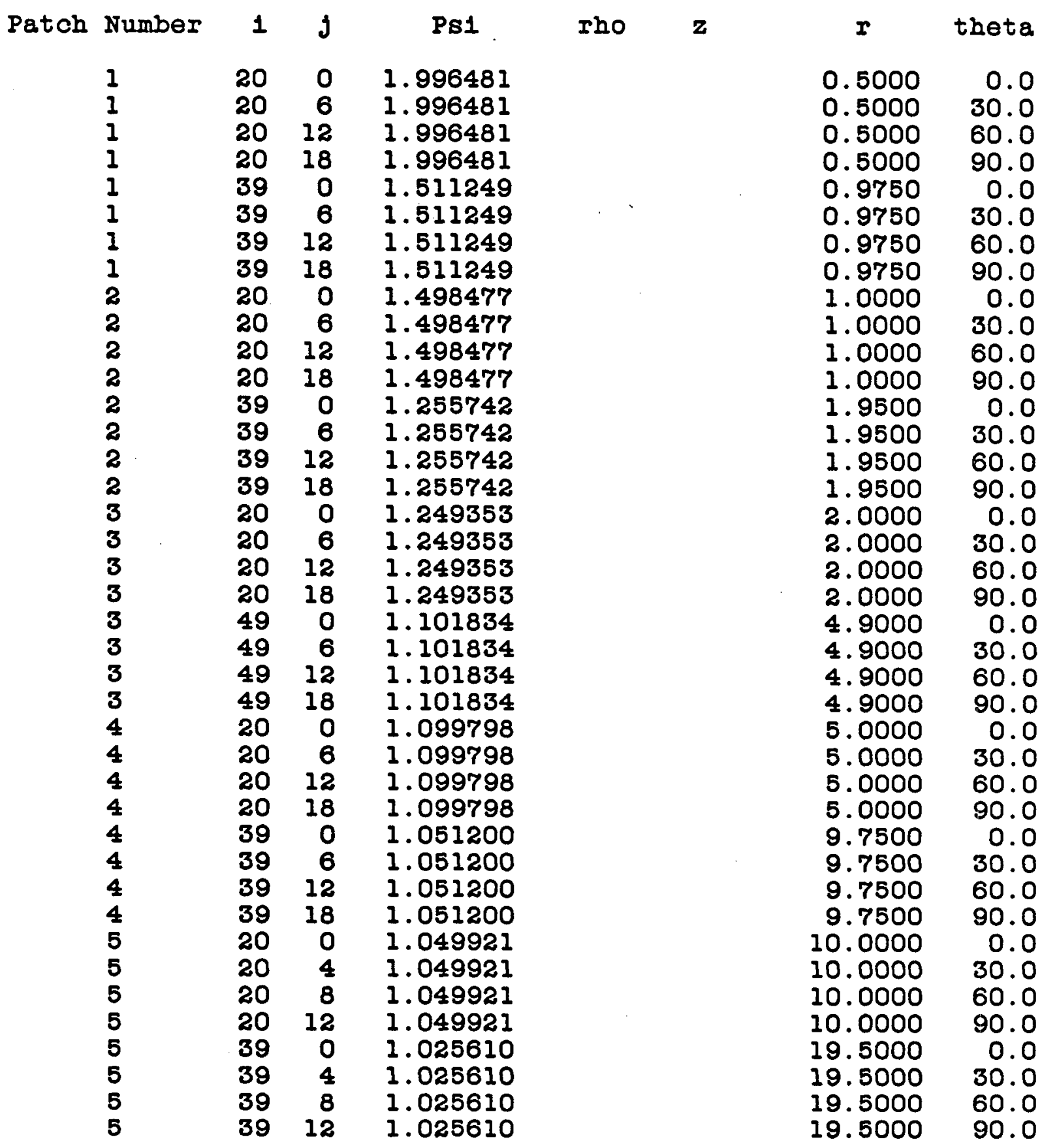

This table gives values of $\Psi$ at selected points in the space. These points are identified by triples (patch number, $i, j$ ), where $(i, j)$ are the generic integer patch coordinates defined in the text. Depending on the patch type, either $(\rho, z)$ or $(r, \theta)$ are also shown. 
Schwarzschild test series

OPO. PAR; 2

$18-O C T-1985 \quad 06: 41$

Page 1

t1tle-"Schwarzsch1ld Solut1on"

N_black_hole=1 Grid-"opo"

;

black_hole-1

rho=0

mass $=1$

$\mathbf{z}=0$

momentum $=0$

surround1ng_patoh_flag=1

$\operatorname{sp} 1 n=0$

reflection_term_sign=0

reflect1on_radius $=0.5$

;

; mkgen opo

; directog opo $1.00-10$ (2737.25 cpu seconds) 


\section{Schwarzschild test series}

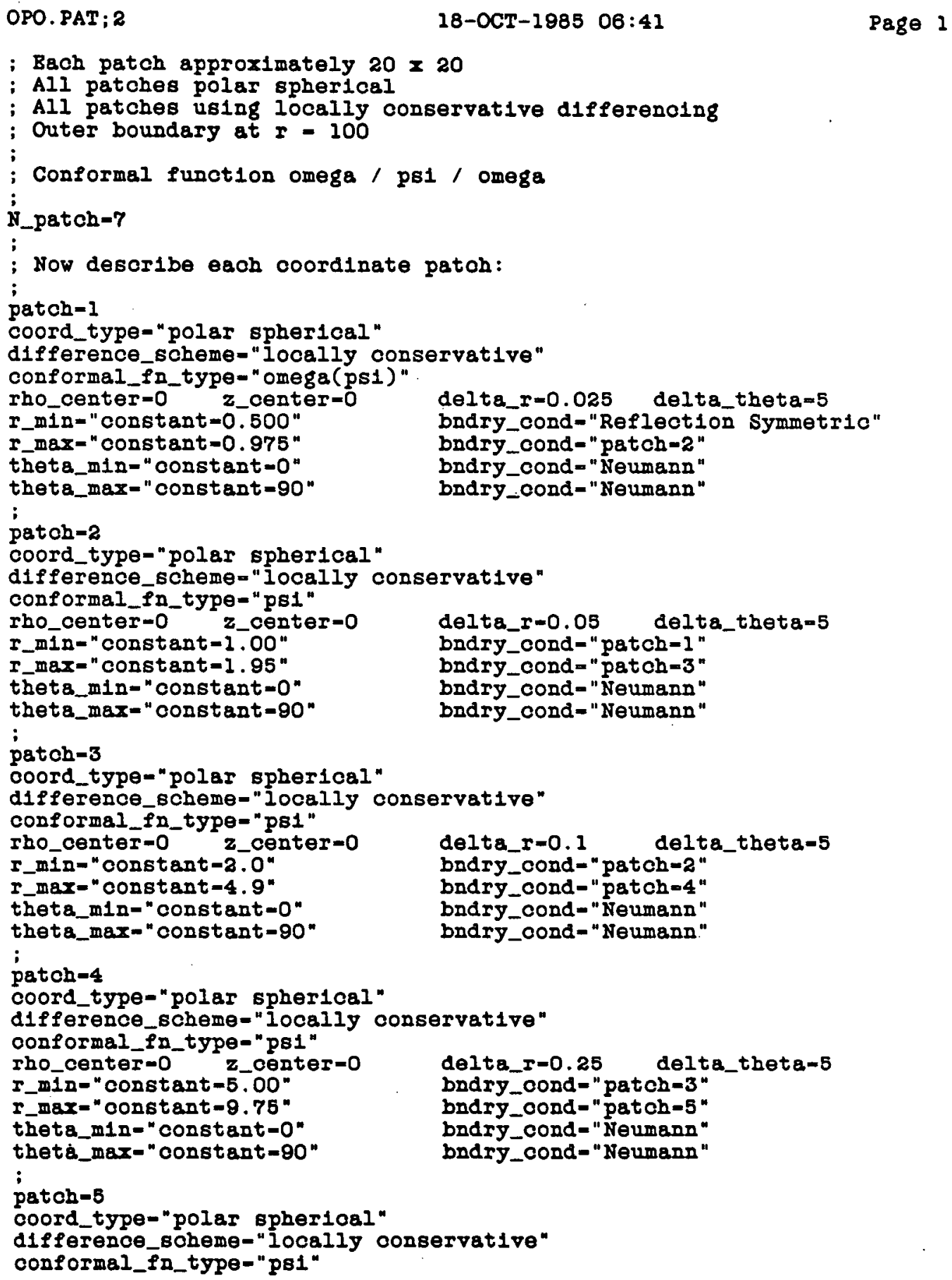




\section{Schwarrschild test series}

OPO. PAT; 2

rho_center=0 z_center=0

$r \_m 1 n="$ constant $=10.0$ "

r_max= "constant $=19.5$ "

theta_min="constant $=0 "$

theta_mar="constant-90"

;

patch $=6$

coord_type="polar spher1cal"

difference_scheme= "I008liy conservative"

conformal_fa_type-"psi"

rho_center $=0$ z_center $=0$

I_mIn=" constant $-20^{\prime \prime}$

r_max= "constant -49 "

theta_min="constant $=0$ "

theta_max $="$ constant $=90$ "

;

patch-7

coord_type-"polar spher1cal"

difference_scheme=" Iocally conservat1ve"

conformal_fn_type="omega(psi)"

rho_center $=0$ z_center $=0$

$r$ mI $=$ "constant $=5 \overline{0}^{\circ}$

r_max="constant-100"

theta min=" constant $=0$ "

theta_max="constant $=90$ "
18-OCT-1885 06:41

Page 2

delta_r=0.5 delta_theta=7.5

bndry_cond = "patch-4"

badry_oond = "patch=6"

bndry_cond = "Neumann"

bndry_cond- "Neumann"

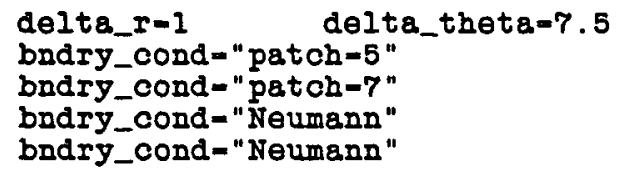


Table 19 - Schwarzschild test series, opo selected $\Psi$ values

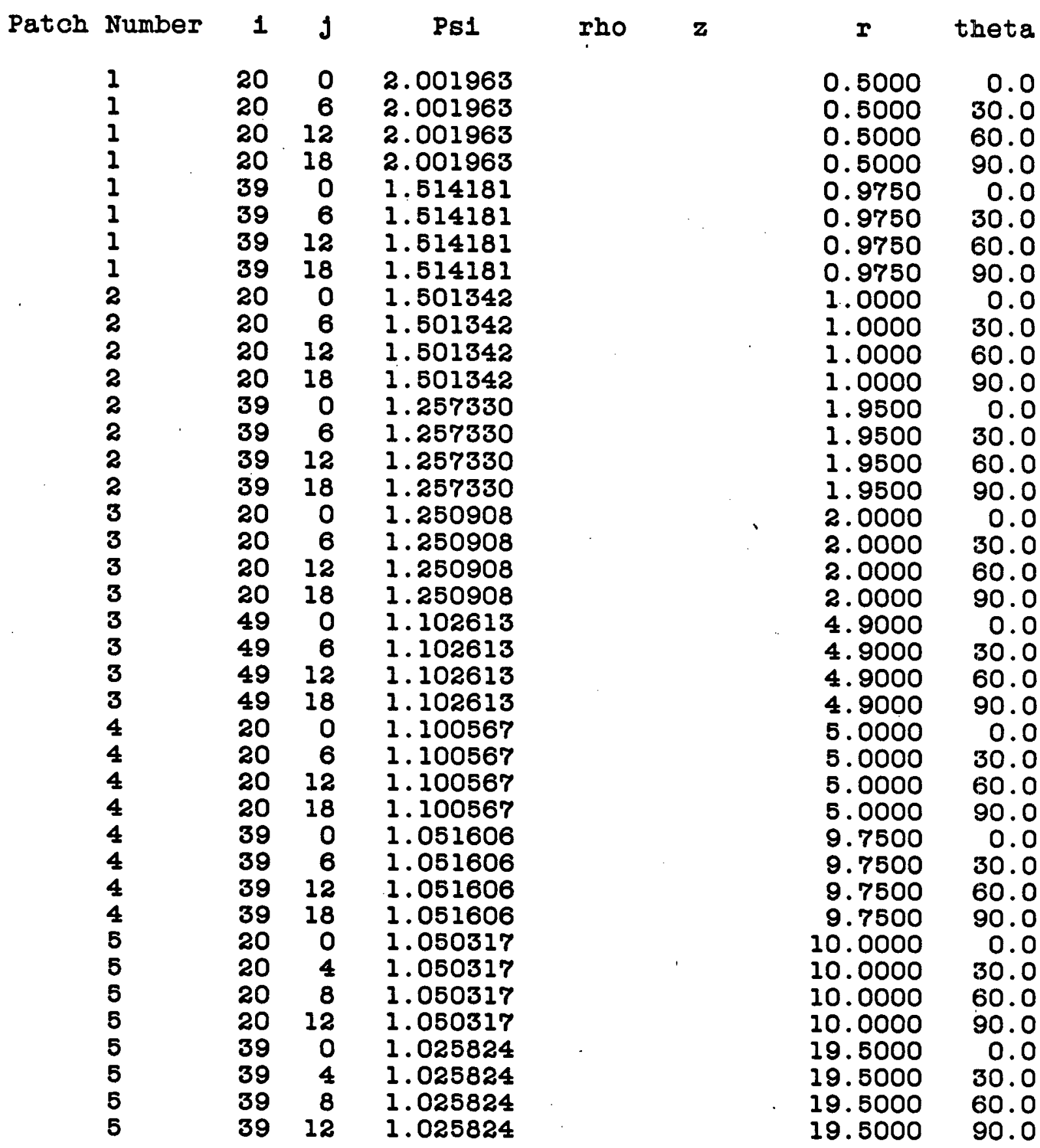

This table gives values of $\Psi$ at selected points in the space. These points are identified by triples (patch number, $i, j$ ), where $(i, j)$ are the generic integer patch coordinates defined in the text. Depending on the patch type, either $(\rho, z)$ or $(r, \theta)$ are also shown. 
Schwarzschild test series

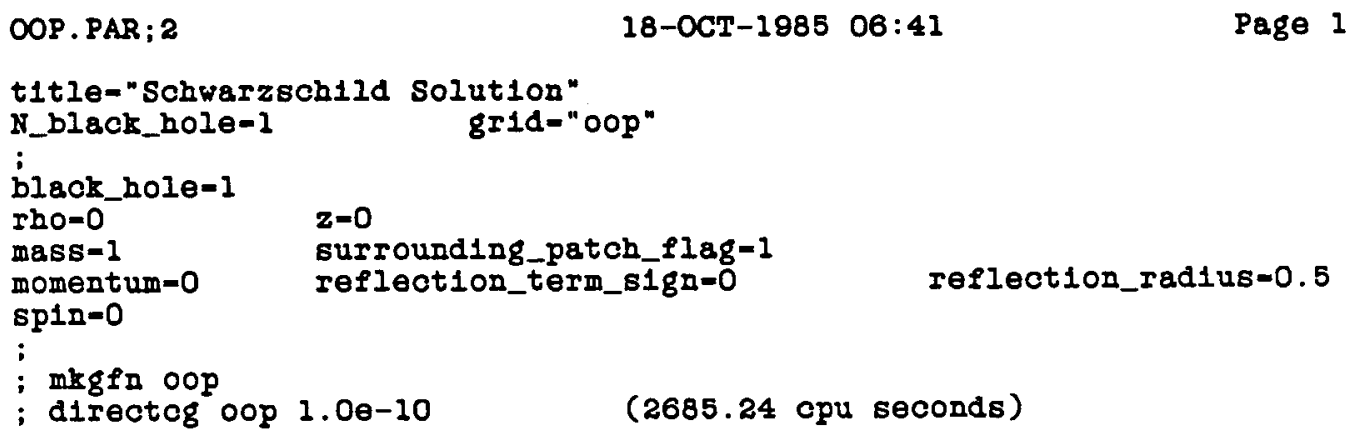




\section{Schwarzschild test series}

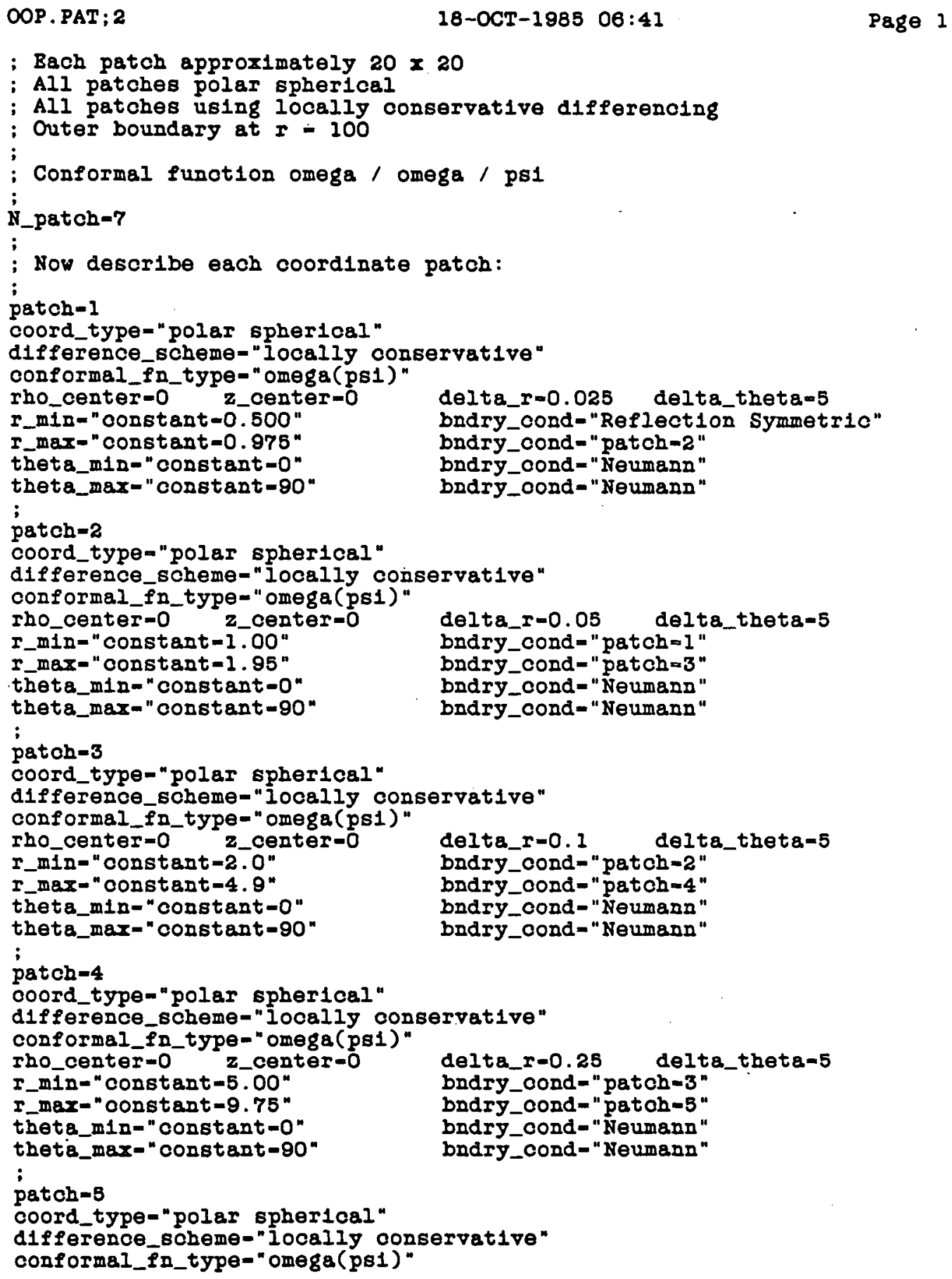


Schwarzschild test series

OOP. PAT ; 2

rho_center-0 z_center $=0$

I_min=" constant $=1 \overline{0}$. 0 "

I_mex-"constant-19.5"

theta_m1n="constant $=0$ "

theta_max-" constant-90"

;

patch $=6$

coord_type-"polar spherical"

difference_scheme= "local1y conservat1ve"

conformal_fn_type-"omega(ps1)"

rho_center $=0 \quad z$ center-0

I_min- "oonstant $=20^{\circ}$

$r$ max $=$ "constant $=49$ "

theta_m1n=" constant $=0$ "

theta_max = "constant-90"

;

patch -7

coord_type="polar spher1cal"

difference_scheme-" Iocally conservative"

conformal_fn_type-"psi"

rho_center-0 z_center-0

$r$ mIn=" constant $=5 \overline{0}$ "

I_max=" constant $=100$ "

theta_m1n="constant $=0$ "

theta_max-" constant $=90$ "
18-OCT-1985 06:41

Page 2

delts_I-0.3 delta_thets-7.5

bndry_cond = "patch $=4$ "

bndry_oond = "patch=6"

bndry_cond- "Neumann"

bndry_cond - "Neumann"
delta_I-1 delta_theta-7.5

bndry_cond = "patch $=5$ "

bndry_cond-"patch $=7$ "

bodry_oond - "Neumenn"

bndry_cond- "Neumann"

delta_r-2.8 delta_theta-7.5

bndry_cond = "patch-6"

bndry_cond = "Rob1n"

bndry_cond = "Neumann"

bndry_cond- "Neumann" 
Table 20 - Schwarzschild test series, oop selected $\Psi$ values

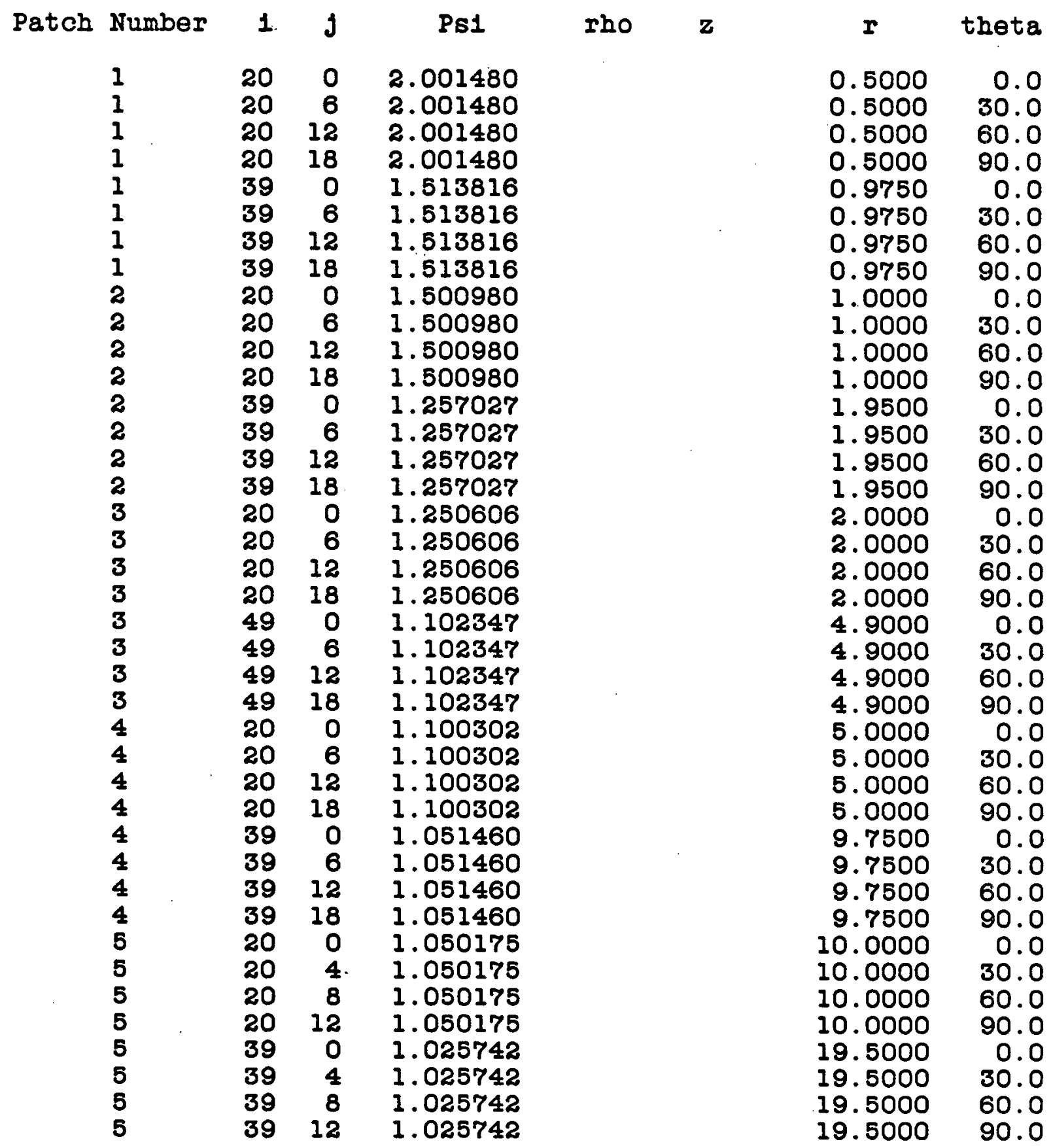

This table gives values of $\Psi$ at selected points in the space. These points are identified by triples (patch number, $i, j$ ), where $(i, j)$ are the generic integer patch coordinates defined in the text. Depending on the patch type, either $(\rho, z)$ or $(r, \theta)$ are also shown. 


\section{Choptuik comparison test series}

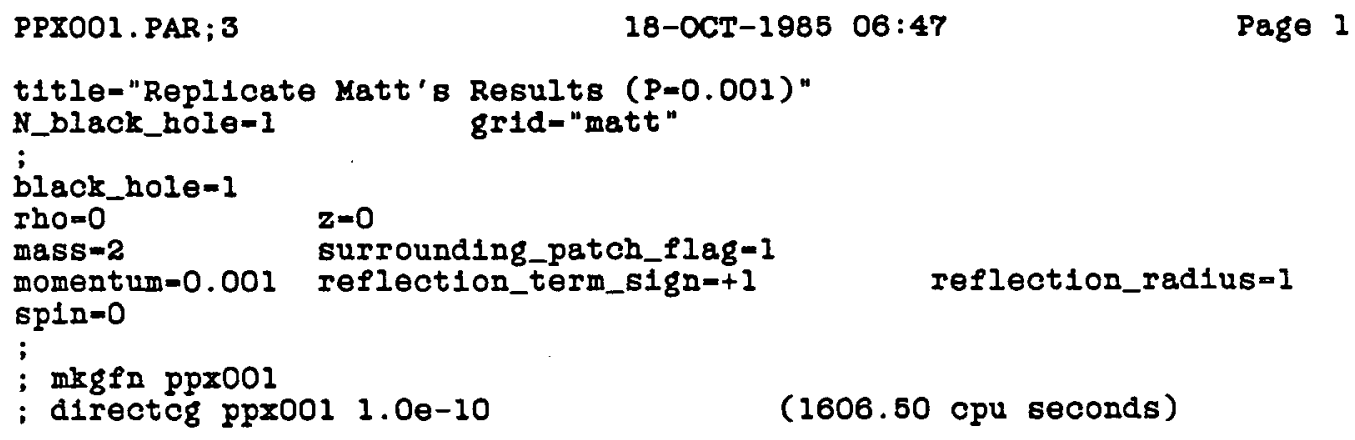


Figure 12 - Choptuik comparison test series, ppx001 quantitative results
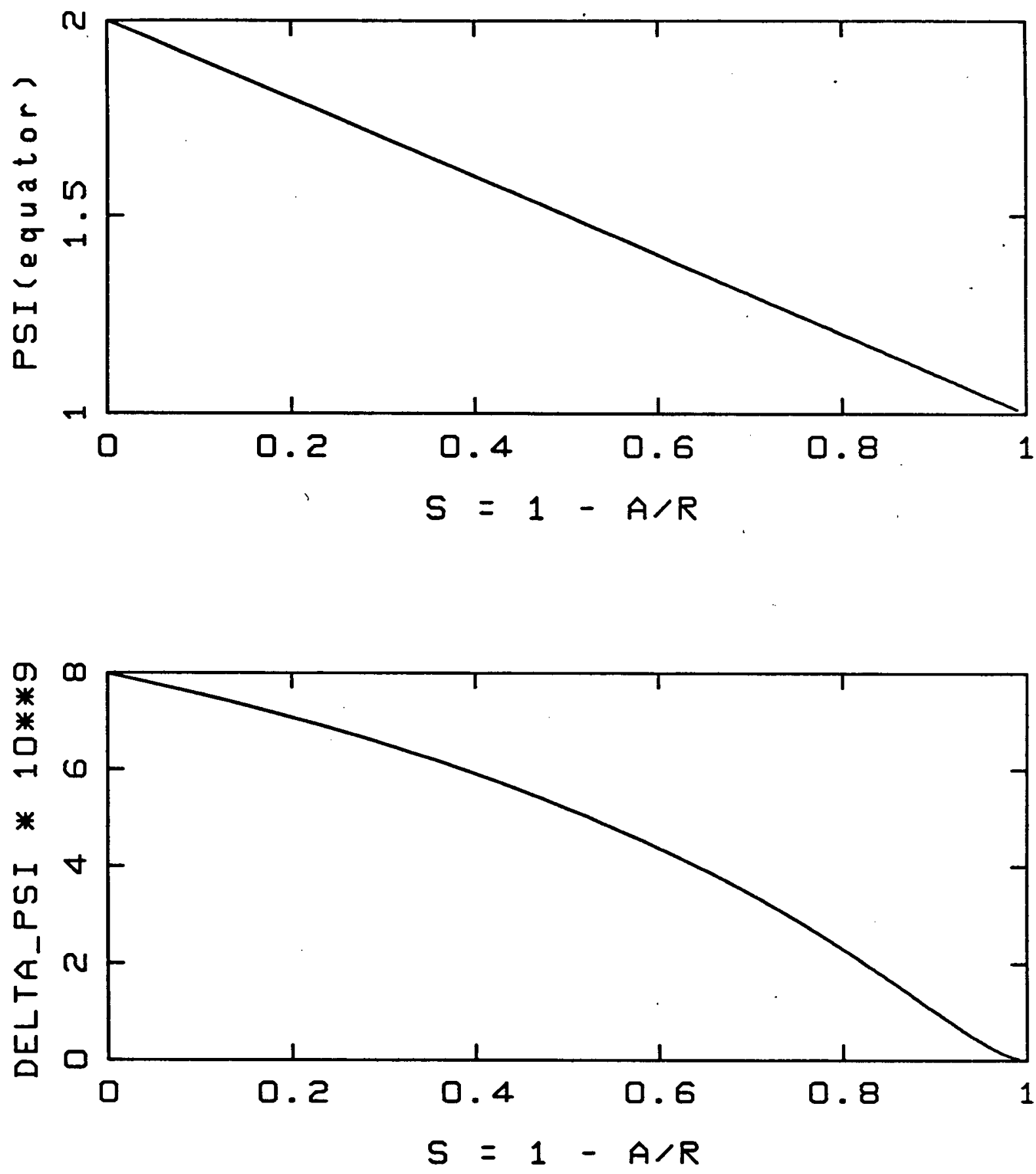

This figure shows $\Psi$ (equator) and $\delta \Psi=\Psi$ (north pole) $-\Psi$ (equator), both as functions of $s=1-a / r$. The former shows the general radial trend of $\Psi$, while the latter is a measure of the deviation of $\Psi$ from spherical symmetry. 


\section{Choptuik comparison test series}

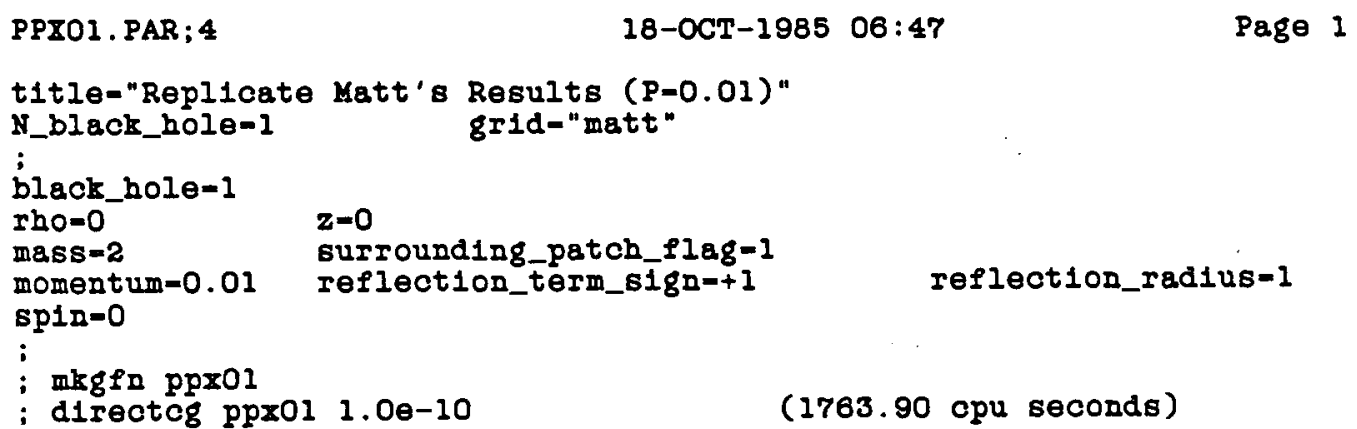


Figure 13 - Choptuik comparison test series, ppr01 quantitative results
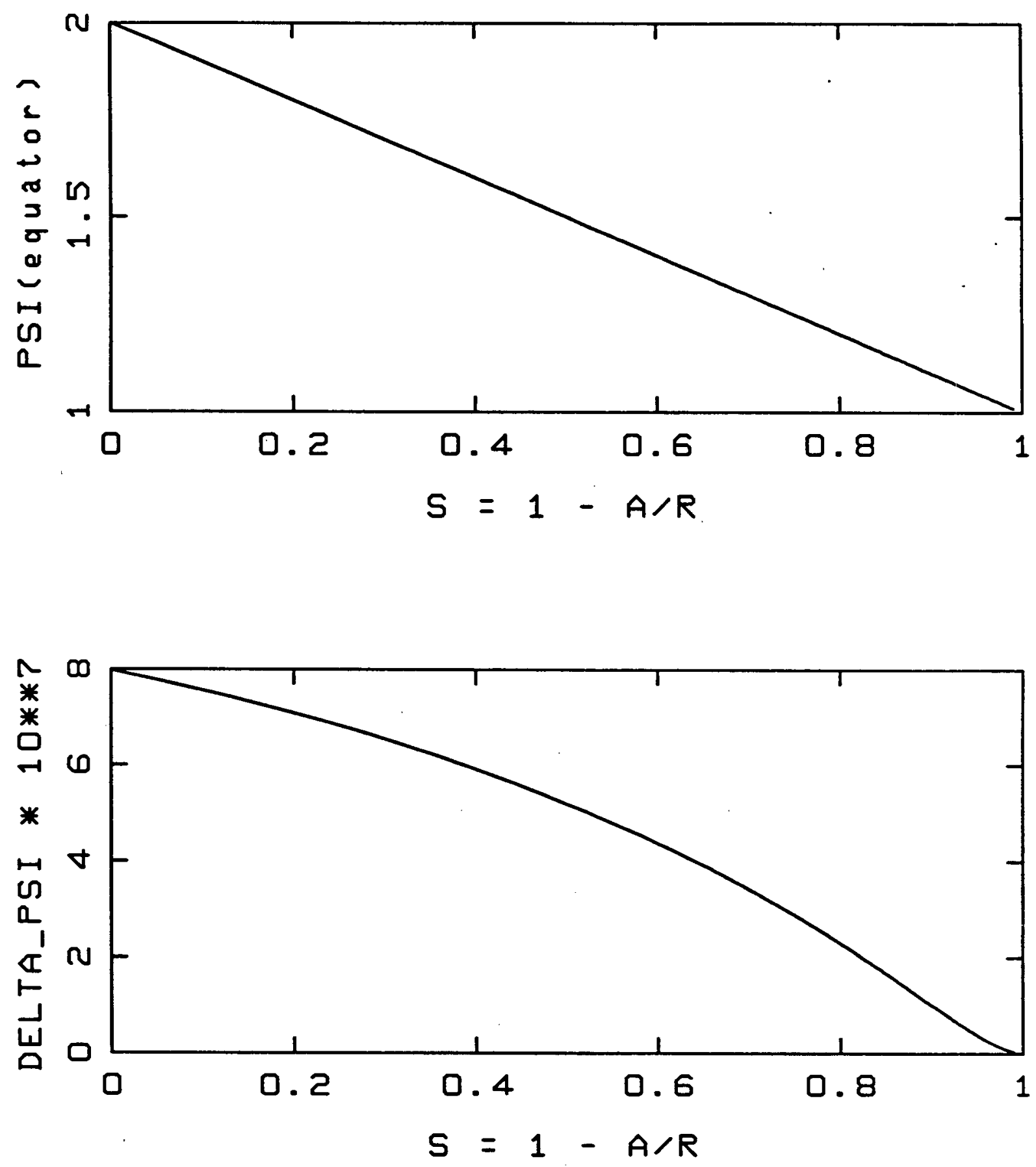

This figure shows $\boldsymbol{\Psi}$ (equator) and $\delta \boldsymbol{\Psi}=\boldsymbol{\Psi}$ (north pole) $-\boldsymbol{\Psi}$ (equator), both as functions of $s=1-a / r$. The former shows the general radial trend of $\Psi$, while the latter is a measure of the deviation of $\Psi$ from spherical symmetry. 
Choptuik comparison test series

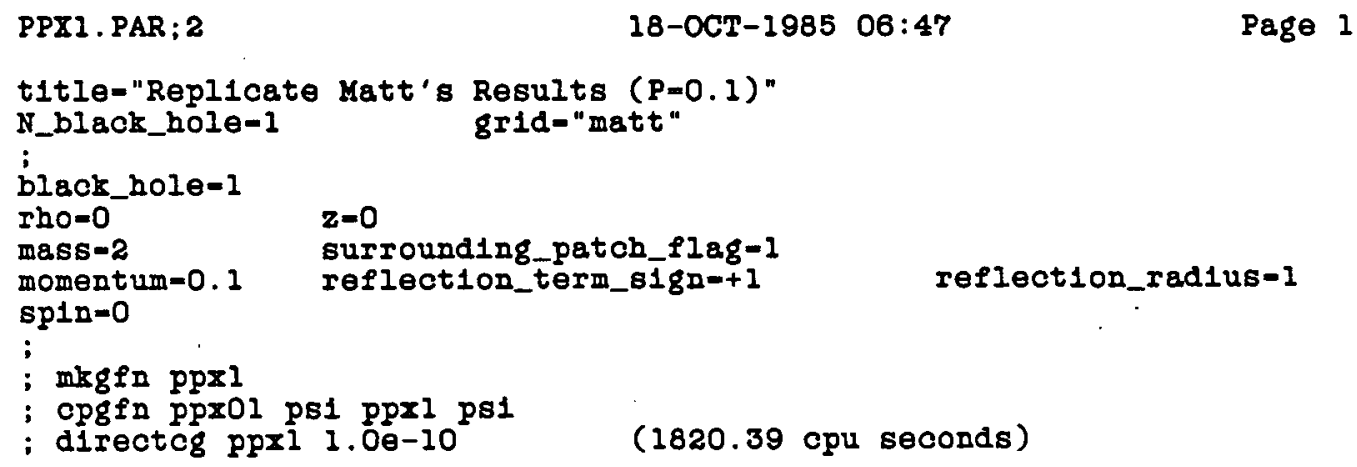


Figare 14 - Choptuik comparison test series, ppr1 quantitative results
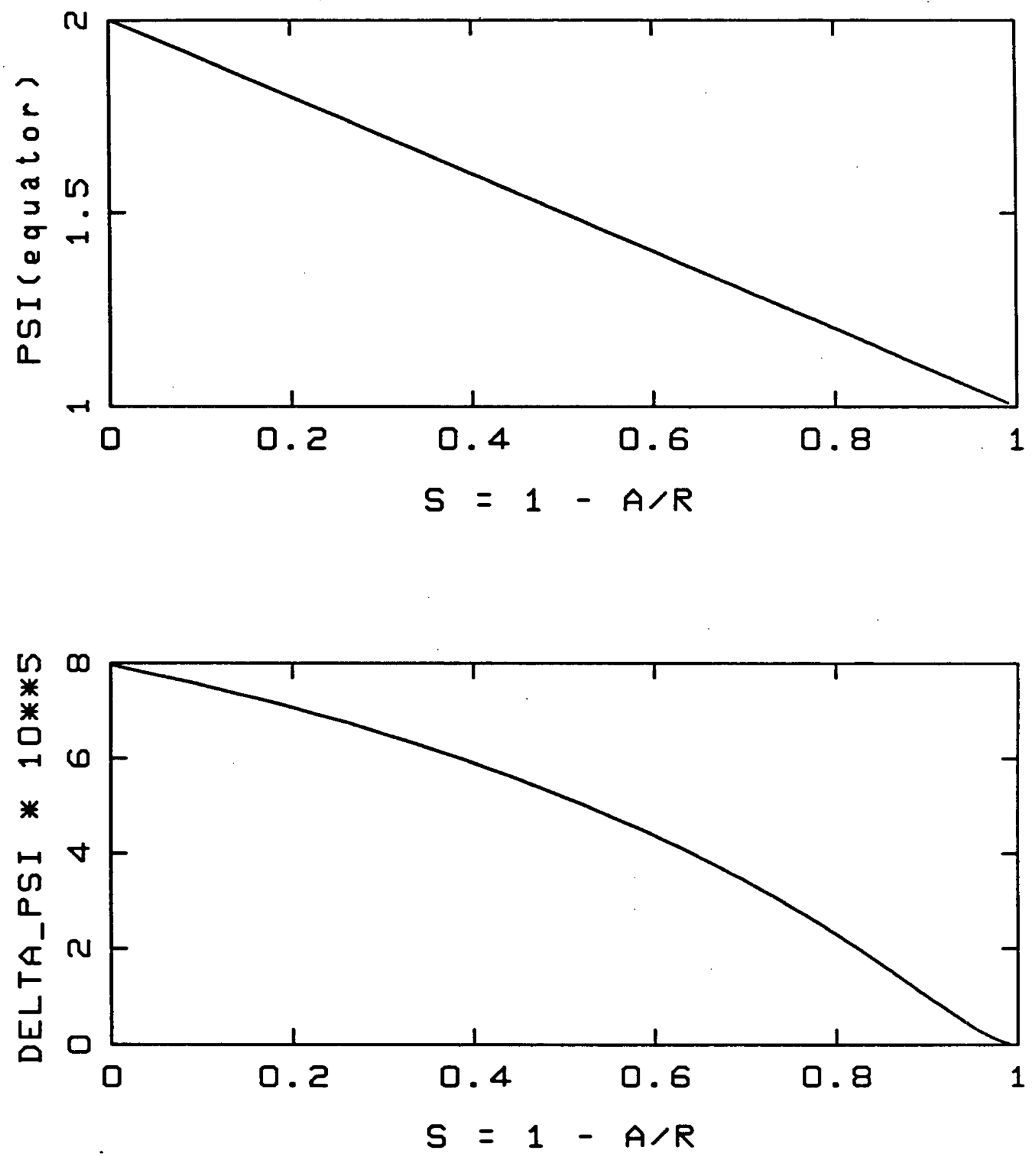

This figure shows $\Psi$ (equator) and $\delta \Psi=\Psi$ (north pole) $-\Psi$ (equator), both as functions of $s=1-a / r$. The former shows the general radial trend of $\Psi$, while the latter is a measure of the deviation of $\Psi$ from spherical symmetry. 
Choptuik comparison test series

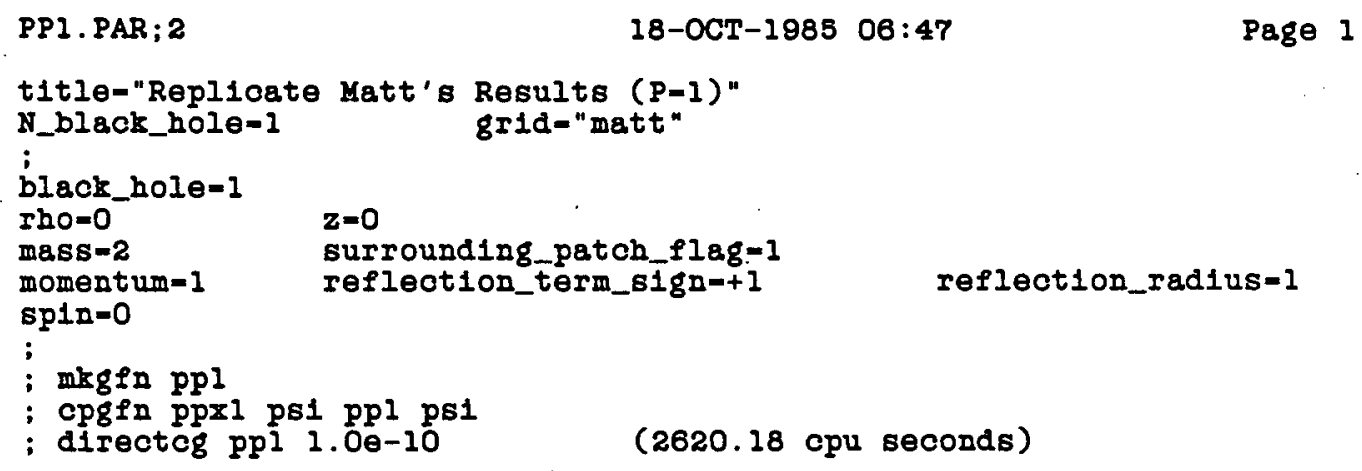


Figure 15 - Choptuik comparison test series, pp1 quantitative results
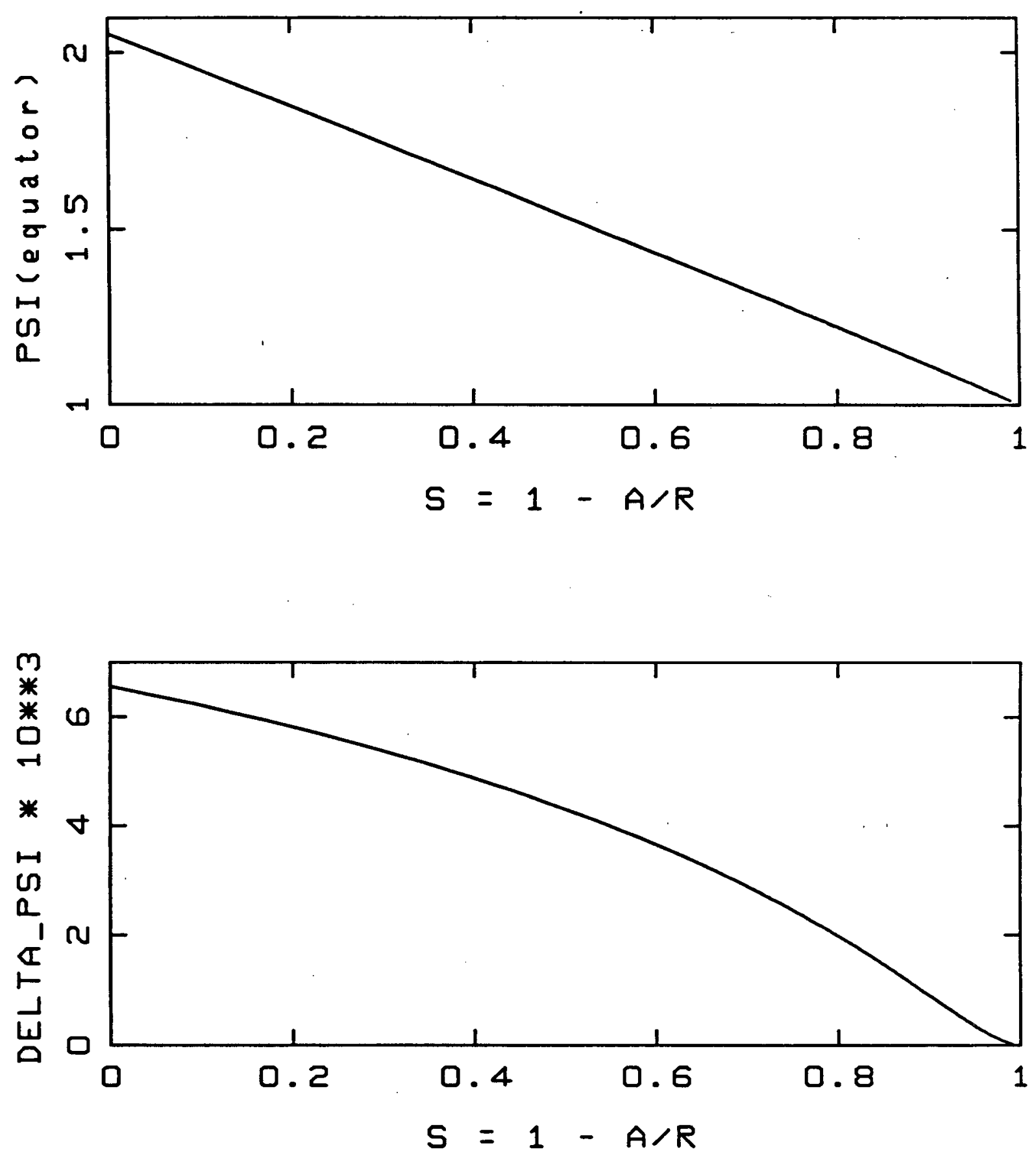

This figure shows $\Psi$ (equator) and $\delta \Psi=\Psi$ (north pole) $-\Psi$ (equator), both as functions of $s=1-a / r$. The former shows the general radial trend of $\Psi$, while the latter is a measure of the deviation of $\Psi$ from spherical symmetry. 


\section{Choptuik comparison test series}

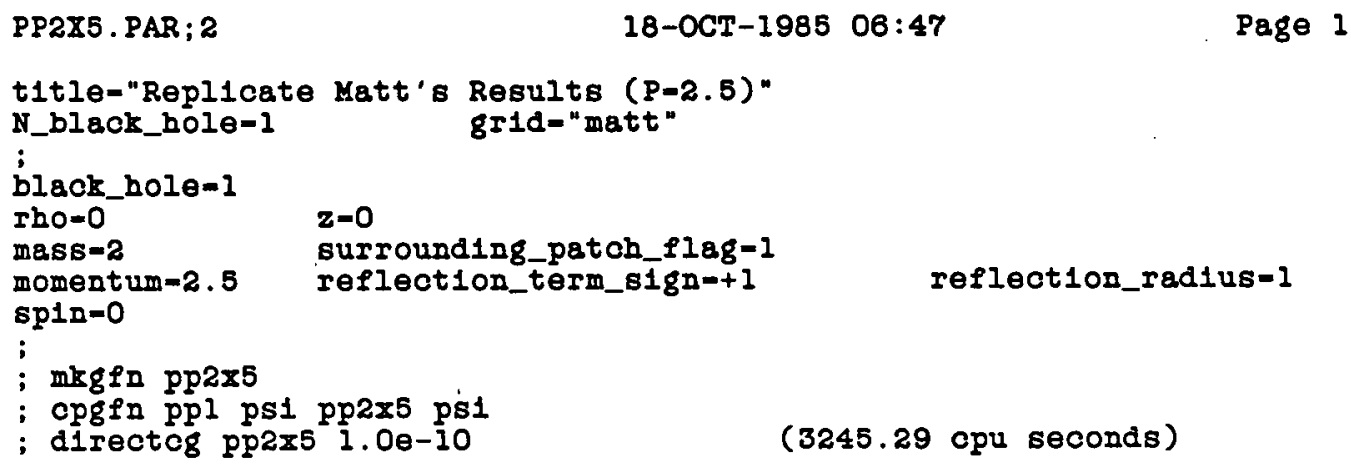


Figure 16 - Choptuik comparison test series, Pp2r5 quantitative results
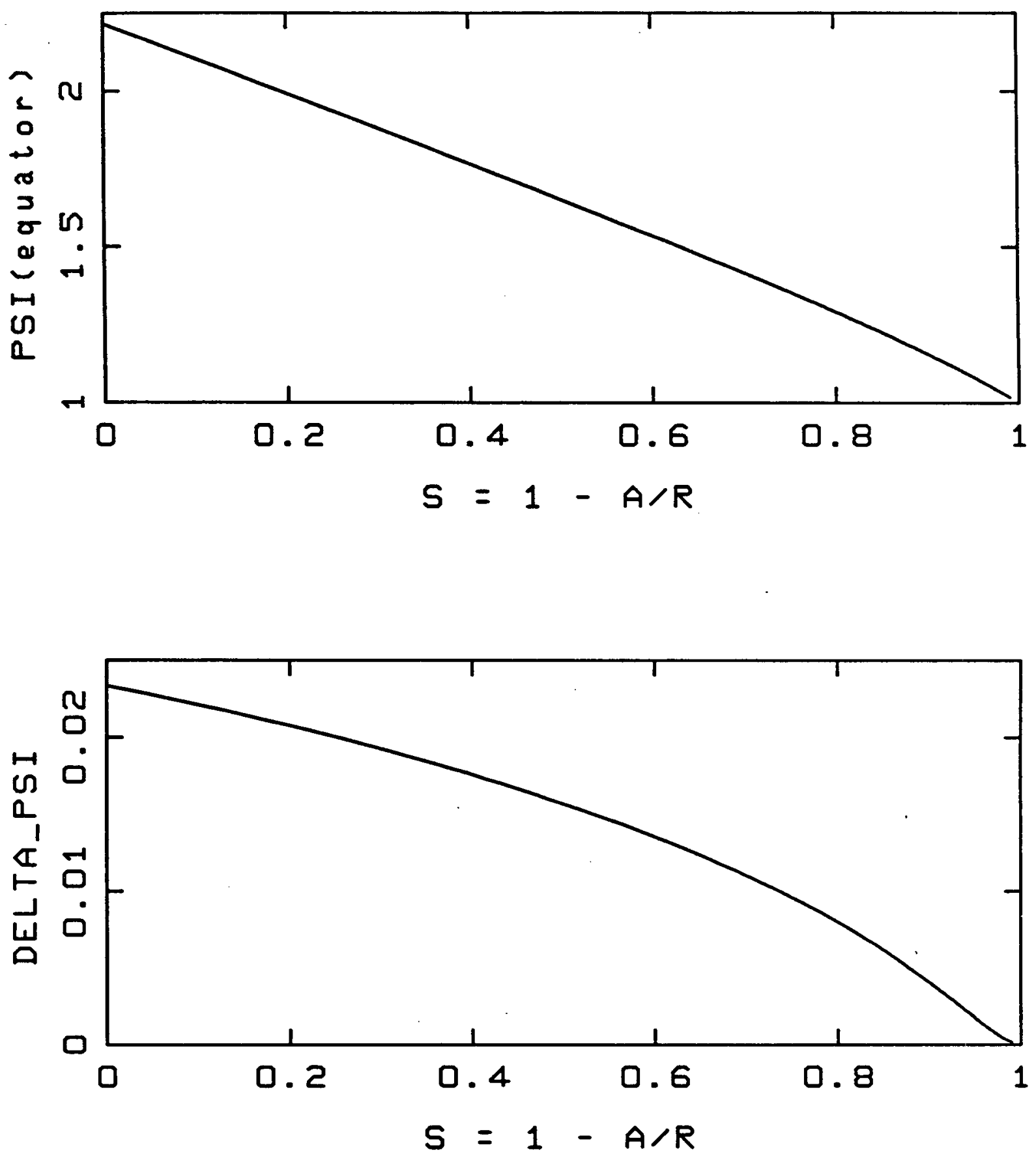

This figure shows $\Psi$ (equator) and $\delta \Psi=\Psi$ (north pole) $-\Psi$ (equator), both as functions of $s=1-a / r$. The former shows the general radial trend of $\Psi$, while the latter is a measure of the deviation of $\Psi$ from spherical symmetry. 
Choptuik comparison test series

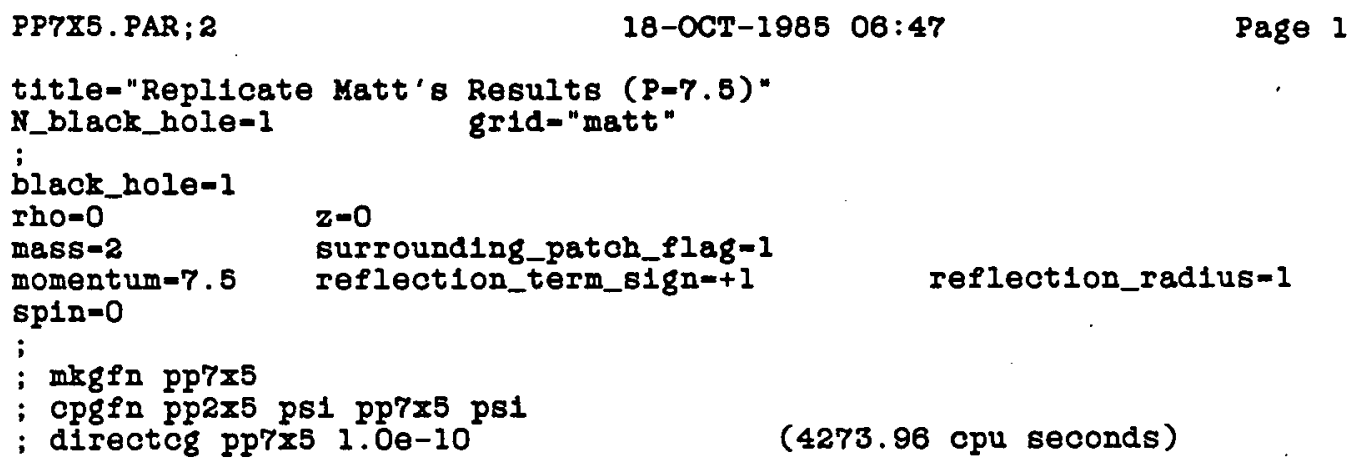


Figure 17 - Choptuik comparison test series, pp7×5 quantitative results
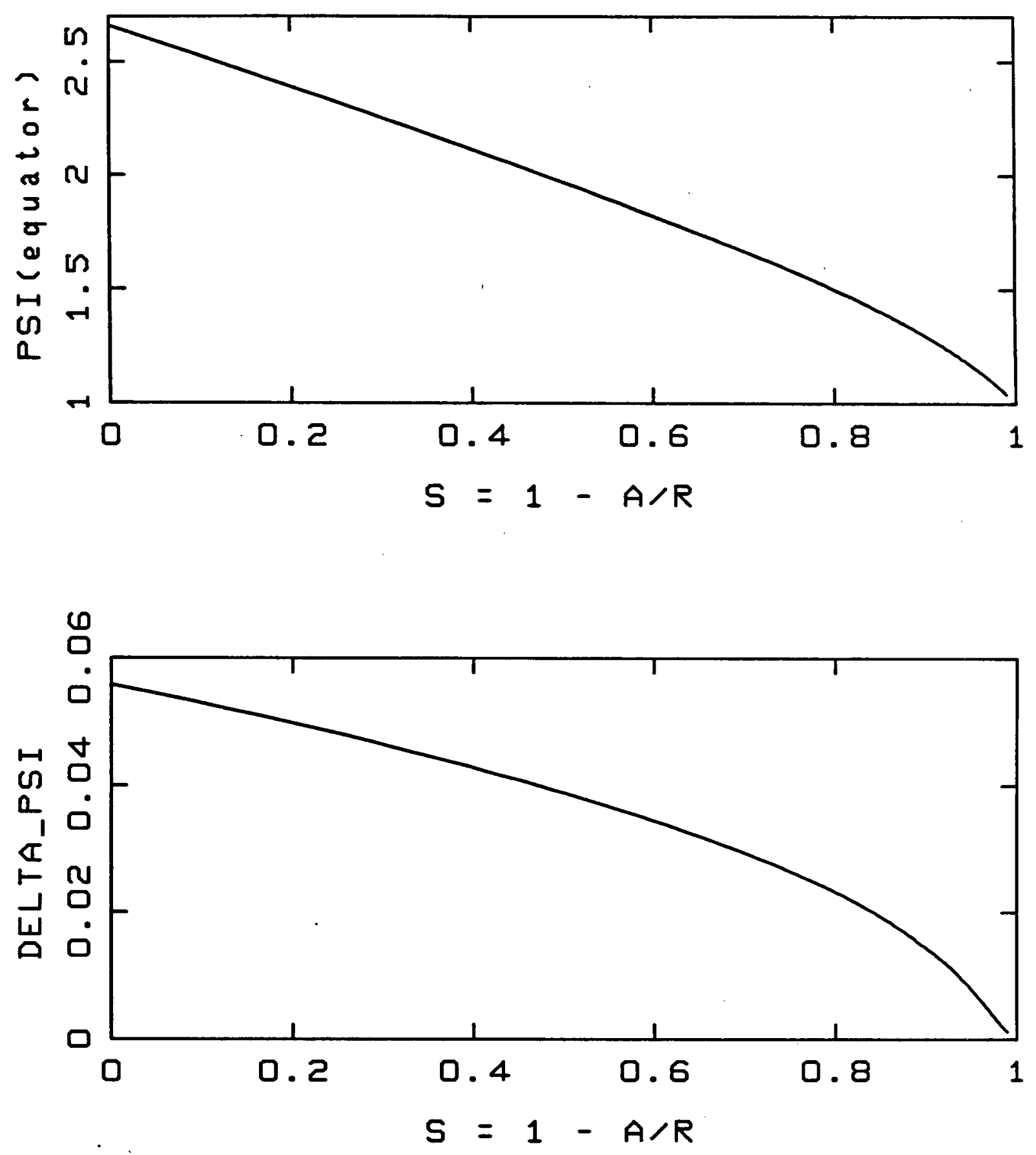

This figure shows $\Psi$ (equator) and $\delta \Psi=\Psi$ (north pole) $-\Psi$ (equator), both as functions of $s=1-a / r$. The former shows the general radial trend of $\Psi$, while the latter is a measure of the deviation of $\Psi$ from spherical symmetry. 
Choptuik comparison test series

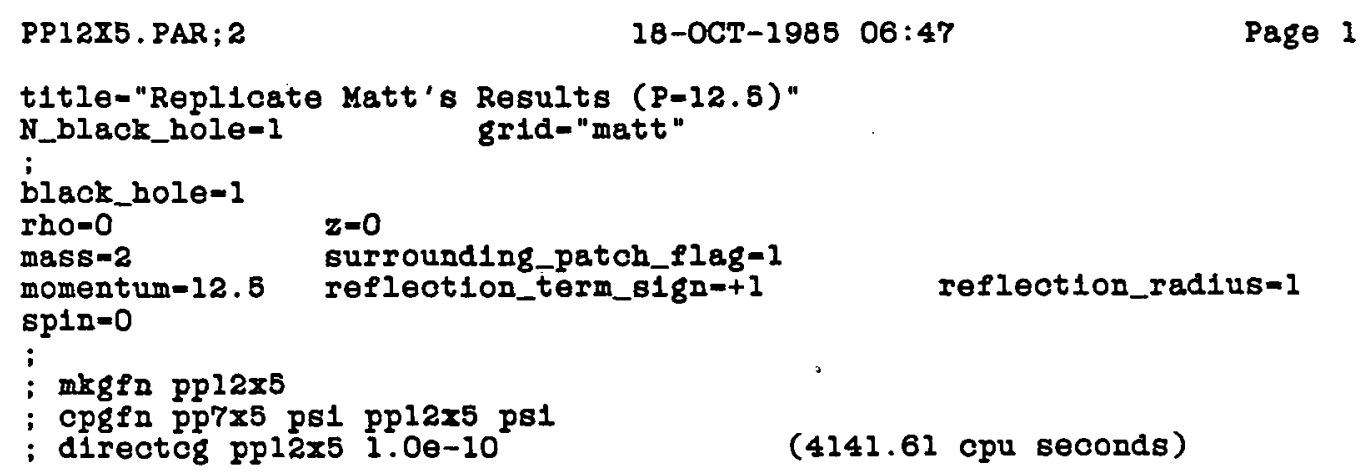


Figure 18 - Choptuik comparison test series, pp12r5 quantitative results
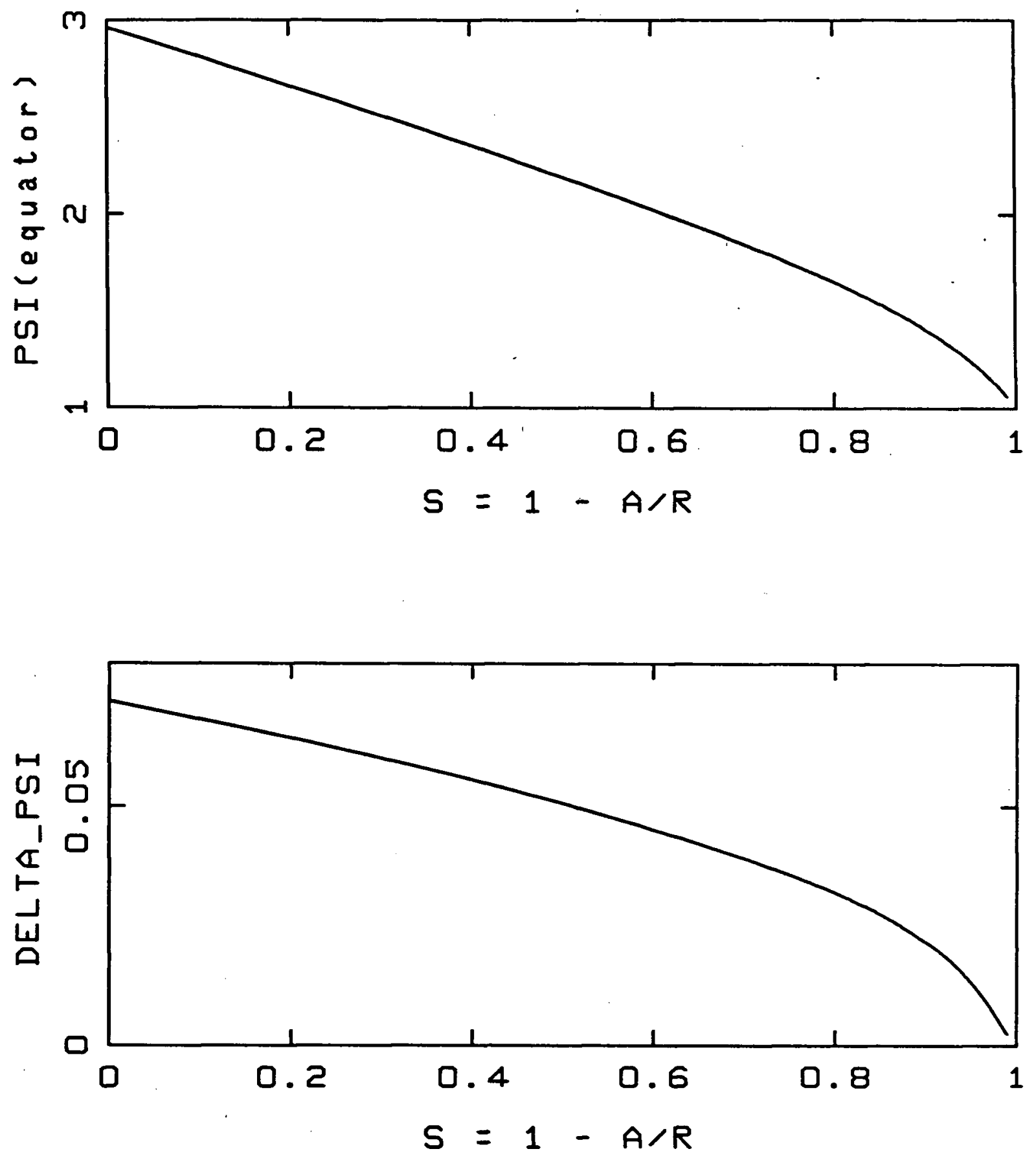

This figure shows $\Psi$ (equator) and $\delta \Psi=\Psi$ (north pole) $-\Psi$ (equator), both as functions of $s=1-a / r$. The former shows the general radial trend of $\Psi$, while the latter is a measure of the deviation of $\Psi$ from spherical symmetry. 


\section{Choptuik comparison test series}

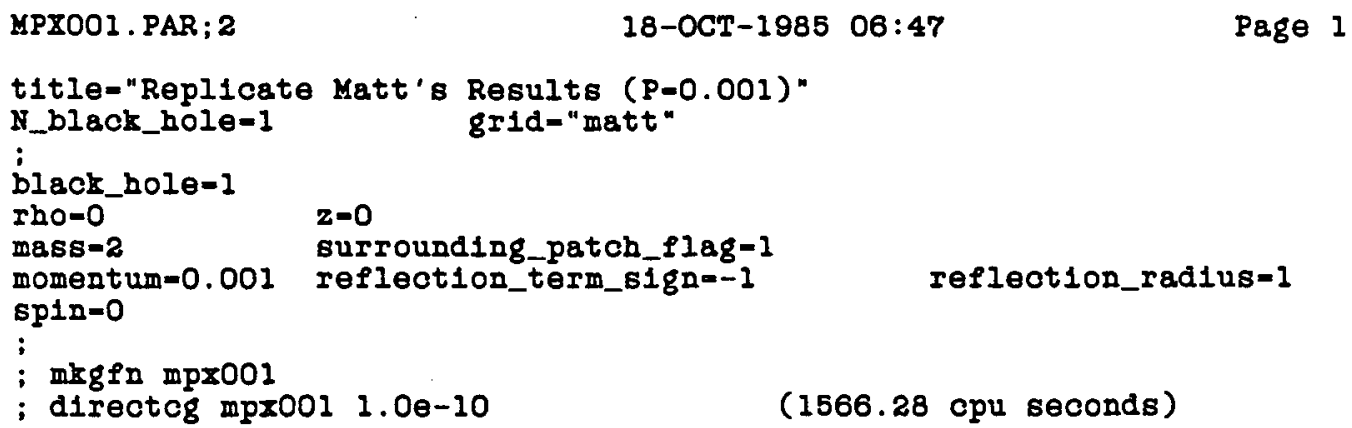


Figure 19 - Choptuik comparison test series, mpx001 quantitative resalts
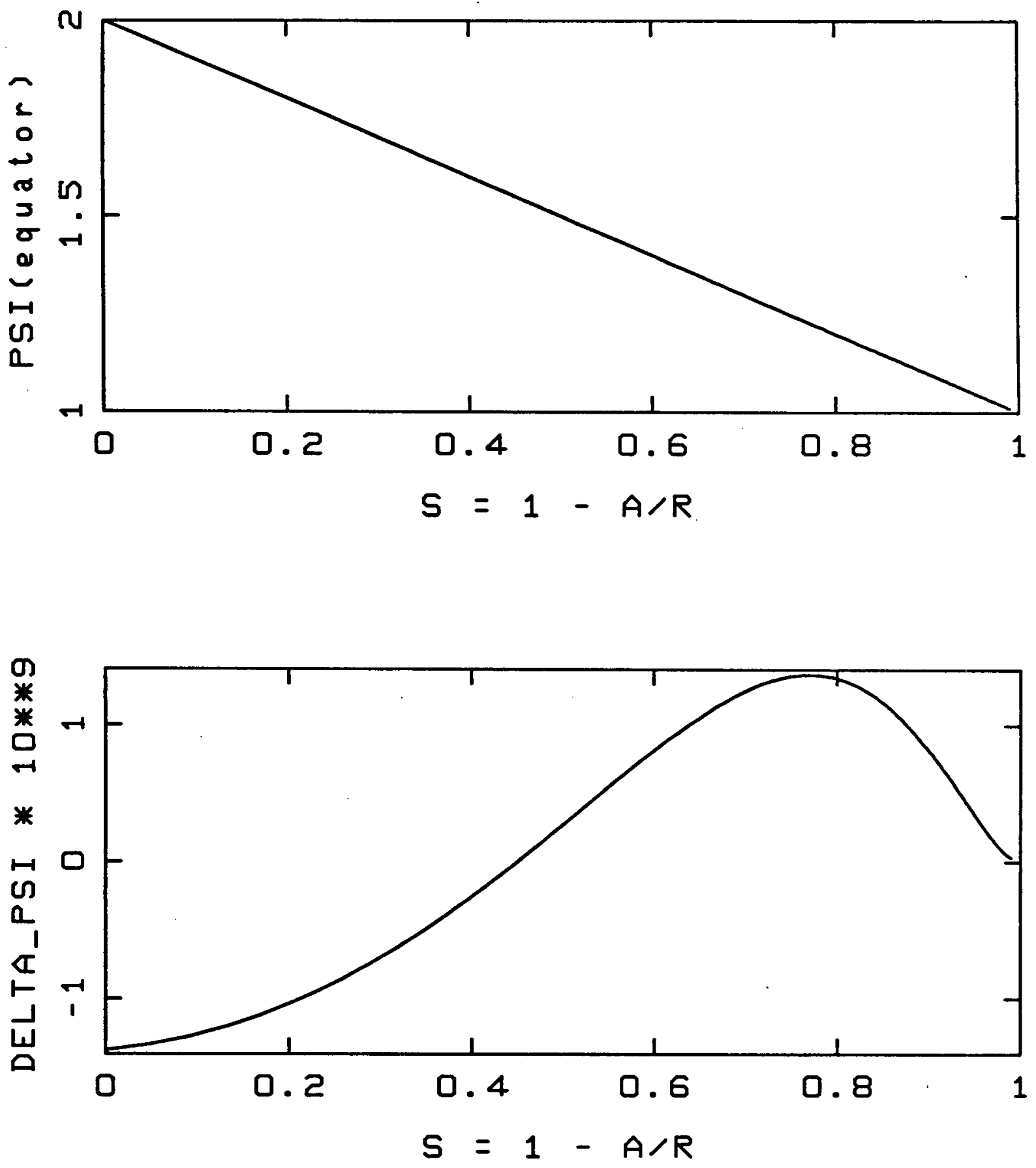

This figure shows $\Psi$ (equator) and $\delta \Psi=\Psi$ (north pole) $-\Psi$ (equator), both as functions of $s=1-a / r$. The former shows the general radial trend of $\Psi$, while the latter is a measure of the deviation of $\Psi$ from spherical symmetry. 
Choptuik comparison test series

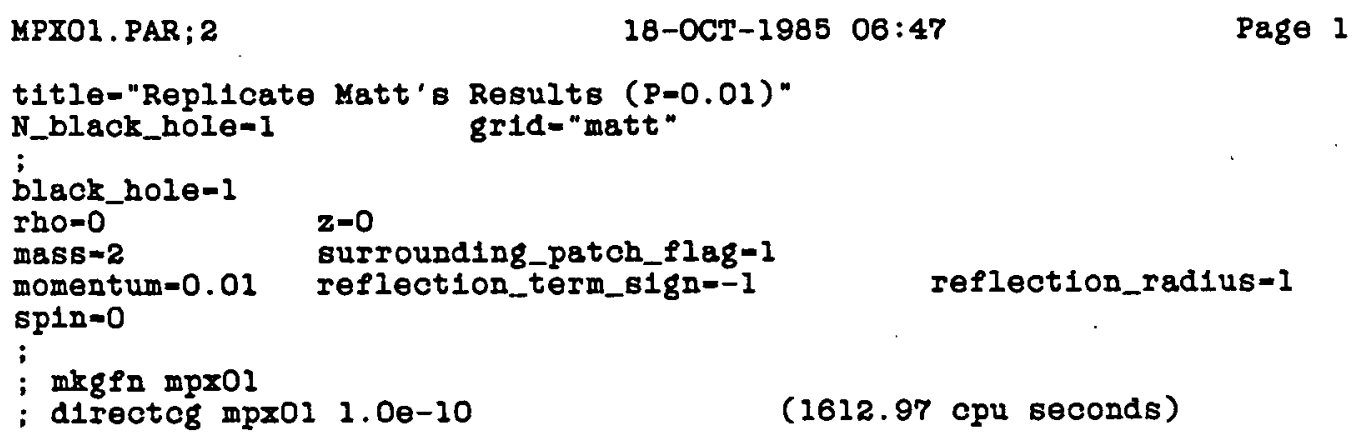


Figare 20 - Choptnik comparison test series, mpx01 quantitative results
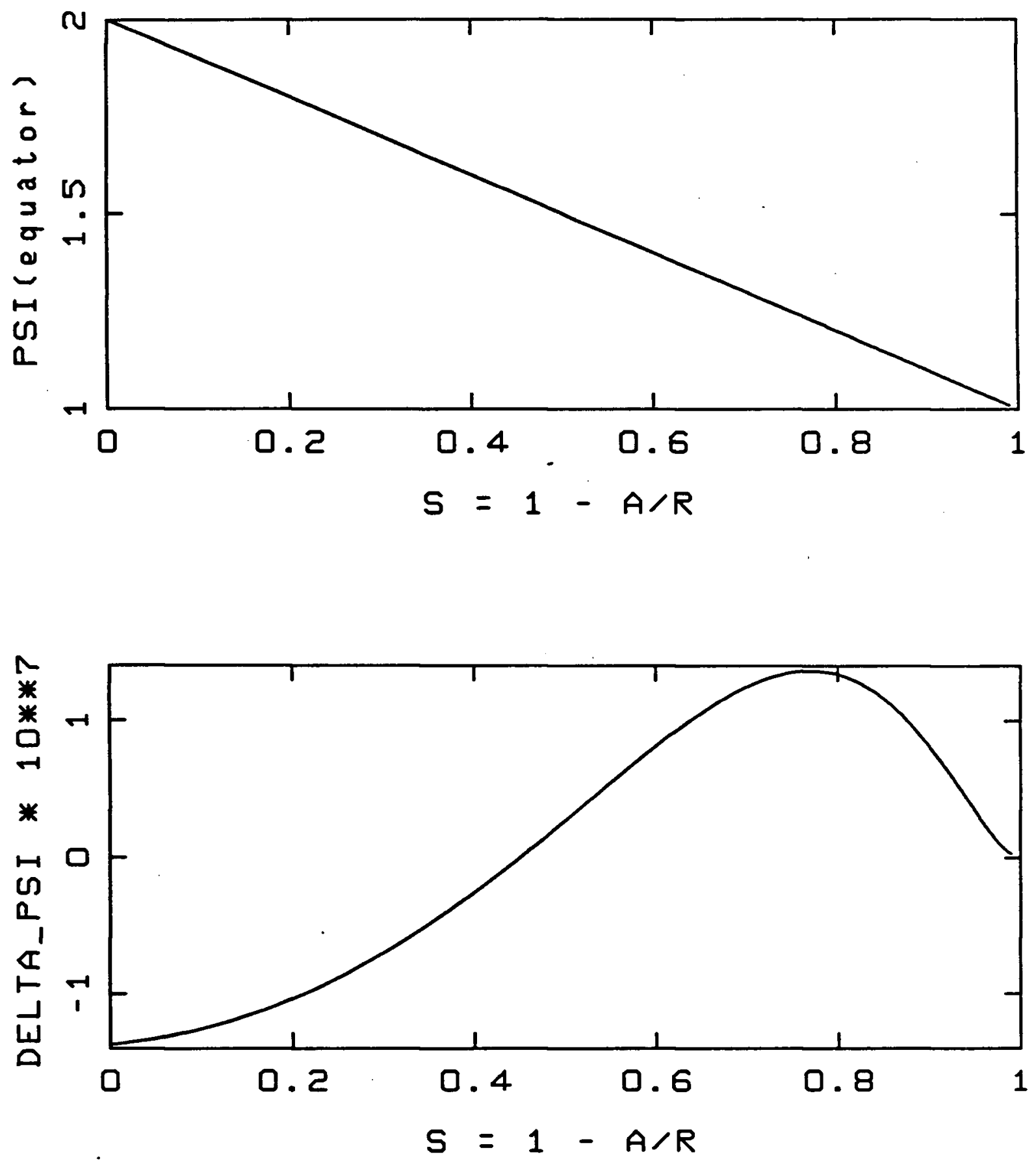

This figure shows $\Psi$ (equator) and $\delta \Psi=\Psi$ (north pole) $-\Psi$ (equator), both as functions of $s=1-a / r$. The former shows the general radial trend of $\Psi$, while the latter is a measure of the deviation of $\Psi$ from spherical symmetry. 
Choptuik comparison test series

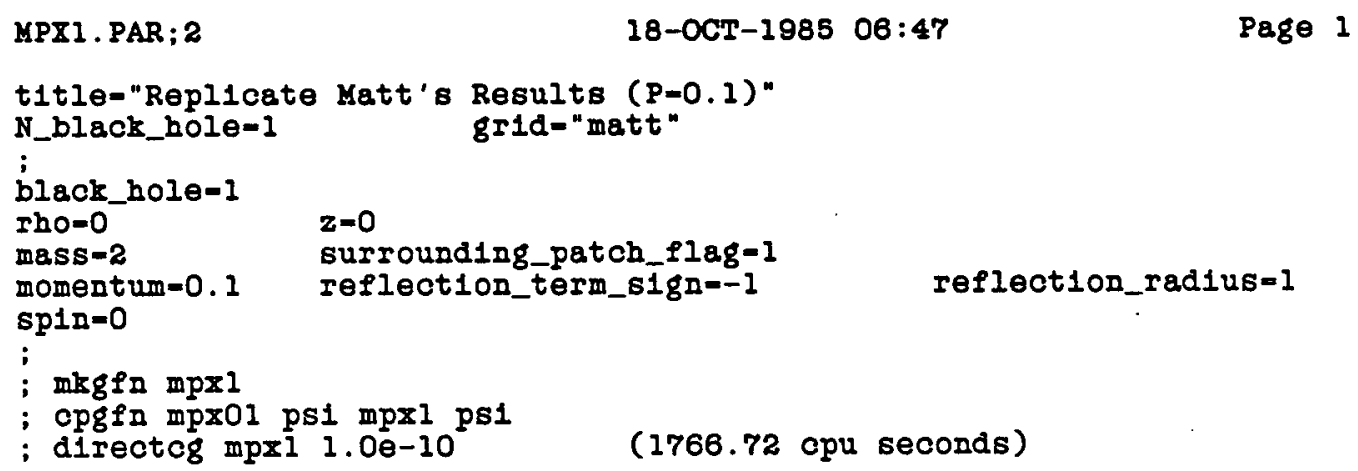


Figure 21 - Choptuik comparison test series, mpx1 quantitative results
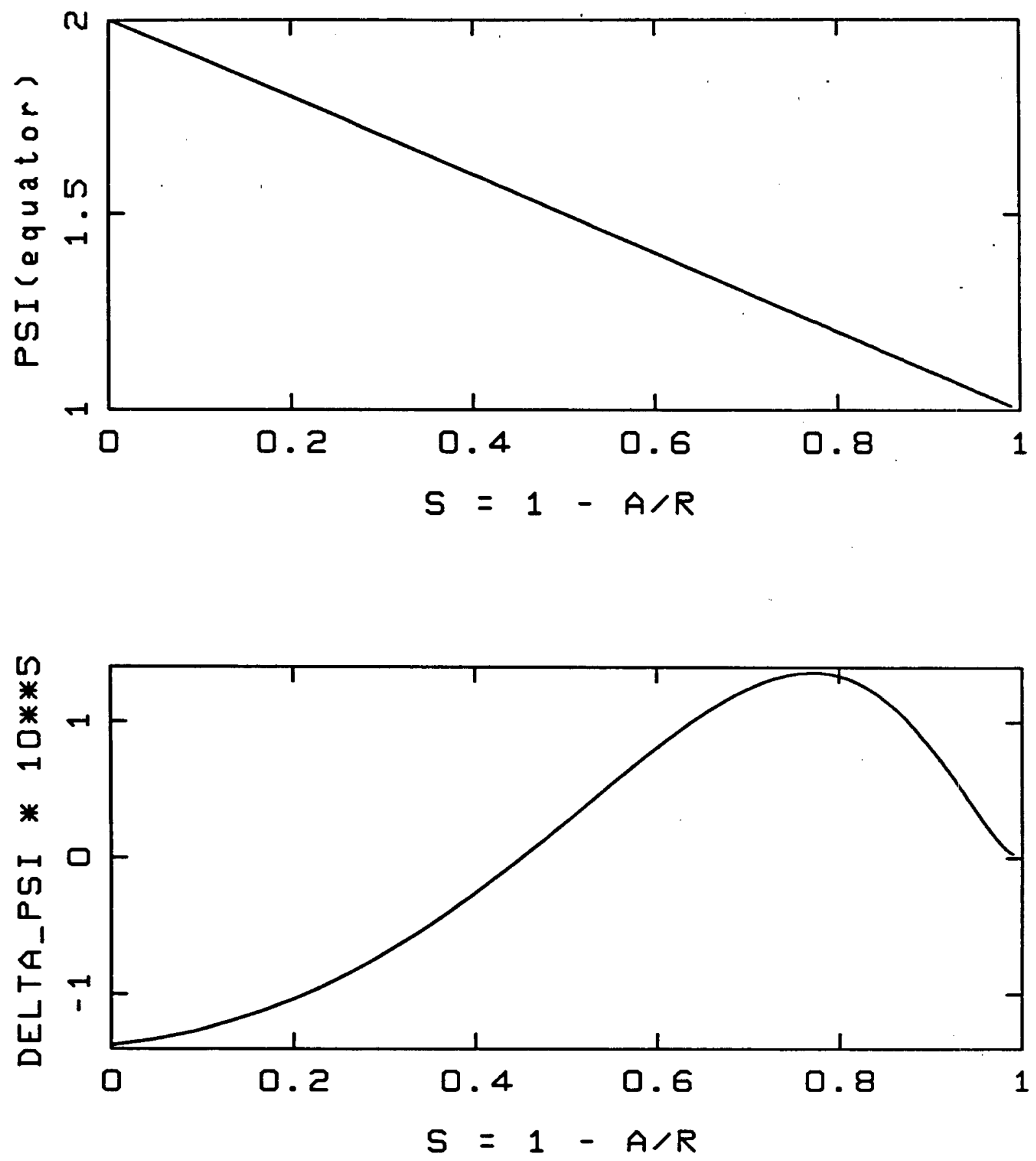

This figure shows $\Psi$ (equator) and $\delta \Psi=\Psi$ (north pole) $-\boldsymbol{\Psi}$ (equator), both as functions of $s=1-a / r$. The former shows the general radial trend of $\Psi$, while the latter is a measure of the deviation of $\Psi$ from spherical symmetry. 
Choptuik comparison test series

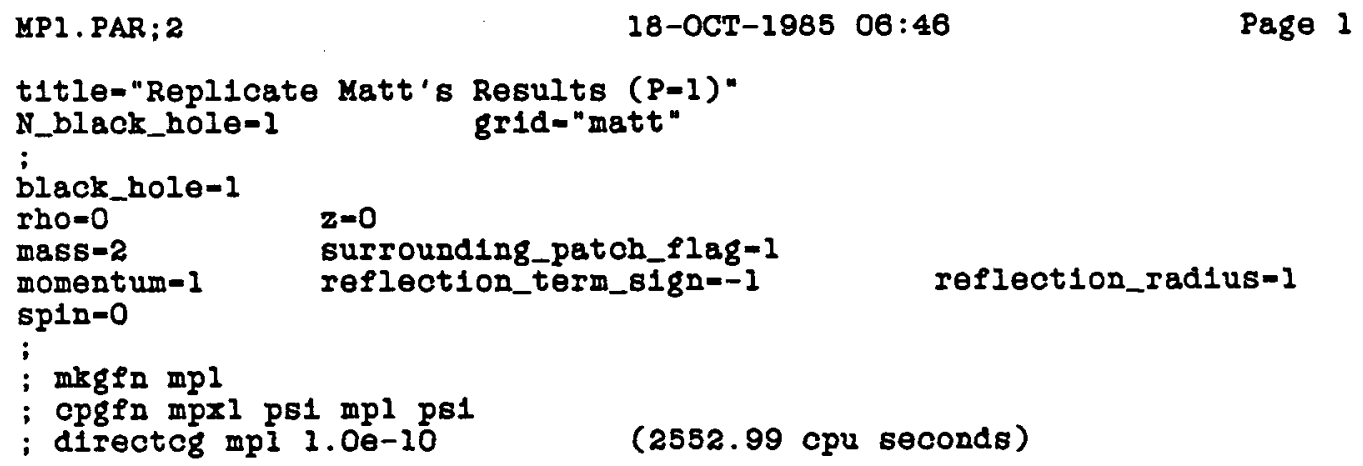


Figure 22 - Choptuik comparison test series, mp1 quantitative results
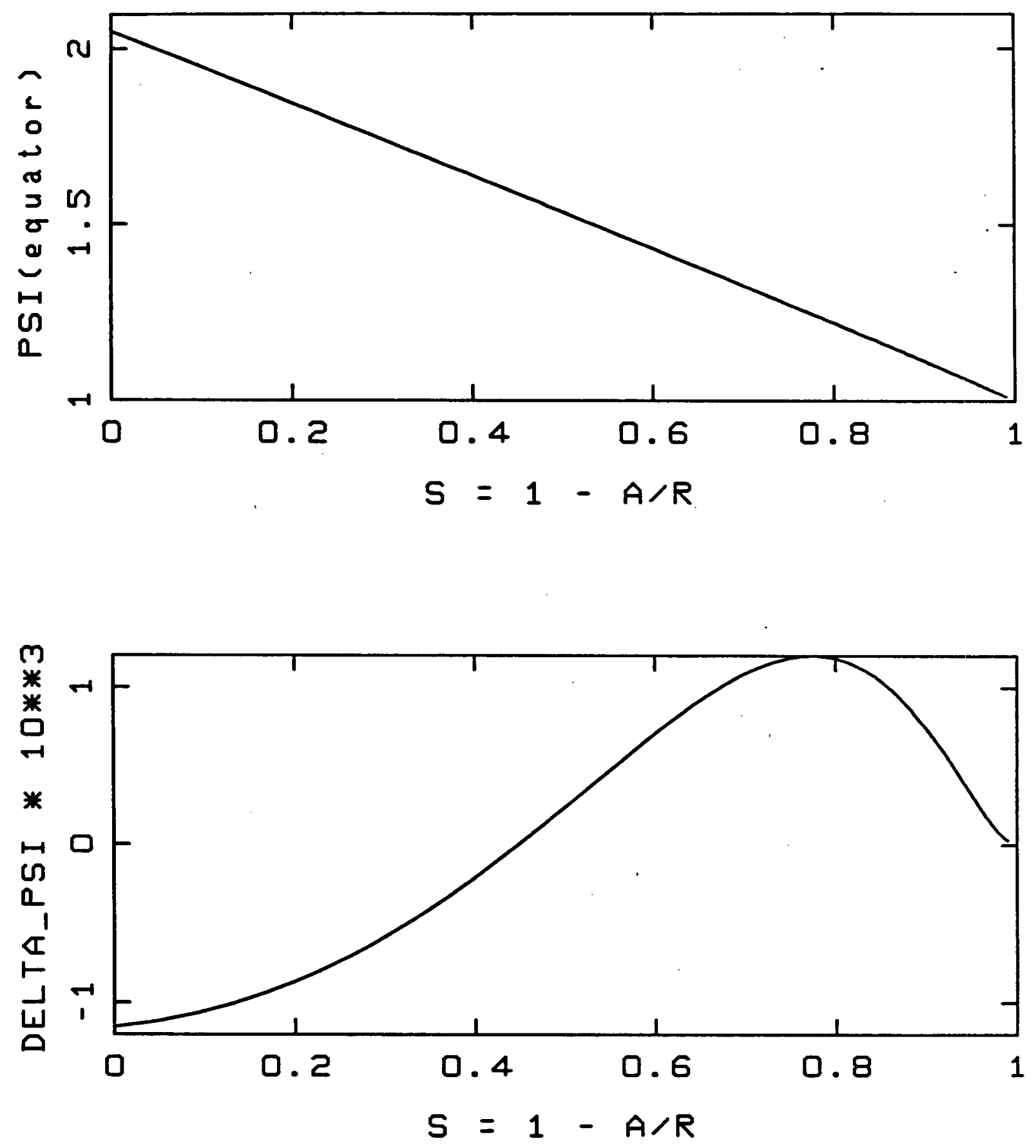

This figure shows $\Psi$ (equator) and $\delta \Psi=\Psi$ (north pole) $-\boldsymbol{\Psi}$ (equator), both as functions of $s=1-a / r$. The former shows the general radial trend of $\Psi$, while the latter is a measure of the deviation of $\Psi$ from spherical symmetry. 


\section{Choptuik comparison test series}

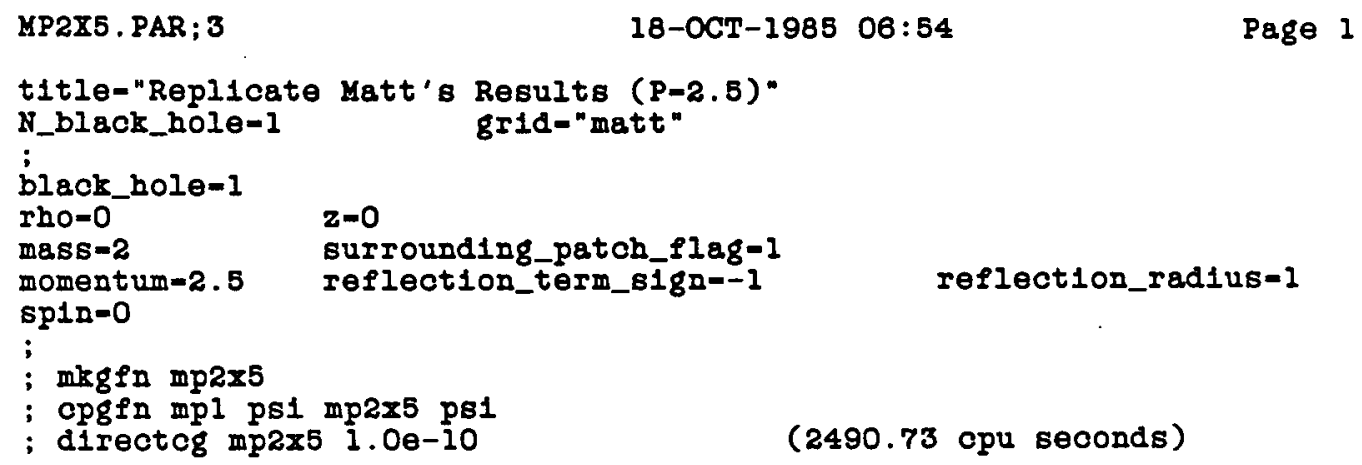


Figure 23 - Choptuik comparison test series, mp2x5 quantitative results
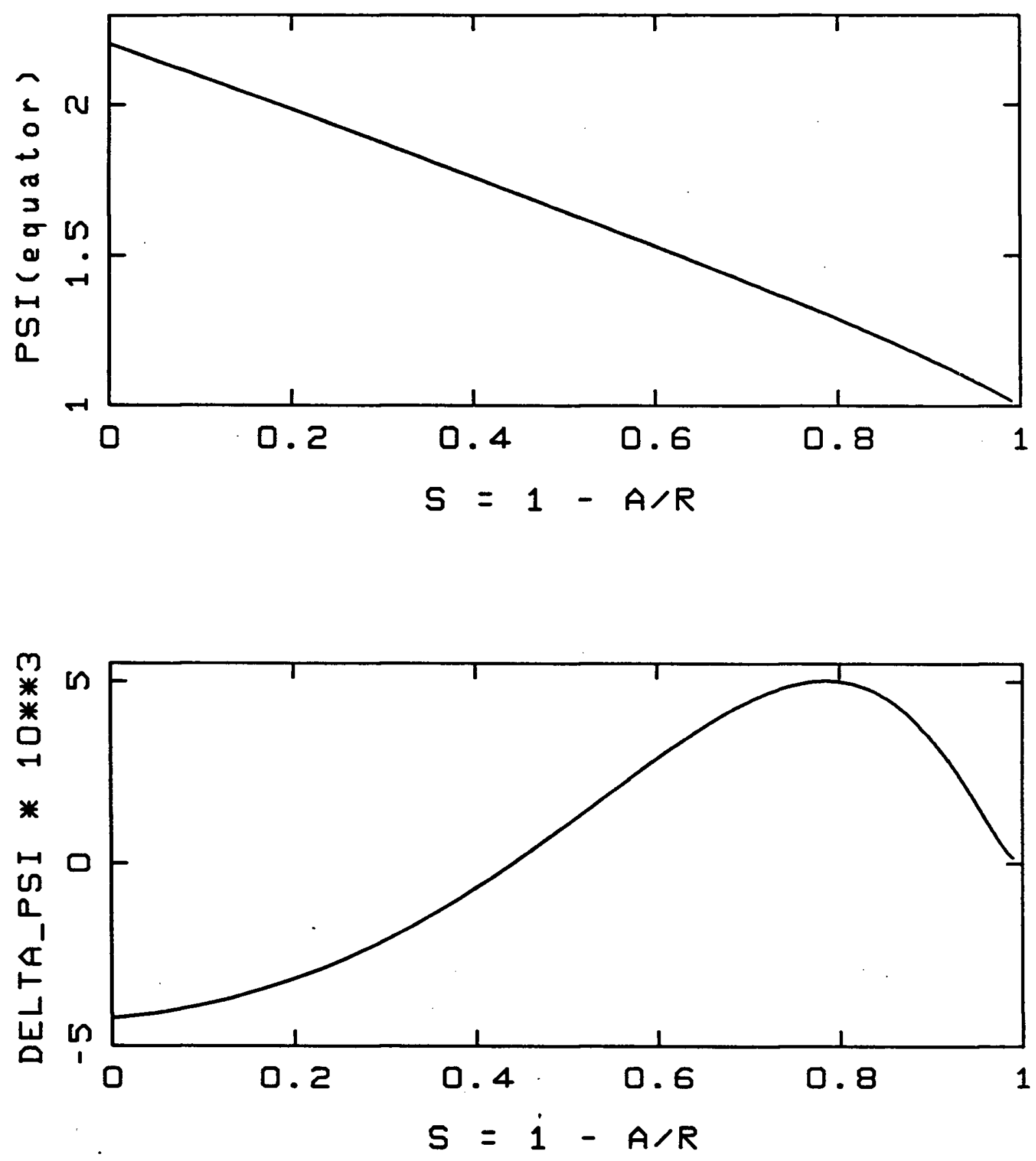

This figure shows $\Psi$ (equator) and $\delta \Psi=\Psi$ (north pole) $-\Psi$ (equator), both as functions of $s=1-a / r$. The former shows the general radial trend of $\Psi$, while the latter is a measure of the deviation of $\Psi$ from spherical symmetry. 


\section{Choptuik comparison test series}

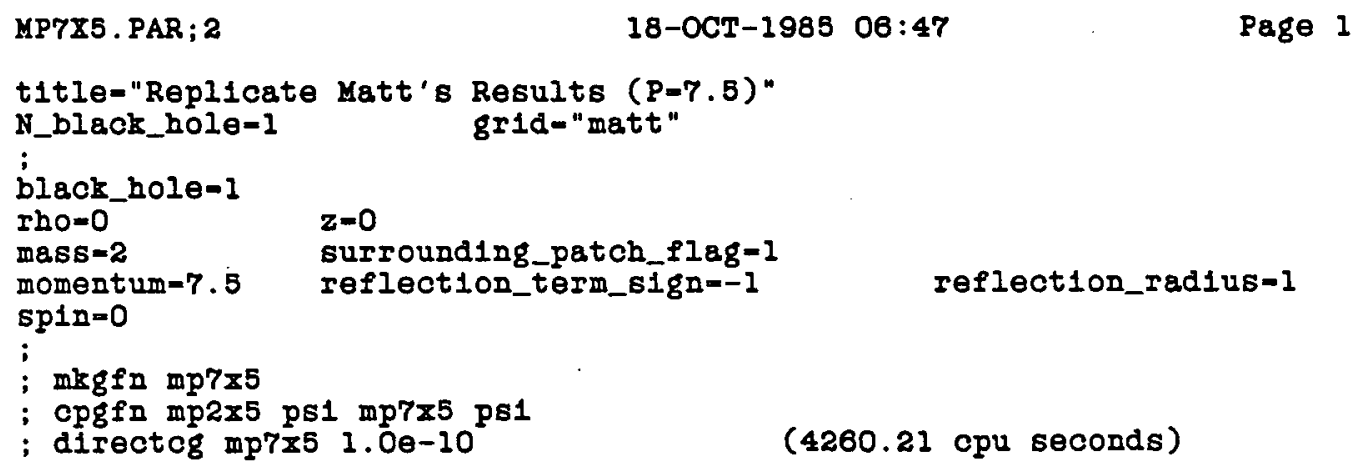


Figure 24 - Choptuik comparison test series, mp7×5 quantitative results
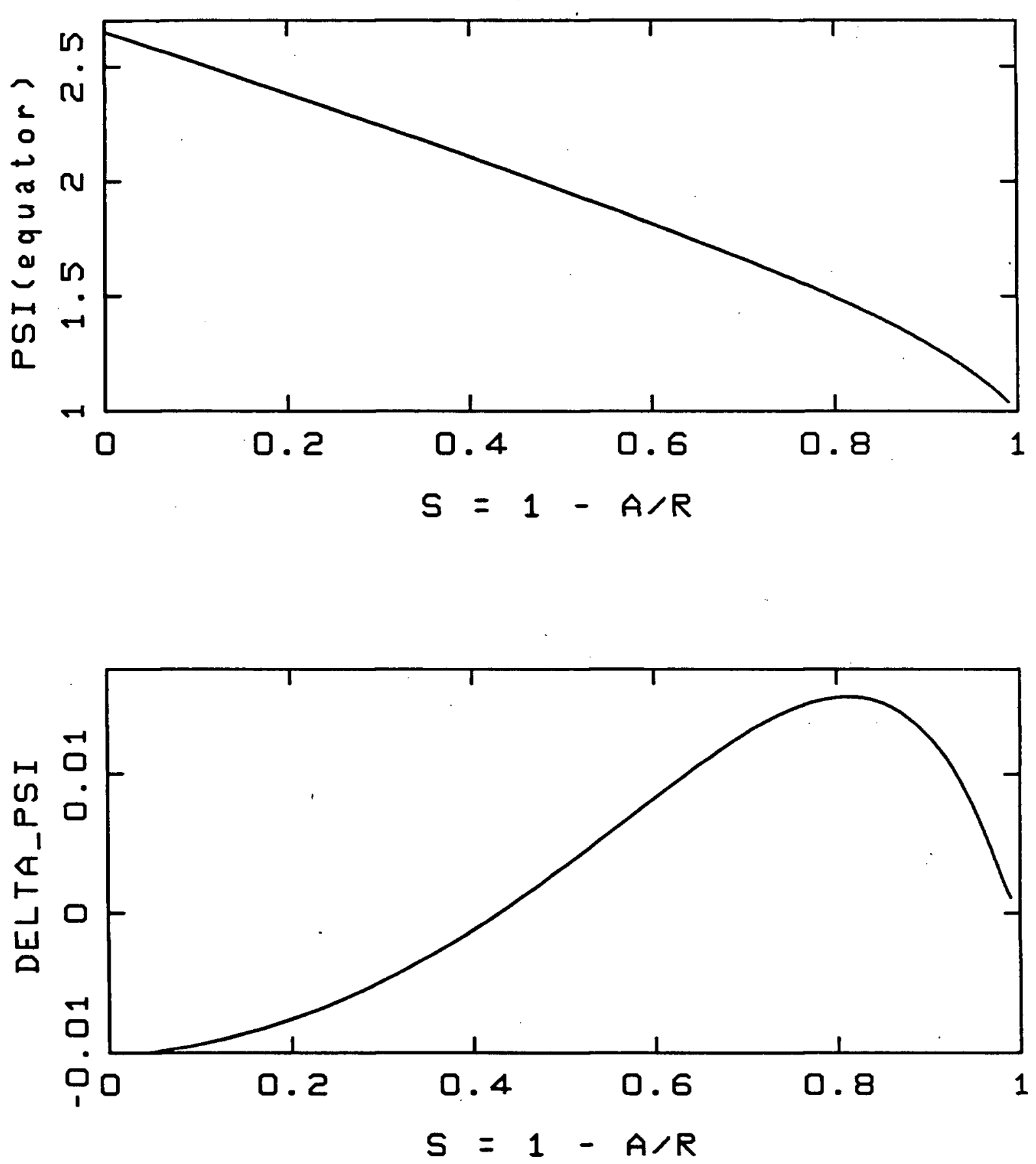

This figure shows $\Psi$ (equator) and $\delta \Psi=\Psi$ (north pole) $-\boldsymbol{\Psi}$ (equator), both as functions of $s=1-a / r$. The former shows the general radial trend of $\Psi$, while the latter is a measure of the deviation of $\Psi$ from spherical symmetry. 
Choptnik comparison test series

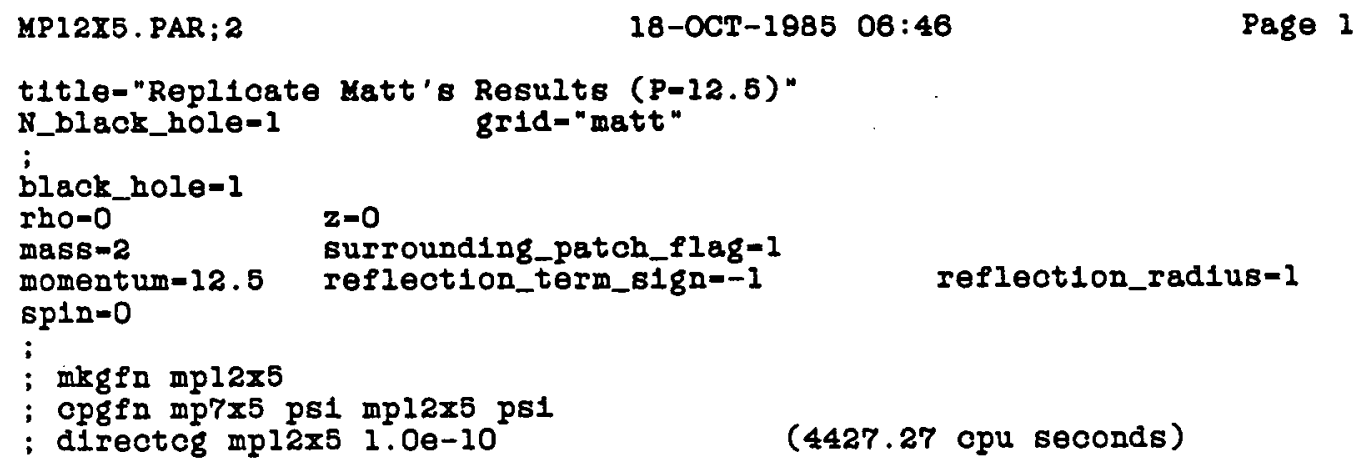


Figure 25 - Choptnik comparison test geries, mp12x5 quantitative results
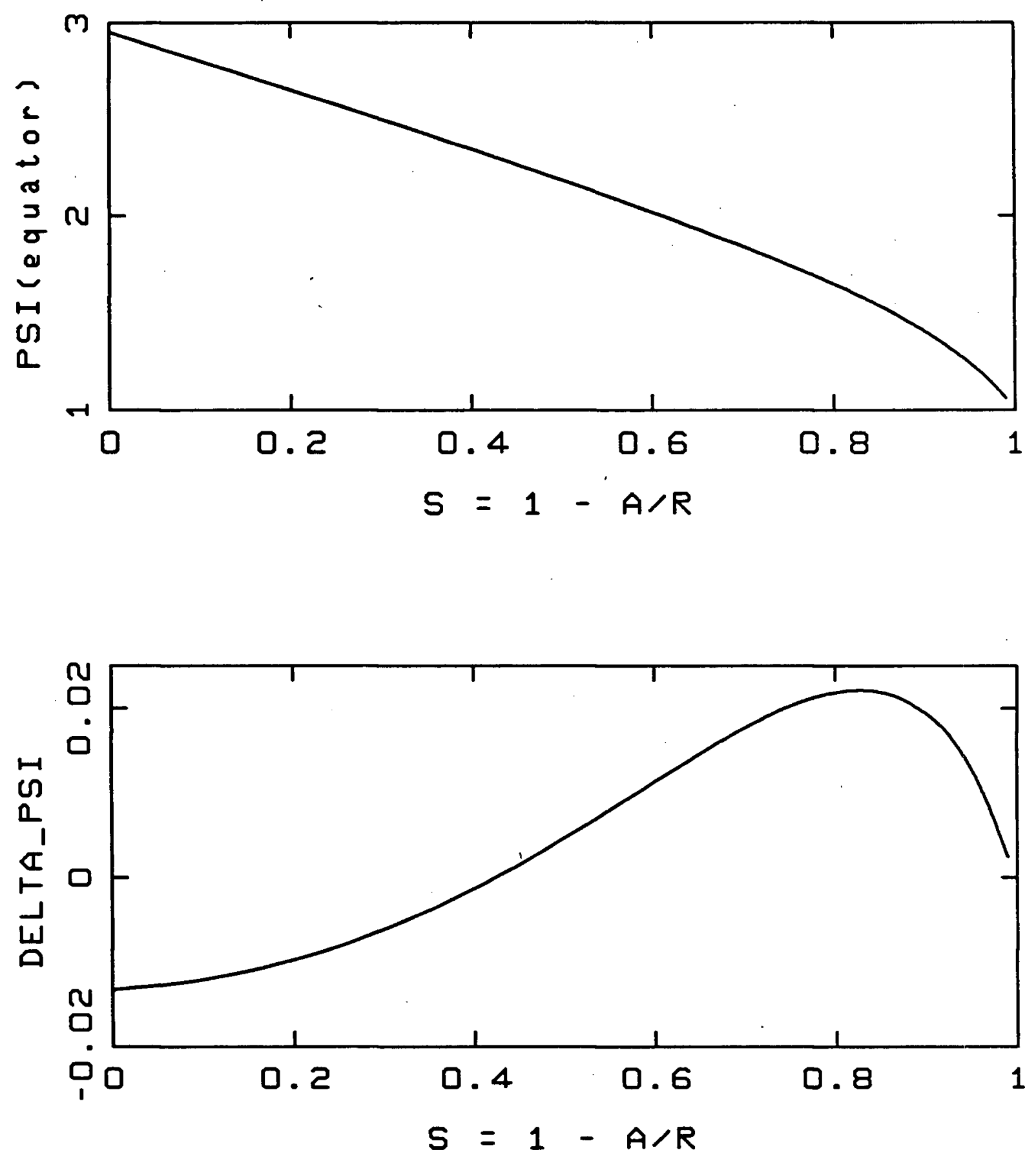

This figure shows $\Psi$ (equator) and $\delta \Psi=\Psi$ (north pole) $-\Psi$ (equator), both as functions of $s=1-a / r$. The former shows the general radial trend of $\Psi$, while the latter is a measure of the deviation of $\Psi$ from spherical symmetry. 


\section{Choptuik comparison test series}

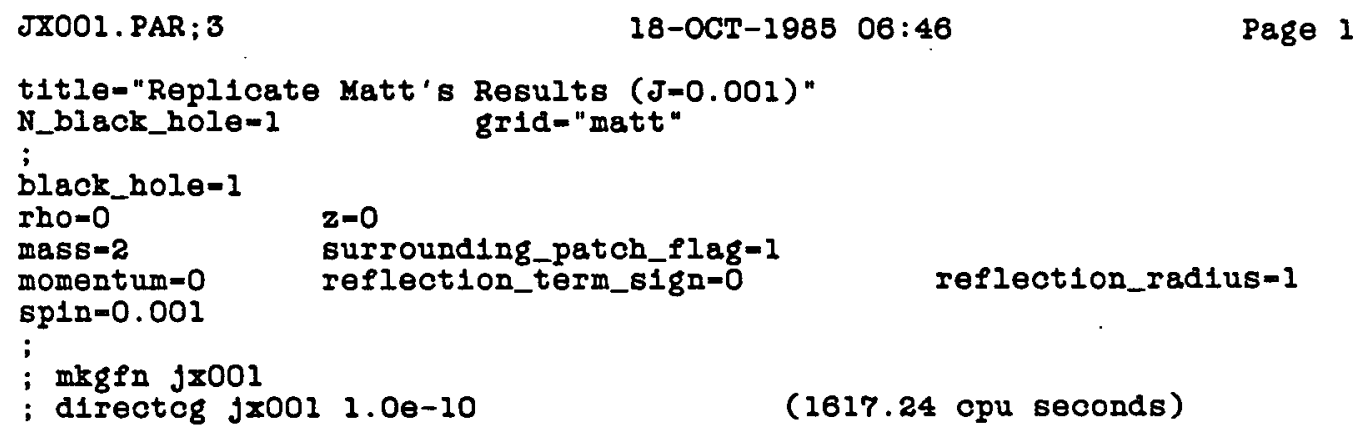


Figure 26 - Choptuik comparison test series, jx001 quantitative results
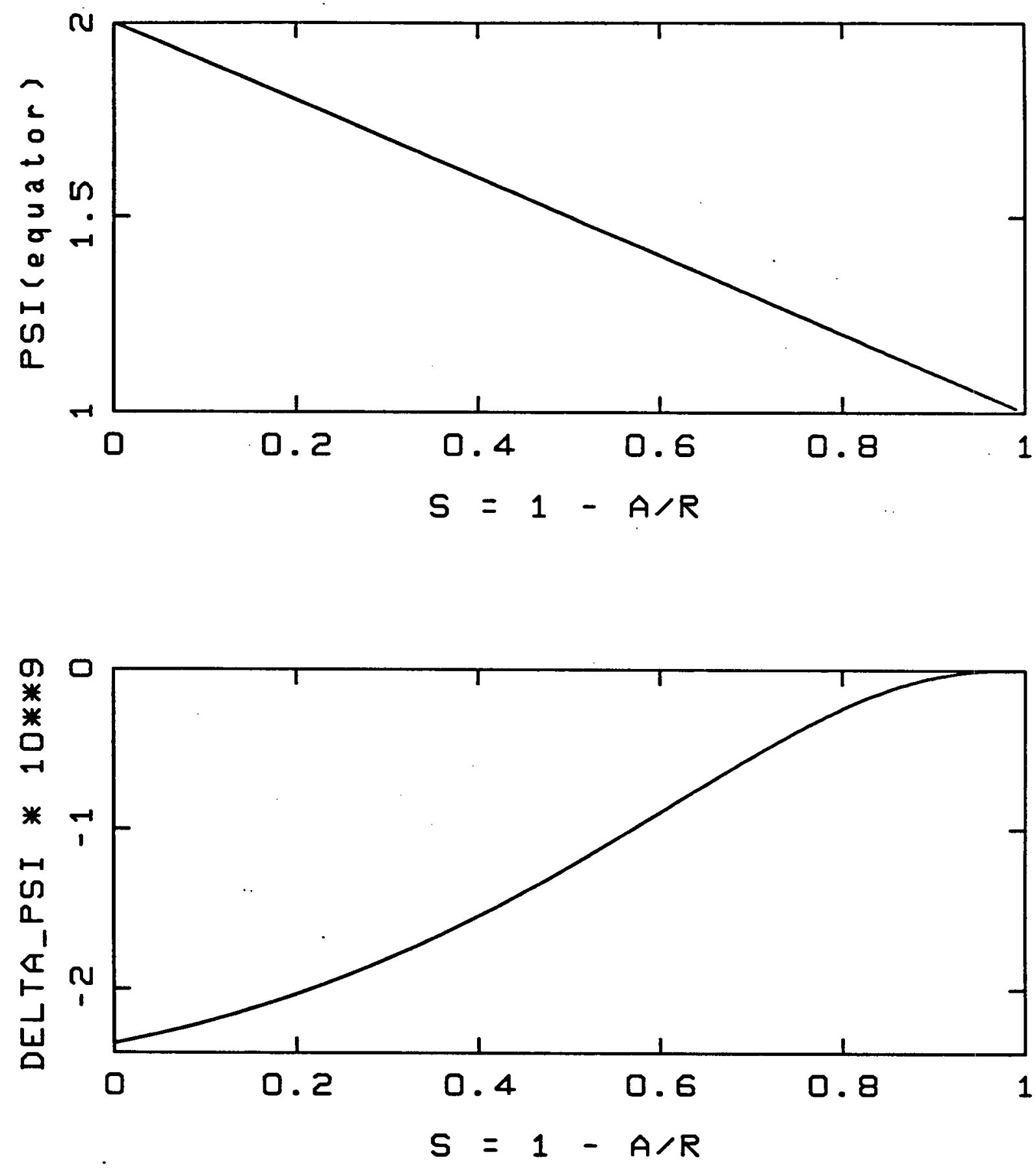

This figure shows $\Psi$ (equator) and $\delta \Psi=\Psi$ (north pole) $-\Psi$ (equator), both as functions of $s=1-a / r$. The former shows the general radial trend of $\Psi$, while the latter is a measure of the deviation of $\Psi$ from spherical symmetry. 


\section{Choptuik comparison test series}

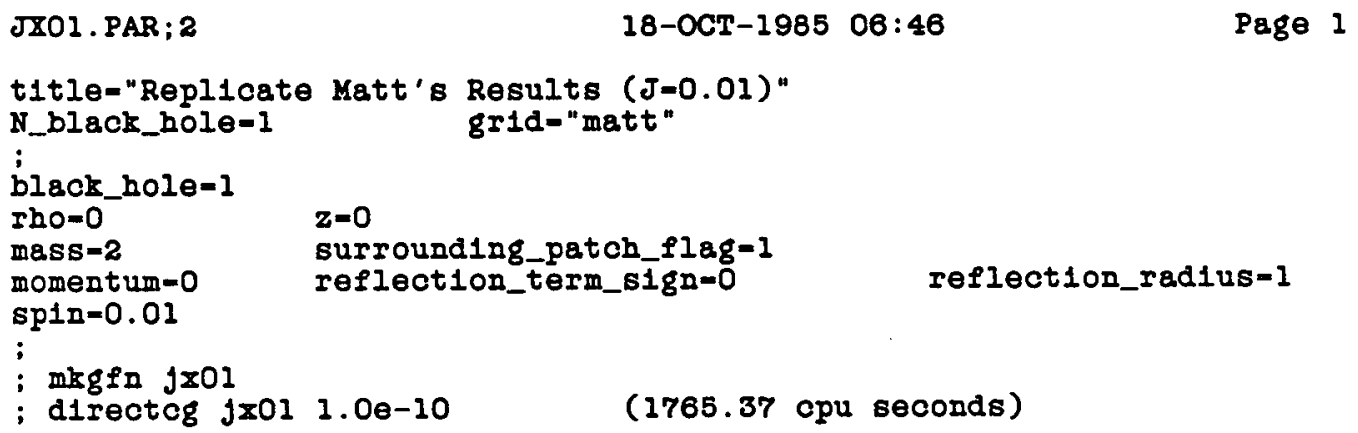


Figure 27 - Choptuik comparison test series, jx01 quantitative results
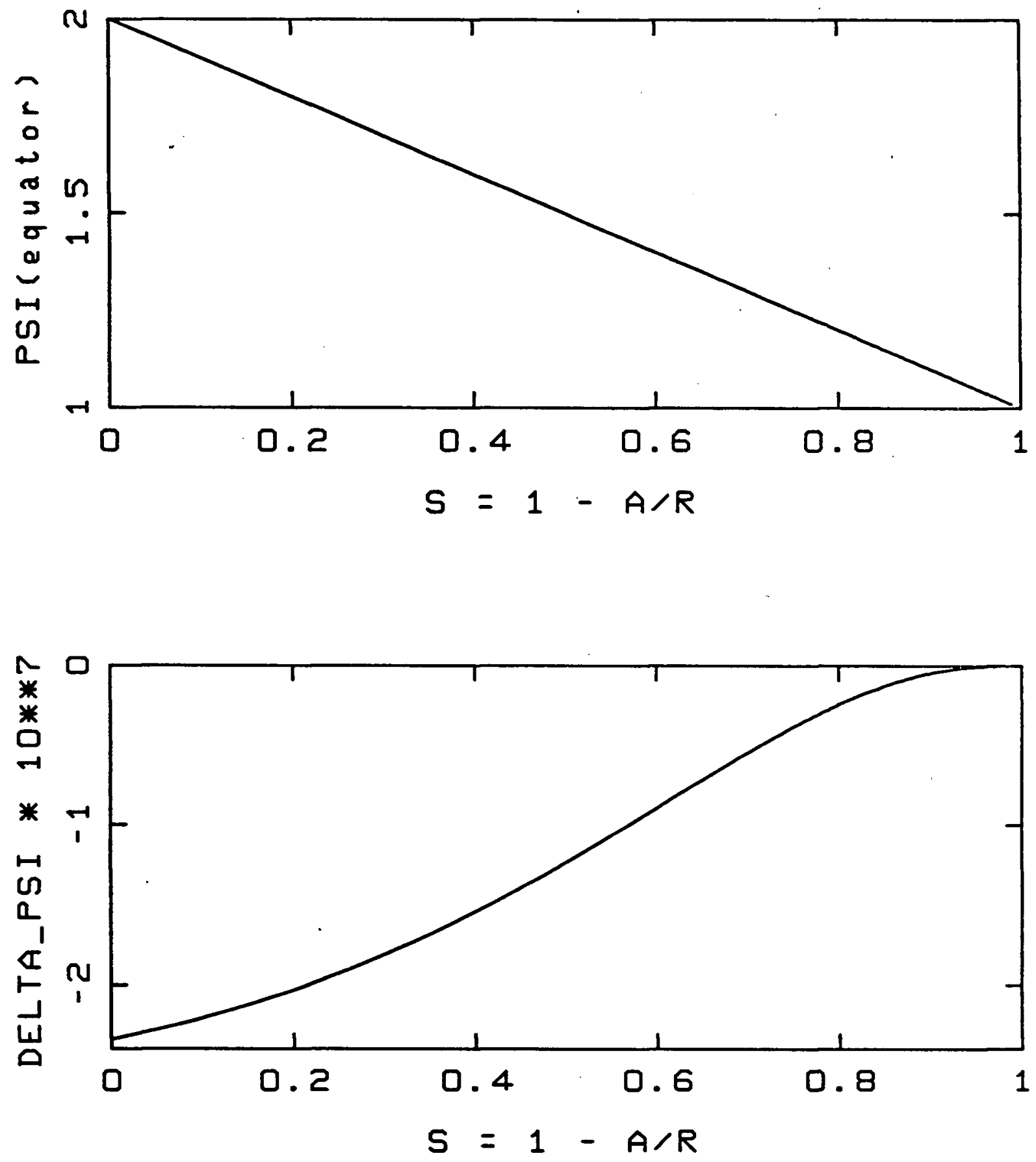

This figure shows $\Psi$ (equator) and $\delta \Psi=\Psi$ (north pole) $-\Psi$ (equator), both as functions of $s=1-a / r$. The former shows the general radial trend of $\Psi$, while the latter is a measure of the deviation of $\Psi$ from spherical symmetry. 
Choptuik comparison test series

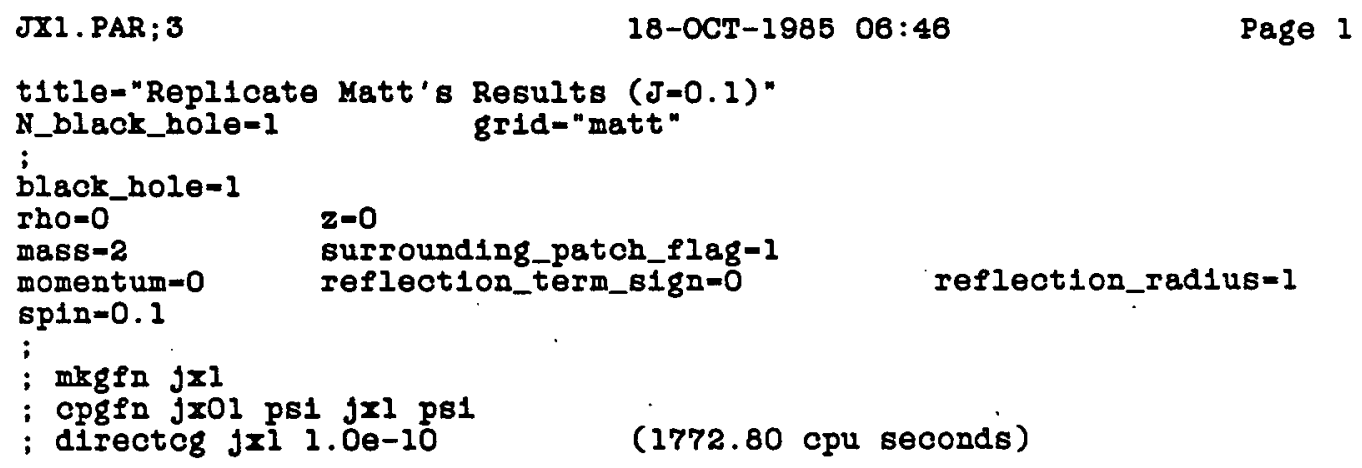


Figure 28 - Choptnik comparison test series, jx1 quantitative results
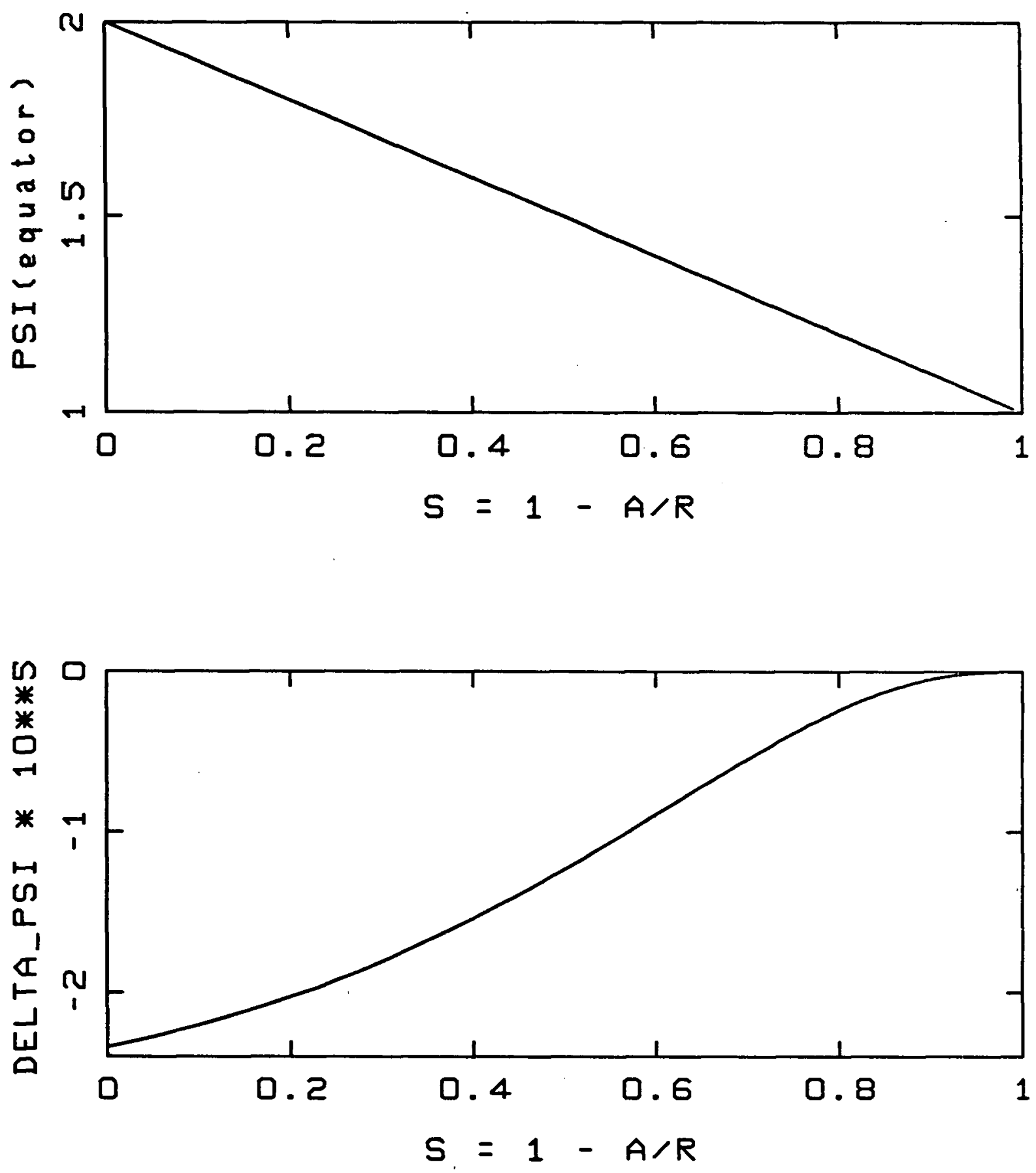

This figure shows $\Psi$ (equator) and $\delta \Psi=\Psi$ (north pole) $-\Psi$ (equator), both as functions of $s=1-a / r$. The former shows the general radial trend of $\Psi$, while the latter is a measure of the deviation of $\Psi$ from spherical symmetry. 


\section{Choptuik comparison test series}

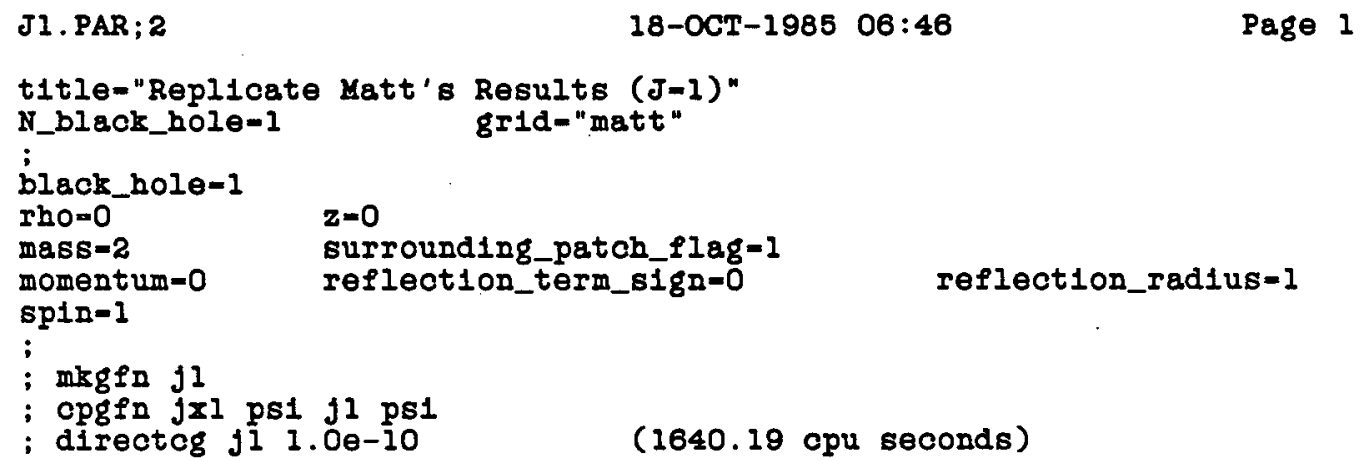


Figure 29 - Choptuils comparison test geries, 11 quantitative results
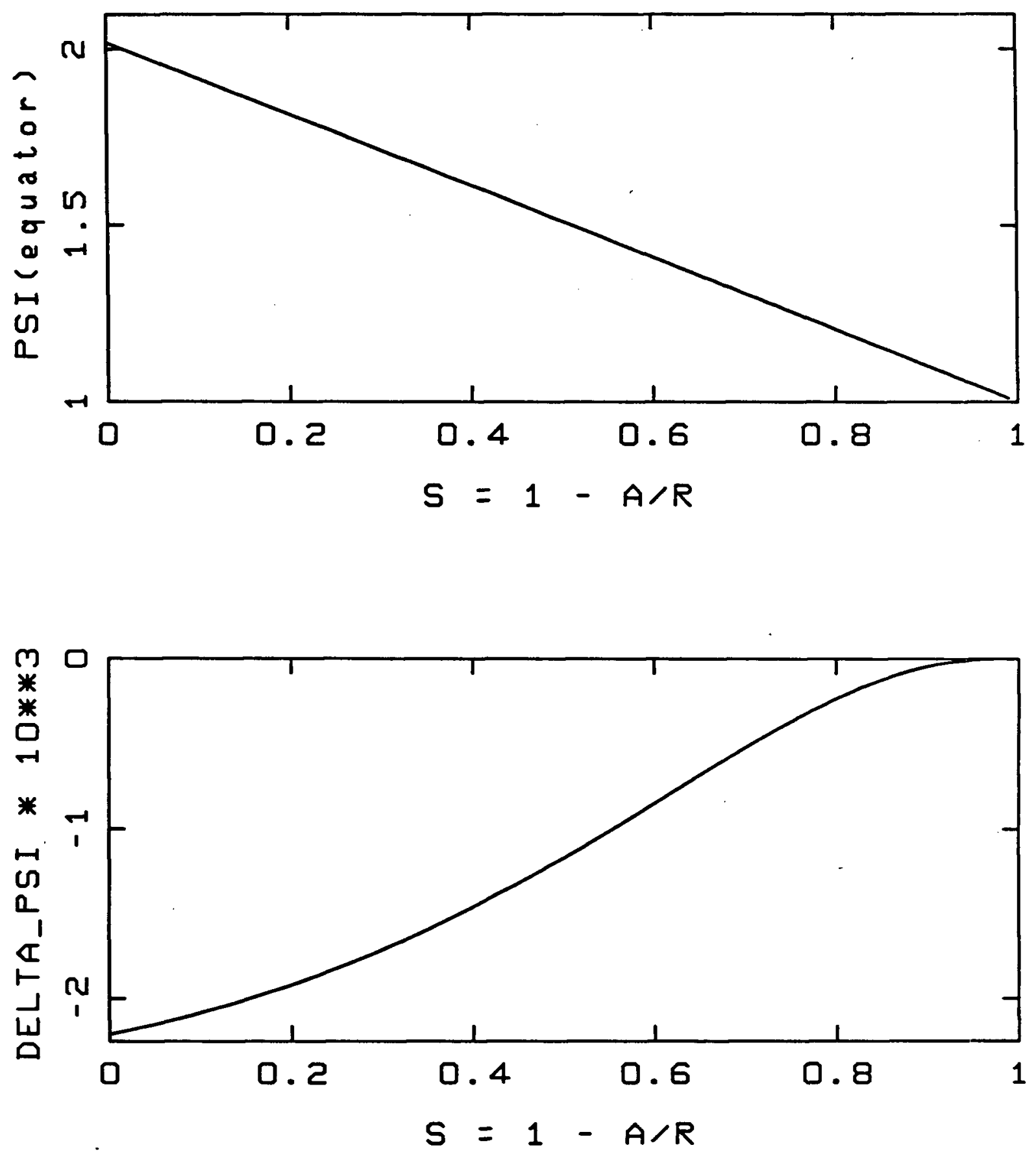

This figure shows $\Psi$ (equator) and $\delta \Psi=\Psi$ (north pole) $-\Psi$ (equator), both as functions of $s=1-a / r$. The former shows the general radial trend of $\Psi$, while the latter is a measure of the deviation of $\Psi$ from spherical symmetry. 
Choptuik comparison test series

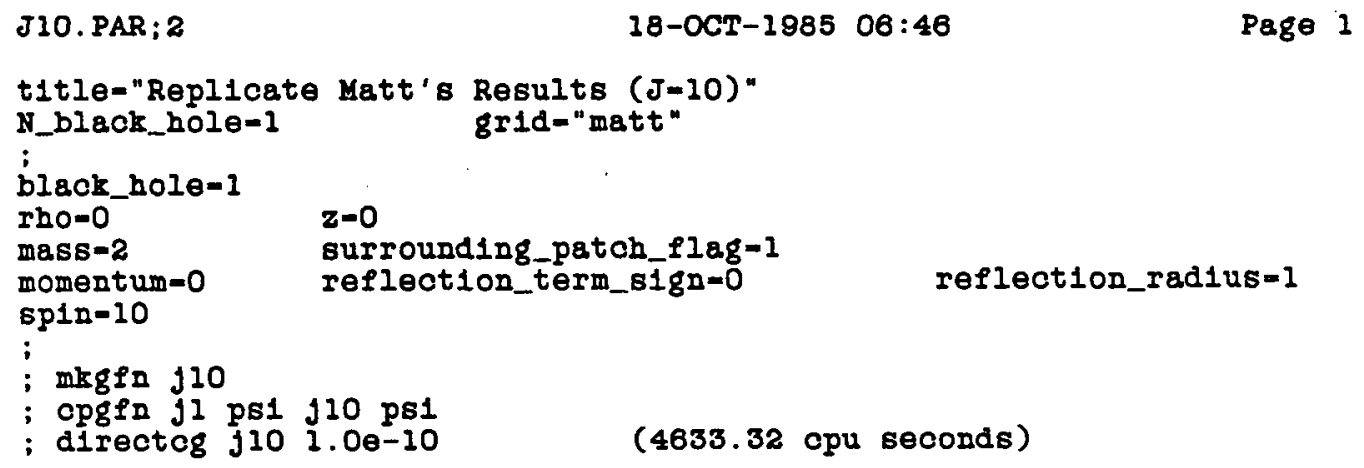


Figure 30 - Choptnik comparison test series, $j 10$ quantitative results
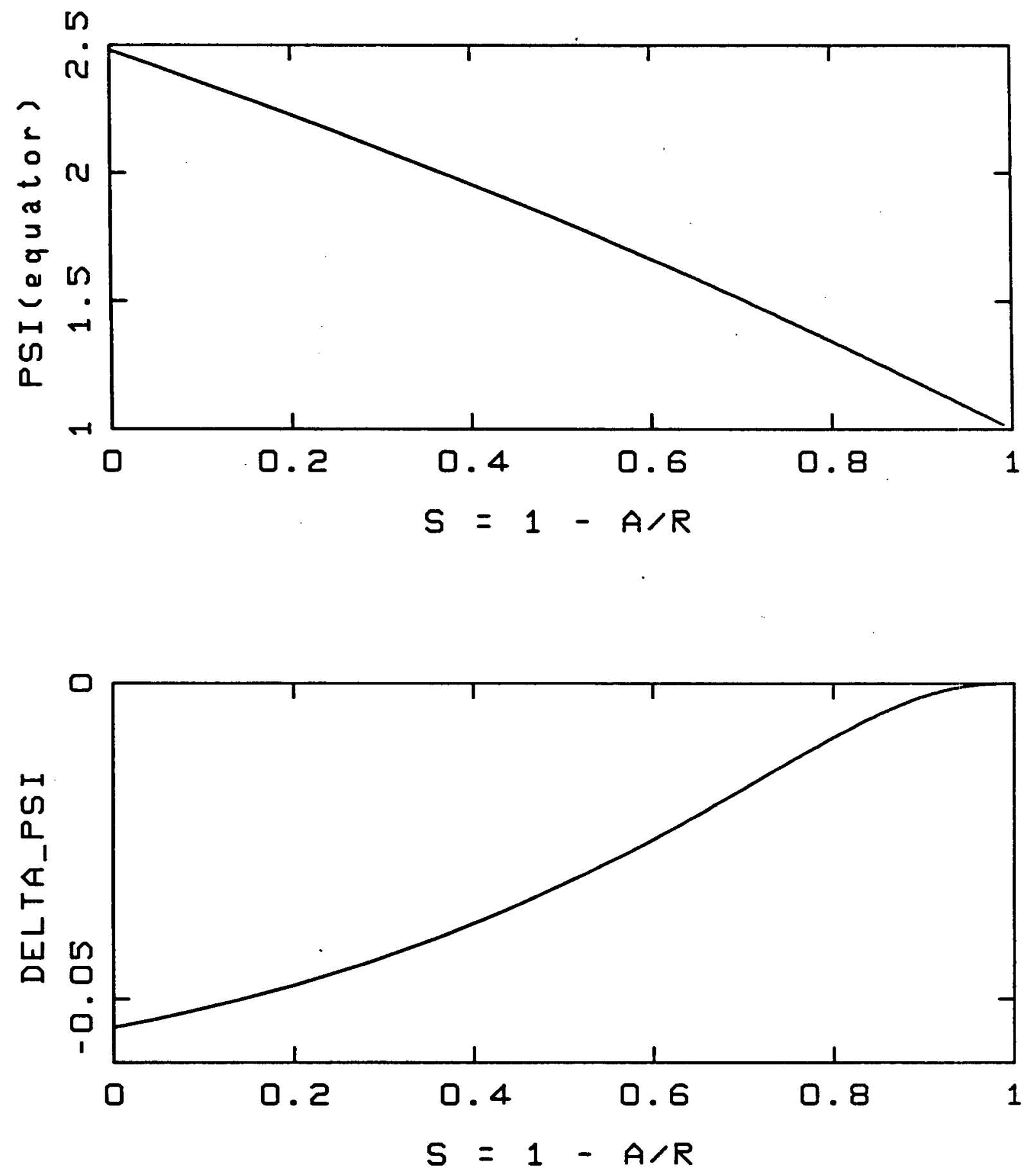

This figure shows $\Psi$ (equator) and $\delta \Psi=\Psi$ (north pole) $-\Psi$ (equator), both as functions of $s=1-a / r$. The former shows the general radial trend of $\Psi$, while the latter is a measure of the deviation of $\Psi$ from spherical symmetry. 
Choptrilk comparison test series

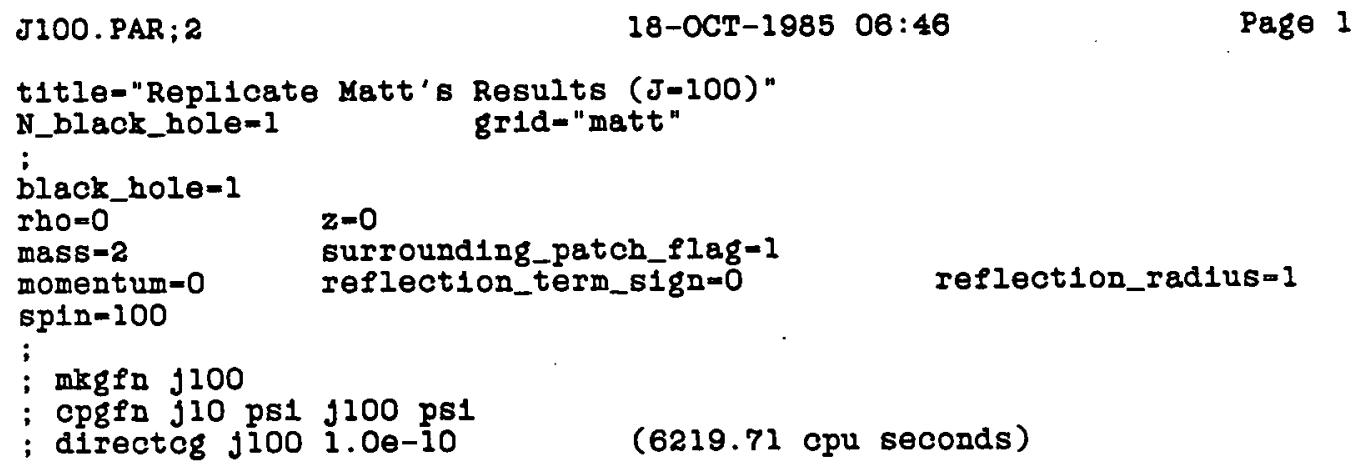


Figure 31 - Choptuik comparison test series, J100 quantitative results
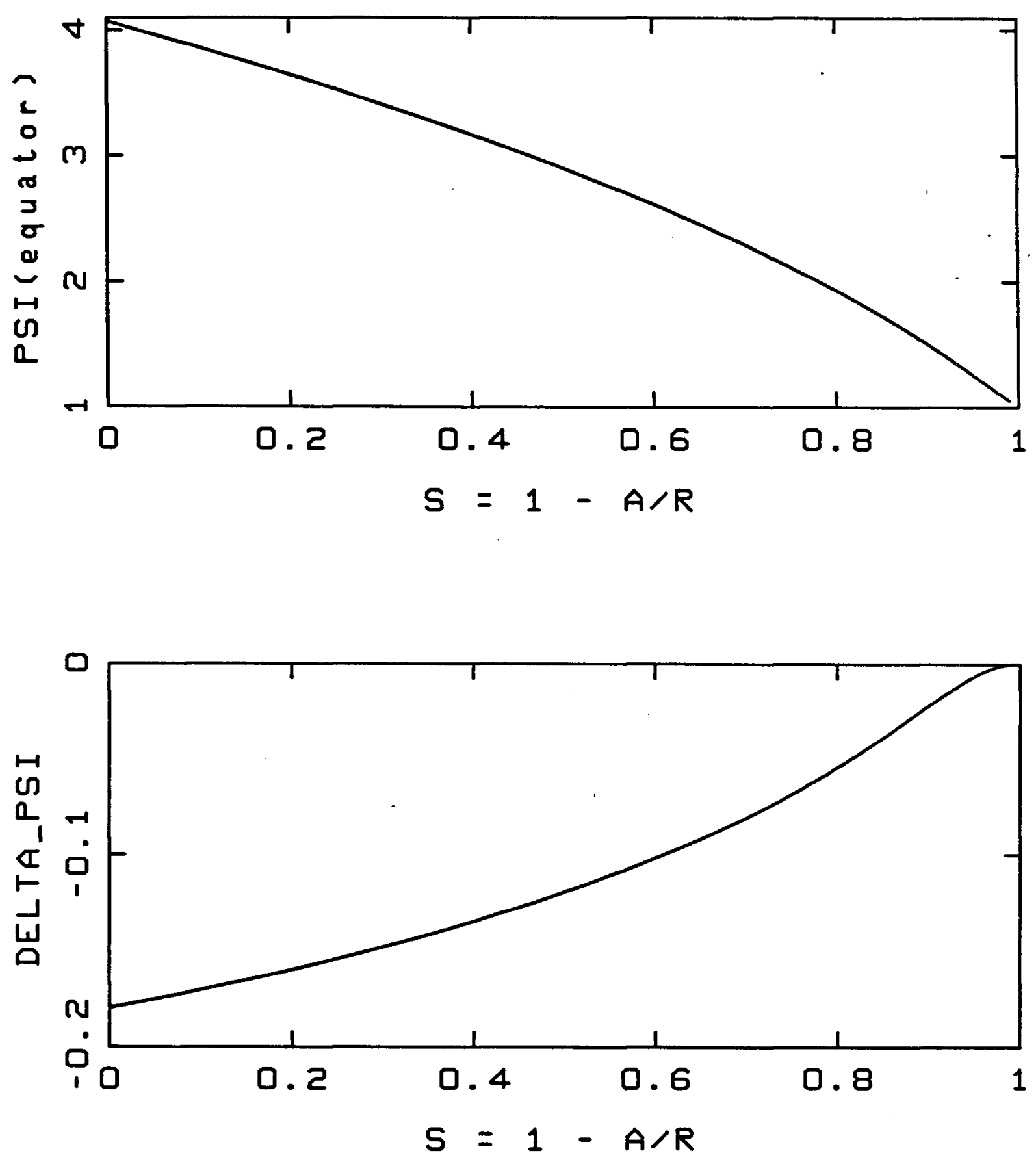

This figure shows $\Psi$ (equator) and $\delta \Psi=\Psi$ (north pole) $-\Psi$ (equator), both as functions of $s=1-a / r$. The former shows the general radial trend of $\Psi$, while the latter is a measure of the deviation of $\Psi$ from spherical symmetry. 


\section{Choptuik comparison test series}

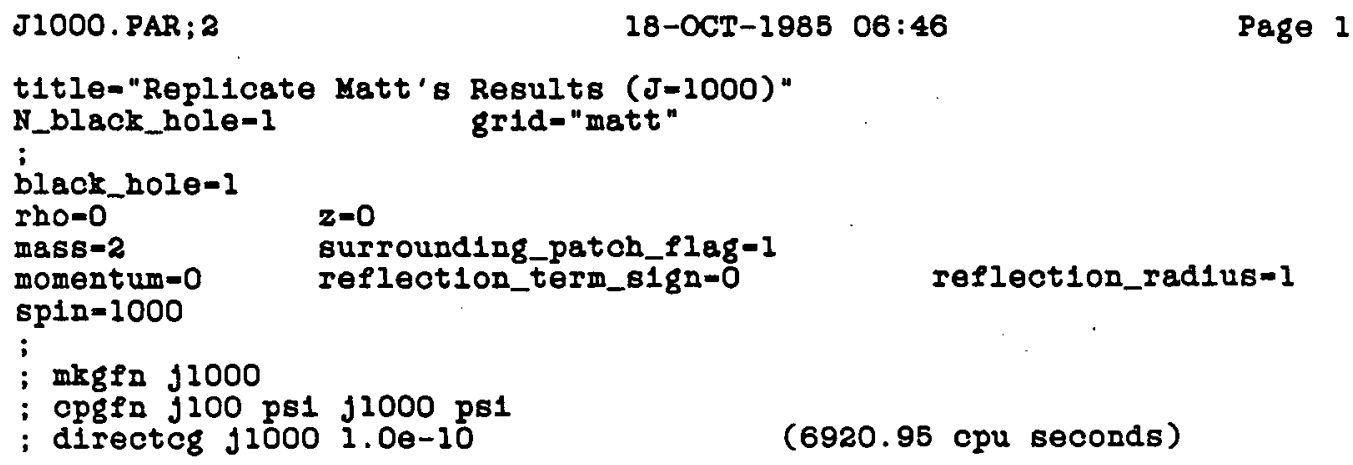


Figure 32 - Choptaik comparison test series, $j 1000$ quantitative results
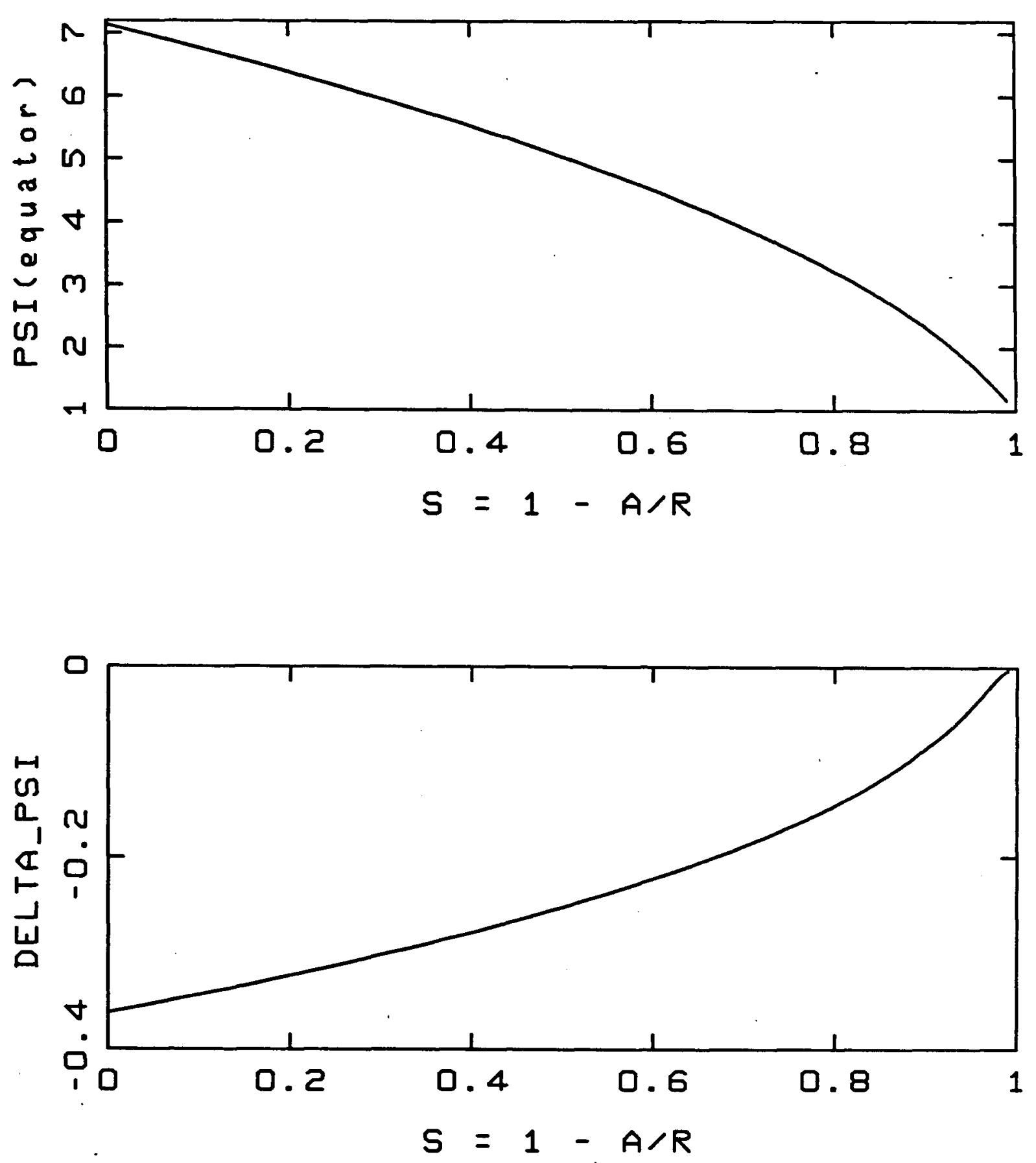

This figure shows $\Psi$ (equator) and $\delta \Psi=\Psi$ (north pole) $-\Psi$ (equator), both as functions of $s=1-a / r$. The former shows the general radial trend of $\Psi$, while the latter is a measure of the deviation of $\Psi$ from spherical symmetry. 


\section{Choptnik comparison test series}

MATT. PAT; 6

;

;

N_patch-6

; Now describe each coordinate patch:

patch-1

coord_type="polar spher1cal"

difference_scheme-" locally conservat1ve"

conformal_fn_type="omega(ps1)"

rho_center $=0$ z_center $=0$

$r$ min="constant $=1.00 "$

r_max-"constant-1.95"

theta_min=" constant $=0$ "

theta_max $=$ " constant $=90$ "

delta_r-0.05 delta_theta $=5$ bndry_cond="Reflection Symmetric"

bndry_cond = "patch $=2$ " bndry_oond = "Neumann" ;

patch=2

coord_type="polar spher1cal"

difference_scheme-"locally conservative"

conformal_fn_type-"omega(ps1)"

rho_center $=0$ z_center $=0$

$r \min ="$ constant $=2.0$ "

I_mar="constant -4.9 "

theta_m1n=" constant $=0$ "

bndry_cond= "Neumann"

theta_max-"constant $=90$ "

delta_r=0.1

delta_theta-5

patch-3

coord_type-"polar spher1cal"

difference_scheme="Iocaliy conservat1ve"

conformal_fn_type-"omega(ps1)"

rho_center $=0$ z_center $=0$

$r$ min-" constant -5.0 "

bndry_cond= "patch $=1$ "

bndry_cond = "patch $=3$ "

bndry_cond- "Neumann"

bndry_cond= "Neumenn"

I_max= "constant $=9.8$ "

theta_min-"constant $=0$ "

theta_max-" constant $=90$ "

delta_r=0.2 delta_theta-5

bndry_cond = "patch-2"

bndry_cond=" patch-4"

bndry_cond = "Neumann"

patoh $=4$

coord_type="polar spher1cal"

difference_scheme-"iocally conservat1ve"

conformal_fn_type="omega(ps1)"

rho_center=0 z_center=0

I_min=" constant $=10.0$ "

I_max= "constant -19.5 "

theta_min="constant $=0$ "

theta_max= " constant $=90$ "

bndry_oond = "Neumann"

patch $=5$

coord_type="polar spherical"

difference_scheme-"Iocalis conservat1ve"

conformal_fn_type-"omega(psi)"

rho_center $=0$ z_center $=0$

$I$ min-" constant $-2 \bar{O}^{\prime \prime}$

$r$ max=" constant $=49$ "

theta min=" constant -0 "

thetr_max-" constant $=90 "$

delta_r=0.5 delta_theta=10

bndry cond = patch $=3$ "

bndry_cond= "patch $=5$ "

bndry_cond- "Neumann"

bndry_oond-" Neumann"

delta_r-1

bndry_cond- "patch $=4$ "

bndry_cond= "patoh=6"

bndry_cond = "Neumann"

bndry_cond- "Neumann" 
Choptuik comparison test series

KATT . PAT ; 6

$18-O C T-1985 \quad 06: 45$

Page 2

;

patch-6

coord_type="polar spherical"

difference_scheme-"Iocally conservative"

conformal_fn_type-"omega(ps1)"

rho_center $=0 \quad$ z_center $=0$

I_mIn="constant $=5 \overline{0}^{\circ}$

deIta_r=2

bndry_cond= "patch -5 "

r_max-"constant $=100$ "

bndry_oond = "Rob1n"

theta min="constant -0 "

bndry_oond- "Neumann"

theta_max=" constant $=90$ "

bndry_cond = "Neumann" 


\section{J test series}

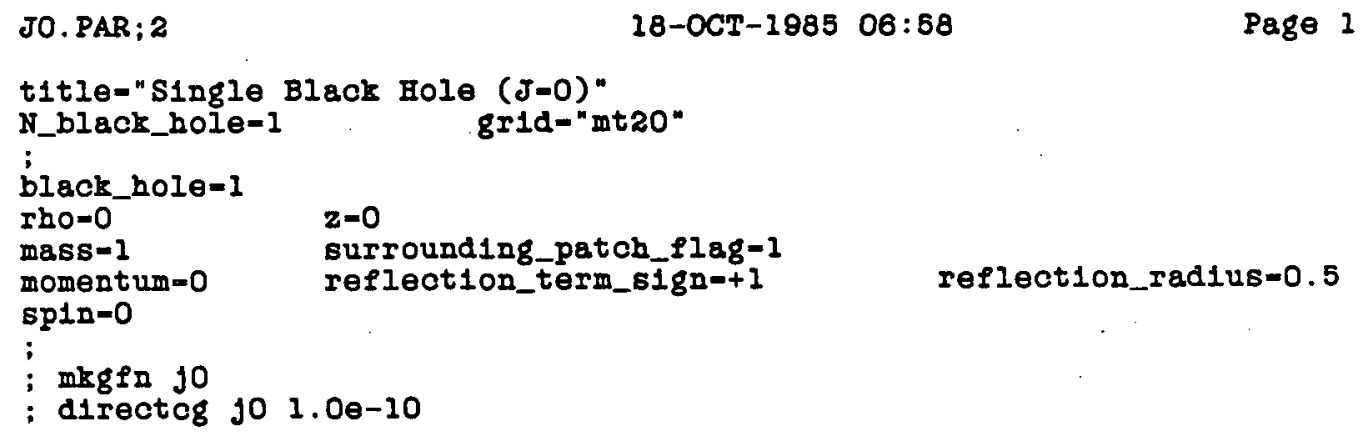


Table 21 - J test series, j0 selected $\Psi$ values

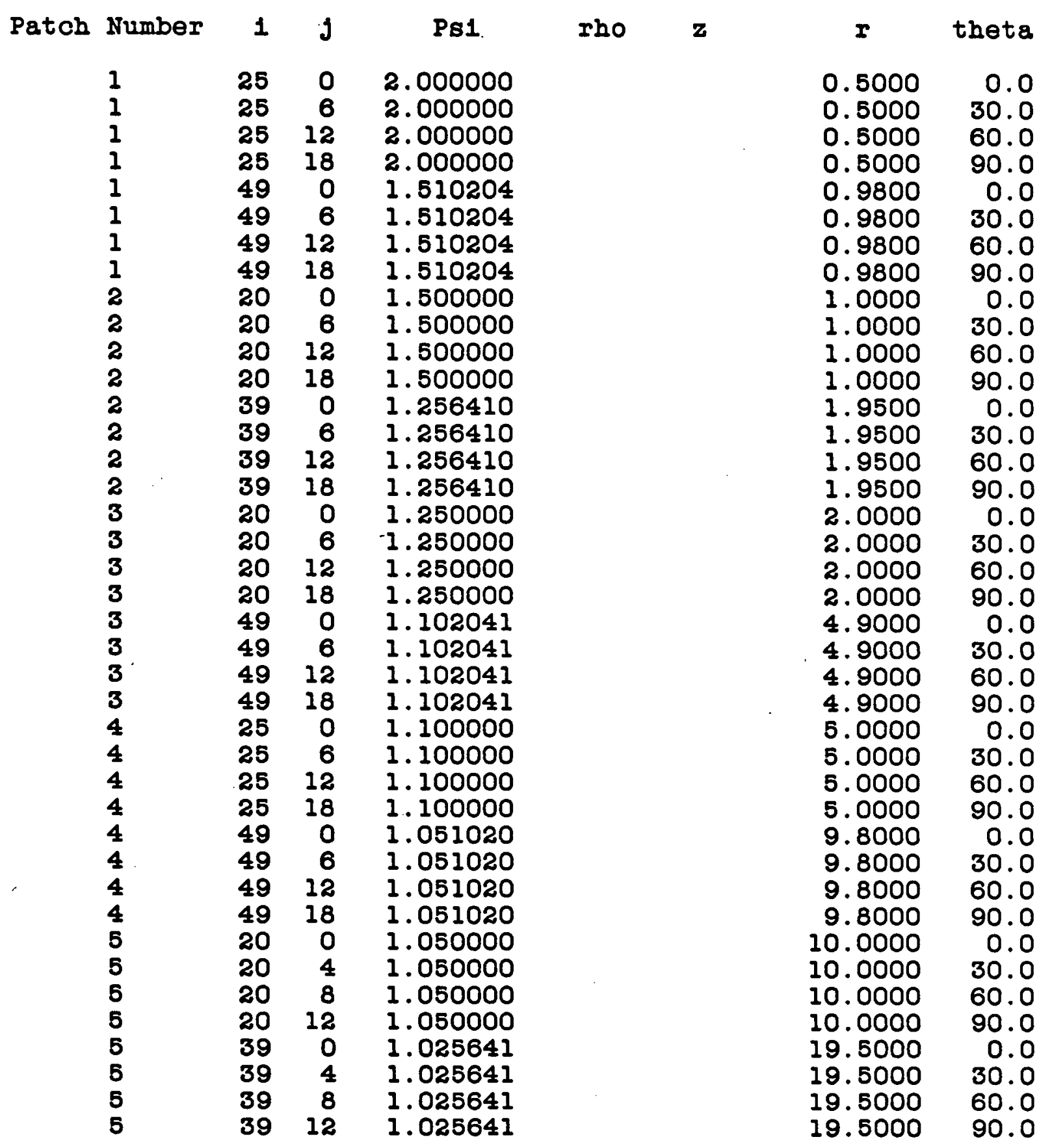

This table gives values of $\Psi$ at selected points in the space. These points are identified by triples (patch number, $i, j$ ), where $(i, j)$ are the generic integer patch coordinates defined in the text. Depending on the patch type, either $(\rho, z)$ or $(r, \theta)$ are also shown. 


\section{$\mathrm{J}$ test series}

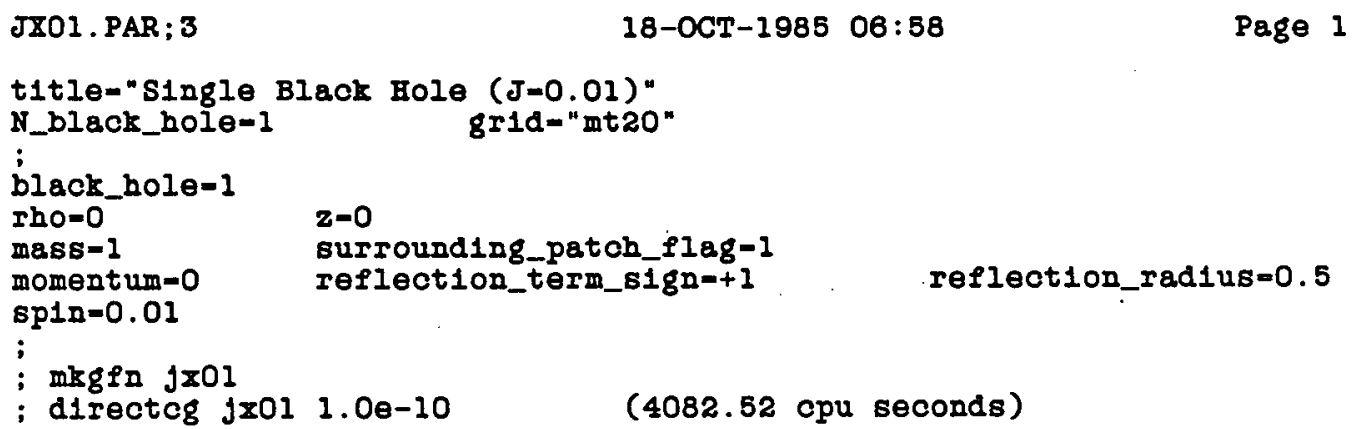


Figure 33 - J test series, jx01 quantitative results
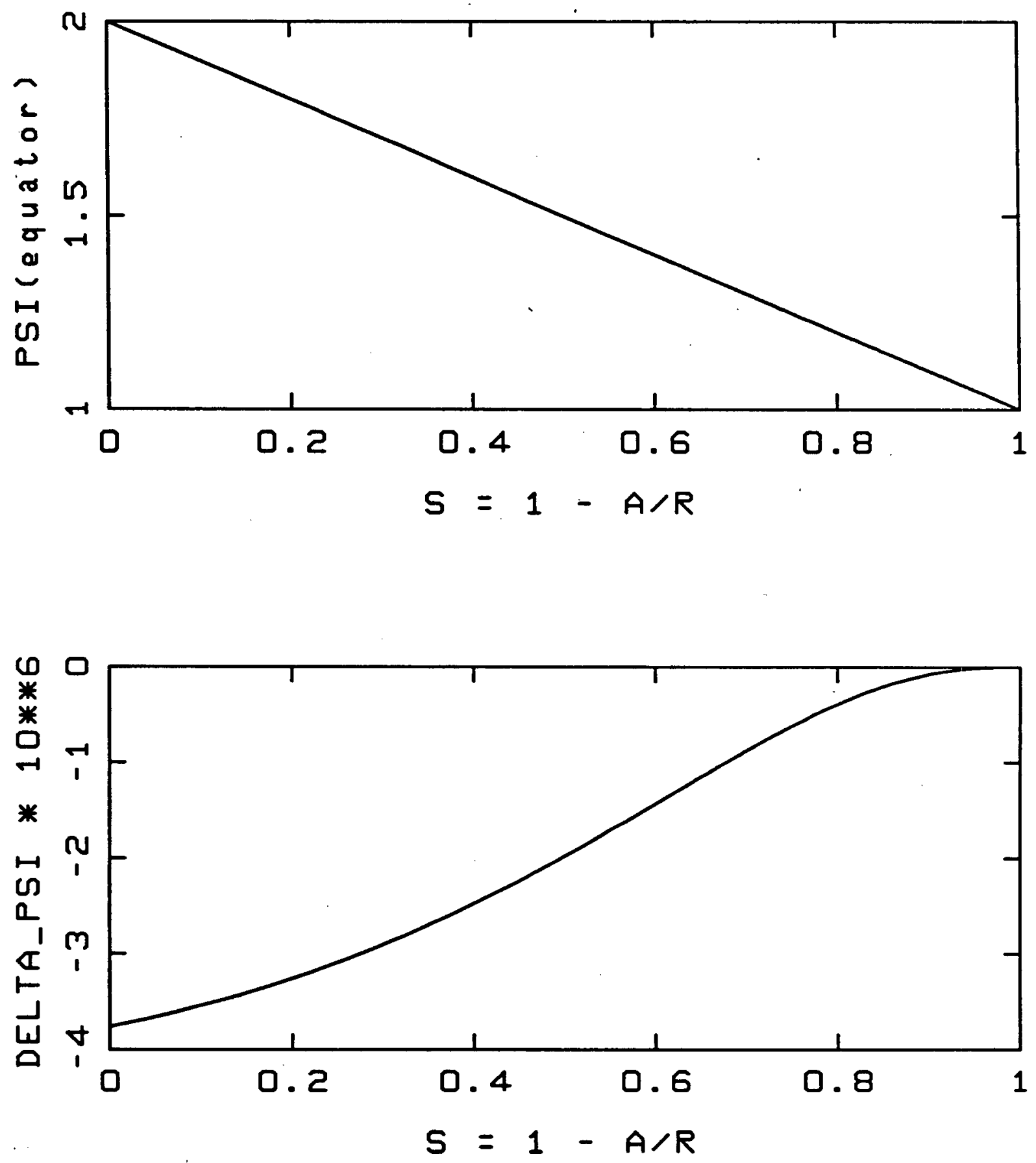

This figure shows $\Psi$ (equator) and $\delta \Psi=\Psi$ (north pole) $-\Psi$ (equator), both as functions of $s=1-a / r$. The former shows the general radial trend of $\Psi$, while the latter is a measure of the deviation of $\Psi$ from spherical symmetry. 
J test series

JZ1 . PAR; 3

$18-O C T-1985 \quad 06: 68$

Page 1

t1tle="Single Black Hole ( J=0.1)"

N_black_hole-1 grid="mt20"

;

bleck_hole-1

rho=0

mass = I

$z=0$

surrounding_patch_flag-1

momentum $=0$

reflection_term_sign=+1

reflect1on_radius $=0.6$

$\operatorname{sp1n}=0.1$

$\operatorname{mrg} \rho \mathrm{j} j \mathrm{l}$

cpgfn jxOl psi jxl psi

directc8 Jxi $1.0 e-10$

(6278.99 cpu seconds) 
Figure 34 - J test series, $j \times 1$ quantitative results
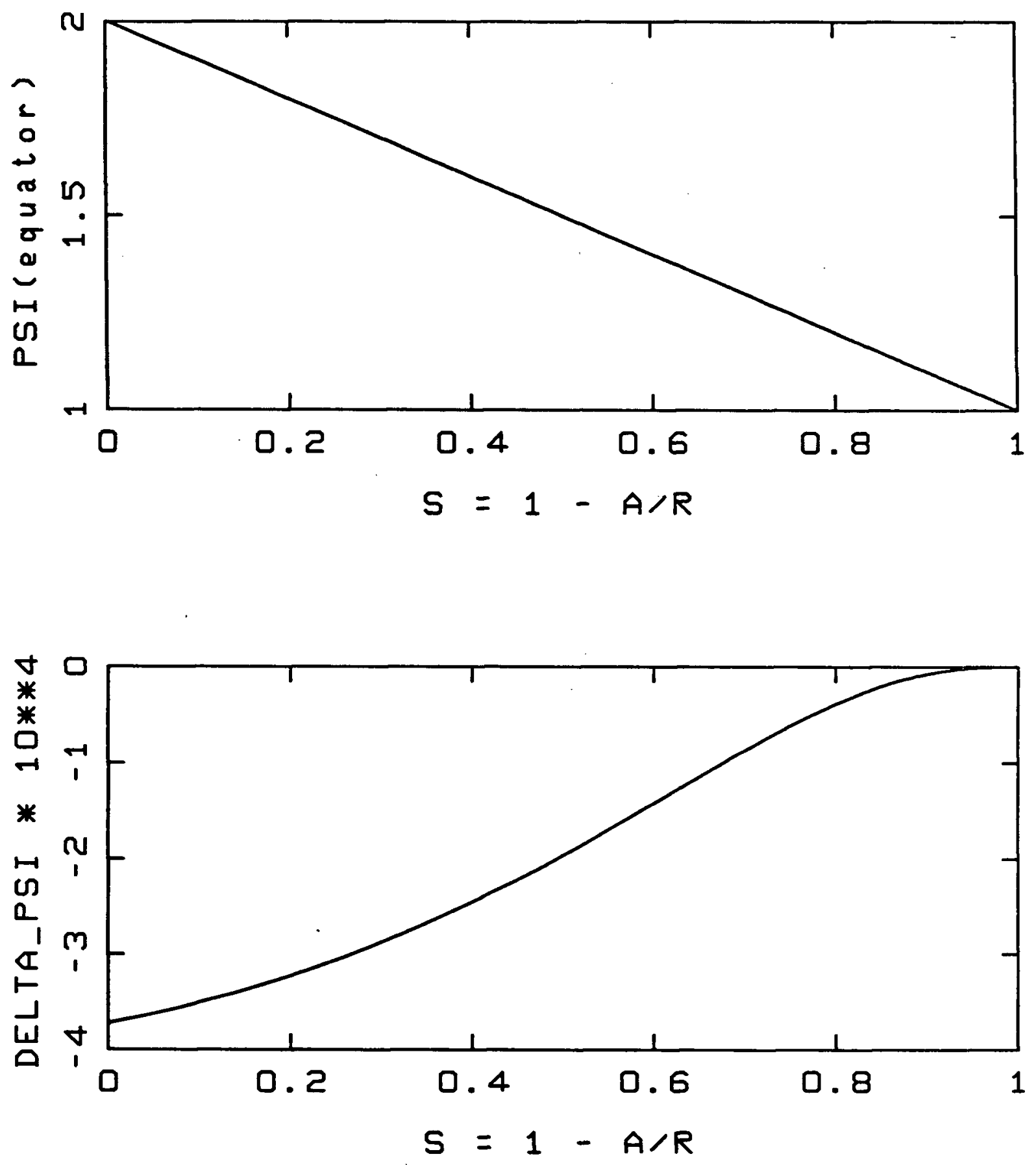

This figure shows $\Psi$ (equator) and $\delta \Psi=\Psi$ (north pole) $-\Psi$ (equator), both as functions of $s=1-a / r$. The former shows the general radial trend of $\Psi$, while the latter is a measure of the deviation of $\Psi$ from spherical symmetry. 


\section{$\mathrm{J}$ test series}

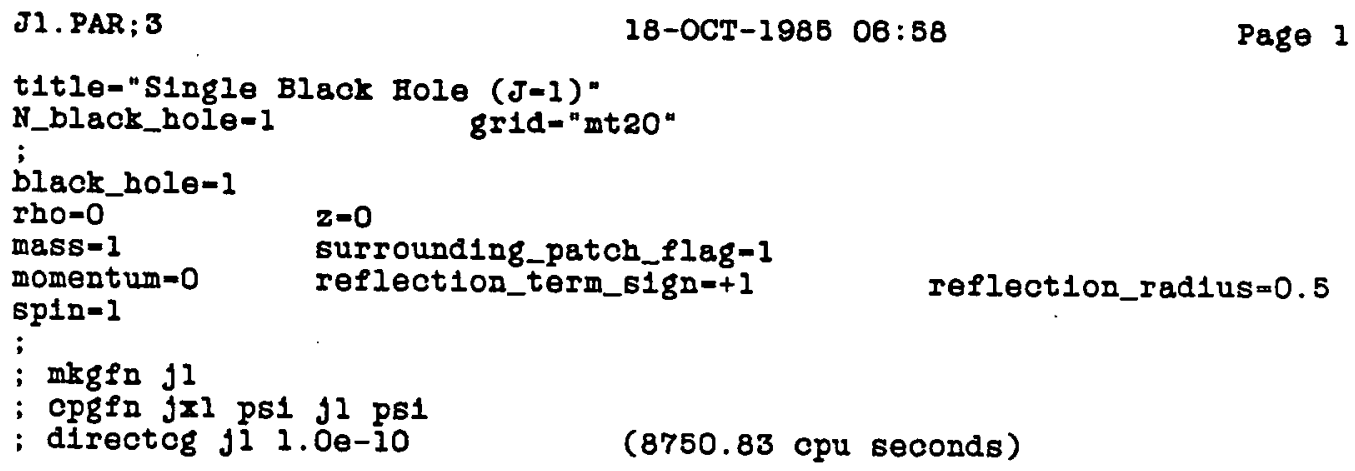


Figure 35 - J test series, $j 1$ quantitative results
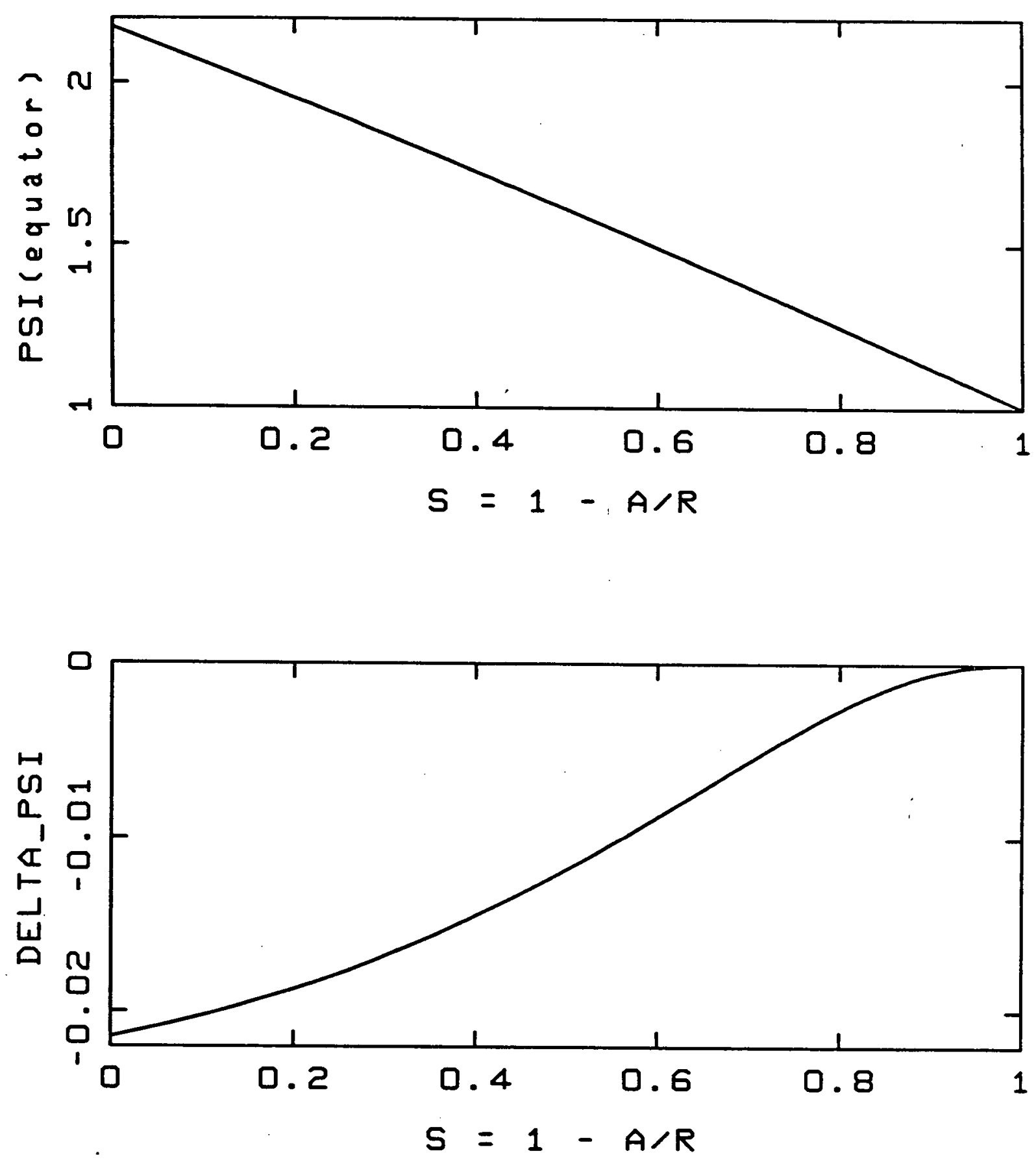

This figure shows $\Psi$ (equator) and $\delta \Psi=\Psi$ (north pole) $-\Psi$ (equator), both as functions of $s=1-a / r$. The former shows the general radial trend of $\Psi$, while the latter is a measure of the deviation of $\boldsymbol{\Psi}$ from spherical symmetry. 


\section{$\mathrm{J}$ test series}

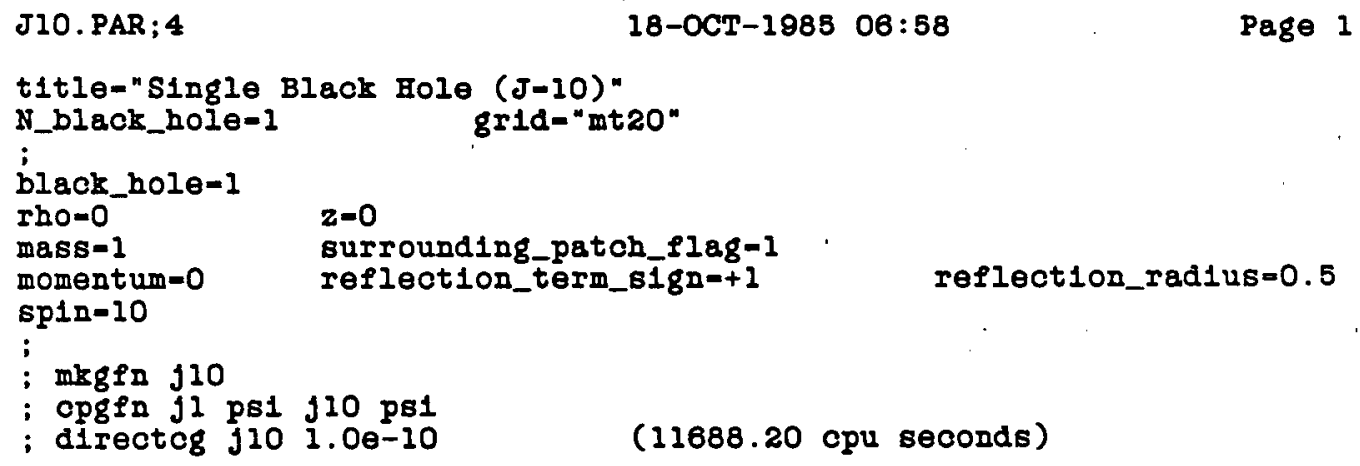


Figure 36 - J test series, $j 10$ quantitative results
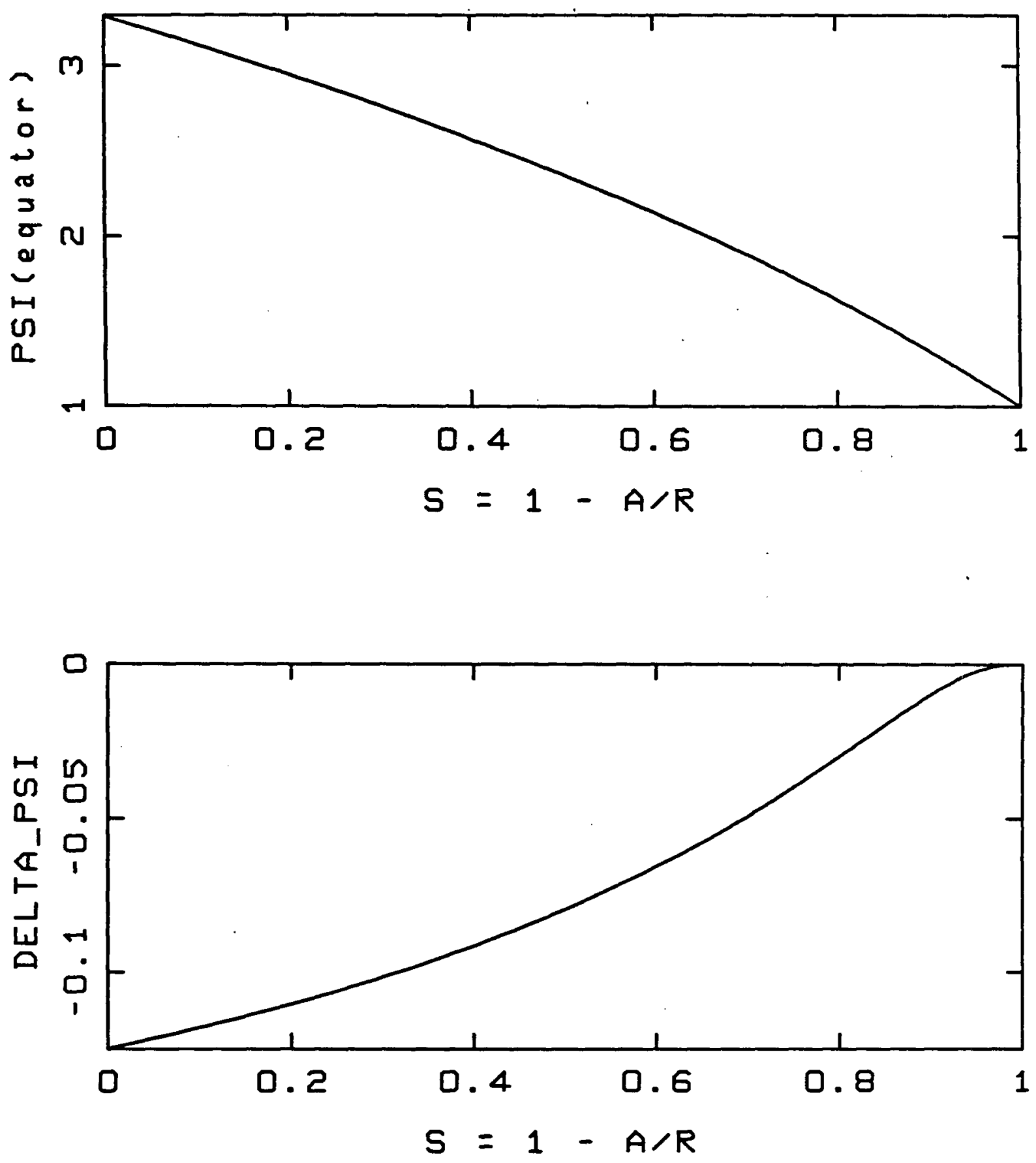

This figure shows $\Psi$ (equator) and $\delta \Psi=\Psi$ (north pole) $-\Psi$ (equator), both as functions of $s=1-a / r$. The former shows the general radial trend of $\Psi$, while the latter is a measure of the deviation of $\Psi$ from spherical symmetry. 


\section{$\mathrm{J}$ test series}

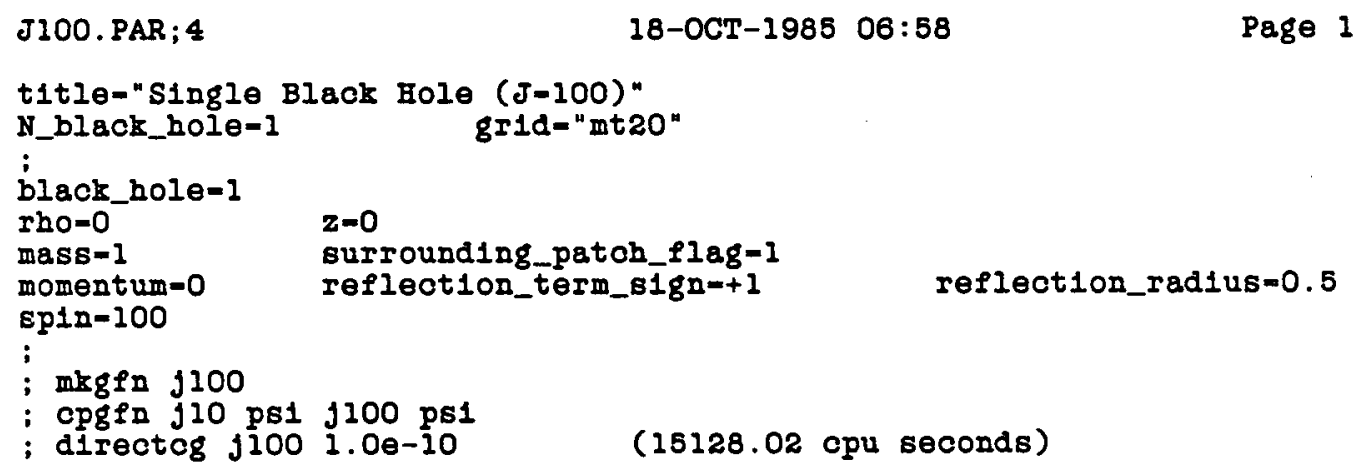


Figure 37 - J test series, $\mathrm{J} 100$ quantitative results
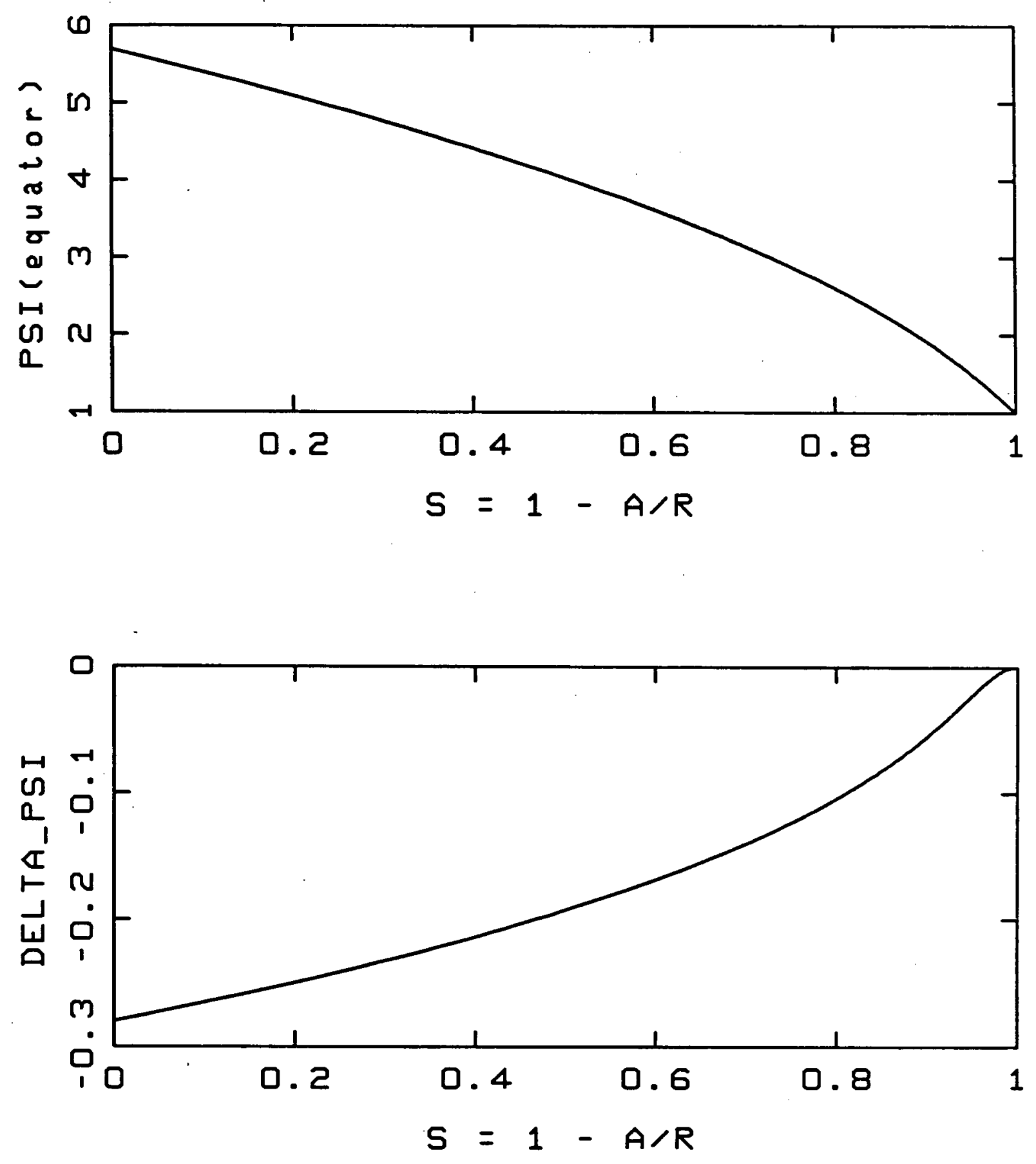

This figure shows $\Psi$ (equator) and $\delta \Psi=\Psi$ (north pole) $-\boldsymbol{\Psi}$ (equator), both as functions of $s=1-a / r$. The former shows the general radial trend of $\Psi$, while the latter is a measure of the deviation of $\Psi$ from spherical symmetry. 


\section{$\mathrm{J}$ test series}

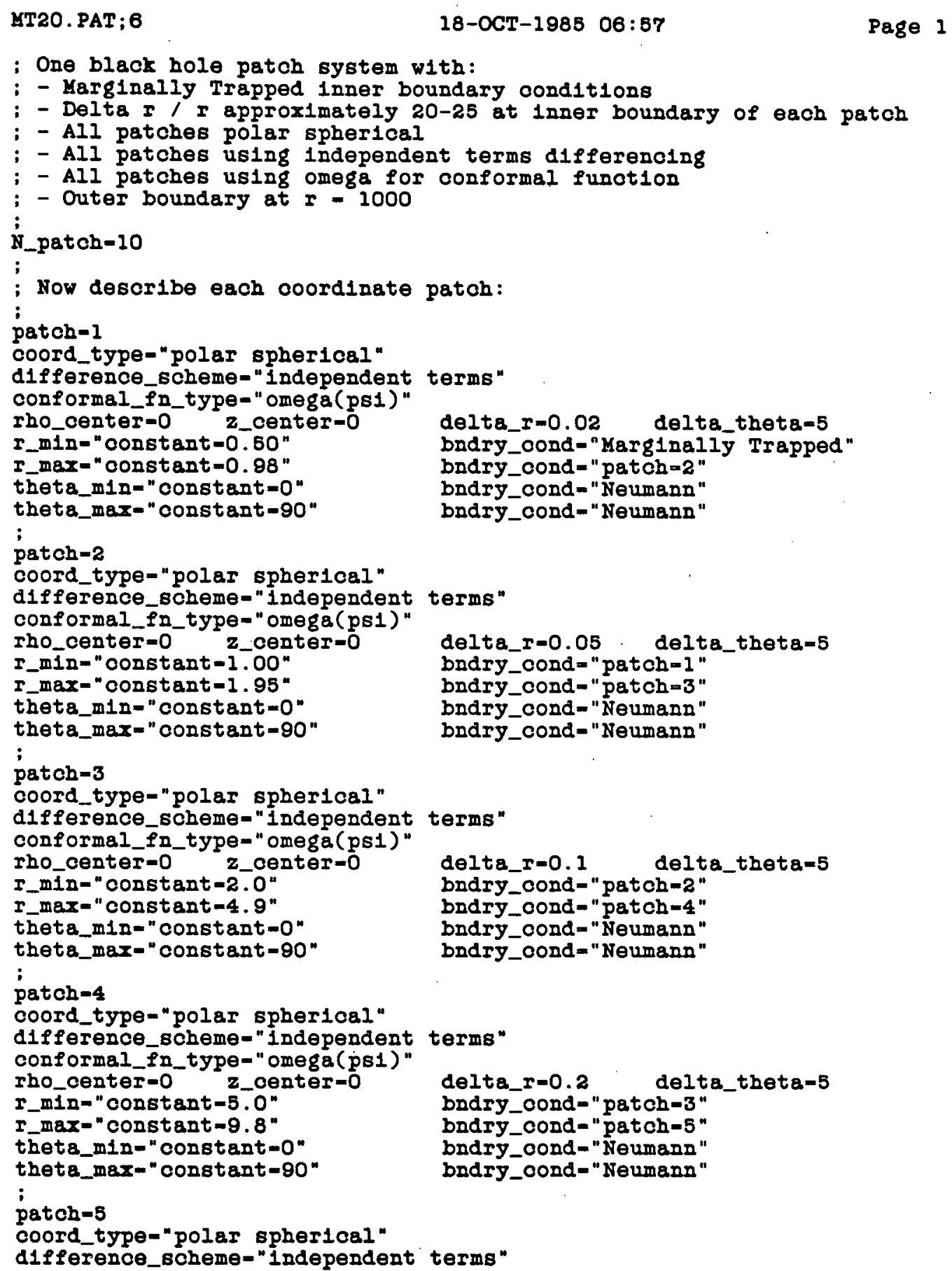


J test series

MT2O . PAT; 6

18-OCT-1985 06:57

Page 3

theta_max="constant-90"

bndry_cond-" Neumenn" 


\section{RSP test geries}

PO. PAR; 12

title-"Single Black Hole $(P-0)$ "

N_black_bole-1

gr1d-"I820"

black_hole=1

rho=0

mass $=1$

$z=0$

momentum $=0$

surrounding_patch_fleg-1 reflection_term_sign=+1

reflection_radius $=0.5$

sp1n=0

; mkgen po

; directcg po $1.0 e-10$ 
Table 22 - RSP test series, p0 selected $\Psi$ values

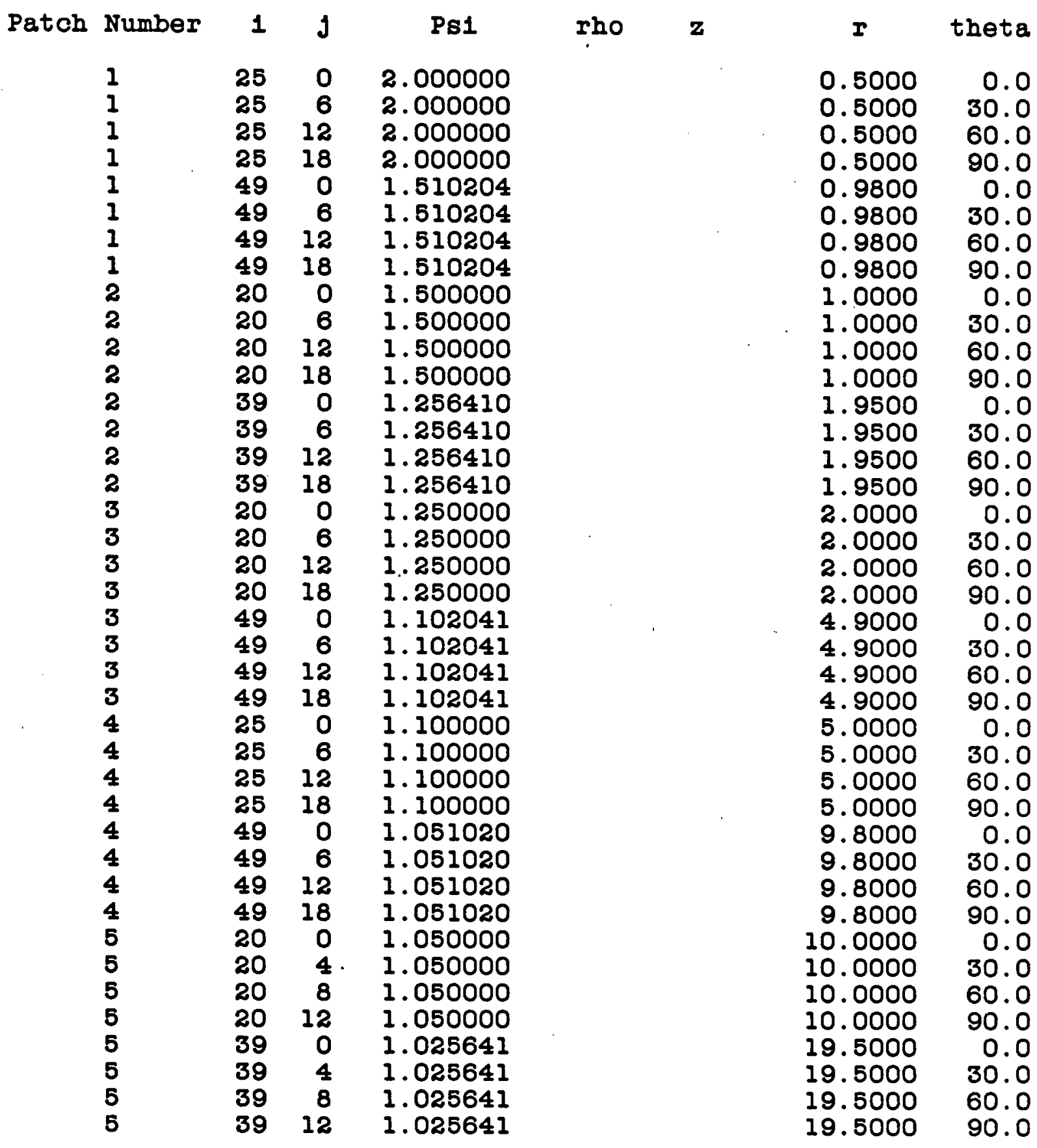

This table gives values of $\Psi$ at selected points in the space. These points are identified by triples (patch number, $i, j$ ), where $(i, j)$ are the generic integer patch coordinates defined in the text. Depending on the patch type, either $(\rho, z)$ or $(r, \theta)$ are also shown. 


\section{RSP test series}

PXO2. PAR; 8

10-NOV-1985 $22: 46$

Page 1

title-"Single Black Hole ( $P=0.01) "$

N_black_hole=1 grid-" Is20"

;

black_hole-1

sho $=0$

$z=0$

mass -1

surround1ng_patch_flag=1

momertum $=0.01$

reflection_term_sign=+1

$\operatorname{sp} 1 \mathrm{n}=0$

reflect1on_radius $=0.5$

; mkgen pxol

directcg pxO1 1.0e-10

(4253.86 cpu seconds) 
Figure 38 - RSP test series, px01 quantitative results
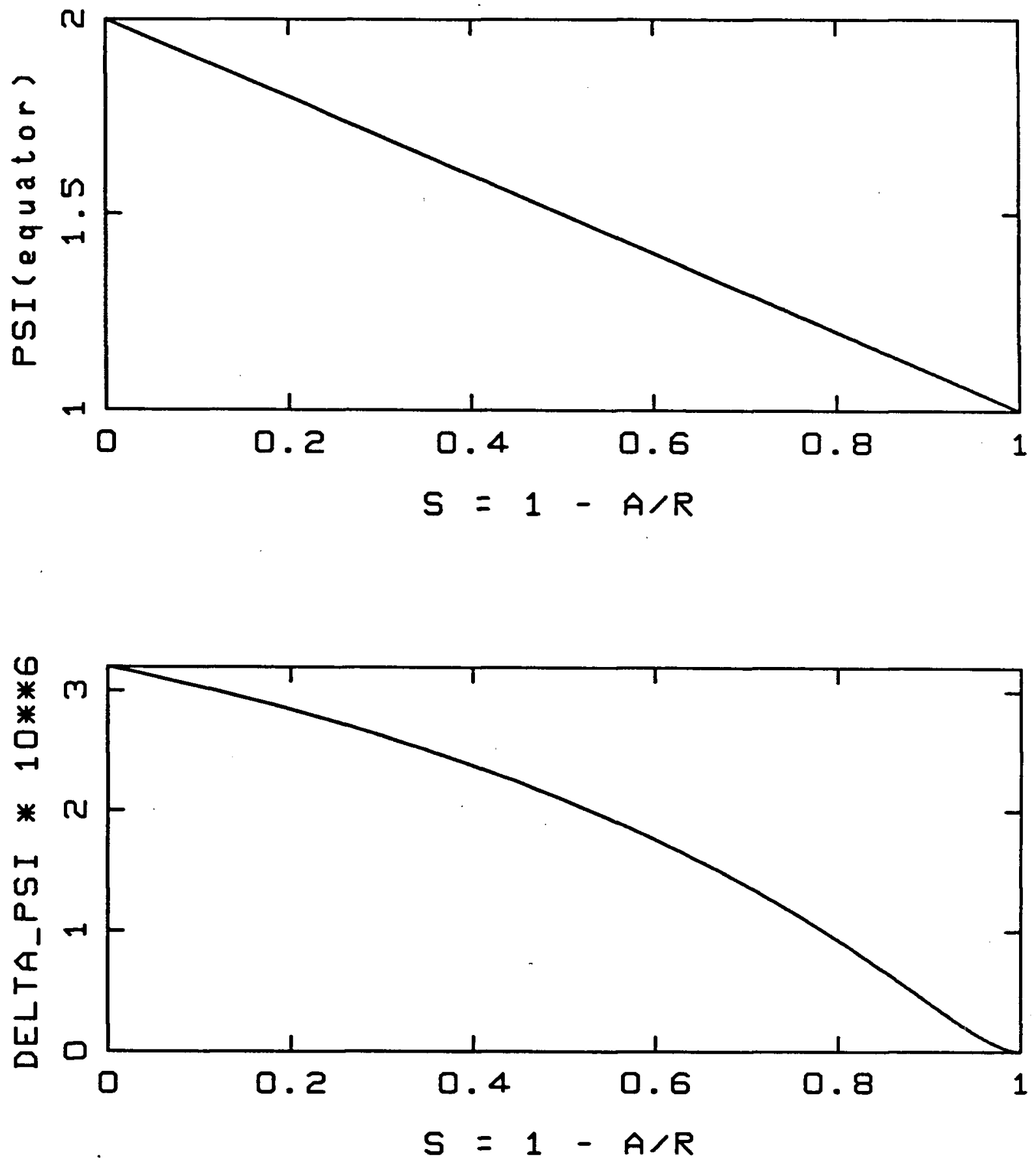

This figure shows $\Psi$ (equator) and $\delta \Psi=\Psi$ (north pole) $-\Psi$ (equator), both as functions of $s=1-a / r$. The former shows the general radial trend of $\Psi$, while the latter is a measure of the deviation of $\Psi$ from spherical symmetry. 


\section{RSP test series}

PXI. PAR; 6

10-NOV-1985 $22: 46$

Page 1

title-"Single Black HOle (P-0.1)"

N_black_hole- 1 grid- "rs20"

;

block_hole-l

rho $=0$

mass $=1$

$\mathbf{z}=0$

surrounding_patch_flag-1

momentum=0.1 reflect1on_term_sign-+1

reflection_radius $=0.5$

$\operatorname{sp} 1 \mathrm{n}=0$

; mkgfn pxl

cpgfa pxOl ps1 pri ps1

i directog pxi $1.00-10$

(4198.79 cpu seconds) 
Figure 39 - RSP test series, px1 quantitative results
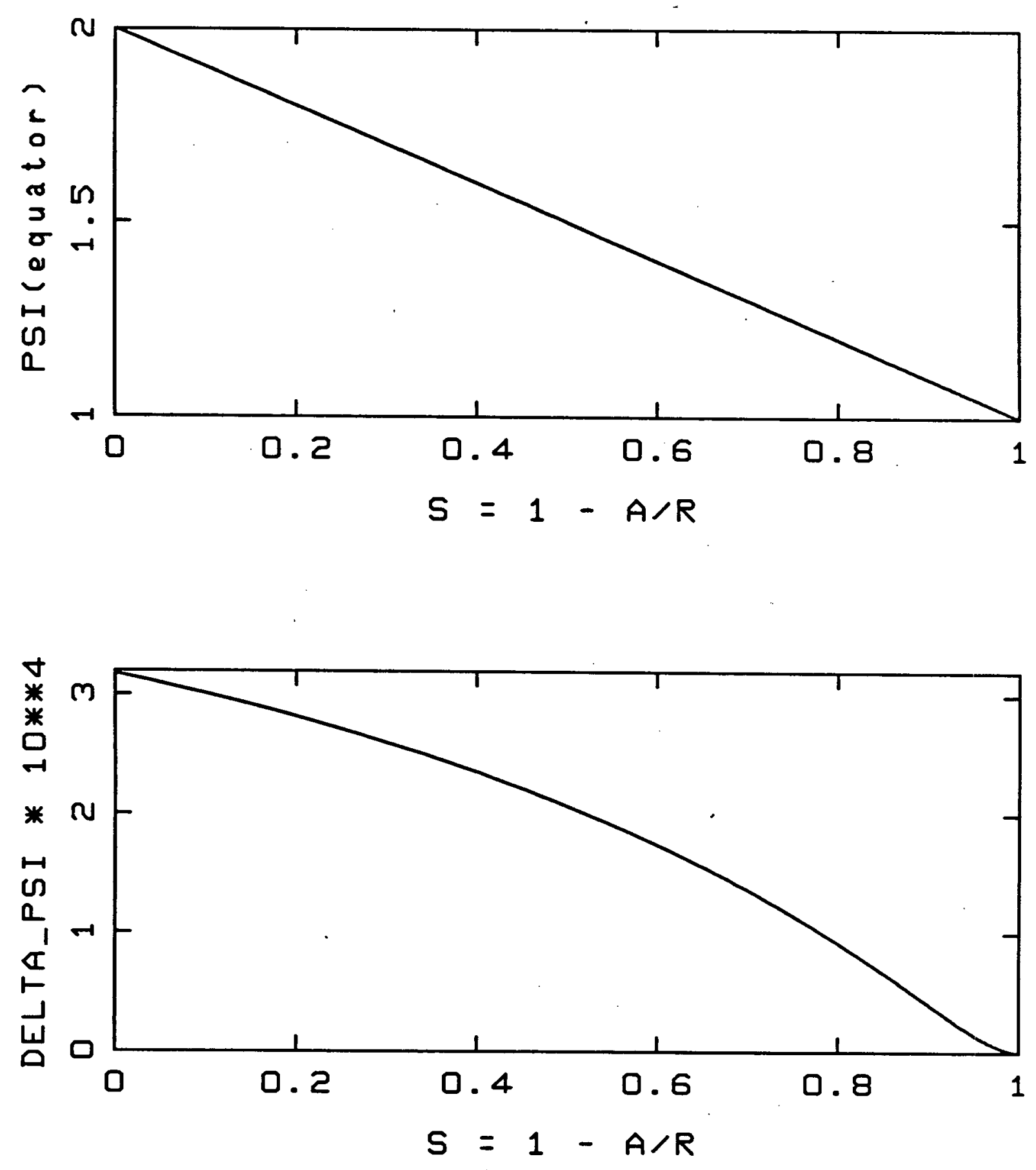

This figure shows $\Psi$ (equator) and $\delta \Psi=\Psi$ (north pole) $-\Psi$ (equator), both as functions of $s=1-a / r$. The former shows the general radial trend of $\Psi$, while the latter is a measure of the deviation of $\Psi$ from spherical symmetry. 


\section{RSP test series}

PI. PAR; 5

10-NOV-1985 22:46

Page 1

title="Single Black Hole $(P=1)$ "

N_black_hole=1 Gr1d="Is20"

i

black_hole=l

rho $=0$

$z=0$

mass $=1$

surrounding_patch_flag-1

momentum $=1$

reflection_term_sign=+1

reflection_radius $=0.5$

spin $=0$

; mkgfn pl

: cpgen pxl psi pl psi

; directeg pi $1.00-10$

(8308.23 cpu seconds) 
Figure 40 - RSP test series, P1 quantitative results
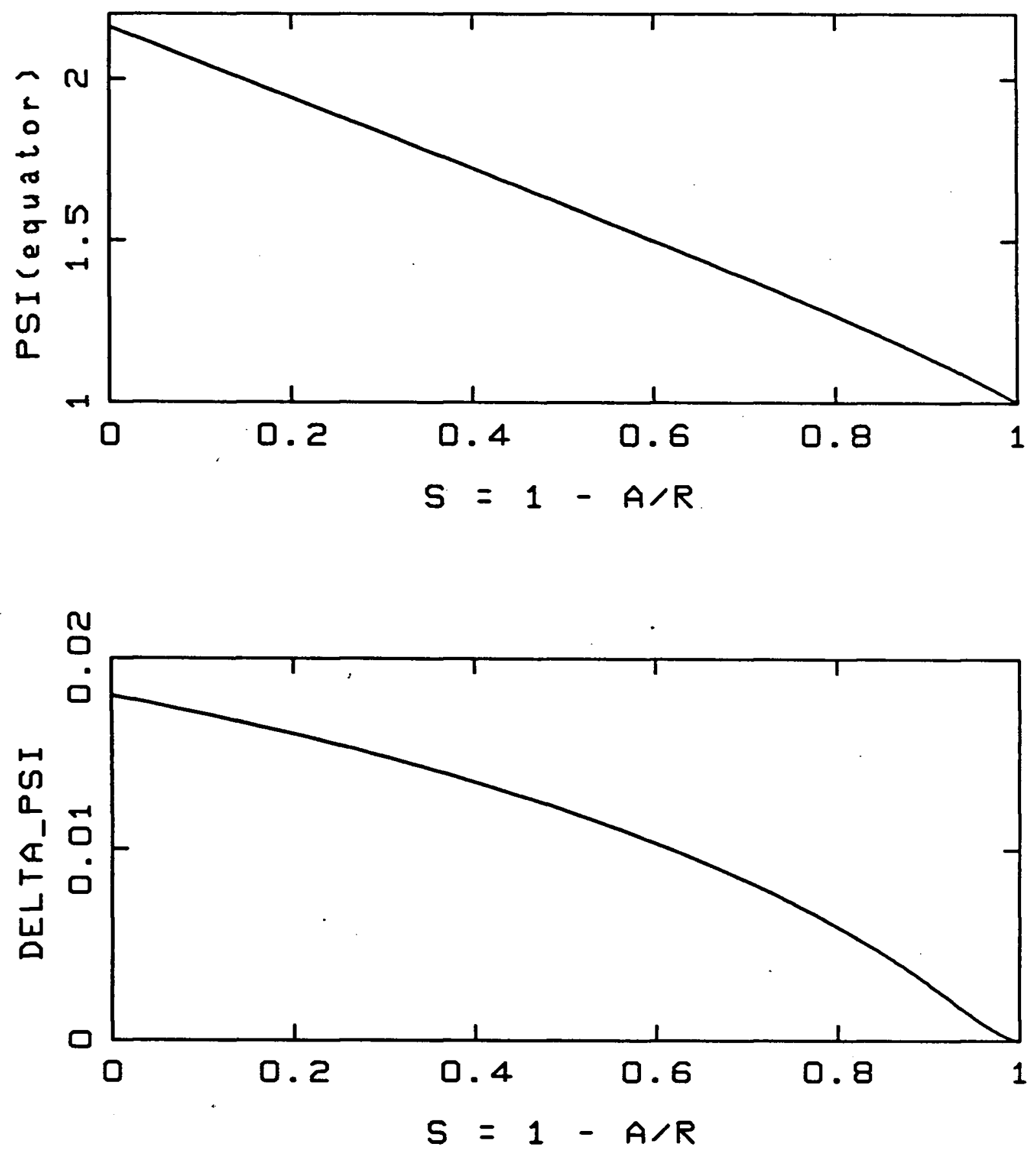

This figure shows $\Psi$ (equator) and $\delta \Psi=\Psi$ (north pole) $-\Psi$ (equator), both as functions of $s=1-a / r$. The former shows the general radial trend of $\Psi$, while the latter is a measure of the deviation of $\Psi$ from spherical symmetry. 
RSP test series

P1O. PAR; 5

$10-$ NOV-1985 $22: 46$

Page 1

t1tle-"Single Black Hole $(p-10)$ "

N_black_hole=l grid=" Is20"

bleck_hole-1

rho=0

mass $=1$

$z=0$

momentum 10

Burrounding_patch_flag=1

$\operatorname{sp} 1 \mathrm{n}=0$ reflection_term_sign=+1

refleotion_rad1us=0.5

;

; mkgfn p10

- cpgen pl psi p10 psi

; directcg p10 1.0e-10

(11212.95 cpu seconds) 
Figure 41 - RSP test series, p10 quantitative results
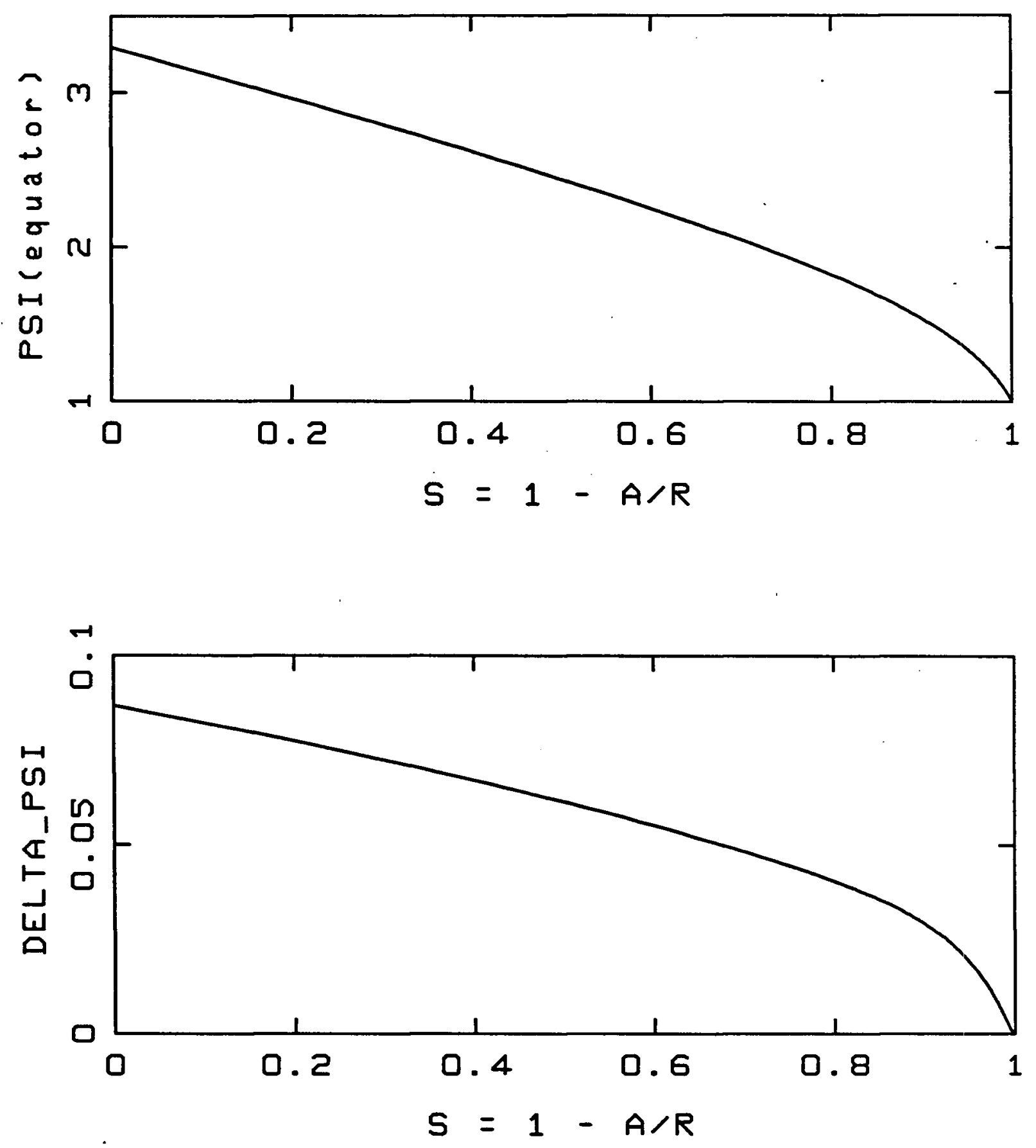

This figure shows $\Psi$ (equator) and $\delta \Psi=\Psi$ (north pole) $-\Psi$ (equator), both as functions of $s=1-a / r$. The former shows the general radial trend of $\Psi$, while the latter is a measure of the deviation of $\Psi$ from spherical symmetry. 
RSP test series

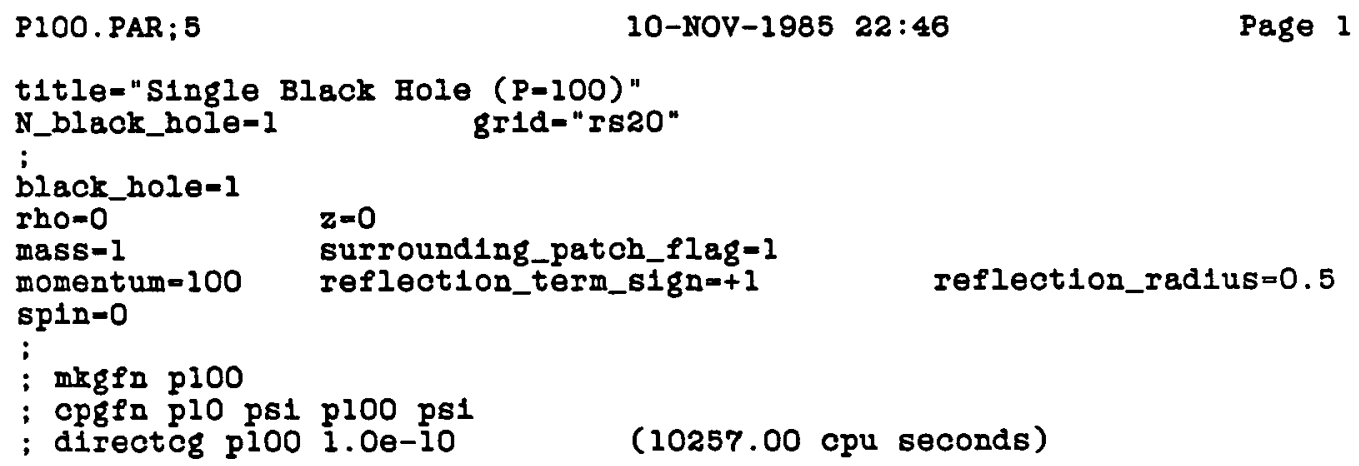


Figure 42 - RSP test series, p100 quantitative results
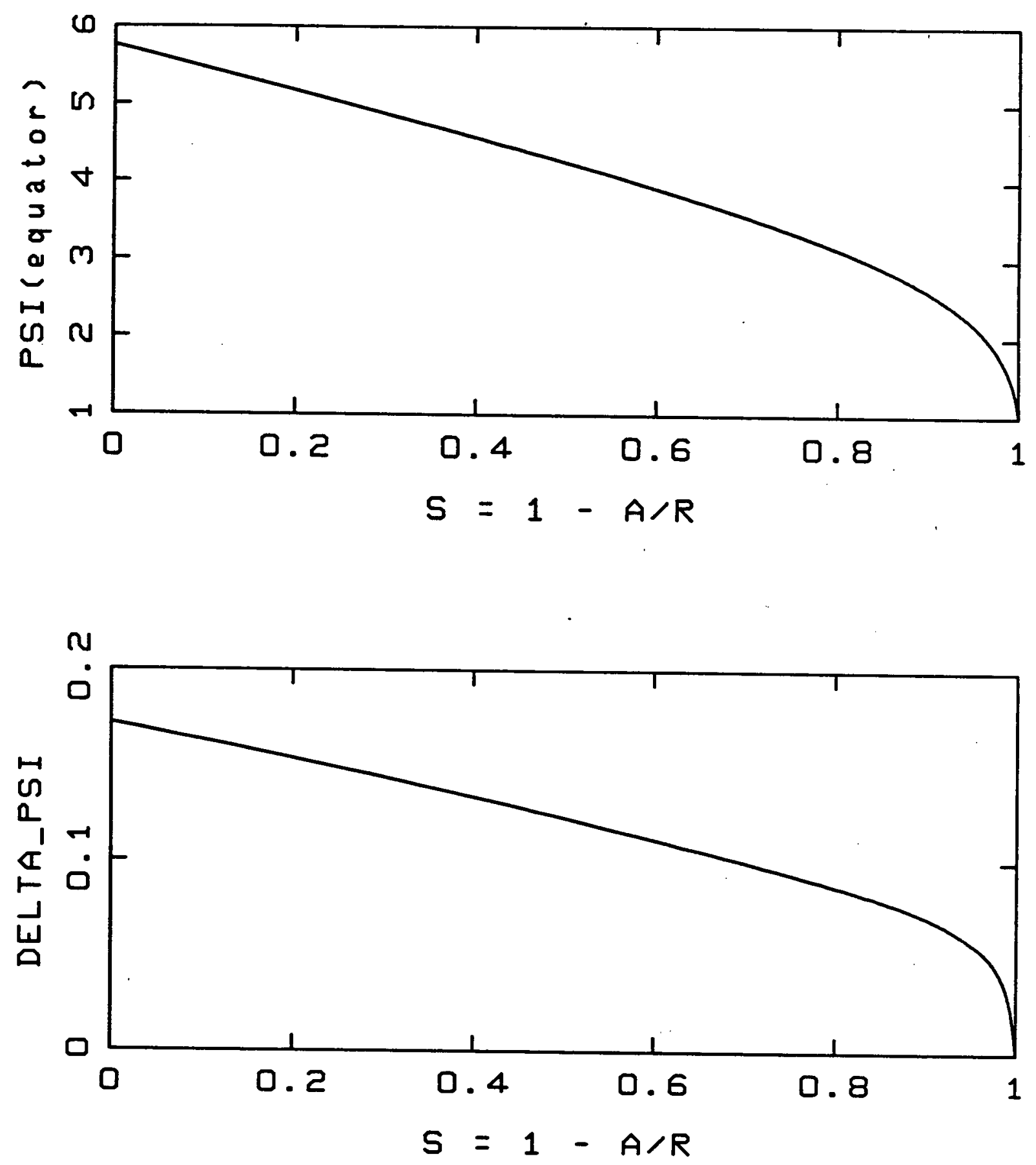

This figure shows $\Psi$ (equator) and $\delta \Psi=\Psi$ (north pole) $-\Psi$ (equator), both as functions of $s=1-a / r$. The former shows the general radial trend of $\Psi$, while the latter is a measure of the deviation of $\Psi$ from spherical symmetry. 


\section{RSP test series}

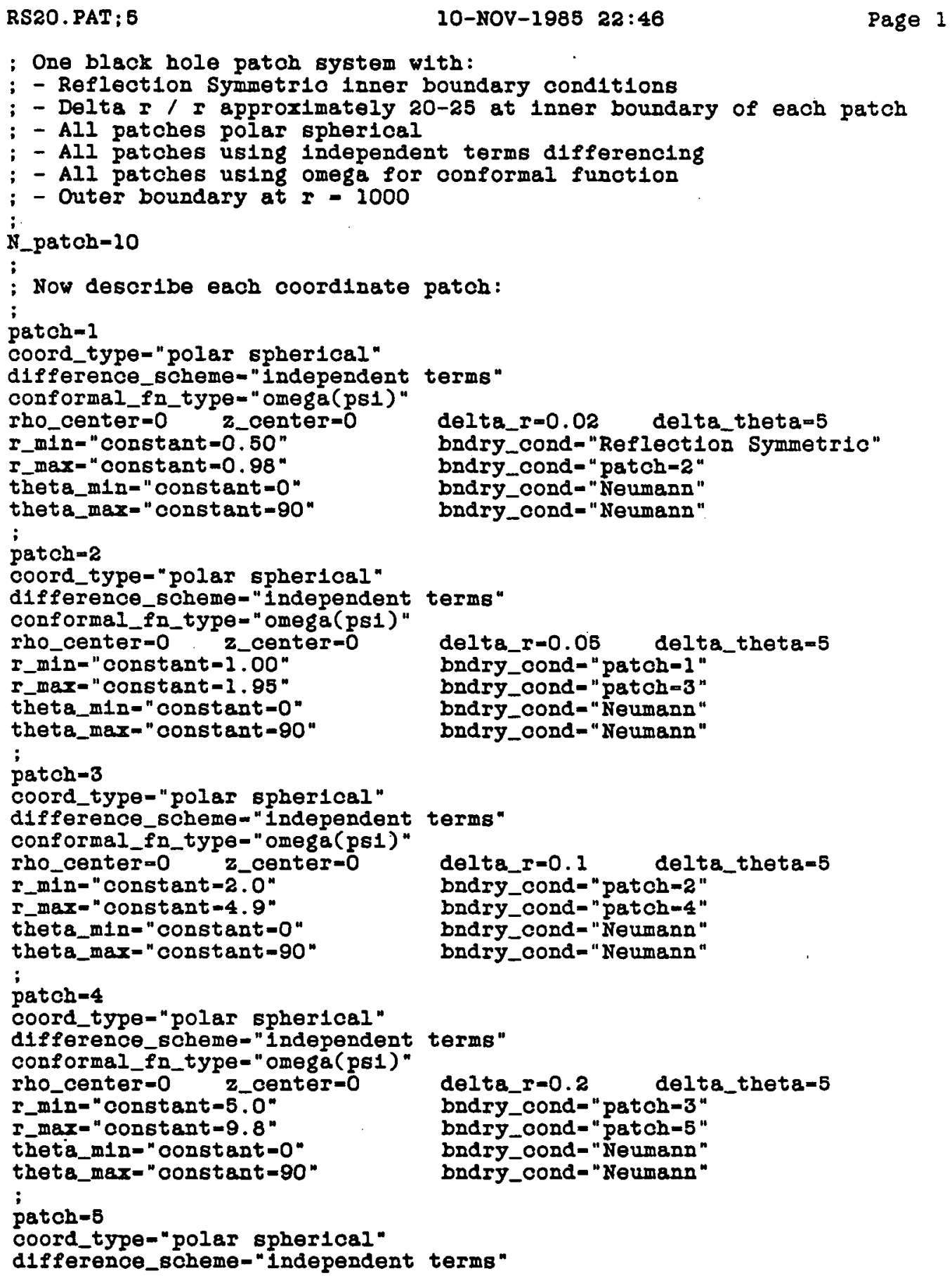

delta_r=0.02 delta_theta=5 bndry_cond-"Reflect1on Symmetric" bndry_cond = "patch-2" bndry_cond- " Neumann" bndry_cond = "Neumann"

\section{delta_r=0.1 delta_theta=5}

bndry_cond= "patch $=2$ " bndry cond - "patch-4" bndry_cond = "Neumann" bndry_cond- "Neumenn" 


\section{RSP test series}

RS2O. PAT; 6

conformal_fn_type="omega(ps1)" rho_center $=0 \quad z$ center $=0$

I_min= "constant $=10.0$ "

r_max-" constant-19.5"

theta_min=" constant-0"

theta_max=" constant $=90$ "

;

patch $=6$

coord_type="polar spher1cal"

difference_scheme="1ndependent terms"

conformal_fn_type-"omega(psi)"

rho_center $=0$ z_center $=0$

I_min="constant $=20$ "

$r$ max=" constant $=49$ "

theta_min="constant $=0$ "

theta_max=" constant $=90$ "

;

patch-7

coord_type-"polar spherical"

difference_scheme-"Independent terms"

conformal_fn_type-"omega (psi)"

rho_center=0 z_center=0

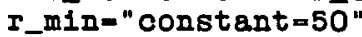

$r$ max- "constant $=98$ "

theta_min="constant $=0$ "

theta_max = "constant $=90$ "

;

patch-8

coord_type-"polar spherical"

difference_scheme-"Independent terms"

conformal_fn_type-"omega(ps1)"

rho_center $=0$ z_center $=0$

r_min=" constant $=1 \overline{0} 0$ "

r_mar-" constant-195"

theta_m1n-"constant $=0$ "

theta_max= "constant $=90$ "

;

patoh-9

coord_type="polar spherical"

difference_scheme-"1ndependent
conformal fn type-"omega(psi)"

rho_center $=0$ z_center=0

I_min-"constant $=200$ "

$r$ max $=$ "constant $=490$ "

theta_min="constant $=0$ "

theta_max="constant $=90$ "

;

patch $=10$

coord_type-"polar spherical"

difference_scheme-"independent terms"

conformal_fn_type="omega(psi)"

rho_center $=0$ z_center $=0$

I_mIn= "constant $=500$ "

$r$ max $=$ " constant $=1000$ "

theta_min-" constant-o"
10-NOV-1985 $22: 46$

Page 2

delta_I=0.5 delta_theta=7.5

bndry_cond= "patch-4"

bndry_cond = "patch-6"

bndry_cond = "Neumann"

bndry_cond=" Neumann"

delta_r-1 delta_theta $=7.5$

bndry_cond = "patch=5"

bndry_cond = "patch=7"

bndry_cond- "Neumann"

bndry_cond = "Neumann"

delta_r-2 delta_theta-7.5

bndry_cond = " patch $=6$ "

bndry_cond= "patch=8"

bndry_cond-" "Neumann"

bndry_cond = "Neumann"

delta_r-5 delta_theta $=10$

bndry_cond = " patch=7"

bndry_cond=" patch=9"

bndry_cond- "Neumann"

bndry_cond= "Neumann"

terms"

delta_r-10 delta_theta $=10$

bndry_cond= "patch $=8$ "

bndry_cond = "patch $=10 "$

bndry_cond = "Neumann"

bndry_cond-"Neumann"

\author{
delta_r-20 delta_theta-10 \\ bndry_cond " patch=9" \\ bndry_cond= "Rob1n" \\ bndry_cond- "Neumenn"
}


RSP test geries

RS2O. PAT; 5

theta_max=" constant $=90$ "
10-NOV-1985 22:46

Page 3

bndry_cond = "Neumann" 
RSN test series

PO. PAR; 13

title="Single Black Hole $(P-0)$ "

N_black_hole-1

grid=" rs20"

;

black_hole=l

Iho=0

mass-1

$z=0$

momentum $=0$

surround1ng_patch_flag-1

spin=0

reflection_term_sign--1

reflection_radius -0.5

; mkgen po

; directog po 1.0e-10 
Table 23 - RSM test series, p0 selected $\Psi$ values

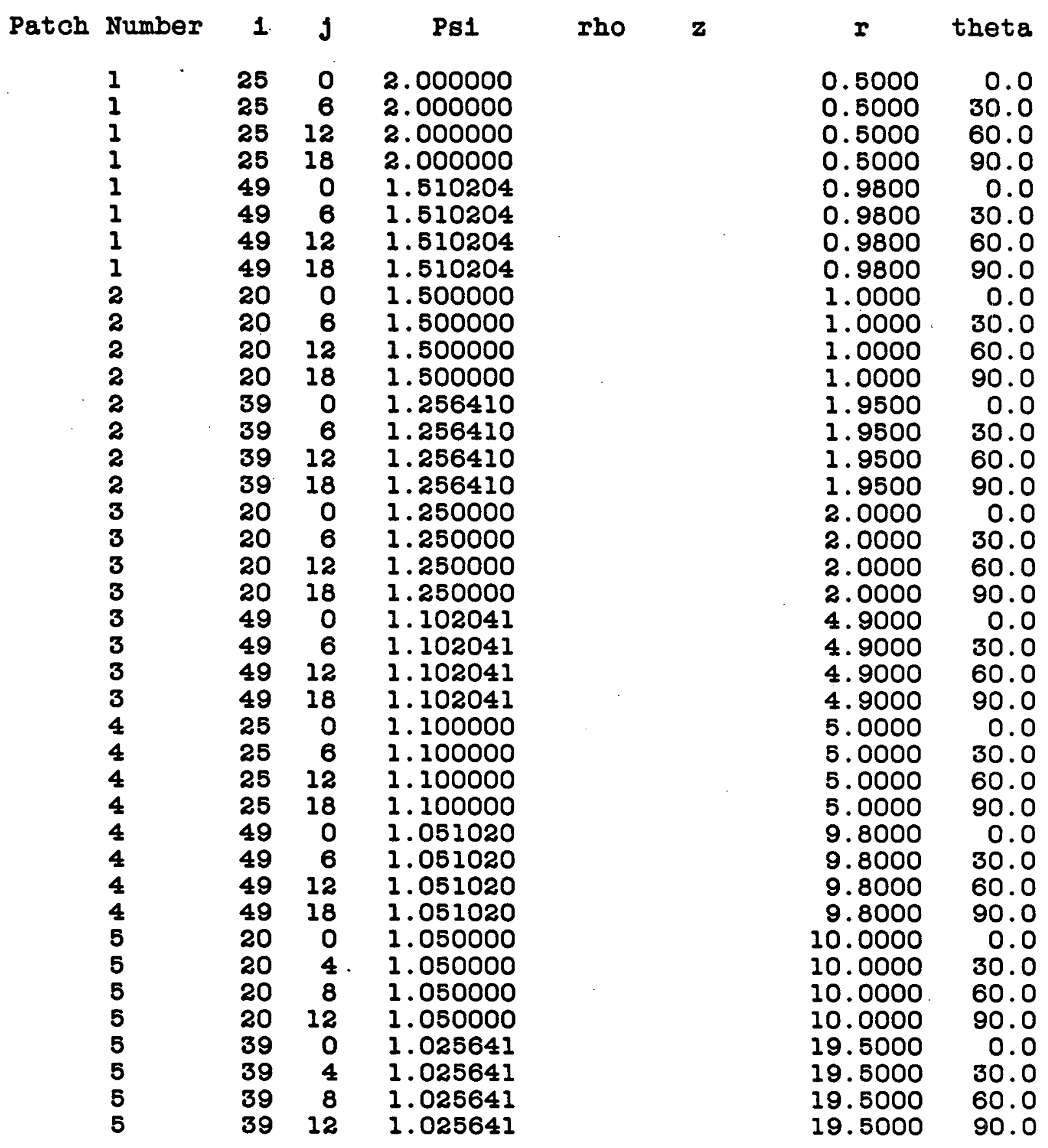

This table gives values of $\Psi$ at selected points in the space. These points are identified by triples (patch number, $i, j$ ), where $(i, j)$ are the generic integer patch coordinates defined in the text. Depending on the patch type, either $(\rho, z)$ or $(r, \theta)$ are also shown. 
RSN test geries

PXO1 . PAR; 9

title-"Single Black Hole ( $P=0.01) "$

N_black_hole-1

grid=" rs2o"

;

black_hole=

Iho=0

mass $=1$

$\mathbf{z}=0$

momentum -0.01

spin=0

: migen pxol

directco pxO1 $1.00-10$

(4310.60 cpu seconds) 
Figure 43 - RSN test series, pr01 quantitative results
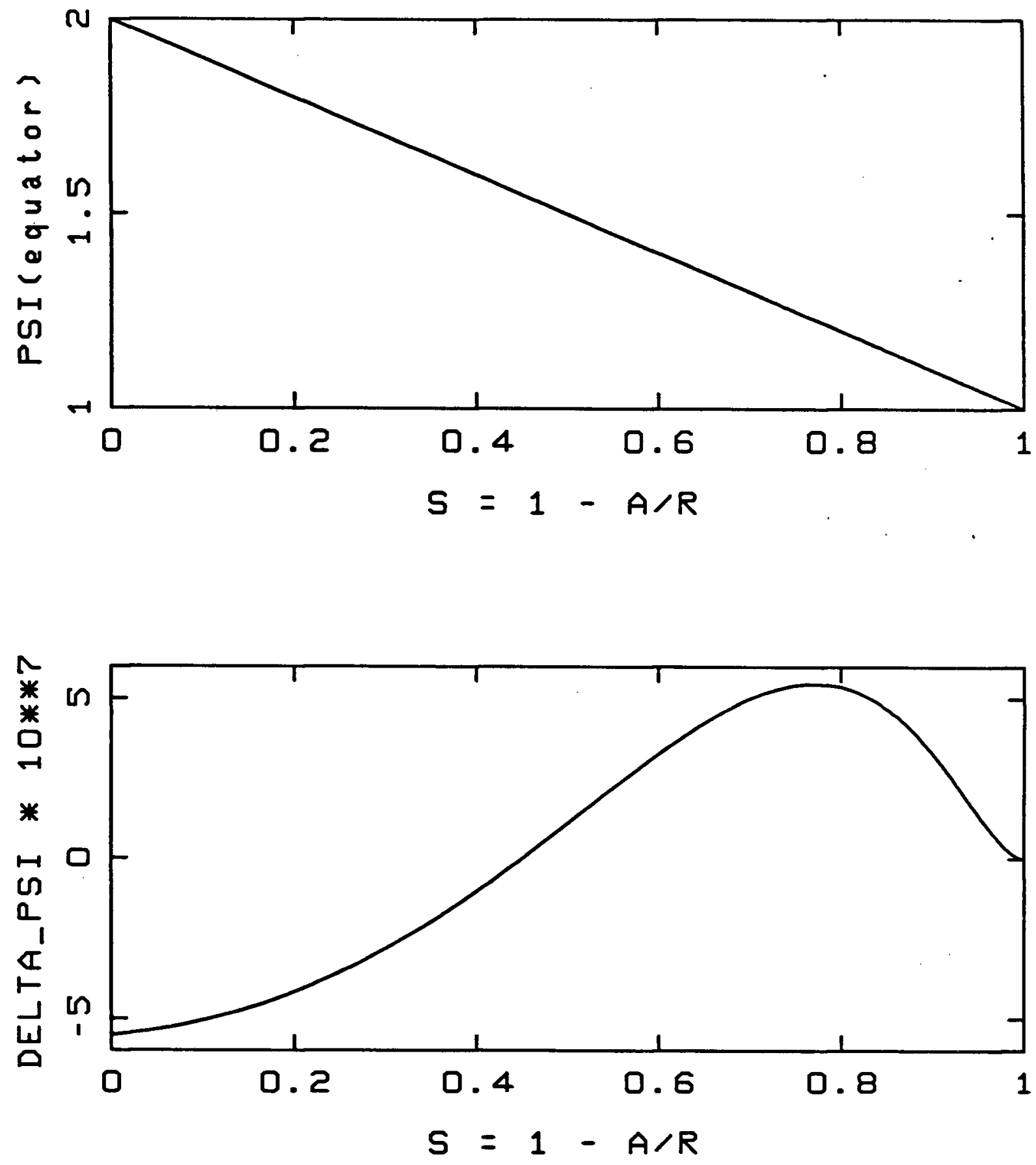

This figure shows $\Psi$ (equator) and $\delta \Psi=\Psi$ (north pole) $-\Psi$ (equator), both as functions of $s=1-a / r$. The former shows the general radial trend of $\Psi$, while the latter is a measure of the deviation of $\Psi$ from spherical symmetry. 


\section{RSN test series}

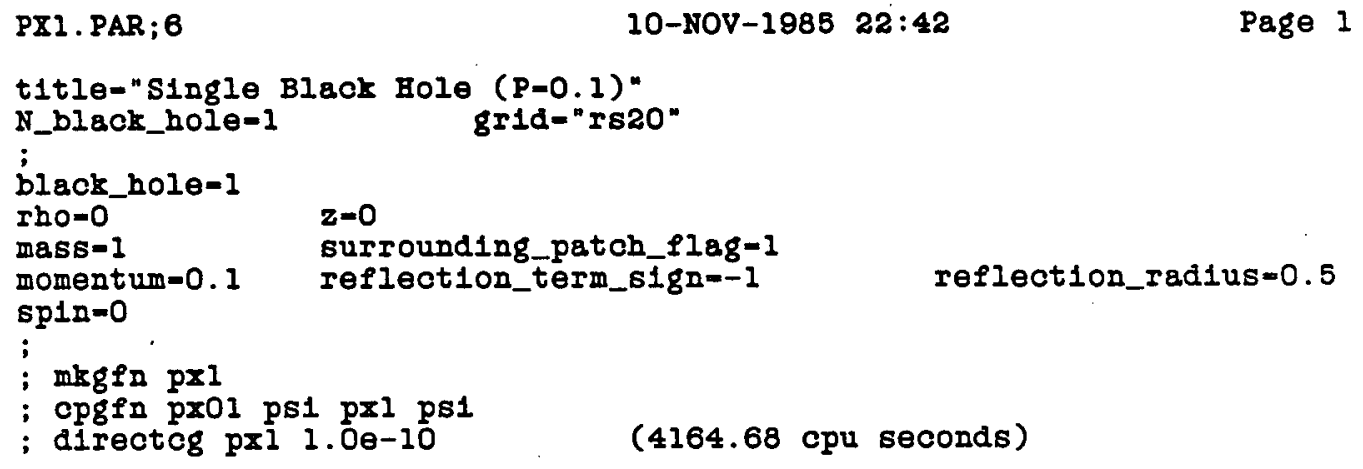


Figure 44 - RSM test series, px1 quantitative resalts
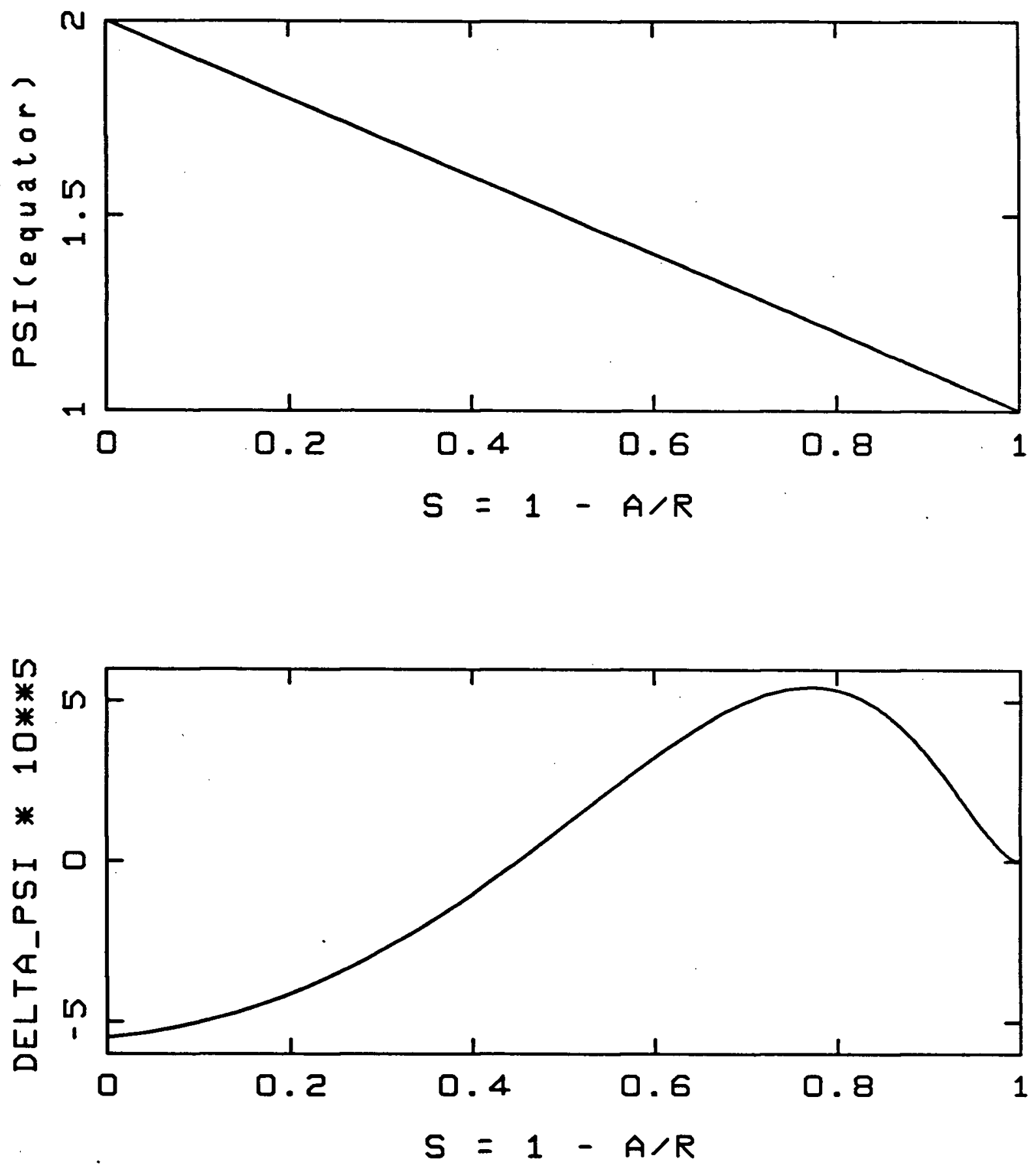

This figure shows $\Psi$ (equator) and $\delta \Psi=\Psi$ (north pole) $-\Psi$ (equator), both as functions of $s=1-a / r$. The former shows the general radial trend of $\Psi$, while the latter is a measure of the deviation of $\Psi$ from spherical symmetry. 


\section{RSM test series}

P1. PAR; 8

$10-$ NOV-1985 $22: 42$

Page 1

title-"Single Black Hole $(p-1)$ "

N_black_hole=1 8I1d-" 5820 "

black_hole-1

rho-0 $z=0$

m858-1 surrounding_patch_flag-1

momentum-1 reflect1on_term_sign=-1 reflect1on_Iadius=0.5

$\operatorname{sp} 12=0$

;

; migfn pl

: Cpgfn pxl psi pl psi

; directos pl $1.00-10$

(7422.40 cpu seconds) 
Figure 45 - RSN test series, p1 quantitative results
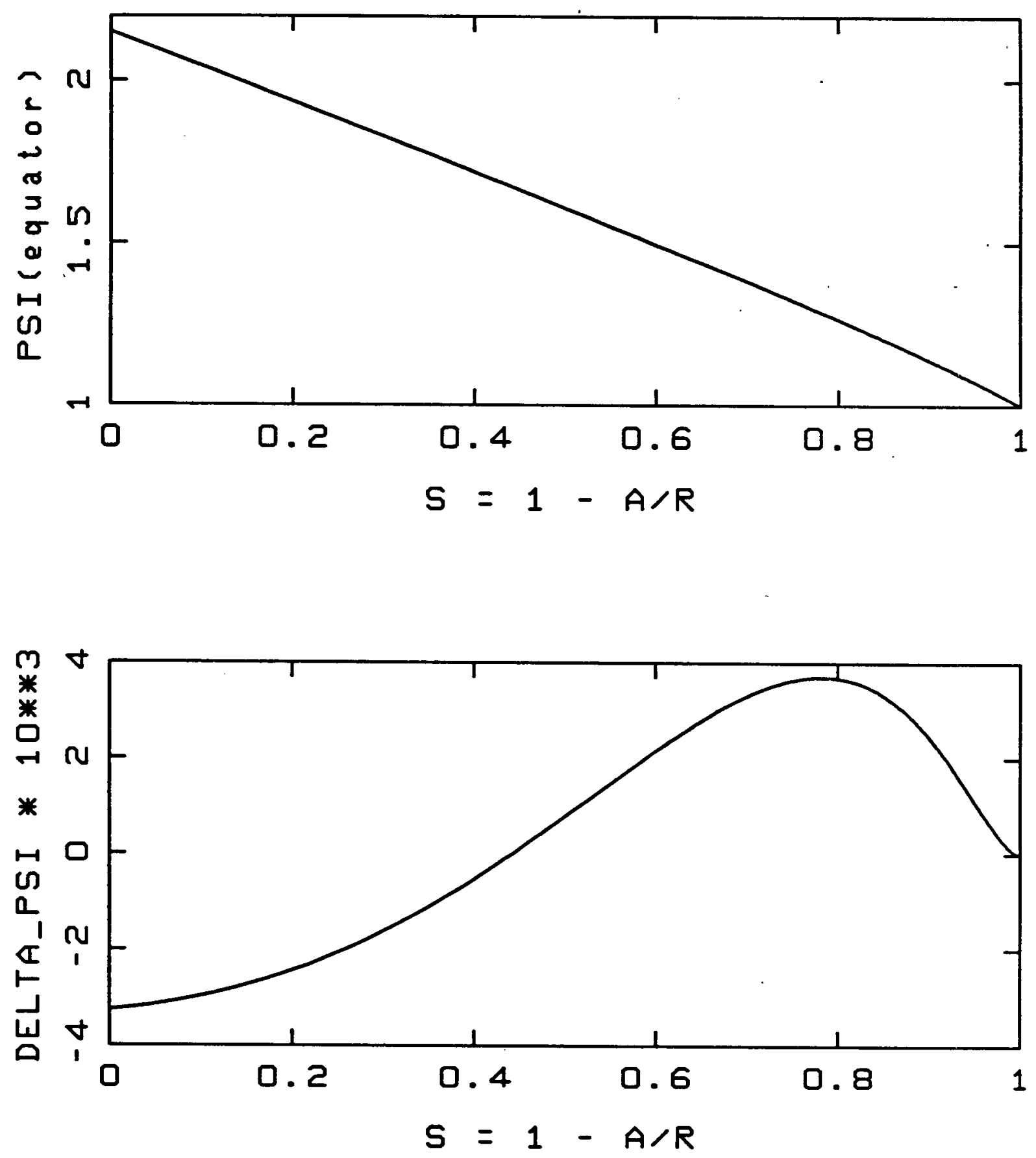

This figure shows $\Psi$ (equator) and $\delta \Psi=\Psi$ (north pole) $-\Psi$ (equator), both as functions of $s=1-a / r$. The former shows the general radial trend of $\Psi$, while the latter is a measure of the deviation of $\Psi$ from spherical symmetry. 


\section{RSM test Beries}

P10. PAR; 5

10-NOV-1985 22:42

Page 1

t1tle="Single Black Hole (P-10)"

N_black_hole=1 grid="rs2o"

;

black_hole=1

rho=0

$z=0$

mass-1

surrounding_patch_flag=1

momentum-10 reflection_term_sign=-1

reflect1on_radius $=0.5$

spin=0

; migen p10

opgin pl psi p10 psi

ilfecteg plo $1.0 e-10$

(11970.08 opu seconds) 
Figure 46 - RSM test series, p10 quantitative result3
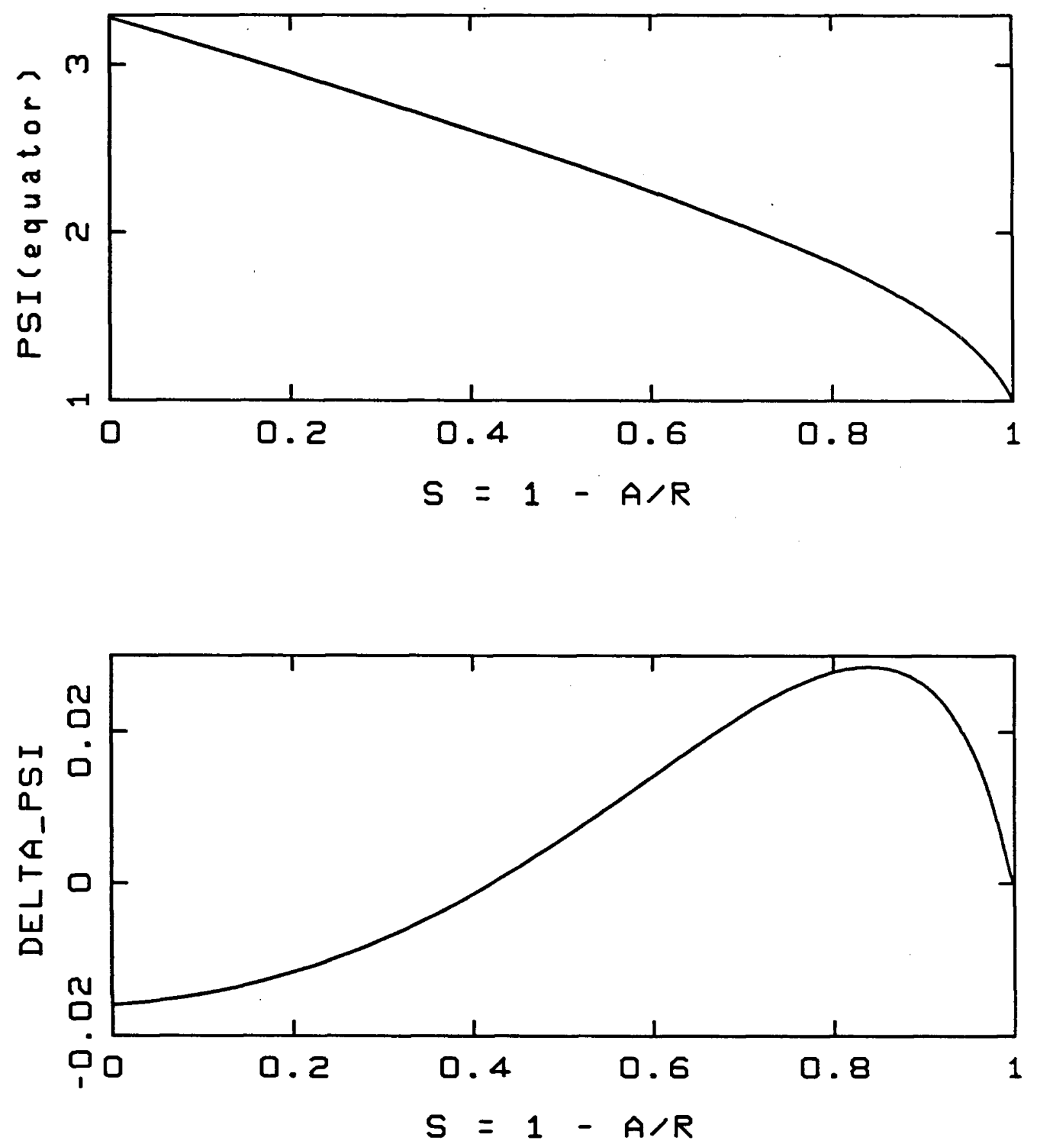

This figure shows $\Psi$ (equator) and $\delta \Psi=\Psi$ (north pole) $-\Psi$ (equator), both as functions of $s=1-a / r$. The former shows the general radial trend of $\Psi$, while the latter is a measure of the deviation of $\Psi$ from spherical symmetry. 


\section{RSN test series}

P100. PAR; 3

10-NOV-1985 $22: 42$

Page 1

t1tle="Single Black Bole $(p=100)$ "

N_black_hole-1 8r1d="Is20"

;

black_hole-1

Iho-0

mass $=1$

$\mathbf{z}=0$

momentum-100

surround1ng_patch_flag-1

$\operatorname{sp} 1 \mathrm{n}=0$

reflection_term_s1gn=-1

reflection_radius $=0.5$

;

; mkgfn p100

; cpgen p10 ps1 p100 ps1

; directog p100 1.00-10

(10439.14 cpu seconds) 
Figure 47 - RSN test series, p100 quantitative results
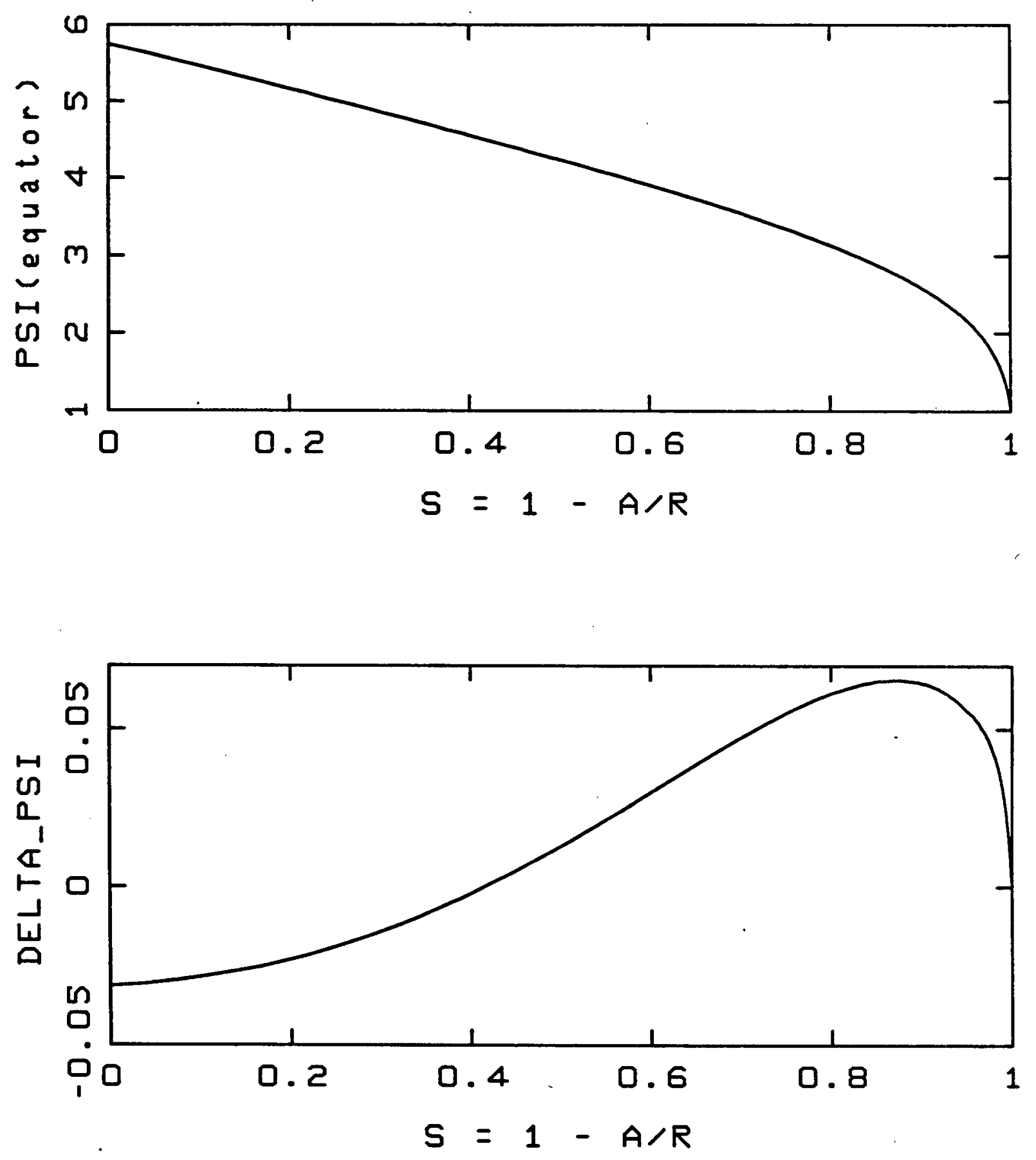

This figure shows $\Psi$ (equator) and $\delta \Psi=\Psi$ (north pole) $-\Psi$ (equator), both as functions of $s=1-a / r$. The former shows the general radial trend of $\Psi$, while the latter is a measure of the deviation of $\Psi$ from spherical symmetry. 


\section{RSM test series}

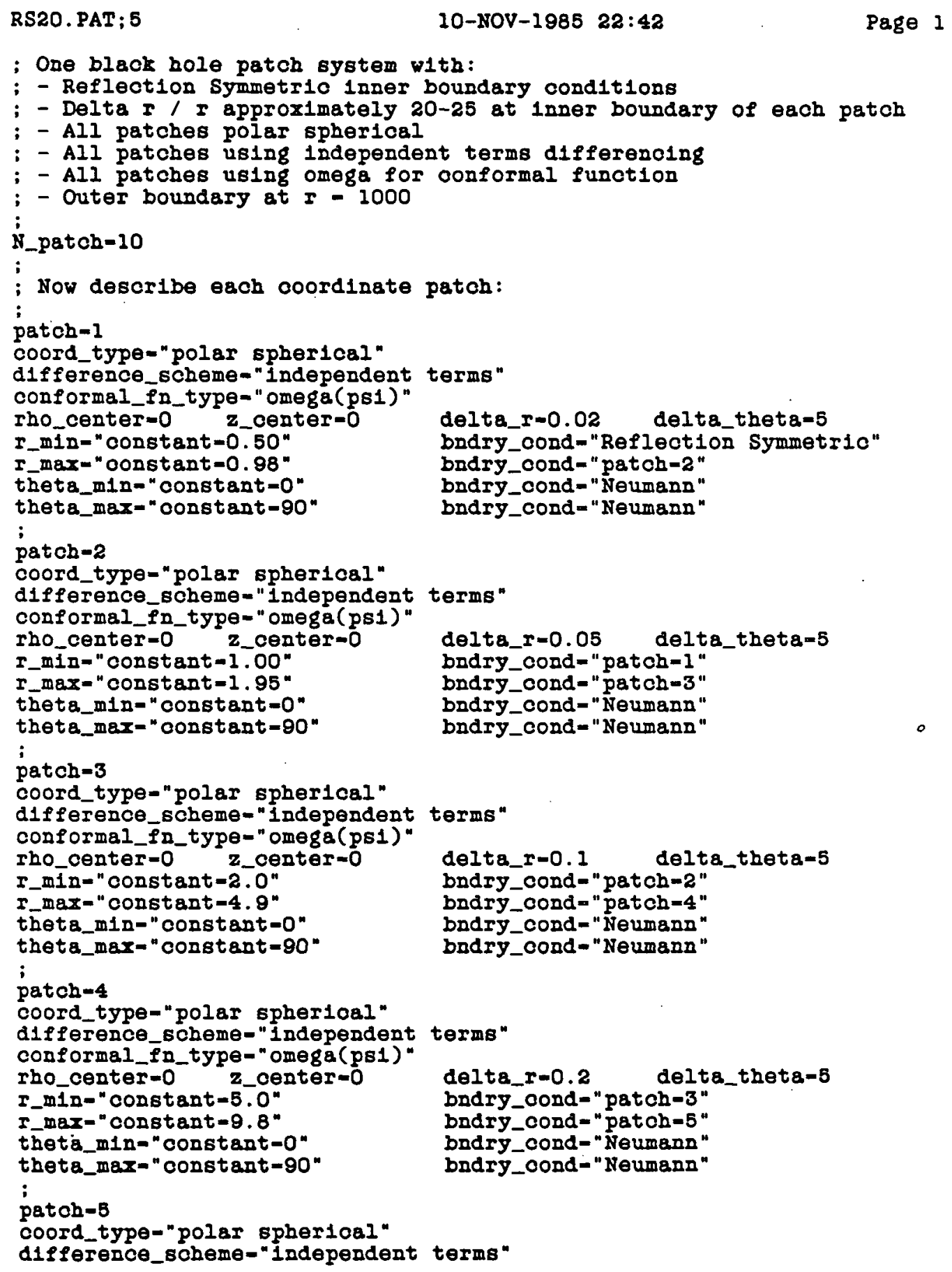


RSN test series

RS20. PAT; $\mathbf{5}$

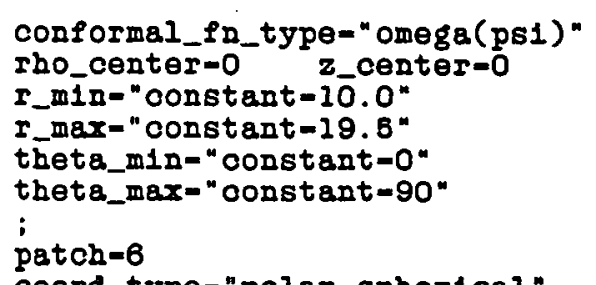

coord_type="polar spher1cal"

difference_scheme="1ndependent terms"

conformal_fn_type="omega(psi)"

rho_center $=0$ z_center $=0$

I_min=" constant $=20^{\text {" }}$

I max- "constant $=49$ "

theta_m1n-"constant-0"

thete_max-" constant $=90$ " ;

patch $=7$

coord_type-"polar spher1cal"

difference_scheme-"Independent terms"

conformal_fn_type="omega(ps1)"

rho_center=0 z_center $=0$

I_min-" constant $=5 \overline{0}$ "

$r$ max-" constant-98"

theta_m1n="constant $=0$ "

theta_mar-" constant-90"

delta_I-0.5 delta_theta-7.5

bndry_cond- "patch-4"

bndry_cond " patch-6"

bndry_cond = "Neumann"

bndry_cond= "Neumana"

;

patch-8

coord_type="polar spherical"

difference_scheme="Independent terms"

conformal_fn_type-"omega(psi)"

rbo_center $=0$ z_center=0

I_min="oonstant $=100$ "

$r$ max- " constent $=195$ "

theta_m1n=" constant $=0$ "

theta_max-"constant $=90$ "

delta_r-1 delta_theta $=7.5$

bndry_cond = "patch=5"

brdry_cond = "patch $=7$ "

bndry_cond = "Neumang"

bndry_cond= "Neumang"

;

patch-9

coord_type="polar spherical"

difference_scheme-"Independent terms"

conformal_fn_type="omega(psi)"

rho_center-o z_center $=0$

I_min=" constant $=200$ "

$r$ max-" constant $=490$ "

theta_m1n="constant $=0$ "

theta_max-" oonstant-90"

delta_r-2 delta_theta-7.5

bndry_cond = "patch-6"

bndry_cond = "patch $=8$ "

bndry_cond = "Neumann"

bndry_cond- "Neumann"

i

patch $=10$

coord_type-"polar spher1cal"

conformal_fn_type-"omege(ps1)"

rho_center $=0$ z_center=0

I_mIn = constent $=5 \overline{0} 0$ "

$I$ max = " constant -1000 "

theta_min="constant=0"

delta_r=5 delta_theta $=10$

bndry_cond- "patch-7"

bndry_cond = "patch-9"

bndry_cond = "Neumann"

bndry_oond= "Neumann"

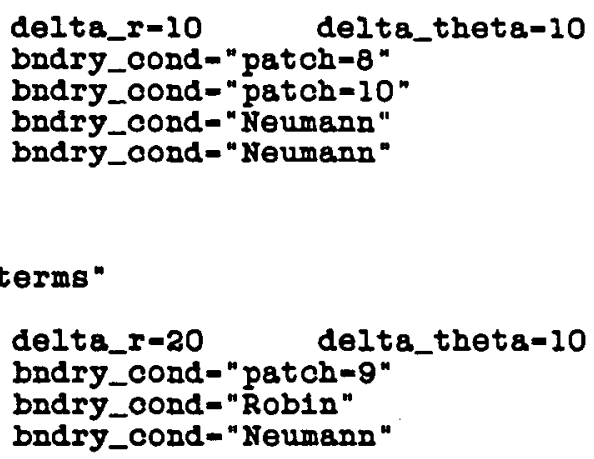


RSN test series

RS2O. PAT; 5

theta_max= "constant $=90$ "
10-NOV-1985 $22: 42$

Page 3 
MTO test series

PO. PAR; 9

10-NOV-1985 22:35

Page 1

title="Single Black Hole (P-O)"

N_black_hole-1 grid-"mt20b1g"

black_hole=1

rho $=0$

mass $=1$

momentum $=0$

$z=0$

$\operatorname{spin}=0$

surrounding_patch_flag=1

reflection_term_sign=0

reflection_radius $=0.5$

mikgin po

directeg po $1.00-10$ 
Table 24 - NTO test series, pO selected $\Psi$ values

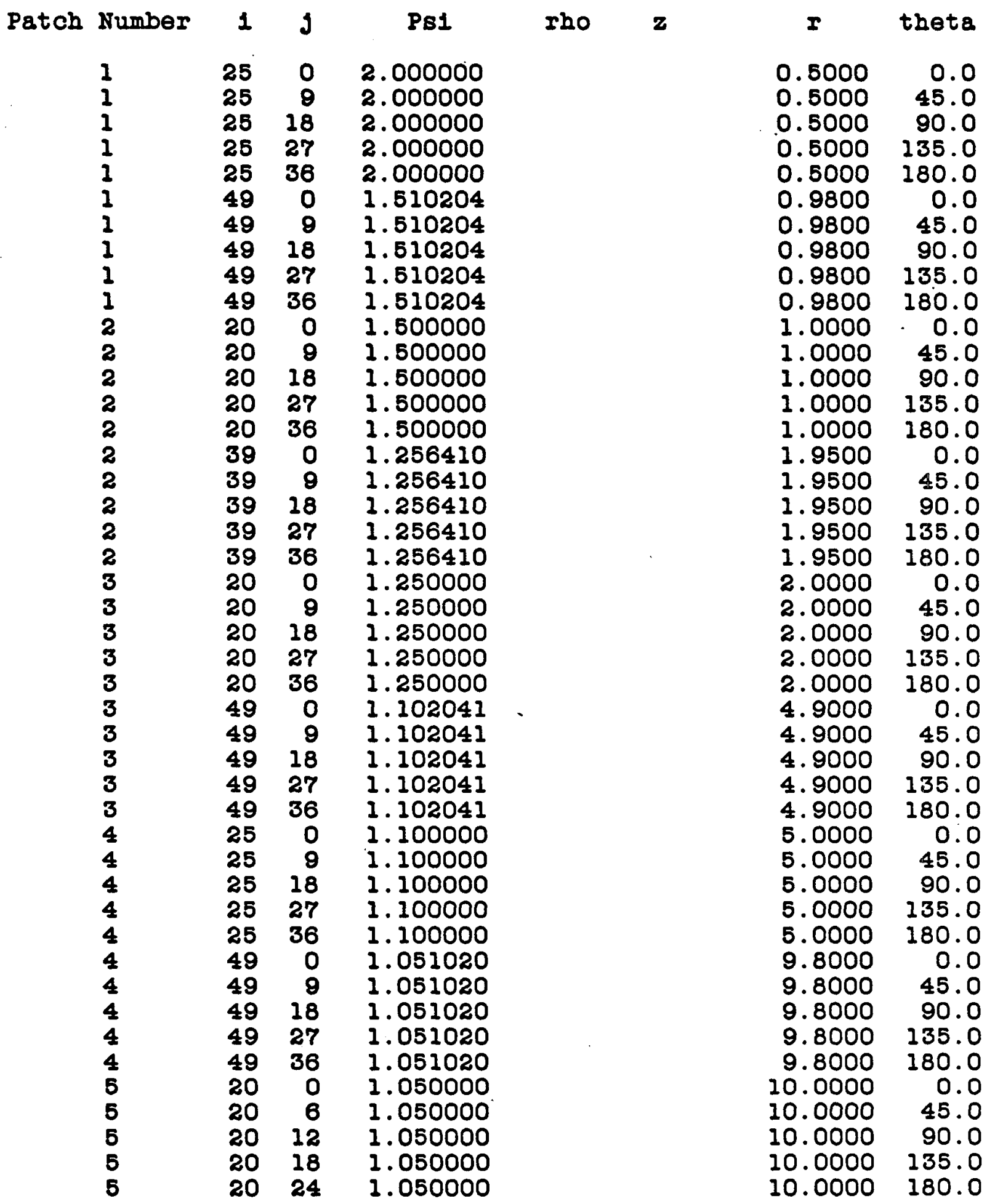

This table gives values of $\Psi$ at selected points in the space. These points are identified by triples (patch number, $i, j$ ), where $(i, j)$ are the generic integer patch coordinates defined in the text. Depending on the patch type, either $(\rho, z)$ or $(r, \theta)$ are also shown. 
NTO test series

PXO1. PAR ; 6

10-NOV-1985 $22: 35$

Page 1

title="Single Black Hole ( $P=0.01)$ "

N_black_hole=l

gr1d-"mt2Ob18"

black_hole-l

rho $=0$

mass $=1$

$\mathrm{z}=0$

momentum $=0.01$

spin $=0$

surrounding_petoh_flag=1

reflection_term_s1gn=0

reflection_radius $=0.5$

; mkgin prol

alrecteg pxO1 $1.00-10$

(28784.57 opu seconds) 
Figure 48 - NTO test series, px01 quantitative results
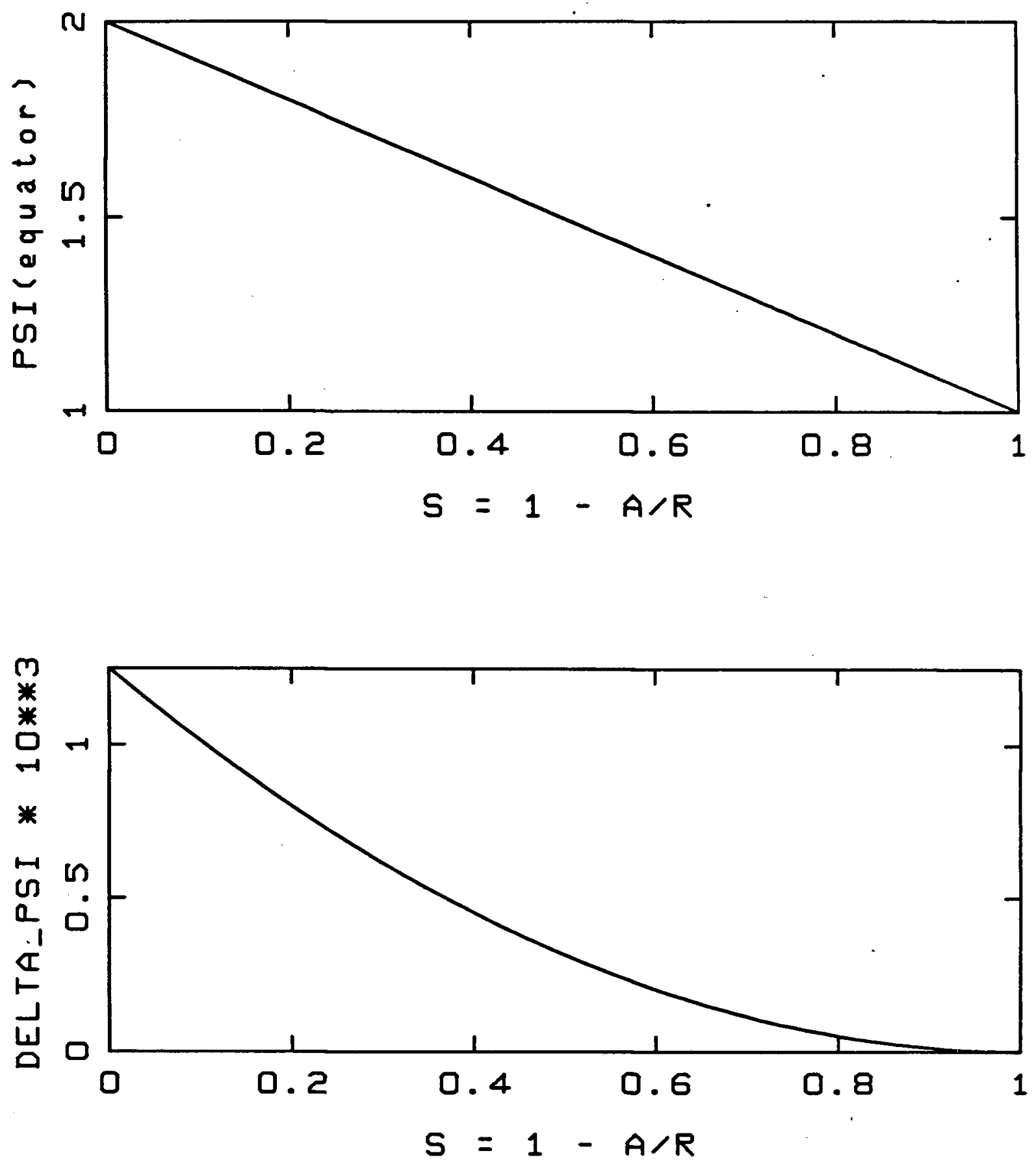

This figure shows $\Psi$ (equator) and $\delta \Psi=\Psi$ (north pole) $-\Psi$ (equator), both as functions of $s=1-a / r$. The former shows the general radial trend of $\Psi$, while the latter is a measure of the deviation of $\Psi$ from spherical symmetry. 


\section{NTO test series}

PXI. PAR; 4

$10-N O V-1985 \quad 22: 35$

Page 1

title="Single Black Hole ( $P=0.1)$ "

N_black_hole=1 grid="mt20b18"

;

black_hole-1

rho $=0$

$z=0$

mass=1 surrounding_patch_plag=1

momentum=0.1 reflection_term_s $1 g n=0$

reflection_radius $=0.5$

$\operatorname{spin}=0$

mkgfn pxl

cpgfn pxOl psi pxl psi

directog pxl $1.00-10$ (36840.61 cpu seconds) 
Figure 49 - MTO test series, p 11 quantitative results
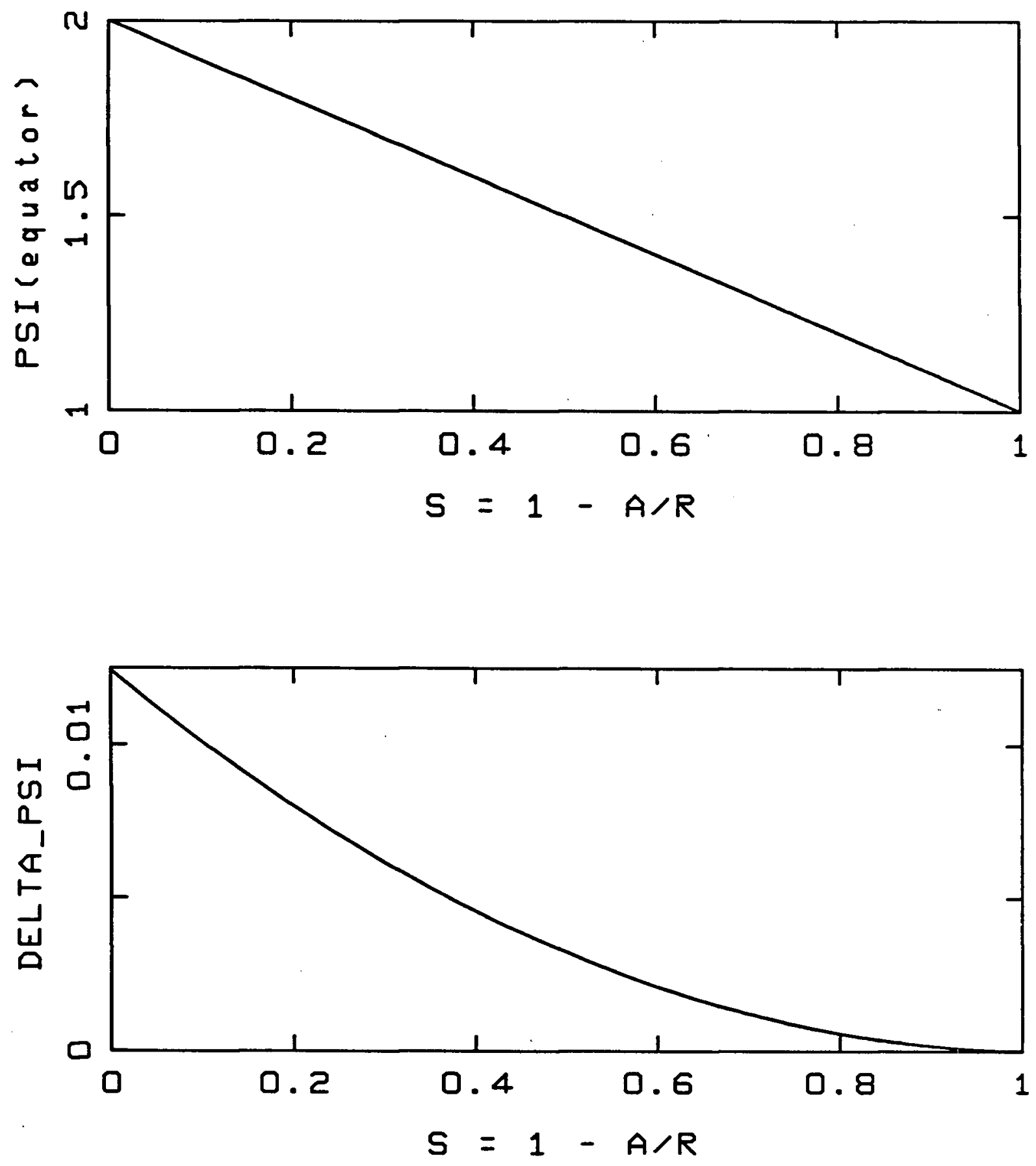

This figure shows $\Psi$ (equator) and $\delta \Psi=\Psi$ (north pole) $-\Psi$ (equator), both as functions of $s=1-a / r$. The former shows the general radial trend of $\Psi$, while the latter is a measure of the deviation of $\Psi$ from spherical symmetry. 


\section{MTO test series}

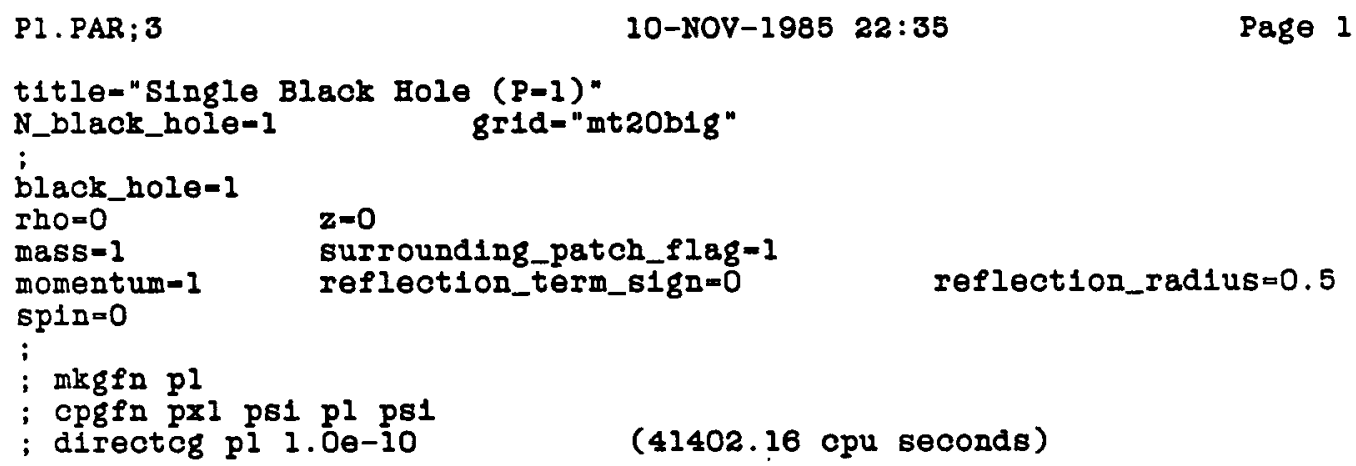


Figure 50 - NTO test series, p1 quantitative results
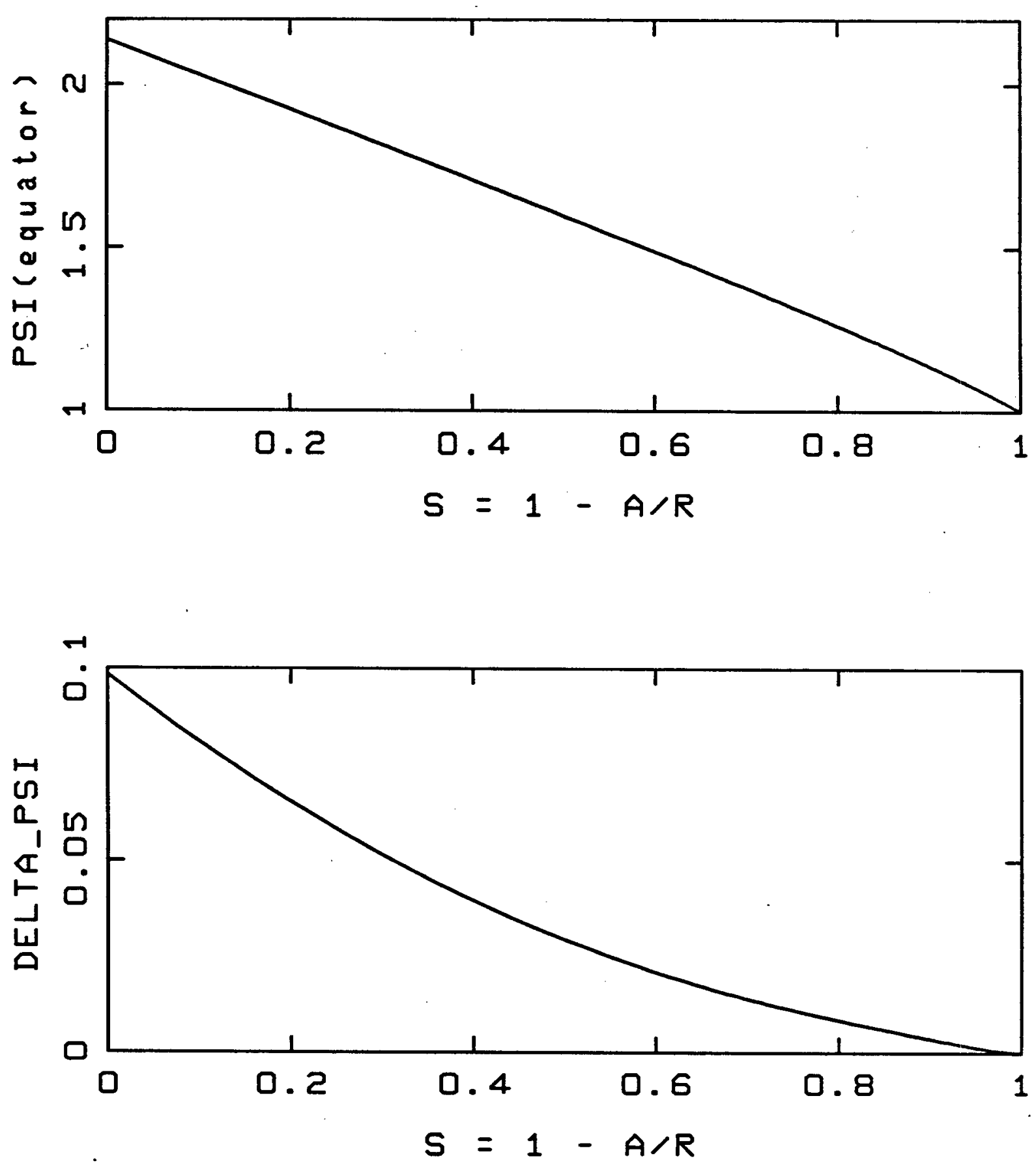

This figure shows $\Psi$ (equator) and $\delta \Psi=\Psi$ (north pole) $-\Psi$ (equator), both as functions of $s=1-a / r$. The former shows the general radial trend of $\Psi$, while the latter is a measure of the deviation of $\Psi$ from spherical symmetry. 


\section{NTO test series}

P10. PAR; 3

10-NOV-1985 22:35

Page 1

title="Single Black Hole (P-10)"

N_black_hole-1 grid="mt20b1g"

; bंlack_hole=1

$\mathrm{rho}=0$

mass $=1$

$\mathbf{z}=0$

momentum $=10$

surrounding_patch_flag=1

spin $=0$

reflection_term_sign=0

reflect1on_radius $=0.5$

;

; mkgfa p10

; cpgfn pl ps1 p10 ps1

; directcg $\mathrm{p} 10 \mathrm{1} 1.00-10$

(47072.84 cpu seconds) 
Figure 51 - NTO test series, p10 quantitative results
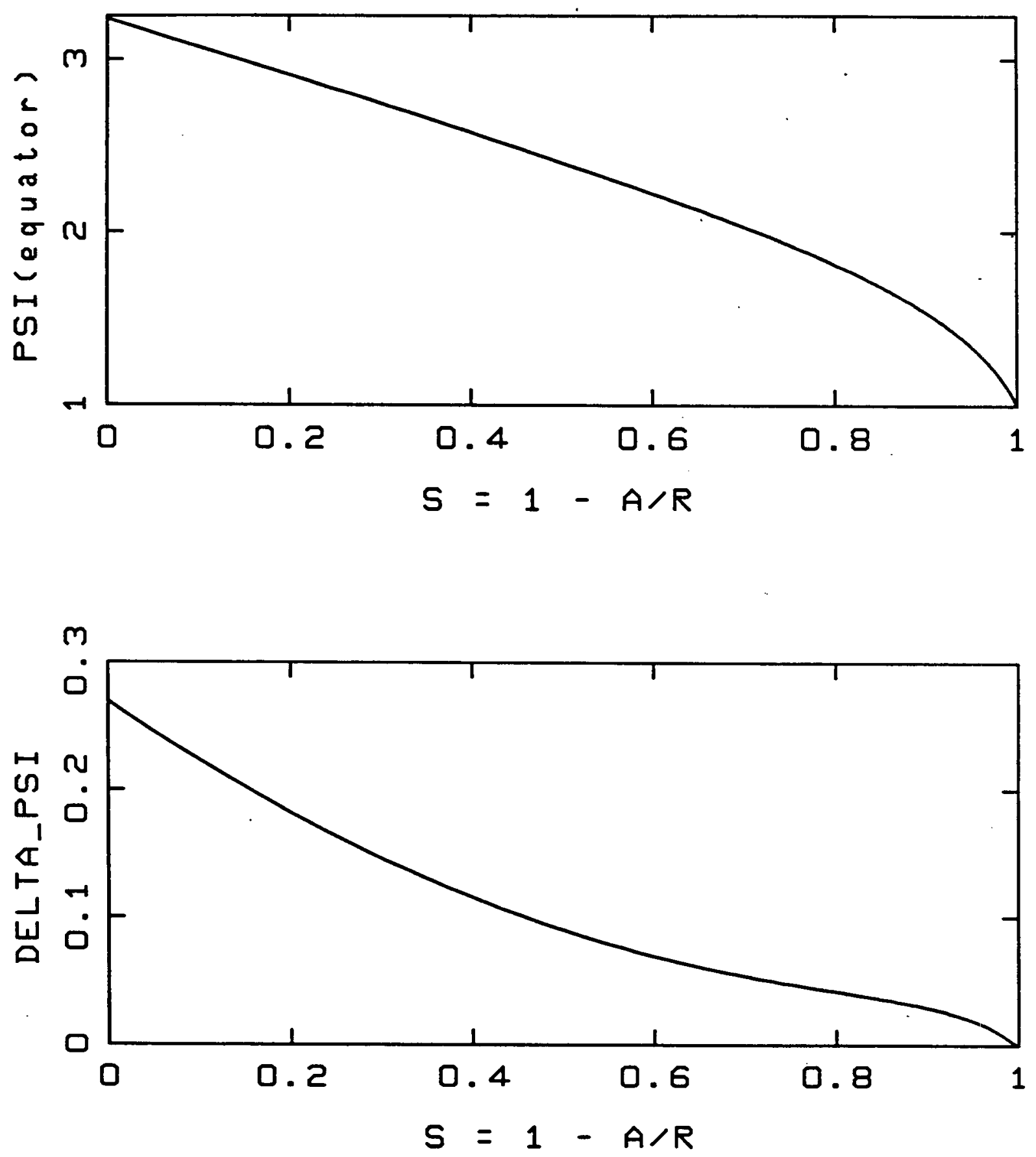

This figure shows $\Psi$ (equator) and $\delta \Psi=\Psi$ (north pole) $-\Psi$ (equator), both as functions of $s=1-a / r$. The former shows the general radial trend of $\Psi$, while the latter is a measure of the deviation of $\Psi$ from spherical symmetry. 


\section{MTO test series}

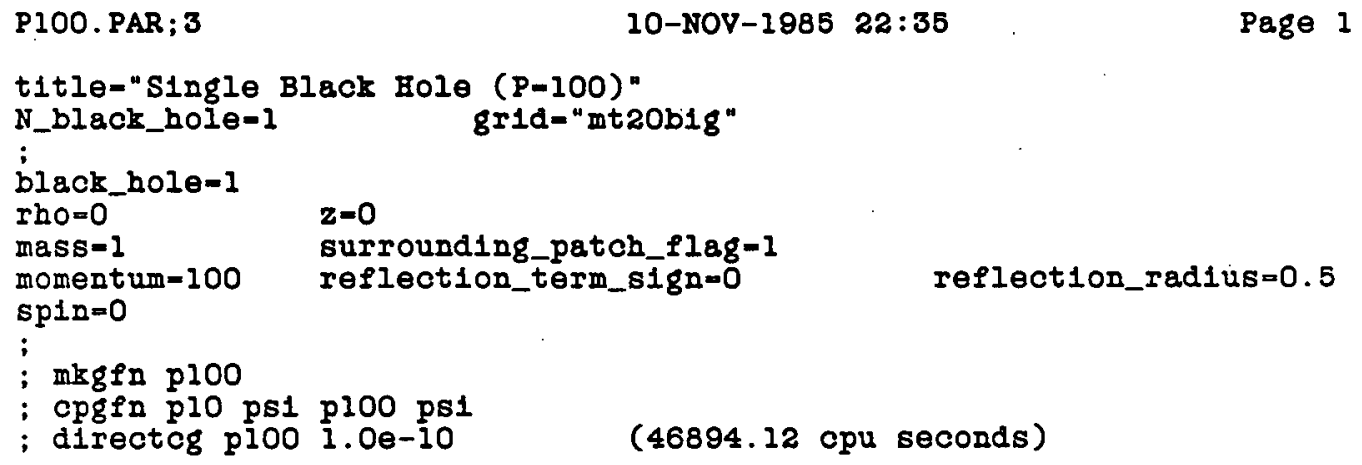


Figure 52 - MTO test series, p100 quantitative results
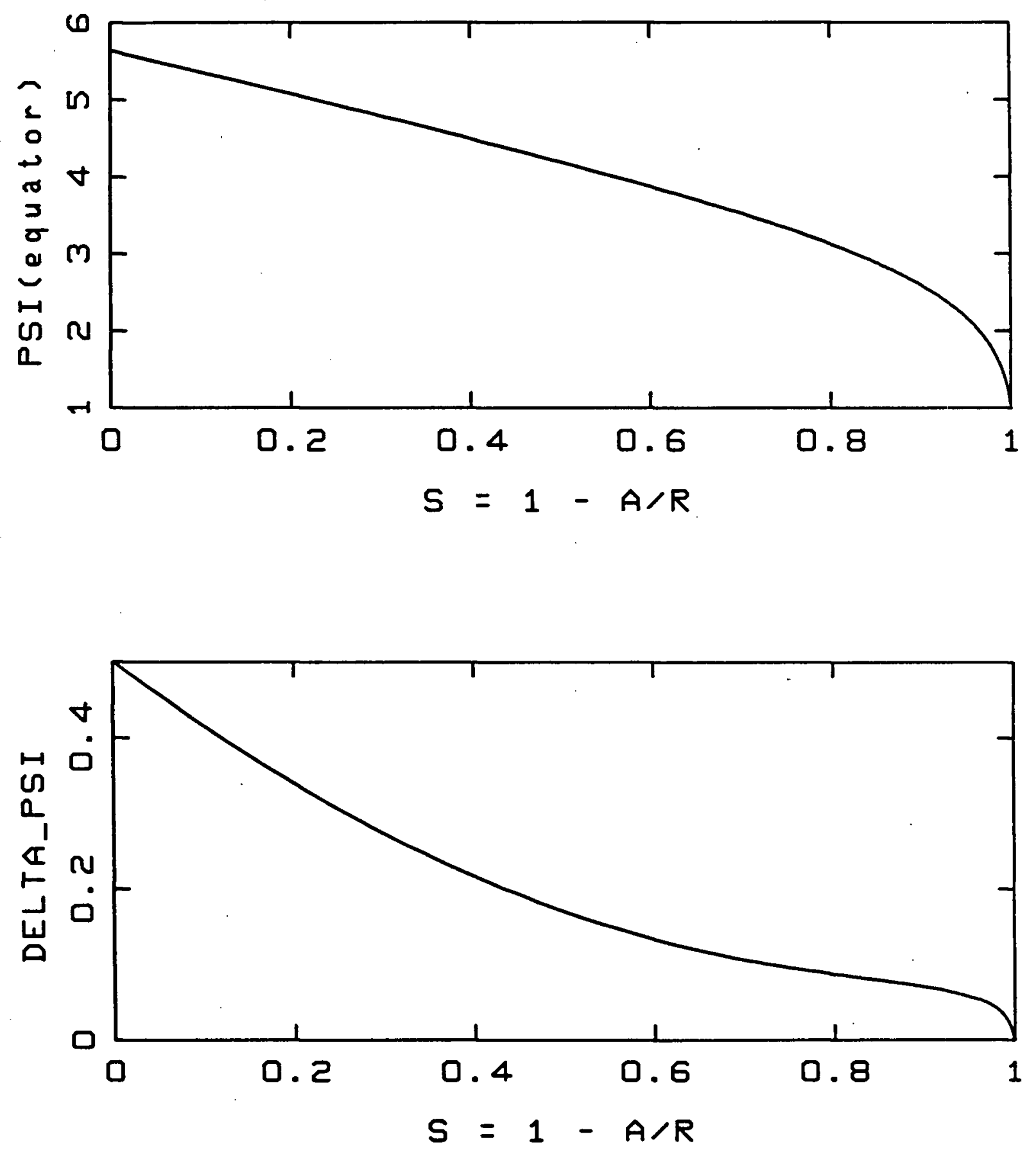

This figure shows $\Psi$ (equator) and $\delta \Psi=\boldsymbol{\Psi}$ (north pole) $-\boldsymbol{\Psi}$ (equator), both as functions of $s=1-a / r$. The former shows the general radial trend of $\Psi$, while the latter is a measure of the deviation of $\Psi$ from spherical symmetry. 


\section{NTO test series}

; One black hole patch system w1th:

- Marginaliy Trapped inner boundary conditions

- Delta I / r approximately 20-25 at Inner boundary of each patch

- All patohes polar spherical

- All patches using independent terms differenoing

- All patches using omega for conformal function

- Outer boundary at $\mathbf{r}=1000$

N_patch-10

Now describe each coordinate patch:

patch-1

coord_type-"polar spherical"

difference_scheme-"Independent terms"

conformal_fn_type="omega(psi)"

rho_center $=0$ z_center $=0$

r_min-"constant $=0.50$ "

I_max = "constant -0.98 "

theta_m1n="constant $=0$ "

delta_r=0.02 delta_theta-5

theta_max-"constant-180"

bndry_cond=" patch=2"

bndry_cond = "Neumann"

;

patch-2

coord_type-"polar spher1cal"

difference_scheme="Independent terms"

conformal_fn_type-"omega(psi)"

rho_center $=0$ z_center $=0$

I_min=" constant $=1.00$ "

I_max-" constant $=1.95$ "

theta_mln-"constant $=0$ "

bodry_cond- "Neumann"

theta_max="constant=180"

delta_r=0.05 delta_theta=5

budry_cond = "patch=1"

badry_cond = "patoh=3"

bndry_cond = "Neumann"

;

patch-3

coord_type-"polar spher1cal"

difference_scheme-"Independent terms"

conformal_fn_type-"omega(psi)"

rho_center $=0$ z_center $=0$

r_min=" constant $=2.0$ "

I_max-" constant $=4.9$ "

theta_min=" constant $=0$ "

bndry_cond= "Neumen"

theta_mar= "constant $=180$ "

delta_I=0.1 delta_theta-5

bndry_cond-"patch-2"

bndry_cond- " patch $=4$ "

bndry_cond-" Neumann"

;

patch $=4$

coord_type="polar spher1cal"

difference_scheme-"1ndependent terms"

conformal_fn_type-"omega(ps1)"

rho_center $=0$ z_center $=0$

I_mIn=" constant $=5.0$ "

I_max="constant $=9.8$ "

theta_m1n-"constant=0"

theta_max="constant $=180$ "

bndry_cond= "Neumenn"

;

patch-5

coord_type-"polar spherical"

difference_Bcheme-"Independent terms"

delta_r=0.2 delta_theta=5

bndry_cond = "patch=3"

bndry_cond= "patch-5"

bndry_cond = "Neumann"

bndry_cond = "Neumenn" 


\title{
MTO test geries
}

KT2OBIG.PAT; 4

conformal_fn_type="omega(ps1)"

rho_center $=0$ z_center=0

r_min-" constant -10.0 "

r_max- "constant $=19.6$ "

theta_min="constant $=0$ "

theta_max=" constant $=180$ "

;

patch $=6$

coord_type="poler spherical"

difference_scheme-"Independent

conformal_fn_type= "omega(pat)"

rho_center $=0 \quad$ z_center $=0$

I_mIn=" constant $-20^{\circ}$

$r$ max-" constant $=49$ "

theta_m1n=" constant $=0$ "

theta_max="constant $=180$ "

;

patch $=7$

coord_type-"poler spher1cal"

difference_scheme-"Independent terms"

conformal_fn_type-"omega(psi)"

rho_center=0 z_center $=0$

I_mIn=" constant $=5 \overline{0}$ "

I_max= " constant -98 "

theta_min="constant $=0$ "

theta_max = "constant $=180$ "

;

patch $=8$

coord_type="poler spher1cal"

difference_scheme-"Independent

conformal_fn_type= "omega(ps1)"

rho_center-0 z_center $=0$

I_mIn="constant $=100$ "

r_max-"constant-195"

theta_m1n-"constant-0"

theta_max-" constant $=180$ "

;

patch $=9$

coord_type="polar spher1cal"

difference_scheme="Independent terms"

conformal_fn_type-"omega(ps1)"

rho_center-0 z_center-0

r_min="constant $=200$ "

$r$ max = "constant -490 "

theta_min-" constant $=0$ "

theta_max=" oonstant-180"

;

patch $=10$

coord_type-"polar spherical"

difference_scheme-"Independent terms"

conformal_fn_type-"omega(p81)"

rho_center $=0$ z_center $=0$

I_mIn="constant $-500 "$

$r$ max-" oonstant $=1000$ "

theta_m1n-"constant-0"

terms"
10-HOV-1985 22:35

Page 2

delta_r=0.5 delta_theta-7.5

bndry_cond = "patch $=4$ "

bndry_cond = "patch=6"

bndry_cond = "Neumann"

bndry_cond = "Neumann"

terms"

delta_r-1 delta_theta=7.5

bndry_cond= "patch-5"

bndry_cond = "patch-7"

bndry_cond = "Neumann"

bndry_cond=" Neumann"

delta_r=2 delta_theta=7.5

bndry_cond- "patch $=6$ "

bndry_cond= "patch-8"

bndry_cond- "Neumann"

bndry_cond = "Neumann"

delta_r=5 delta_theta $=10$

bndry_cond = "patch-7"

bndry_cond = "patch $=9 "$

bndry_cond= "Neumann"

bndry_cond = " Neumann"

\author{
delta_r-10 delta_theta-10 \\ bndry_cond- "patoh-8" \\ bndry_cond=" patch-10" \\ bndry_cond- "Neumann" \\ bndry_cond- "Neumann"
}

delta_r-20 delta_theta-10

bndry_cond-" patch=9"

bndry_cond- "Rob1n"

bidry_cond- "Neumenn" 
MTO test series

MTZOBIG. PAT; 4

theta_max $=$ " constant $=180 "$
10-NOV-1985 22:36

Page 3 
PP test series

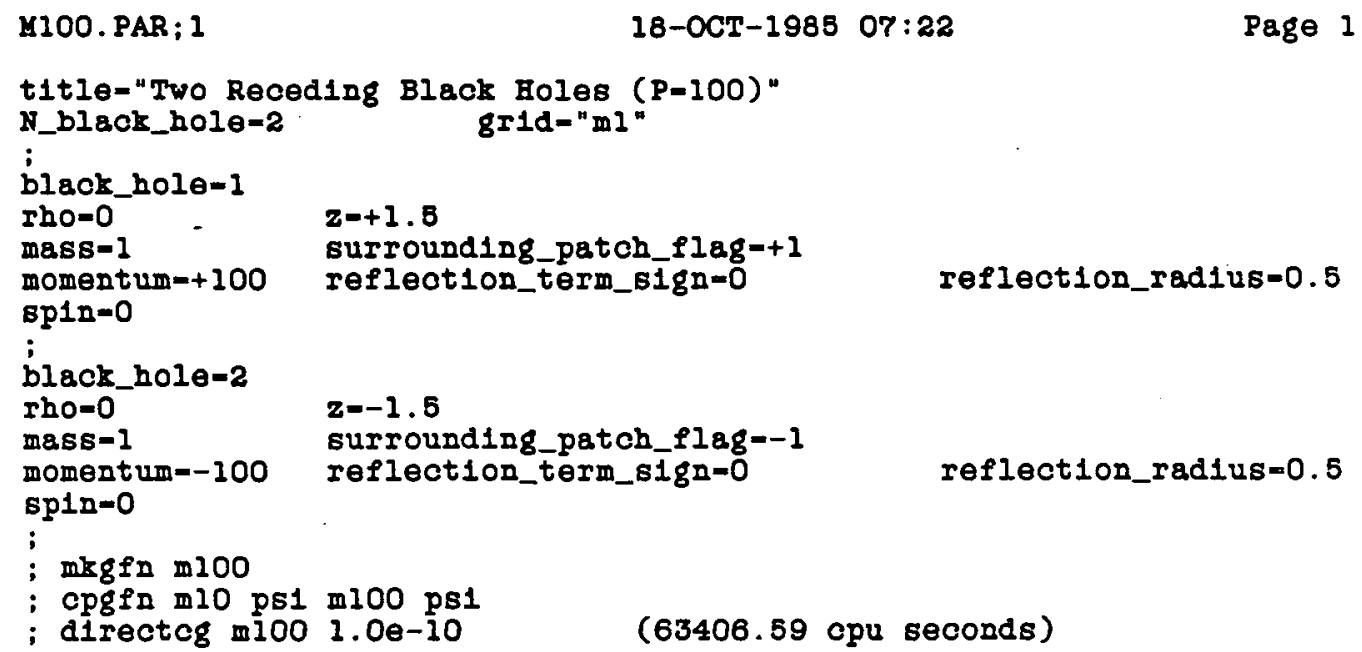


Table 25 - PP test series, m100 selected $\Psi$ values

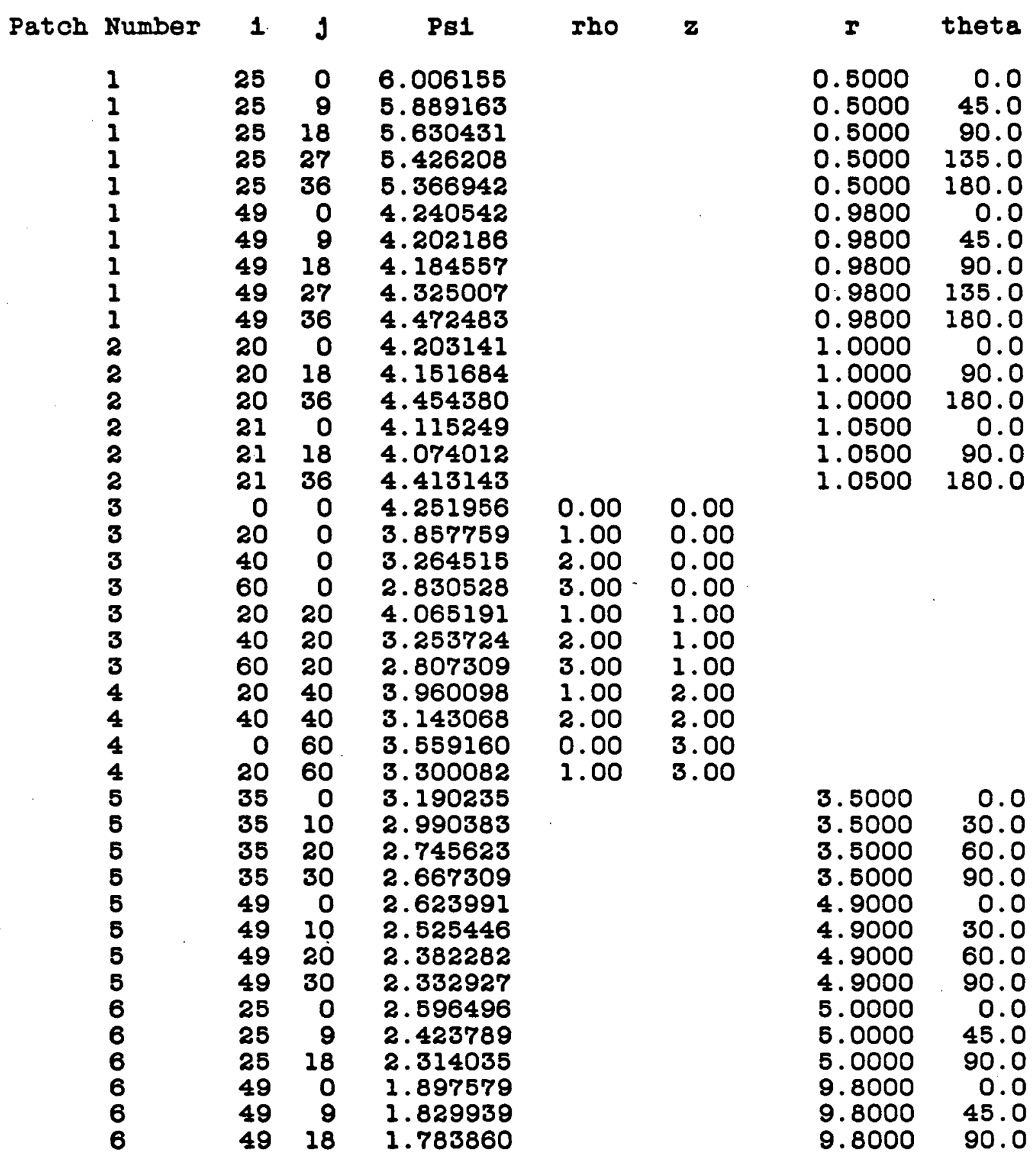

This table gives values of $\Psi$ at selected points in the space. These points are identified by triples (patch number, $i, j$ ), where $(i, j)$ are the generic integer patch coordinates defined in the text. Depending on the patch type, either $(\rho, z)$ or $(r, \theta)$ are also shown. 


\section{PP test series}

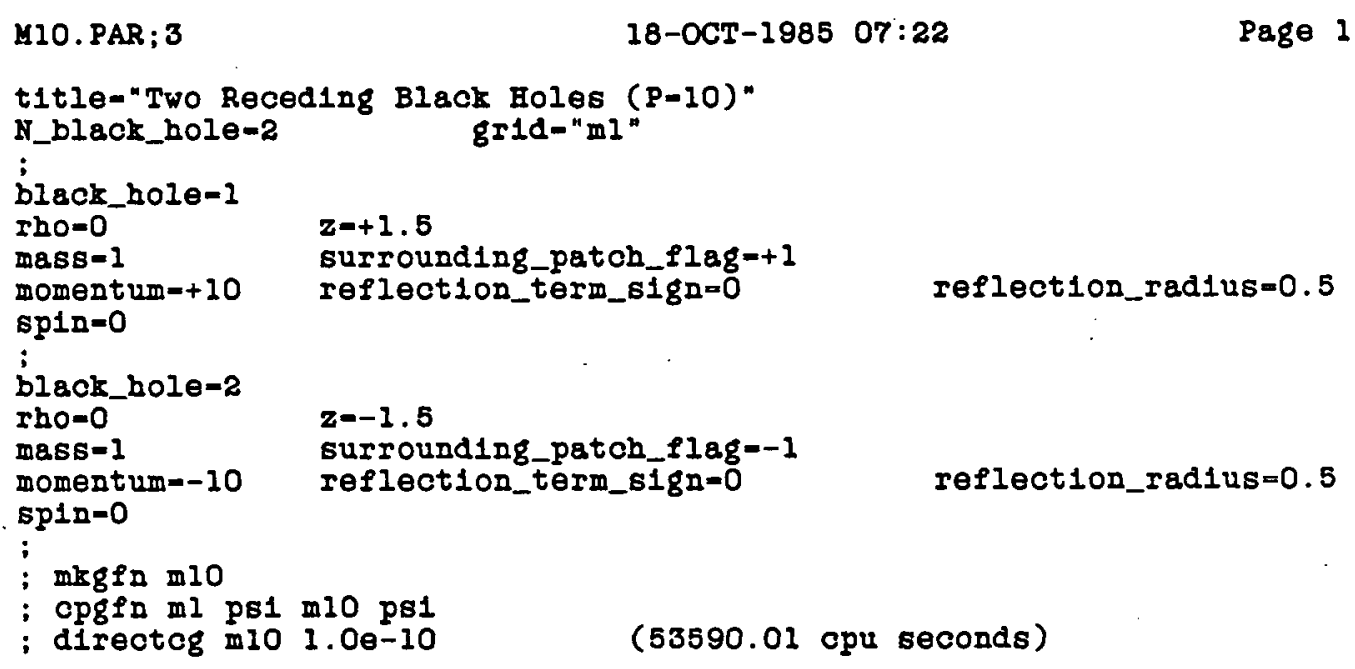


Table 26 - PP test Beries, m10 Belected $\Psi$ valnes

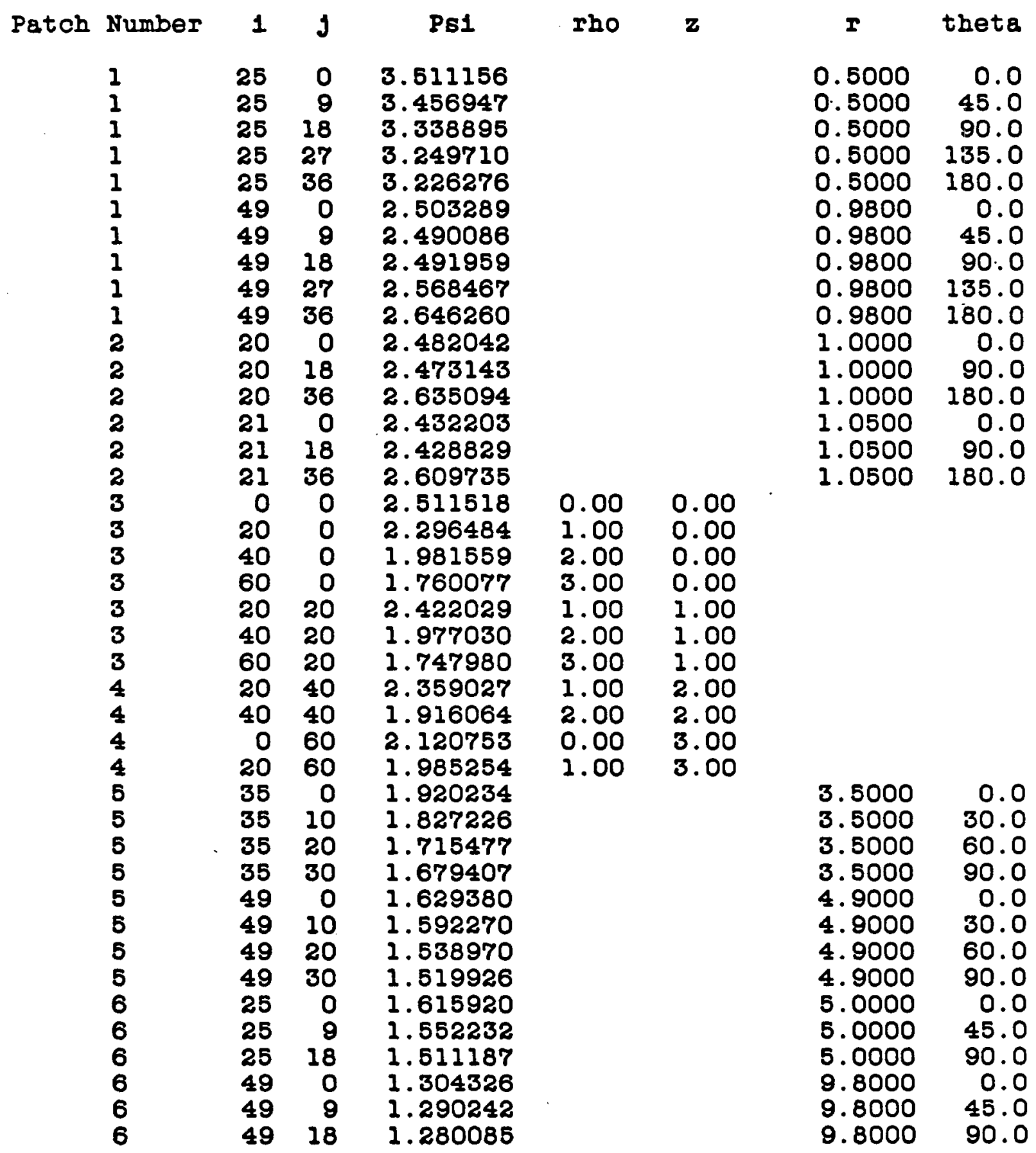

This table gives values of $\Psi$ at selected points in the space. These points are identified by triples (patch number, $i, j$ ), where $(i, j)$ are the generic integer patch coordinates defined in the text. Depending on the patch type, either $(\rho, z)$ or $(r, \theta)$ are also shown. 
PP test series

Y1. PAR;2

18-OCT-1985 $07: 22$

Page 1

t1tle-"Two Receding Black Holes ( $P=1)$ "

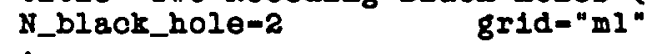

;

black_hole=1

Iho=0

mass $=1$

momentum $=+1$

$\operatorname{sp} 1 \mathrm{n}=0$

;

black_hole-2

rho $=0$

mass $=1$

momentum=-1

spin=0

$z=+1.5$

surrounding_patch_flag-+1

reflect1on_term_sign=0

reflect1on_radius $=0.5$

;

mkgfn ml

cpgfn mxl psi ml psi

- directos mi 1.0e-10

(47018.38 cpu seconds)

$z=-1.6$

surround1ng_patch_flag=-1

reflection_term_s1gn=0

reflection_radius $=0.5$ 
Table 27 - PP test series, m1 selected $\Psi$ values

$\begin{array}{rrrcrlrr}\text { Patch Number } & 1 & 1 & \text { Ps1 } & \text { rho } & \text { Z } & \text { I } & \text { theta } \\ 1 & 25 & 0 & 2.476853 & & & 0.5000 & 0.0 \\ 1 & 25 & 9 & 2.470882 & & & 0.5000 & 45.0 \\ 1 & 25 & 18 & 2.464894 & & & 0.5000 & 90.0 \\ 1 & 25 & 27 & 2.477059 & & & 0.5000 & 135.0 \\ 1 & 25 & 36 & 2.490642 & & & 0.5000 & 180.0 \\ 1 & 49 & 0 & 1.821982 & & & 0.9800 & 0.0 \\ 1 & 49 & 9 & 1.827481 & & & 0.9800 & 45.0 \\ 1 & 49 & 18 & 1.852638 & & & 0.9800 & 90.0 \\ 1 & 49 & 27 & 1.911104 & & & 0.9800 & 135.0 \\ 1 & 49 & 36 & 1.961148 & & & 0.9800 & 180.0 \\ 2 & 20 & 0 & 1.808130 & & & 1.0000 & 0.0 \\ 2 & 20 & 18 & 1.839552 & & & 1.0000 & 90.0 \\ 2 & 20 & 36 & 1.951830 & & & 1.0000 & 180.0 \\ 2 & 21 & 0 & 1.775717 & & & 1.0500 & 0.0 \\ 2 & 21 & 18 & 1.808897 & & & 1.0500 & 90.0 \\ 2 & 21 & 36 & 1.930789 & & & 1.0500 & 180.0 \\ 3 & 0 & 0 & 1.850764 & 0.00 & 0.00 & & \\ 3 & 20 & 0 & 1.710494 & 1.00 & 0.00 & & \\ 3 & 40 & 0 & 1.515366 & 2.00 & 0.00 & & \\ 3 & 60 & 0 & 1.385855 & 3.00 & 0.00 & & \\ 3 & 20 & 20 & 1.806741 & 1.00 & 1.00 & & \\ 3 & 40 & 20 & 1.513728 & 2.00 & 1.00 & & \\ 3 & 60 & 20 & 1.378632 & 3.00 & 1.00 & & \\ 4 & 20 & 40 & 1.750647 & 1.00 & 2.00 & & \\ 4 & 40 & 40 & 1.473670 & 2.00 & 2.00 & & \\ 4 & 0 & 60 & 1.577118 & 0.00 & 3.00 & & \\ 4 & 20 & 60 & 1.500688 & 1.00 & 3.00 & & \\ 5 & 35 & 0 & 1.455685 & & & 3.5000 & 0.0 \\ 5 & 35 & 10 & 1.412833 & & & 3.5000 & 30.0 \\ 5 & 35 & 20 & 1.358943 & & & 3.5000 & 60.0 \\ 5 & 35 & 30 & 1.340448 & & & 3.5000 & 90.0 \\ 5 & 49 & 0 & 1.294193 & & & 4.9000 & 0.0 \\ 5 & 49 & 10 & 1.281592 & & & 4.9000 & 30.0 \\ 5 & 49 & 20 & 1.261733 & & & 4.9000 & 60.0 \\ 5 & 49 & 30 & 1.253732 & & & 5.9000 & 90.0 \\ 6 & 25 & 0 & 1.287189 & & & 5.0000 & 0.0 \\ 6 & 25 & 9 & 1.265370 & & & 5.0000 & 90.0 \\ 6 & 25 & 18 & 1.249105 & & & 9.8000 & 0.0 \\ 6 & 49 & 0 & 1.136699 & & & 9.8000 & 45.0 \\ 6 & 49 & 9 & 1.133951 & & & 9.8000 & 90.0 \\ 6 & 49 & 18 & 1.131489 & & & & \end{array}$

This table gives values of $\Psi$ at selected points in the space. These points are identified by triples (patch number, $i, j$ ), where $(i, j)$ are the generic integer patch coordinates defined in the text. Depending on the patch type, either $(\rho, z)$ or $(r, \theta)$ are also shown. 


\section{PP test series}

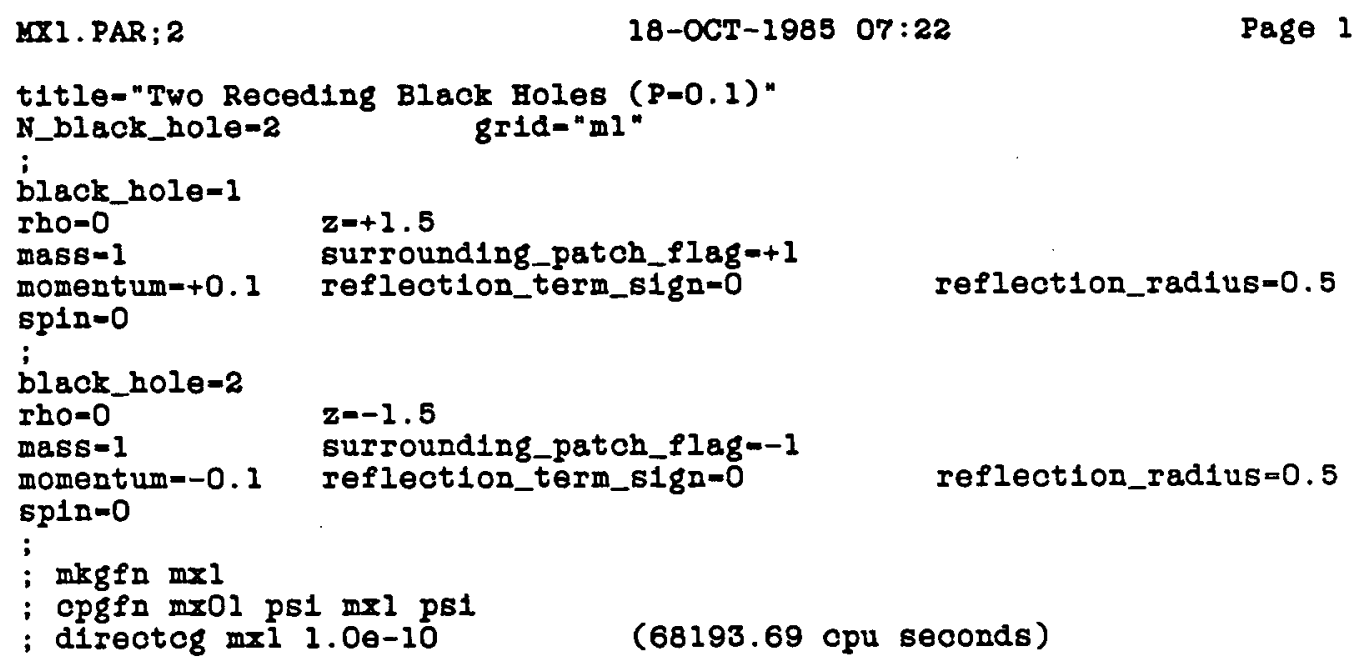


Table 28 - PP test series, $m \times 1$ selected $\Psi$ values

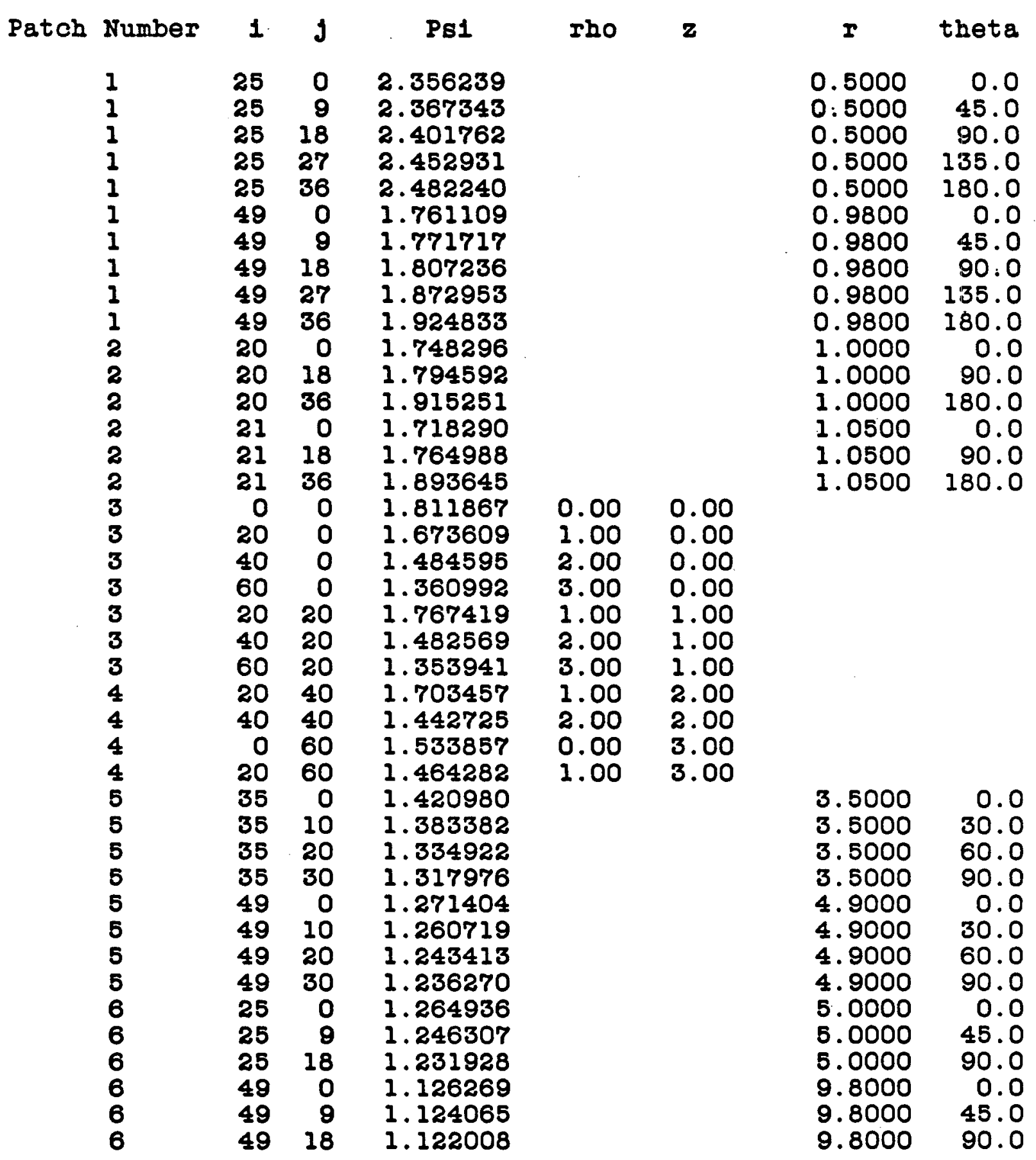

This table gives values of $\Psi$ at selected points in the space. These points are identified by triples (patch number, $i, j$ ), where $(i, j)$ are the generic integer patch coordinates defined in the text. Depending on the patch type, either $(\rho, z)$ or $(r, \theta)$ are also shown. 


\section{PP test series}

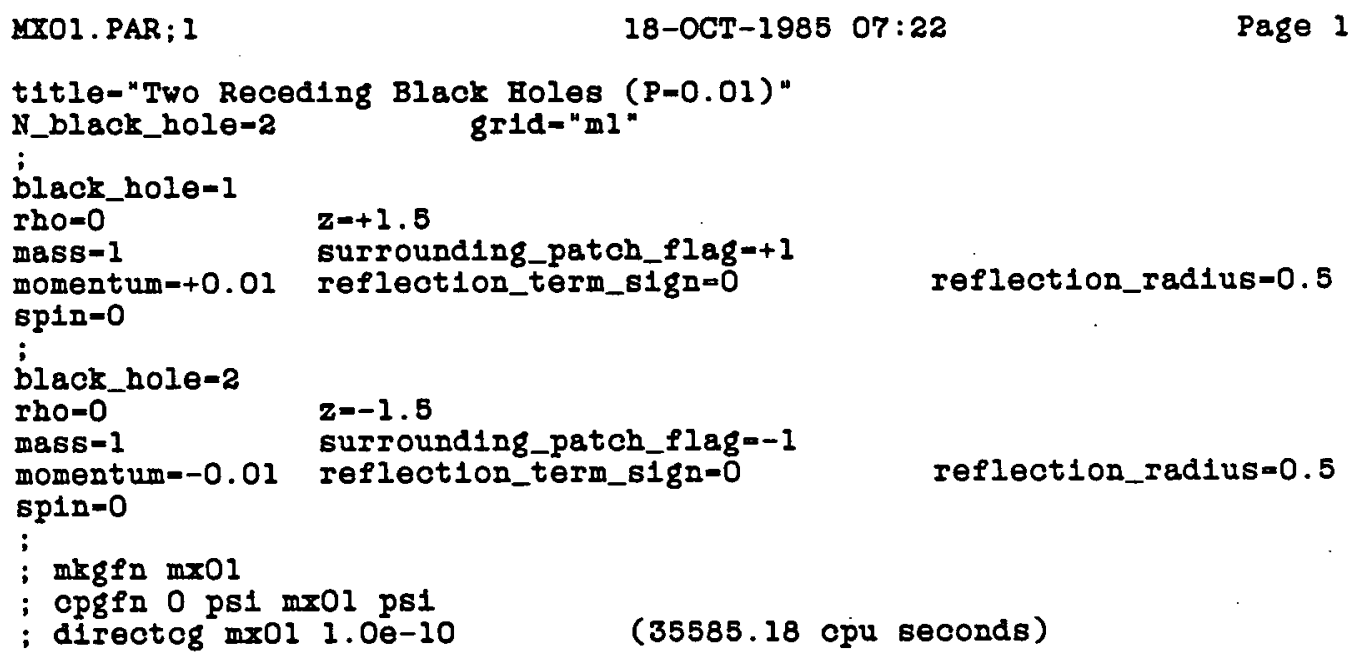


Table 29 - PP test series, mx01 selected $\Psi$ values

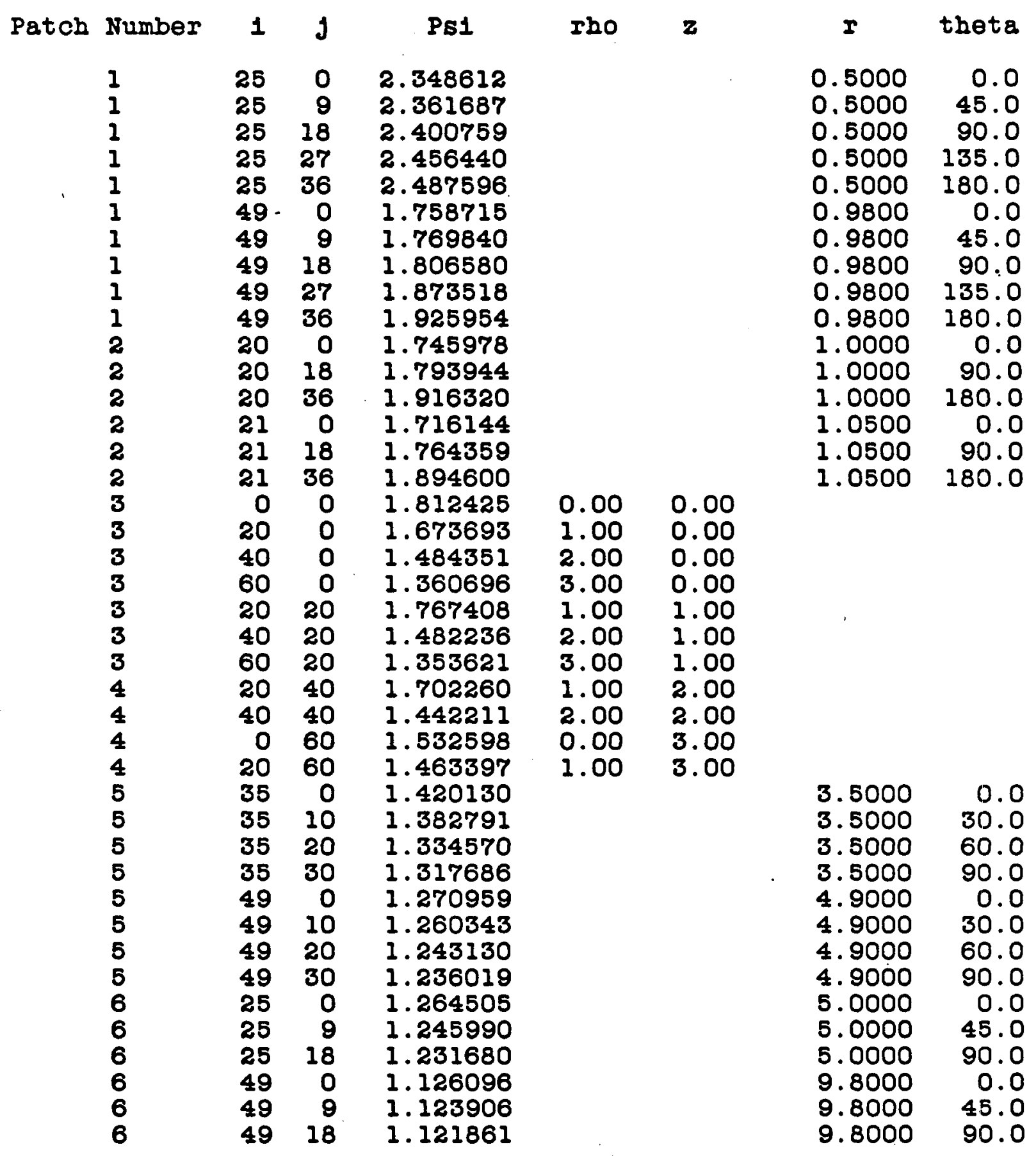

This table gives values of $\Psi$ at selected points in the space. These points are identified by triples (patch number, $i, j$ ), where $(i, j)$ are the generic integer patch coordinates defined in the text. Depending on the patch type, either $(\rho, z)$ or $(r, \theta)$ are also shown. 
PP test series

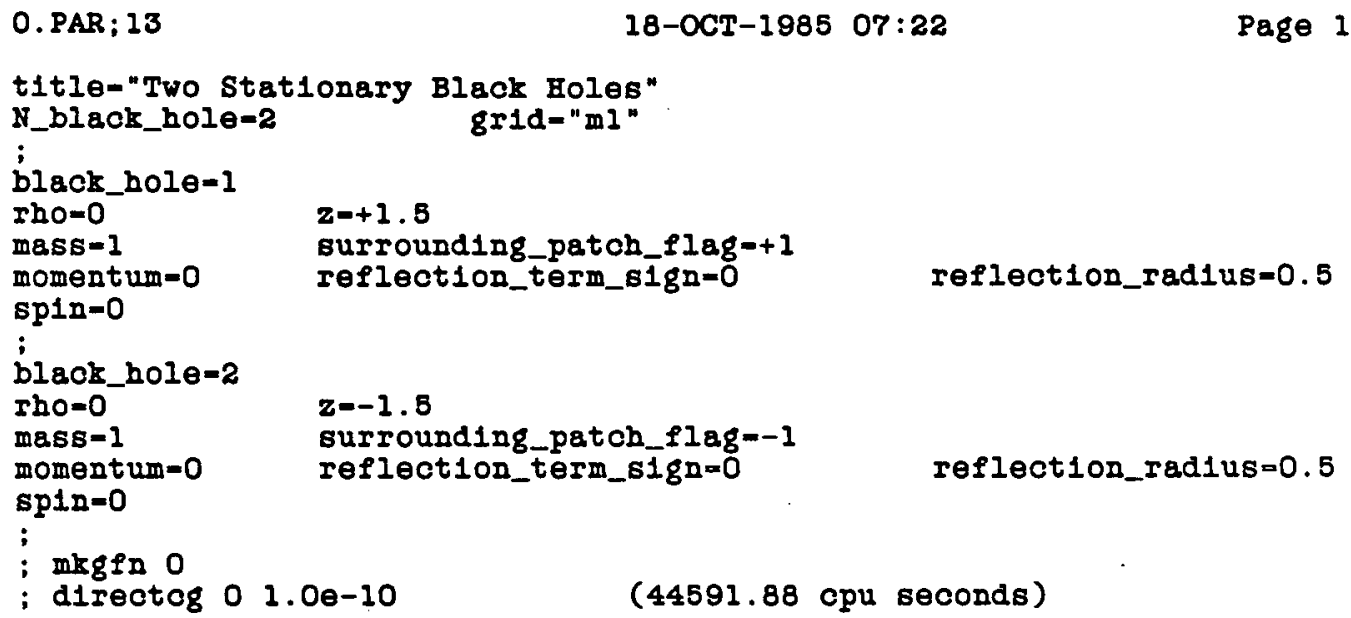

(44591.88 cpu seconds) 
Table 30 - PP test series, 0 selected $\Psi$ values

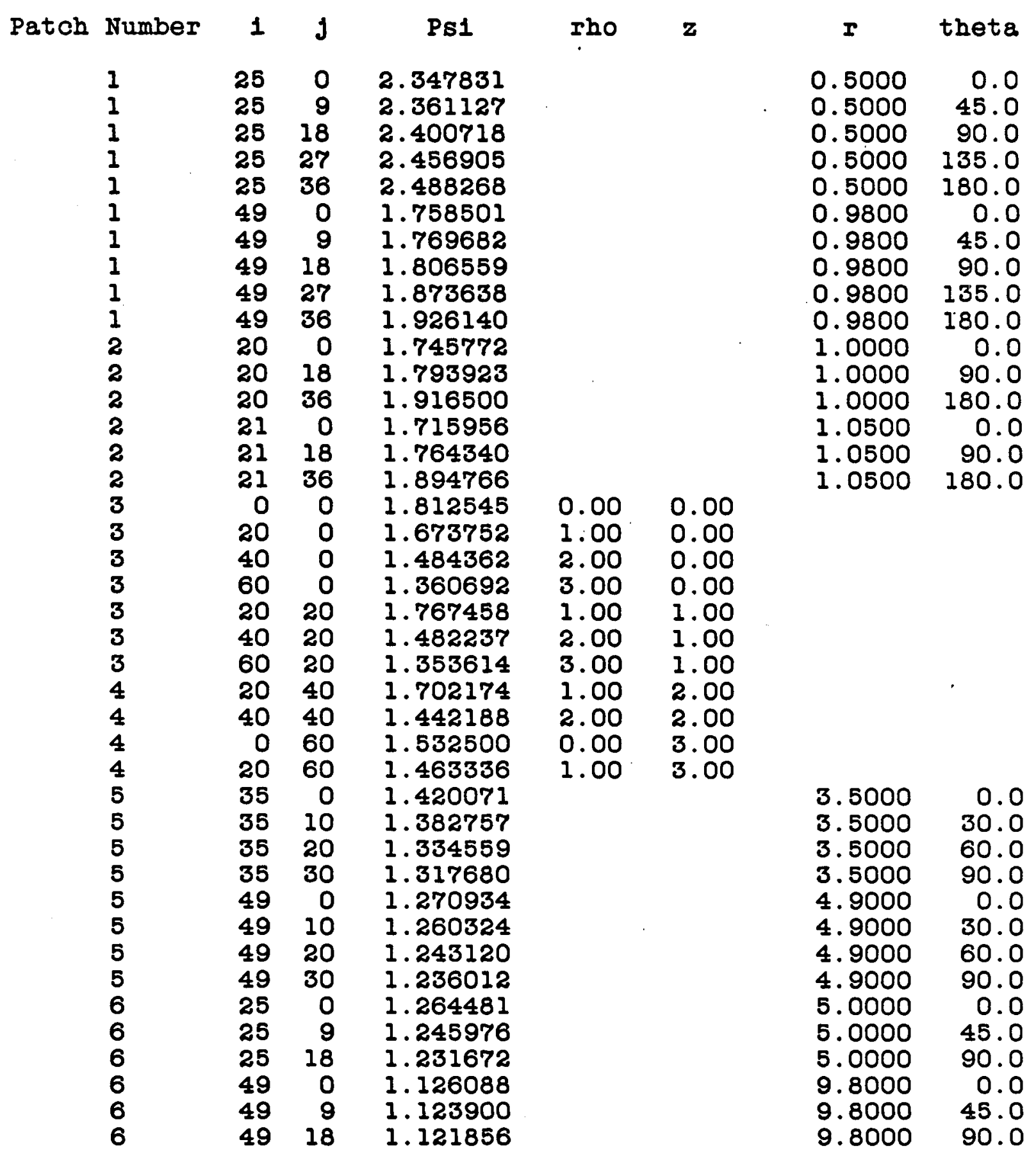

This table gives values of $\Psi$ at selected points in the space. These points are identified by triples (patch number, $i, j$ ), where $(i, j)$ are the generic integer patch coordinates defined in the text. Depending on the patch type, either $(\rho, z)$ or $(r, \theta)$ are also shown. 


\section{PP test series}

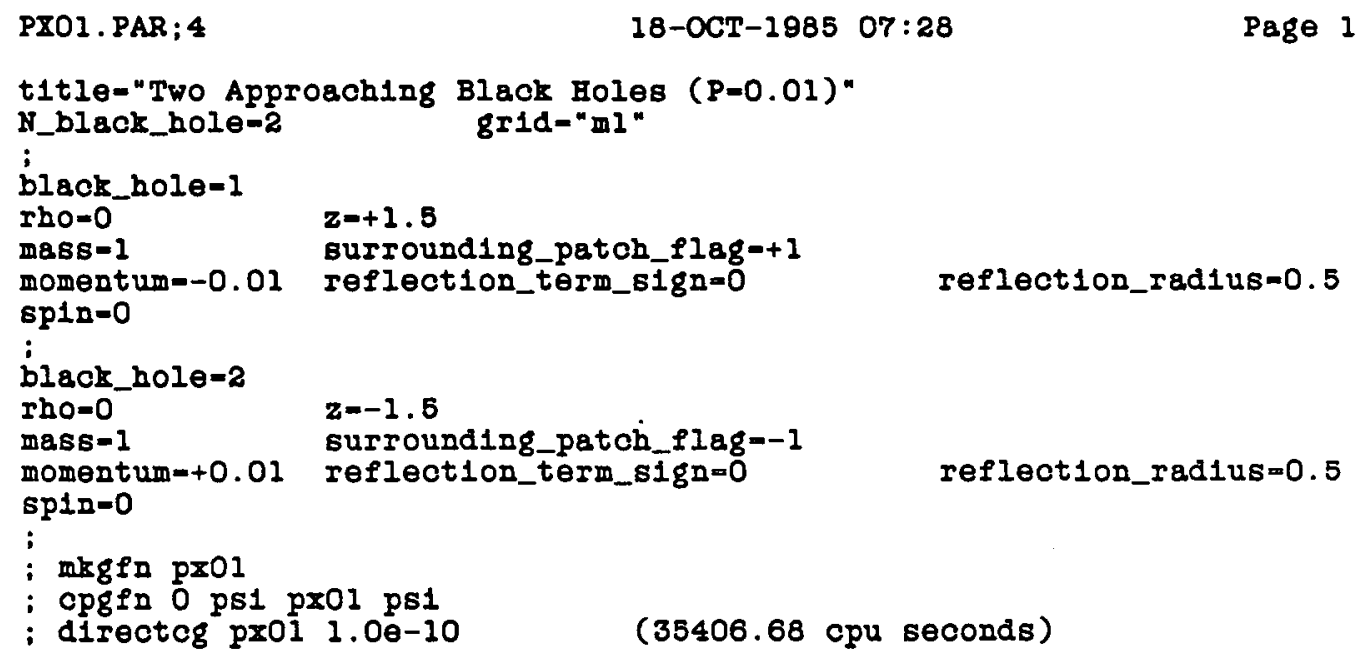


Table 31 - PP test series, px01 selected $\Psi$ valnes

$\begin{array}{rrrcccrr}\text { Patch Number } & 1 & \mathrm{j} & \text { Ps1 } & \text { rho } & \mathbf{z} & \mathbf{r} & \text { theta } \\ & 25 & 0 & 2.347064 & & & 0.5000 & 0.0 \\ 1 & 25 & 9 & 2.360580 & & & 0.5000 & 45.0 \\ 1 & 25 & 18 & 2.400692 & & & 0.5000 & 90.0 \\ 1 & 25 & 27 & 2.457385 & & & 0.5000 & 135.0 \\ 1 & 25 & 36 & 2.488955 & & & 0.5000 & 180.0 \\ 1 & 49 & 0 & 1.758297 & & & 0.9800 & 0.0 \\ 1 & 49 & 9 & 1.769535 & & & 0.9800 & 45.0 \\ 1 & 49 & 18 & 1.806549 & & & 0.9800 & 90.0 \\ 1 & 49 & 27 & 1.873769 & & & 0.9800 & 135.0 \\ 1 & 49 & 36 & 1.926339 & & & 0.9800 & 180.0 \\ 2 & 20 & 0 & 1.745576 & & & 1.0000 & 0.0 \\ 2 & 20 & 18 & 1.793913 & & & 1.0000 & 90.0 \\ 2 & 20 & 36 & 1.916693 & & & 1.0000 & 180.0 \\ 2 & 21 & 0 & 1.715777 & & & 1.0500 & 0.0 \\ 2 & 21 & 18 & 1.764330 & & & 1.0500 & 90.0 \\ 2 & 21 & 36 & 1.894945 & & & 1.0500 & 180.0 \\ 3 & 0 & 0 & 1.812677 & 0.00 & 0.00 & & \\ 3 & 20 & 0 & 1.673821 & 1.00 & 0.00 & & \\ 3 & 40 & 0 & 1.484380 & 2.00 & 0.00 & & \\ 3 & 60 & 0 & 1.360694 & 3.00 & 0.00 & & \\ 3 & 20 & 20 & 1.767519 & 1.00 & 1.00 & & \\ 3 & 40 & 20 & 1.482244 & 2.00 & 1.00 & & \\ 3 & 60 & 20 & 1.353613 & 3.00 & 1.00 & & \\ 4 & 20 & 40 & 1.702099 & 1.00 & 2.00 & & \\ 4 & 40 & 40 & 1.442173 & 2.00 & 2.00 & & \\ 4 & 0 & 60 & 1.532411 & 0.00 & 3.00 & & \\ 4 & 20 & 60 & 1.463283 & 1.00 & 3.00 & & \\ 5 & 35 & 0 & 1.420020 & & & 3.5000 & 0.0 \\ 5 & 35 & 10 & 1.382730 & & & 3.5000 & 30.0 \\ 5 & 35 & 20 & 1.334552 & & & 3.5000 & 60.0 \\ 5 & 35 & 30 & 1.317679 & & & 3.5000 & 90.0 \\ 5 & 49 & 0 & 1.270914 & & & 4.9000 & 0.0 \\ 5 & 49 & 10 & 1.260310 & & & 4.9000 & 30.0 \\ 5 & 49 & 20 & 1.243113 & & & 4.9000 & 60.0 \\ 5 & 49 & 30 & 1.236008 & & & 5.9000 & 90.0 \\ 6 & 25 & 0 & 1.264462 & & & 5.0000 & 0.0 \\ 6 & 25 & 9 & 1.245966 & & & 5.0000 & 45.0 \\ 6 & 25 & 18 & 1.231668 & & & 9.8000 & 0.0 \\ 6 & 49 & 0 & 1.126083 & & & 9.8000 & 45.0 \\ 6 & 49 & 9 & 1.123895 & & & 9.8000 & 90.0 \\ 6 & 49 & 18 & 1.121852 & & & & \end{array}$

This table gives values of $\boldsymbol{\Psi}$ at selected points in the space. These points are identified by triples (patch number, $i, j$ ), where $(i, j)$ are the generic integer patch coordinates defined in the text. Depending on the patch type, either $(\rho, z)$ or $(r, \theta)$ are also shown. 


\section{PP test series}

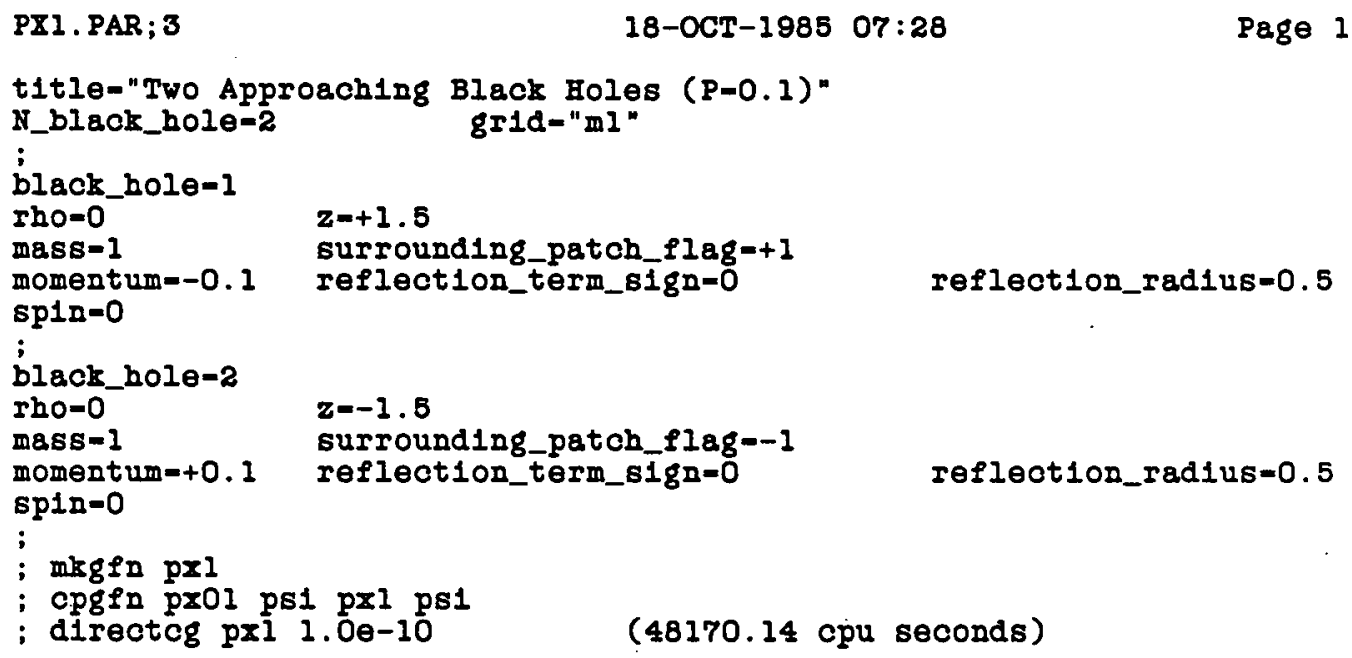


Table 32 - PP test series, P 11 selected $\Psi$ values

$\begin{array}{rrrcccrr}\text { Patch Number } & 1 & \mathrm{j} & \text { Ps1 } & \text { rho } & \text { z } & \text { r } & \text { theta } \\ 1 & 25 & 0 & 2.340766 & & & 0.5000 & 0.0 \\ 1 & 25 & 9 & 2.356280 & & & 0.5000 & 45.0 \\ 1 & 25 & 18 & 2.401102 & & & 0.5000 & 90.0 \\ 1 & 25 & 27 & 2.462366 & & & 0.5000 & 135.0 \\ 1 & 25 & 36 & 2.495811 & & & 0.5000 & 180.0 \\ 1 & 49 & 0 & 1.756930 & & & 0.9800 & 0.0 \\ 1 & 49 & 9 & 1.768673 & & & 0.9800 & 45.0 \\ 1 & 49 & 18 & 1.806924 & & & 0.9800 & 90.0 \\ 1 & 49 & 27 & 1.875466 & & & 0.9800 & 135.0 \\ 1 & 49 & 36 & 1.928674 & & & 0.9800 & 180.0 \\ 2 & 20 & 0 & 1.744276 & & & 1.0000 & 0.0 \\ 2 & 20 & 18 & 1.794287 & & & 1.0000 & 90.0 \\ 2 & 20 & 36 & 1.918971 & & & 1.0000 & 180.0 \\ 2 & 21 & 0 & 1.714628 & & & 1.0500 & 0.0 \\ 2 & 21 & 18 & 1.764700 & & & 1.0500 & 90.0 \\ 2 & 21 & 36 & 1.897096 & & & 1.0500 & 180.0 \\ 3 & 0 & 0 & 1.814382 & 0.00 & 0.00 & & \\ 3 & 20 & 0 & 1.674888 & 1.00 & 0.00 & & \\ 3 & 40 & 0 & 1.484881 & 2.00 & 0.00 & & \\ 3 & 60 & 0 & 1.360975 & 3.00 & 0.00 & & \\ 3 & 20 & 20 & 1.768532 & 1.00 & 1.00 & & \\ 3 & 40 & 20 & 1.482647 & 2.00 & 1.00 & & \\ 3 & 60 & 20 & 1.353860 & 3.00 & 1.00 & & \\ 4 & 20 & 40 & 1.701852 & 1.00 & 2.00 & & \\ 4 & 40 & 40 & 1.442344 & 2.00 & 2.00 & & \\ 4 & 0 & 60 & 1.531987 & 0.00 & 3.00 & & \\ 4 & 20 & 60 & 1.463140 & 1.00 & 3.00 & & \\ 5 & 35 & 0 & 1.419875 & & & 3.5000 & 0.0 \\ 5 & 35 & 10 & 1.382767 & & & 3.5000 & 30.0 \\ 5 & 35 & 20 & 1.334743 & & & 3.5000 & 60.0 \\ 5 & 35 & 30 & 1.317905 & & & 3.5000 & 90.0 \\ 5 & 49 & 0 & 1.270956 & & & 4.9000 & 0.0 \\ 5 & 49 & 10 & 1.260388 & & & 4.9000 & 30.0 \\ 5 & 49 & 20 & 1.243240 & & & 4.9000 & 60.0 \\ 5 & 49 & 30 & 1.236151 & & & 4.9000 & 90.0 \\ 6 & 25 & 0 & 1.264508 & & & 5.0000 & 0.0 \\ 6 & 25 & 9 & 1.246070 & & & 5.0000 & 45.0 \\ 6 & 25 & 18 & 1.231808 & & & 9.8000 & 90.0 \\ 6 & 49 & 0 & 1.126139 & & & 9.8000 & 45.0 \\ 6 & 49 & 9 & 1.123956 & & & 9.8000 & 90.0 \\ 6 & 49 & 18 & 1.121917 & & & & \end{array}$

This table gives values of $\Psi$ at selected points in the space. These points are identified by triples (patch number, $i, j$ ), where $(i, j)$ are the generic integer patch coordinates defined in the text. Depending on the patch type, either $(\rho, z)$ or $(r, \theta)$ are also shown. 
PP test series

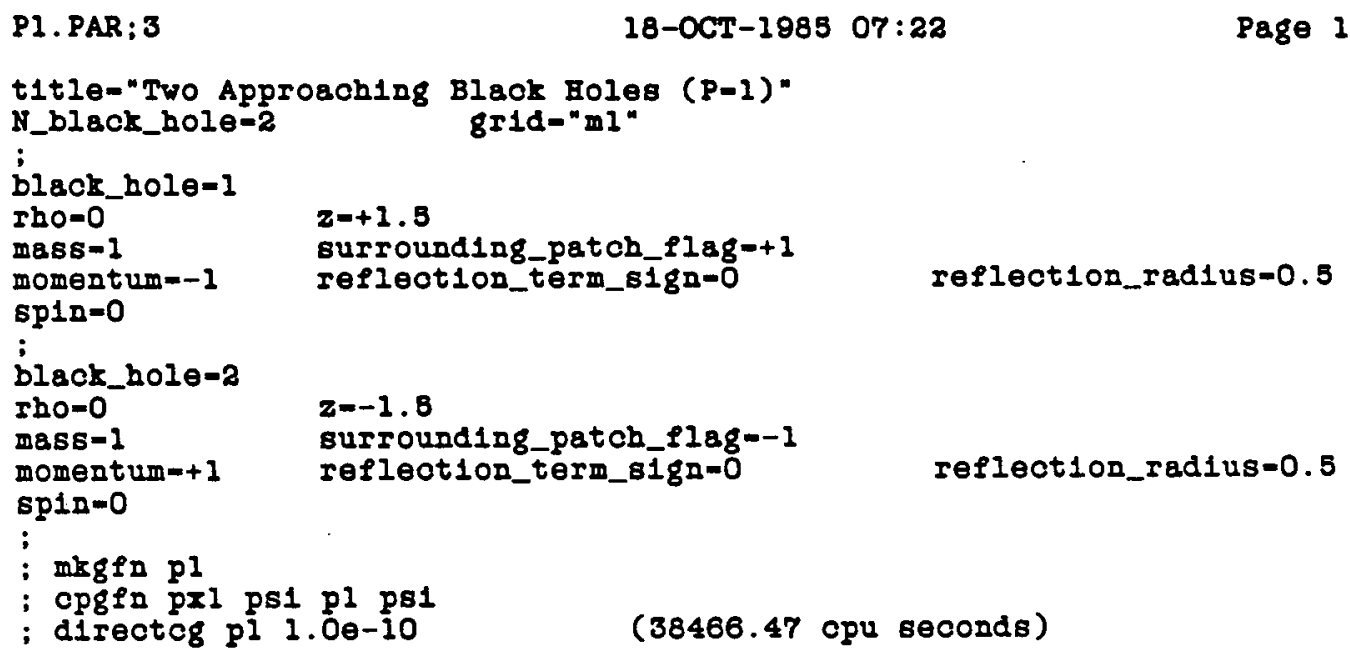


Table 33 - PP test series, P1 selected $\Psi$ values

$\begin{array}{rrrcccrr}\text { Patch Number } & 1 & j & \text { Ps1 } & \text { rho } & \text { z } & \text { r } & \text { theta } \\ 1 & 25 & 0 & 2.334259 & & & 0.5000 & 0.0 \\ 1 & 25 & 9 & 2.369034 & & & 0.5000 & 45.0 \\ 1 & 25 & 18 & 2.459010 & & & 0.5000 & 90.0 \\ 1 & 25 & 27 & 2.564154 & & & 0.5000 & 135.0 \\ 1 & 25 & 36 & 2.615783 & & & 0.5000 & 180.0 \\ 1 & 49 & 0 & 1.784179 & & & 0.9800 & 0.0 \\ 1 & 49 & 9 & 1.799919 & & & 0.9800 & 45.0 \\ 1 & 49 & 18 & 1.849937 & & & 0.9800 & 90.0 \\ 1 & 49 & 27 & 1.934115 & & & 0.9800 & 135.0 \\ 1 & 49 & 36 & 1.996160 & & & 0.9800 & 180.0 \\ 2 & 20 & 0 & 1.771787 & & & 1.0000 & 0.0 \\ 2 & 20 & 18 & 1.836919 & & & 1.0000 & 90.0 \\ 2 & 20 & 36 & 1.985722 & & & 1.0000 & 180.0 \\ 2 & 21 & 0 & 1.742668 & & & 1.0500 & 0.0 \\ 2 & 21 & 18 & 1.806419 & & & 1.0500 & 90.0 \\ 2 & 21 & 36 & 1.962210 & & & 1.0500 & 180.0 \\ 3 & 0 & 0 & 1.873554 & 0.00 & 0.00 & & \\ 3 & 20 & 0 & 1.722127 & 1.00 & 0.00 & & \\ 3 & 40 & 0 & 1.518051 & 2.00 & 0.00 & & \\ 3 & 60 & 0 & 1.385800 & 3.00 & 0.00 & & \\ 3 & 20 & 20 & 1.816994 & 1.00 & 1.00 & & \\ 3 & 40 & 20 & 1.514541 & 2.00 & 1.00 & & \\ 3 & 60 & 20 & 1.377993 & 3.00 & 1.00 & & \\ 4 & 20 & 40 & 1.736197 & 1.00 & 2.00 & & \\ 4 & 40 & 40 & 1.470350 & 2.00 & 2.00 & & \\ 4 & 0 & 60 & 1.560439 & 0.00 & 3.00 & & \\ 4 & 20 & 60 & 1.490559 & 1.00 & 3.00 & & \\ 5 & 35 & 0 & 1.445917 & & & 3.5000 & 0.0 \\ 5 & 35 & 10 & 1.407431 & & & 3.5000 & 30.0 \\ 5 & 35 & 20 & 1.357425 & & & 3.5000 & 60.0 \\ 5 & 35 & 30 & 1.339889 & & & 3.5000 & 90.0 \\ 5 & 49 & 0 & 1.290289 & & & 4.9000 & 0.0 \\ 5 & 49 & 10 & 1.278718 & & & 4.9000 & 30.0 \\ 5 & 49 & 20 & 1.260251 & & & 4.9000 & 60.0 \\ 5 & 49 & 30 & 1.252731 & & & 4.9000 & 90.0 \\ 6 & 25 & 0 & 1.283461 & & & 5.0000 & 0.0 \\ 6 & 25 & 9 & 1.263329 & & & 5.0000 & 45.0 \\ 6 & 25 & 18 & 1.248098 & & & 9.8000 & 90.0 \\ 6 & 49 & 0 & 1.135581 & & & 9.8000 & 45.0 \\ 6 & 49 & 9 & 1.133012 & & & 9.8000 & 90.0 \\ 6 & 49 & 18 & 1.130708 & & & & \end{array}$

This table gives values of $\Psi$ at selected points in the space. These points are identified by triples (patch number, $i, j$ ), where $(i, j)$ are the generic integer patch coordinates defined in the text. Depending on the patch type, either $(\rho, z)$ or $(r, \theta)$ are also shown. 


\section{PP test series}

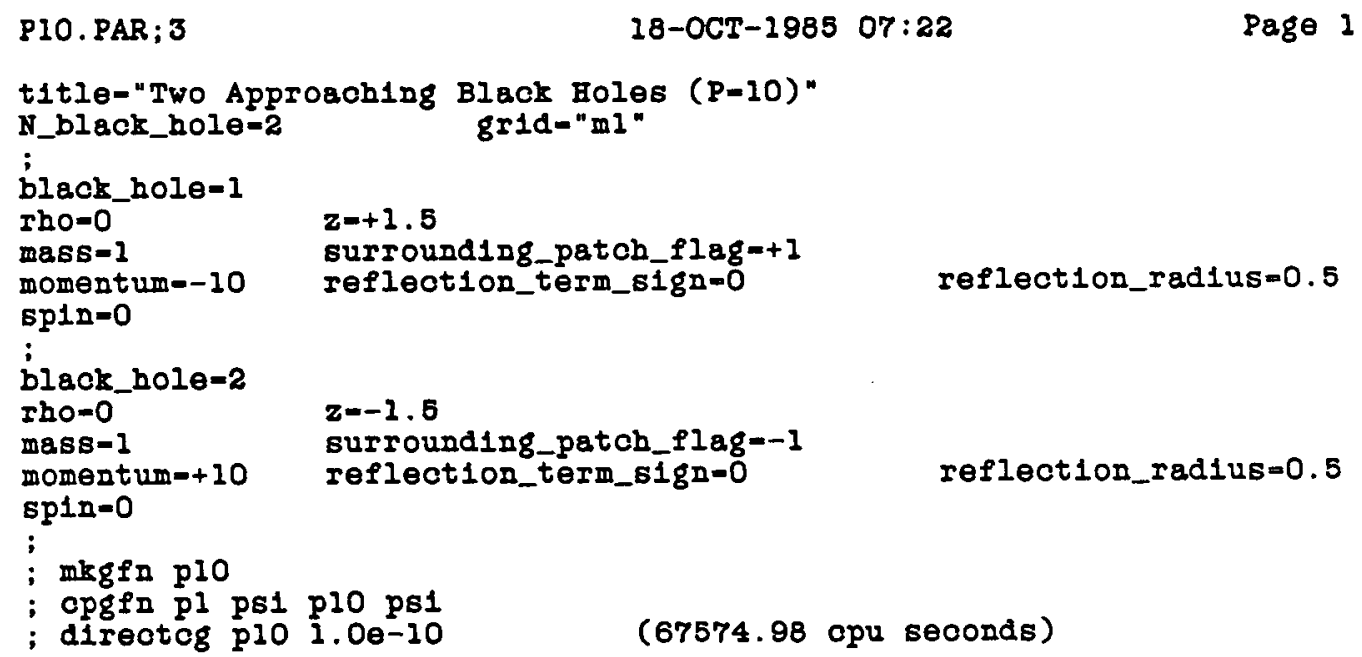

(67574.98 opu seoonds) 
Table 34 - PP test series, p10 selected $\Psi$ valnes

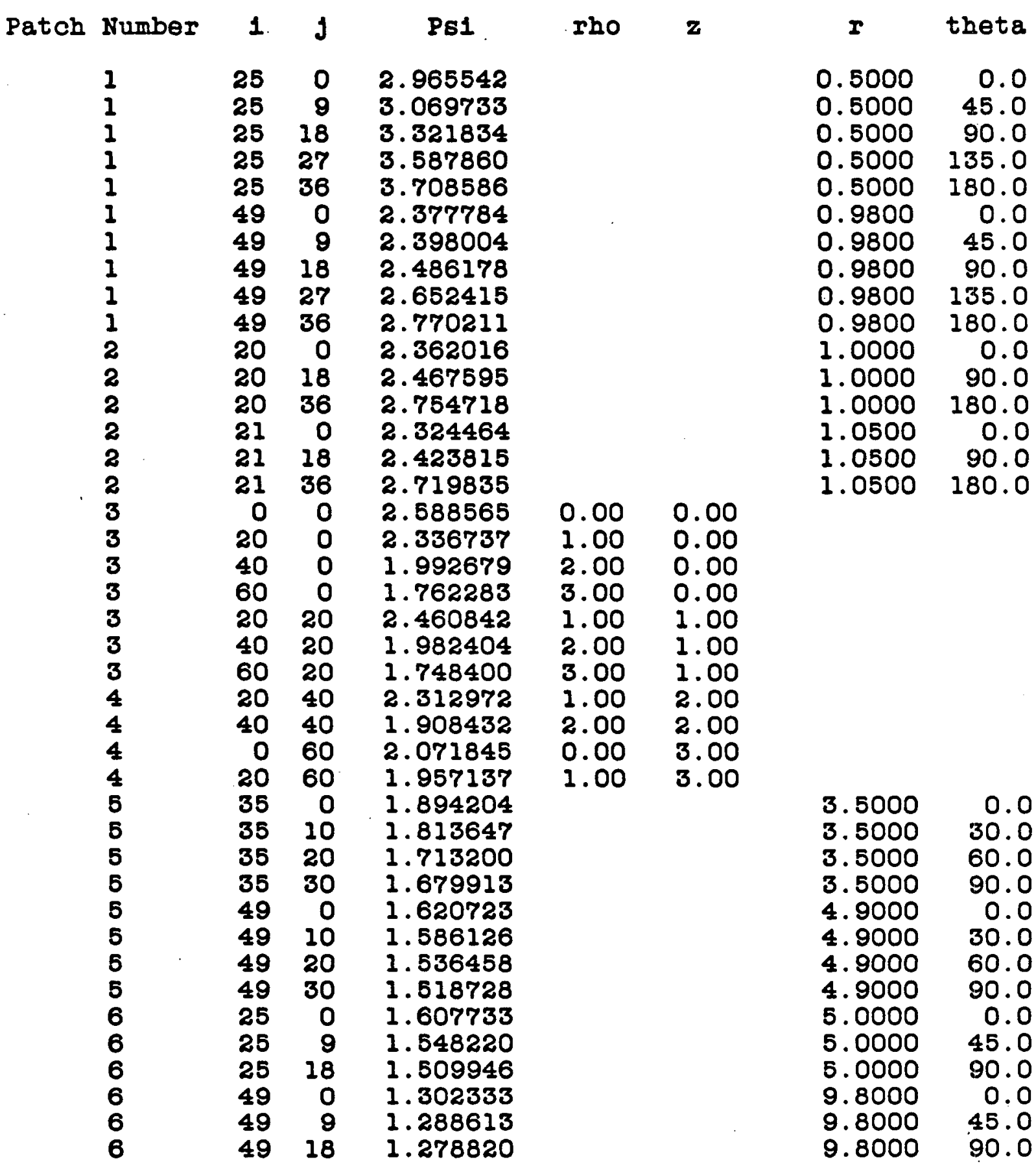

This table gives values of $\Psi$ at selected points in the space. These points are identified by triples (patch number, $i, j$ ), where $(i, j)$ are the generic integer patch coordinates defined in the text. Depending on the patch type, either $(\rho, z)$ or $(r, \theta)$ are also shown. 
PP test series

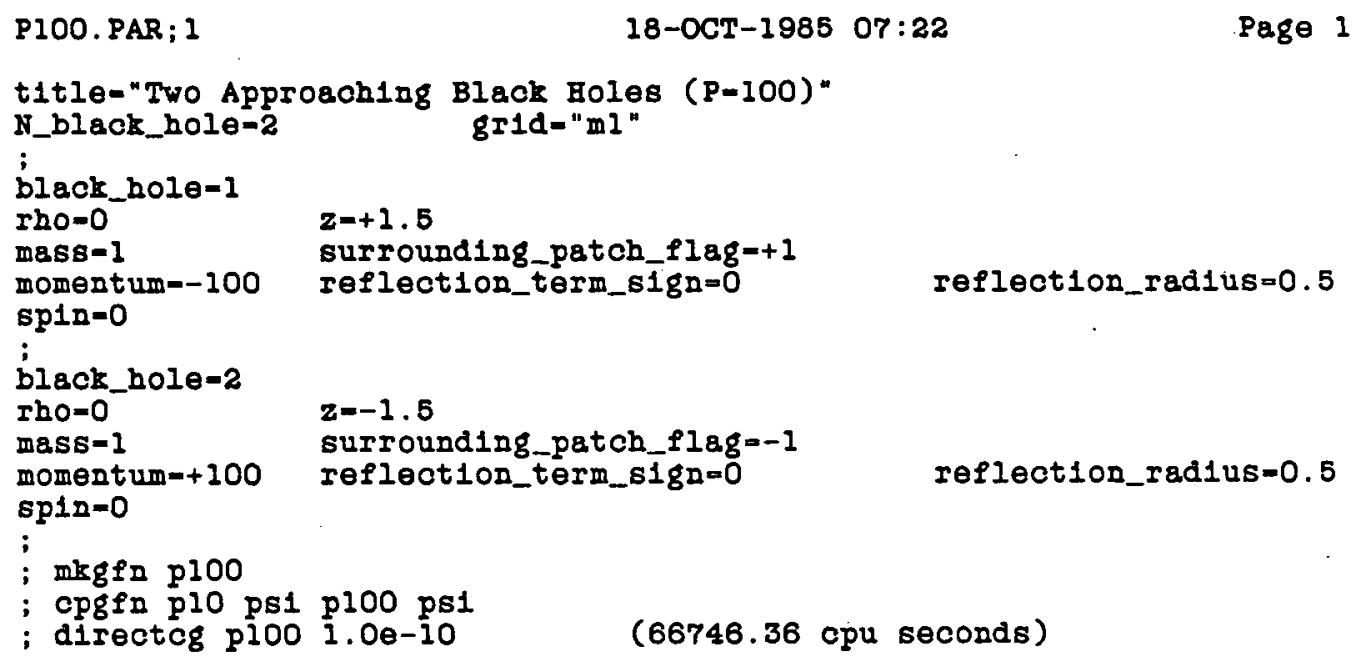


Table 35 - PP test series, p100 selected $\Psi$ values

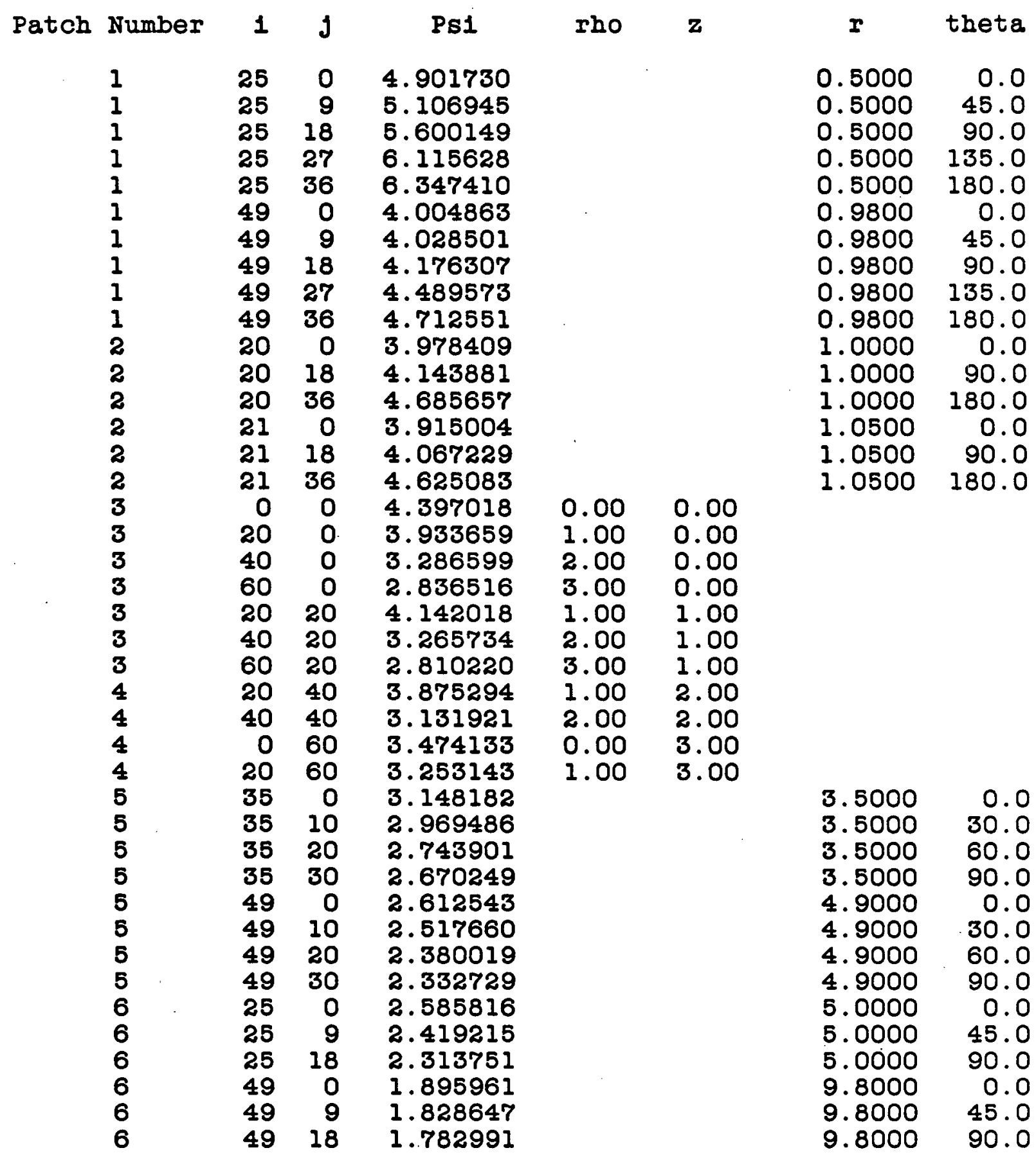

This table gives values of $\Psi$ at selected points in the space. These points are identified by triples (patch number, $i, j$ ), where $(i, j)$ are the generic integer patch coordinates defined in the text. Depending on the patch type, either $(\rho, z)$ or $(r, \theta)$ are also shown. 


\section{PP test geries}

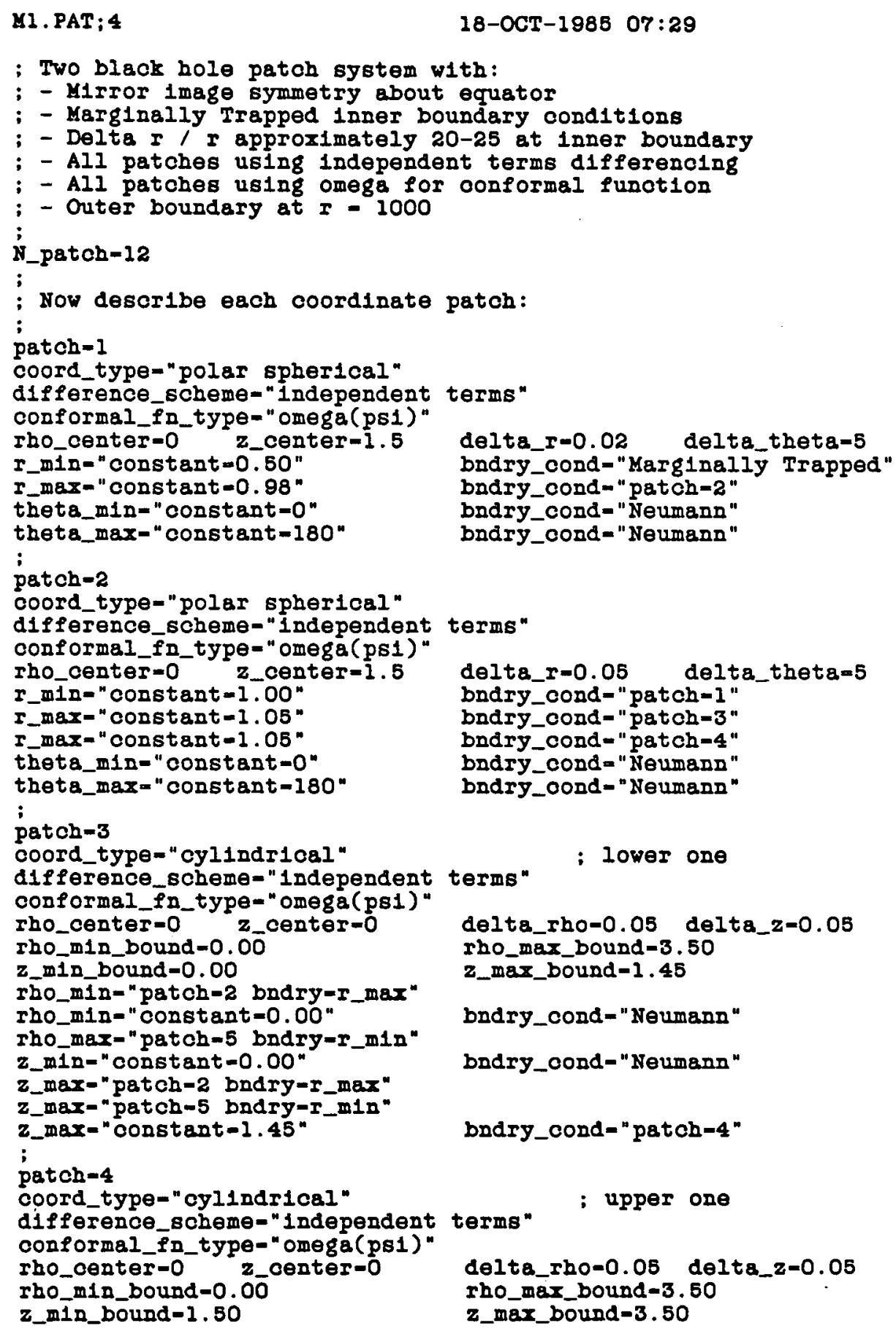




\section{PP test series}

\section{YI. PAT;4}

rho_m1n="patch-2 bndry=r_max"
rho_m1n="constant-0.00"
rho_max-"patch-5 bndry=r_min"
z_m1n-"patch-2 bndry-r_max"
z_m1n-"constant-1.60"
z_max="patch-5 bndry=r_m1n"
; patoh-5
coord_type="polar spher1cal"

difference_scheme="Independent conformsi_fn_type-"omega (psi)" rho_center $=0$ z_center $=0$

r_min="constant-3.5"

r_m1n="constant $=3.3$ "

r_max-"constant $-4.9^{\prime \prime}$

theta_m1n="constant $=0$ "

theta_max=" constant $=90$ "

;

patch-6

coord_type-"polar spher1cal" difference_scheme="1ndependent rho_center $=0$ z_center $=0$

r_min-" constant -5.0 "

r_mar= " constant $=9.8$ "

theta_min=" constant $=0$ "

theta_max = " constant -90 "

;

patch=7

coord_type="polar spher1oal"

difference_scheme-"Independent conformal_fn_type-"omega(psi)" rho_center $=0$ z_center-0

I_m1n-" constant $=10.0$.

r_max-" constant-19.5"

theta_min="constant $=0 "$

theta_max=" constant $=90$ "

;

patoh-8

coord_type-"polar spher10al"

difference_scheme-"1ndependent terms"

conformal_fn_type-"omega(psi)"

rho_center=0 z_center $=0$

r.mIn=" constant $=20^{\prime \prime}$

I_max-" constant $=49$ "

theta_min="constant=0"

theta_max=" constant -90 "

patch -8

coord_type-"polar spher10al"

difference_scheme-"Independent terms"

conformal_fn_type-"omega(p81)"

rho_center $=0$ z_center-0

I.m1n-" constant $-8 \overline{0}$ "

I_mar- "oonstant-98"
18-OCT-1885 07:29

Page 2

bndry_cond- "Neumann"

bndry_oond= "patch $=3$ "

terms"

delta_I-0.1 delta_theta=3

bndry_cond = "patch=3"

badry_cond= "patch $=4$ "

bndry_cond = "patch=6"

bndry_cond = "Noumann"

bndry_cond-"Neumann"

terms *

delta_r-0.2 delta_theta=5

bndry_cond= " patch-5"

bndry_cond-"patch-7"

bndry_cond = "Neumann"

bndry_cond=" Neumann"

terms"

delta_r=0.5 delta_theta-5

bndry_cond-"patoh-6"

bndry_cond = "patch=8"

bndry_cond = "Neumann"

bndry_cond = "Neumann"

delta_r=1

delta_theta-7.5

bndry_cond = " patch=7"

bndry_cond-" patch-9"

bndry_cond-"Neumann"

bndry_cond = "Neumann"

de1ta_r-2

delta_theta-7.5

bndry_cond-" patch $=8$ "

bndry_cond- " patch=10" 


\section{PP test series}

Y1. PAT; 4

theta_m1n="constant $=0$ " theta_max-"oonstant $=90$ " ;

patch $=10$

coord_type-"polar spherioal"

difference_soheme-"1ndependent terms"

conformal_fn_type="omega(psi)"

rho_center $=0 \quad$ z_center=0

r_m1n=" constant $=100$ "

r_max-"oonstant-195"

theta_m1n="constant $=0$ "

theta_max $=$ "oonstant $=90$ "

;

patch=11

coord_type="polar spherical"

difference_scheme-"Independent terms"

conformal_fn_type-"omega(psi)"

rho_center $=0$ z_center $=0$

$r \_m i n="$ constant $=200$ "

r_max= "constant $=490$ "

theta_m1n="constant $=0$ "

theta_max-"constant $=90$ "

;

patch $=12$

coord_type="polar spher1cal"

differenoe_scheme-"independent terms"

conformal_fn_type-"omega(ps1)"

rho_center $=0$ z_center $=0$

I_min=" constant $=5 \overline{0} 0$ "

r_max-" constant-1000"

theta_min-"constant $=0$ "

theta_max="constant $=90$ "
$18-O C T-1885 \quad 07: 29$

Page 3

bndry_cond = "Neumann"

bndry_cond = "Neumann"

\section{delta_I $=5$}

bndry_cond = "patch-9"

bndry_cond = "patoh-11"

bndry_cond- "Neumann"

bndry_cond- "Neumann"

delta_I=10

delta_theta-10

bndry_cond= "patch=10"

bndry_cond " patch-12"

bndry_cond = "Neumenn"

bndry_cond = "Neumann"

delta_r $=20$

delta_theta=10

bndry_cond - "patch-11"

bndry_cond = "RobIn"

bndry_cond = "Neumann"

bndry_cond- "Neumann" 


\section{Appendix 2}

This appendix contains the source listings of the numerical GR code described in this thesis. The bulk of the code is written in the $\mathrm{C}$ programming language (Kernighan and Ritchie (1978)). The "ILUCG" sparse matrix subroutine package used to solve the linearized algebraic equations is written in FORTRAN. There is one short assembler routine, used to support the passing of variable numbers of arguments to $\mathrm{C}$ functions.

The code is written for (and runs on) a VAX/750 with floating point accelerator, running the VMS operating system, version 3.2. Double precision arithmetic is used throughout, giving about 17 decimal digits (56 bits) of precision in floating point quantities. The time for a floating point add/multiply pair is about 20 microseconds.

The $\mathbf{C}$ dialect used extends the Kernighan and Ritchie standard in only one significant way-enumerated data types are allowed, with semantics similar to those of (for example) PASCAL. The author uses a number of private "language extensions", via the header file "util:stdc.h". The most significant of these are the definition of "new" data types rold and boolean, and the definition of a then keyword for use in the C if-else statement.

Most of the source files begin with a list of the external objects (functions and variables) they contain. Those items preceded by a single asterisk are "public" (visible outside the file), while those preceded by a double asterisk are declared as static and hence have scope limited to the file.

The code resides in 4 distinct directories, which have been given "logical names" so files in them may be easily referred to from other subdirectories. The syntax of such a reference is that the logical name precedes the filename, separated by a colon. The 


\section{Table 36 - Directories where the code resides}

Logical Name
main
fortran
patchut1l
ut1l

Logical Name

main

patchut1l

ut1l

\author{
Contents \\ Routines to set up and solve equation (21) \\ "ILUCG" Sparse Matrix package \\ Patch utility routines \\ General purpose utility routines
}

directories and their contents are listed in table 36.

The next 4 pages give indices to the listings for each of these directories, giving the page numbers on which each file's listing starts. The listings themselves follow. Within each directory, header files are listed first, then "normal" source code files. Within these groupings, the files are ordered alphabetically. In general, the name of an executable program is the same as that of the file holding it's main function. The sole exception to this is the executable program directcg, derived from the main function in the file direct.c, both in the main directory (the name comes from "DIRECT solver via Conjugate Gradient method").

The reader wishing a general understanding of how the code works is advised to start by reading the header files in the patchutil directory, especially patch.h. After this, the mkgfn.c and direct.c files, together with the functions they call, are probably the next place to look. The directcg executable program derived from direct.c was used to obtain all the model solutions in this thesis. 
Table 37 - File index for the main directory

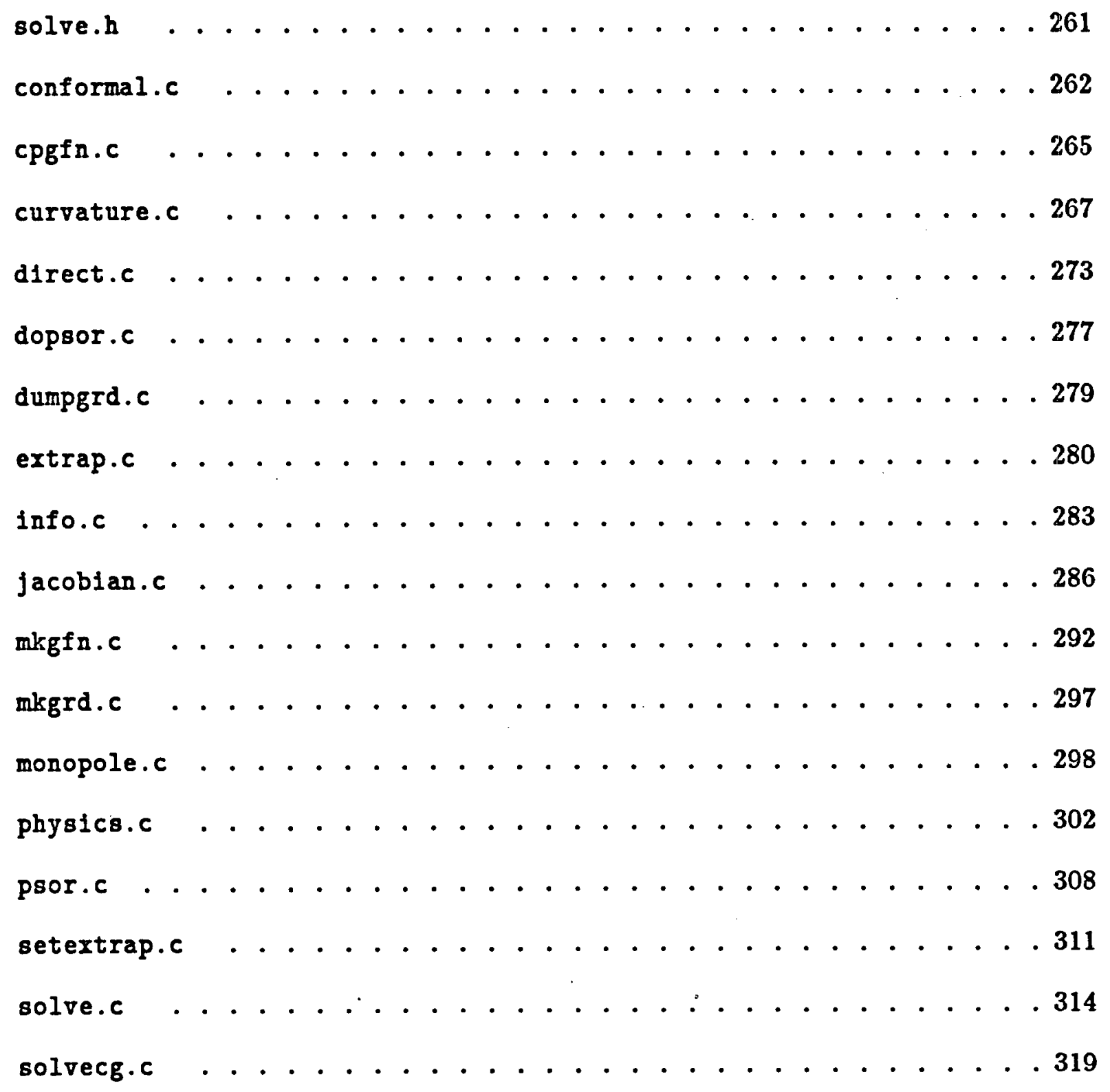


Table 38 - File index for the fortran directory

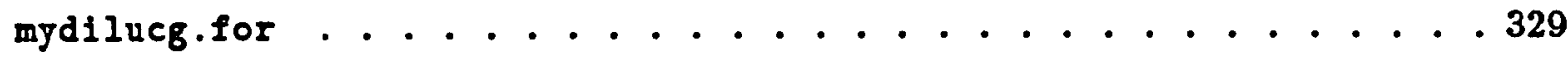

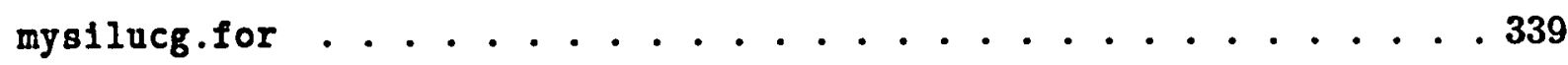


Table 39 - File index for the patchutil directory

blackhole.h ...... . . . . . . . . . . . 349

cot.h.......................... 350

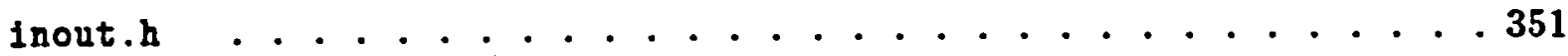

patch.h ...................... 352

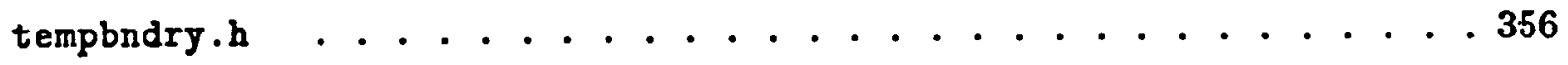

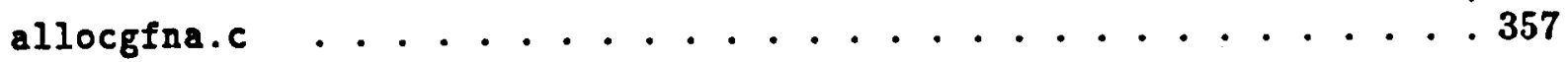

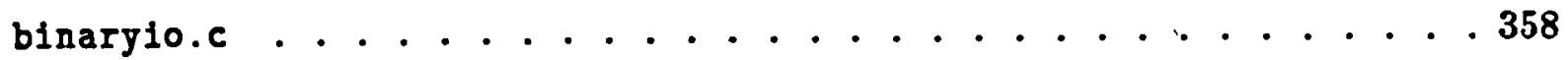

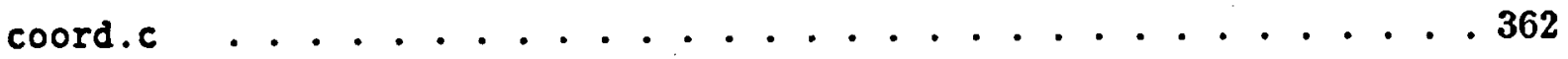

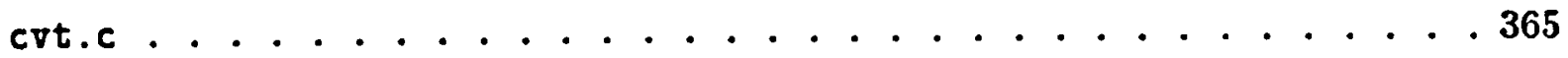

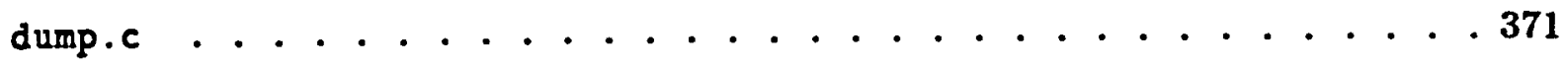

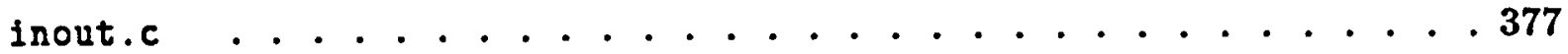

inputpar.c ...................... 379

Inputpat.c ..................... 382

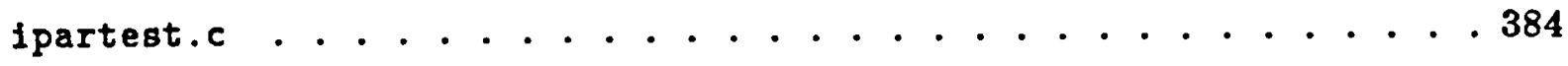

ipattest.c ...................... 385

ngridpts.c ..................... 386

parlog.c ...................... 387

readpat.c ...................... 388

setbndry.c . . . . . . . . . . . . . . . . 398

setpatch.c ..................... 405 
Table 40 - File index for the util directory

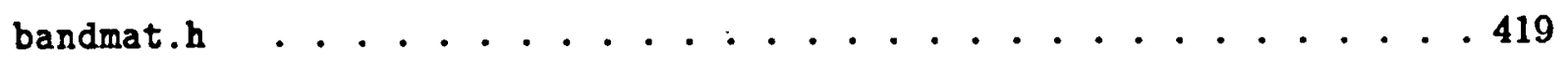

keyword.h ...................... 420

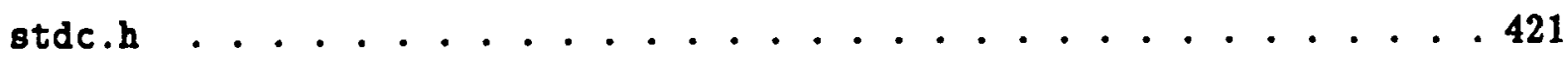

arrayio.c ..................... 422

bgec1.c ....................... 423

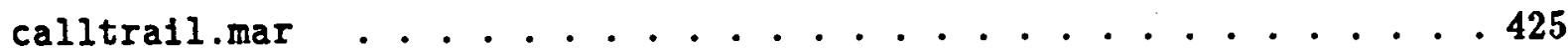

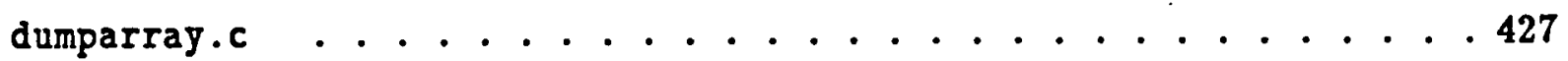

errorexit.c ............................ 430

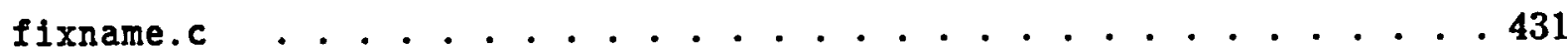

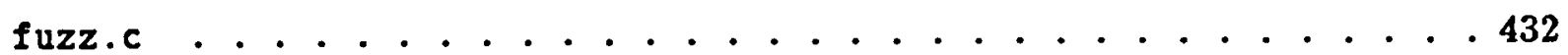

keyword.c .....................4455

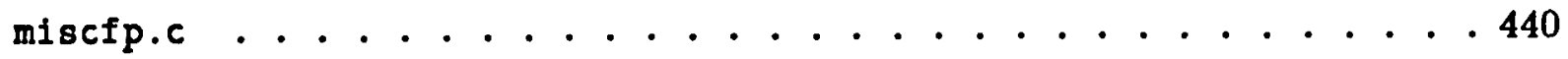

round.c .................................. 441

spline.c ......................... 442

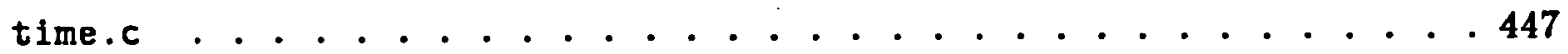




\section{main directory}

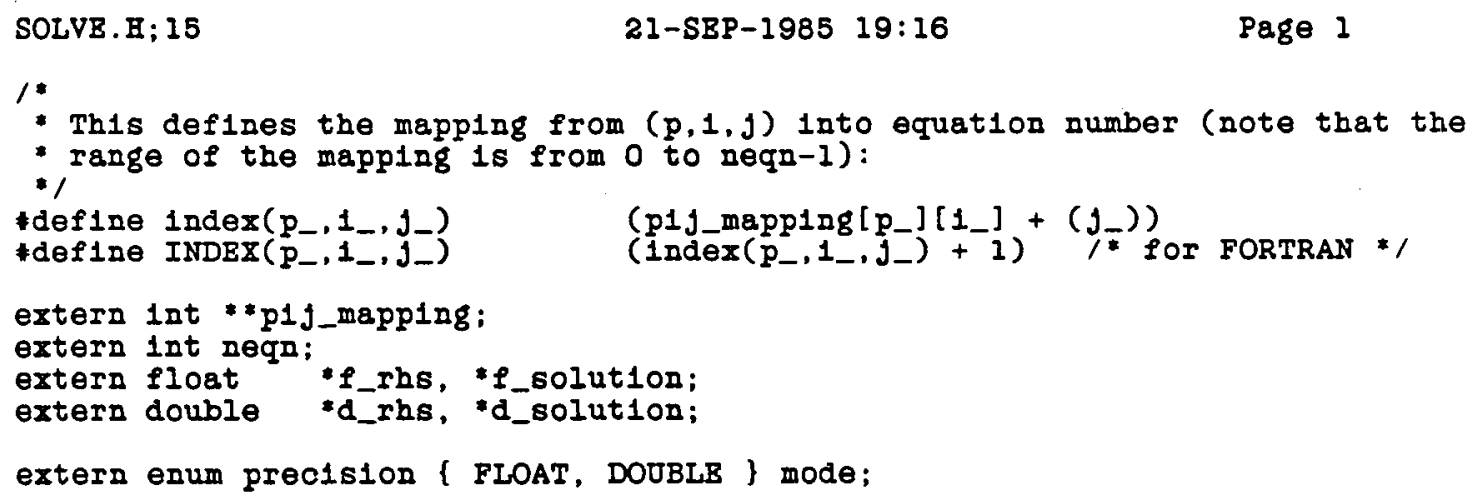




\section{main directory}

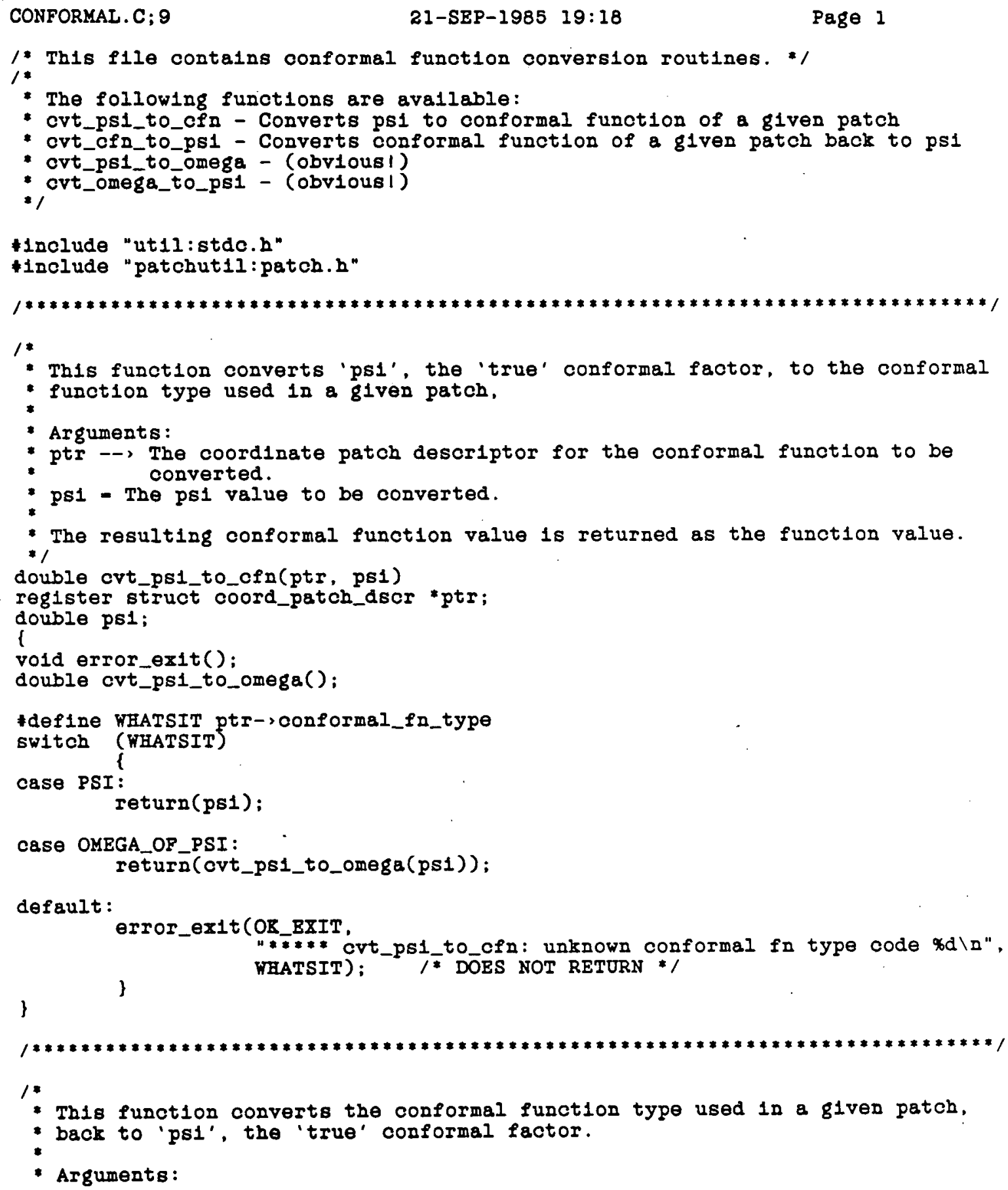




\section{main directory}

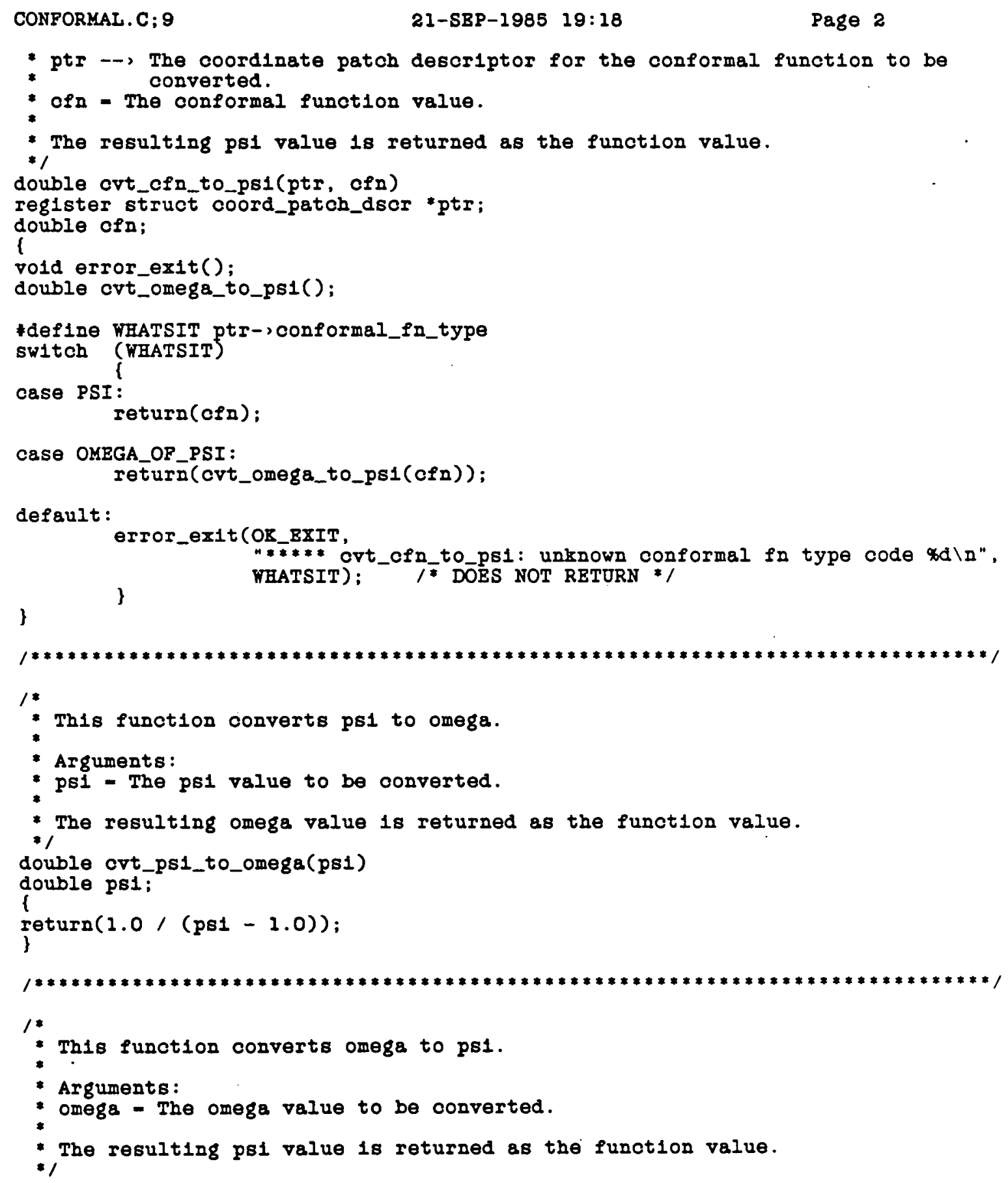




\section{main directory}

CONFORMAL.C; 9

21-SBP-1985 19:18

Page 3

double crt_omega_to_ps1 (omega)

double omega; \{

return( $1.0+1.0 /$ omege $)$; 


\section{main directory}

CPGFN.C; 15

21-SBP-1985 19:18

Page 1

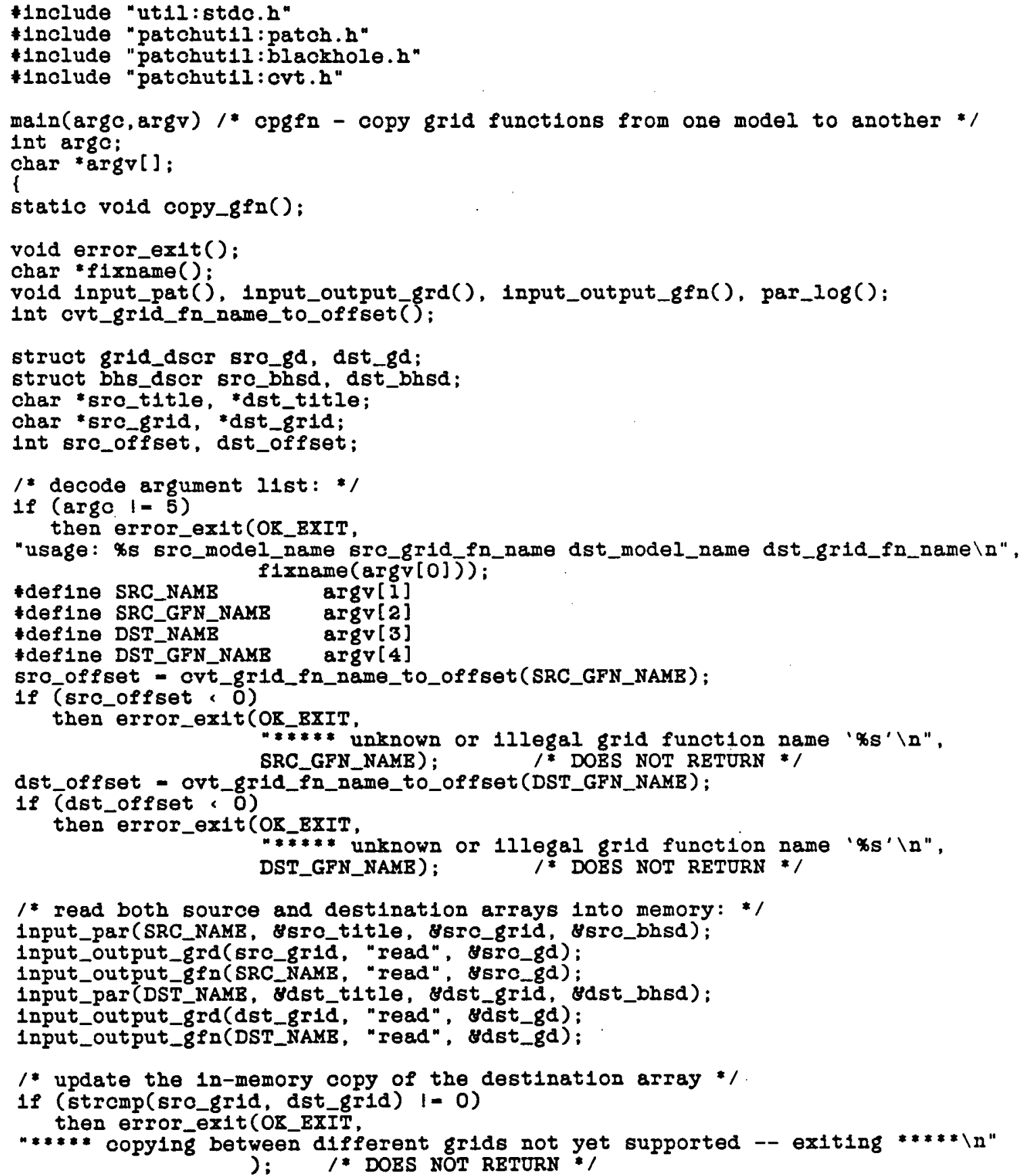




\section{main directory}

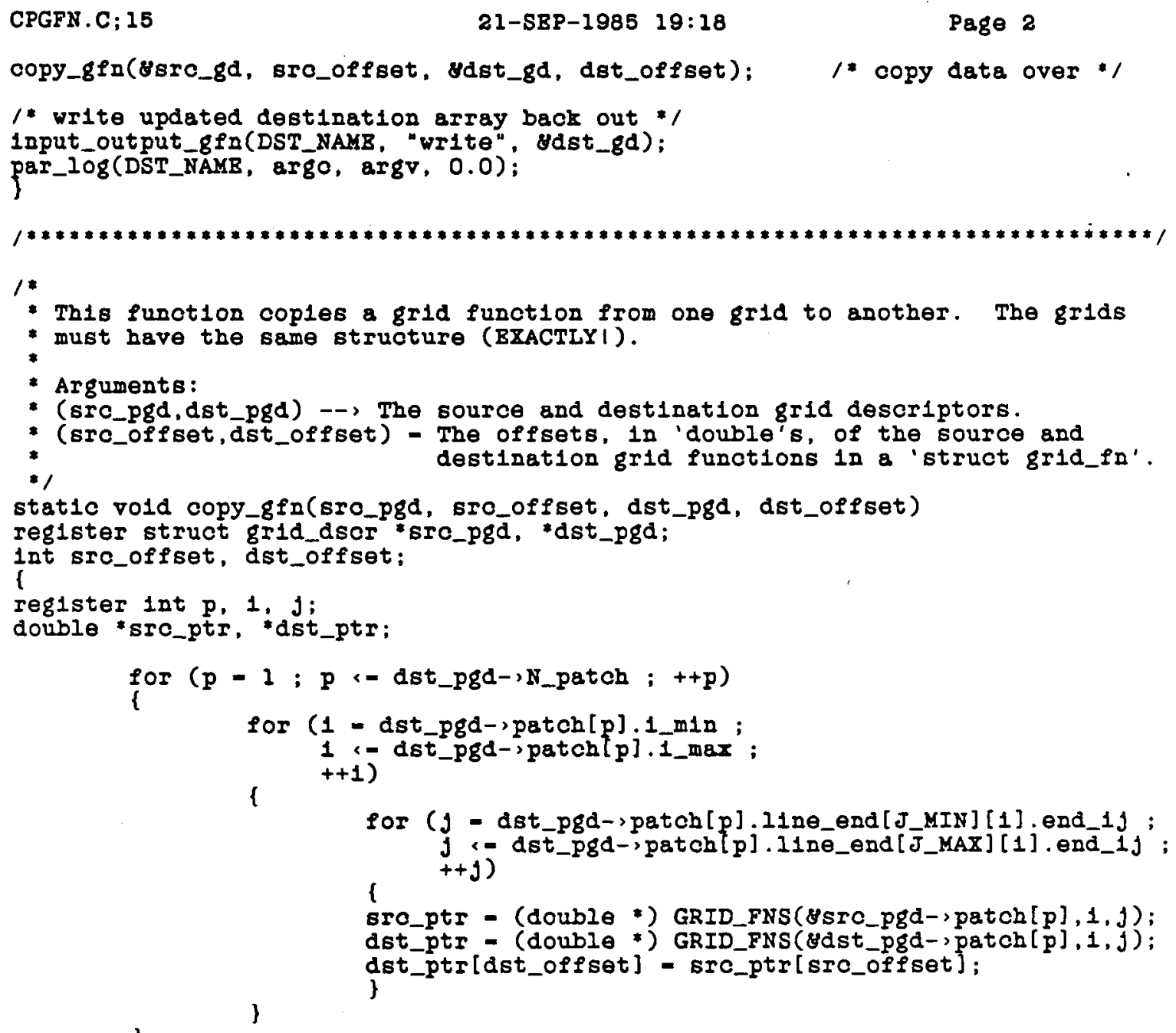




\section{main directory}

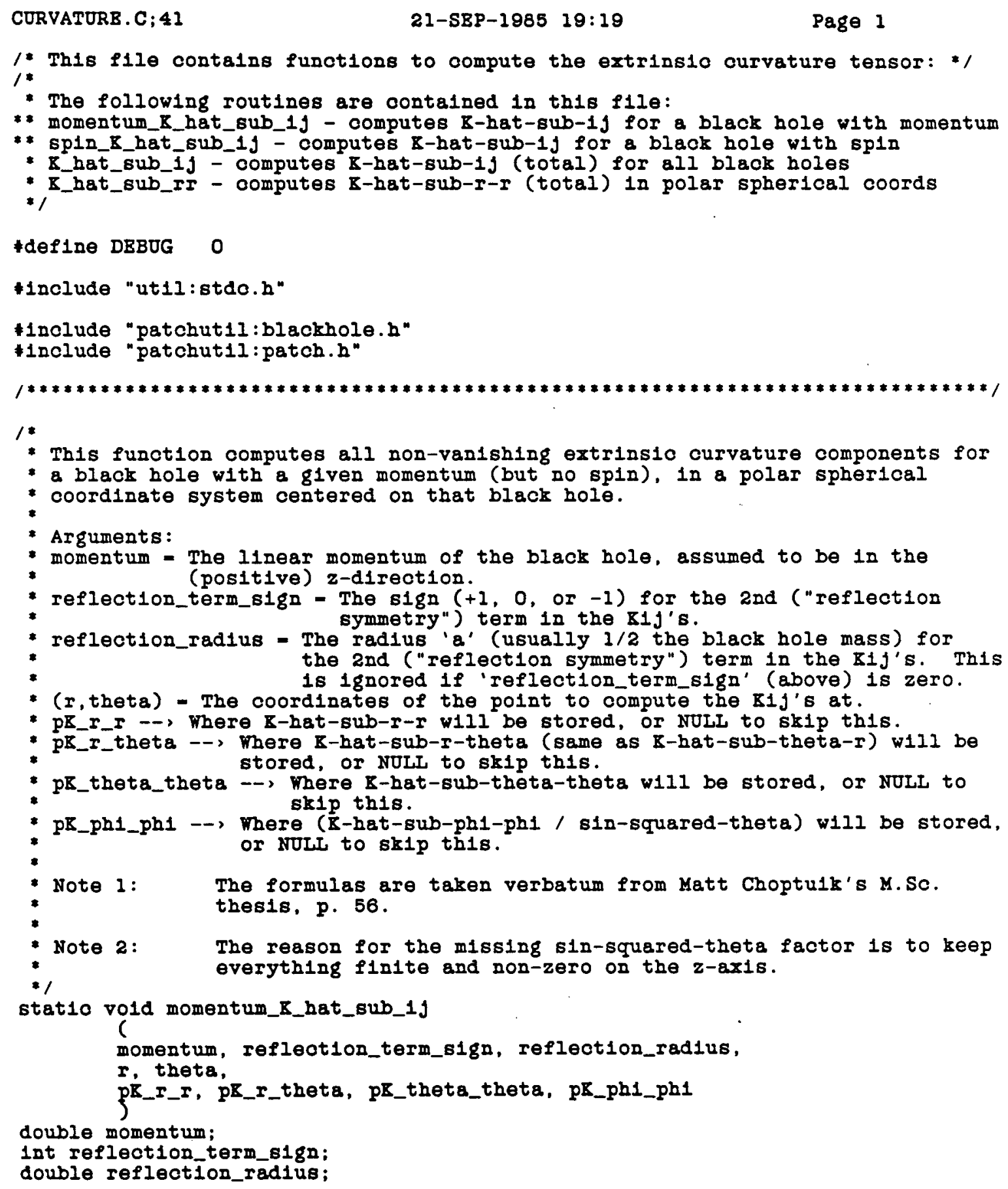




\section{main directory}

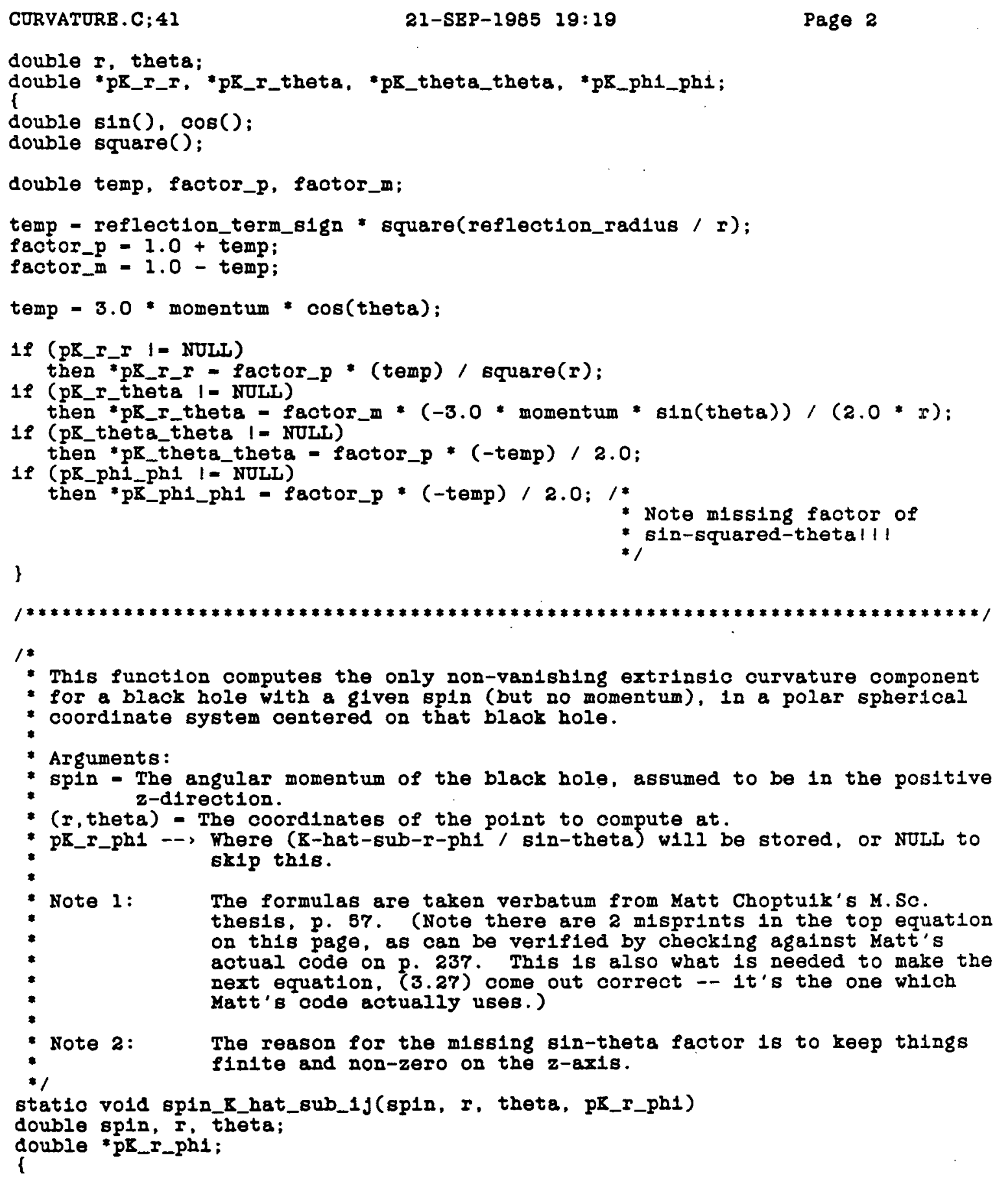




\section{main directory}

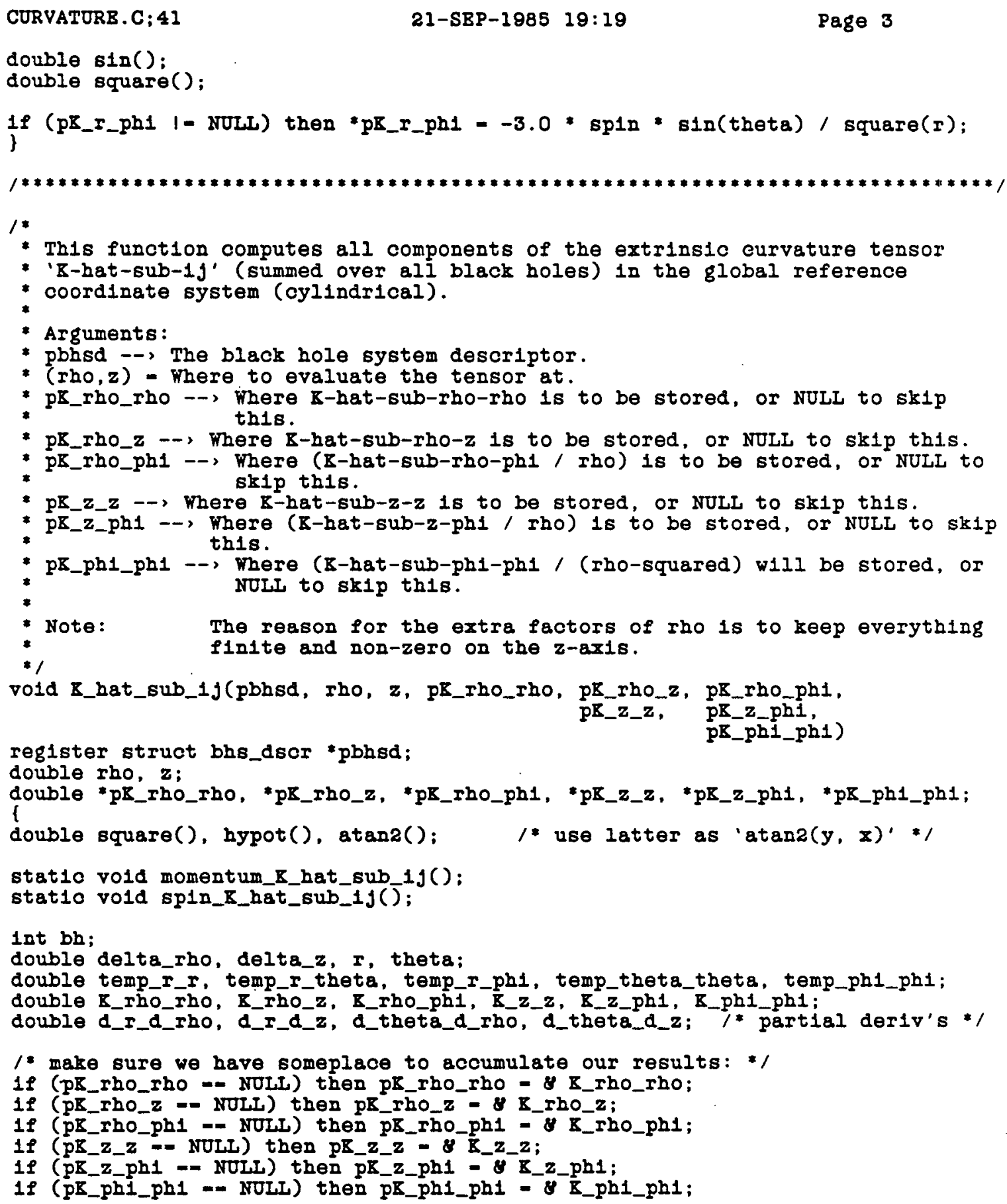




\section{main directory}

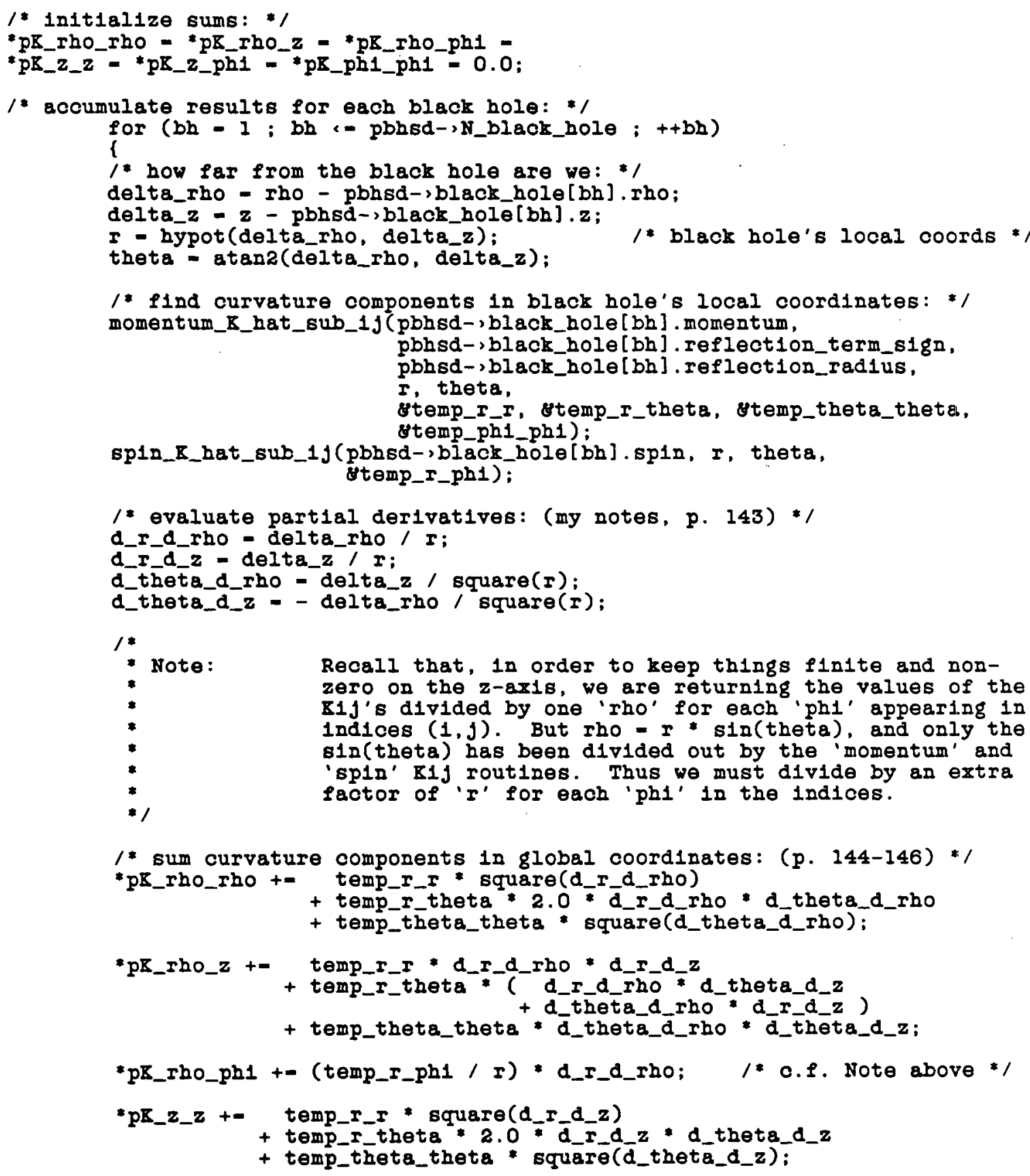




\section{ma1n directory}

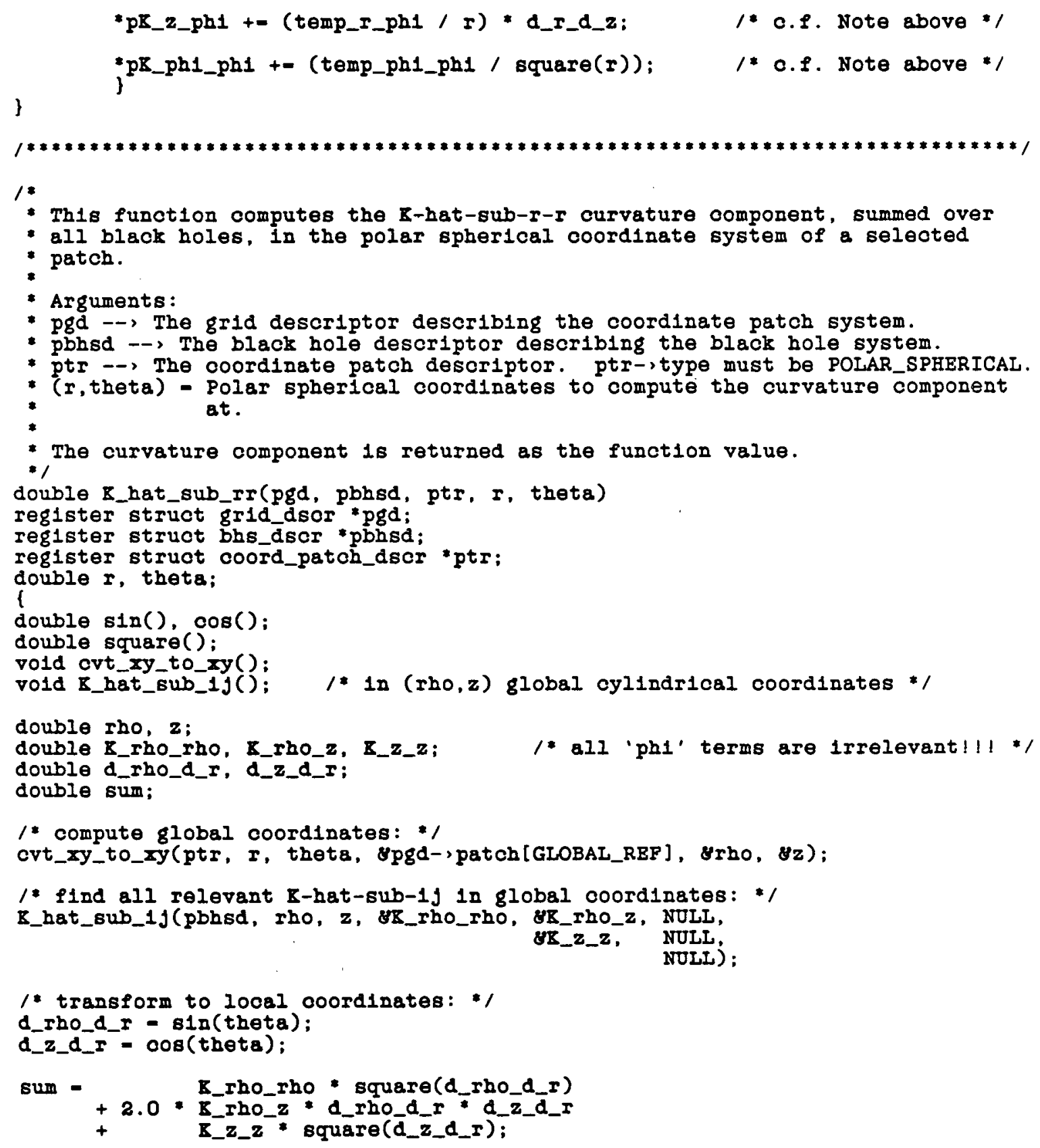


main directory

CURVATURB.C 41

21-SEP-1985 19:19

Page 6

return(sum);

$$
\text { \} }
$$




\section{main directory}

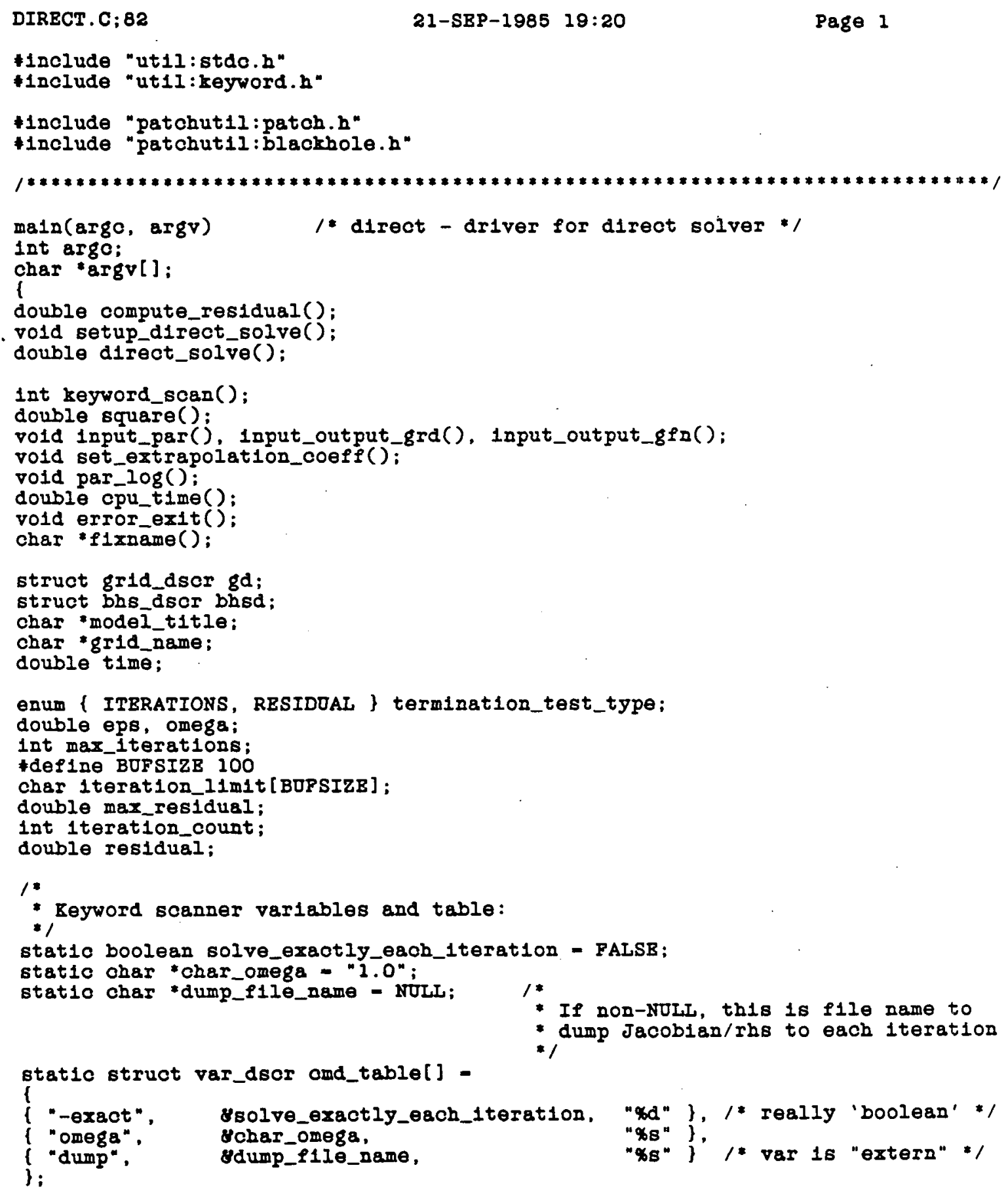




\section{main directory}

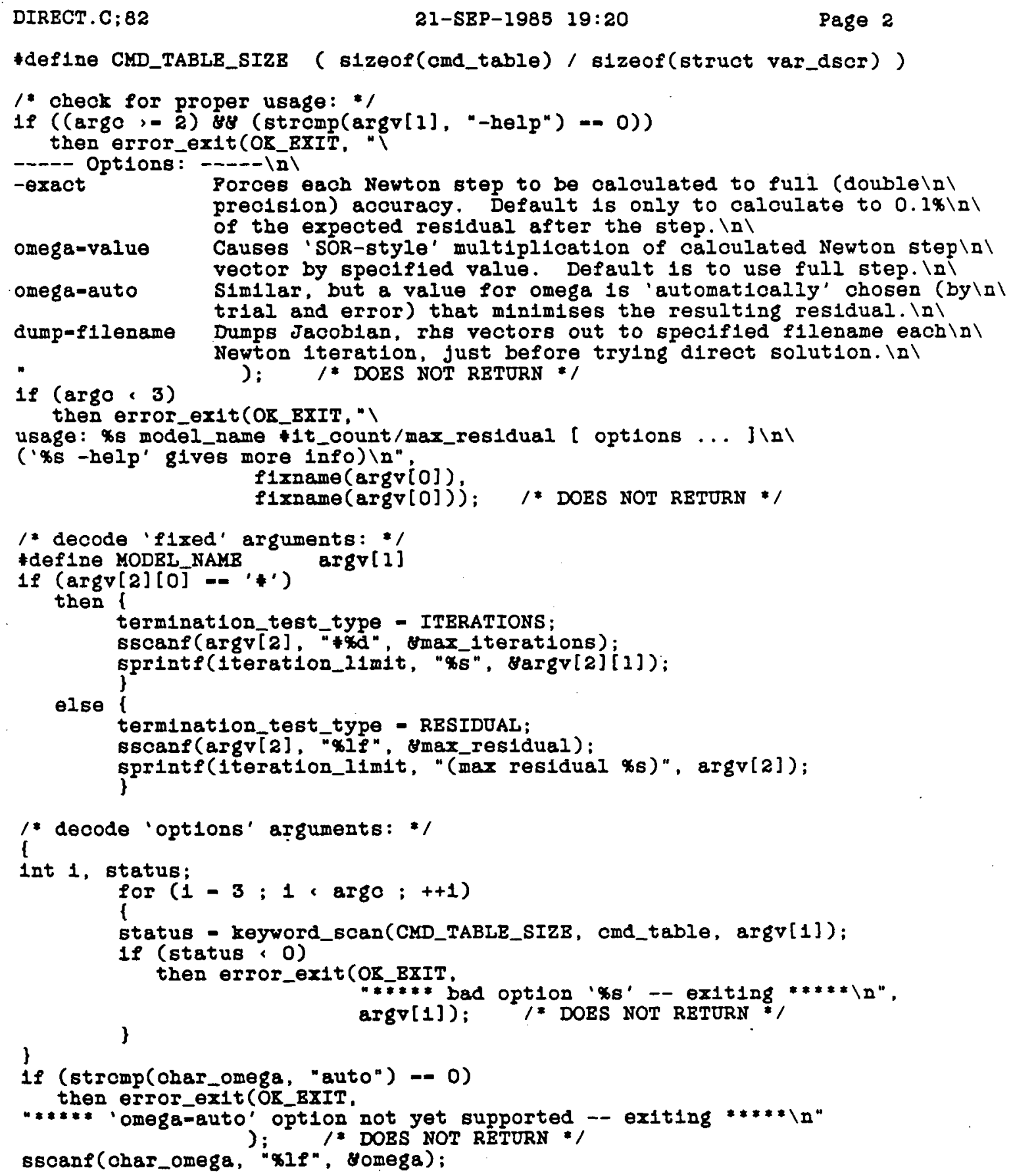




\section{main directory}

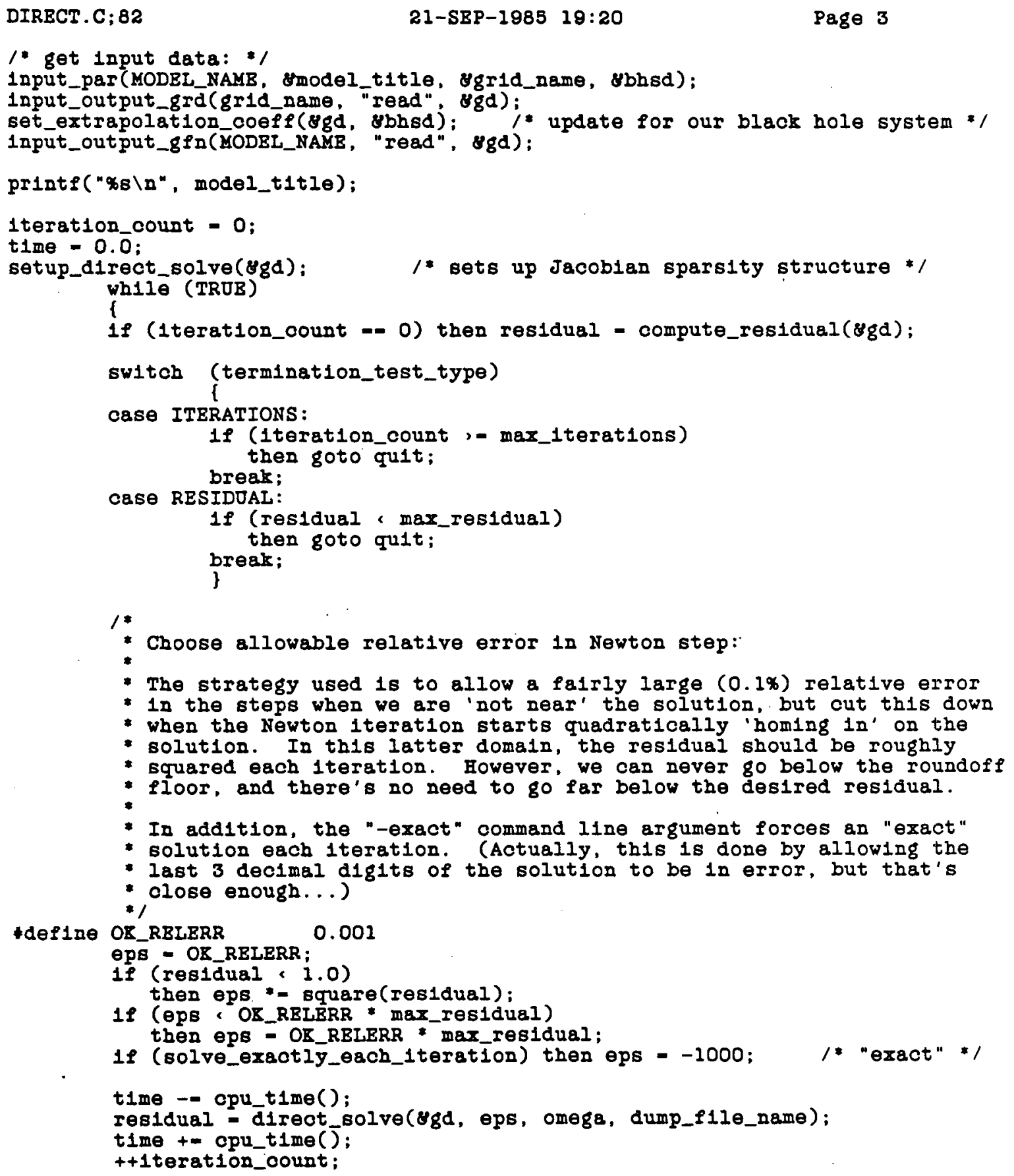


main directory

DIRECT.C; 82

21-SEP-1985 19:20

Page 4

printf("Iteration xd of xs: Maximum residual = $.210 \backslash n "$, 1terat1on_count, 1terat1on_limit, residual);

printe(", (disk update ");

input_output_gfn(MODEL_NAMB, "write", 8gd); printé("done?,

quit :

printf("xd 1terations / x.2f cpu seconds", 1terat1on_count, t1me);

if (1teration_count 1- 0)

then printf(" -X) $.2 f$ cpu sec/1teration", time/1teration_count); printf ("n");

1* f1nal disk update */

1nput_output_gfn(MODEL_NAMB, "write", ggd);

1* log to '.par' f1le: */

par_log(MODBL_NAMB, argc, argv, t1me); 


\section{main directory}

DOPSOR.C; 16

\#1nclude "ut1l:stdo.h"

\#1nclude "patchut1l: patoh.h"

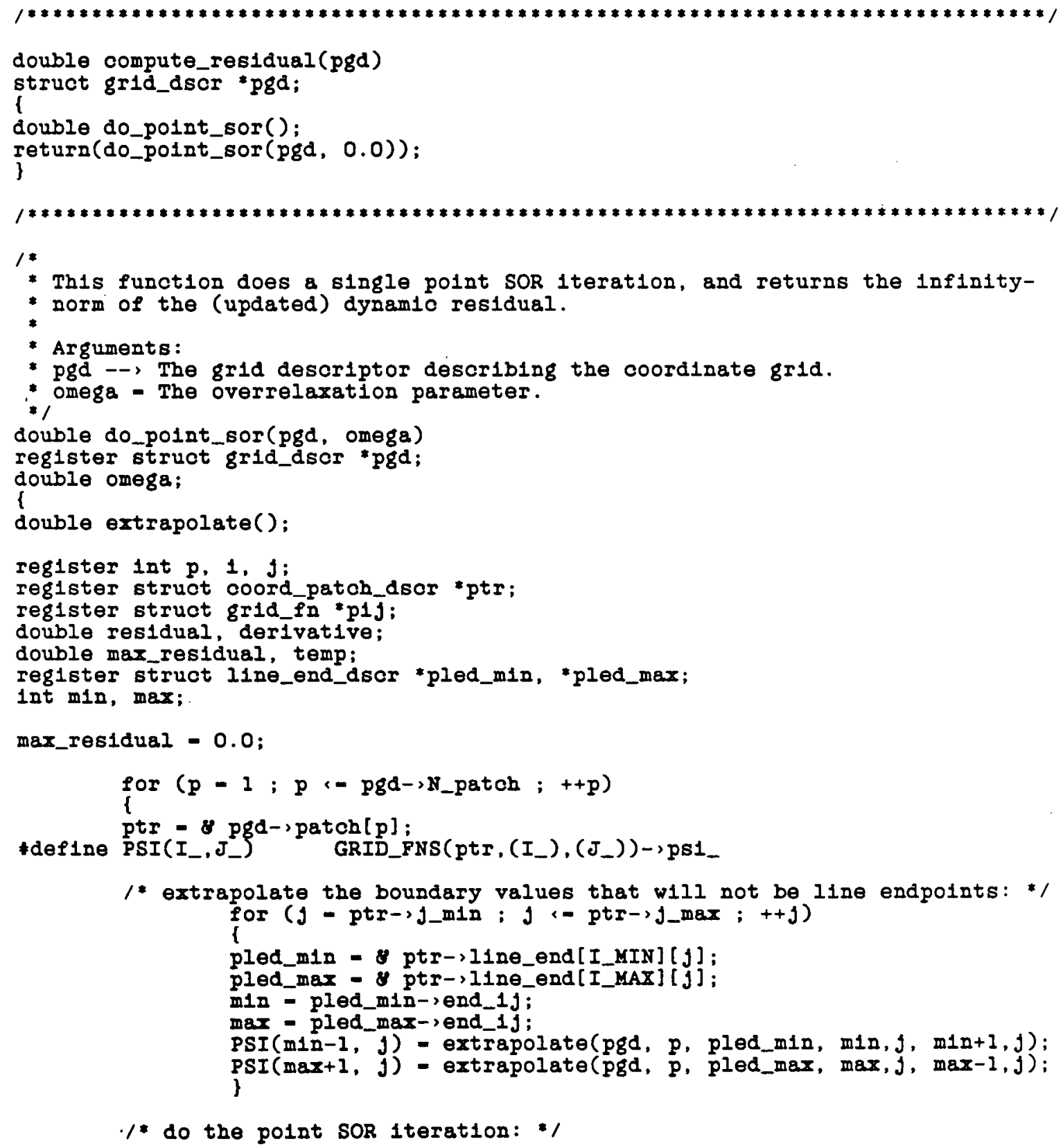




\section{main directory}

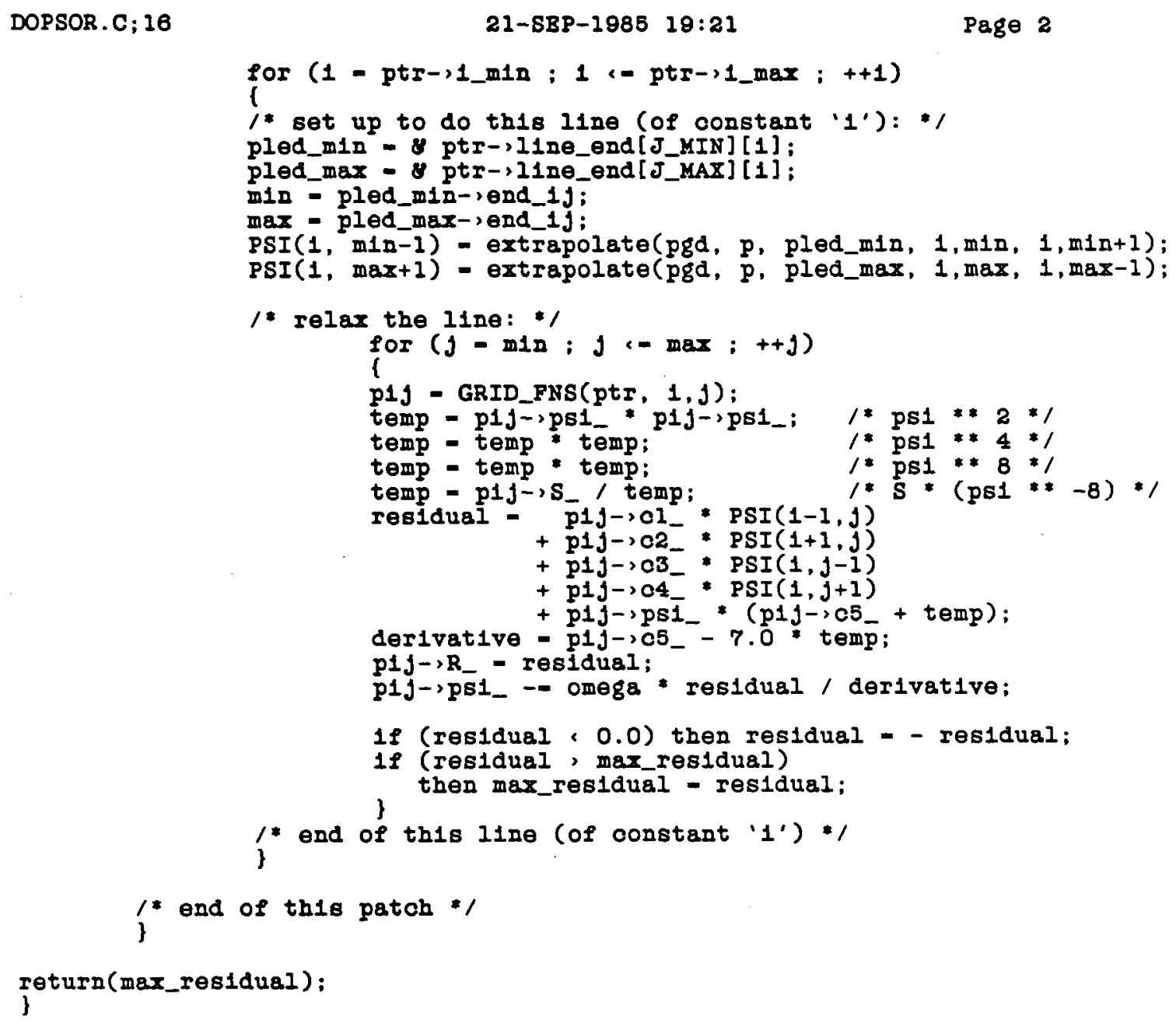




\section{main directory}

DUMPGRD. $C ; 6$

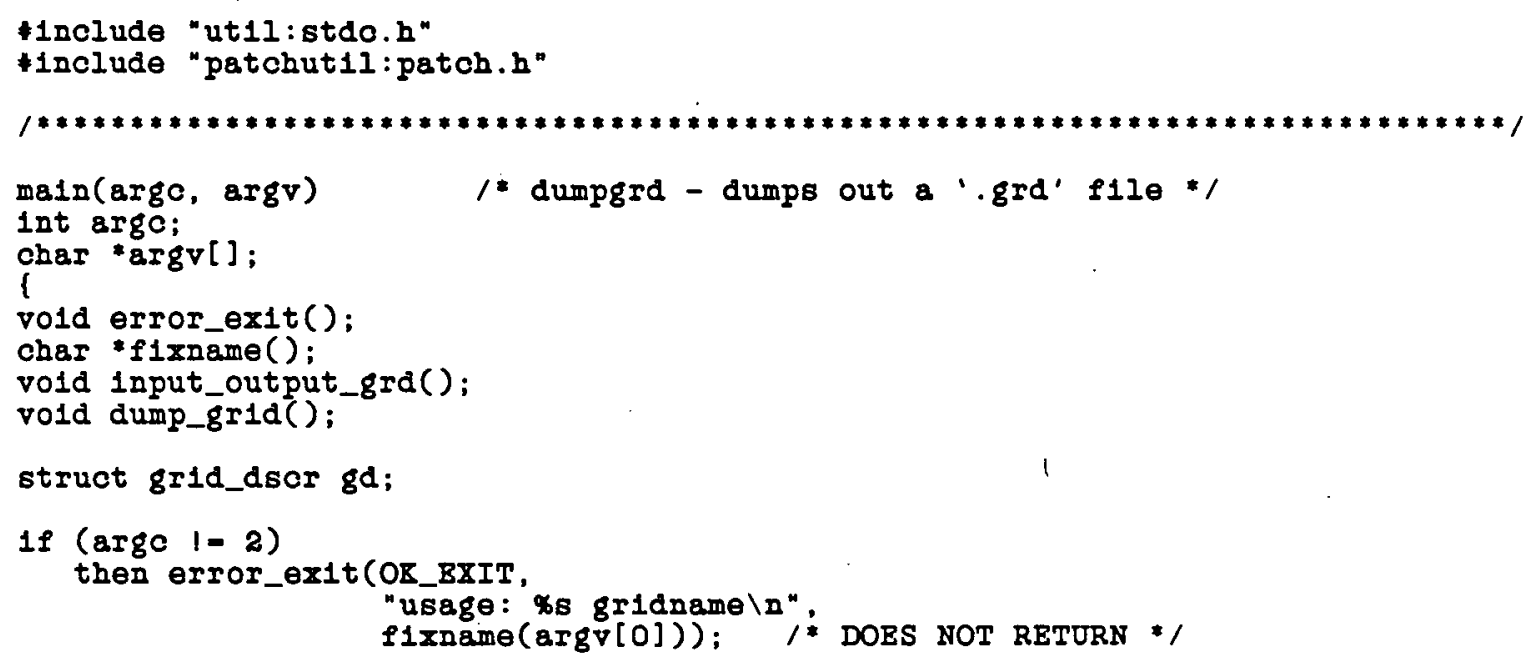




\section{main directory}

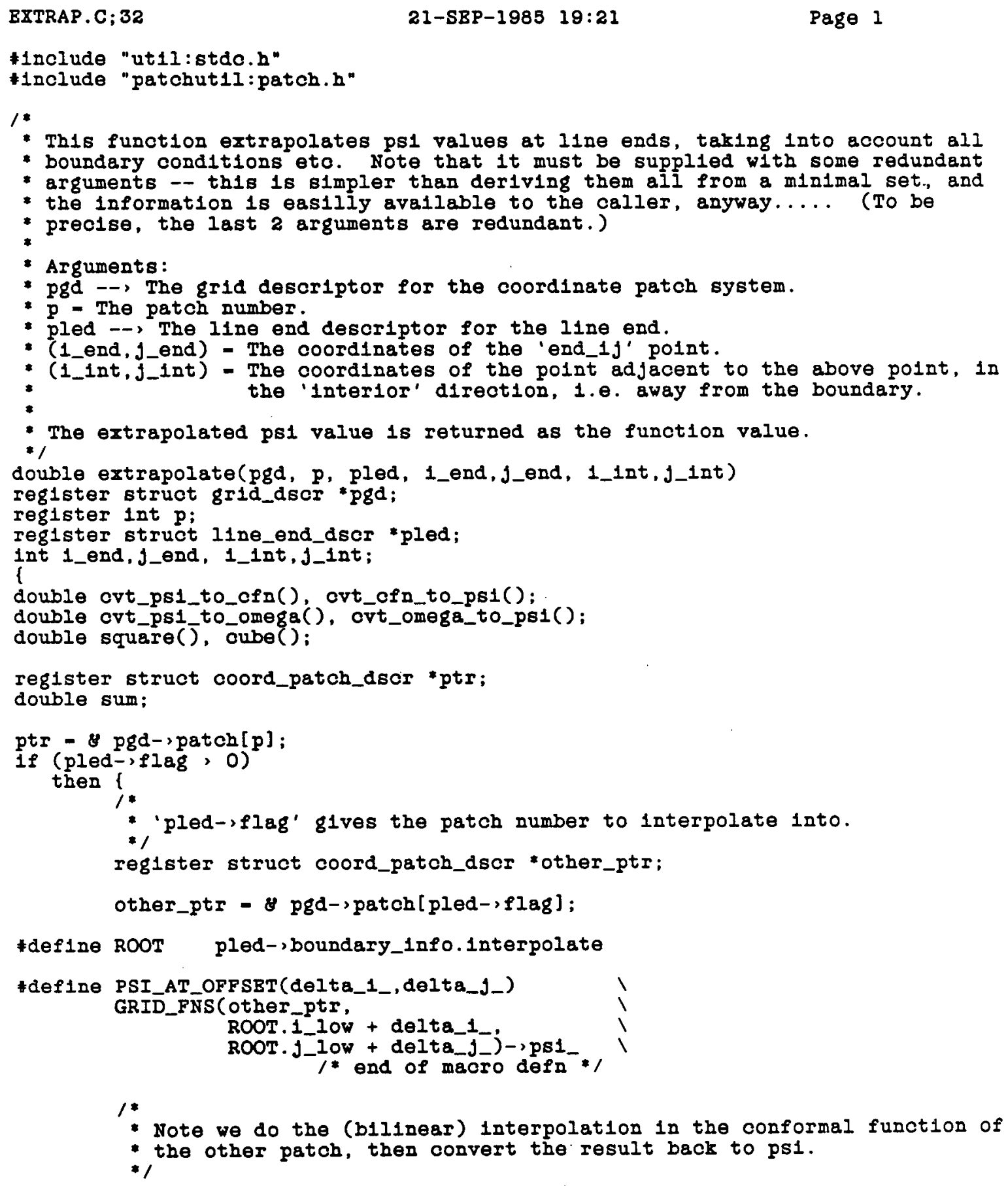

*1nclude "ut1l:stdo.h"

*1nclude "patchut1l:patch. $h$ "

$1 *$

- Th1s function extrapolates psi values at line ends, taking 1nto account all

- boundary conditions etc. Note that it must be supplied with some redundant

- arguments -- this is simpler than deriving them ali from a minimal set., and

- the information is easiliy avallable to the caller, anyway.... (To be

- precise, the last 2 arguments are redundant.)

- Arguments:

- pgd - The grid descriptor for the coordinate patch system.

- $p$ - The patch number.

- pled - The line end descriptor for the line end.

- (1_end,j_end) - The coordinates of the 'end_1j' point.

- (1_int,j_int) - The coordinates of the point adjacent to the above point, in

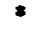

$*$

- The extrapolated psi value is returned as the function value.

1

double extrapolate (pgd, p, pled, 1_end,j_end, 1_int,j_int)

register struct grid_dsor *pgd;

register int $p$;

reg1ster struct 11ne_end_dscr *pled;

int 1_end, J_end, 1_int, J_int; \{

double cvt_ps1_to_ofn( ), ovt_cfn_to_psi();

double cvt_psi_to_omega( ), cvt_omega_to_psi();

double square( ), cube( );

register struct coord_patch_dscr *ptr;

double sum;

ptr $=\&$ pgd-spatch $[p]$;

if (pled-iflag, o)

then \{

* 'pled-slag' gives the patch number to interpolate into.

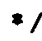

reg1ster struct coord_patch_dscr other_ptr;

other_ptr - \&gd->patch[pled->flag];

*define ROOT pled->boundary_info.interpolate

*def1ne PSI_AT_OFFSET(delta_1_,delta_l_)

GRID_FNS(other_ptr,

ROOT.1_low + delta_1

ROOT.J_low + delta_j_j-ipsi

$1 *$

- Note we do the (bilinear) 1nterpolation in the conformal function of

* the other patch, then convert the result back to psi.

$* 1$ 


\section{main directory}

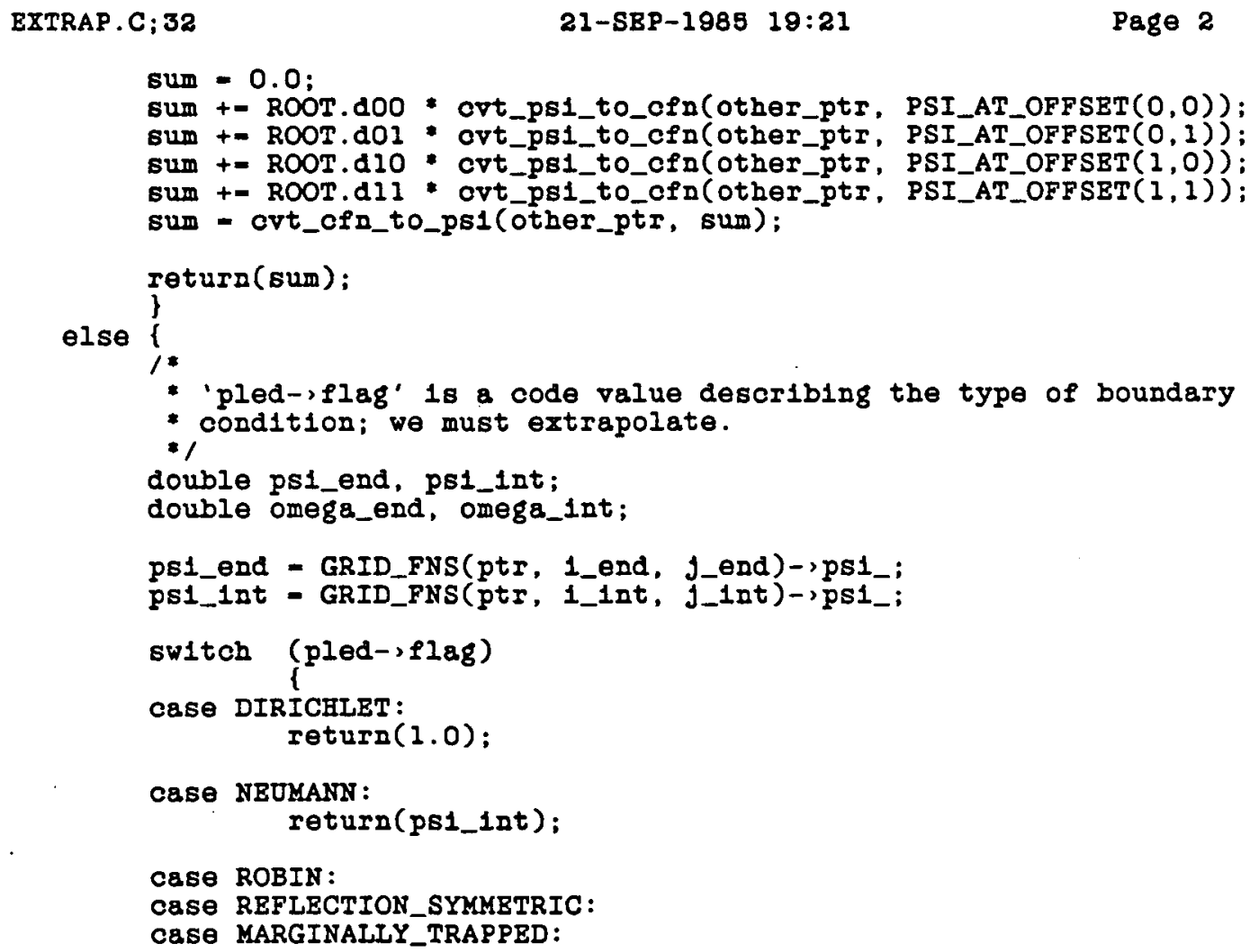
WHATSIT, 


\section{main directory}

EXTRAP.C; 32

return(sum);

default :

error_ex1t(OR_BXIT,

"**** extrapolate: bad pled->1lag-\%d 1n patch *\%d at (\%d,\%d)\n", pled->1lag,

$p$,

1_end, j_end); $\quad /$ DOES NOT RETURN *

/ end of 'switch' on type of boundary condition */

$j$ end of pled-, 12 ag $; 0$ log10 *

\} 


\section{main directory}

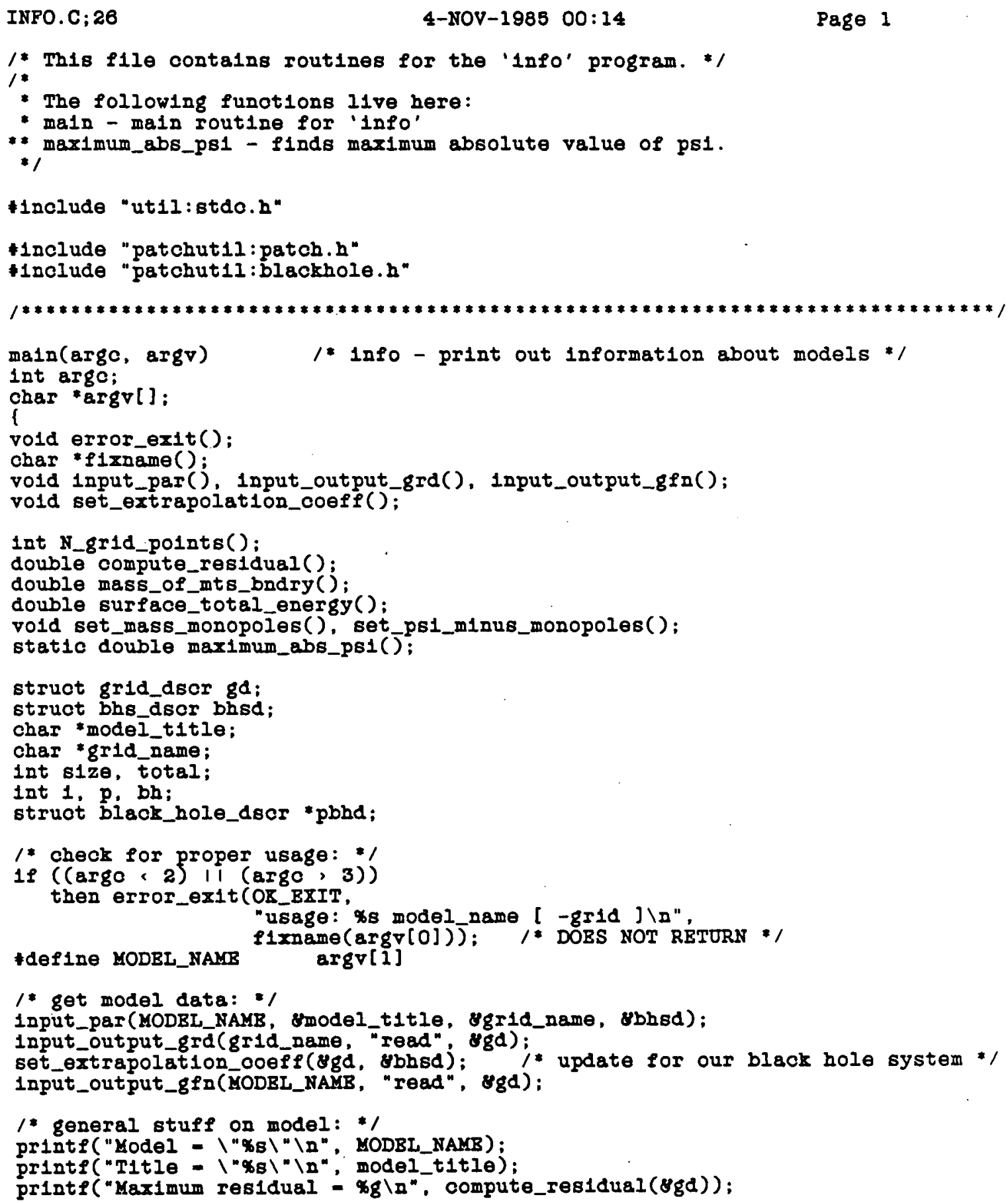




\section{main directory}

INPO.C;26

4-NOV-1985 00:14

Page 2

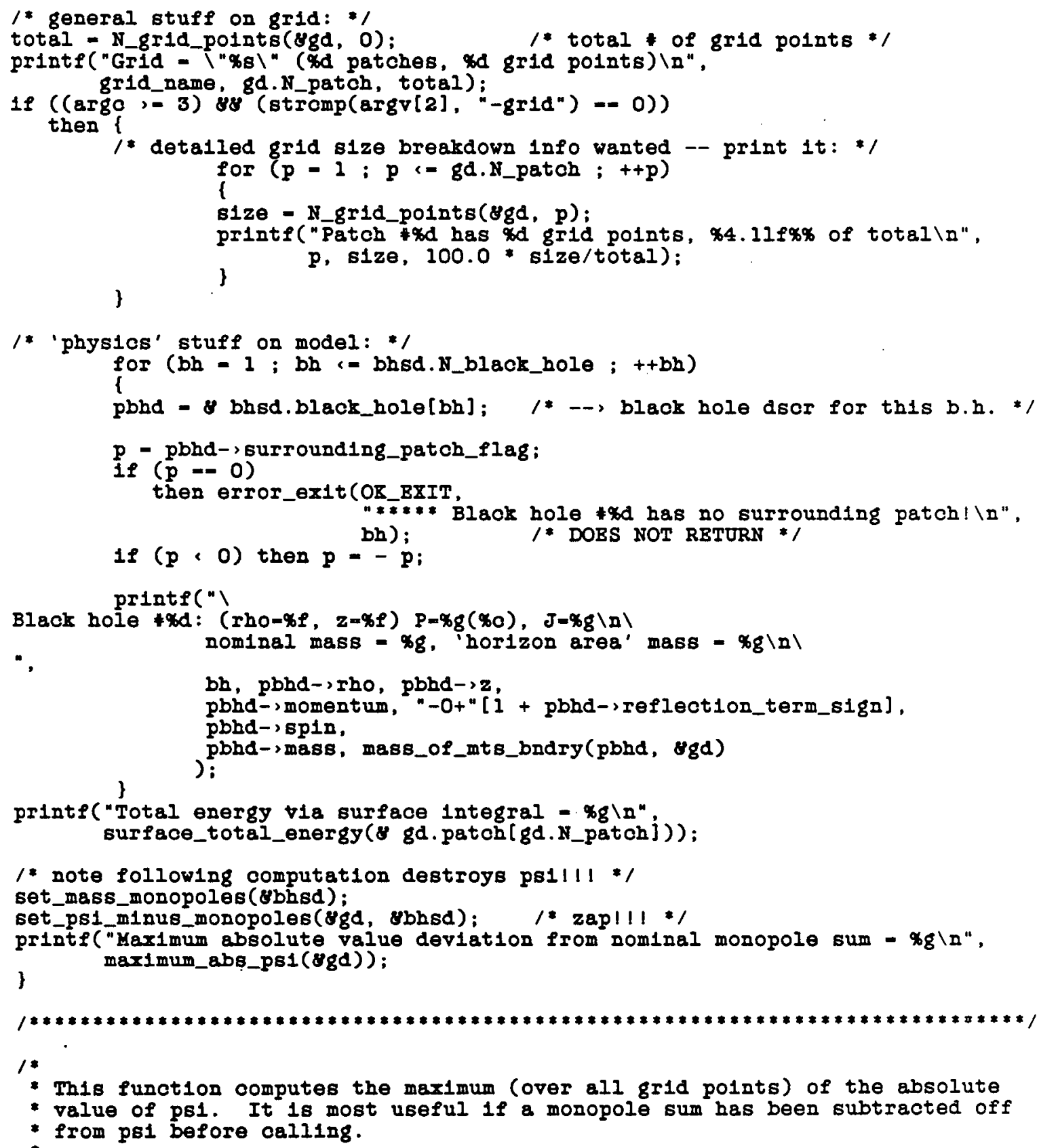

- This function computes the maximum (over all grid polnts) of the absolute

- value of psi. It is most useful if a monopole sum has been subtracted off

* from psi before calling. 
main directory

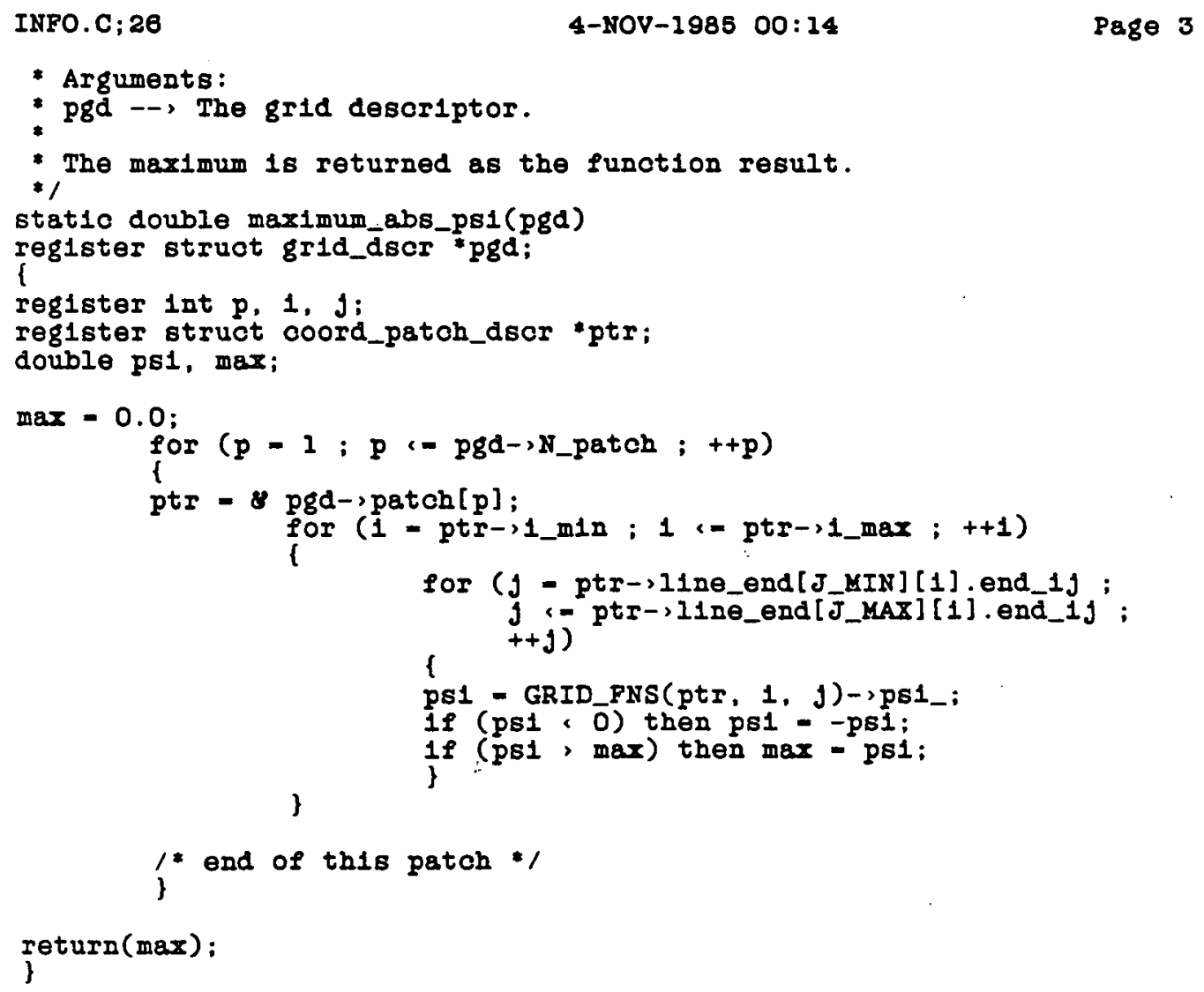




\section{main directory}

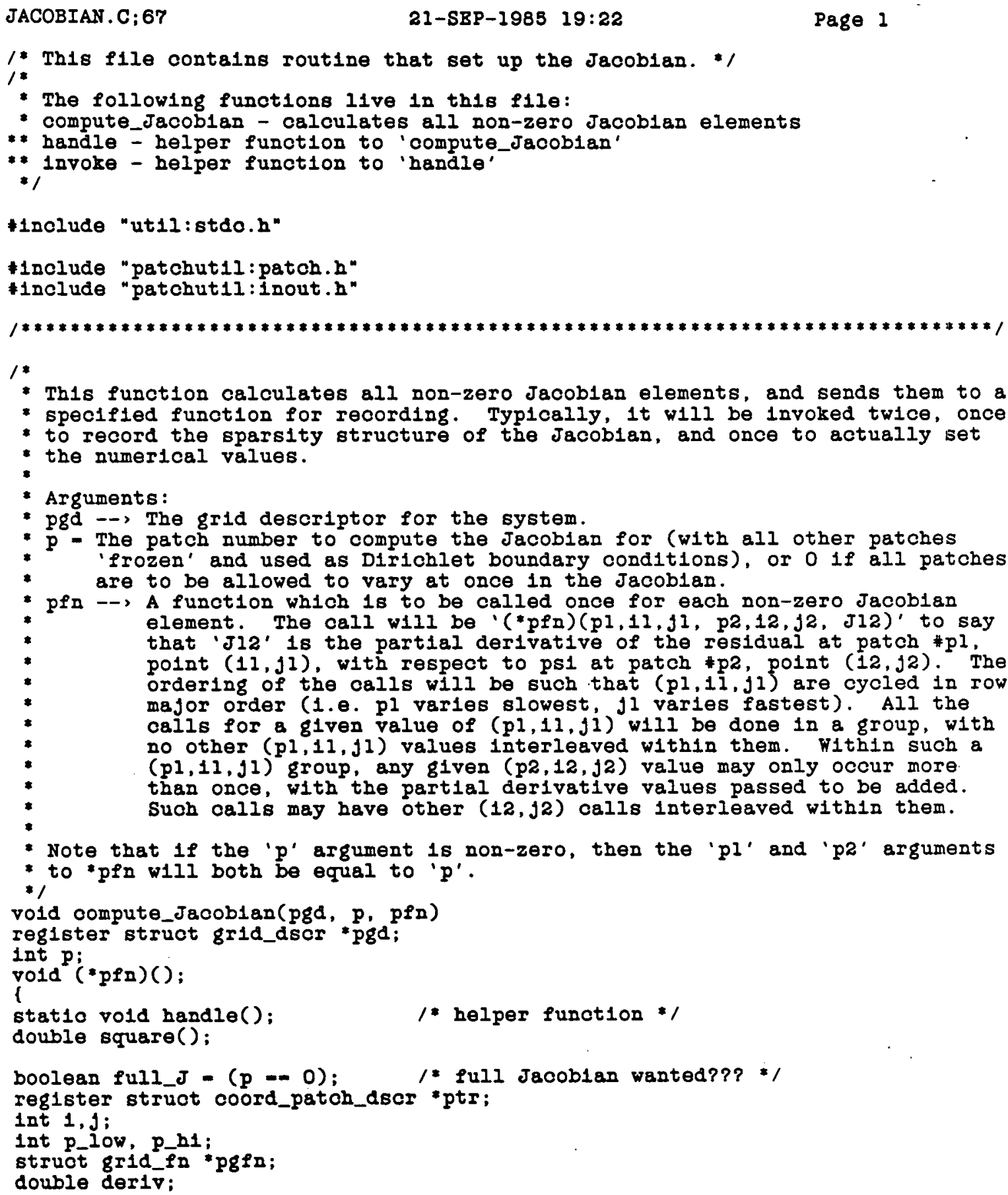




\section{main directory}

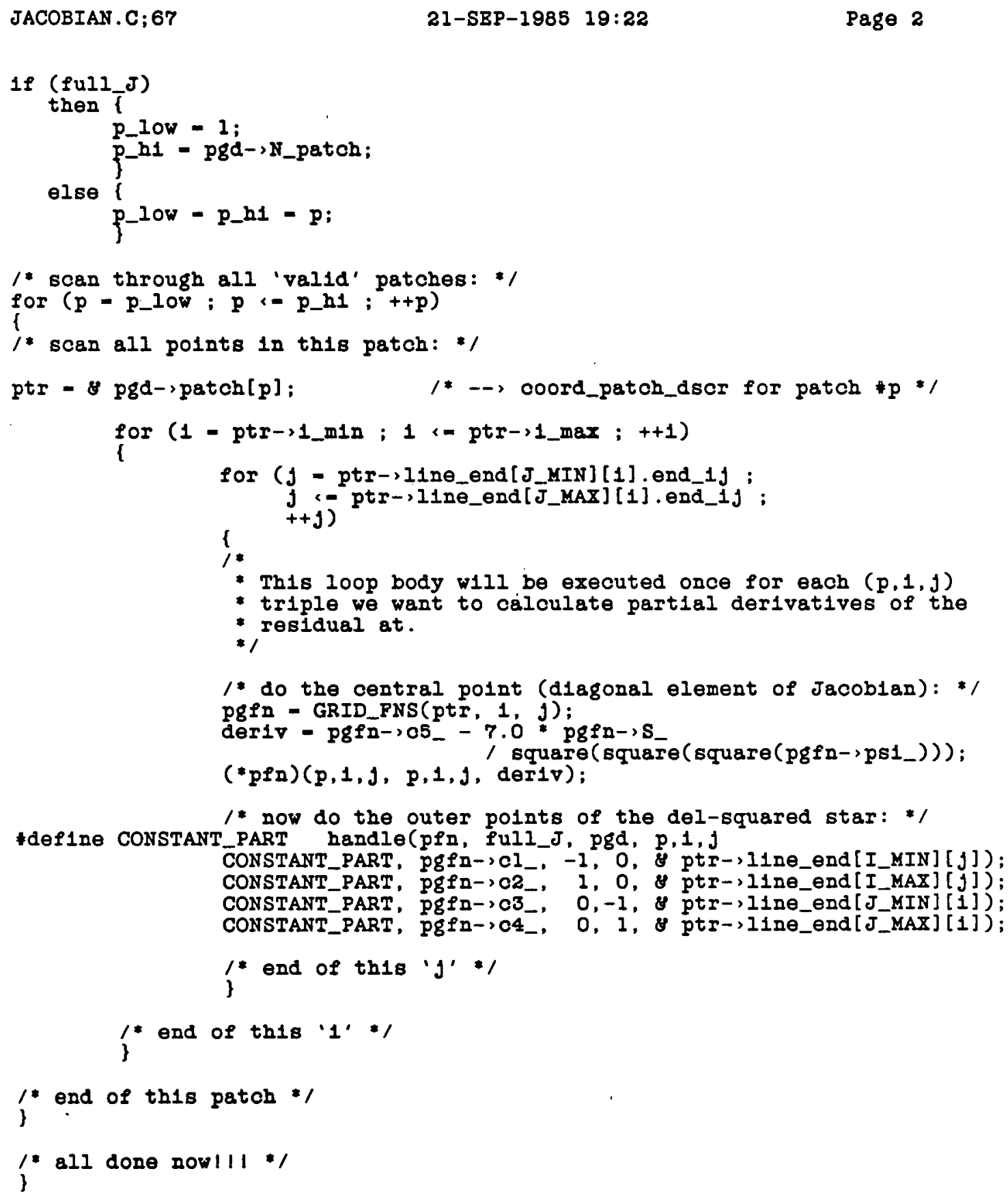




\section{ma1n directory}

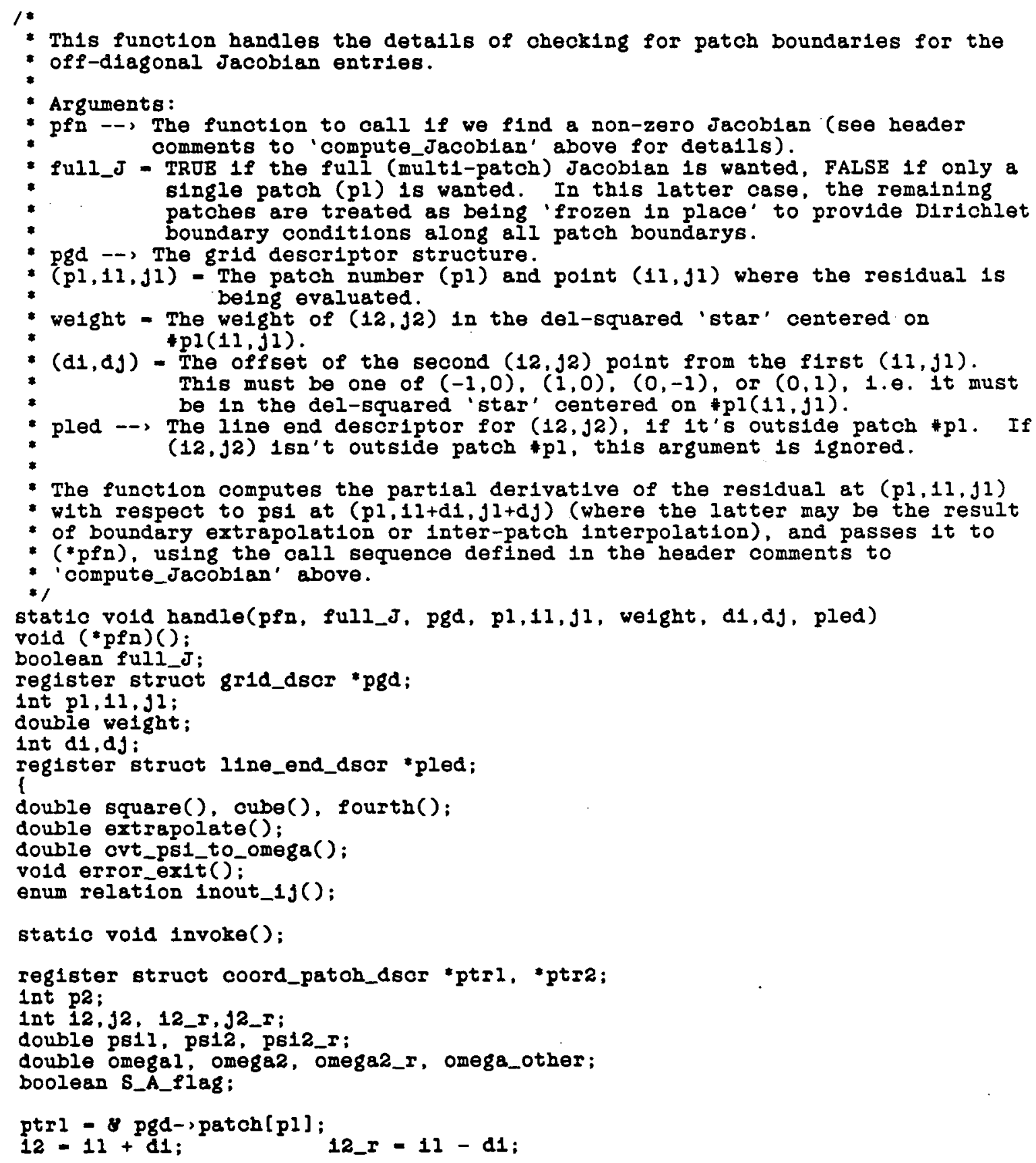




\section{main directory}

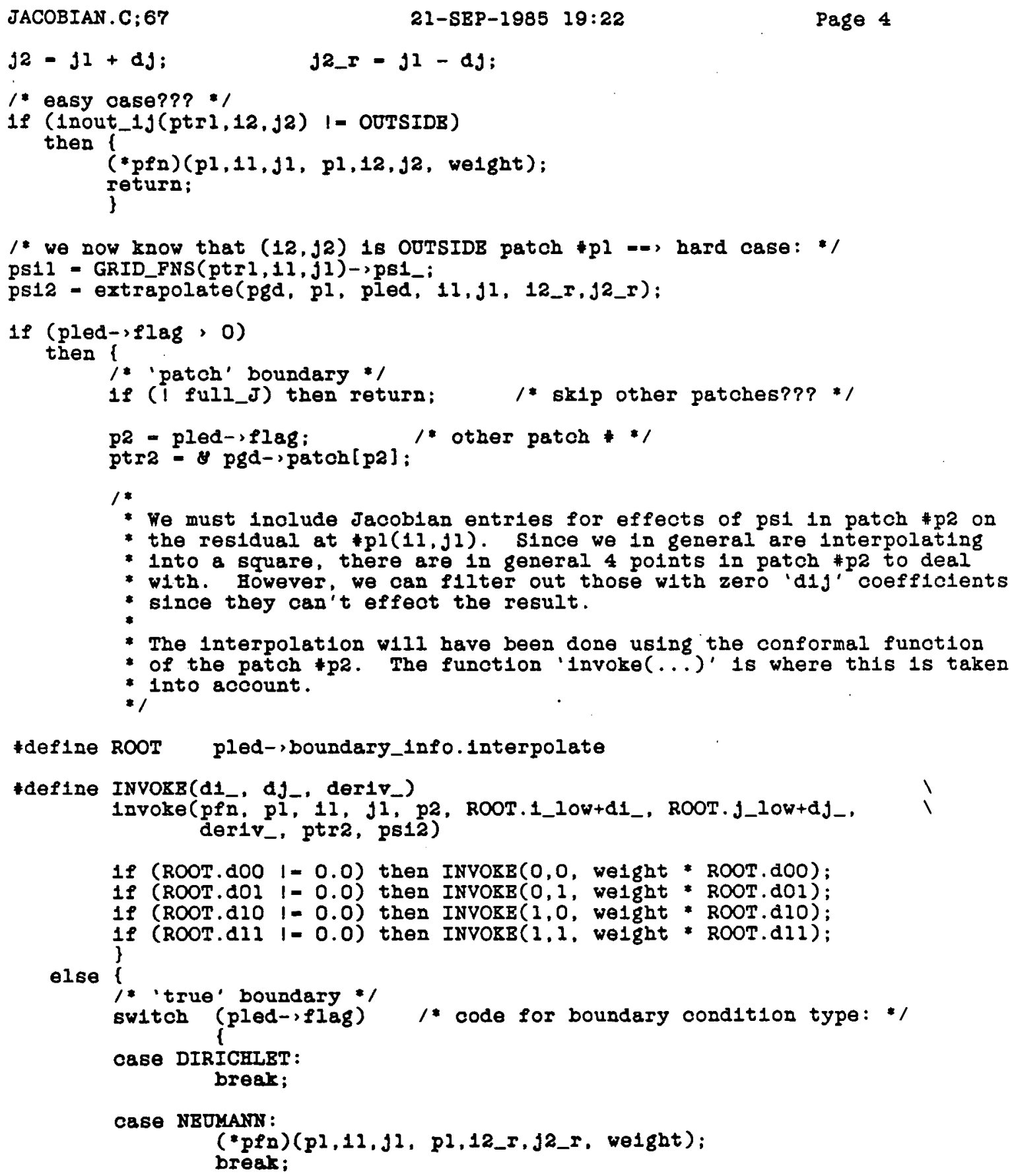




\section{main directory}

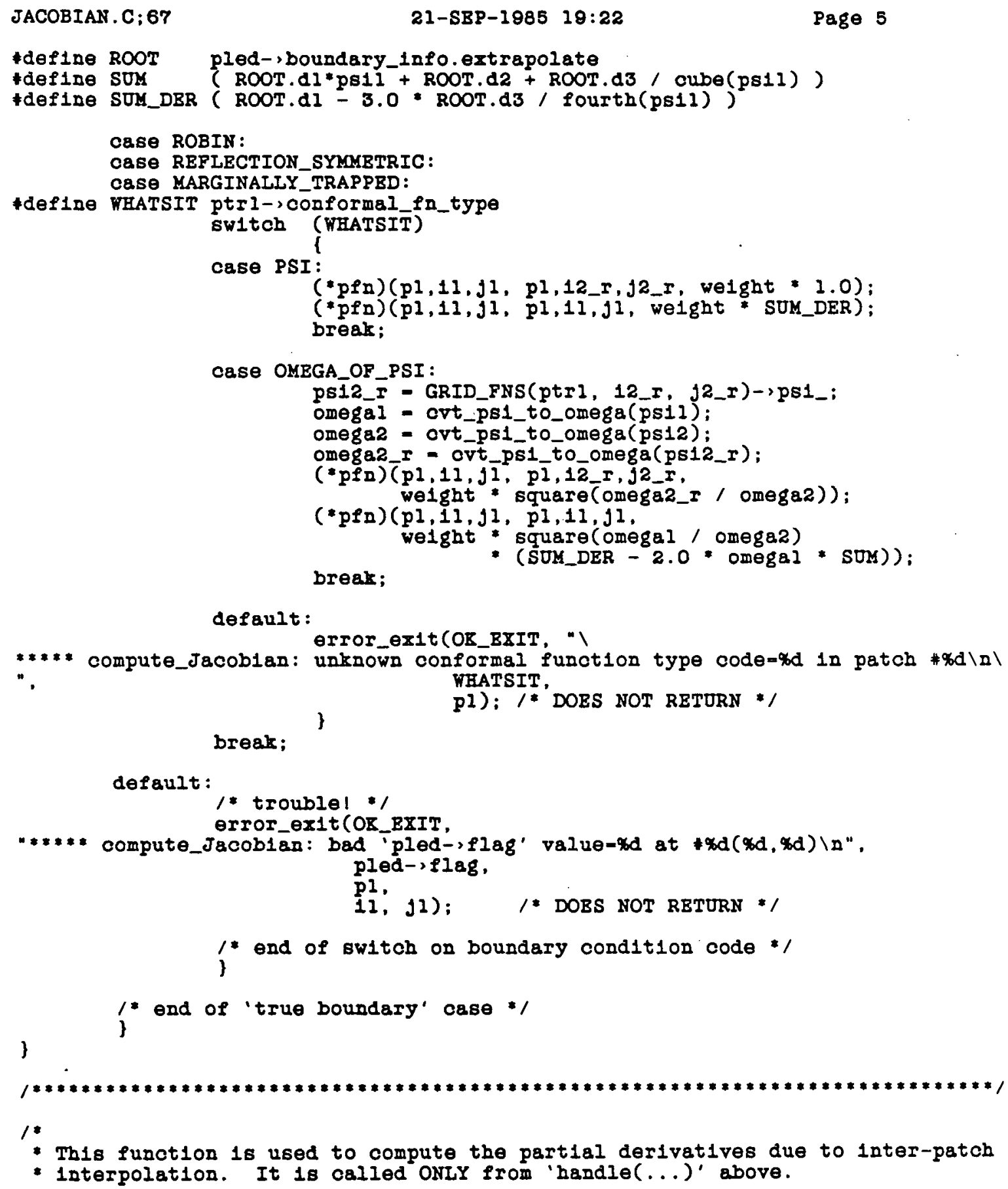




\section{ma1n directory}

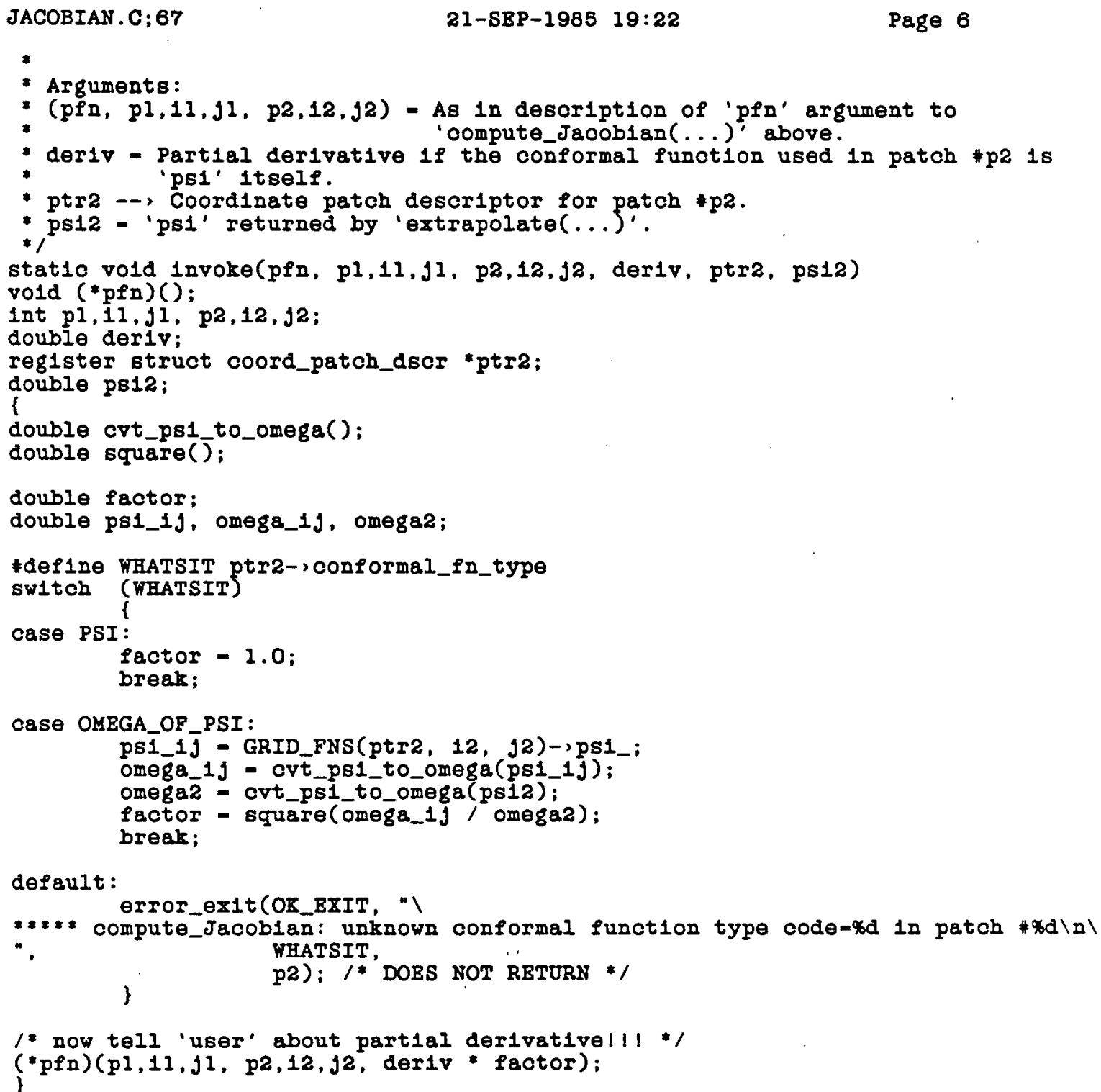

$\bullet$

* Arguments:

* (pfn, pl,11,jl, p2,12,j2) = As in description of 'pfn' argument to

- 'compute_Jacob1an(...)' above.

- deriv - Partial derivative if the conformal function used 1 patch \#p2 is

- 'psi' itself.

- ptra -- Coordinate patch descriptor for patch $\neq$ p2.

* psiz - 'psi' returned by 'extrapolate(...)'.

stat1o vold 1nvoke(pfn, p1,11,j1, p2,12,j2, der1v, ptr2, ps12)

vold (*pen)( );

lat p1,11,j1, p2,12,j2;

double deriv;

reg1ster struct coord_patch_dscr *ptra; double psi2;

f

double crt_ps1_to_omega( ) :

double square ();

double factor:

double psi_1j, omega_1j, omegaz;

*def1ne WHATSIT ptr2->conformal_fn_type

switch (WHATSIT)

case PSI :

factor $=1.0$;

break;

case OMEGA_OF_PSI :

psi_1 $\bar{j}$ - GRID_FNS(ptr2, 12, j2)->psi_;

omega_1j - cvt_ps1_to_omega(ps1_1j);

omega2 = cvt_ps1_to_omega(ps12):

factor - square(omega_1j/ omegaz);

break;

default:

error_exitCOR_BXIT, ")

**:* compute_Jacoblan: unknown conformal function type code-xd in patch *xd $\backslash n \backslash$

$\cdots$ WHATSIT,

\} p2): $/$ * DOBS NOT RETURN *I

1* now tell 'user' about partial derivativelll */

(*ppn)(pl,11,j1, p2,12,j2, der1v factor); 


\section{main directory}

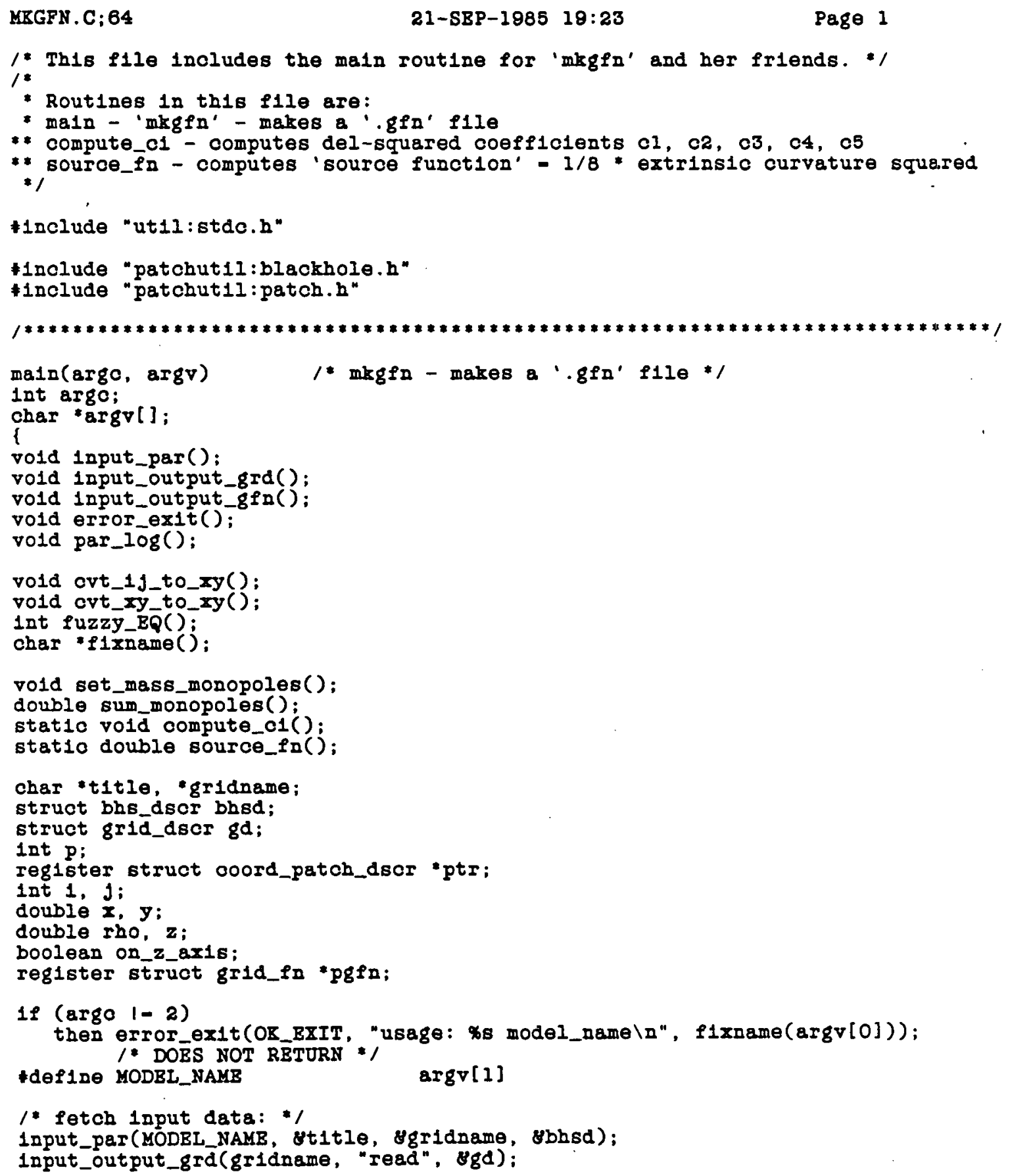




\section{main directory}

MRGFN.C; 64

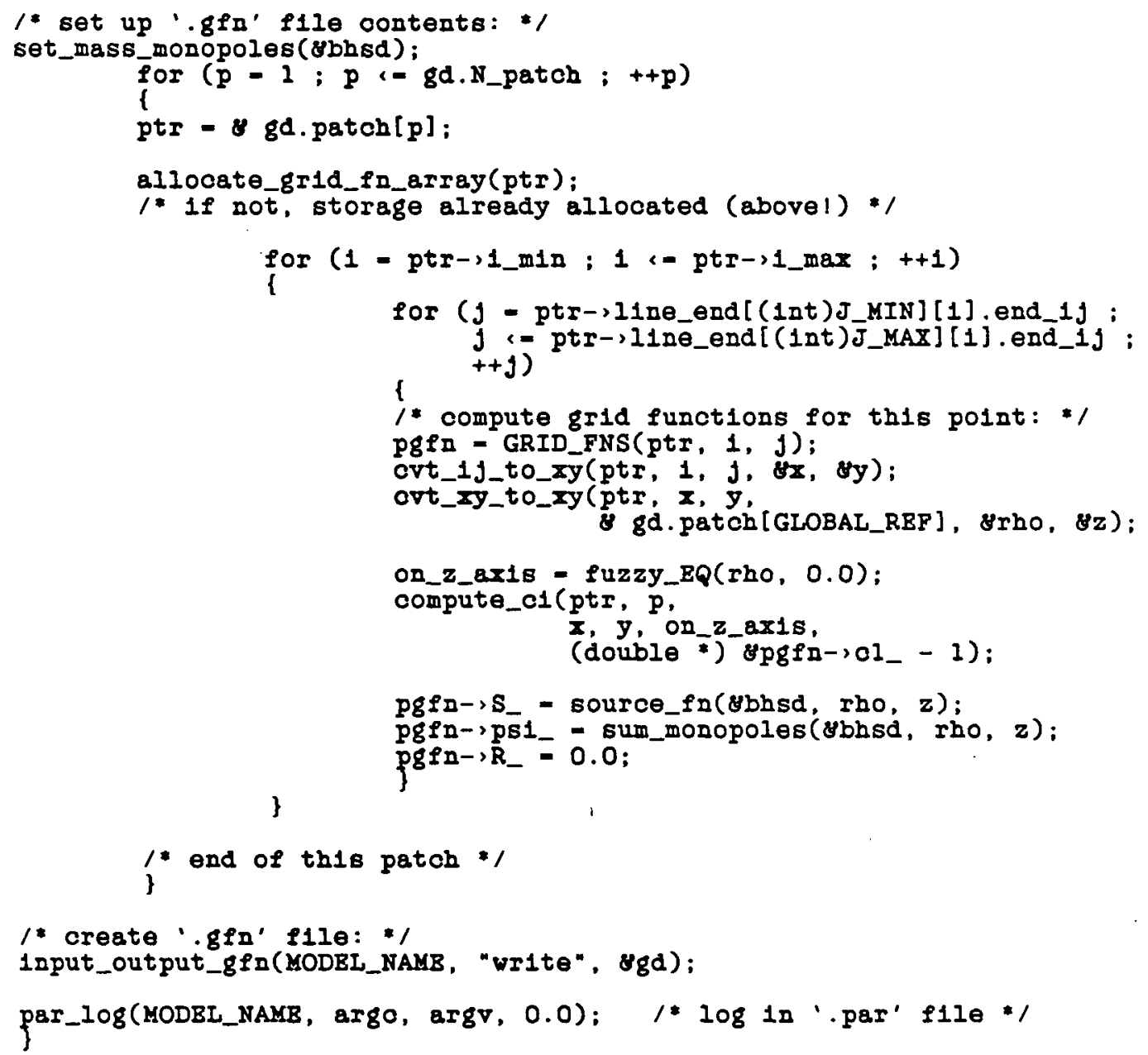




\section{main directory}

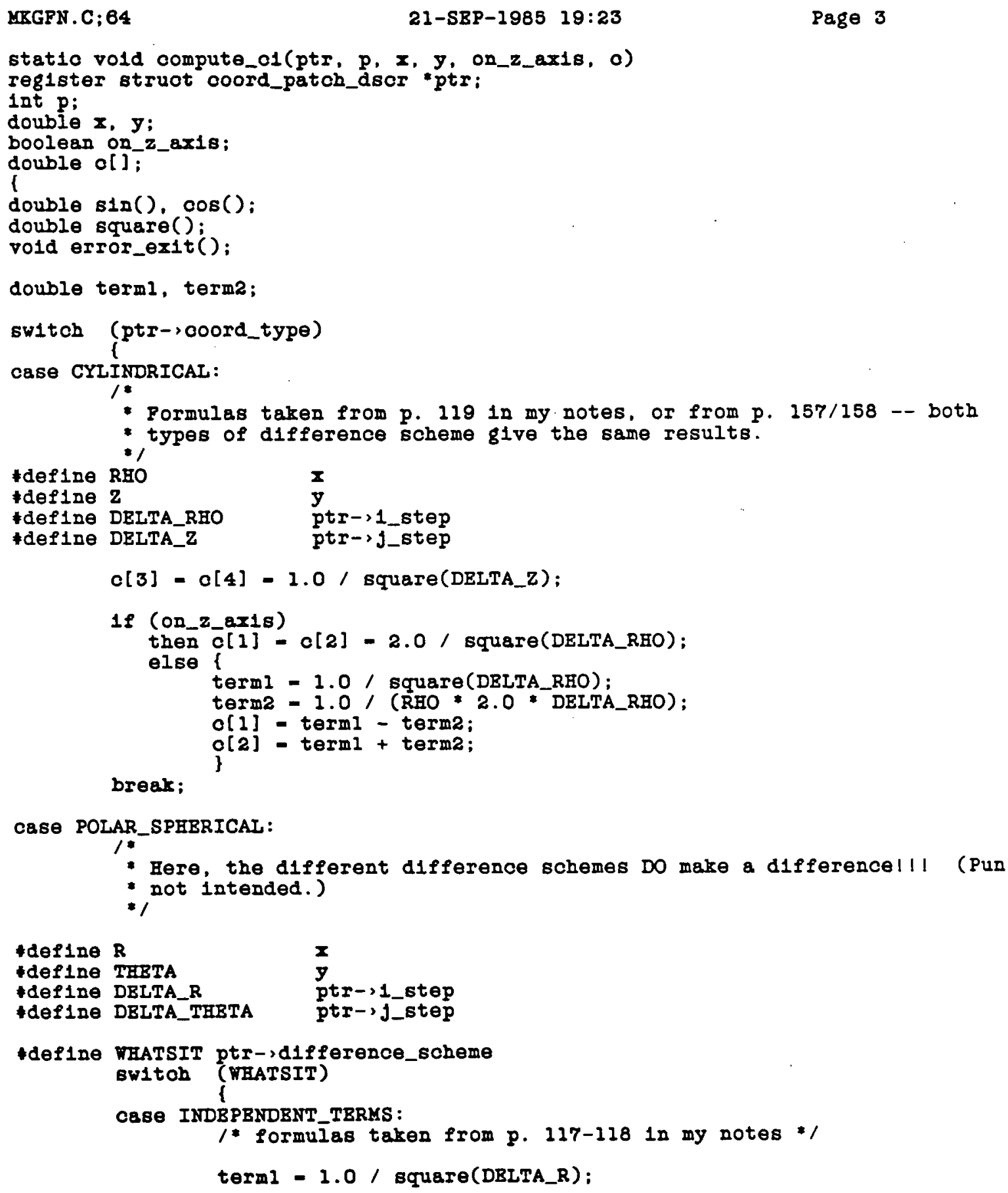




\section{main directory}

MBGFN . C ; 64

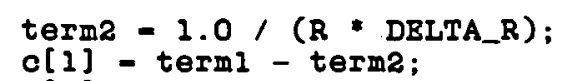

break;

case LOCALLY_CONSERVATIVB:

1 * formulas taken from p. 159-162 in my notes */

term1 = $1.0 /$ square (DBLTA_R);

term2 - DBLTA R / (2.0 R);

c[1] = terml square(1.0 - term2):

$o[2]=\operatorname{term}$ * square $(1.0+\operatorname{term} 2)$;

if (on_z_oxis)

then $\bar{c}[3]-c[4]-2.0 / \operatorname{square}(R *$ DELTA_THETA);

else

terml $=1.0 /($ square $(R *$ DELTA_THETA $) * \sin ($ THETA $))$;

term2 $=0.5 *$ DELTA_TEETA:

$o[3]=$ term] * sin(THETA - term2);

$o[4]=\operatorname{term} 1$ s1n(THETA + term2);

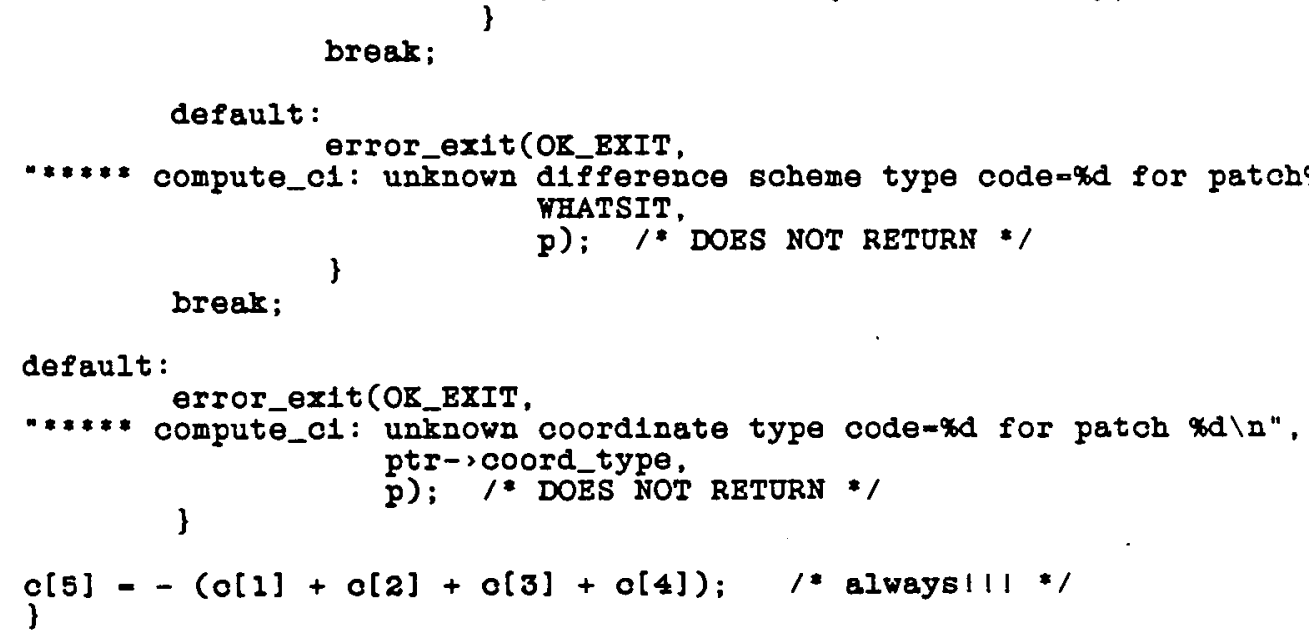

break; 


\section{main directory}

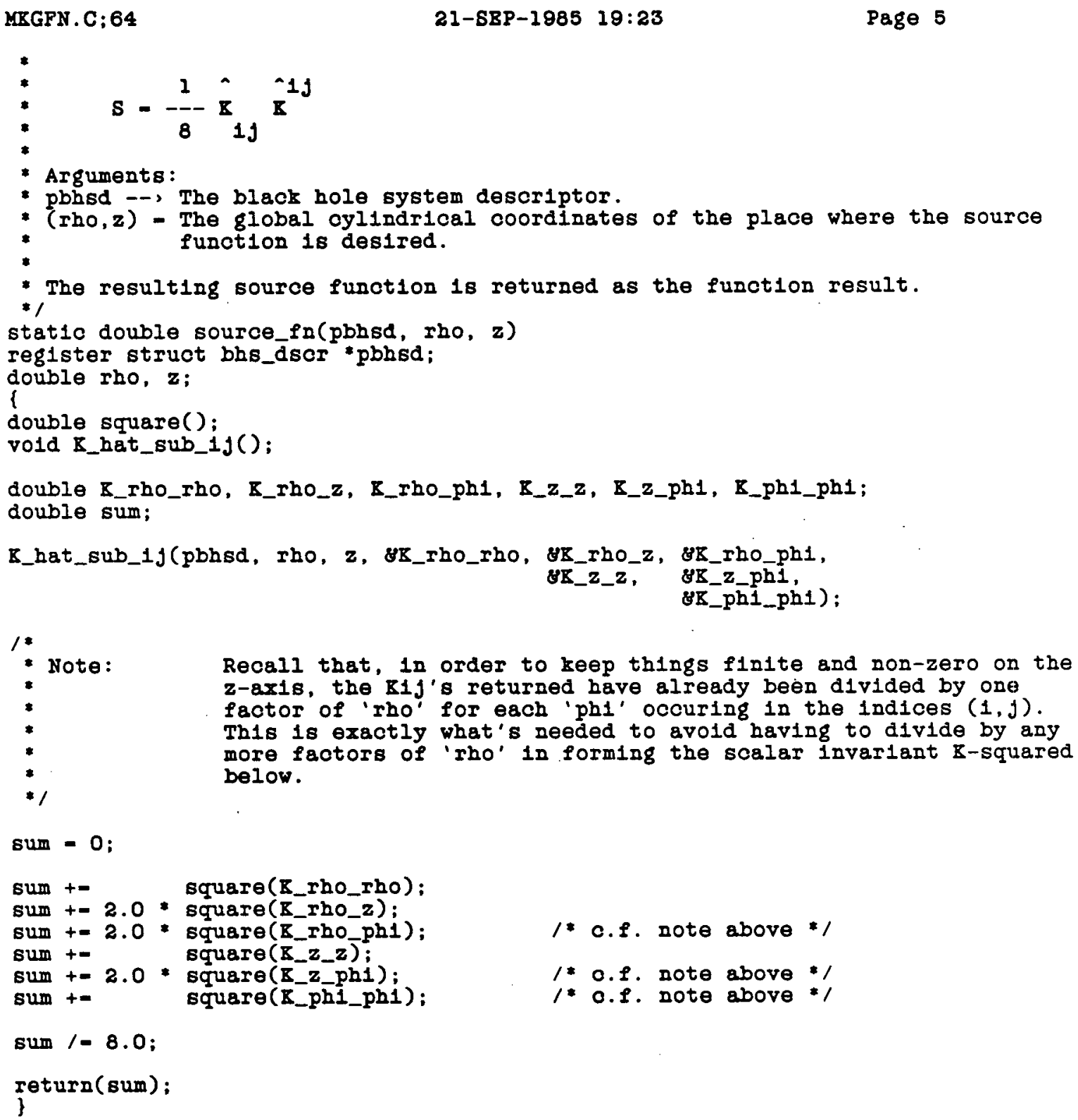

$$
s=-\frac{1}{8} \hat{x}_{1 J} \mathrm{~K}^{-1 \mathrm{~J}}
$$




\section{main directory}

KEGRD.C: 6

\#1nclude "ut1l:stdc.h"

Hnclude "patchut1l:patch.h"

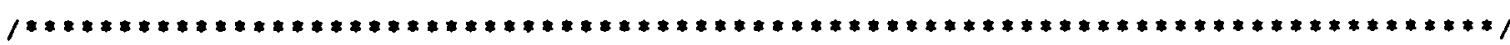

main(argo,argv) /* mkgrd -- makes a '.grd' flle from a '.pat' flle *I

int arge;

char argiv[]:

\{

vo1d 1nput_pat();

vo1d input_output_grd();

char efirname ();

struct grid_dscr gd:

if ( $\operatorname{argc} 1=2$ )

then error_exit(OR_BXIT,

"usage: Xs gridname $\backslash n$ "

f1xname(aTgvioj)); , DOES NOT RETURN *

1nput_pat (argv[1], 8gd);

input_output_grd(argv[1], "wr1te", \&gd); 


\section{main directory}

MONOPOLB.C; 28

4-NOV-1885 $00: 14$

Page 1

/* This file contains routines that deal with 'monopoles' in psi */

$1 *$

* Routines in this flle are:

* sum_monopoles - Sums current monopoles for specified position

- set_mass_monopoles - Sets monopole strengths to one half b.h. masses

- set_area_monopoles - Sets monopole strengths to one half horizon areas

- nornal1se_monopoles - normalises monopoles so they sum to specifled amount

* set_psi_minus_monopoles - computes 'psi -- (monopole sum)'

1

"1nclude "ut1l:stdo.h"

\#1nclude "patchut1l:blackhole.h"

\#1nclude "patchut1l:patch.h"

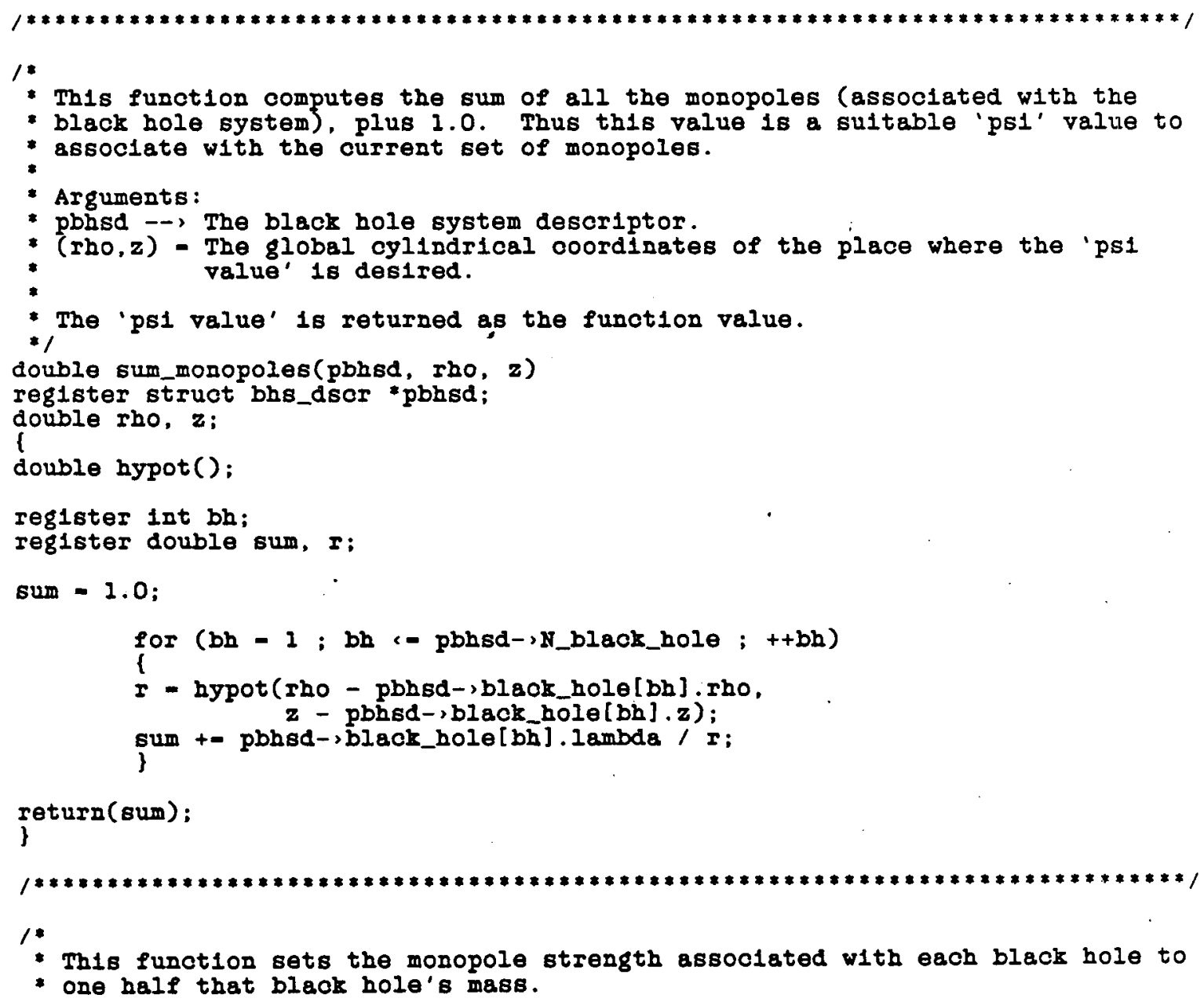




\section{main directory}

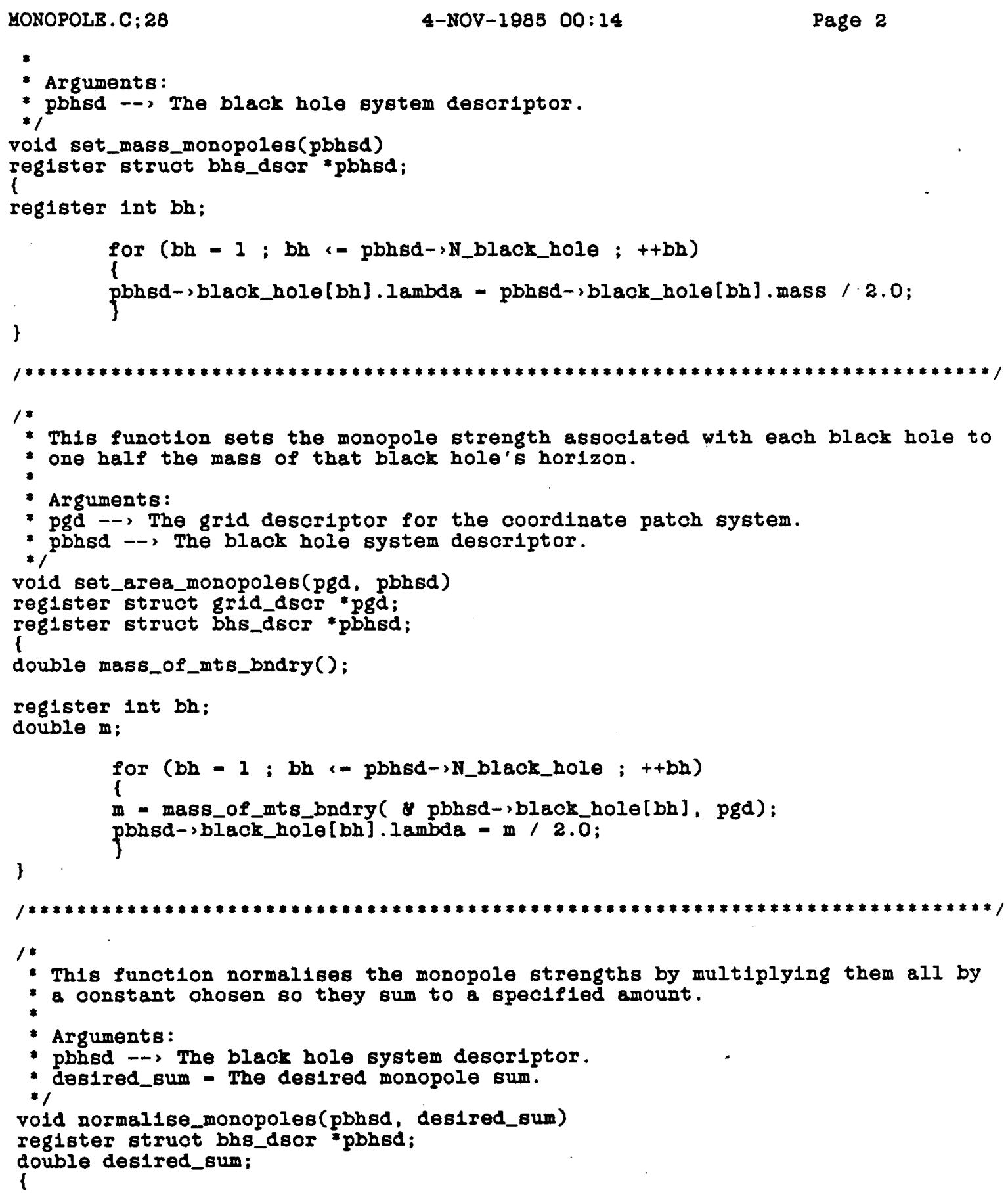




\section{main directory}

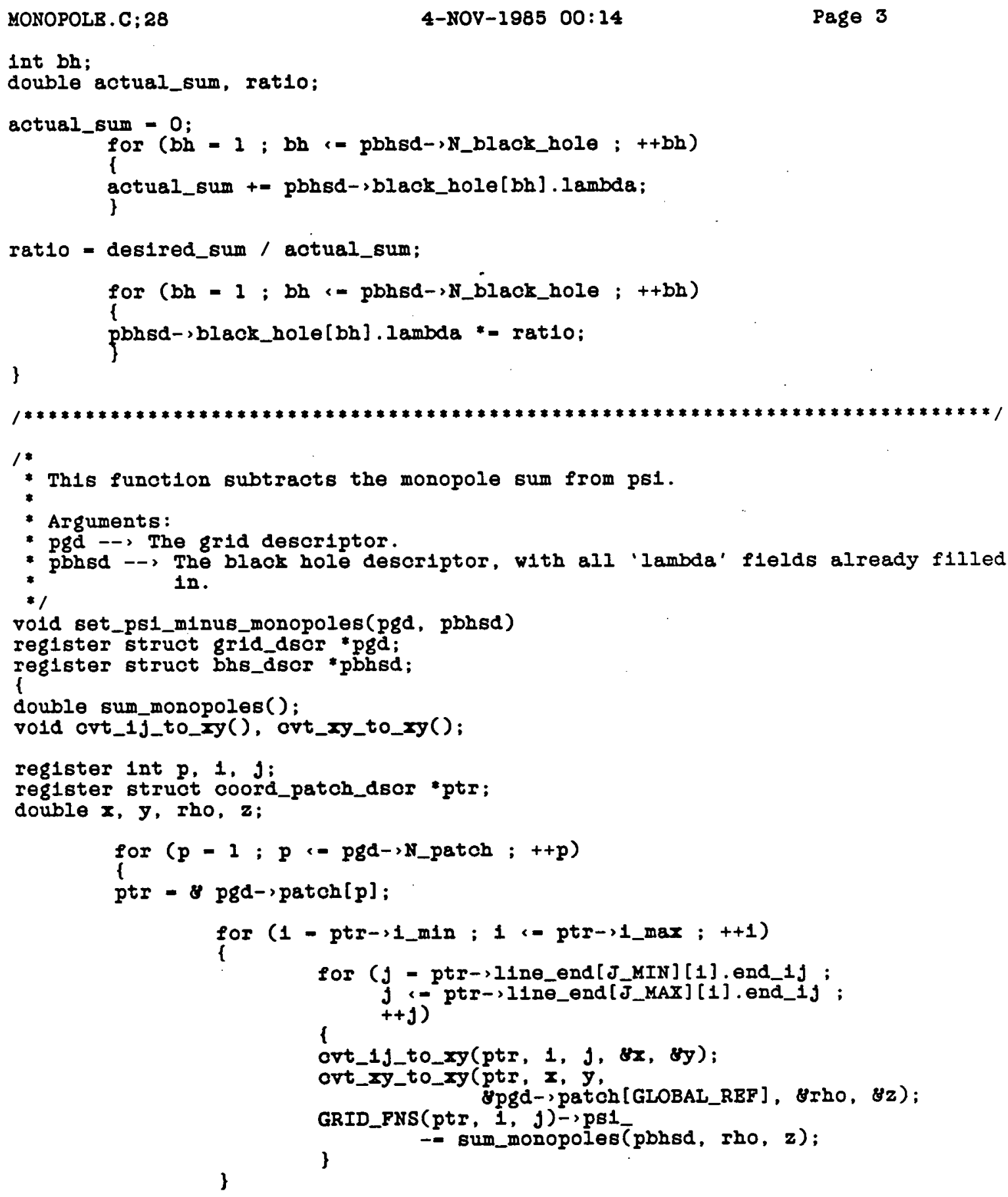


main directory

KONOPOLB.C;28

4-NOV-1986 00:14

Page 4

I end of this patch *I

)

\} 


\section{main directory}

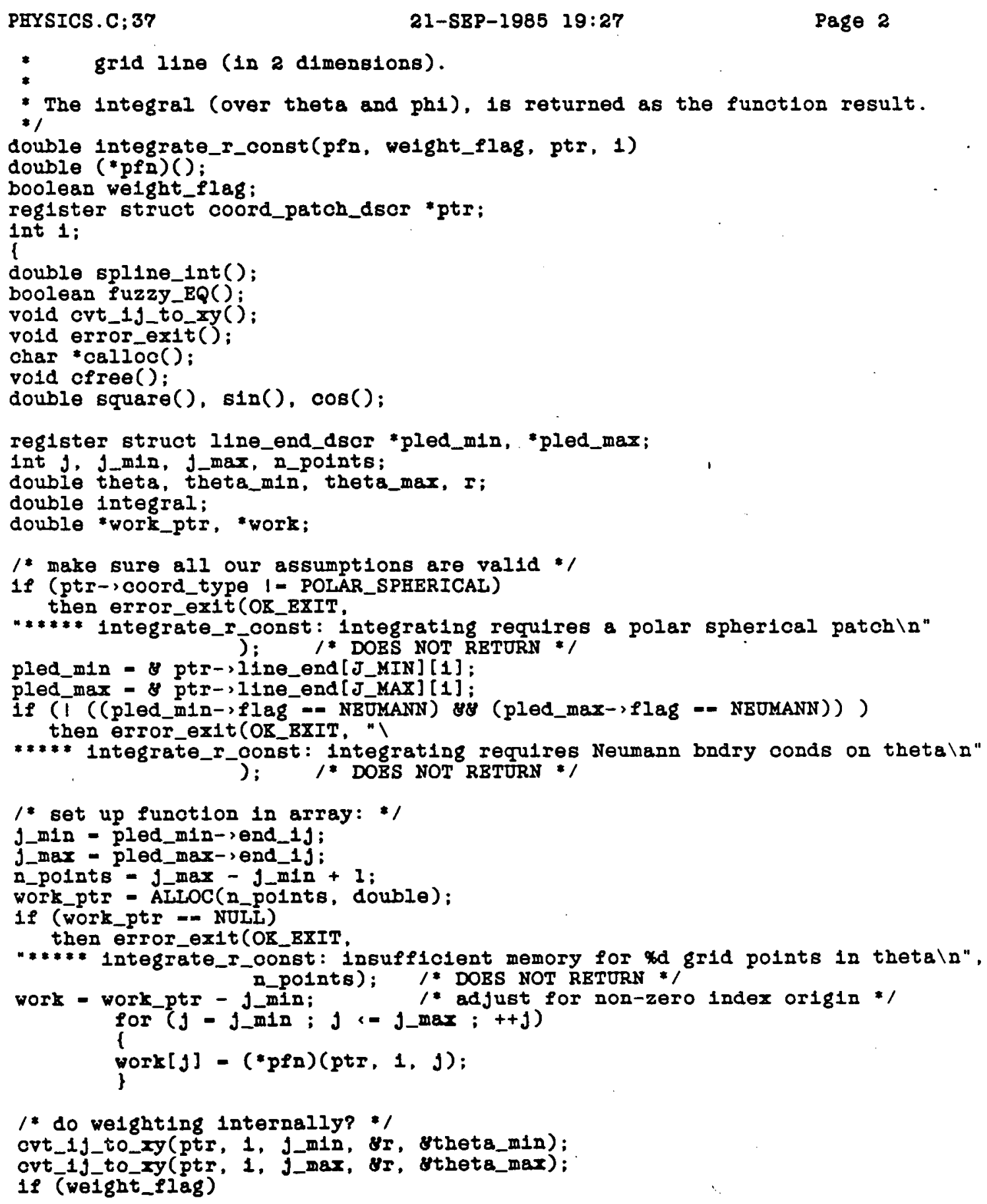




\section{main directory}

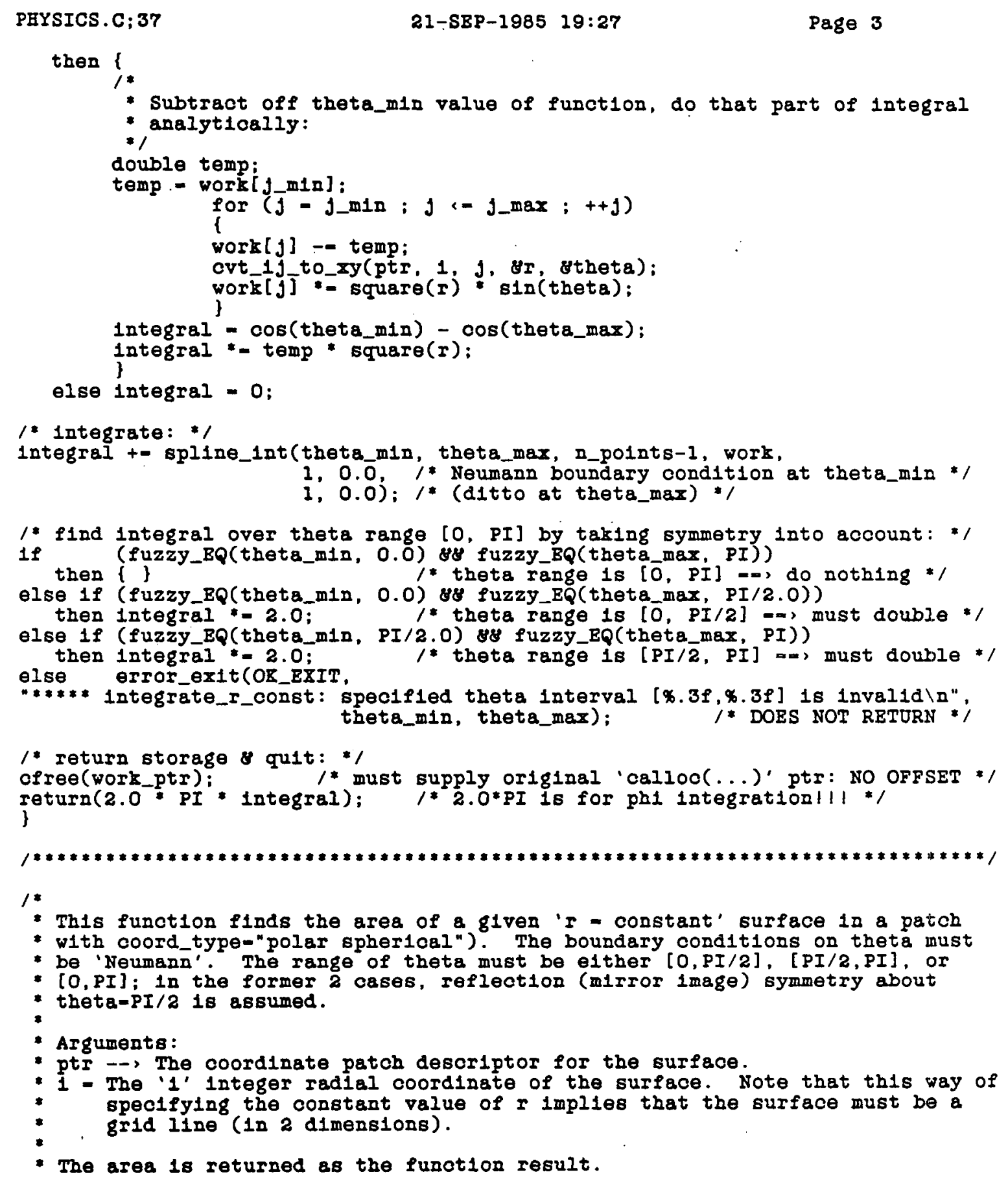




\section{main directory}






\section{main directory}

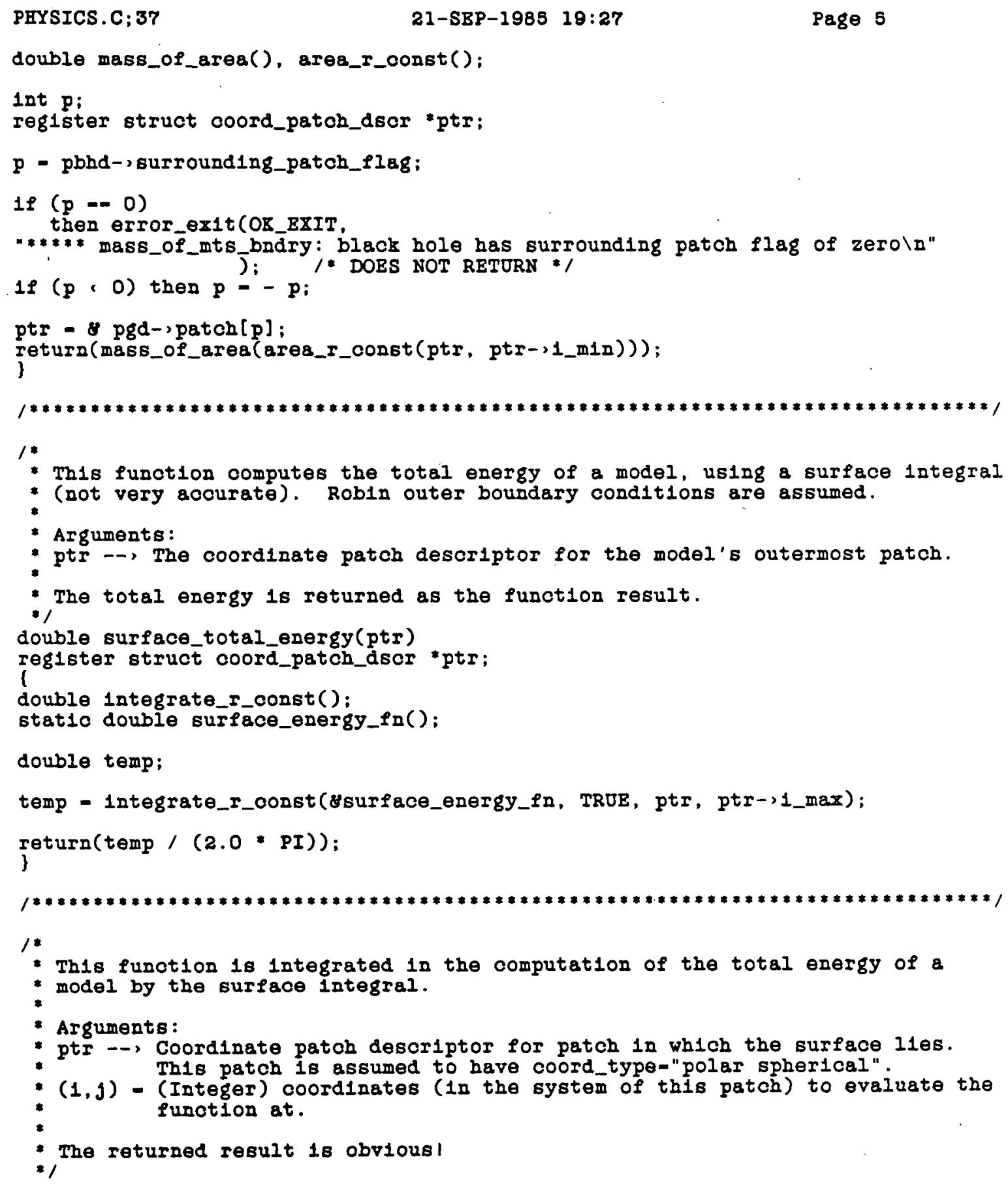




\section{main directory}

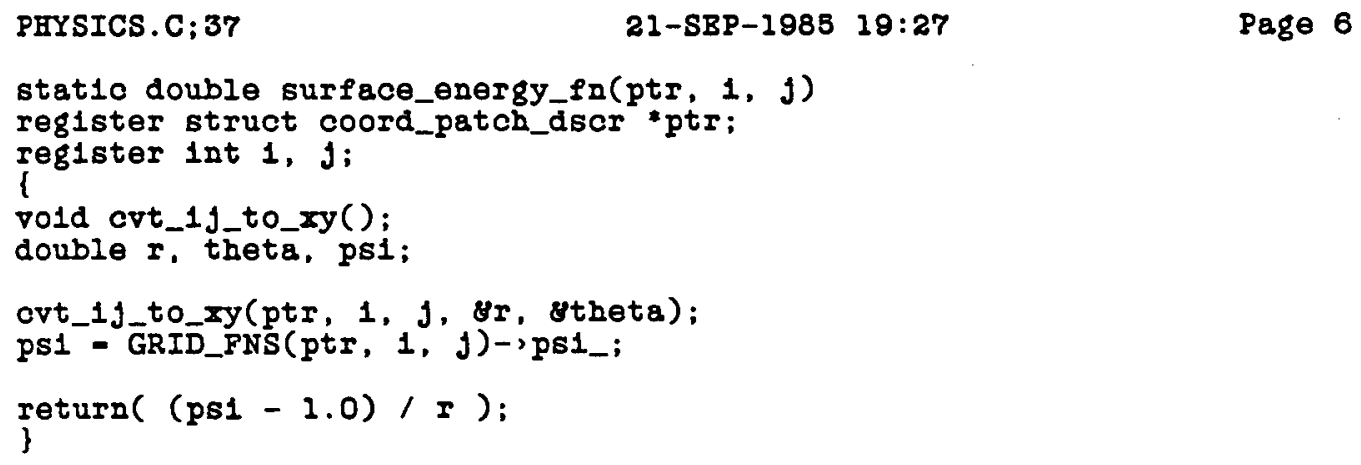




\section{main directory}

PSOR.C; 38

+1nolude "ut1l:stdo.h"

*1nclude "patchut11:patch.h"

*1nclude "patchut1 : blackhole.h"

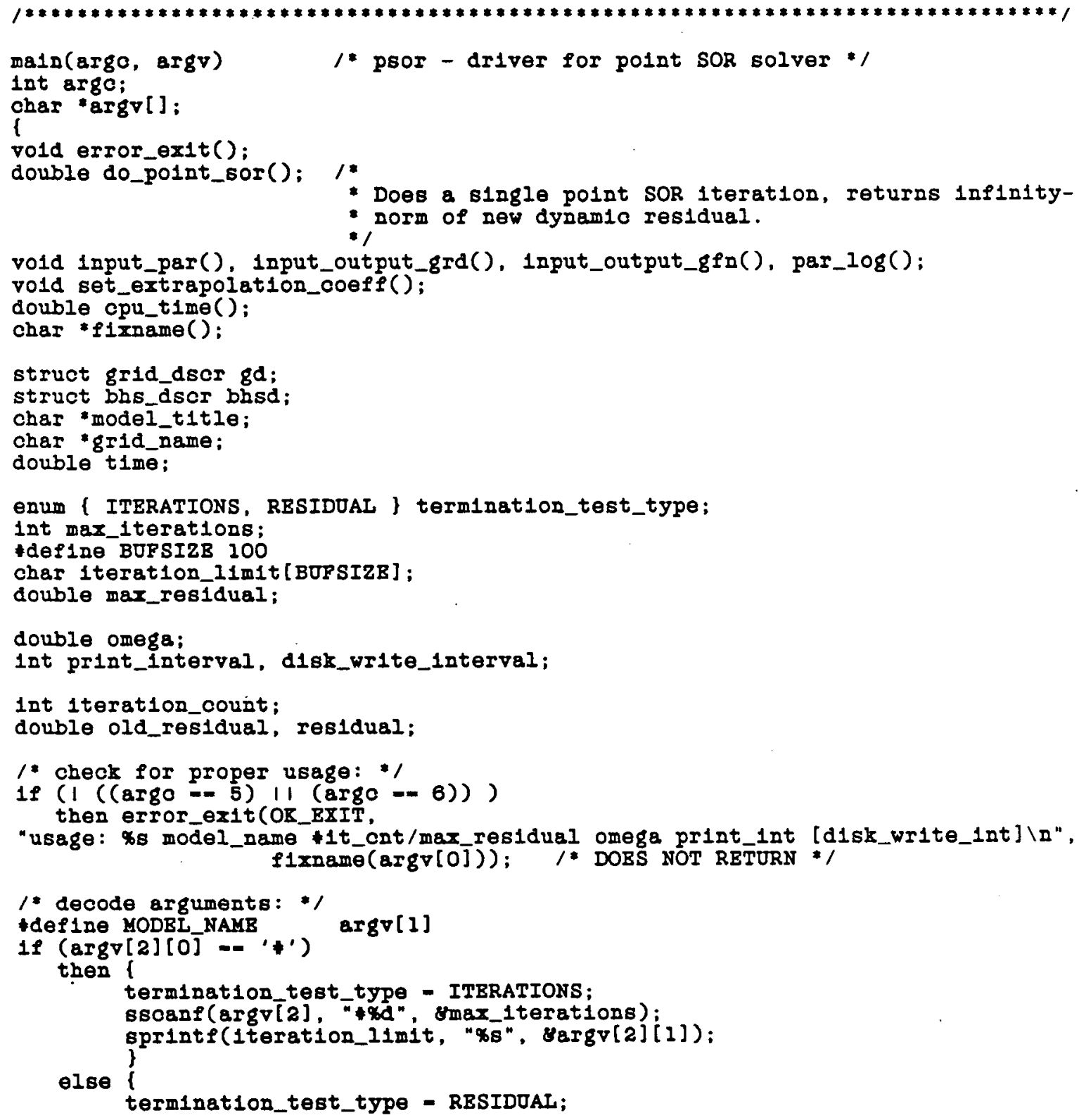




\section{main directory}

PSOR.C; 38

21-SEP-1985 19:28

Page 2

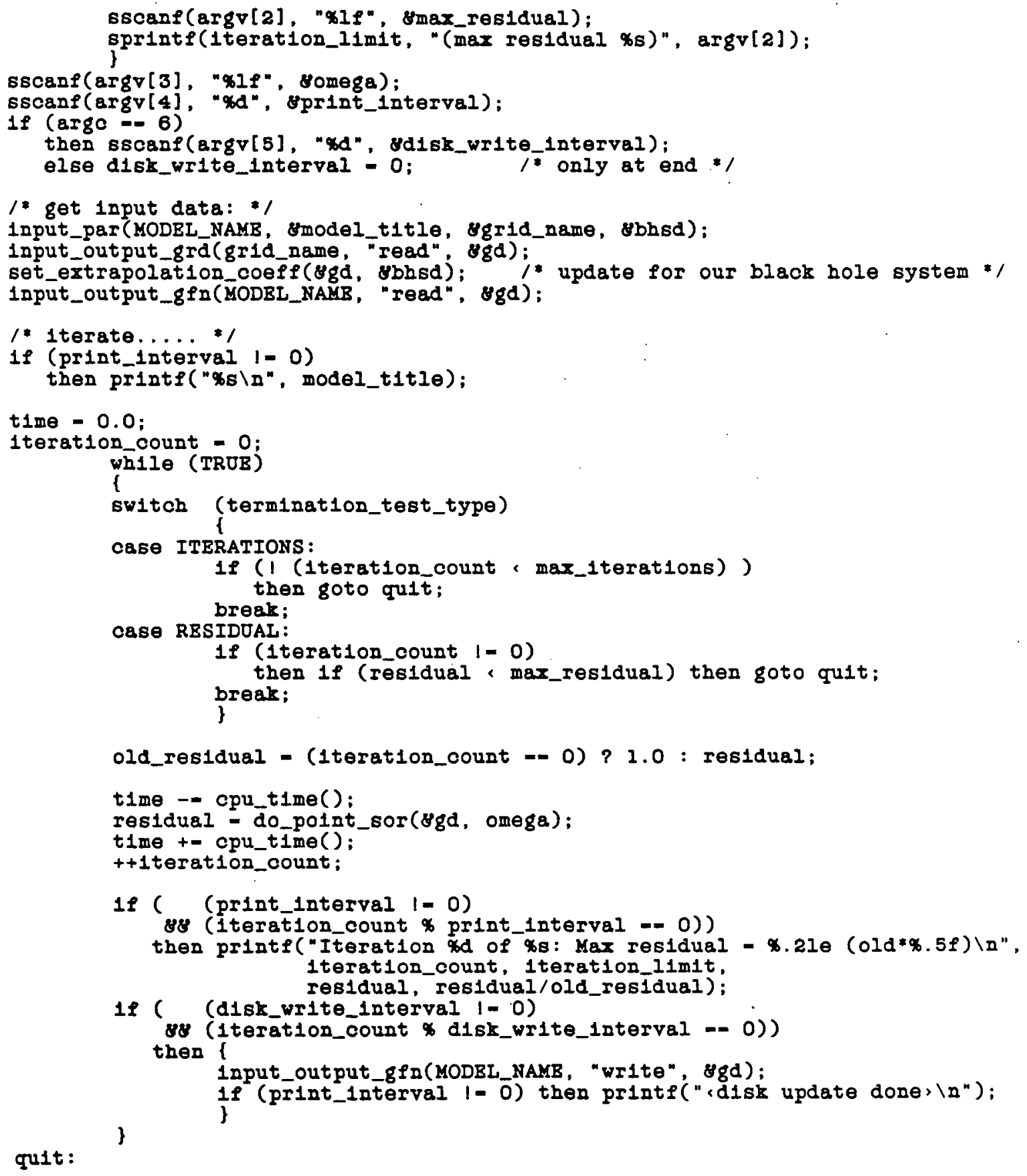




\section{main directory}

PSOR.C; 38

$1 f\left(\left(p r i n t \_1 n t e r v a l 1-0\right) \& \&(1\right.$ teration_count $1=0)$ )

then printf("\%d 1terations/x.2f cpu seconds --) \%.2f cpu msec/1teration $\backslash$ ", iteration_count, time, $1.003 *$ time/1teration_count);

/ IInal disk update */

1nput_output_gfn(MODEL_NAMB, "write", 8gd);

/ $\log$ to ' par' f1le: */

$p^{a r} \log \left(M O D E I \_N A M B\right.$, argo, argv, $\left.t 1 m e\right)$; 


\section{main directory}

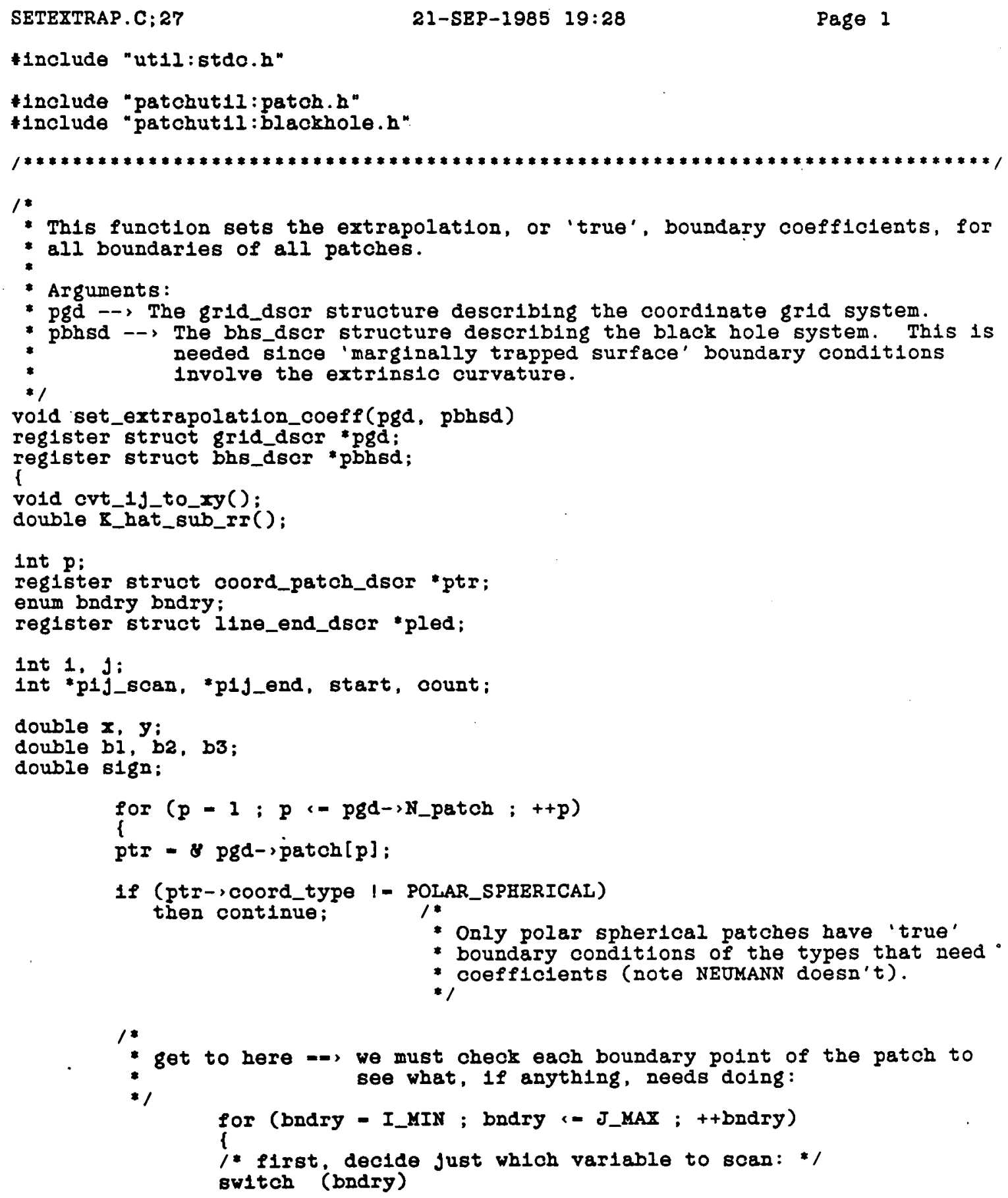




\section{main directory}

SETEXTRAP.C;27

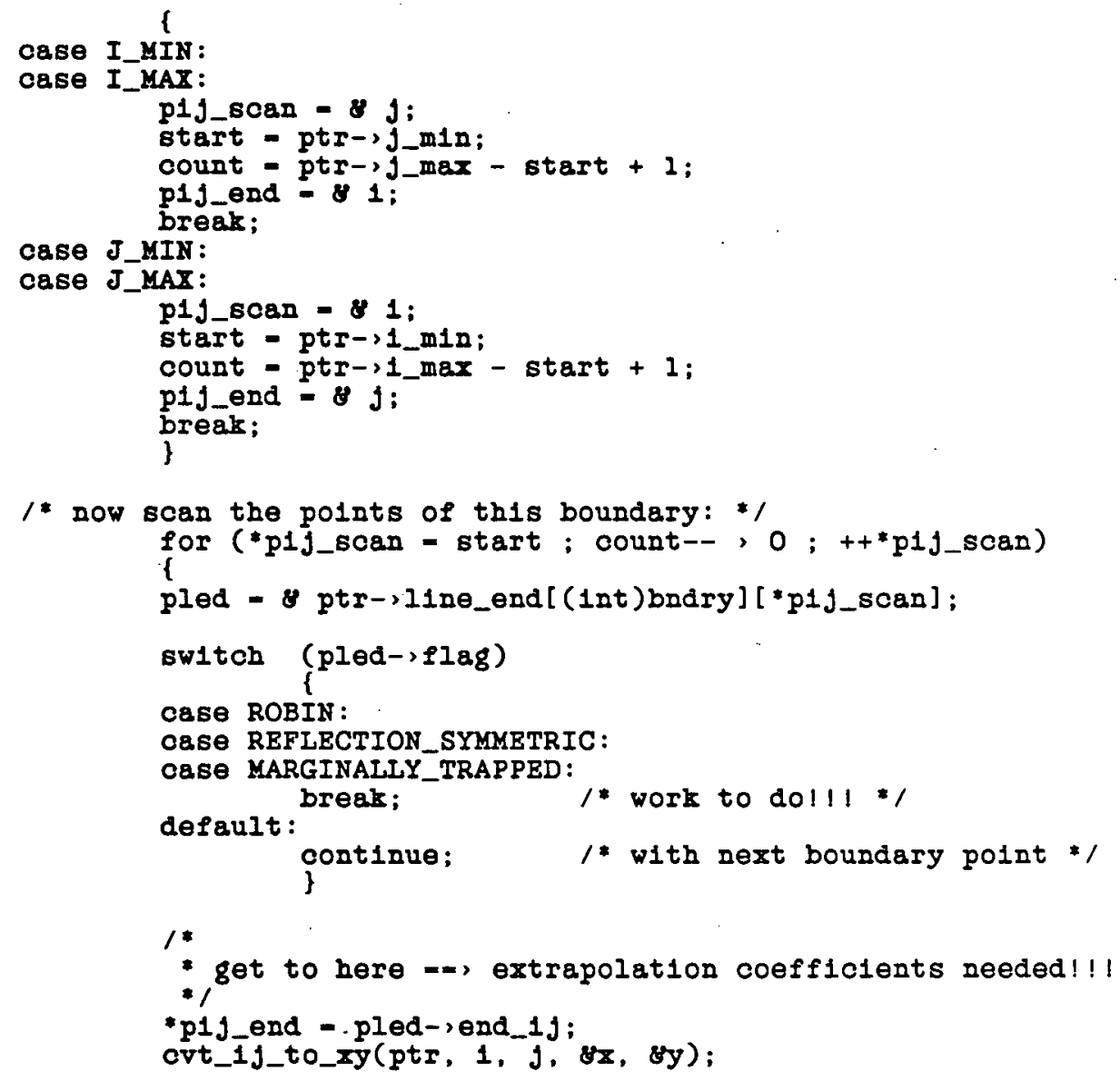




\section{main directory}

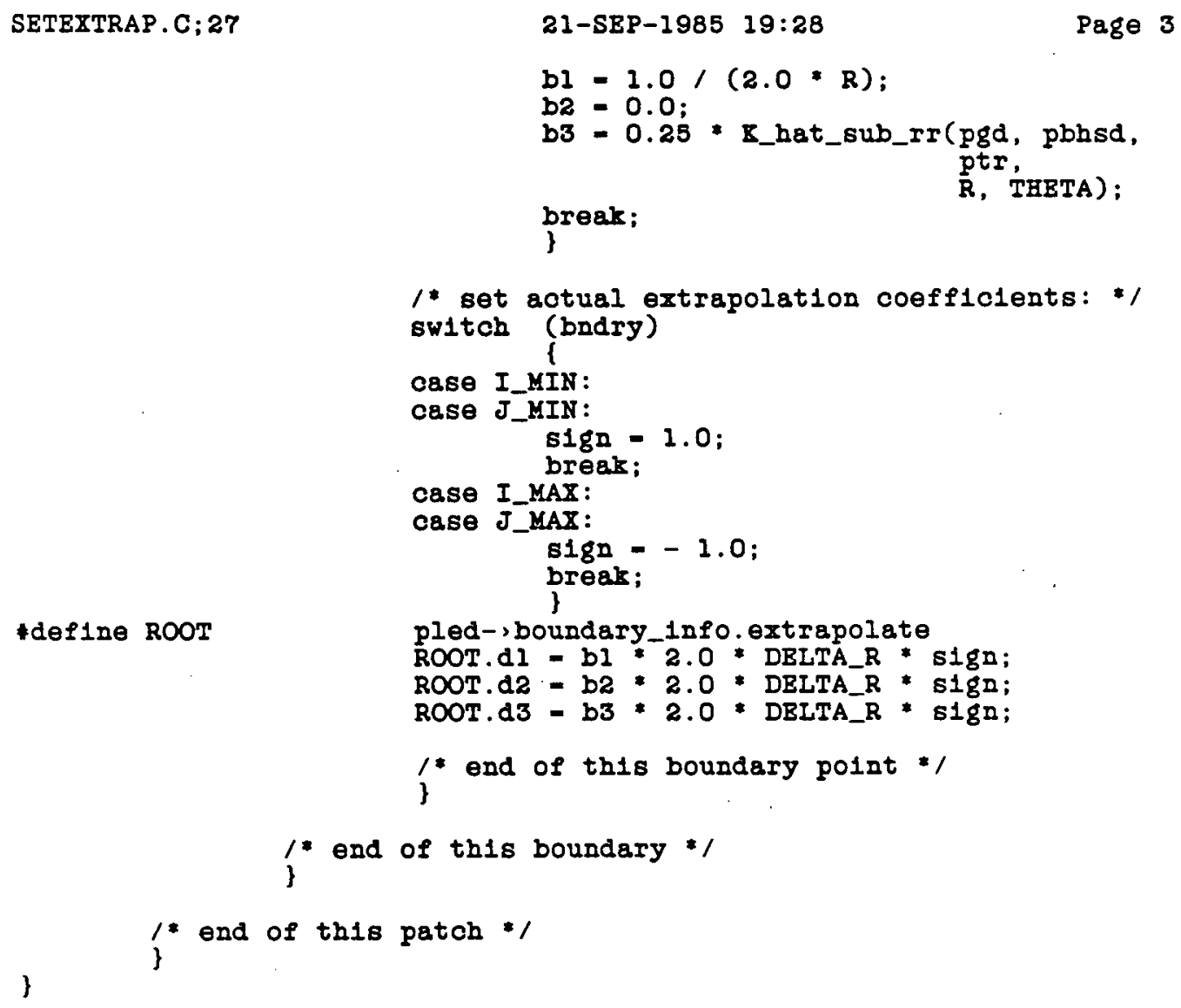




\section{main directory}

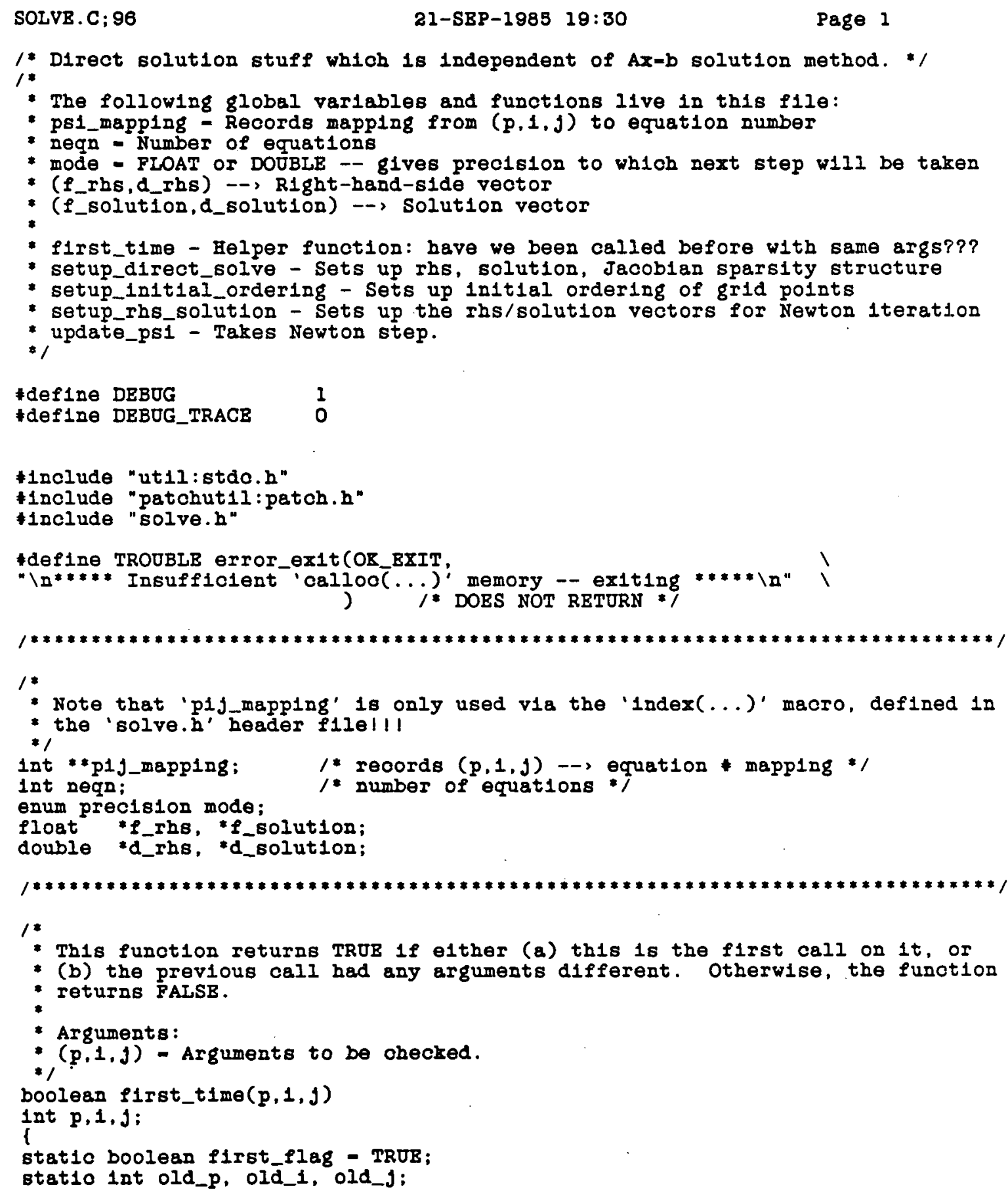




\section{main directory}

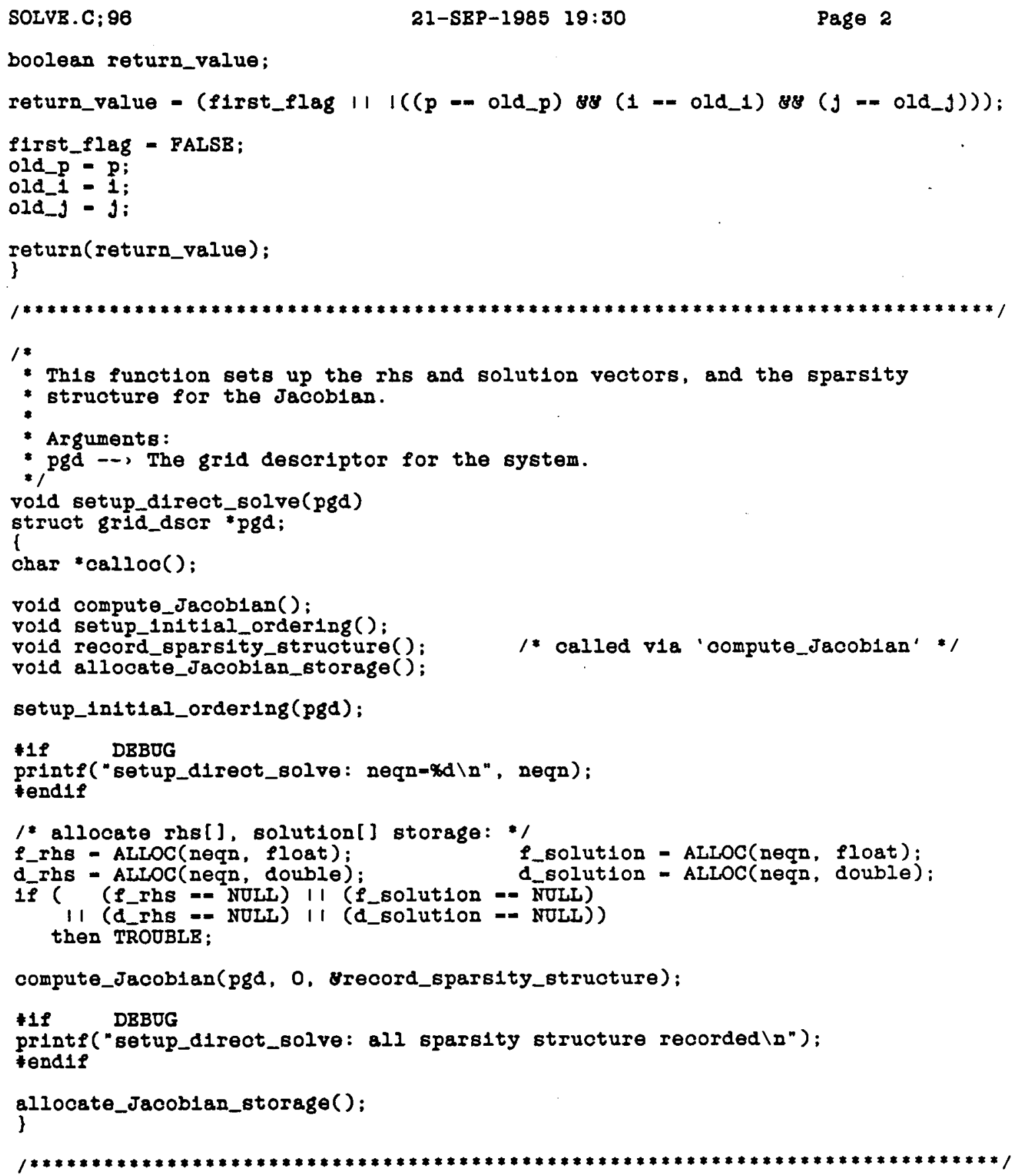




\section{main directory}

SOLVB.C; 98

21-SBP-1983 19:30

Page 3

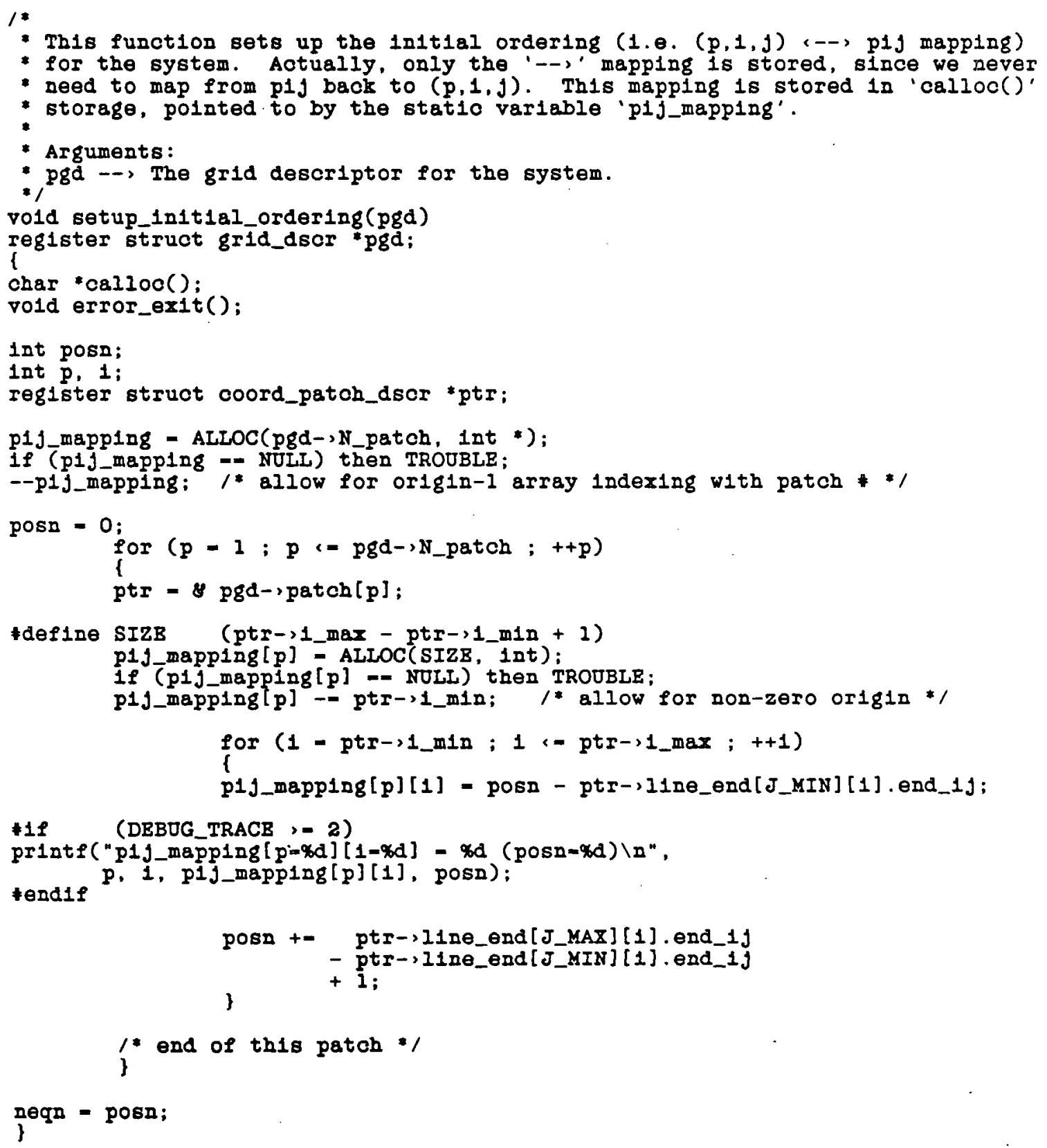




\section{main directory}

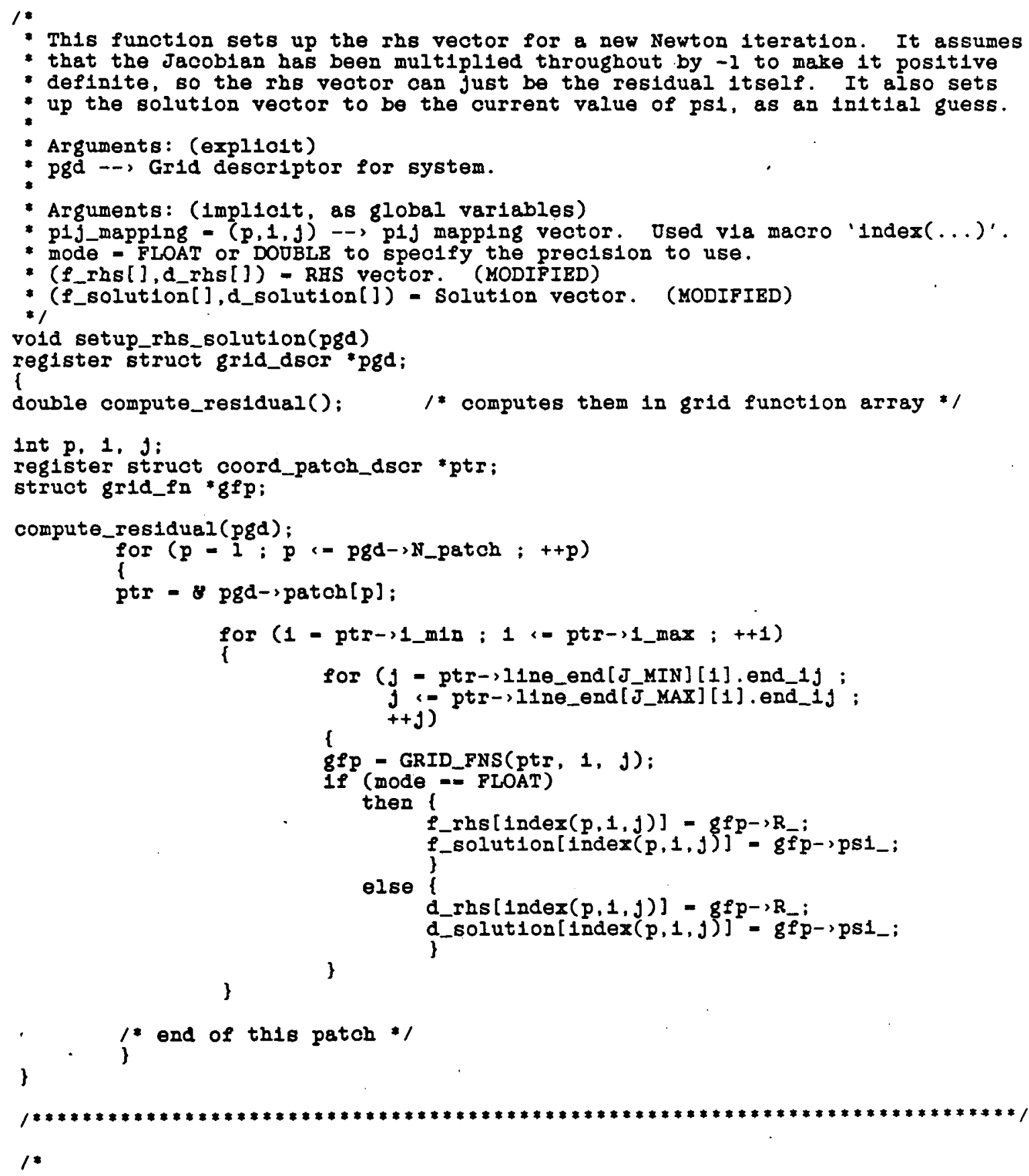




\section{main directory}

SOLVB.C; 96

21-SBP-1985 $19: 30$

Page 5

- Th1s function uses the computed solution to the linear system (with negated

- Jacobian) to update psi in the grid funotion array by the Newton step s1ze.

- Arguments (explic1t):

- pgd - The grid descriptor for the system.

* omega - Fraction of the computed step to take. Standard Newton 1teration is * omega-1.0.

- Arguments (1mplicit, as global variables):

- mode - E1ther FLOAT or DOUBLE as appropriate.

* (f_solution[],d_solution[]) = The vector giving the Newton step amount.

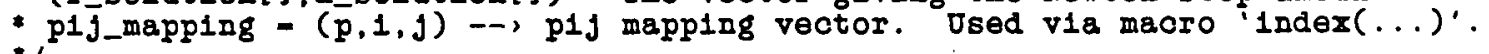
$\%$ vold update_psi (pgd, omega)

register struct grid_dscr pgd;

double omega;

I

register int $p, 1, j$;

register struct coord_petch_dscr "ptr;

double calc_step;

for $(p=1 ; p<$ pgd-,N_patch $;++p)$

ptr $=\& p g d-$ patch $[\mathrm{p}]$;

for $\left(1=p t r->1 \_m 1 n ; 1<-p t r->1 \_m a x ;++1\right)$

I

for ( $J$ - ptr->11ne_end[J_MIN][1] end_1J ; $j,=$ ptr->IIne_end $\left[\bar{J} \_\right.$KAX $][1]$. end_ij;

\{ $++j)$

oalo_step - (mode -- FLOAT)

? P_solution[1ndex $(p, 1, j)]$

: d_solution $[1$ index $(p, 1, j)]$;

GRID_FNS( 8 pg-ipatch $[p], 1, j)$-'ps1_

\}

+ omega *alc_step; 


\section{main directory}

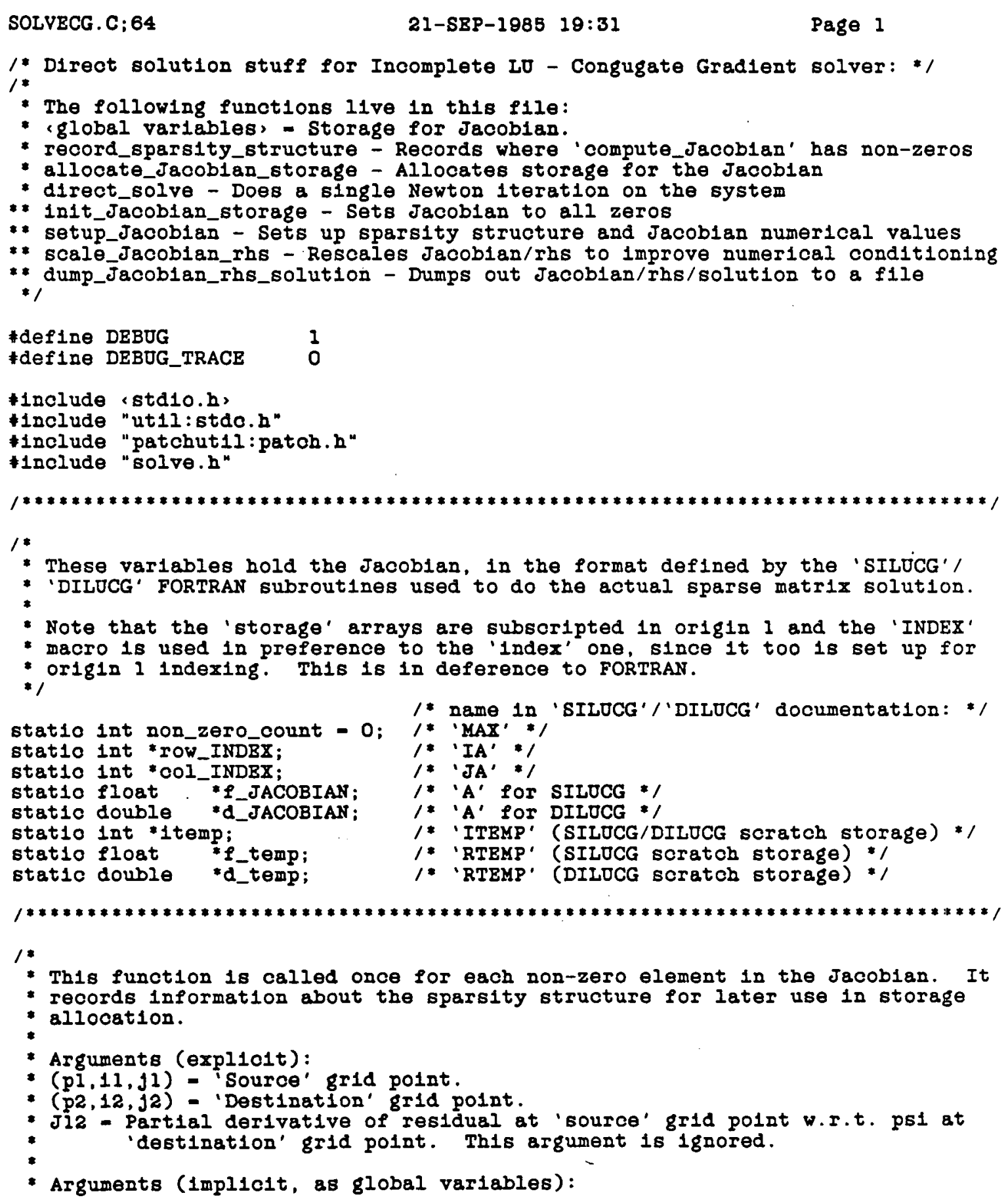


main directory

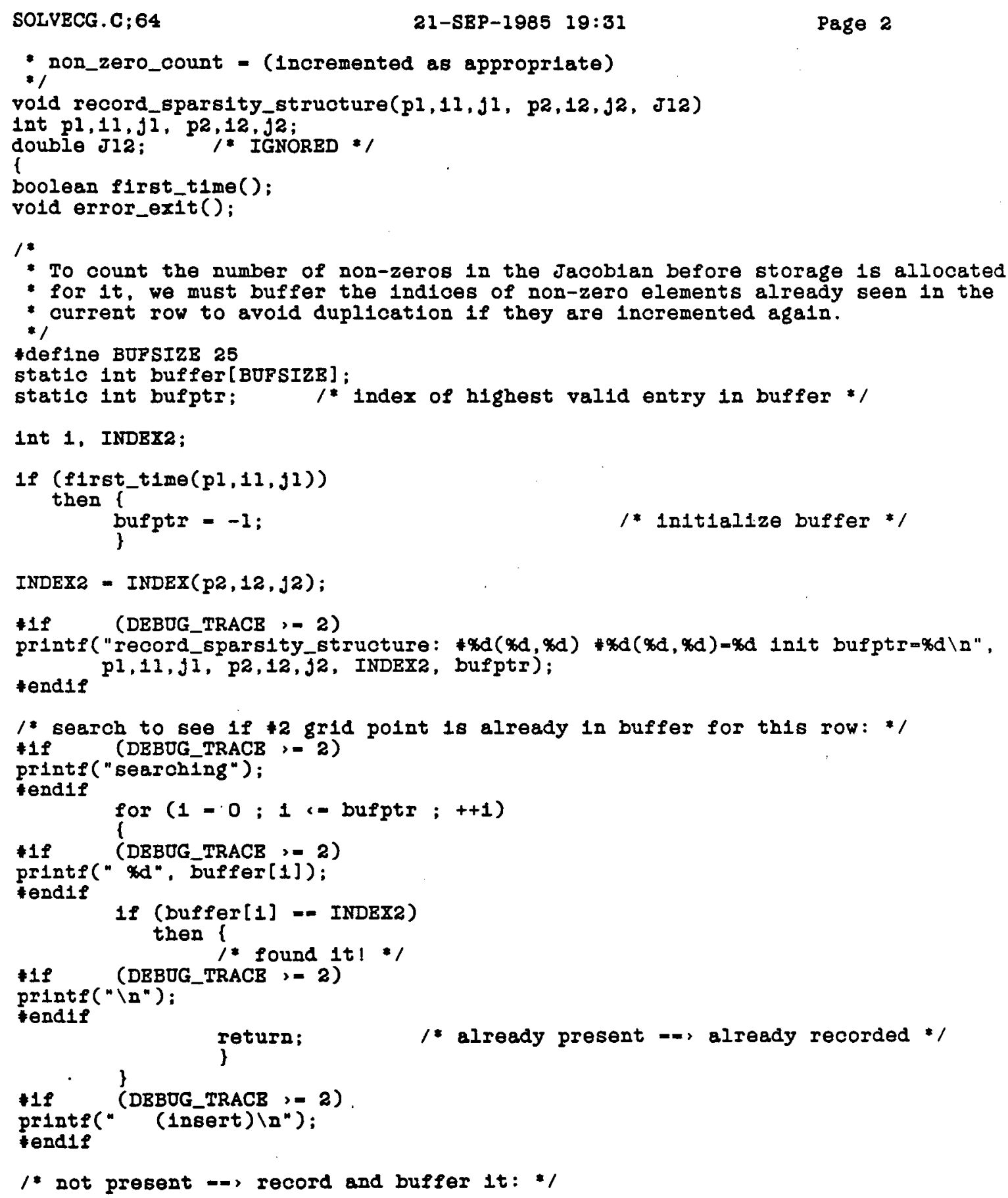


main directory

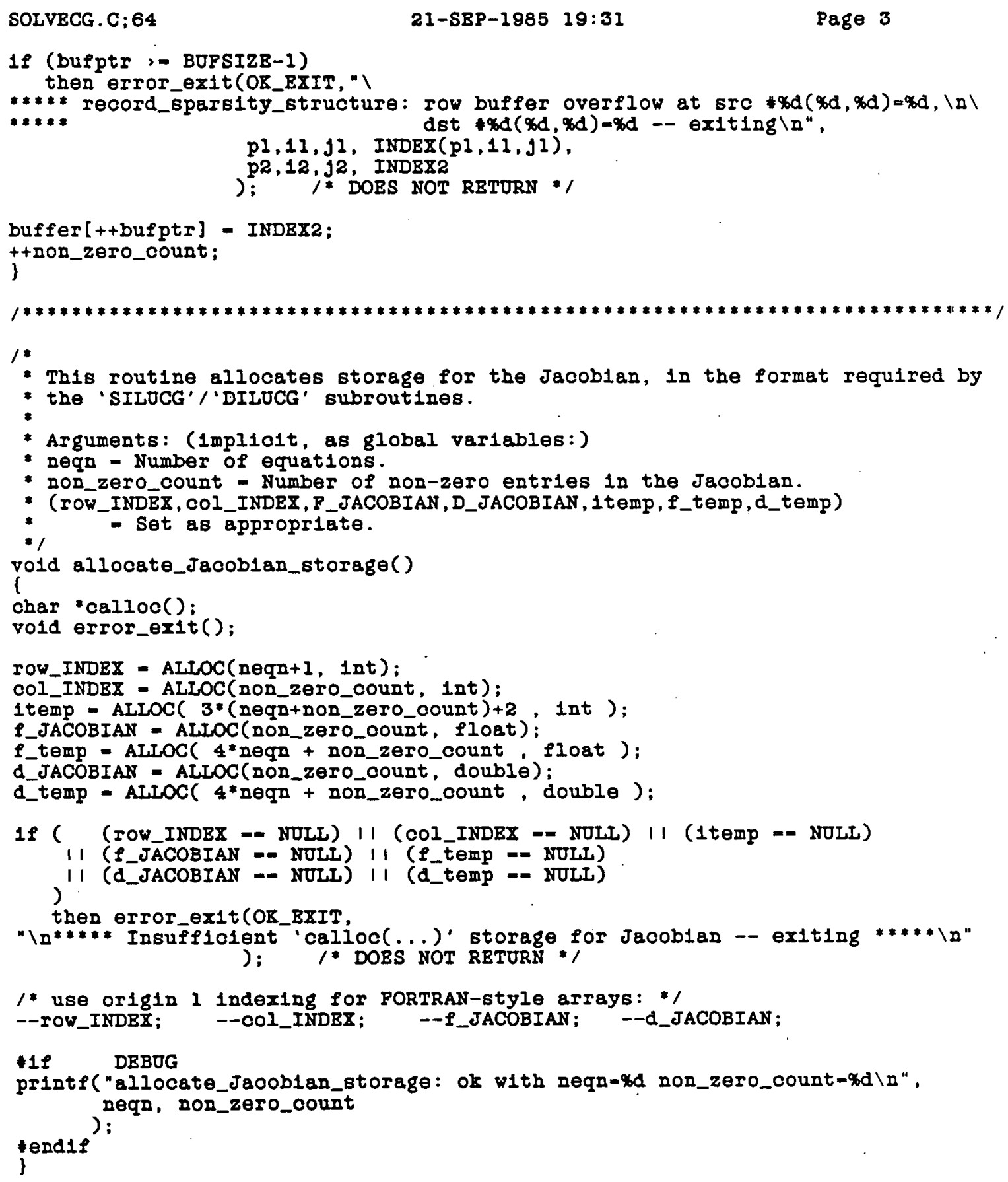




\section{main directory}

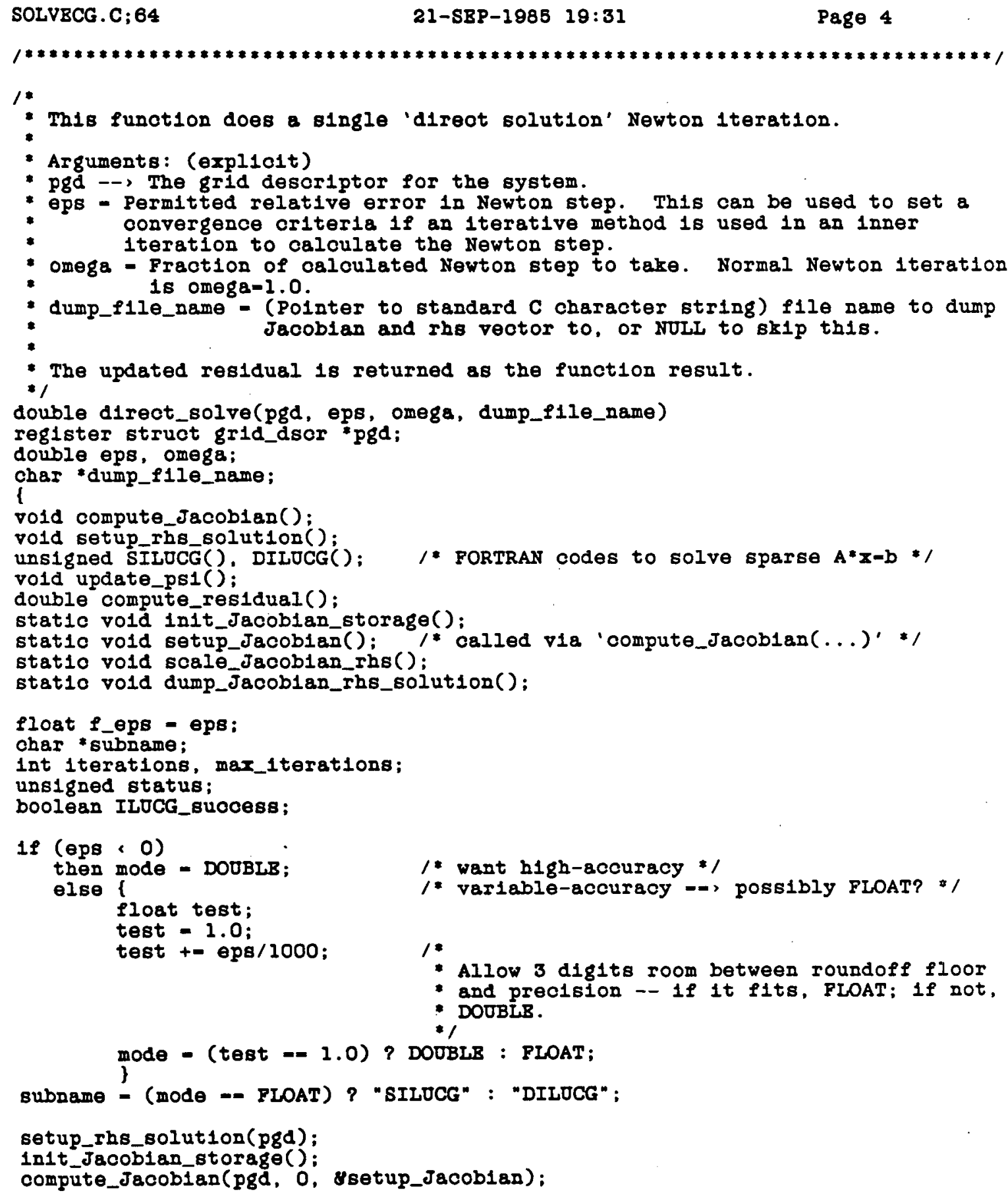




\section{main directory}

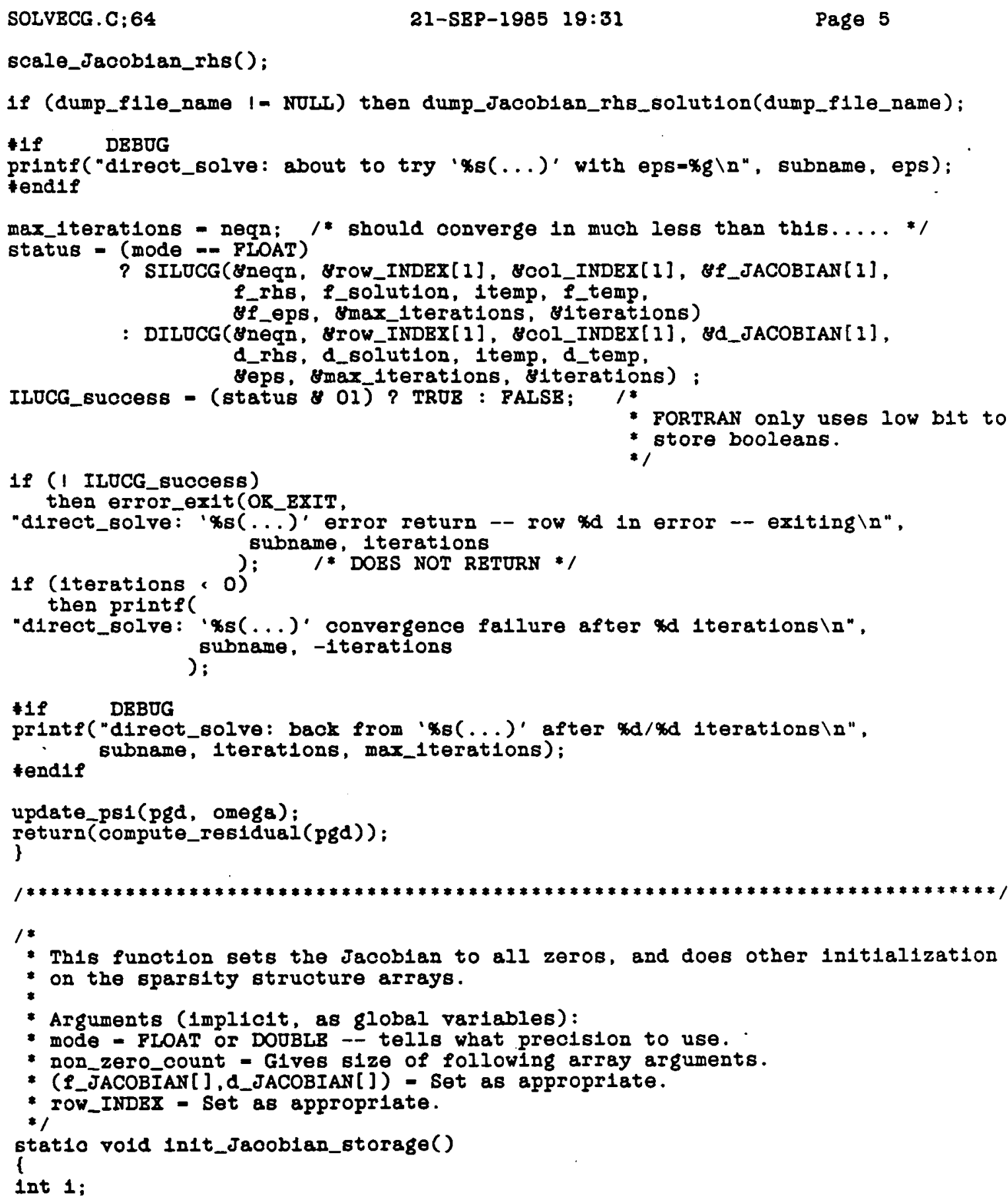




\section{main directory}

SOLVBCG.C;64

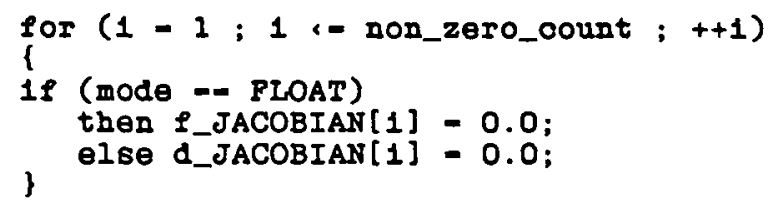

row_INDEX[1] $=1$;

1* where f1rst row w1ll start */

\section{\}}

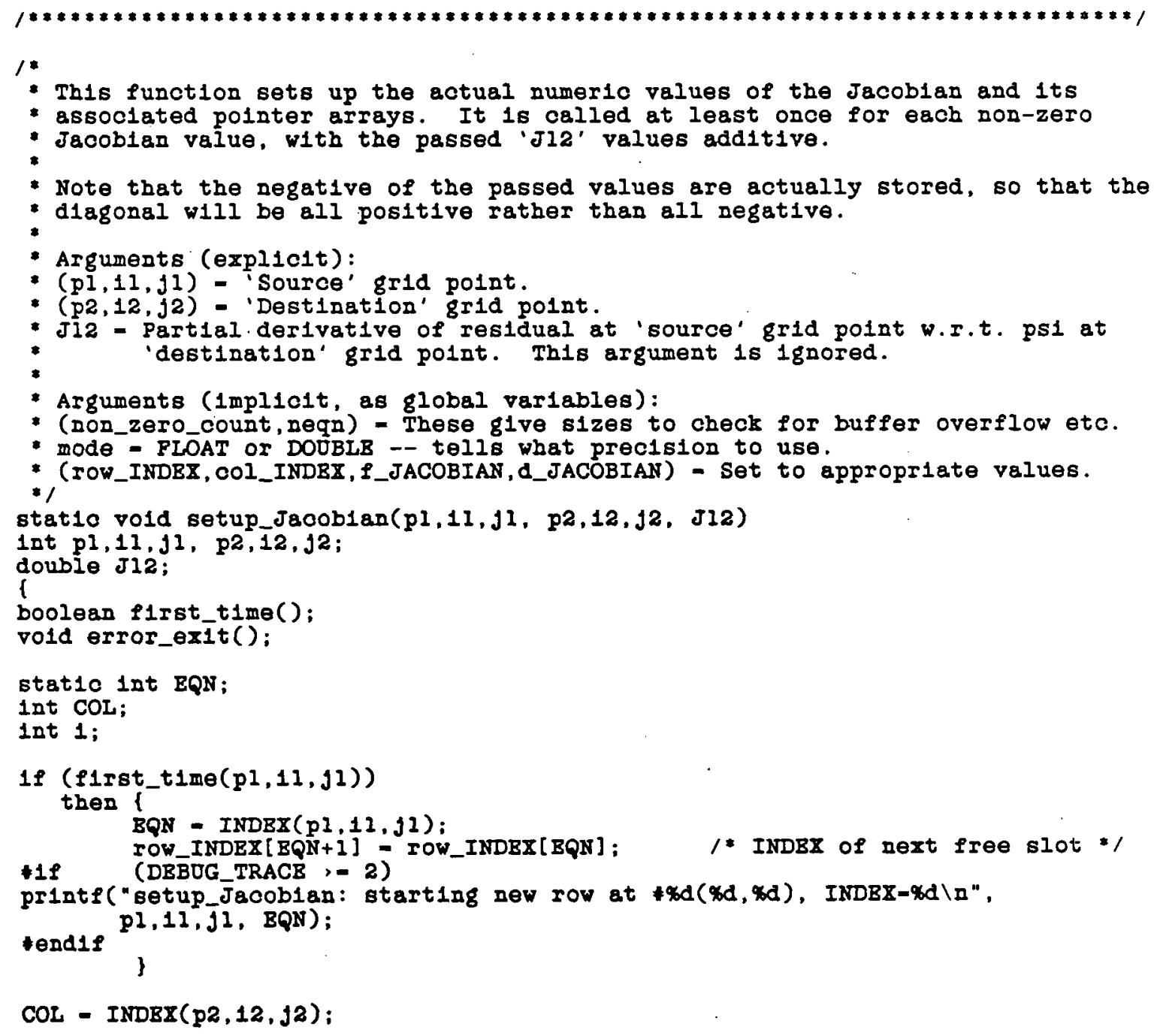




\section{main directory}

SOLVECG.C;64

21-SBP-1985 19:31

Page 7

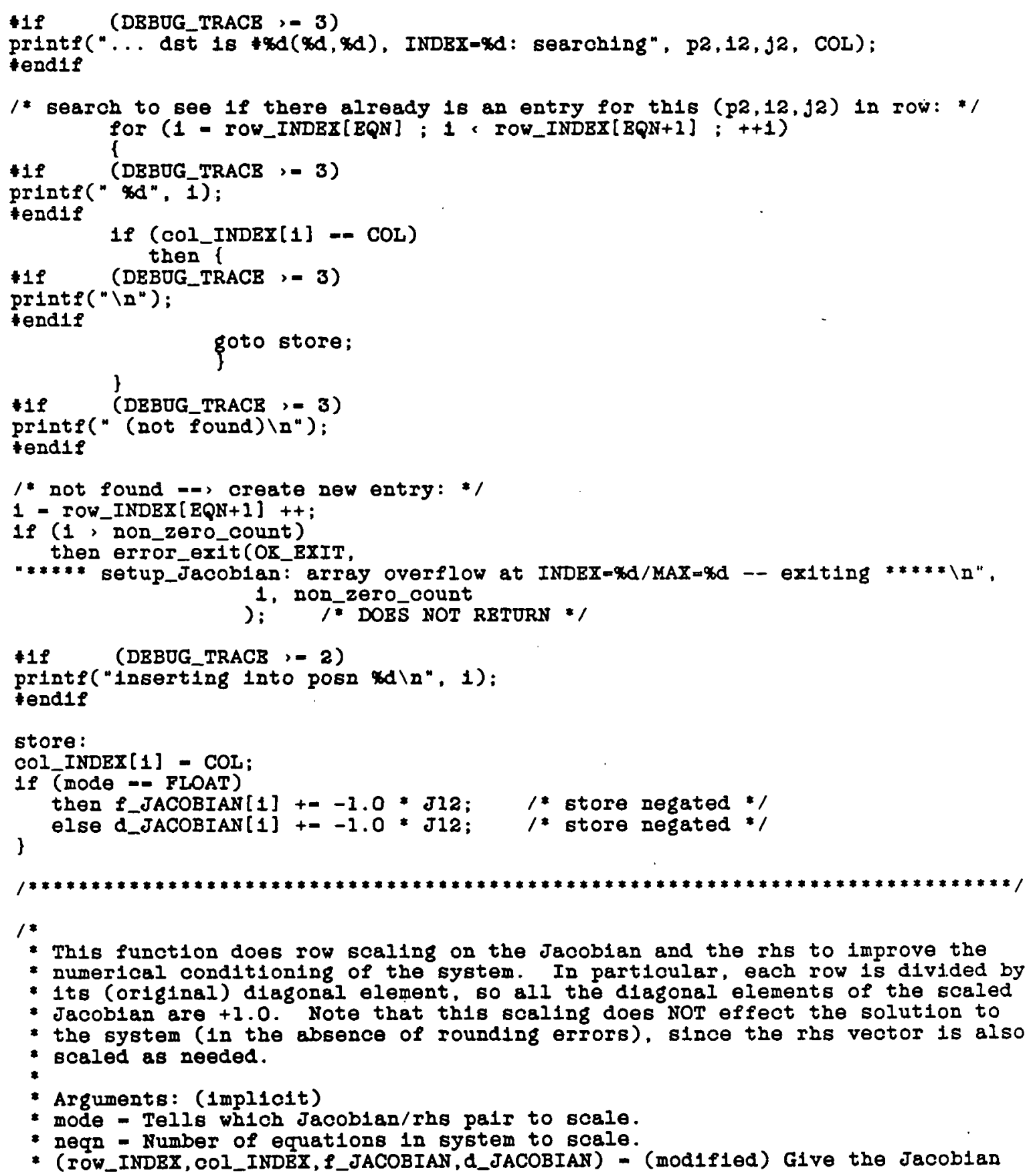




\section{main directory}

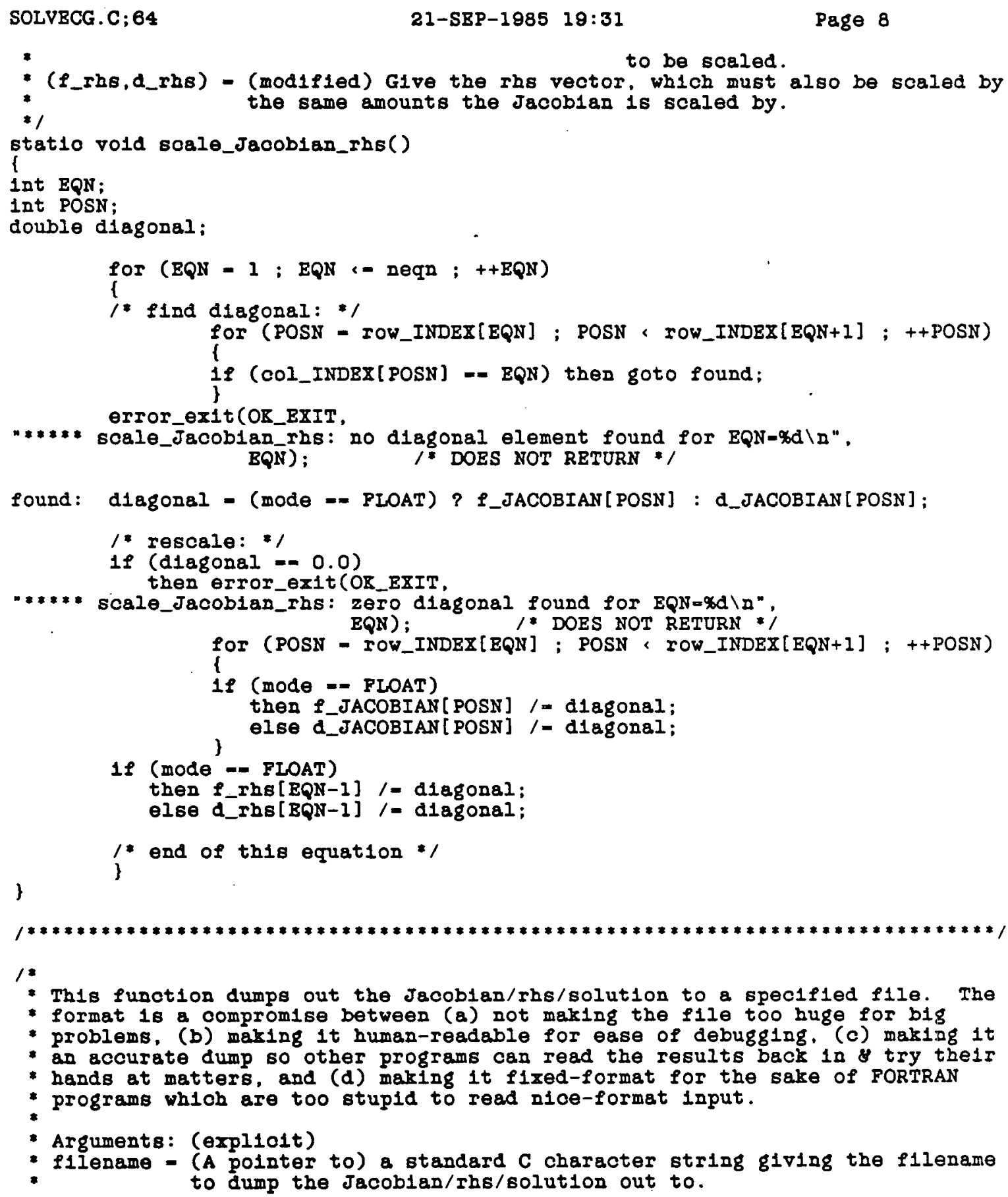




\section{main directory}

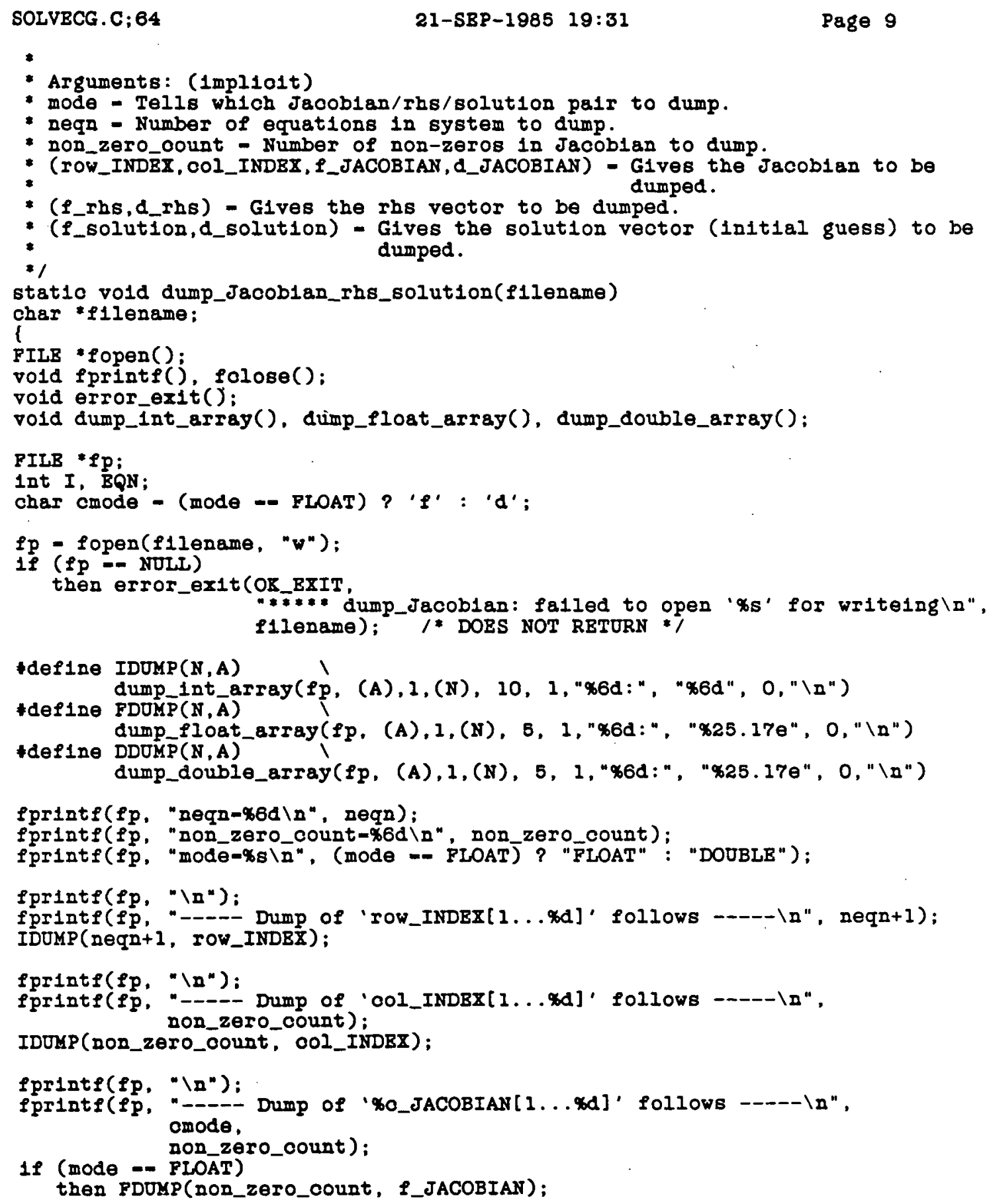




\section{main directory}

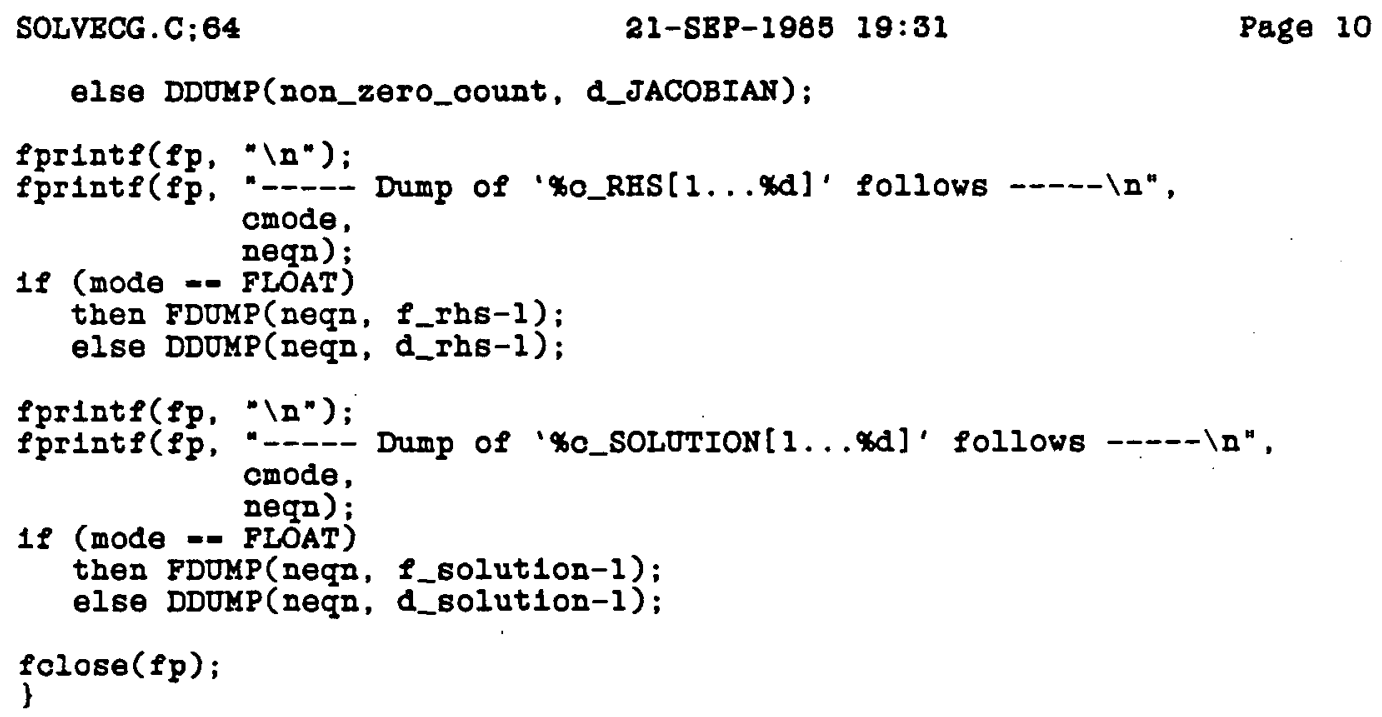




\section{fortran directory}

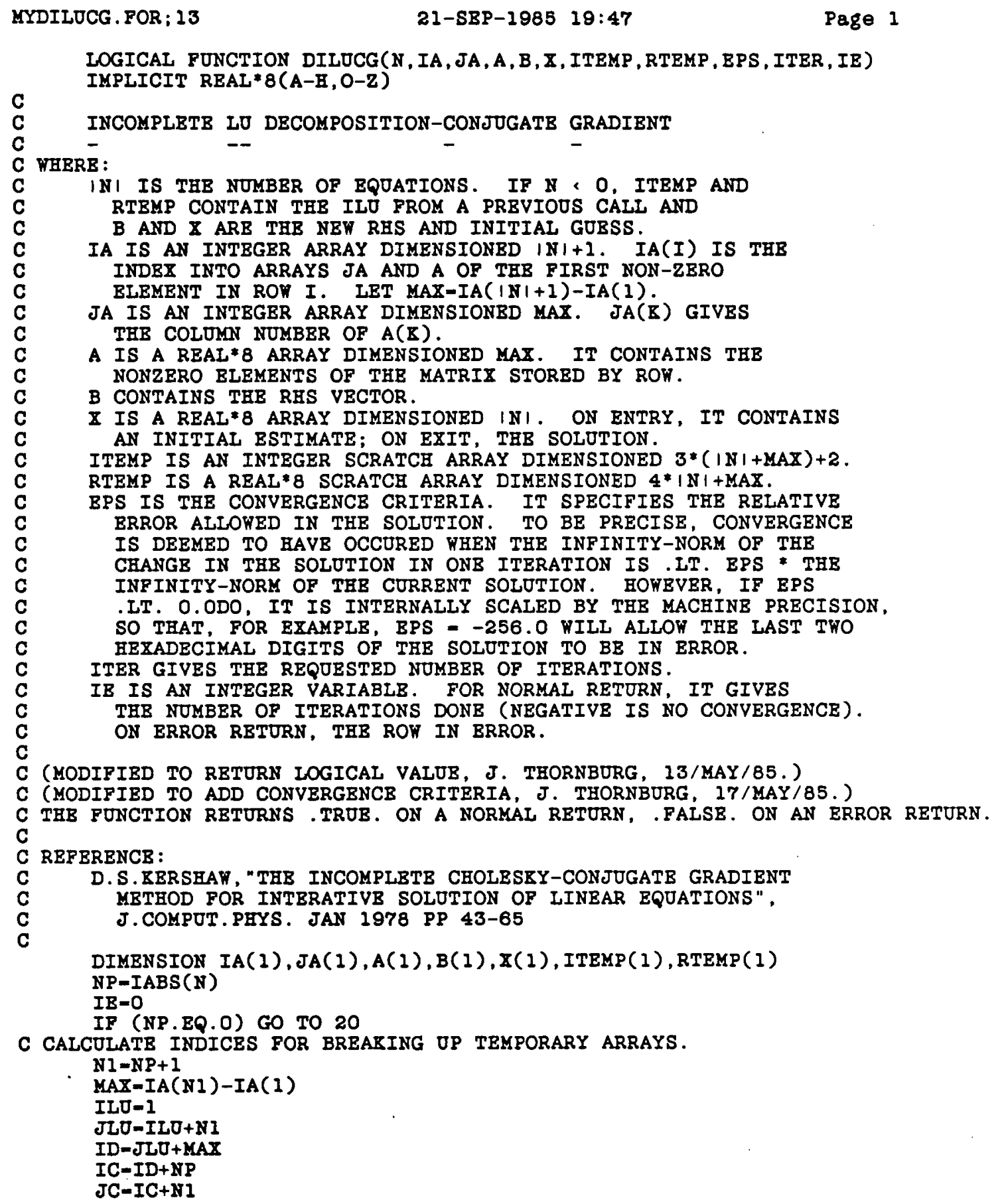




\section{fortran directory}

KYDILOCG. FOR; 23

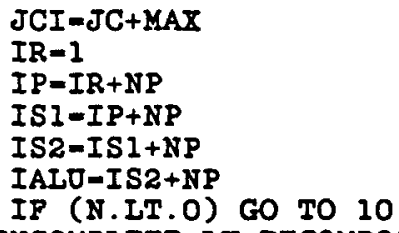




\section{fortran directory}

MYDILUCG.FOR; 13

10 CONTINOB

DO $30 \quad I-1, N$

RS-IA(I)

$K B=I A(I+1)-1$

NODIAG=. TROB.

DO $20 \mathrm{~K}-\mathrm{RS}, \mathrm{KB}$

J-JA(K)

IP (J.LT.I.OR.J.GT.N) GO TO 210

IC (J) $-I C(J)+I$

IP (J.BQ.I) NODIAG-.FALSB.

20 CONTINOB

IF (NODIAG) GO TO 210

30 CONTINOB

C MABE IC INTO INDICES

KOID-IC( 1 )

IC $(1)=1$

DO $40 \mathrm{I}=1, N$.

RNEW-IC $(I+1)$

IP (BOID.BQ.O) GO TO 210

$I C(I+1)=I C(I)+$ KOID

40 CONTINOB

$$
\text { BOLD=KNEW }
$$

C SET JC AND JCI FOR COLUMN STRUCTURE

DO $60 \mathrm{I}=1, \mathrm{~N}$

BS-IA(I)

$\mathrm{EB}=I \mathrm{~A}(I+1)-1$

DO $50 \mathrm{~K}=\mathrm{RS}, \mathrm{KB}$

J-JA( $\mathrm{B})$

$\mathrm{L}=\mathrm{IC}(\mathrm{J})$

$\operatorname{IC}(J)-I+1$

$J C(L)-I$

50 JCI (L) $=5$

60 CONTINUB

C FIX UP IC

KOID-IC(1)

IC(1) -1

DO $70 I-1, N$

KNBW-IC $(I+1)$

IC $(I+1)$ - - DOL D

BOID-RNBW

70 CONTINOB

C FIND SORTBD ROH STROCTURB PROM SORTED COLUMN STROCTURE $\mathrm{NP}=\mathrm{N}+1$

DO 80 I-1, NP

ILU(I)-IA(I)

C MOVB BLEMBNTS, SBT JLO AND ID

DO $100 \mathrm{~J}=\mathrm{I}, \mathrm{N}$

BS-IC ( $(J)$

$\mathrm{BB}-I C(J+1)-1$

DO $90 \mathrm{~K}=\mathrm{KS}, \mathrm{KB}$

$I=J C(R)$

$I=I L O(I)$

$\operatorname{ILO}(I)-I+1$ 


\section{fortran directory}

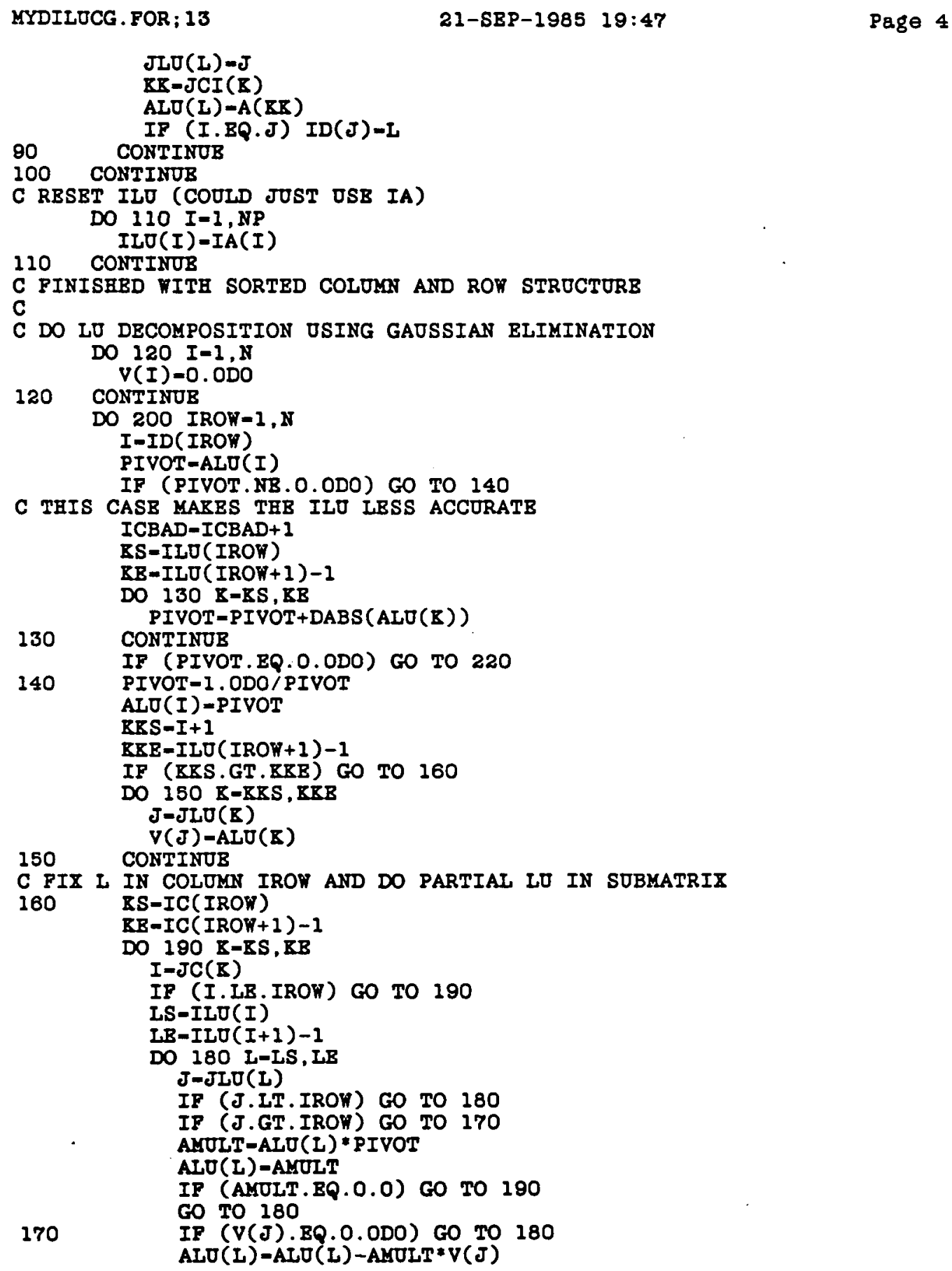




\section{fortran directory}

MYDILUCG.POR; 13

21-SBP-1985 $19: 47$

Page 5

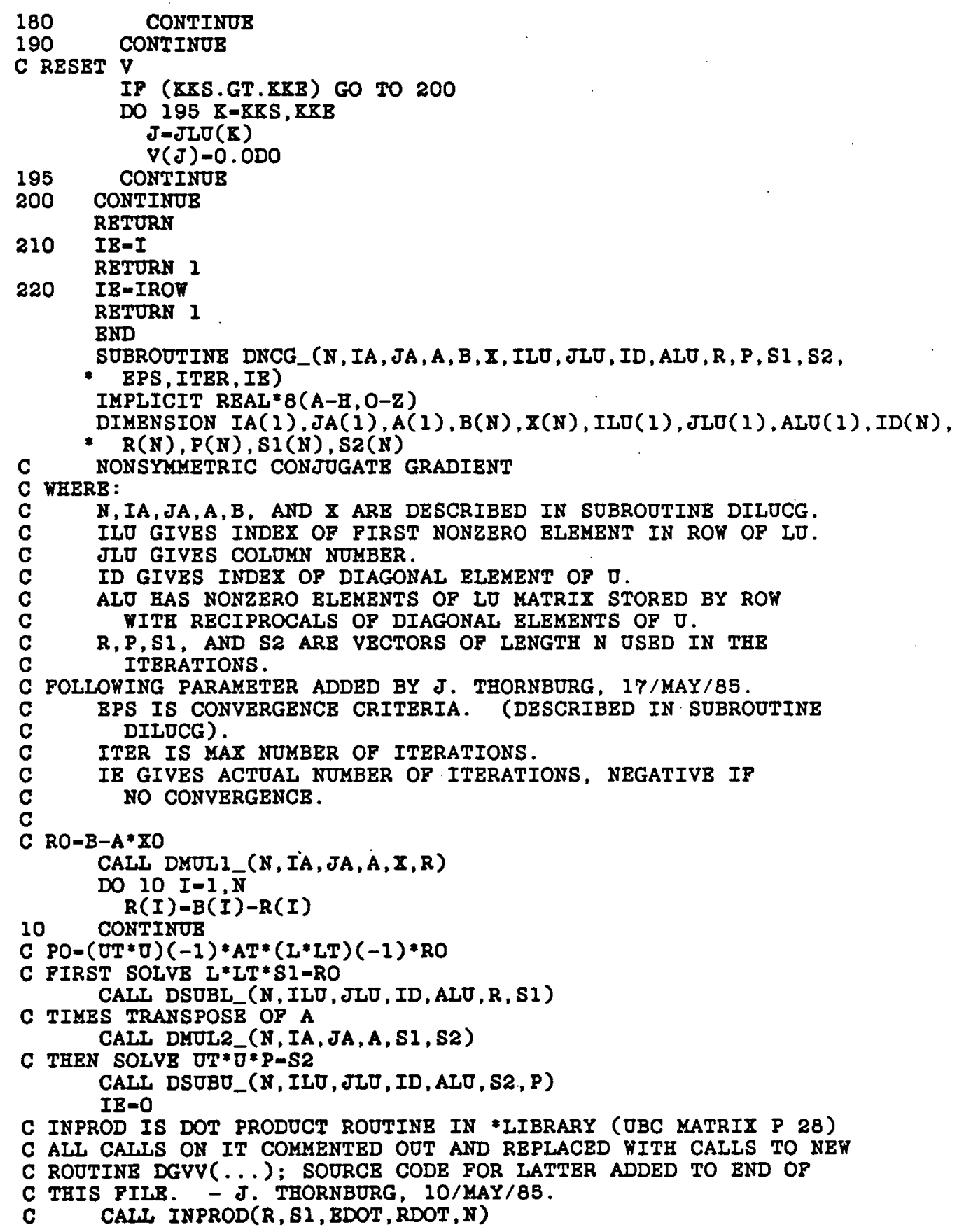




\section{fortran directory}

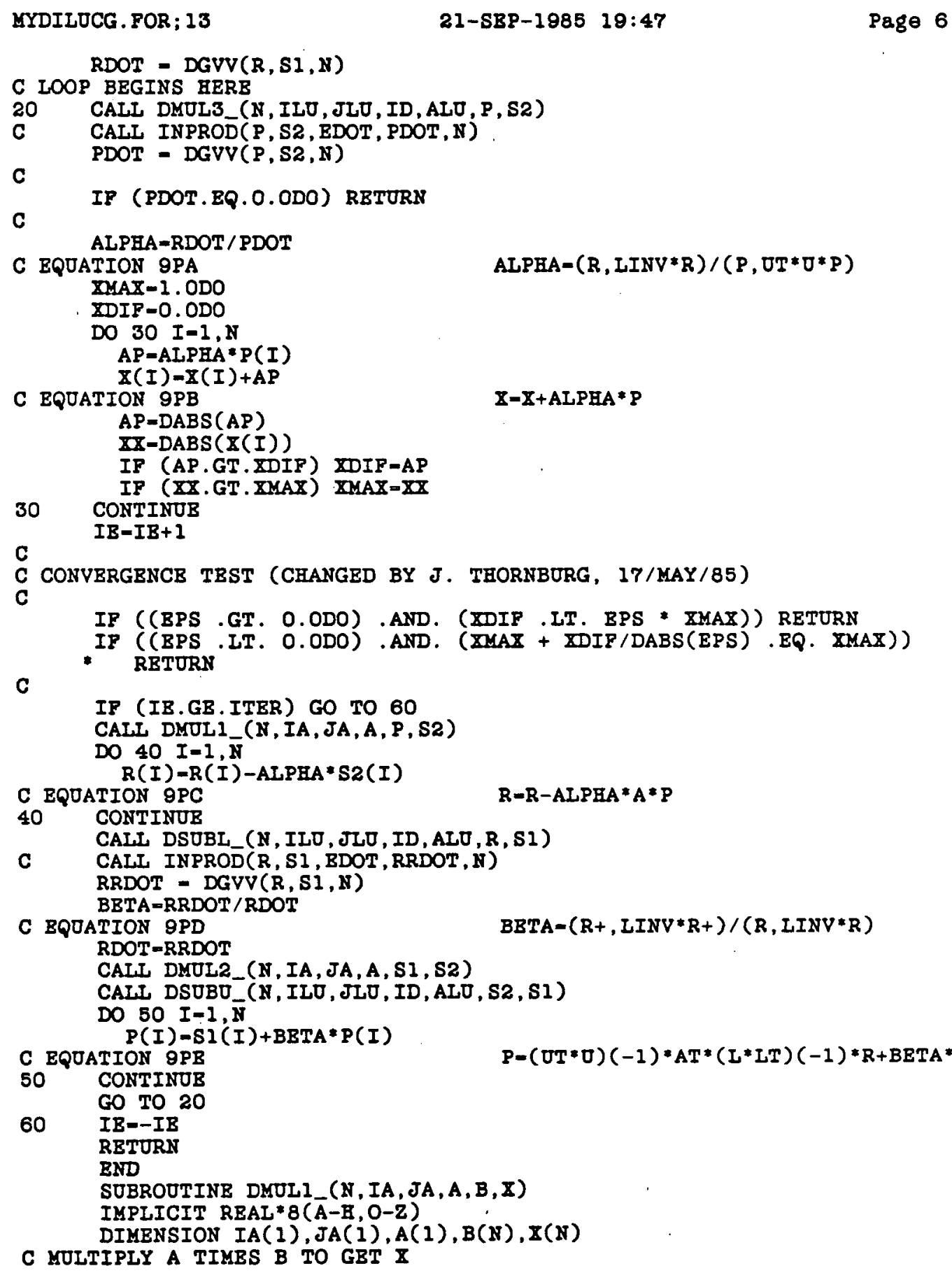




\section{fortran directory}

KYDILUCG.FOR; 13

21-SBP-1985 19:47

Page 7

C WHERB :

C N IS THB ORDER OF THE KATRIX
C IA GIVES INDEX OF FIRST NONZERO BLEMENT IN ROW

C JA GIVBS COLUMN NUMBRR

C A CONTAINS THE NONZERO ELEMENTS OF THE NONSYMMETRIC

C MATRIX STORED BY ROW

C B IS THB VBCTOR

C $\mathrm{X}$ IS THB PRODUCT ( COST BB DIFPERENT FROM B)

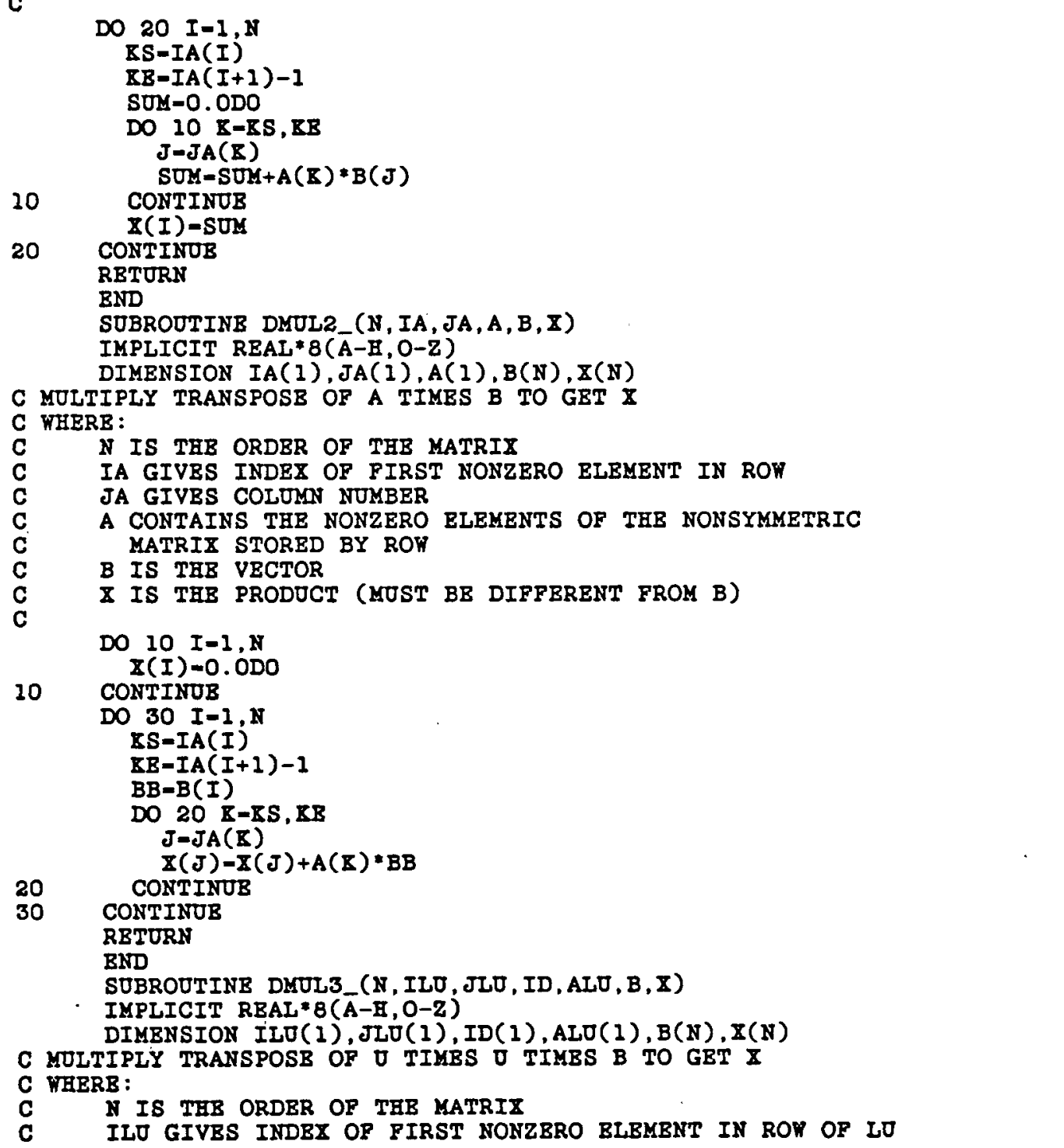




\section{fortran directory}

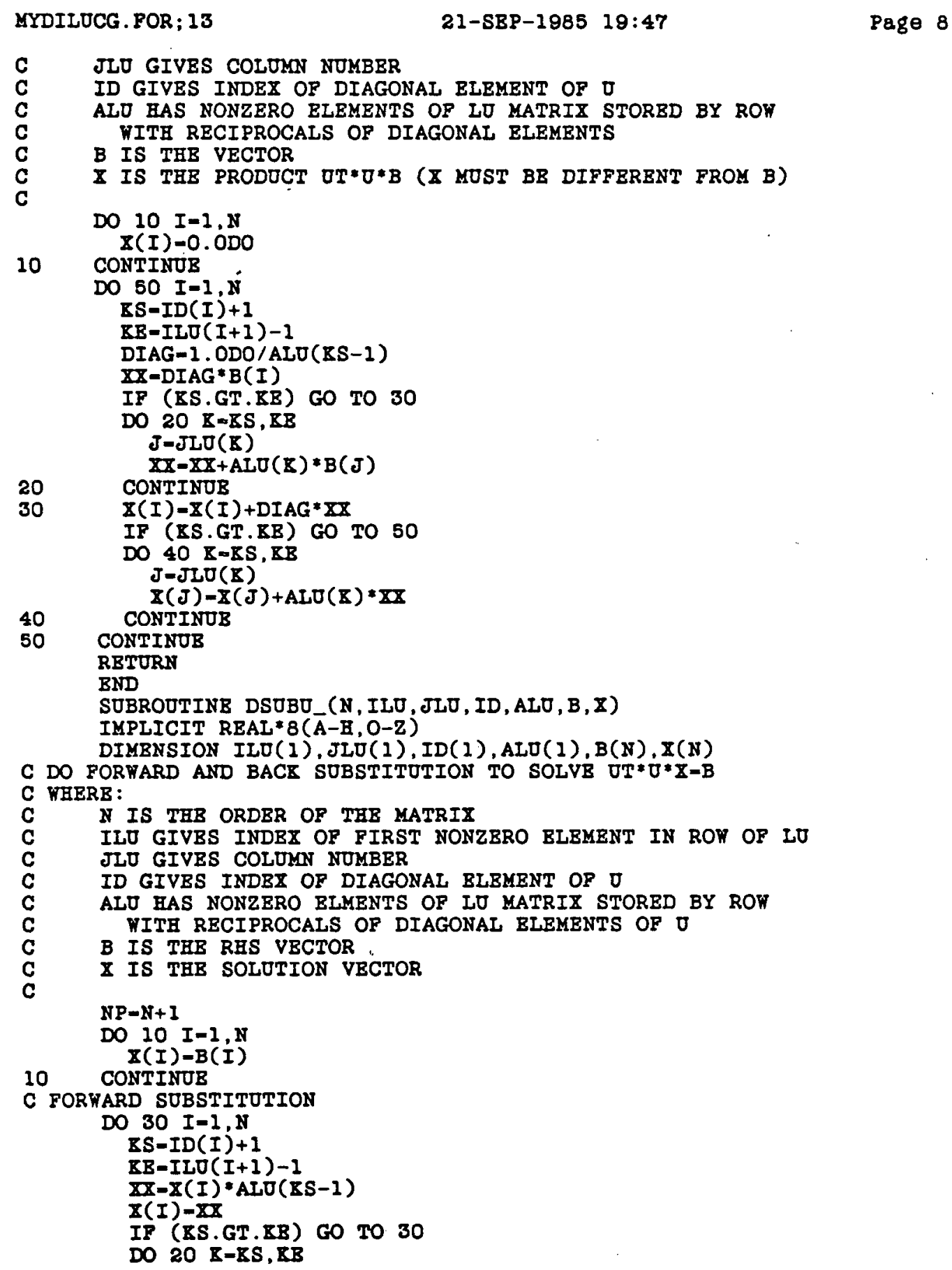

C JLO GIVES COLUMN NOMBER

C ID GIVES INDEX OF DIAGONAL BLEMENT OF $\square$

C ALO HAS NONZERO ELEMENTS OF LU MATRIX STORED BY ROW

C WITH RECIPROCALS OP DIAGONAL ELEMENTS

C B IS THB VECTOR

C $I$ IS THE PRODUCT UT* ${ }^{*} B$ ( $X$ NOST BR DIFFERENT FROM B)

10

DO $10 I=1, N$

$Z(I)=0.0 D 0$

CONTINOB

DO $50 \mathrm{I}=1, \mathrm{~N}$

KS-ID $(I)+1$

$K E-I L U(I+I)-1$

DIAG-1.ODO/ALO(ES-1)

$X X-D I A G * B(I)$

IF (KS.GT.KE) GO TO 30

DO $20 \mathrm{~B}-\mathrm{BS}, \mathrm{RB}$

J-JLO(B)

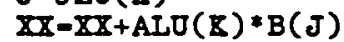

20$$
\text { CONTINOB }
$$

$30 \quad X(I)-X(I)+D I A G * I X$

IF (XS.GT.EB) GO TO 50

DO 40 K-KS, XB

$\mathrm{J}=\mathrm{JLU}(\mathrm{R})$

40 CONTINOB

J) $+A L U(X) * B X$

50 CONTINUB

RBTURN

BND

SUBROJTINB DSOBD_(N, ILO, JLO, ID, ALV, B , X)

IMPLICIT REAL* $8(\bar{A}-\mathrm{H}, 0-\mathrm{Z})$

DIMBNSION ILU(1), JLU(1), ID(1), ALU(1),B(N),X(N)

C DO FORYARD AND BACX SOBSTITUTION TO SOLVB $U T * U * Z-B$

C WHERB:

C N IS THB ORDER OP THE YATRIX

C ILU GIVBS INDBX OF FIRST NONZBRO BLBMENT IN ROW OF LU

C JLO GIVES COLUMN NUMBER

C ID GIVBS INDBX OF DIAGONAL RLBMENT OP $O$

C ALO HAS NONZERO BLMENTS OF LO MATRIX STORED BY ROW

C WITH RECIPROCALS OF DIAGONAL BLEMENTS OF 0

C B IS THB RHS VBCTOR

C $\quad X$ IS THB SOLUTION VECTOR 


\section{fortran directory}

MODIIOCG.FOR; 13

21-SBP-1985 19:47

Page 9

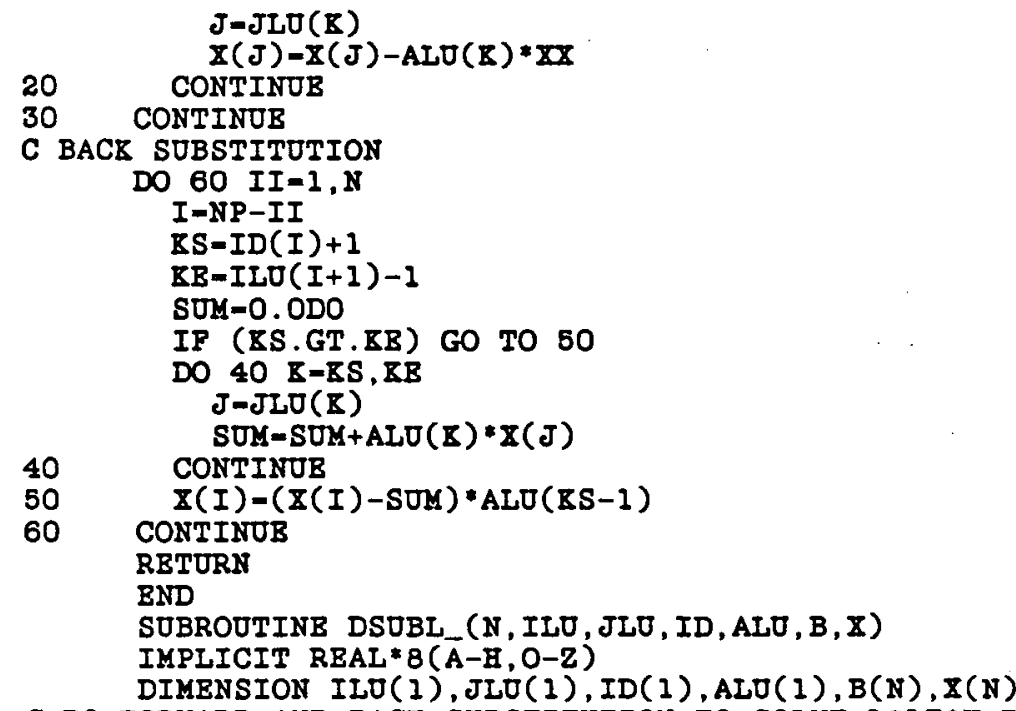

C DO FORWARD AND BACE SOBSTITUTION TO SOLVB $I * I T * Z=B$

C WHERB :

C N IS THB ORDER OF THB MATRIX

C ILU GIVES INDBX OF FIRST NONZBRO BLEMENT IN ROW LU

C JLD GIVBS THB COLUMN NOMBER

C ID GIVBS INDEX OP DIAGONAL ELEMENT OF 0

C ALO HAS NONZERO BLEMENTS OF LO MATRIX STORED BY ROW

C DIAGONAL BLBMENTS OF I ARB 1.0 AND NOT STORED

C B IS THE RHS VECTOR

C $\quad Z$ IS THB SOLOTION VECTOR

c

$\mathrm{NP}-\mathrm{N}+1$

DO $10 \mathrm{I}=1, \mathrm{~N}$

$Z(I)=B(I)$

10 CONTINOB

C FORWARD SUBSTITUTION

DO $30 \quad I=1, N$

BS-ILU(I)

$\mathrm{KB}=\mathrm{ID}(\mathrm{I})-1$

IF (KS.GT.KB) GO TO 30

SUM $=0$. ODO

DO $20 \quad \mathbf{B}=\mathbf{K S}, \mathrm{KB}$

$J=J L U(\mathbf{K})$

SUM $-S U M+A L U(B) * X(J)$

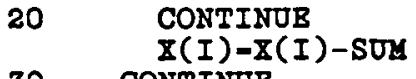

30 CONTINUB

C BACK SUBSTITUTION

DO 50 II=1,

$I=N P-I I$

KS-IIO(I)

$\mathrm{KB}=\mathrm{ID}(\mathrm{I})-1$

IF (KS.GT.KB) GO TO 50 


\section{fortran directory}

MYDILUCG.POR; 13

21-SBP-1988 $19: 47$

Page 10

$X X-X(I)$

IF (XX.BQ.0.0) GO TO 50

DO $40 \mathrm{Z}-\mathrm{KS}, \mathrm{KB}$

$\mathrm{J}=\mathrm{J} L \mathrm{R}(\mathrm{B})$

$X(J)-Z(J)-A L D(K)=X X$

40 CONTINOB

50 CONTINOB

RETURN

BND

DOUBLB PRECISION FUNCTION DGVV(V,N,N)

IMPLICIT DOUBLB PRECISION (A-H,O-Z)

DIMENSION $V(N), W(N)$

C SUBROOTINE TO COMPUTE DOUBLE PRECISION VECTOR DOT PRODUCT.

C

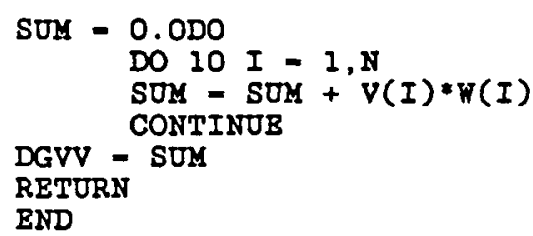$$
\text { CONTINOB }
$$

DGVV = SUM

RETURN

END

10 


\section{fortran directory}

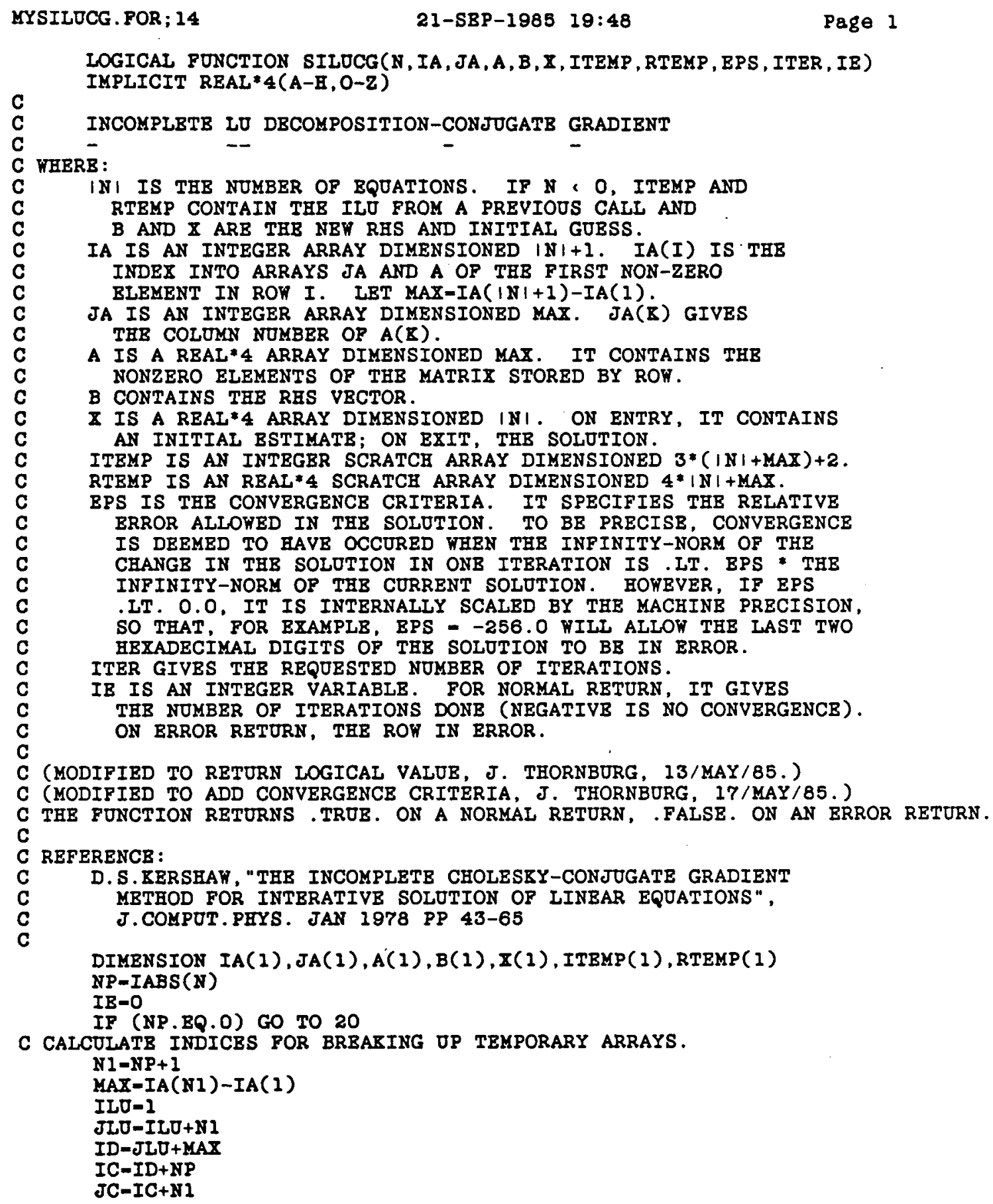


fortran directory

MYSILOCG. FOR; 14

JCI-JC+MAX

$I R=1$

$I P-I R+N P$

$I S I-I P+N P$

IS $2-I S I+N P$

IALO-ISE+NP

IF (N.LT.O) GO TO 10

C DO INCOMPLETB LO DECOMPOSITION

CALL SLU_(NP, IA, JA, A, ITEMP (IC), ITEMP (JC), ITEMP(JCI), RTEMP(IALU),

- ITEMP(IIU), ITEMP(JIU), ITEMP(ID), RTEMP(IR), IB, \&20)

C AND DO CONJUGATB GRADIENT ITERATIONS

C CALL MODIFIED TO ADD ADJUSTABLE CONVERGENCB CRITERIA EPS

C - J. THORNBURG, 17/MAY/85.

10 CALI SNCG_(NP, IA JA, A,B, X, ITEMP(ILU), ITEMP(JLU), ITEMP(ID),

- RTEMP (IALU), RTEMP(IR), RTEMP(IP), RTEMP(ISI), RTEMP(ISZ).

- EPS, ITER,IB)

SILUCG - .TRUB.

RETURS

SILUCG - . FALSB.

RETURN

END

SUBROOTINB SLO_(N, IA , JA , A , IC, JC, JCI , ALO, ILO, JLO, ID, V, IE, *)

IMPIICIT REAL* $\overline{4}(\mathrm{~A}-\mathrm{B}, \mathrm{O}-\mathrm{Z})$

DIMENSION IA(1), JA(1),A(1), IC(1), JC(1), JCI(1),

* ALO(1), ILO(1), JLO(1), ID(N),V(N)

LOGICAL NODIAG

COMYON /ICBD_/ ICBAD

C INCOMPLETB LO DBCOMPOSITION

C WHERB:

C N,IA, JA, AND A ARR DESCRIBED IN FUNCTION SILUCG

c IC IS AN INTBGER ARRAY DIMENSIONED $N+1$, IC (J) GIVES THE

C INDBX OF THB FIRST NONZERO BLEMENT IN COLMN $J$ IN ARRAY JC.

JC IS AN INTBGER ARRAY WITH THE SAMB DIMENSION AS A. JC(B) GIVBS THB ROW NOMBER OF THB $B$ 'TH BLEMENT IN THB COLUMN STRUCTURB.

JCI IS AN INTEGER ARRAY WITH THB SAMB DIMENSION AS A. JCI (B) GIVES THB INDEX INTO ARRAY $A$ OF THB $B$ 'TH ELEMENT OP TEB COLUMN STRDCTURE.

ALD HAS THB SAMB DIMBNSION AS A. ON BXIT, IT WILL CONTAIN THB INCOMPLBTE LO DECOMPOSITION OP A WITH THB RBCIPROCALS OF THB DIAGONAL BLEMENTS OF $\sigma$.

ILO AND JLU CORRBSPONDS TO IA AND JA BUT FOR ALU.

ID IS AN INTBGBR ARRAY DIMBNSIONBD N. IT CONTAINS INDICES TO THB DIAGONAL BLEMENTS OF 0 .

$\checkmark$ IS A REAI SCRATCH VBCTOR OF LBNGTH $N$.

IB GIVBS THB ROF NUMBRR IN BRROR.

NOTB: SLO_ SETS ARGUMBNTS IC THROUGH $v$.

ICBAD-O

C ZERO COUNT OF ZERO DIAGONAL BLEMENTS IN 0 .

C

C PIRST CHBCE STROCTURB OP A AND BUILD COLOMN STROCTURE

DO 10 I-1,N

IC(I) -0 


\section{fortran directory}

MYSILOCG . FOR; 14

21-SBP-1985 $19: 48$

10 CONTINUB

DO $30 I=1, N$

$R S=I A(I)$

$\mathrm{RB}=I A(I+1)-1$

NODIAG= . TRUB .

DO $20 \mathrm{~K}=\mathrm{KS}, \mathrm{KB}$

$J=J A(B)$

IF (J.IT.1.OR.J.GT.N) GO TO 210

IC(J) $-I C(J)+1$

20 CONTINUE

30 CONTINUB

C MAEE IC INTO INDICES

KOLD=IC ( 1 )

$\operatorname{IC}(1)=1$

DO 40 I- $1, N$

KNBWICIC $(I+1)$

IF (KOLD.BQ.O) GO TO 210

$I C(I+I)=I C(I)+$ KOID

KOID-RNBW

40 CONTINUB

C SET JC AND JCI FOR COLUNN STRUCTURB

DO $60 \quad I=1, N$

RS-IA(I)

$\mathrm{BB}-I A(I+1)-1$

DO $60 \mathrm{~K}=\mathrm{KS}, \mathrm{KB}$

$J=J A(8)$

$L=I C(J)$

IC (J) $=I+1$

JC(I) $-I$

50 CONTINUB

60 CONTINUB

C FIX OP IC

EOLD=IC(1)

IC( 1 ) $=1$

DO $70 \mathrm{I}=1, \mathrm{~N}$

KNBW=IC $(I+1)$

$I C(I+1)=$ KOID

KOLD-KNEW

70 CONTINOB

C FIND SORTRD ROF STRUCTURB FROM SORTED COLUMN STRUCTURB $\mathrm{NP}-\mathrm{N}+1$

DO $80 I=1, N P$

80 CONTINUB

$\operatorname{ILO}(I)=\operatorname{IA}(I)$

C KOVE BLBMBNTS, SBT JLD AND ID DO $100 \mathrm{~J}=1, \mathrm{H}$

$\mathrm{IS}=\mathrm{IC}(\mathrm{J})$

$Z B-I C(J+1)-1$

DO $80 \mathrm{~K}=\mathbf{R S}, \mathbf{K B}$

$I-J C(B)$

I-IIU(I)

$I L U(I)=I+I$ 


\section{fortran directory}

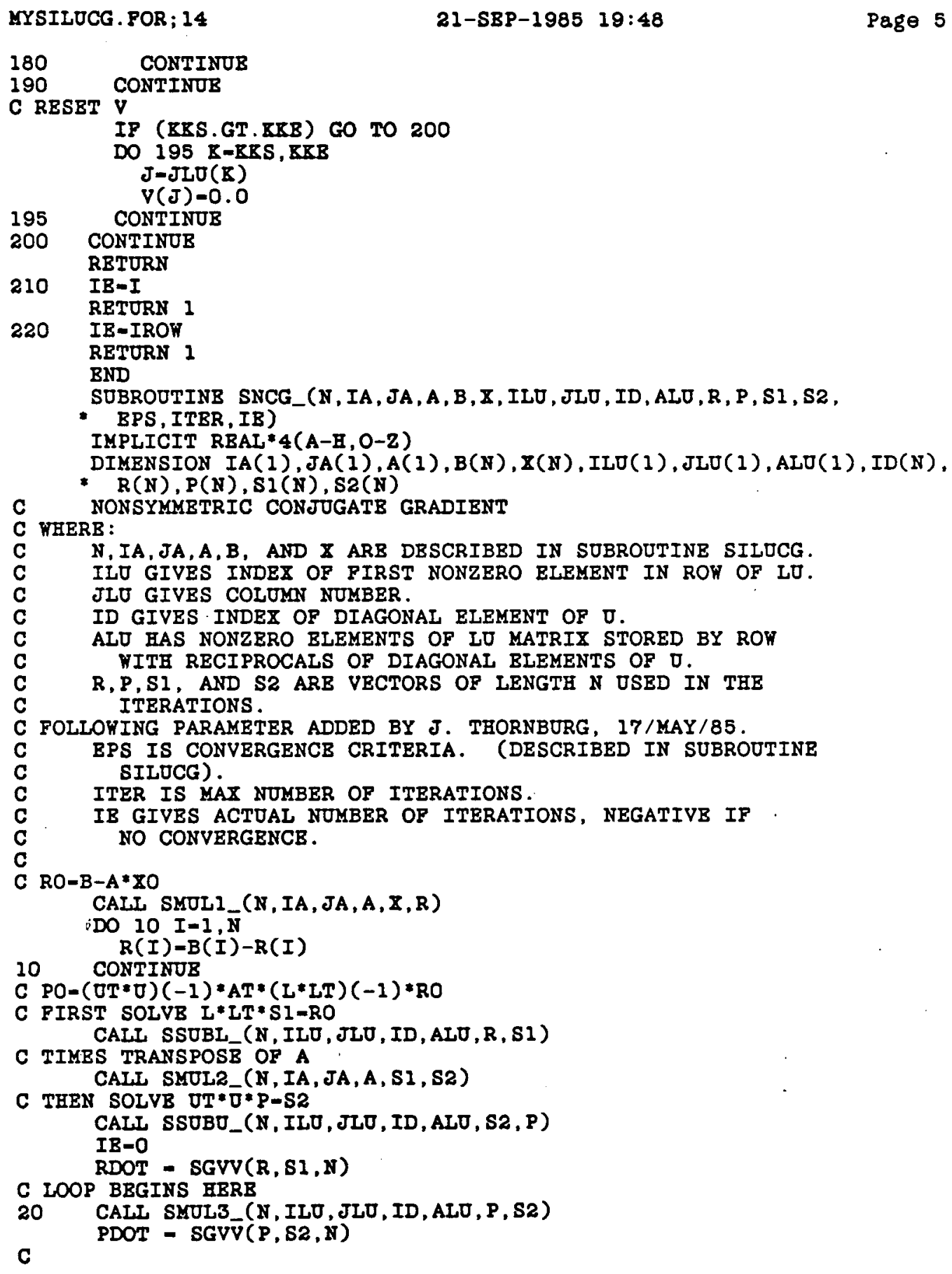


fortran directory

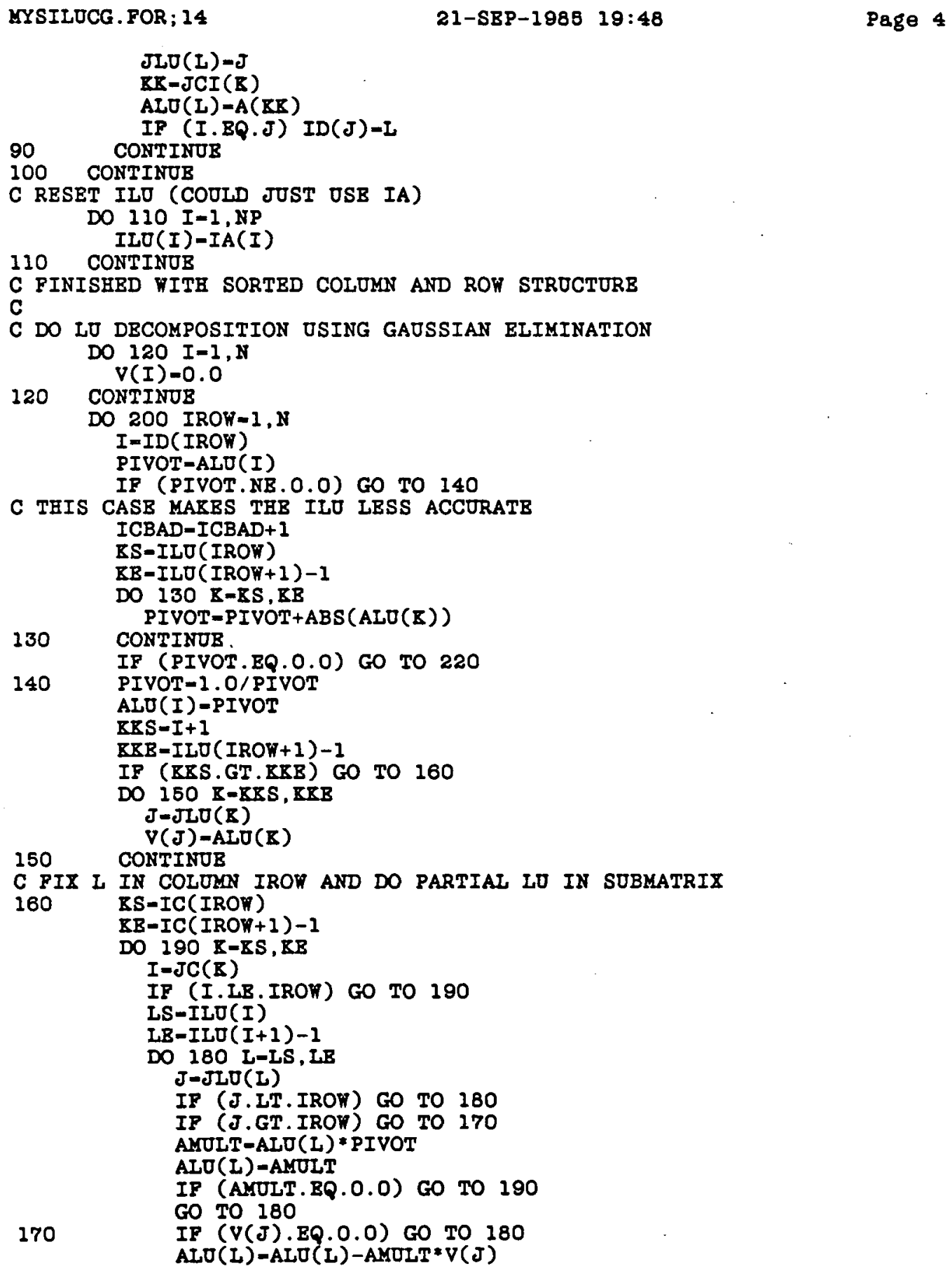




\section{fortran directory}

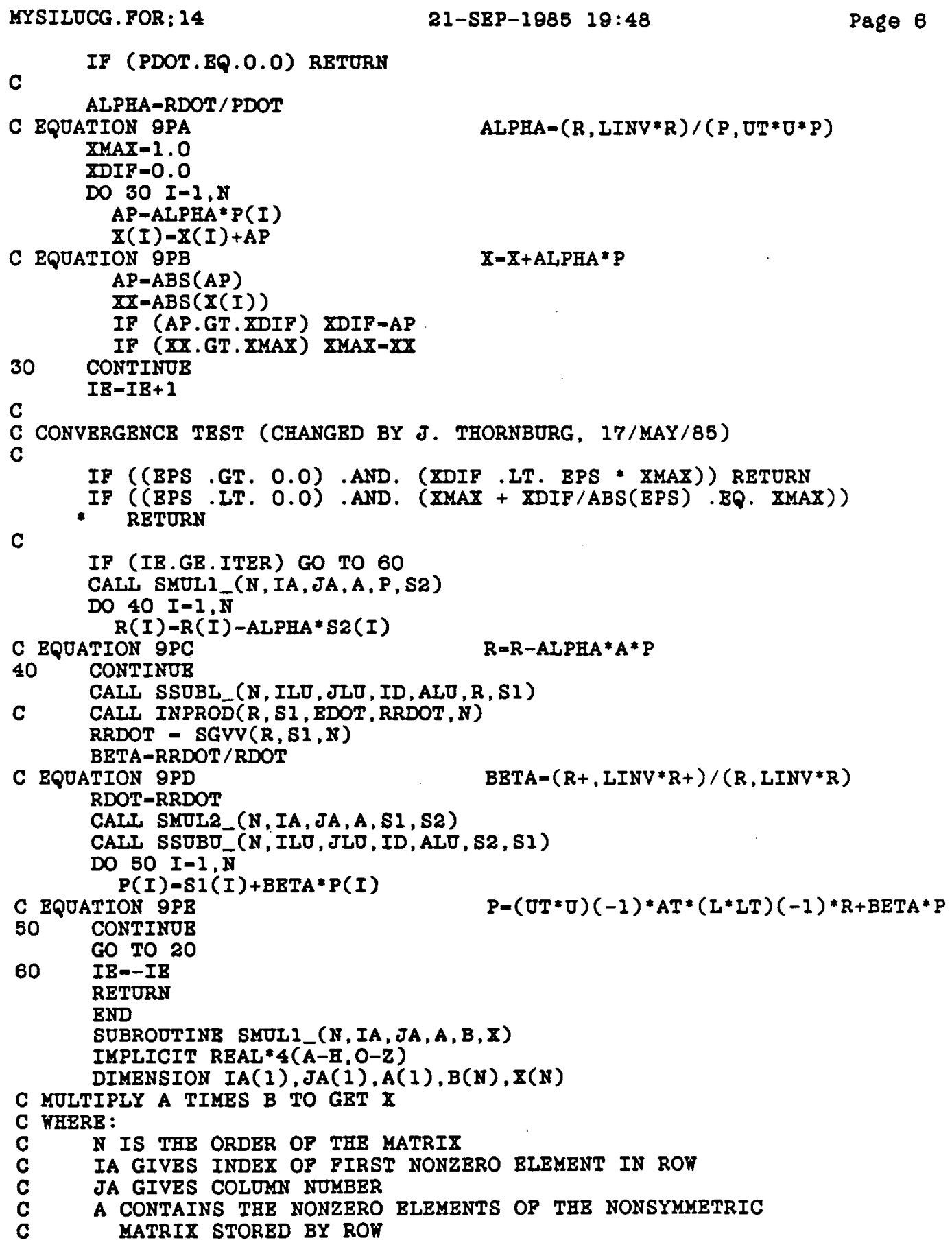




\section{fortran directory}

YYSILUCG. FOR ; 14

22-SEP-1985 19:48

Page 7

C B IS THB VECTOR

C $\mathrm{X}$ IS THB PRODOCT (MOST BB DIFFERENT PROM B)

DO 20 I-I,N

BS-IA(I)

$B B-I A(I+1)-1$

SUM -0.0

DO 10 R-RS, RB

$J=J A(B)$

10 CONTINOB

SUM $=S U M+A(Z) * B(J)$

20 CONTINOB

RBTURN

END

SUBROUTINB SMULZ_(N, IA, JA, A , B, X)

IMPLICIT REAL * $4(A-H, 0-2)$

DIMENSION IA(1),JA(I), A(I), B(N), $X(N)$

C MOLTIPLY TRANSPOSB OF A TIMBS B TO GBT $X$

C WHERB:

C N IS THB ORDER OF THB MATRIX

C IA GIVBS INDEX OF PIRST NONZERO BLBMENT IN ROW

C JA GIVBS COLUMN NOMBER

C A CONTAINS THE NONZERO BLEMENTS OF THE NONSYMMETRIC

C MATRIX STORED BY ROY

C B IS THE VECTOR

C I IS TEB PRODUCT (MUST BB DIFFBRENT FROM B)

C

10

DO $10 I-1, N$

$\mathbf{X}(I)=0.0$

CONTINUB

DO $30 \mathrm{I}=1, \mathrm{~N}$

BS-IA(I)

$\mathbf{K B}-I A(I+1)-1$

$\mathrm{BB}-\mathrm{B}(\mathrm{I})$

DO $20 \mathrm{E}-\mathrm{BS}, \mathrm{KB}$

J-JA (K)

$$
\bar{X}(\mathrm{~J})-\mathbf{X}(\mathrm{J})+A(\mathrm{~K}) * B B
$$

20 CONTINOB

30 CONTINOB

RETURN

END

SOBROUTINB SMUL3_(N,ILO, JLO, ID, ALO, B, X)

IMPLICIT REAL* $4(\bar{A}-\mathrm{H}, 0-Z)$

DIMENSION ILU(1),JLU(1), ID(1), ALU(1), B(N), I (N)

C MULTIPIY TRANSPOSB OF O TIMES $O$ TIMES $B$ TO GET $\mathbb{Z}$

C MHERE:

C N IS THB ORDER OF THB MATRIX

C ILO GIVES INDEX OP FIRST NONZERO BLEMENT IN ROW OF LO

C JLU GIVBS COLUMN NUMBER

C ID GIVBS INDEX OP DIAGONAL BLEMENT OF $D$

C ALO HAS NONZERO BLEMENTS OF LO MATRIX STORED BY RON

C VITE RECIPROCALS OF DIAGONAL BLBMENTS

C B IS THB VBCTOR

C $X$ IS THB PRODOCT UT*O*B ( $X$ YOST BB DIFFBRENT PROM B) 


\section{fortran directory}

C

$$
\text { Do } \begin{gathered}
10 \quad I-1, X \\
X(I)=0.0
\end{gathered}
$$

10

CONTINOR

DO $50 \quad I=1, N$

RS $=I D(I)+1$

ER=ILU $(I+I)-1$

DIAG-1.0/ALU(BS-1)

$X X=D I A G * B(I)$

IF (BS.GT.EB) GO TO 30

DO $20 \mathrm{~K}-\mathrm{BS}, \mathrm{KB}$ $J=J L U(K)$

20 CONTINUE

$$
X X-X X+A L U(\mathbb{R}) * B(J)
$$

$30 \quad \mathrm{Z}(I)=\mathrm{X}(\mathrm{I})+\mathrm{DIAG} * \overline{X X}$

IF (BS.GT.RB) GO TO 50

DO 40 B-BS, BB $\mathrm{J}=\mathrm{JLO}(\mathrm{B})$ $\bar{X}(J)=\bar{X}(J)+A L O(B) * Z X$

40 CONTINUB

50 CONTINUB

RETURN

BND

SUBROOTINE SSUBU_(N,ILU, JLU, ID, ALU, B, Z)

IKPIICIT REAL* $4(\bar{A}-\mathrm{H}, \mathrm{O}-\mathrm{Z})$

DIMENSION ILU(1), JLU(1), ID(1),ALU(1),B(N), $X(N)$

$C$ DO FORWARD AND BACK SUBSTITUTION TO SOLVB UT*U* $\bar{X}=B$

C WHERB :

C N IS THB ORDER OF THB MATRIX

C ILO GIVBS INDEX OF FIRST NONZERO ELEMENT IN ROW OF LU

C JLO GIVES COLUMN NUMBER

C ID GIVES INDEX OF DIAGONAL BLEMENT OF 0

C ALU HAS NONZBRO BIMENTS OF LU MATRIX STORED BY ROW

C WITH RECIPROCALS OF DIAGONAL ELEKENTS OF $D$

C B IS THB RHS VBCTOR

C $\quad Z$ IS THB SOLUTION VBCTOR

C

$N P-N+1$

DO $10 I=1, N$

$Z(I)=B(I)$

10 CONTINUB

C FORTARD SUBSTITUTION

DO 30 I $-1, N$

BS-ID $(I)+1$

$\mathrm{EB}=\mathrm{ILO}(I+1)-1$

$Z=Z(I) * A L D(B S-1)$

$X(I)-X X$

IF (BS.GT.EB) GO TO 30

DO $20 \mathrm{~K}=\mathrm{KS}, \mathrm{KB}$

$J=J L U(R)$

$20 \quad \mathbf{X}(J)-\bar{J}(J)-A L U(K) * X X$

20 CONTINUB

30 CONTINUB

C BACK SUBSTITUTION

Do 60 II $=1, N$ 


\section{fortran directory}

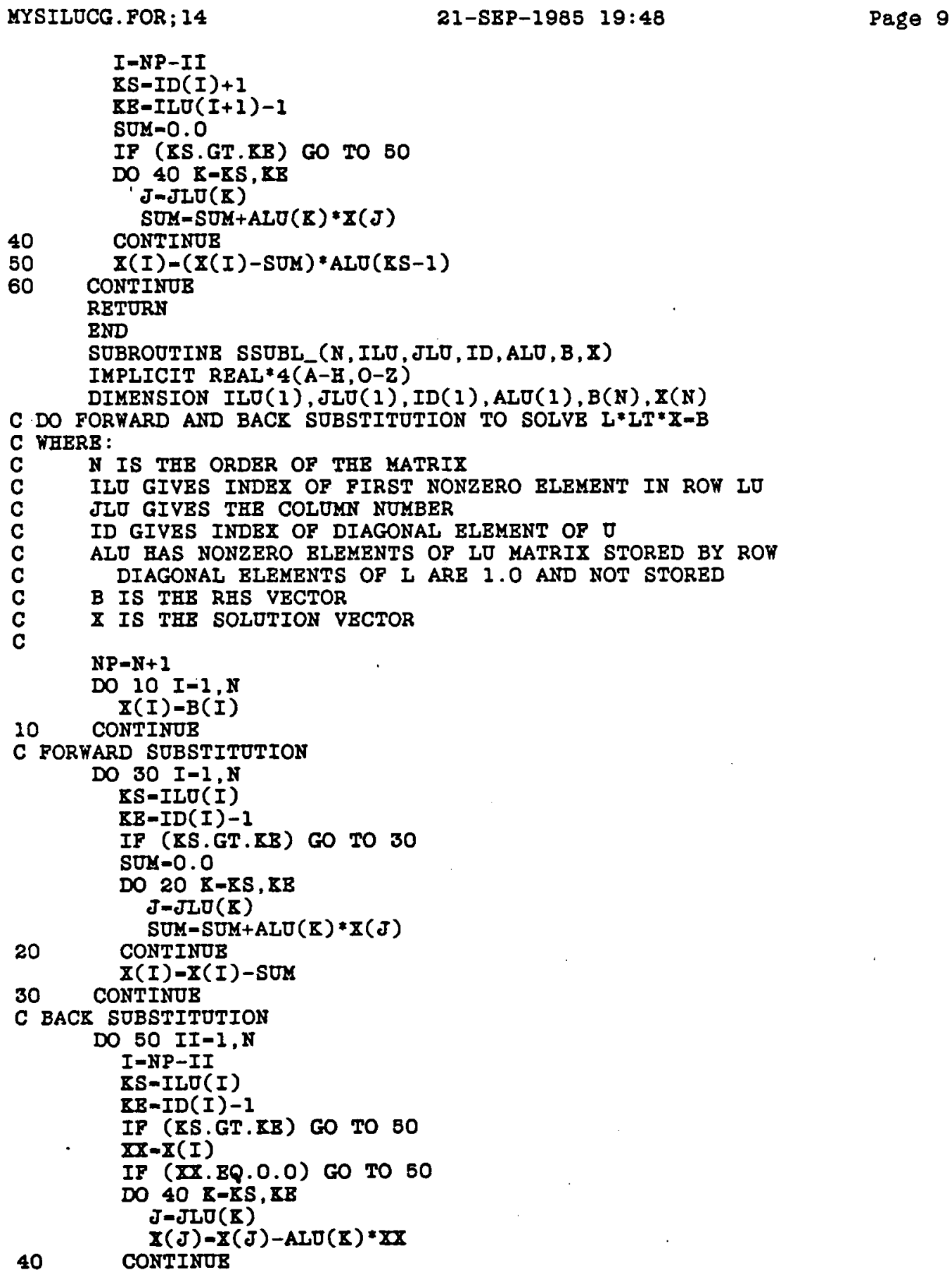




\section{fortran diréctory}

KYSILOCG.FOR; 14

21-SRP-1985 19:48

Page 10

50 CONTINOB

RETURN

\section{RND}

REAL PUNCTION SGVV $(V, N, N)$

IMPLICIT REAL (A-H,O-Z)

DIMENSION $V(N), W(N)$

C SUBROUTINB TO COMPUTB REAL VECTOR DOT PRODUCT.

SUR $=0.0$

DO $10 I=1, N$

10

SUM - SUM
CONTINUB

SGVV = SUL

RETURN

BND 


\section{patchut1l directory}

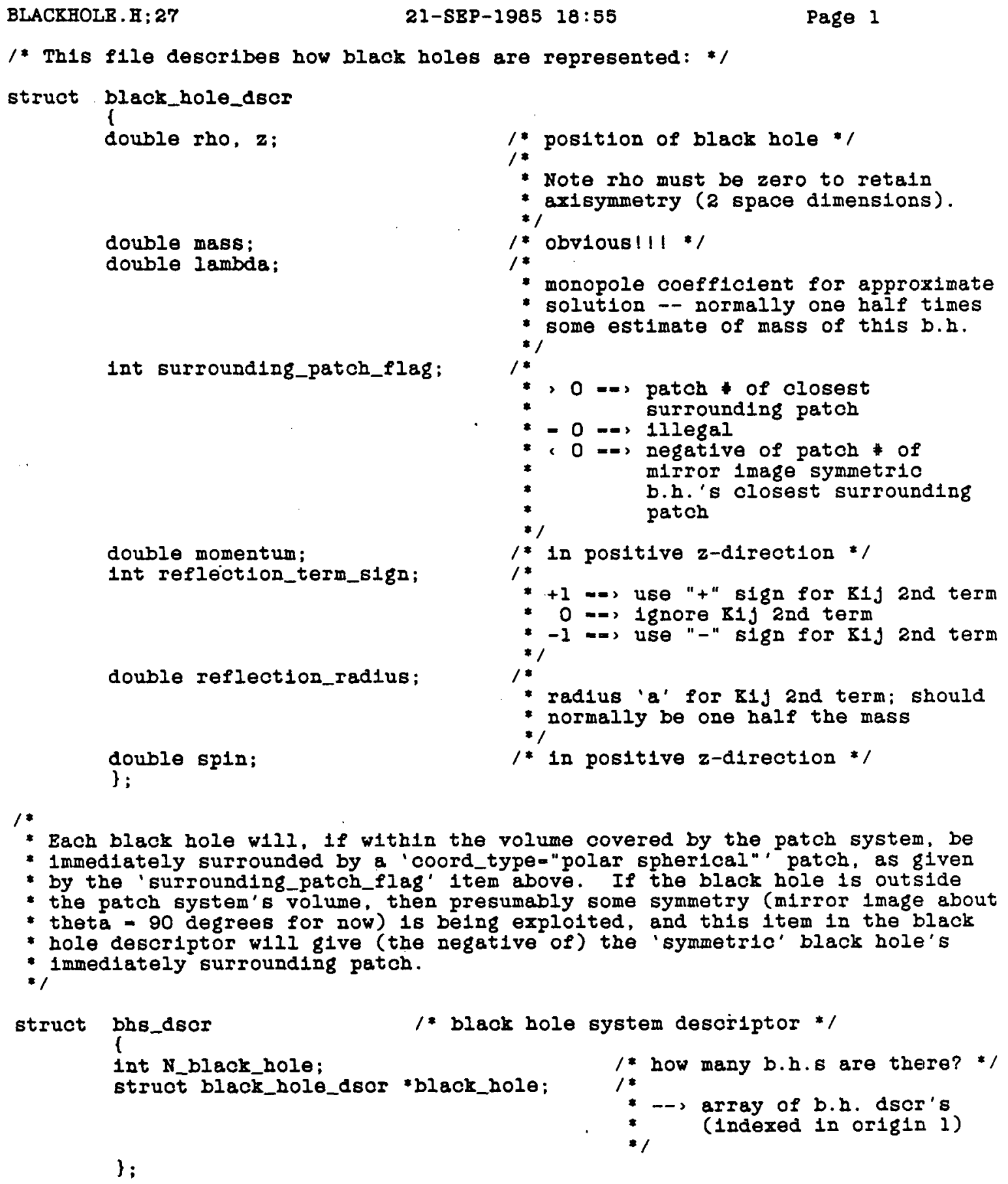




\title{
patchut1l directory
}

CVT. $\mathrm{H} ; 18$

21-SBP-1985 $18: 50$

Page 1

\begin{abstract}
define CVT_ERROR
†define OFFSET_CI

* define OFFSETC2

†define OFFSET_C3

*define OFFSET_C4

*define OFFSET_C5

*define OFFSET S

* define OFFSET_PSI

* def1ne OFFSET_R
\end{abstract}

1* Integer codes for 'cvt_' convertion functions: *I

†def1ne CVT_BNDRY_COND_TYPB_ERROR

$-1$

* def1ne PSI_MINUS_MASS_MONOPOLES

₹ def1ne PSI_MINUS_AREA_MONOPOLES

\#def1ne PSI_MINUS_TOT_B_PER_M_MONOPOLES

*define PSI_MINUS_TOT_E_PER_A_MONOPOLES

*define PSI_MINUS_SPHERICALIY_SYM_MBAN

*define PSI_MINOS_SPHERICALIY_SYM_MIN

\#def1ne PSI_MINUS_Z_AXIS

*def1ne PSI_MINUS_EQUATOR

0

1

2

3

4

6

6

*def1ne TRUE_RESIDUAL

\#defIne R_HAT_RR

$-10$

$-11$

$-12$

$-13$

$-14$

$-15$

$-16$

$-17$

$-25$

$-26$ 
patchutil directory

INOUT.ㅍ; 2

21-SBP-1985 18:50

Page 1

$1 *$

* The following 'enum' is used in classifying points w.r.t. patoh boundaries

* and/or patch boundary terms:

enum relation ( INSIDE, ON_BNDRY, OUTSIDE \}; 
patchutil directory

PATCH. В;79

21-SBP-1985 $18: 48$

Page 1

1. Th1s p1le describes how patches, gr1ds, and functions are represented: *

1

- The basic 1dea 1s that each patoh 18 a 'Manhattan convex' subset of a

- rectangle in the 1-j plane, where ' 1 ' and ' $j$ ' are the 2 integer indices of

- 1t's coordinate system. This subset is defined by the minimum and maximum

- values of 1 on each ine of constant $j$, and of $j$ on each ing of constant 1 .

- (The 'Manhattan convez1ty' condition gaurantees that these will suffice,

- 1.e. that any constant 1 or constant $j$ line will intersect the patch in a

- pinite closed interval.)

- To transform from 1-j coordinates back to 'physical' rho-z coordinates, the - followiag scheme is used:

- x-1:1_step. J-j: J-step

- $1 f$ (polar spherical) then ( 1 - x, theta - y, transform to rho, z \}

- If (cylindrical) then \{ rho $-x, z-y\}$

Iho +- rho_center.

z + z_center

1. 'flag' code values for boundary condition types: " /

\QPIIO DIRICHLET

† DefIIE NEUMANN

* define ROBIN

\#defIIE REFLECTION_SYMMETRIC

\#def1IE MARGINALIY_TRAPPED ,-5

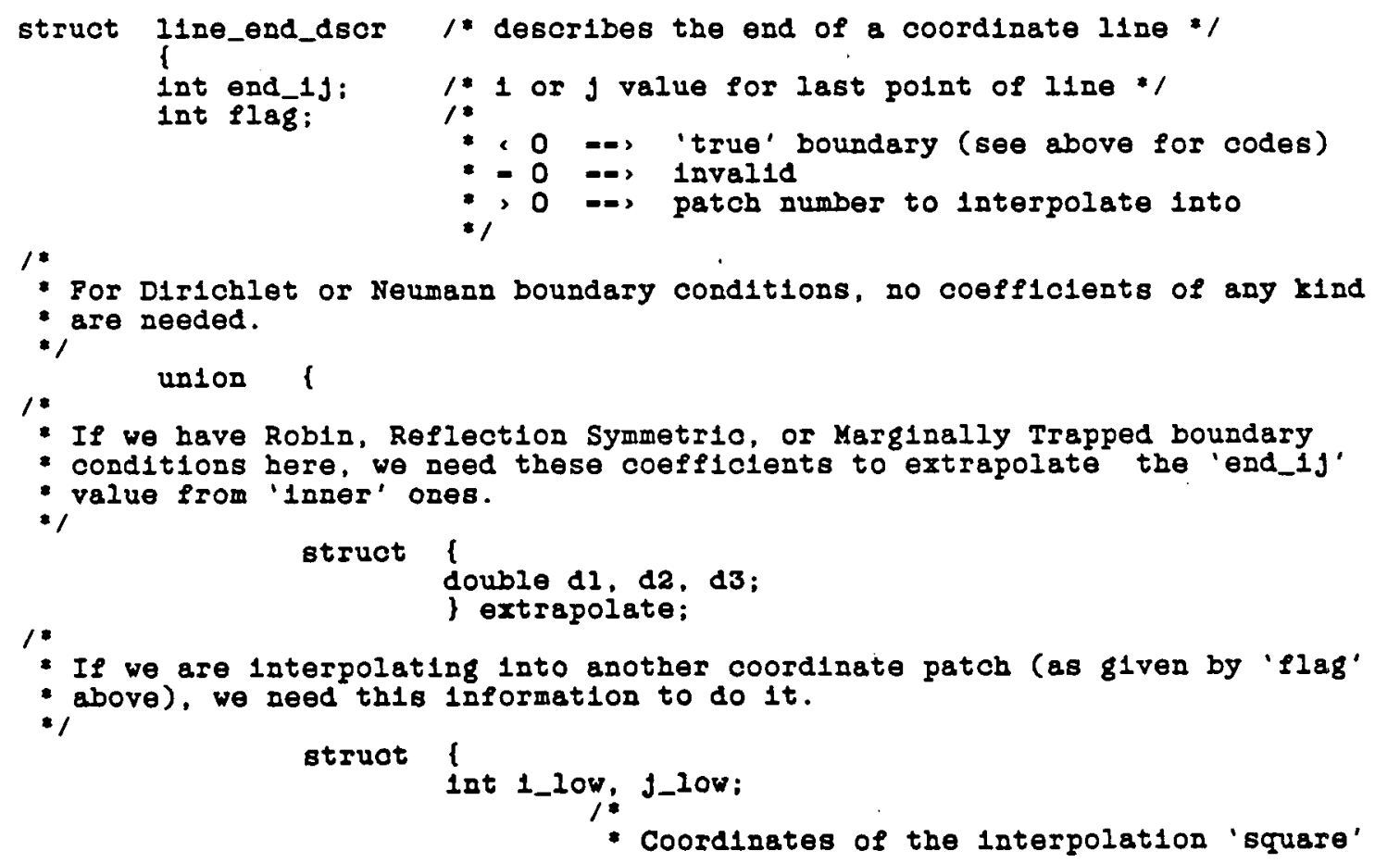




\section{patchutil directory}

PATCH.H; 79

21-SEP-1985 $18: 48$

Page 2

- are (1_low,j_low), (1_low, J_low+1),

- (1_low+1,j_low,$(1$ low $1, j$ j_low+1), in the

* system of the 'other' patch.

double do0, d01, d10, d11;

$1 *$

- The Interpolated value is found by taking a

* 'dot product' of these coefficlents with the

* function values at the 4 coordinates given

- above.

1

\} Interpolate;

\};

\} boundary_1nfo;

$1 *$

* Grid functions are stored as arrays of 'grid_fn' structures, each of which

* holds all the functions defined at that grid polnt. This is convienient to

* use, and also improves reference locality for the program.

* 1

struct grid_fn

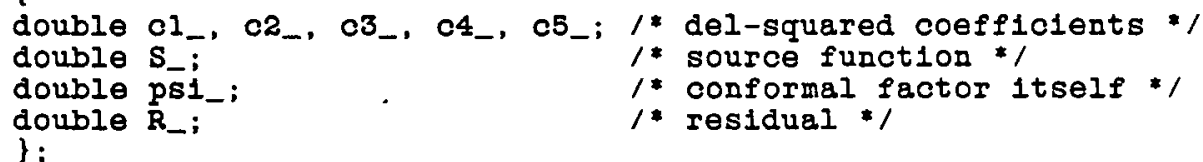

enum bndry \{ I_MIN, I_MAX, J_MIN, J_MAX \};

enum coord_type \{ CYIINDRICAI, POIAĀ_SPHERICAL \};

enum difference_scheme \{ INDEPENDENT_TERMS, IOCALII_CONSERVATIVE \};

enum conformal_fn_type \{ PSI, OMEGA_OF_PSI\};

struct coord_patch_dscr

\{

enum coord_type coord_type;

enum difference_scheme difference_scheme;

enum conformal_fn_type conformal_fn_type;

double rho center, z_center; $/ *$ position for $1=0, j-0 * /$

double 1_step, J_step;

1nt 1_min, 1_max;

1nt J_min, J_max;

struct line_end_dscr * 11ne_end[4]:

/* referred to as 'slze Info'*/

$1 *($ ditto $) * 1$

$1 *$

- This 4-element array is Indezed by (an 'int' type-

- case ofl an 'enum bndry'; each element polnts to an

- array of Iine_end_dsor's to describe that boundary.

- Note these arrays are 1ndexed in origin j_min,

* J_min, 1_min, and 1_min respectively, so the

- polnters are offset as appropriate. The 1 line ends

* are indexed by $g$ and vice versa ( 111$)$.

* Note also that the 'size 1nfo' may be 'downsized'

* after these arrays are allocated $=$ ', don't try to 


\section{patchutil directory}

PATCH.H; 79

21-SEP-1985 18:48

Page 3

1nt fn_1_step,

- deallocate them via 'ofree(...)'.

*

fn_J_step:

- These define the storage layout of the grid function

- arrays in memory -- they are in units of 'struct

* grid_fn's.

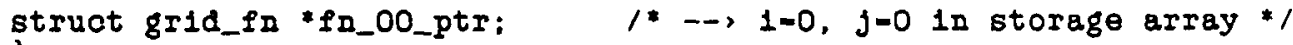

\};

struct

grid_dser

int N_patch; /* 'real' patches are numbered \#1 to \#N_patch */

struct coord_patch_dscr "patch; $\quad,-1$ erray of patch dscrs *

\#define GLOBAL_REF

0

/* patch * to use as global reference coord */

* By convention, the highest numbered patch 1s of coord_type="polar spherical"

and has the model's outer boundary as 1t's J_MAX boundary.

1

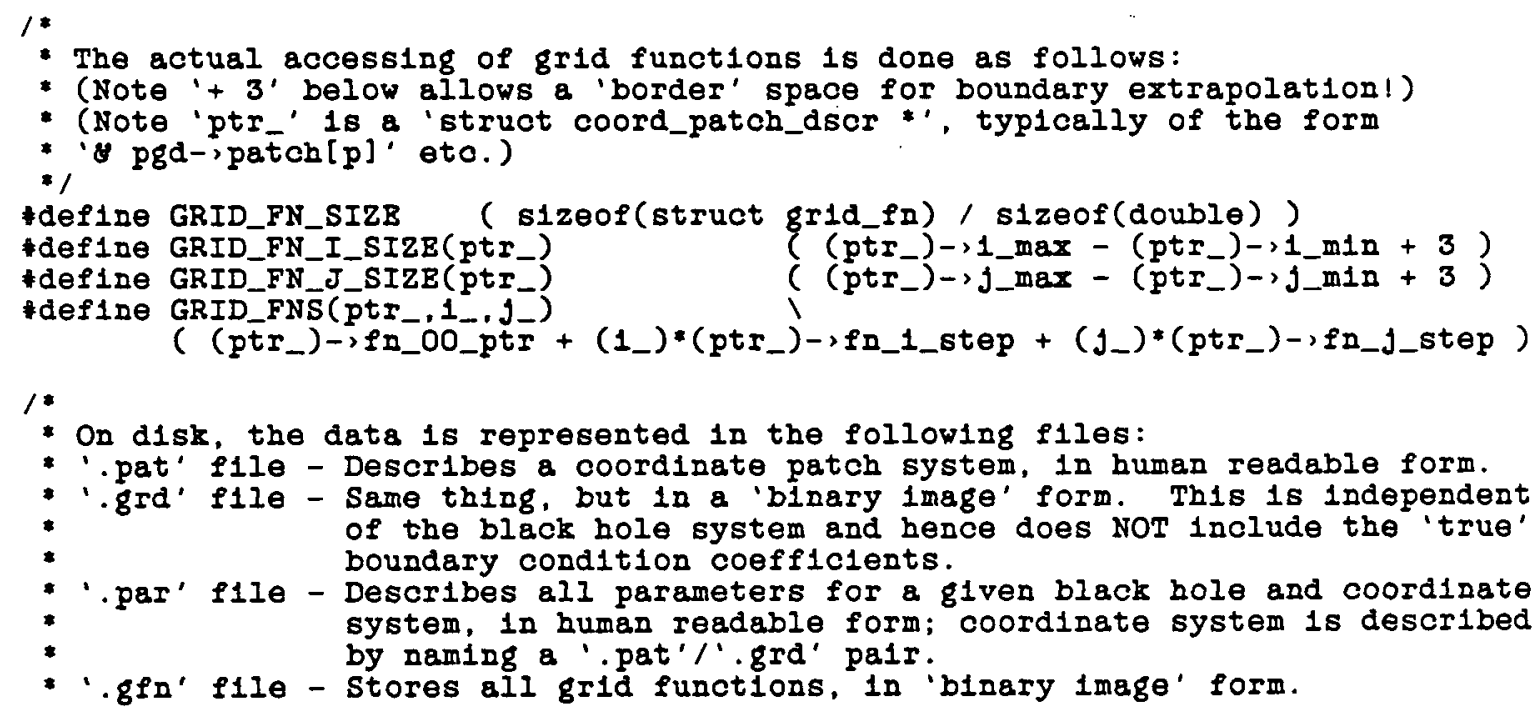

gridname.pat

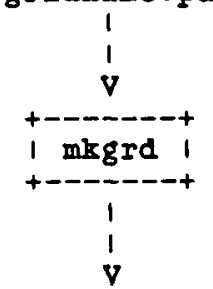


patchutil directory

PATCH.H;79

21-SBP-1985 $18: 48$

Page 4

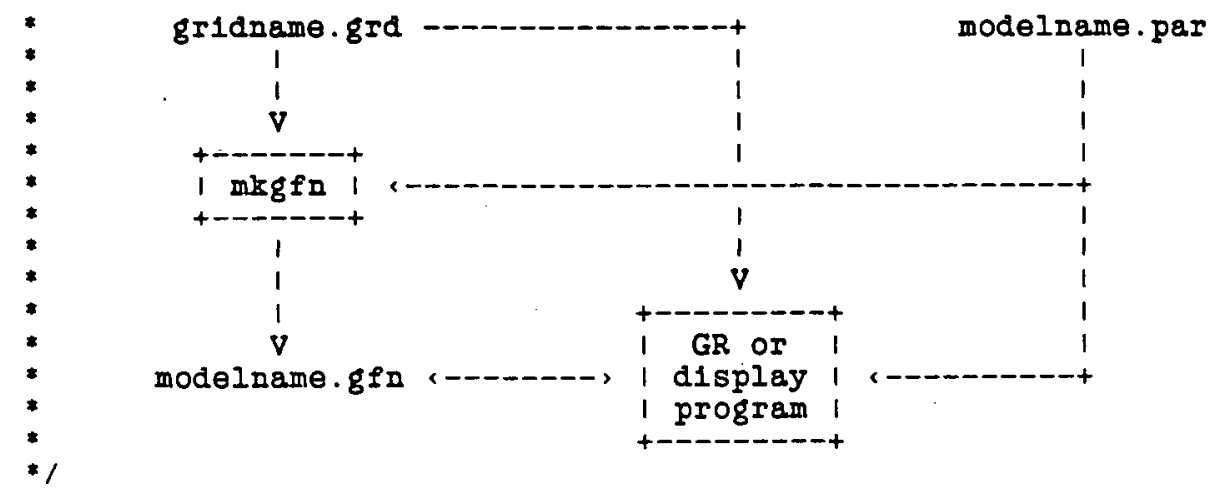




\section{patchut1l directory}

TEMPBNDRY.H; 7

21-SBP-1985 18:61

Page 1

1. Th1s flle describes the temporary patch boundary data structure */

struct bndry_defn_term

l

struct bndry_defn_term "next;

- The terms are in a one-way linked

- I1st. since we have no knowledge in

* advance of how many there will be.

$\because$

1* Pirst define how the boundary location 18 chosen: */

enum \{CONSTANT_BNDRY_TYPB, PATCH_BNDRY_TYPB \} defn_type; union $l$

double velue; $\quad 1$ if constant type *

struct 1 if PATCE type *1

Int patch; $\quad 1$ * the other patch number */

enum bndry bndry;

\} defo value:

\} patch_1nfo;

;* which bndry of 1 to we hit??? */

1. now declde how we w1ll get the boundary values: */

enum \{ TRUE_BNDRY_COND, PATCH_BNDRY_COND \} bRdry_cond_type;

union

char bndry_cond;

1nt patch;

/* If TRUE_BNDRY type */

\};

\} bndry_cond_value;

/* If PATCH type *

struct

patch_bndry

double x_min_bound, x_max_bound, y_m1n_bound, y_max_bound :

struct bndry_defn_term bdt_l1st [4];

(* bounding box of patch */

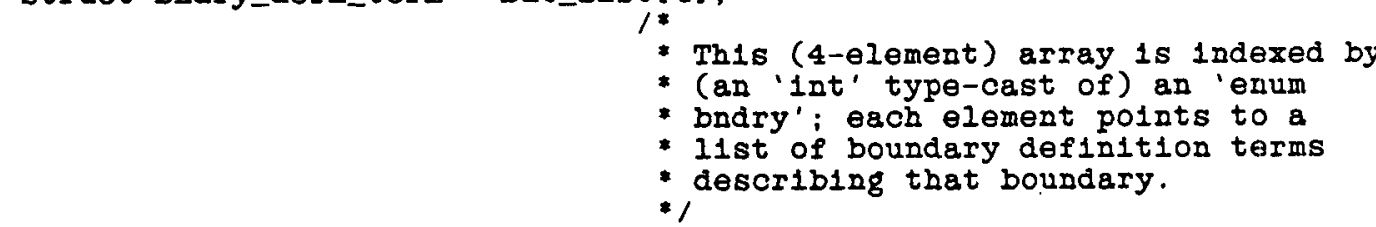

\};

* The overall temporary data structure will be an array ( 1 origin) of

- patch_bndry structures, Indexed by patch number.

* 1 
patchutil directory

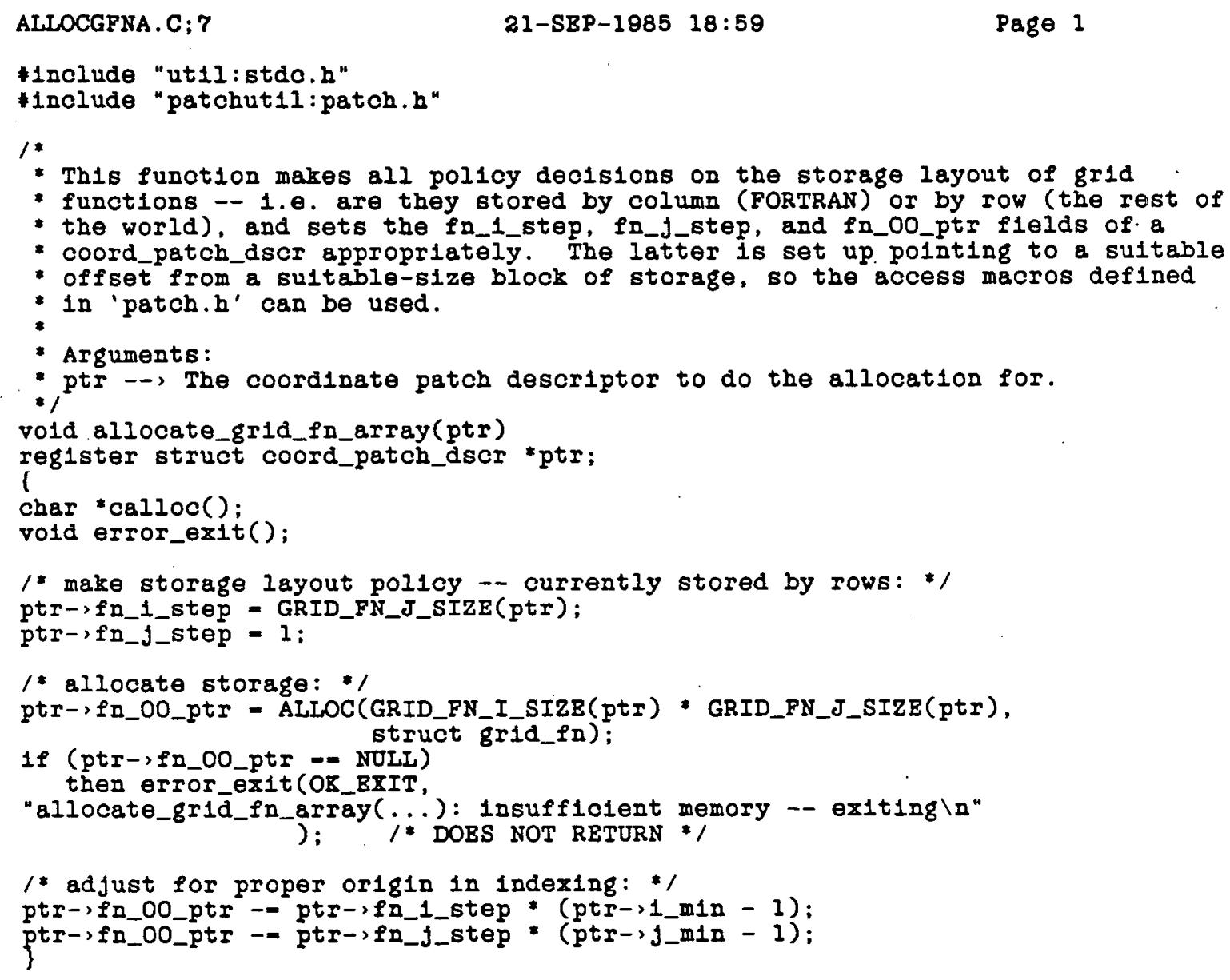


patchutil directory

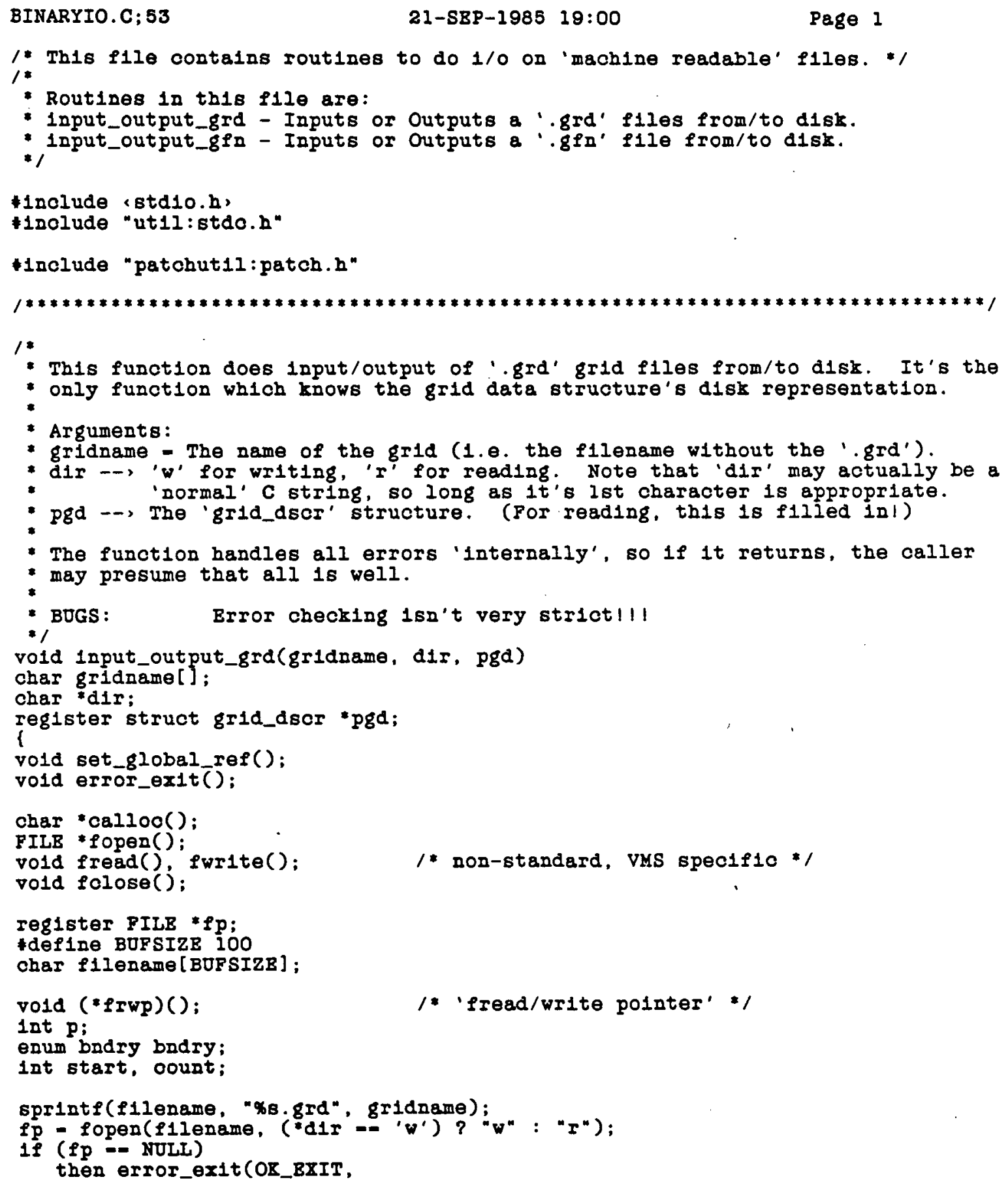


patchutil directory

BINARYIO.C; 53

21-SBP-1985 19:00

Page 2

"1nput_output_grd: unable to open file 'xs' -- exiting",
fllename):

†efine NOMEM

error_ex1t(OR_EXIT. "Input_output_grd: 1nsufflcient memory for grid '\%s' -- exiting".
gridname)

/ END OP MACRO DEFN *

frwp - (*dir - - 'w') ? fwr1te : fread;

1 * do the grid_dscr structure 1tself: *I

(*frwp)(y pqd->N_patch, sizeof(1nt), l, fp);

if ("dir $1=$ ' $w$ ')

then \{

* read $=$, allocate coord_patch_dscr's and set up global ref. patch *I pgd->patch - ALLOC (pgd->N_patch + 2, struct coord_patch_dscr);

if (pgd->patch =- NULL) then NOMEM;

set_globaI_ref(\& pgd->patch[GLOBAL_REF]);

\}

/* do the coord_patch_dsor's themselves: */

(*frwp)(\& pgd-ipatch[i].

sizeof (struct coord_patch_dscr), pgd->N_patch, fp):

/ finally, do the 11ne_end_dscr arrays: *

for $\left(p=1 ; p ;-p g d->N_{-}\right.$patch ; $\left.++p\right)$

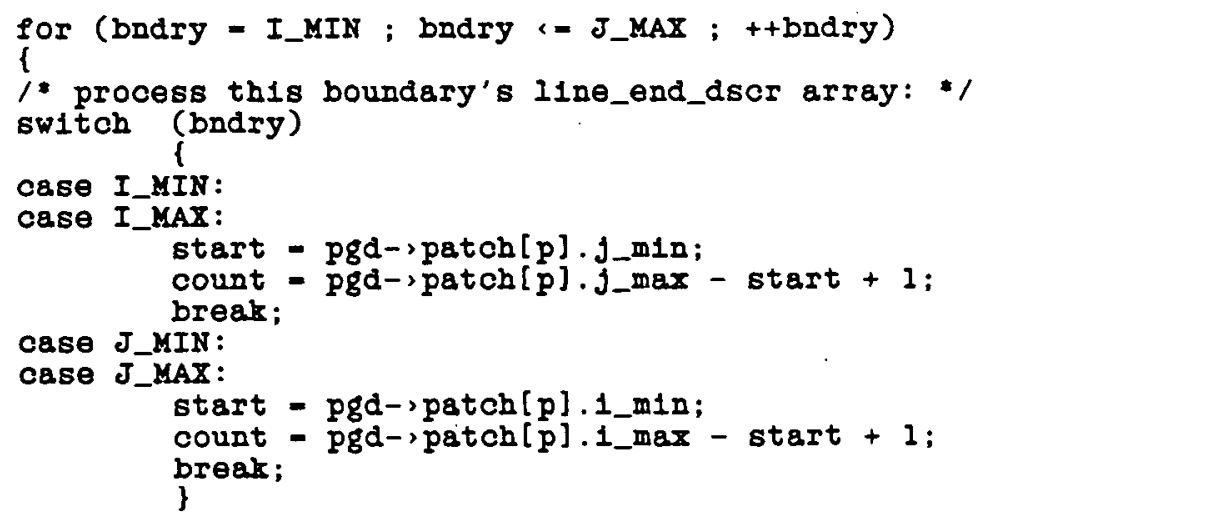

ldefine PTR pgd->patch[p].11ne_end[(1nt)bndry]

$1 f(* d 1 r 1=' w ')$

then 1

/* read --> allocate 11ne_end_dscr array: */

PTR - ALIOC(count, struct IIne_end_dscr);

$1 f$ (PTR - NULL) then NOMBK;

PTR - start; , adjust index origin*/ 


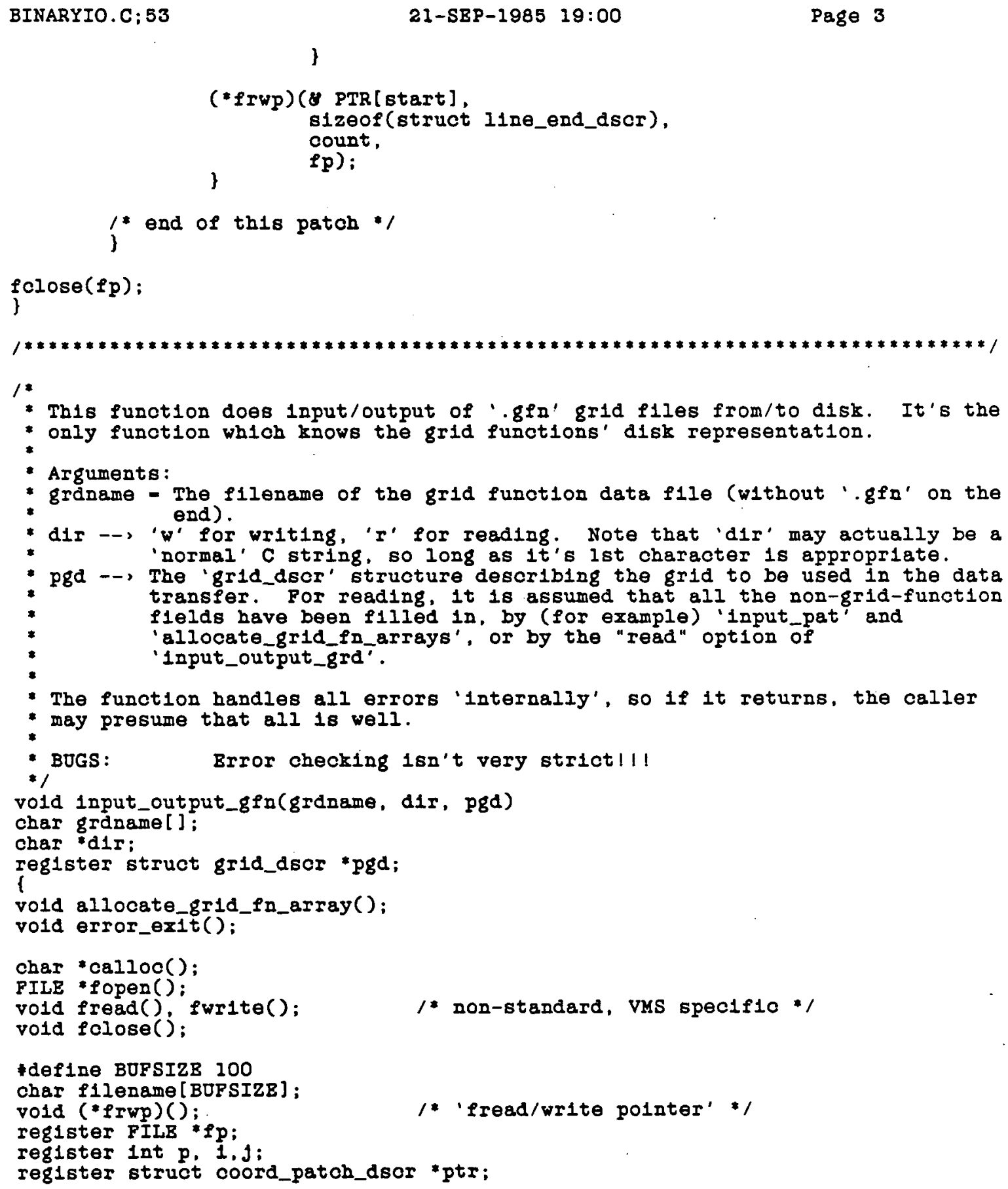




\section{patchut1l directory}

BINARYIO.C; 53

21-SBP-1985 19:00

Page 4

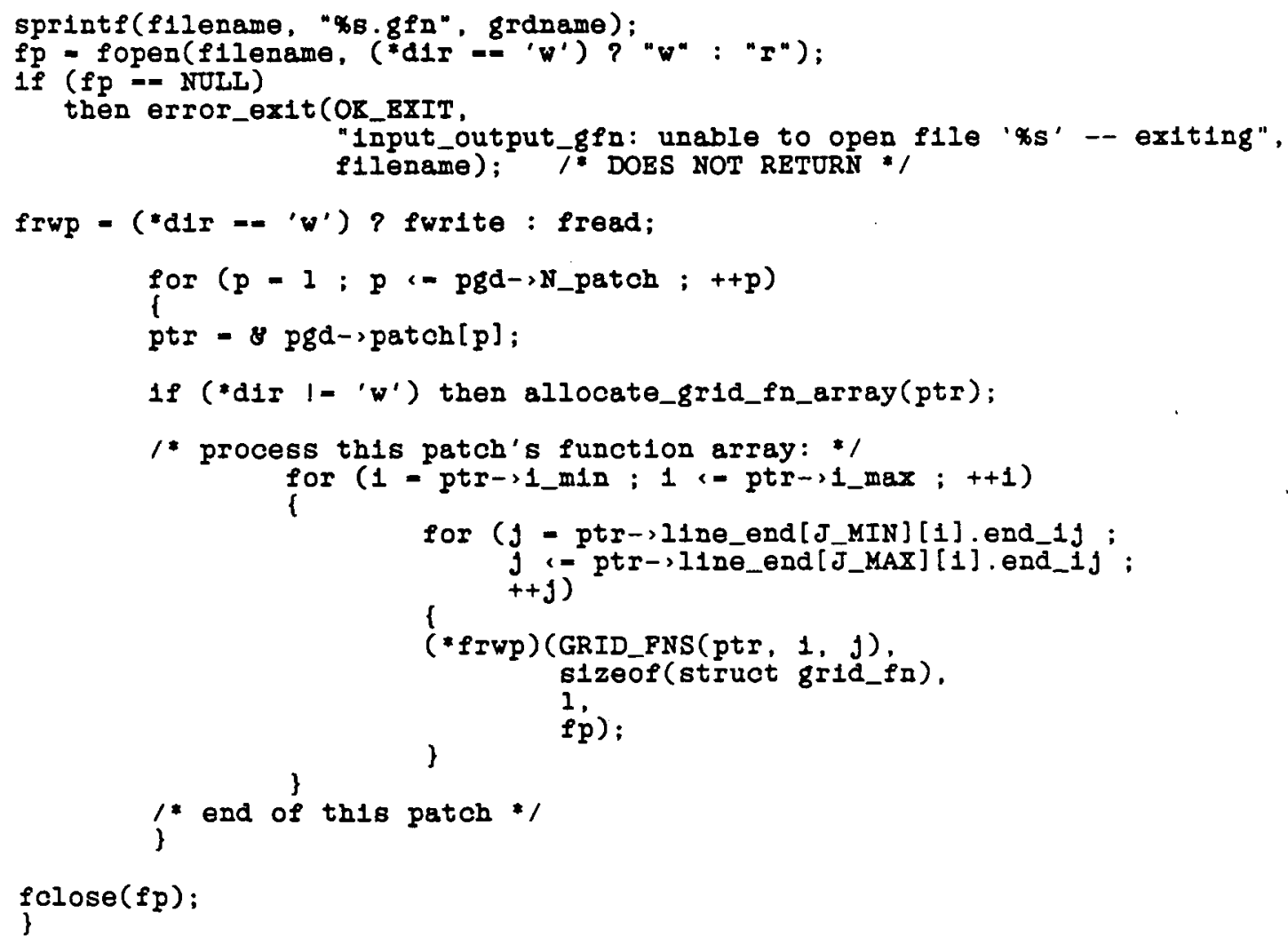




\section{patchutil directory}

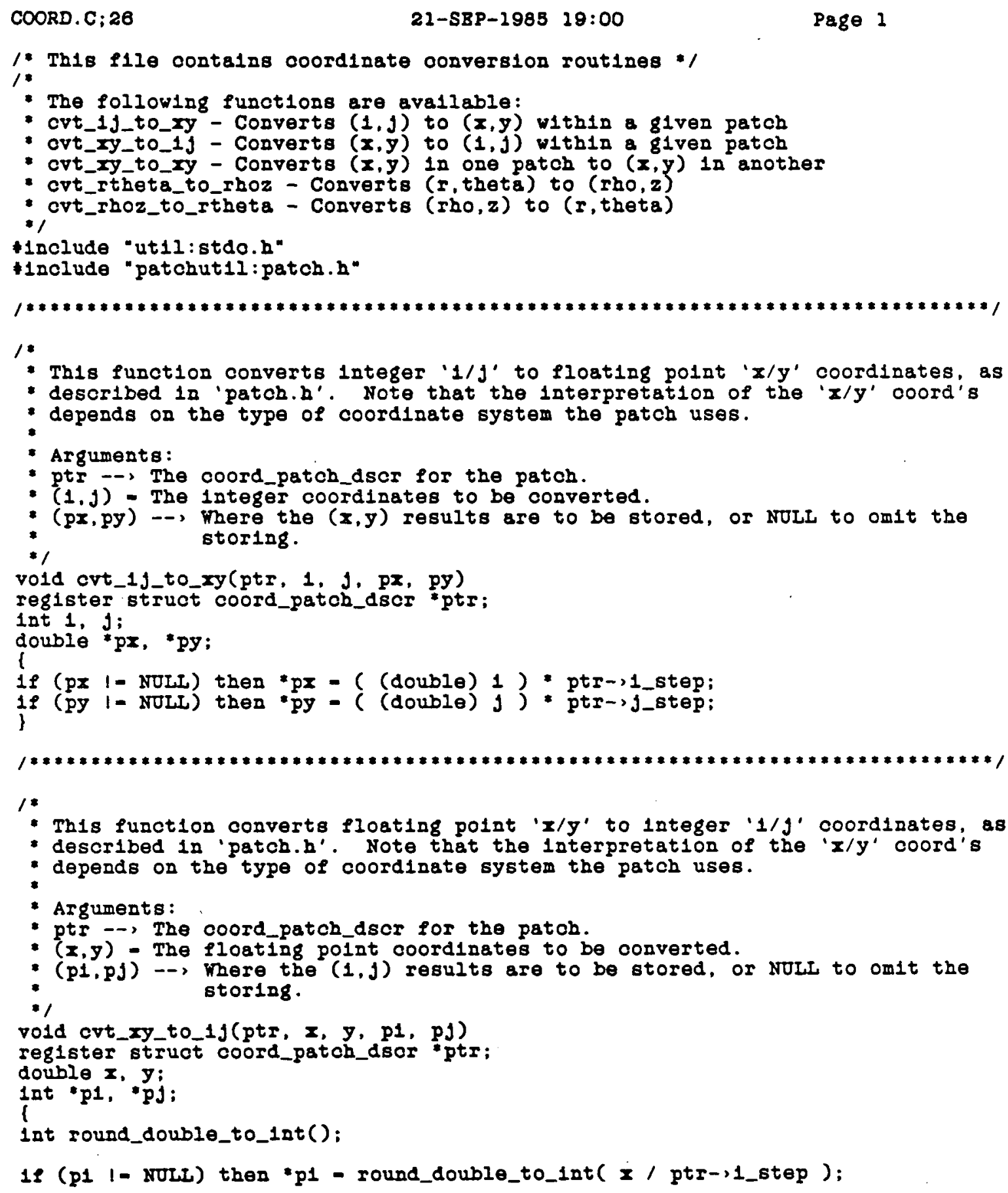




\section{patchutil directory}

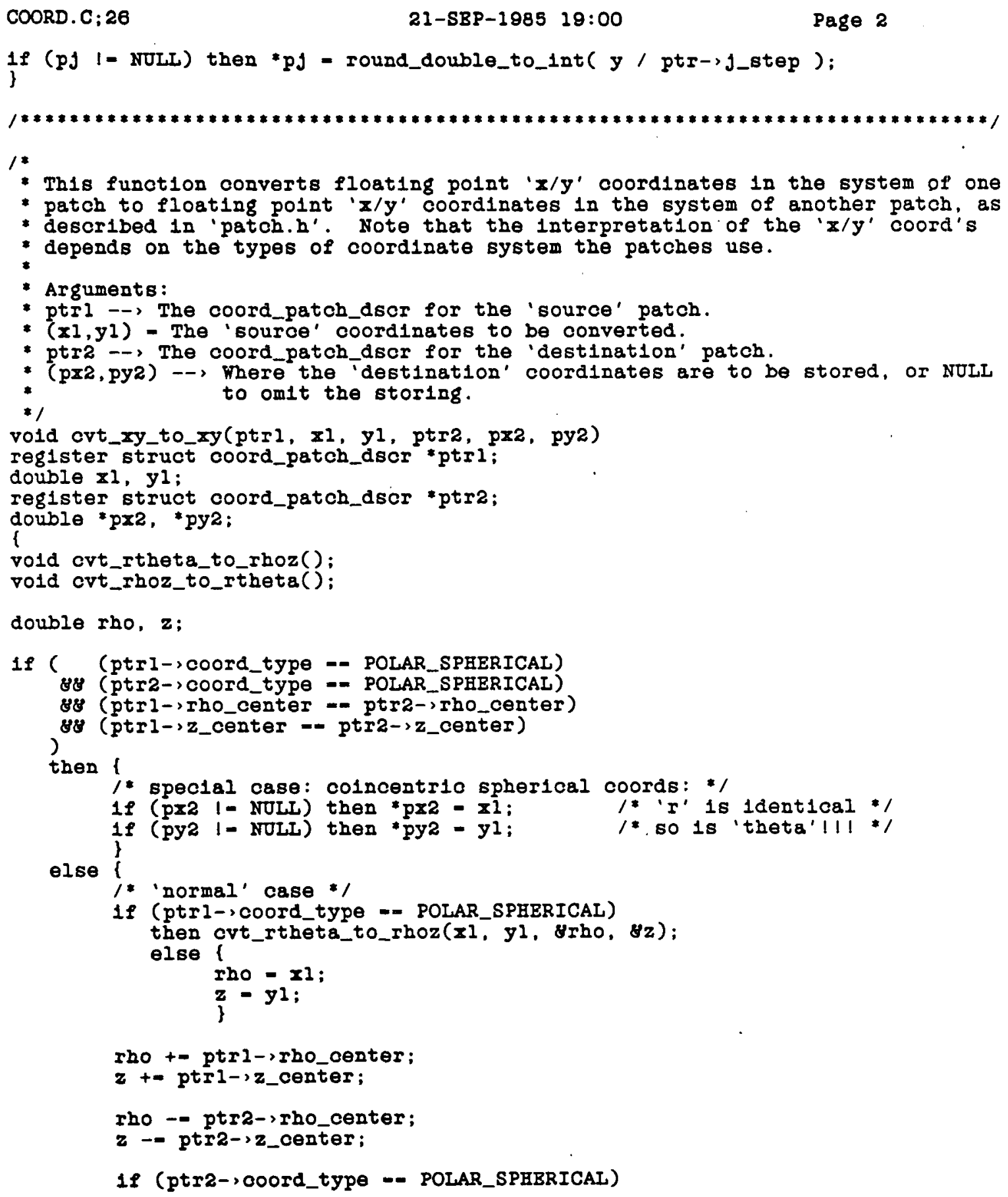


patchutil directory

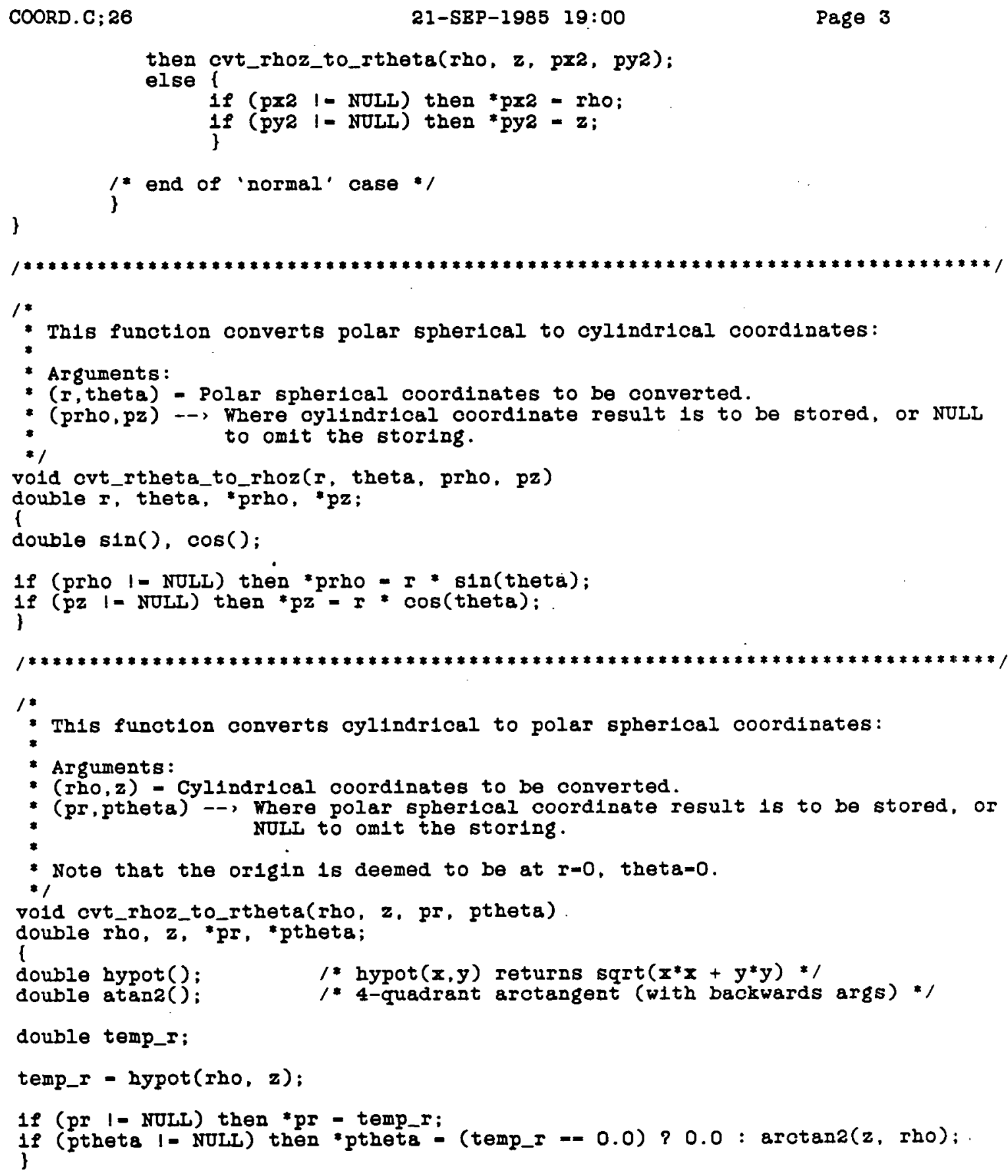


patchut1l directory

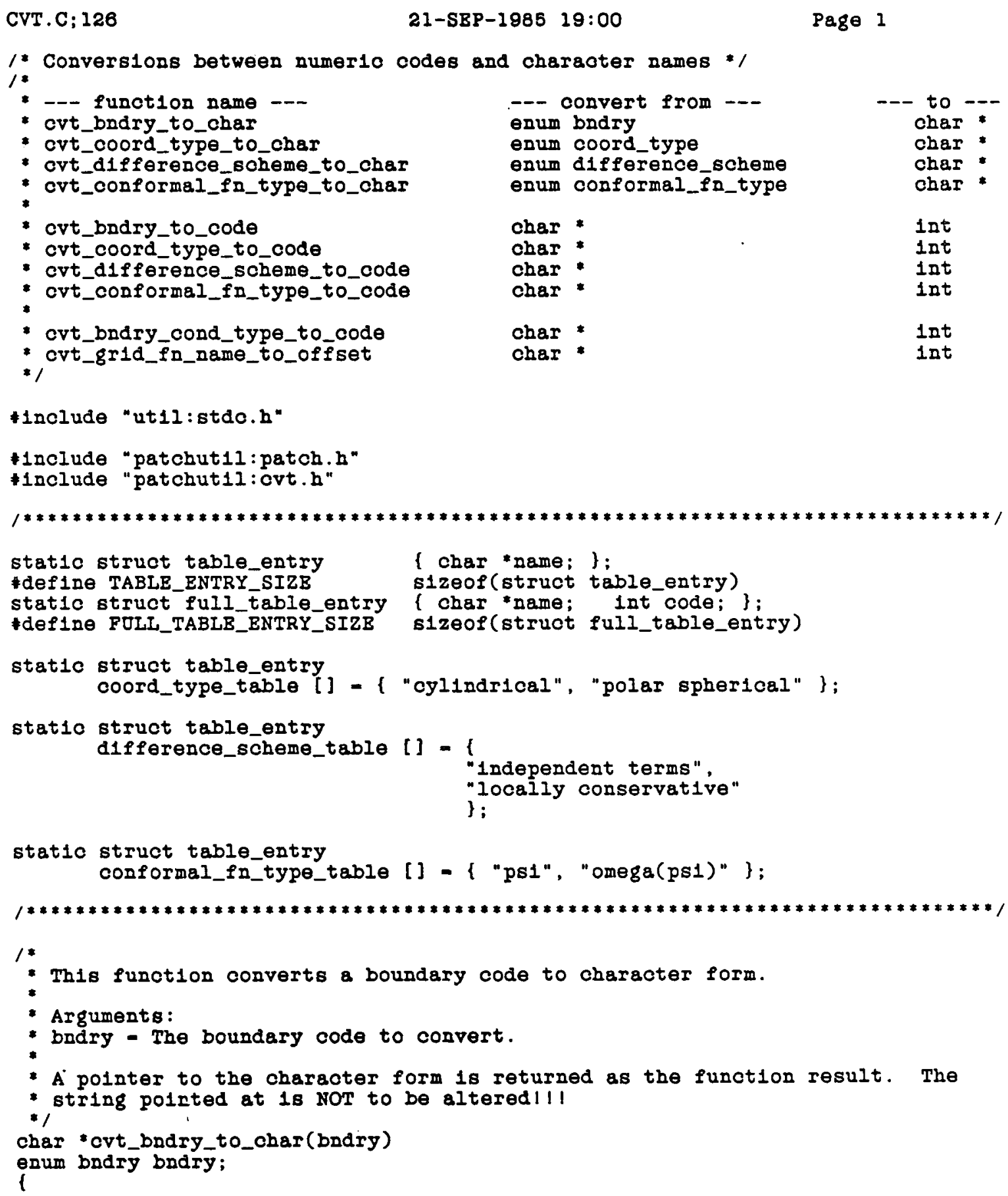


patchutil directory

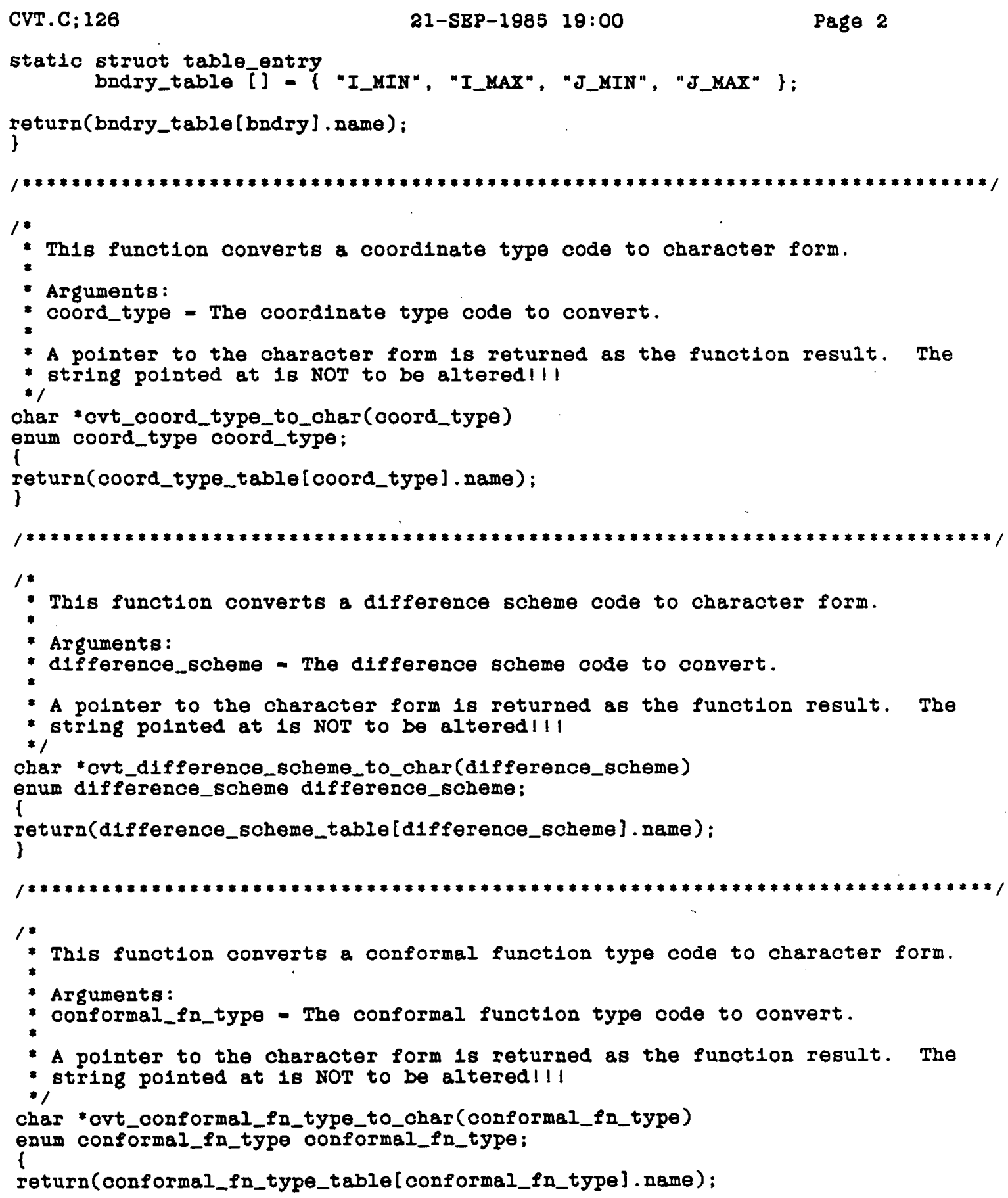


patchutil directory

CVT.C; 126

21-SBP-1985 19:00

Page 3

\}

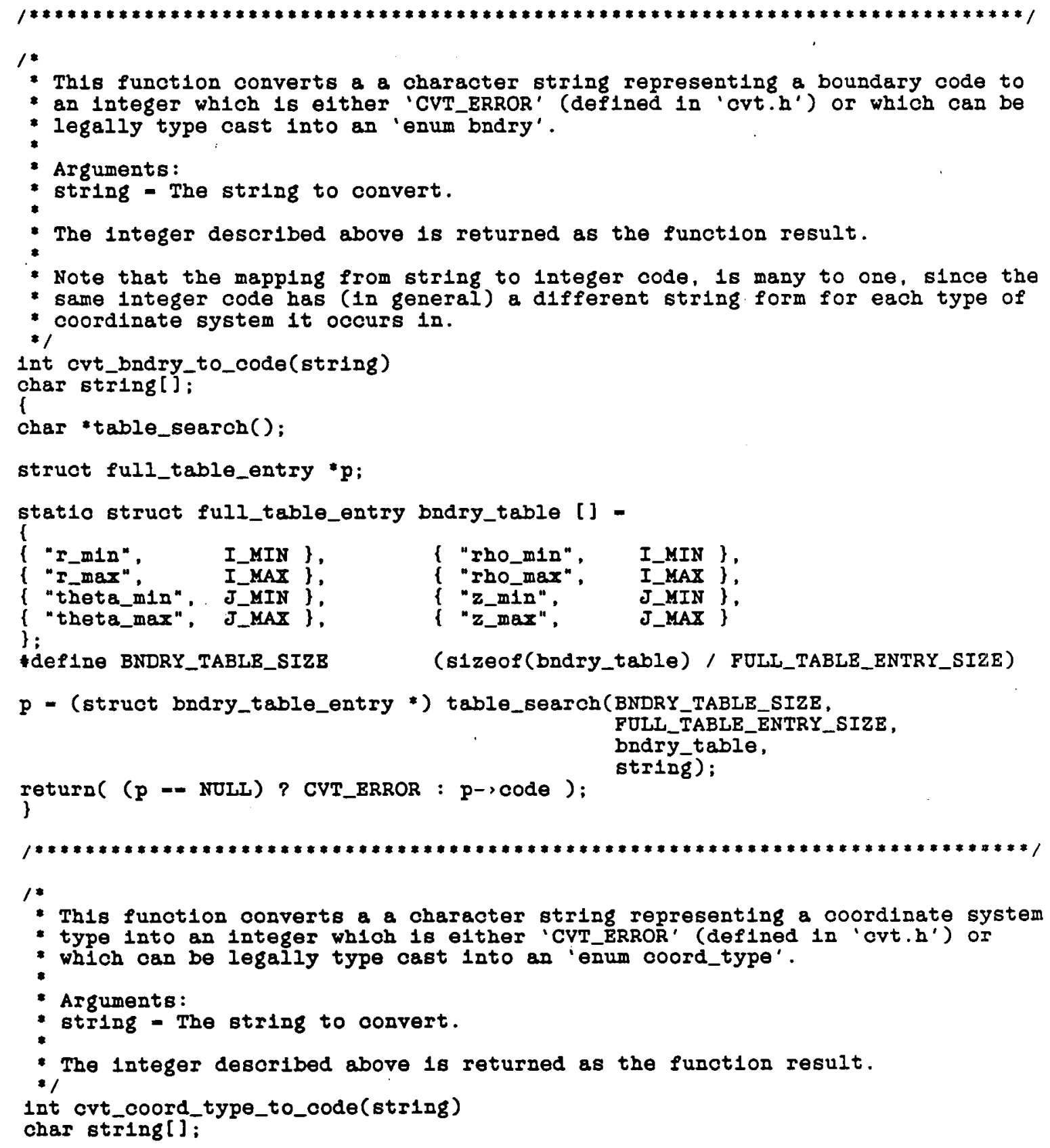




\section{patchutil directory}

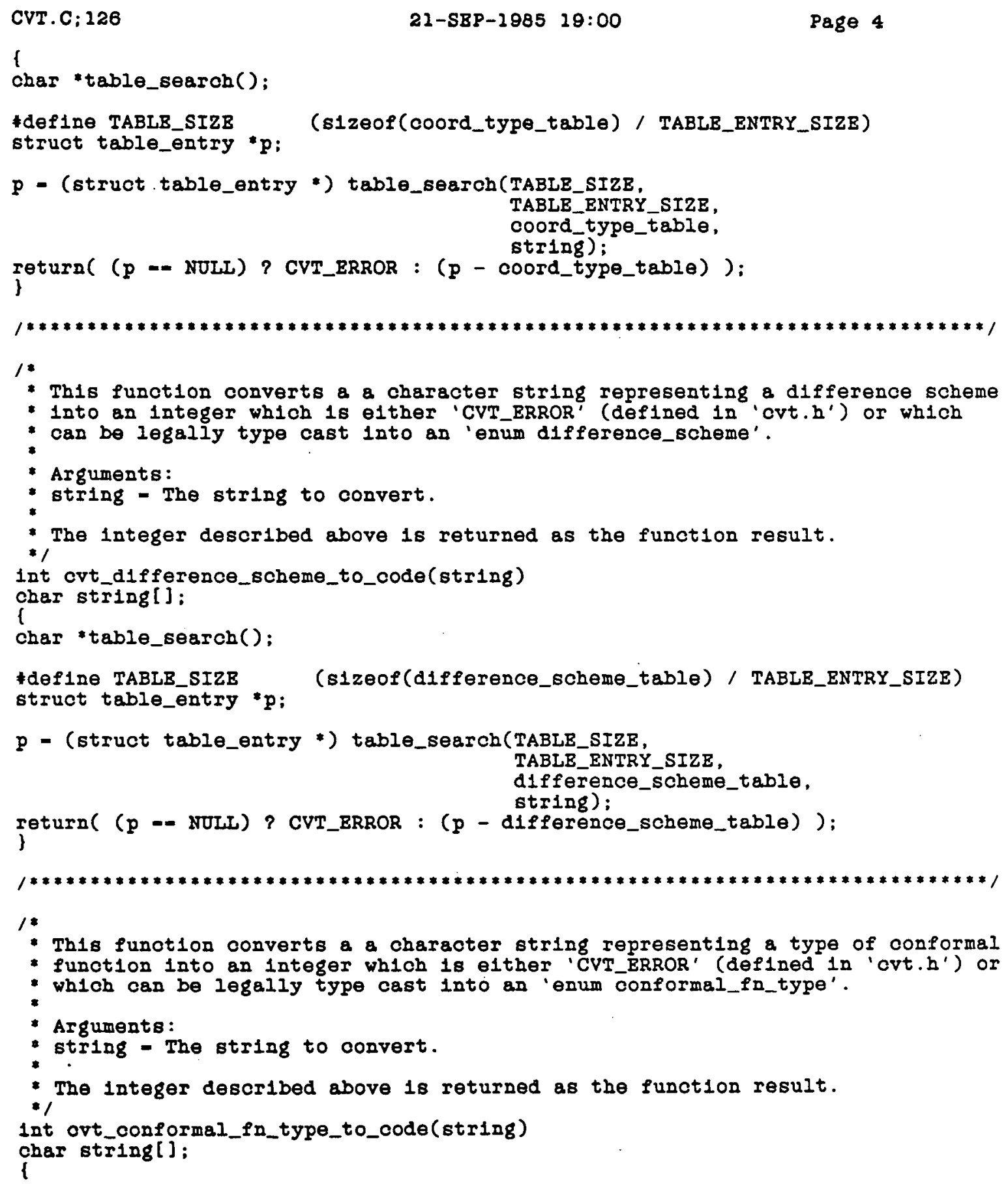


patchutil directory

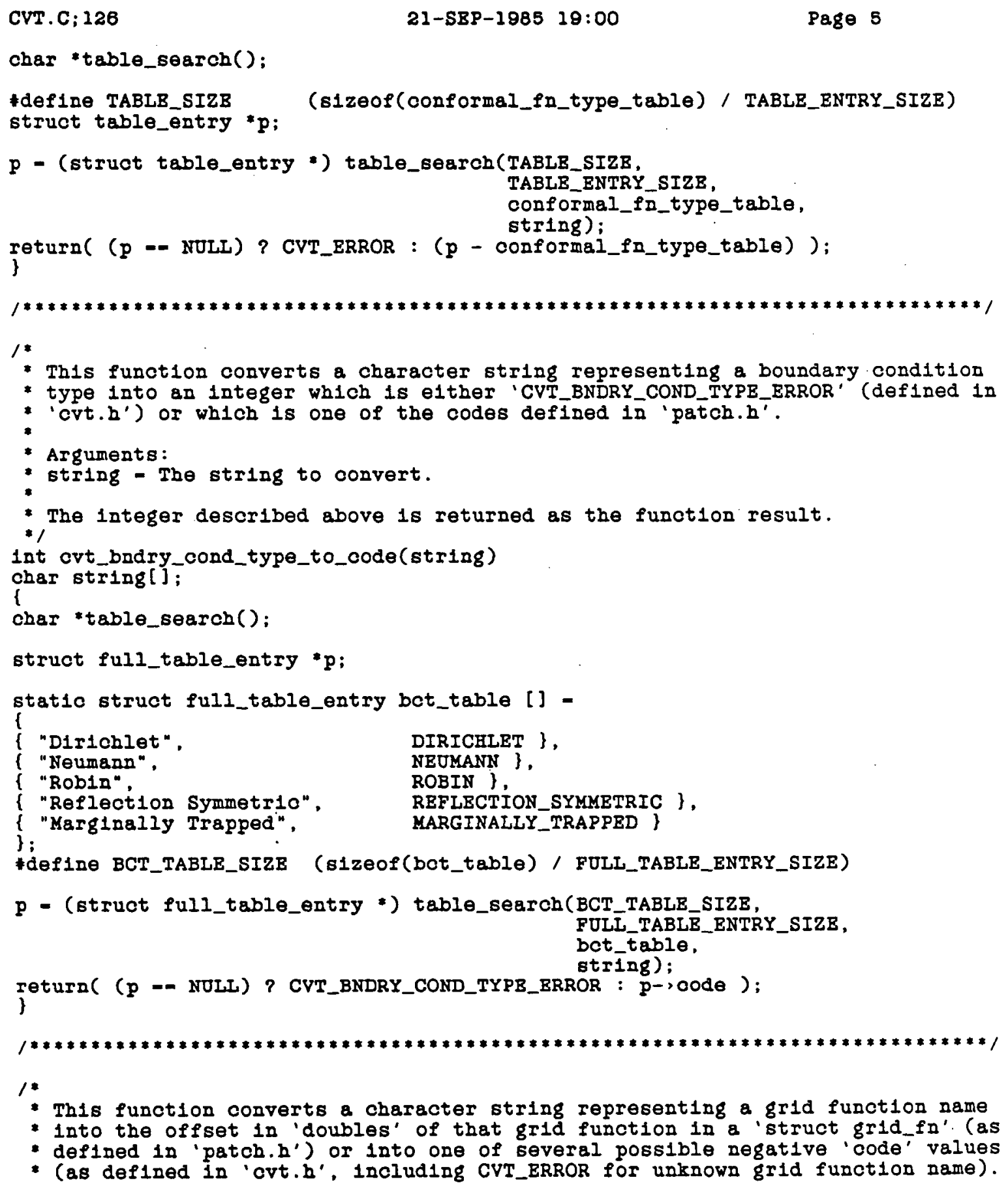




\section{patchutil directory}

CVT.C; 126

21-SBP-1985 19:00

Page 6

$*$

- Arguments :

- string - The string to convert.

- The integer desoribed above 18 returned as the function result.

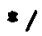

int crt_grid_fn_name_to_offset(string)

char string[];

1

char table_search ();

struct full_table_entry *p;

static struct full_table_entry grid_en_name_table [] -

I "cl",

\{ "c2".

( "C3"

\{ "04",

$\{" 05 n$,

\{ $\mathrm{s}$ " 。

"psi",

\{ "T",

\{ "psi-mm".

1 "psi-am",

( "ps1-tem",

\{ "psi-tea",

f "psi-ssmean".

("psi-ssm1n",

1 "ps1-zar1s".

( "psi-equator",

( "I (true)",

( "krr".

OFFSET_CI \},

OFFSET_C2 \},

OFFSET_C3 \},

OFFSET_C4 \},

OFFSET_C5 \},

OFFSET_S \}

OFPSBT_PSI \},

OFFSBT_R \},

PSI_MINUS_MASS_MONOPOLES \},

PSI_MINOS_AREA_MONOPOLES \},

PSI_MINUS_TOT_E_PER_M_MONOPOLES \},

PSI_MINUS_TOT_E_PER_A_MONOPOLES $\}$,

PSI_MINOS_SPEERICAIIY_SYM_MEAN \},

PSI_MINUS_SPHERICALIY_SYM_MIN \},

PSI_MINUS_Z_AXIS \},

PSI_MINUS_EQDATOR \},

TROE_RESIDOAL\}, B_HAT_RR \}

;

\#define GFN_TABLE_SIZB (s1zeof(gr1d_fn_name_table) / FULL_TABLE_ENTRY_SIZE)

$p=$ (struct full_table_entry *) table_search(GFN_TABLE_SIZE, FULL_TABLE_ENTRY_SIZE. grid_fn_name_table,

return( ( $p-$ NOLL) ? CVT_BRROR : $p-\operatorname{cod} \theta)$; string); \} 
patchut1l directory

DUMP.C;63

21-SBP-1985 $19: 02$

Page 1

1* This file conteins routines to dump out the various data structures */

$1 *$

- The following functions are contained in this file:

* dump_bhs - Dumps 'bhs' data structure.

- dump_grid - (a1tto for 'grid')

- dump_patch_bndry - (d1tto for 'patch_bndry')

$* 1$

*1nclude "util:stdc.h"

\#1nclude "patchut1l:blackhole.h"

1 Include "patchutil:patch.h"

"Include "patchut1l: tempbndry.h"

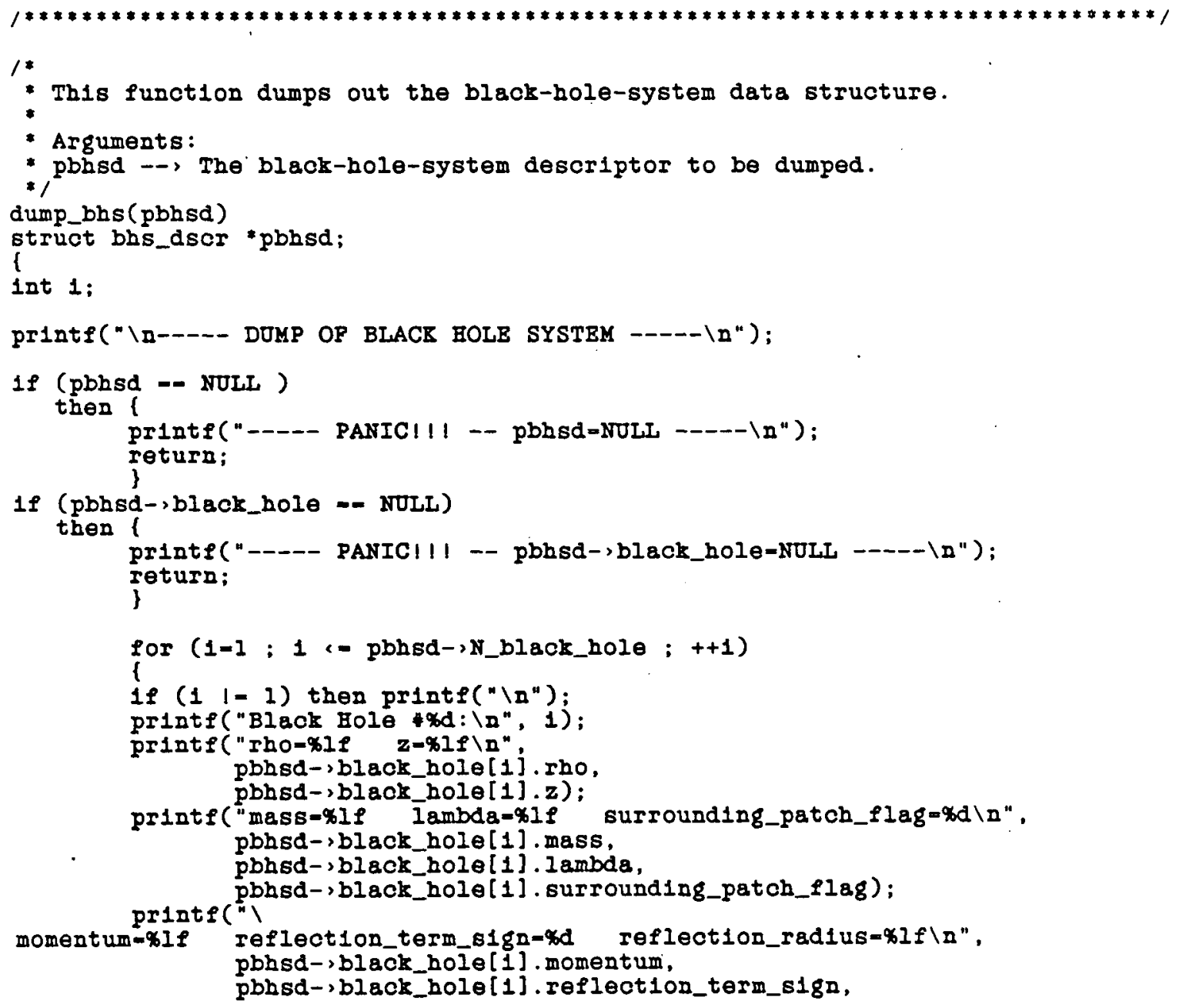


patchutil directory

DUMP.C; 63

$$
\text { 21-SBP-1985 19:02 }
$$

pbhsd-, black_hole[1].reflection_rad1us);

printe ("spin=xIf\n",

\}

pbhsd->black_bole[1].sp1n);

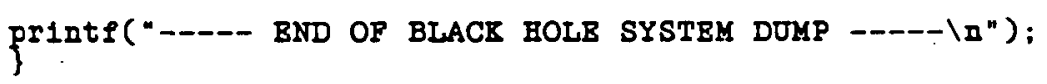

" Th1s function dumps out the 'grid' data structure.

- Arguments:

* pgd - The grid data structure to be dumped.

dump_gr1d(pgd)

struct grid_dscr pgd;

f

int $p$;

enum bndry bndry:

1nt 1J;

char *1j_string:

lot 1j_mIn, 1j_max;

reglster struct coord_patch_dsor *ptr;

register struct line_end_dscr "pled;

int N_grid_points();

char cvt_coord_type_to_char();

char cvt_difference_scheme_to_char();

char cvt_conformal_fn_type_to_char();

char cvt_bndry_to_char():

printe("\n---- DUMP OF GRID SYSTEM ----1n");

if (pgd $=-$ NOLL)

then \{

printe(" return:

PANICIII -- Pgd-NOLI $-(n)$

j

$1 f$ (pgd->patch -- NOLL)

then 1

printe("---- PANICIII -- pgd->patch-NOLI -----\n");

return;

\}

printf("Th1s Grid System has a total of sod patches, sd grid points $\backslash n$ ", pgd-iN_patch, N_grid_points (pgd, 0));

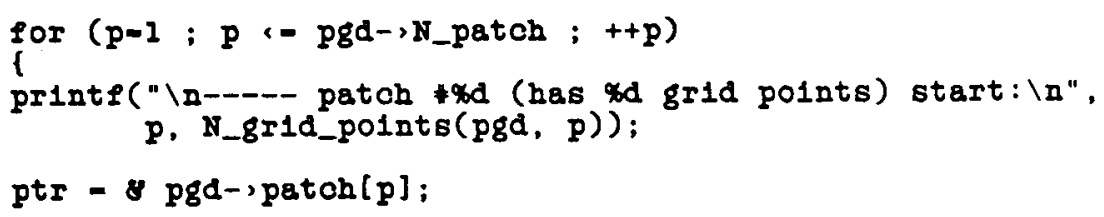




\section{patchutil directory}

DUMP.C; 63

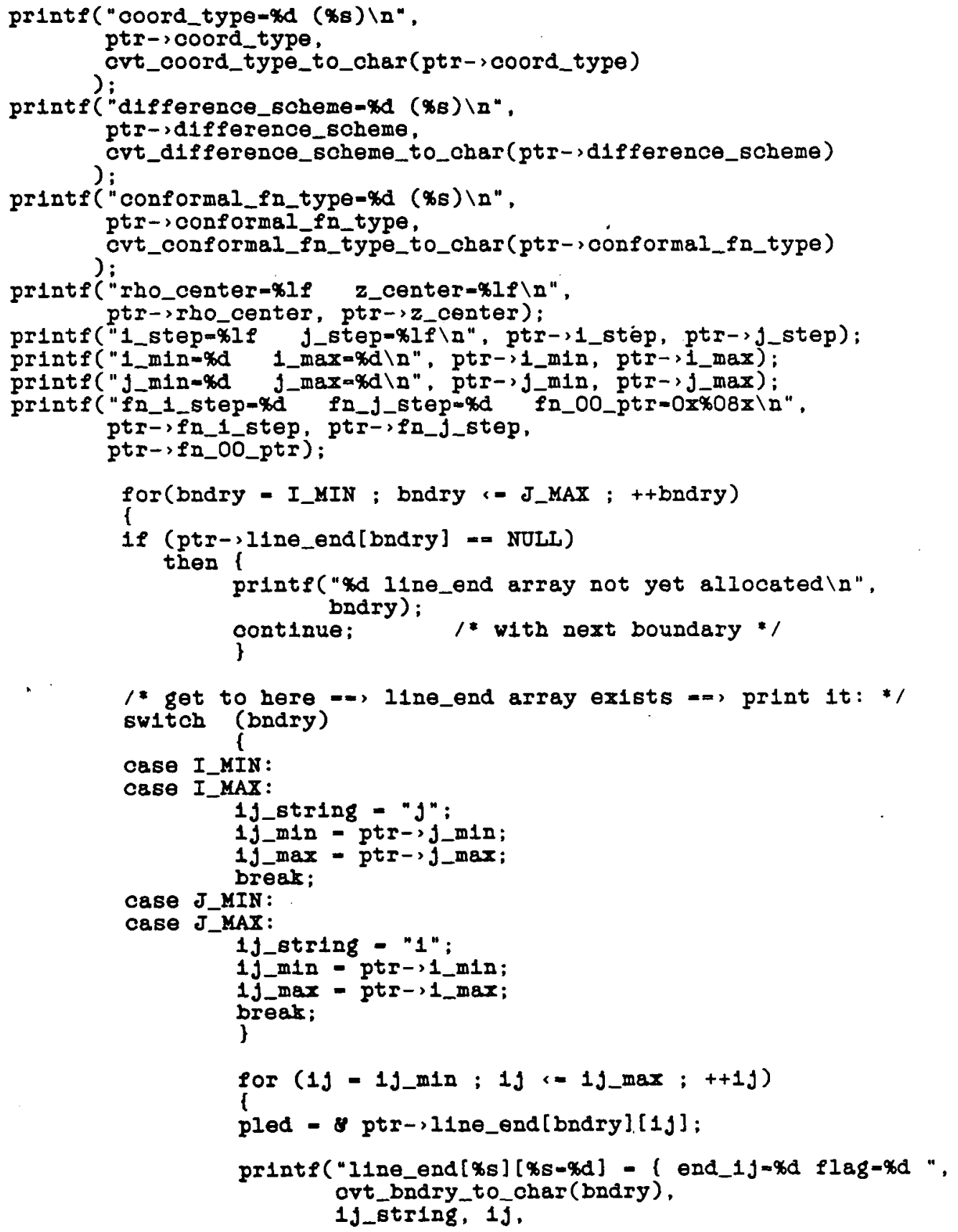




\section{patchutil directory}

DUMP.C;63

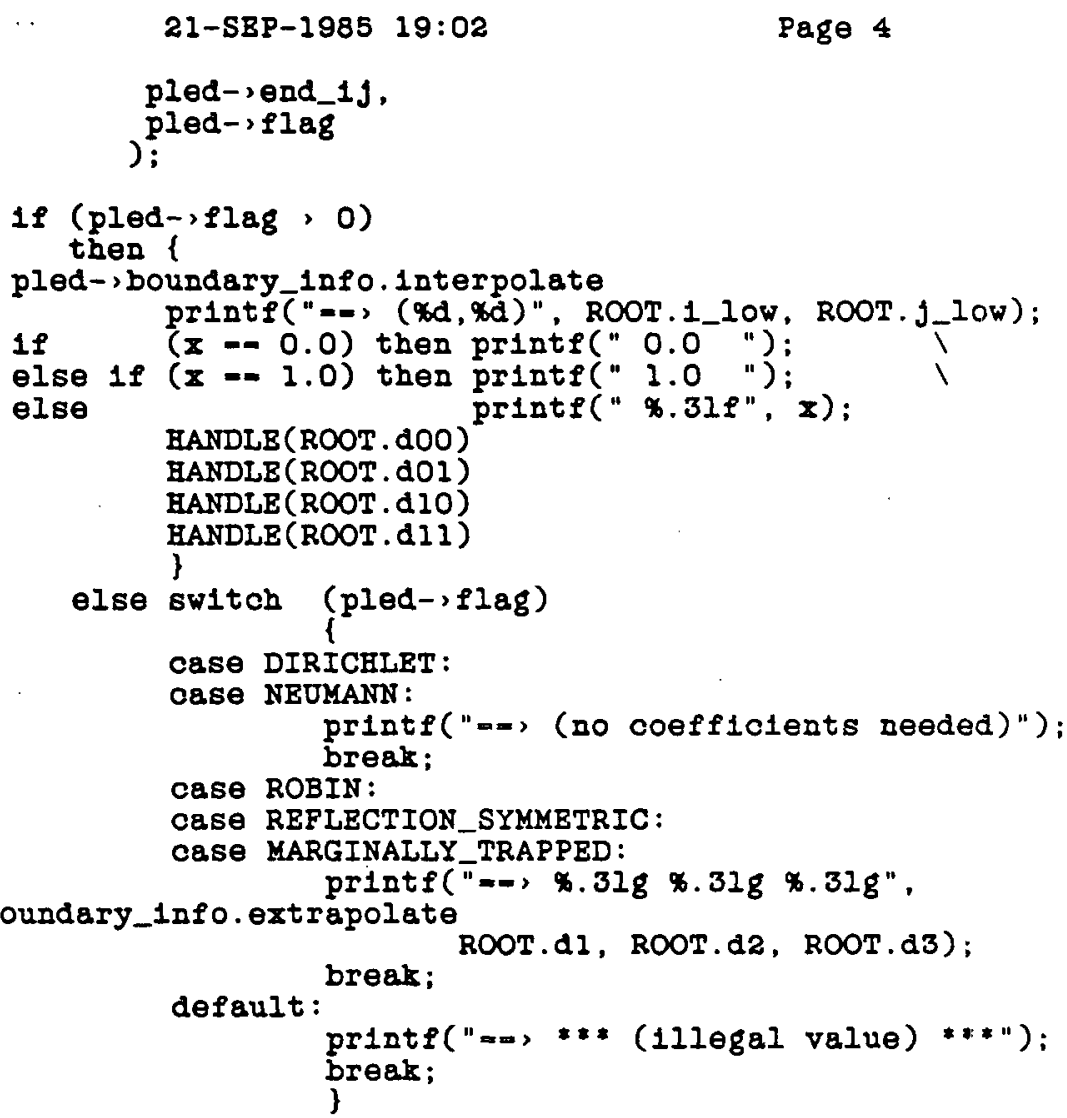

$1 *$

- This function dumps out the temporary patch boundary data structure.

- Arguments :

- N_patch - The number of coordinste patches.

* patch_bndry -- (1 or181n) The patch boundary data structure to dump. 
patchutil directory

DUMP.C;63

dump_patch_bndry (N_patch, patch_badry)

Int $\bar{N}_{-}$patch;

reg1ster struct patch_bndry *patch_bndry;

1

reg1ster struct bndry_defn_term "ptr;

Int p;

enum bndry bndry;

printf(" $\backslash n----$ DUMP OF PATCH BOUNDARY TEMPORARY DATA STROCTURE ---- $\backslash n "$ );

$1 f$ (patch_bndry $=$ - NOLL)

then 1

printf ("patch_bndry-NULL\n");

return;

\}

for $\left(p=1 ; p<-N_{-p a t c h} ;++p\right)$

(

printf("\n----- patch \$sd start: \n", p);

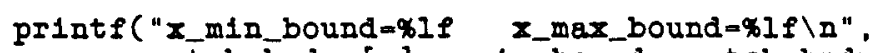
patch_bndry[p].x_min_bound, patch_bndíy[p].x_max_bound);

printe("y_min_bound=xif y_max_bound=xif $\backslash n$ ", patoh_bndry[p].y_m1n_bound, patch_bndry[p].y_max_bound);

1* print out 11st, 1f any: *I

for (bndry = I_MIN ; bndry c- J_MAX ; ++bndry)

\{

ptr = patch_bndry[p].bdt_list[bndry];

If (ptr $=-$ NOLL)

then

printe ("\nxd list --, (NULL) \n", bndry);

continue; /* with next bndry */

\}

printe ("Inxd 11st: $\backslash n$ ", bndry);

for (; ptr 1- NULL ; ptr - ptr-snext)

\{

printe("--) defn_type-xd", ptr->defn_type);

if (ptr->defn_type = CONSTANT_BNDRY_TYPE)

then printe (" defn_value.value=ólf $\backslash n$ ",

else printe (ptr-idefn_value.value);

defn_value.patch_1nfo. ( patch=od

bndry-\%d ) $\backslash n "$ "

ptr-idefn_value.patch_1nfo.patch,

ptr-idefn_value.patch_1nfo.bndry);

printf(" bndry_cond_type-\%d", ptr-,bndry_cond_type);

if (ptr->bndry_cond_type - TRUE_BNDRY_COND)

then printe(" bndry_cond_value.bndry_cond-' $x s^{\prime} "$.

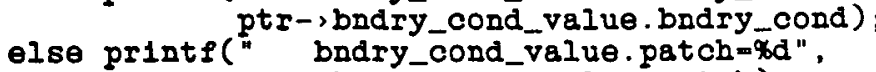

printe(" $\backslash n ")$; ptr->bndry_cond_value.patch); 
patchutil directory

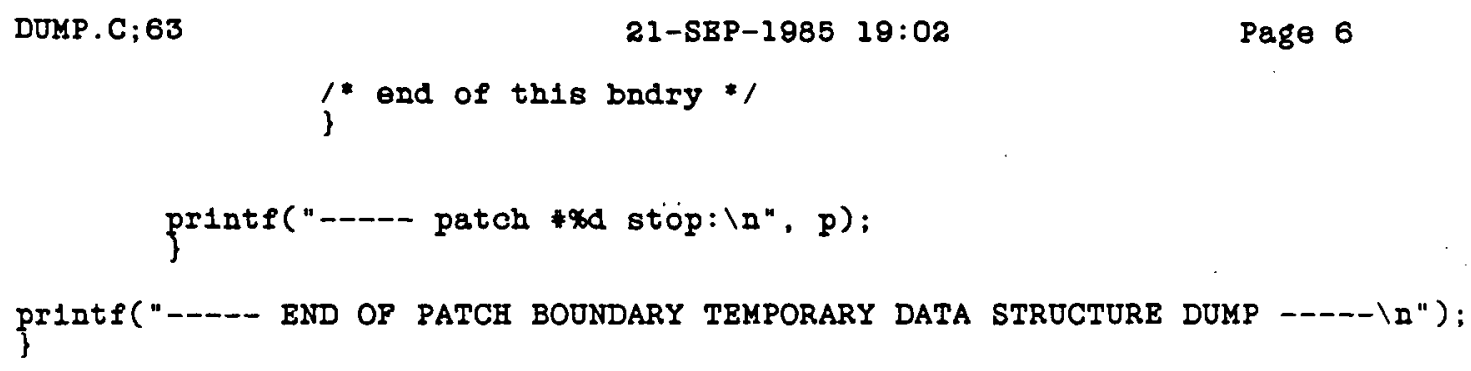


patchutil directory

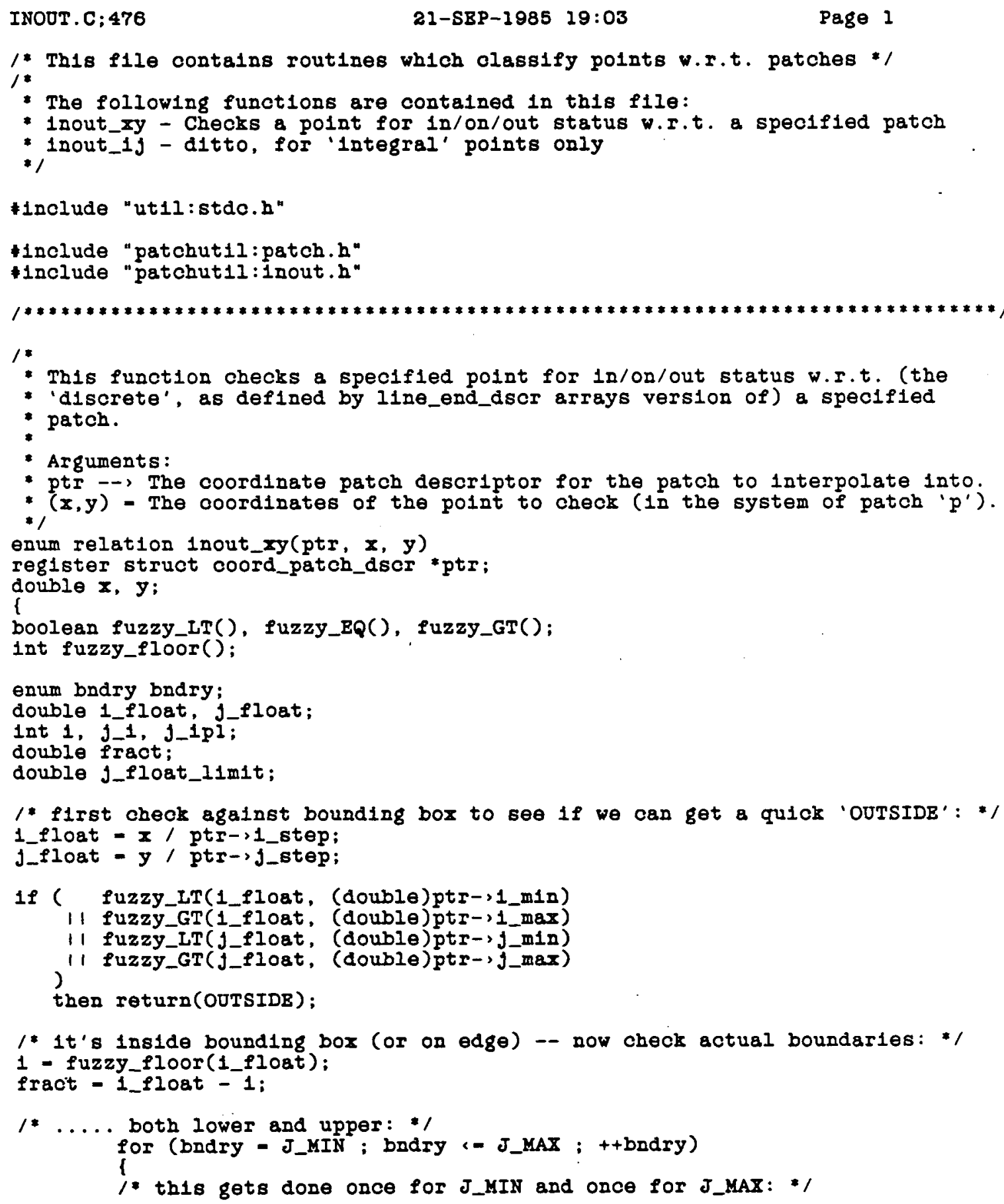


patchutil directory

INOUT.C; 476

$1-1-$ ptr->11ne_end[bndry] [1] .end_1j;

If ( 1 = ptr->1_max)

then j-1pl - j_i;

else $j$ ipl $=$ ptr-11ine_end[bndry $][1+1]$.end_1j;

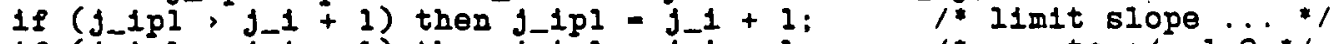

if (j_ipl. j_1 - 1) then j_ipl-j_1-1; $1 * \ldots$ to $+1-1.0 * 1$

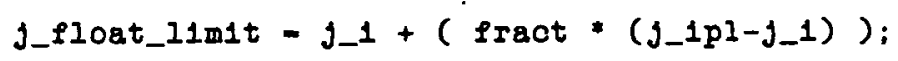

$1 f$ (fuzzy_EQ(j_float, j_float_lim1t)) then return(ON_BNDRY);

$1 f$ ( ( )

11 ((bndry -- J_MAX) \&8 (j_float, j_float_lim1t))

\}

then return(OOTSIDE);

return(INSIDE);

\}

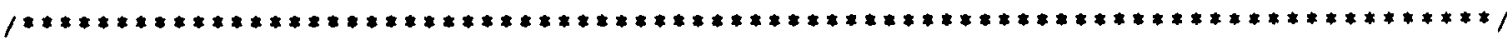

$1 *$

* This function checks a specifled '1ntegral' point for in/on/out status

*.r.t. a specified patch.

* Arguments :

- ptr -- The coordinate patch descriptor for the patch to check.

- $(1, j)$ - The 'integral' coordinates to check.

$\because$

enum relation 1nout_1j(ptr, $1, j)$

reglster struct coord_patch_dscr *ptr;

Int $1, j$ :

l

enum relation 1nout_ry();

vold cvt_1J_to_xy():

double $x, y$;

cvt_1j_to_xy(ptr, 1, j, \&x, vy);

return(1nout_xy (ptr, $x, y))$;

) 
patchut1l directory

INPUTPAR.C; 362

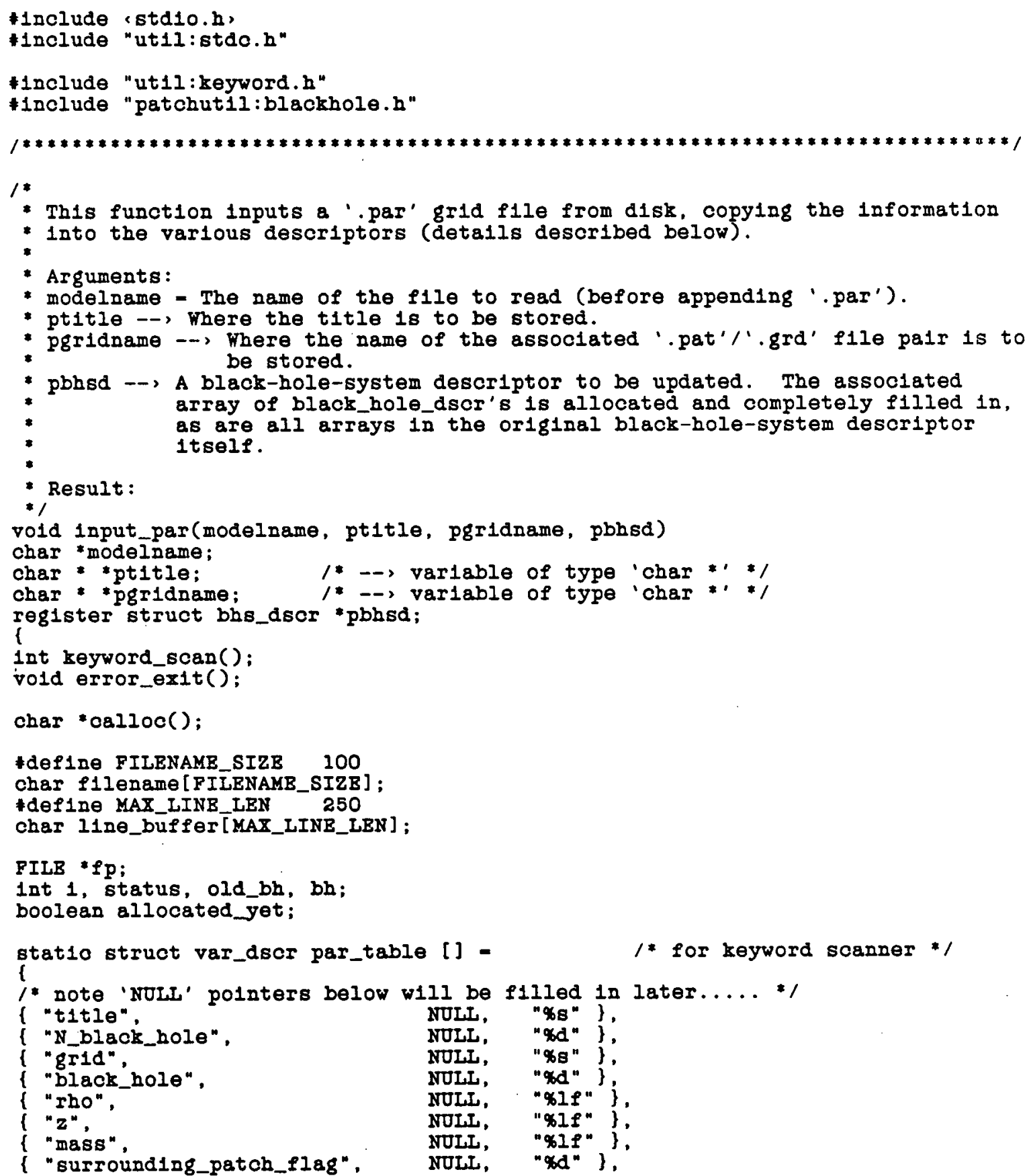


patchutil directory

INPOTPAR. C; 362

$\left\{\begin{array}{l}\text { "momentum", } \\ \text { "reflect1on_term_81ga", } \\ \text { l "seflect10n_radius", }\end{array}\right.$

† def1De PAR_TABLB_SIZB

(sizeof(par_table)/ sizeof(struct var_dscr) )

$1 *$

- BXECUTION STARTS HERE:

$*$

1. 1111 in keyword scanner, table entries, as much as possible: *

1 - 0 ; / offset 1n par_table of lst entry to be fliled 10 *

par_table[1++].value = (char : ptitle;

par_table[1++].value - (1nt *) Epbhsd->N_black_hole;

par_table $[1++]$.value - (char * ) pgridname;

par_table[1++].value - (1nt ) sbh;

1* open elle: *

sprintf(fllename, "Xs.par", modelname);/"form 'full' flle name */

$f p=$ fopen ( 1 llename, "I");

$1 f$ ( $f p=-N U L L)$

then error_exitCOR_EXIT.

**** 1nput_par: could not open $f 11 \theta \backslash " x_{s} \backslash \ldots "$,

fllename); / DOES NOT RETURN */

1 process flle: *

bh = pbhsd->N_block_hole - O;

allocated_yet - FALSB;

while (fgets(11ne_buffer, MAX_LINE_LEN, $f p)$ (- NULL)

l

old_bh = bh;

status - keyword_scan(PAR_TABLB_SIZB, par_table, 11ne_buffer);

$1 f$ (statua, 0)

then error_exitcor_EXIT.

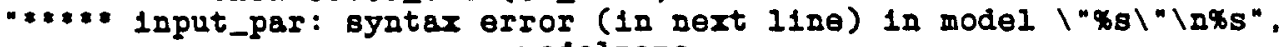
modelname, I1ne_buffer):

/* DOBS NOT RETURN *

$1 f\left(b h I=0 l d \_b b\right)$

then

1* f11l in remalning keyword scanner table entries: *

1 - 4; / offset 1n par_table of lst entry to be fliled in $/$

†def1De LBS

†define RBS_ROOT par_table $[1++]$. $v a 1$ ue

\& phid-iblack_hole[bh]

IES = (double ) RAS_ROOT.rho:

IES - (double $*$ ) RHS_ROOT.z;

IHS - (double *) RHS_ROOT.mass;

LHS - (1nt *) RHS_ROOT. Burround1ng_patch_flag;

IHS - (double *) RHS_ROOT.momentum;

IHS - (1nt *) RHS_ROOT.reflection_term_s1gn;

IHS - (double :) RES_ROOT. reflect 10 D_rad1us;

IES - (double *) RES_ROOT.sp1n;

) 
patchutil directory

INPOTPAR.C; 362

21-SEP-1985 19:03

Page 3

$1 f($ ( 1 allocated_yet) \&8 (pbhsd-2N_black_hole 1-0) ) then \{

1* allocate array of black_hole_dscr's: */ pbhsd->black_hole - ALLOC(pbhsd->N_black_hole,

$1 f$ (pbhsd-, black_hole =- NULL)

then error_exit(OR_EXIT,

***** 1nput_par: 1nsufficient memory for \%d black holes in model $\backslash " \% s \backslash " \backslash n "$, pbhsd-, N_black_hole, modelname); $/$ * DOES NOT RETURN * --pbhsd->black_hole; $\quad /$ adjust for 1 orig1n indering */ aliocated_yet = TROB;

\} \}

folose ( $f p)$;

\} 


\section{patchutil directory}

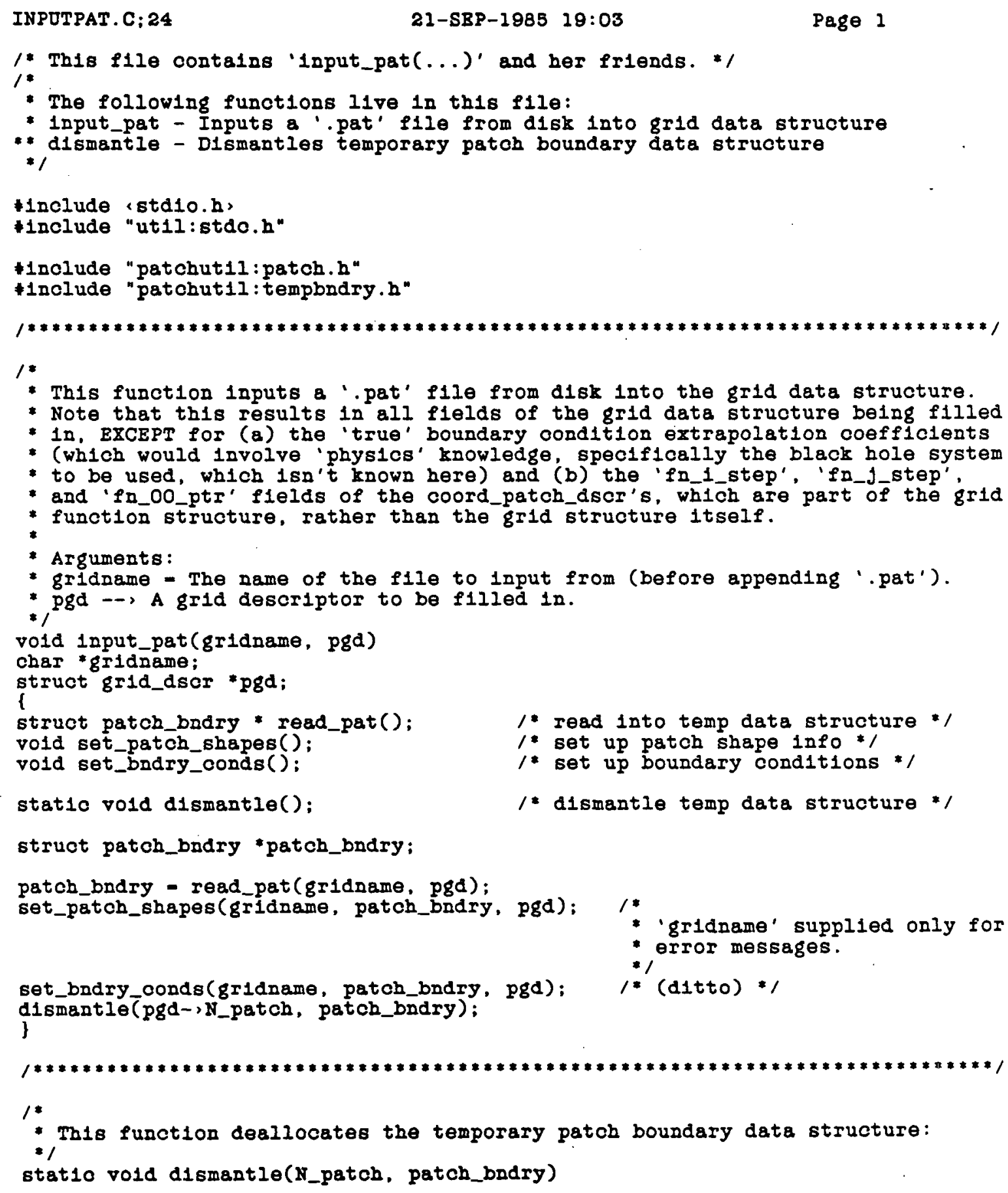


patchutil directory

INPOTPAT.C;24

21-SEP-1985 19:03

Page 2

int N_petch;

register struct patch_bndry patch_bndry:

I

vold cfree();

1nt $p$;

enum bndry bndry;

reglster struct bndry_defn_term *ptr, *next_ptr;

for $(p-1 ; p<=$ N_patch ; $++p)$

for (bndry = I_KIN ; bndry < = J_MAX ; ++bndry)

I* free badry'th chain: */

ptr = patch_bndry[p].bdt_list [(1nt)bndry];

while (ptr $1=$ NULL)

i

next_ptr = ptr->next;

cfree (ptr);

ptr = next_ptr;

\}

\}

cfree(patch_bndry); 
patchutil directory

IPARTEST.C; 12

*1nclude "ut1l:stdo. $h$ "

"1nclude "patchut1l:blackhole.h"

1nclude "patchut1l:patch.h"

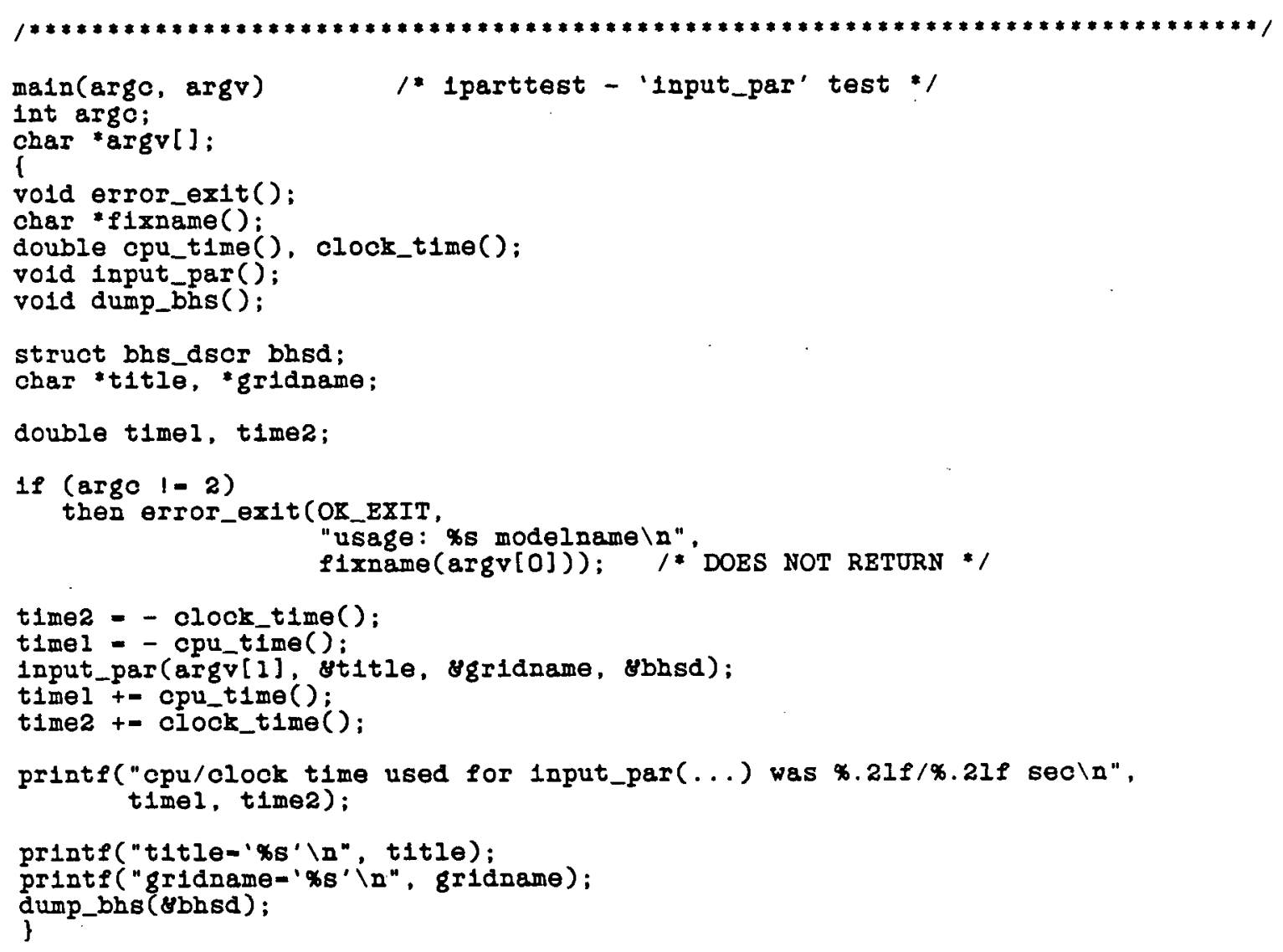


patchutil directory

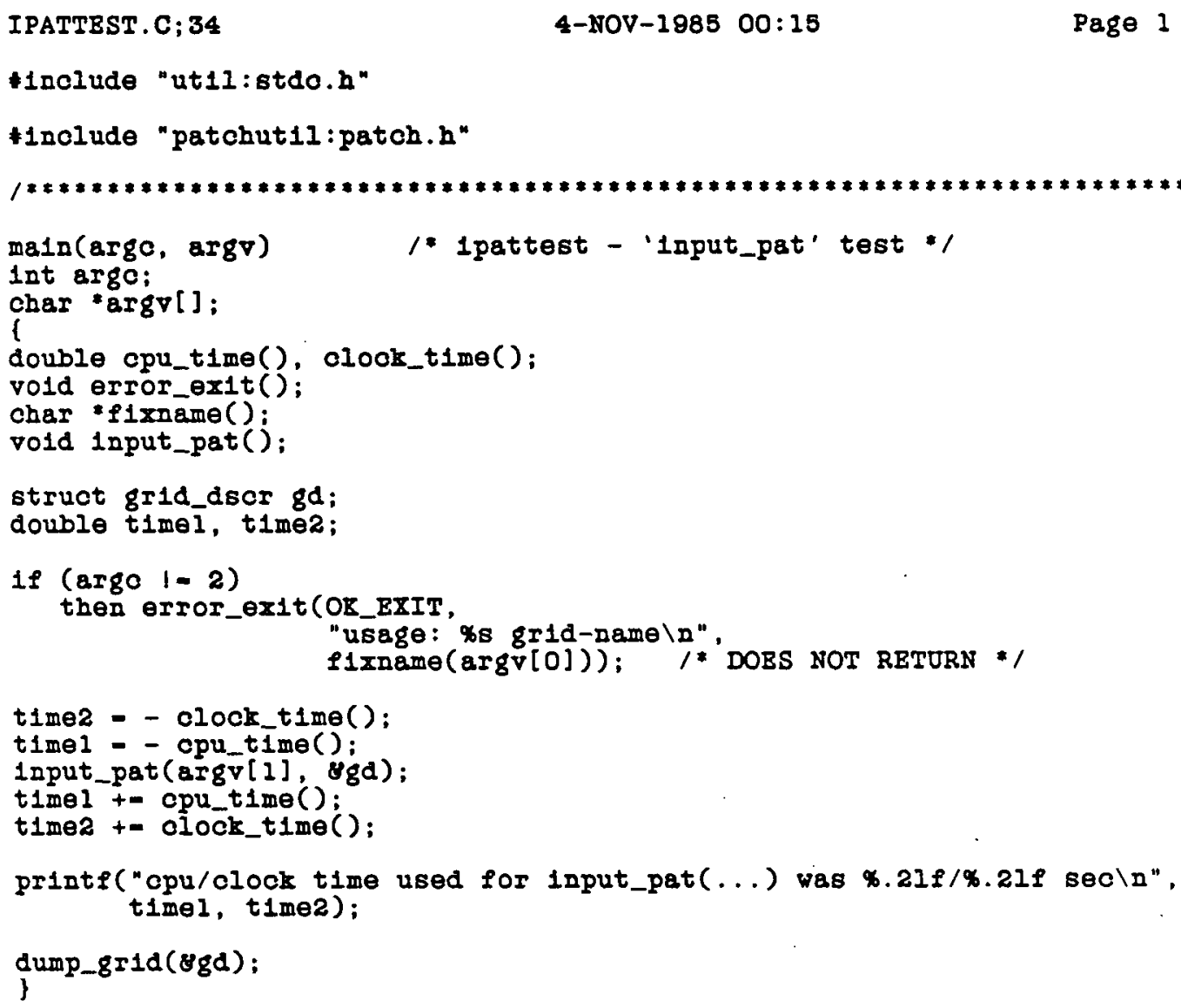

*1nclude "ut1l:stdo.h"

1nclude "patchut1l:patch.h" 
patchutil directory

NGRIDPTS.C; 3

21-SBP-1985 19:04

Page 1

*1nclude "ut1l:stdc.h"

+1nclude "patchut1l: patoh.h"

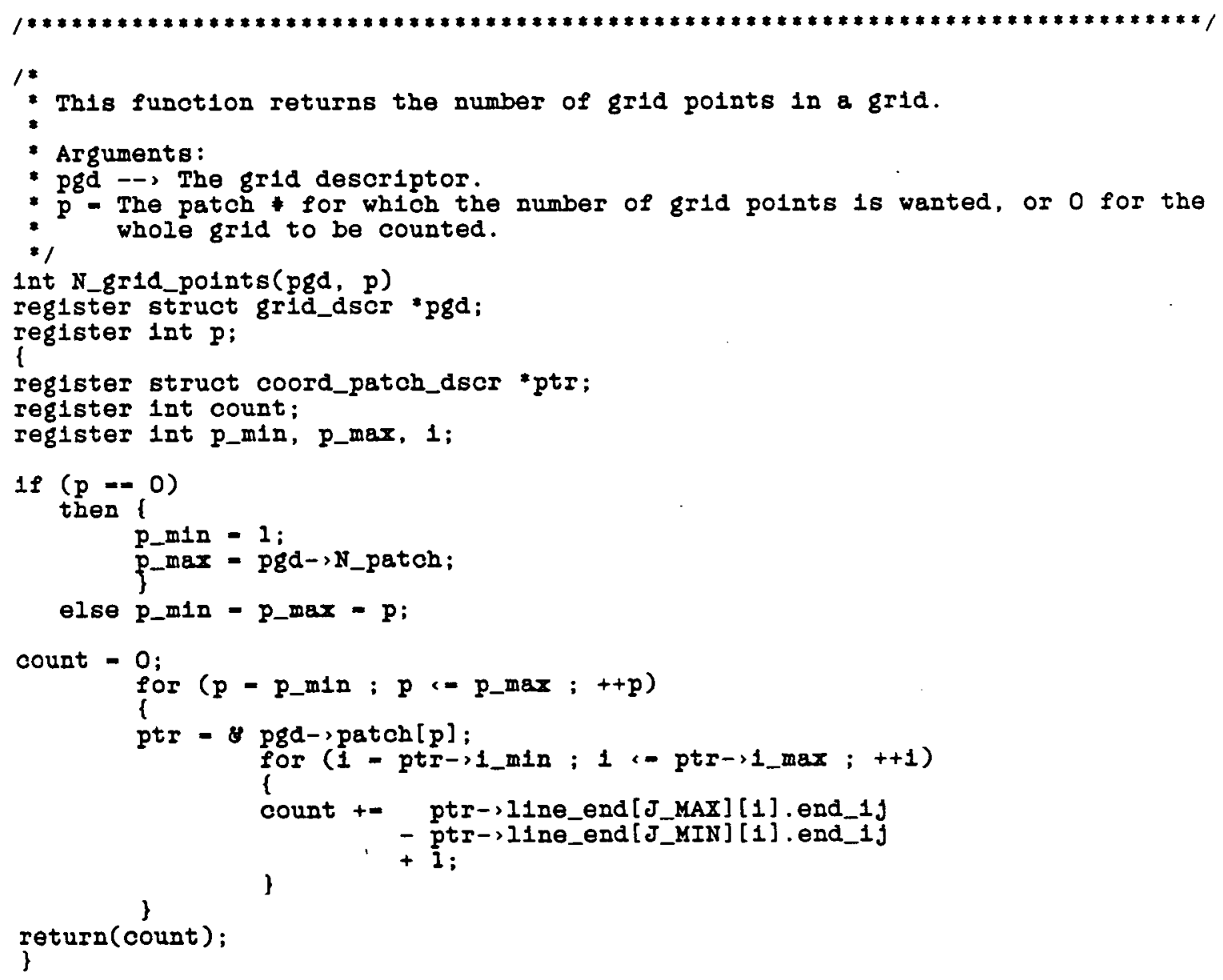




\section{patchutil directory}

PARLOG.C; 12

21-SBP-1985 19:04

Page 1

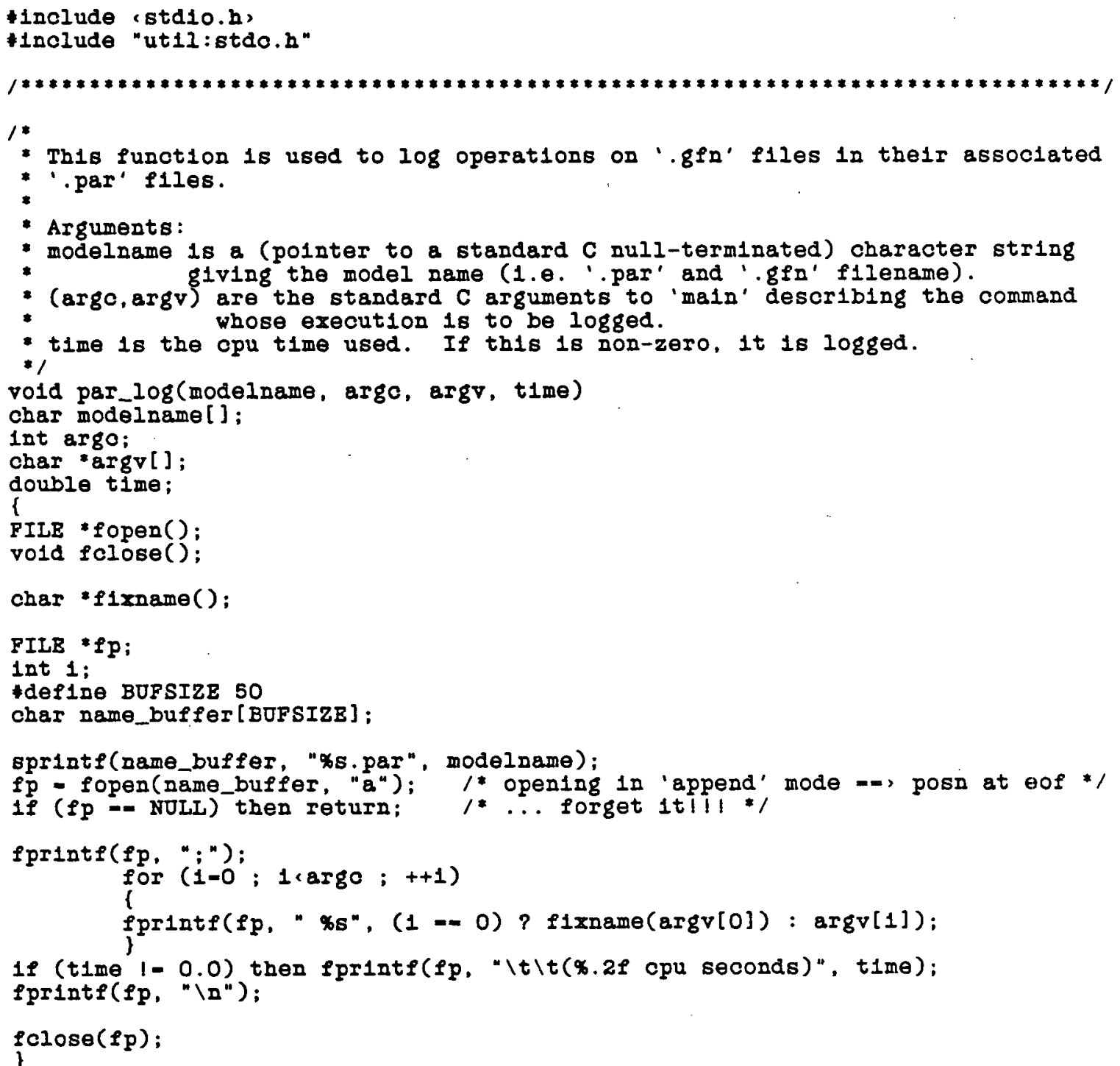


patchutil directory

READPAT.C; 361

21-SBP-1985 19:05

Page 1

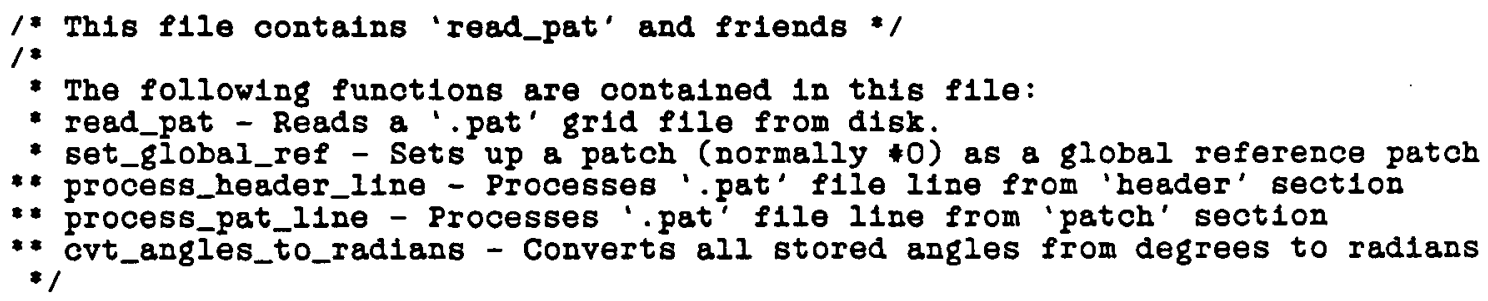

1nclude ista10.h

"1nclude "ut1l:stdc.h"

*1nclude "ut1l:keyword.h"

1nolude "patchut1l:patch.h"

1nclude "patchut11: tempbndry. $h$ "

1nclude "patchut1l: cvt.h"

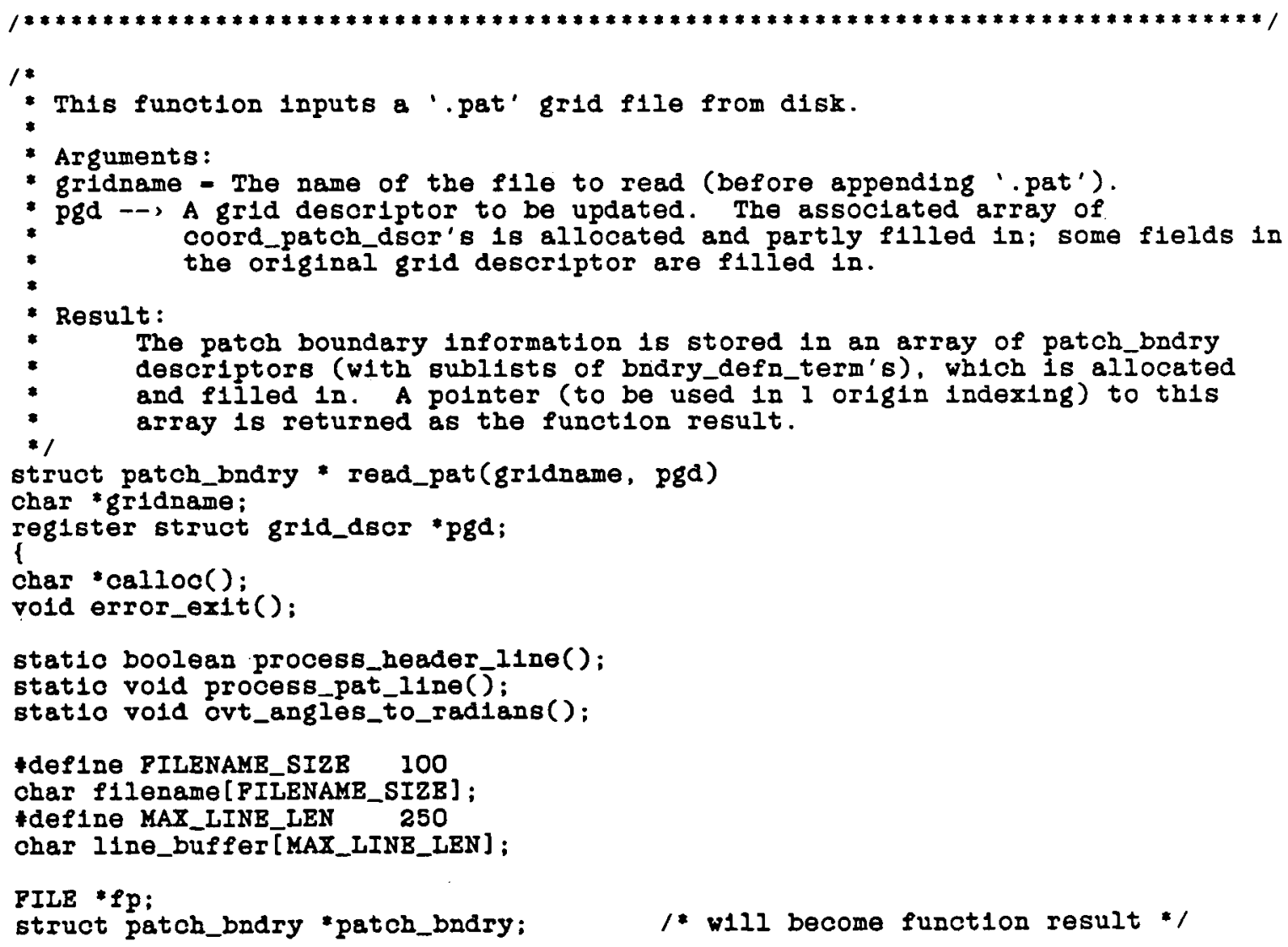


patchutil directory

READPAT . C: 361

enum \{ IN_HEADER, IN_PATCHBS \} stato;

Int done_flag;

1* open flle: *I

sprintf(filename

fp - fopen (fllen

if ( $\rho p=$ NOLL)

then error_exit(OR_EXIT,

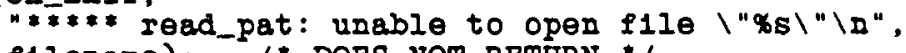

f1lename); /* DOES NOT RETURN */

1* process file: :

state - IN_HEADER;

while (

\{

switch (state)

cese IN_HBADER:

done_flag - process_header_l1ne(gridname, I1ne_buffer, pgd);

1f (done $f 18 \mathrm{~g})$

then \{

* allocate space: */

pgd->patch - ALLOC (pgd->N_patch + 1 ,

struct coord_patch_dscr):

patch_bndry - ALIOC(pgd-, N_patch, struct patch_bndry);

if ((pgd->patch - NULI) 11 (patch_badry = NUII)) then error_exit(OK_EXIT,

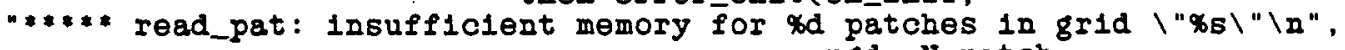
pgd->N_patch,

Gridname; ; $/$ DOES NOT RETURN *

set_global_ref(\& pga-ppatch[0]);

- patch_bndry; $/ *$ shift to origin $1 * /$ state - IN_PATCHES;

break;

\}

case IN_PATCHES :

process_pat_l1ne(gridname, line_buffer, pgd, patch_bndry); break;

\} \}

folose $(p p)$;

cvt_angles_to_radians (pgd, patch_bndry);

1* we're done with the '.pat' flle now! */

return(patch_bndry);

1* f1x stored angles *I

\}

$1 *$ (input in degrees) */

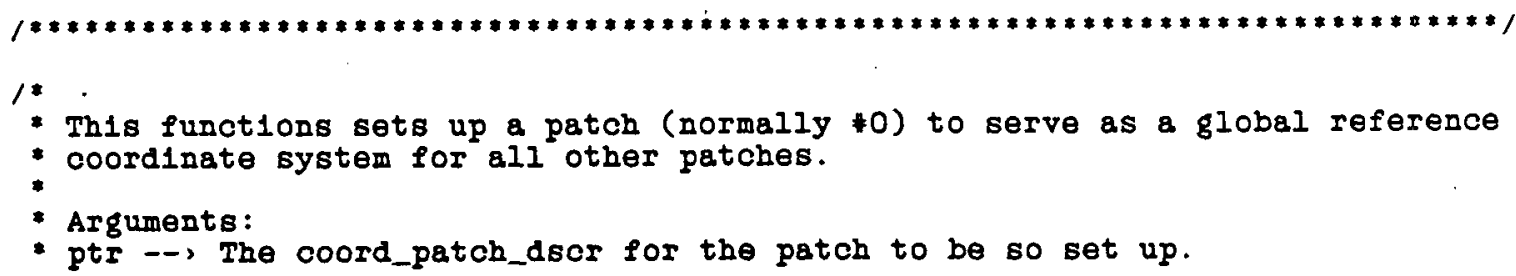




\section{patchut1l directory}

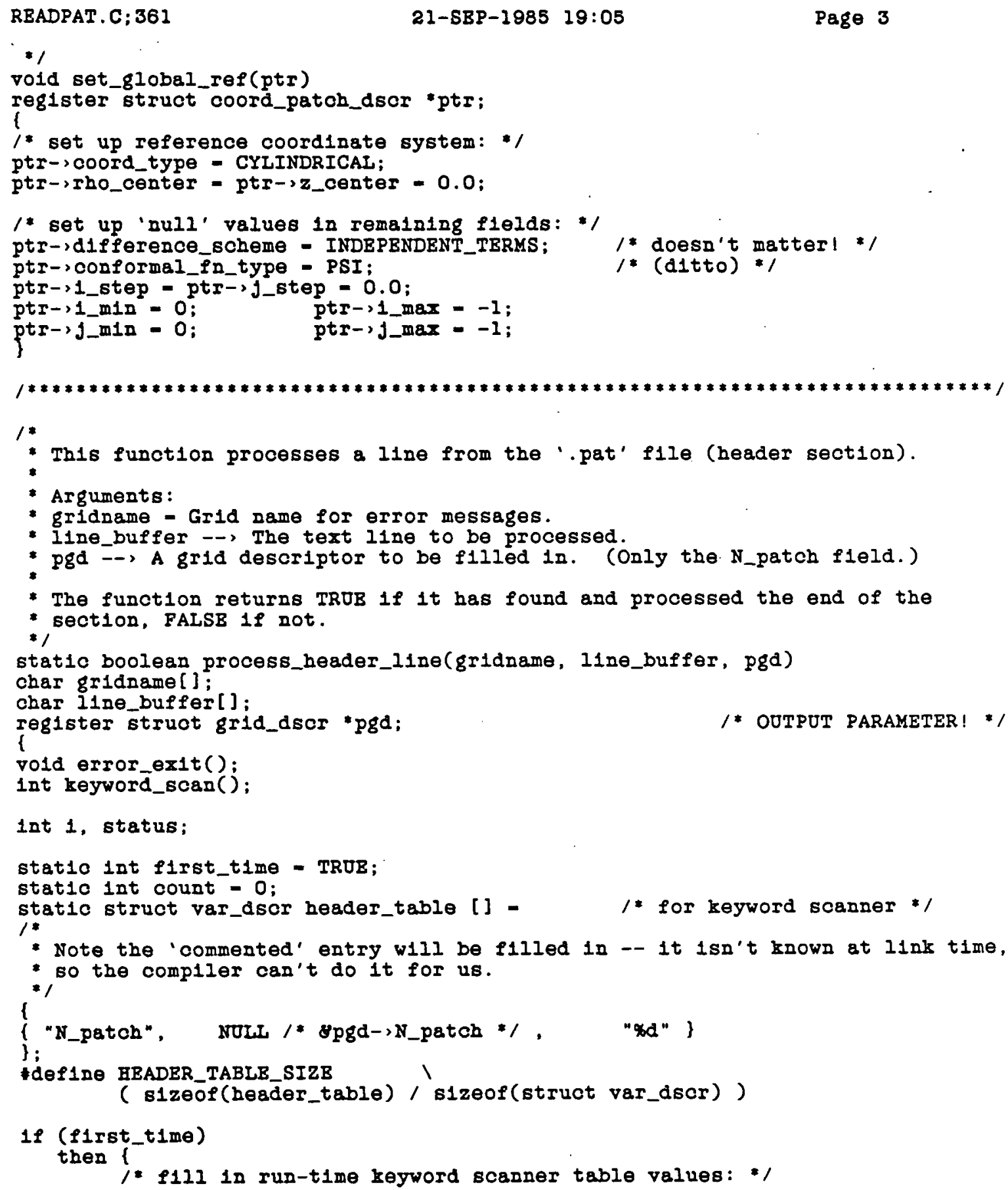


patchutil directory

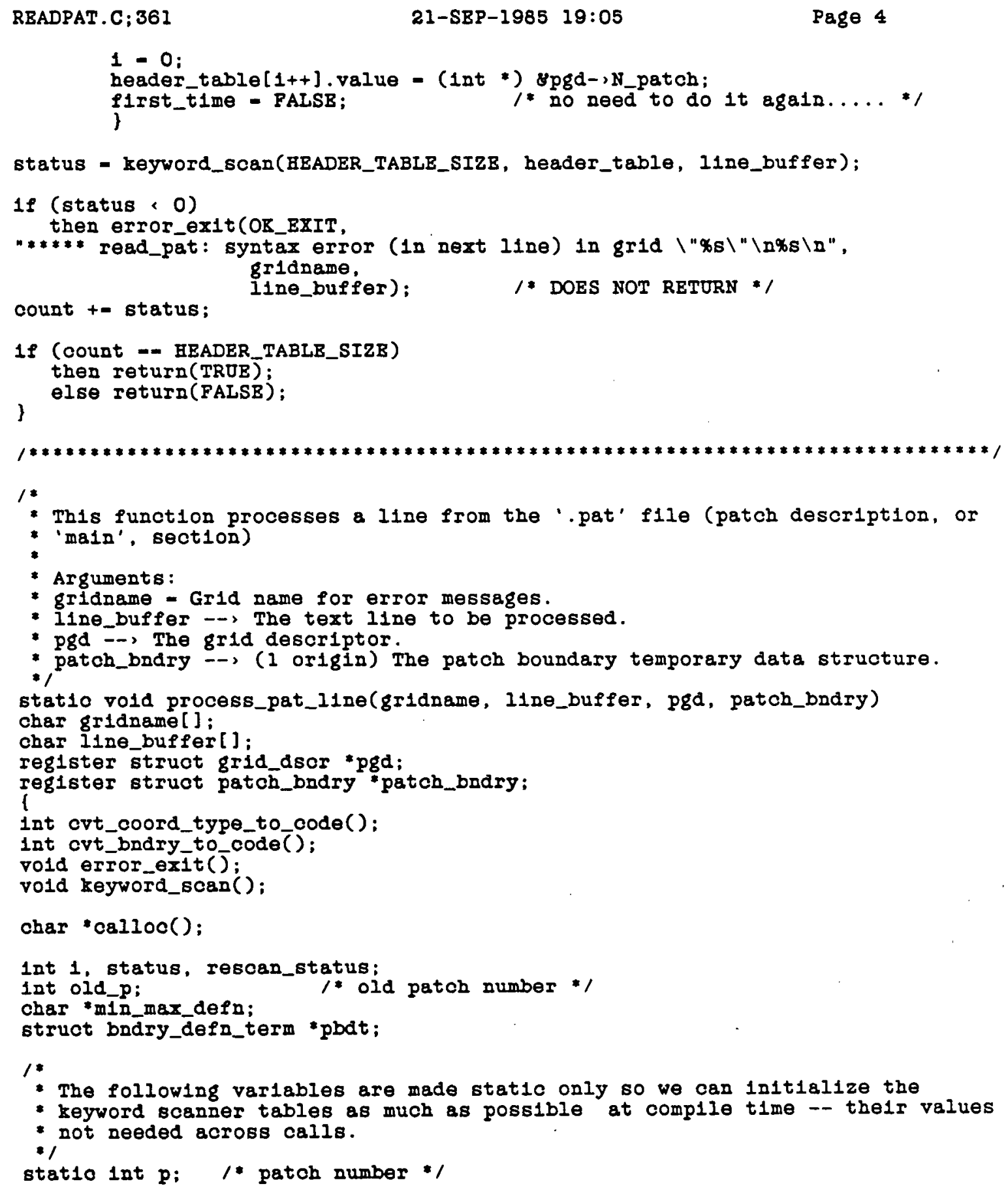


patchutil directory

RBADPAT. C; 361

21-SBP-1985 19:05

Page 5

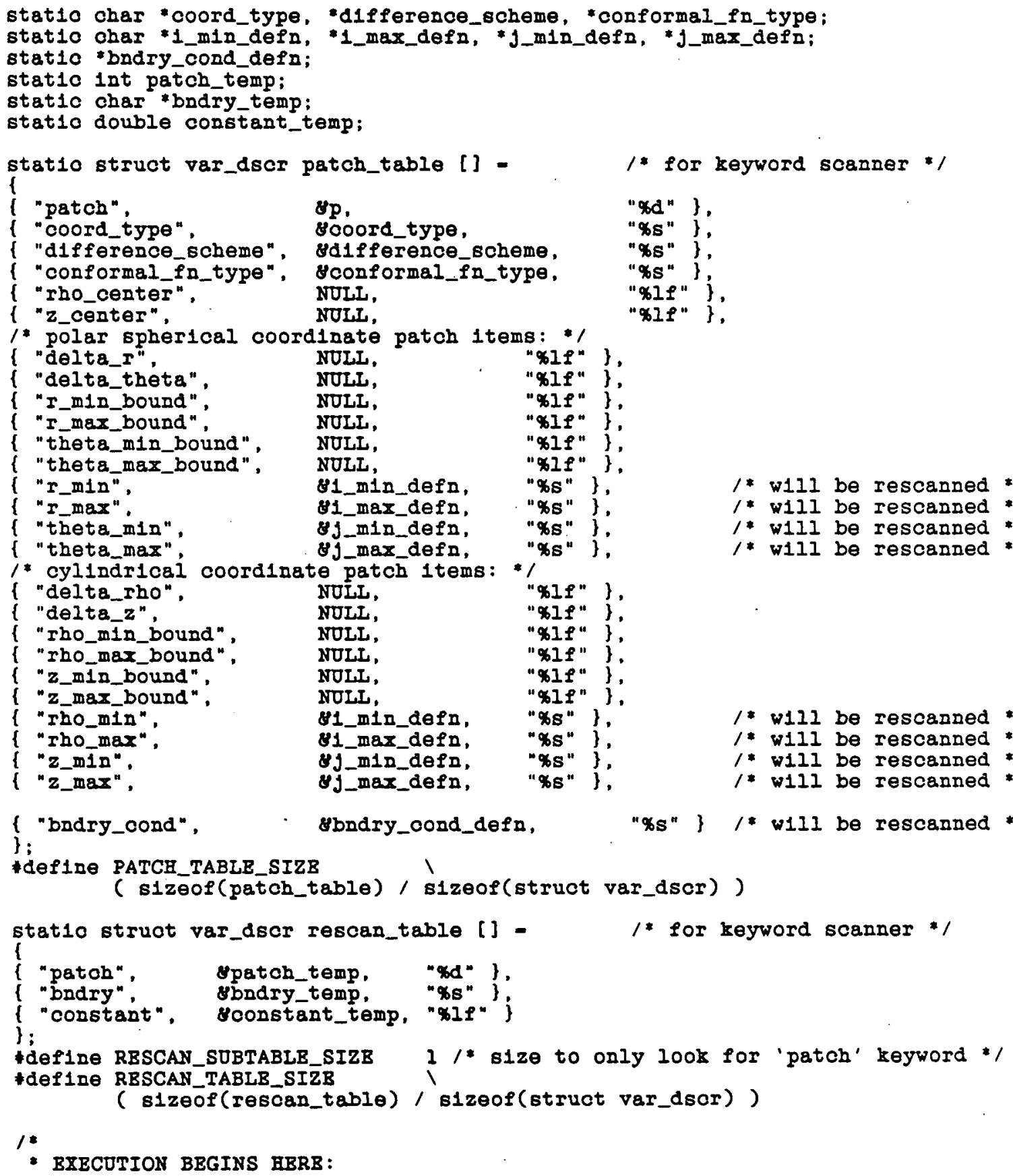


patchutil directory

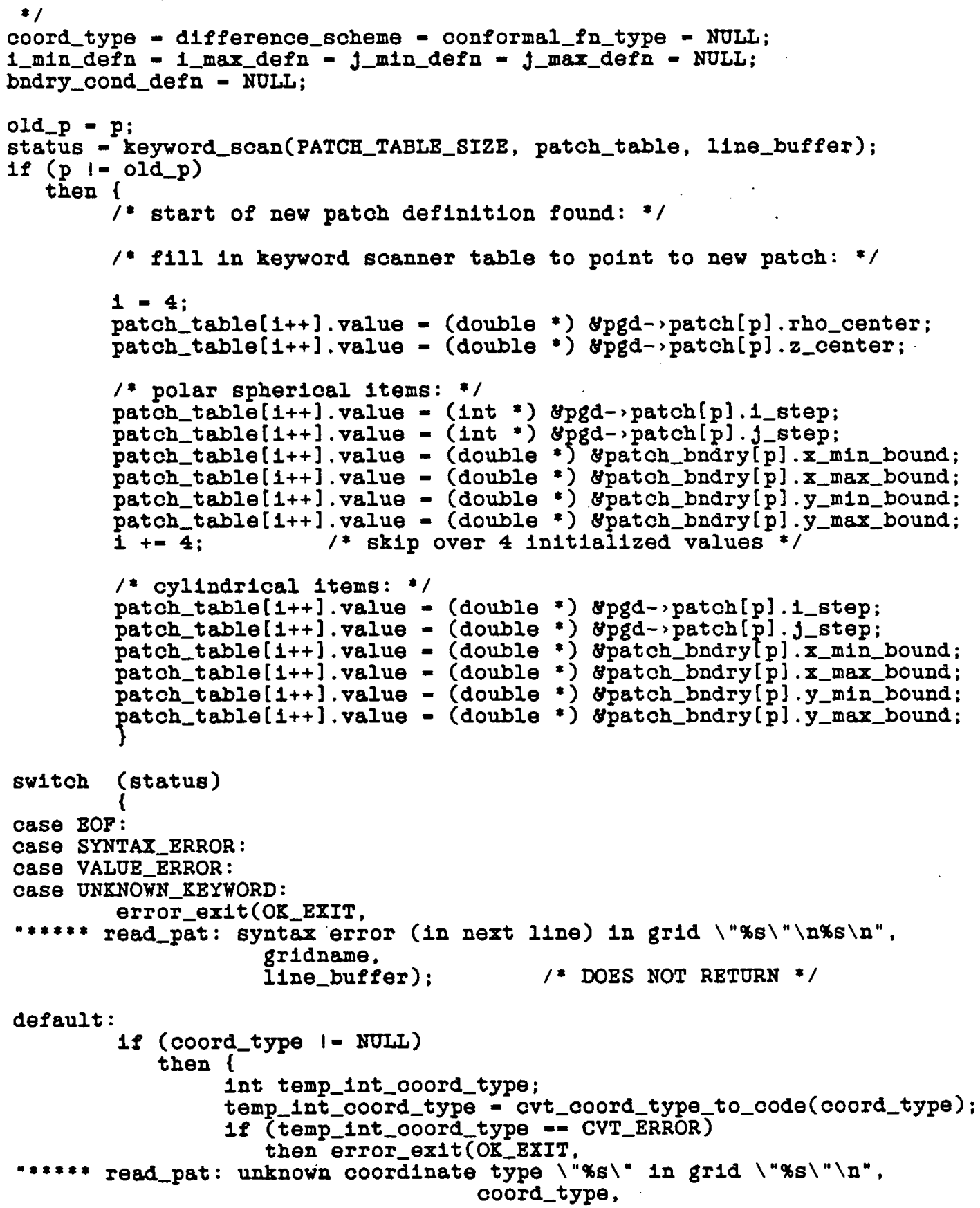


patchutil directory

READPAT.C; 361

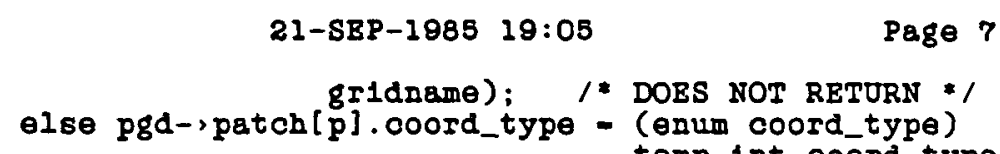

Page 7

READPAT.C:381 
patchutil directory

READPAT.C; 361

$$
\text { 21-SBP-1985 19:05 }
$$

Page 8

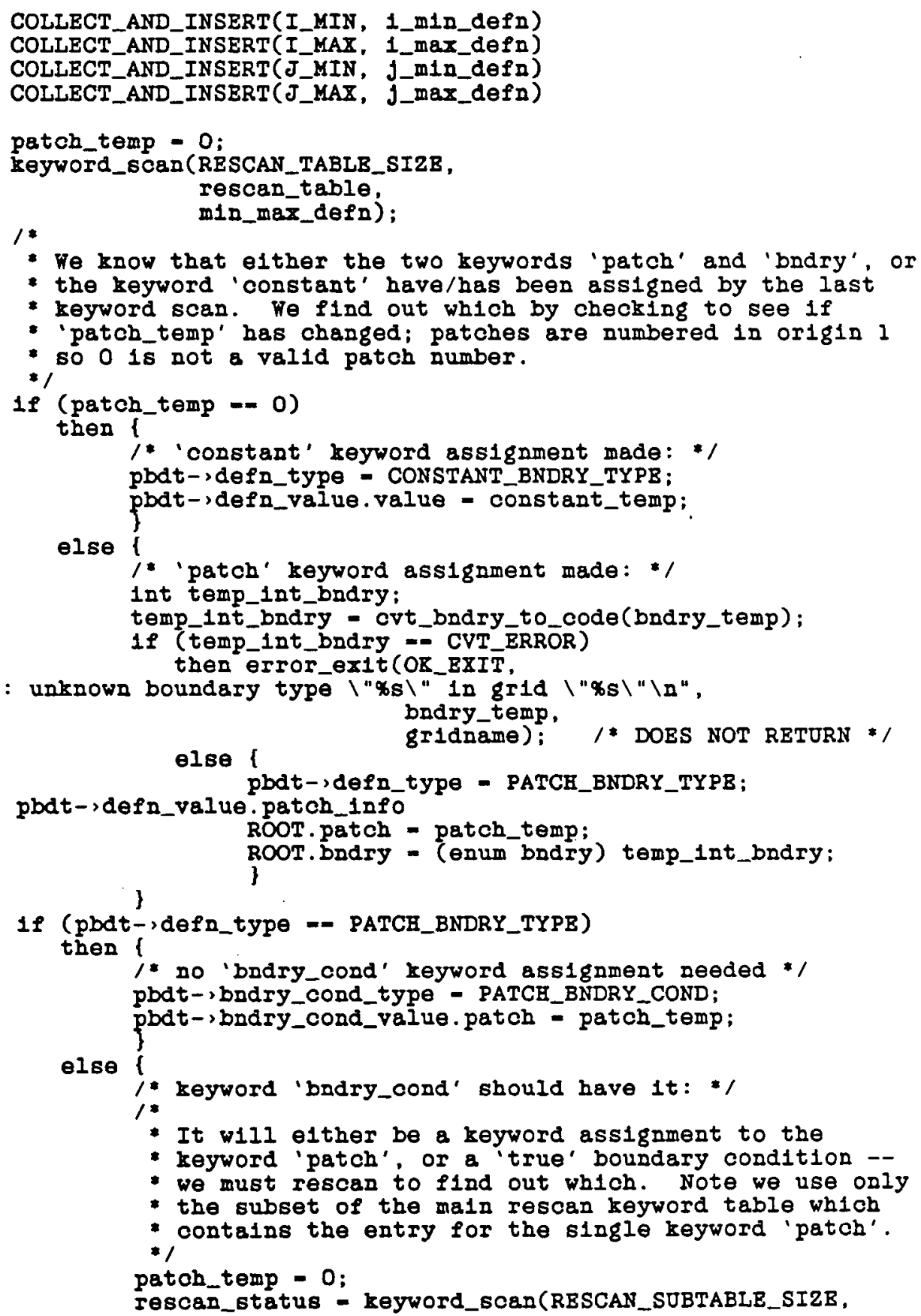

pbdt-,defn_value.patch_info ROOT. patch = patch_temp;

*define ROOT 


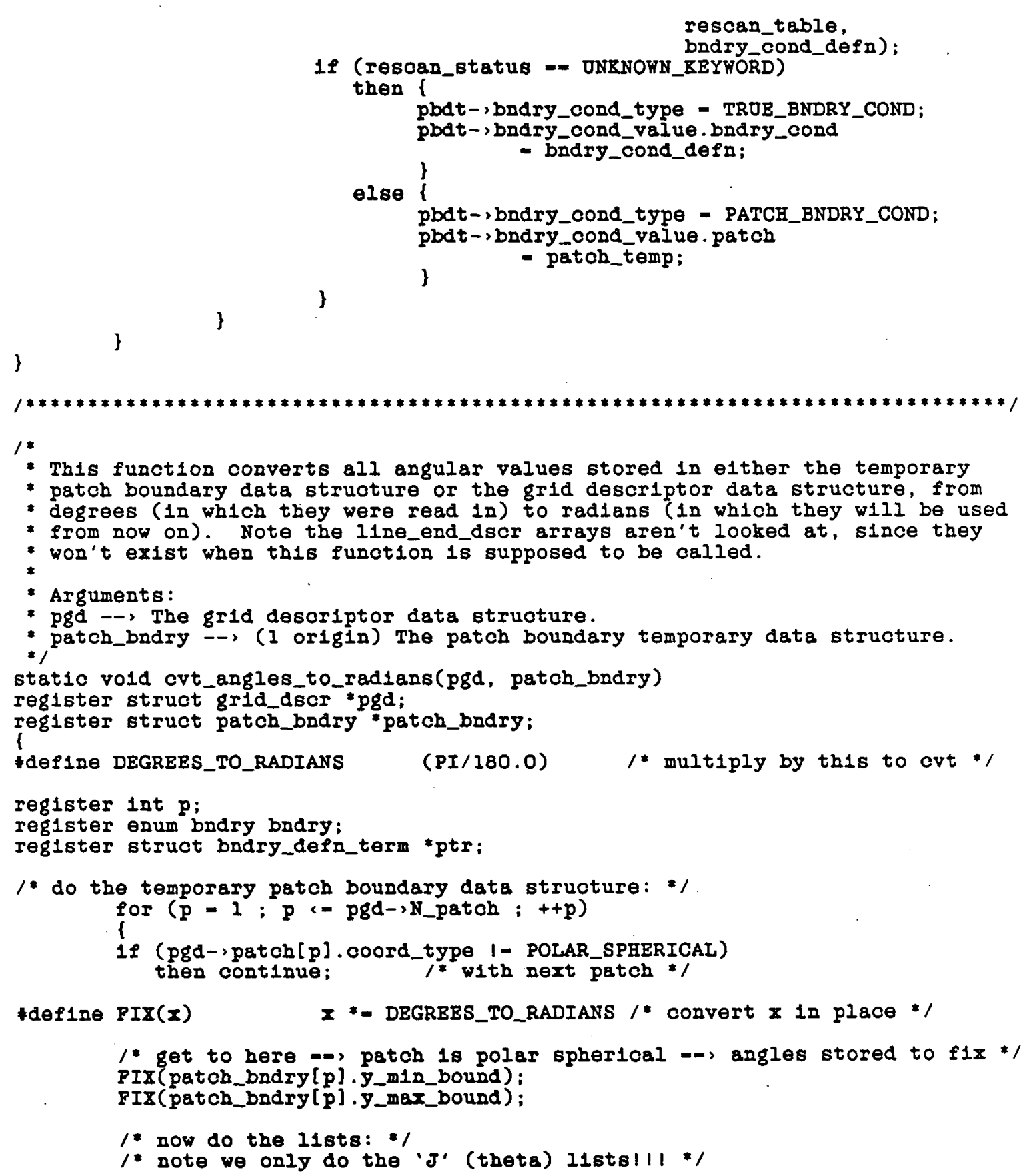




\section{patchutil directory}

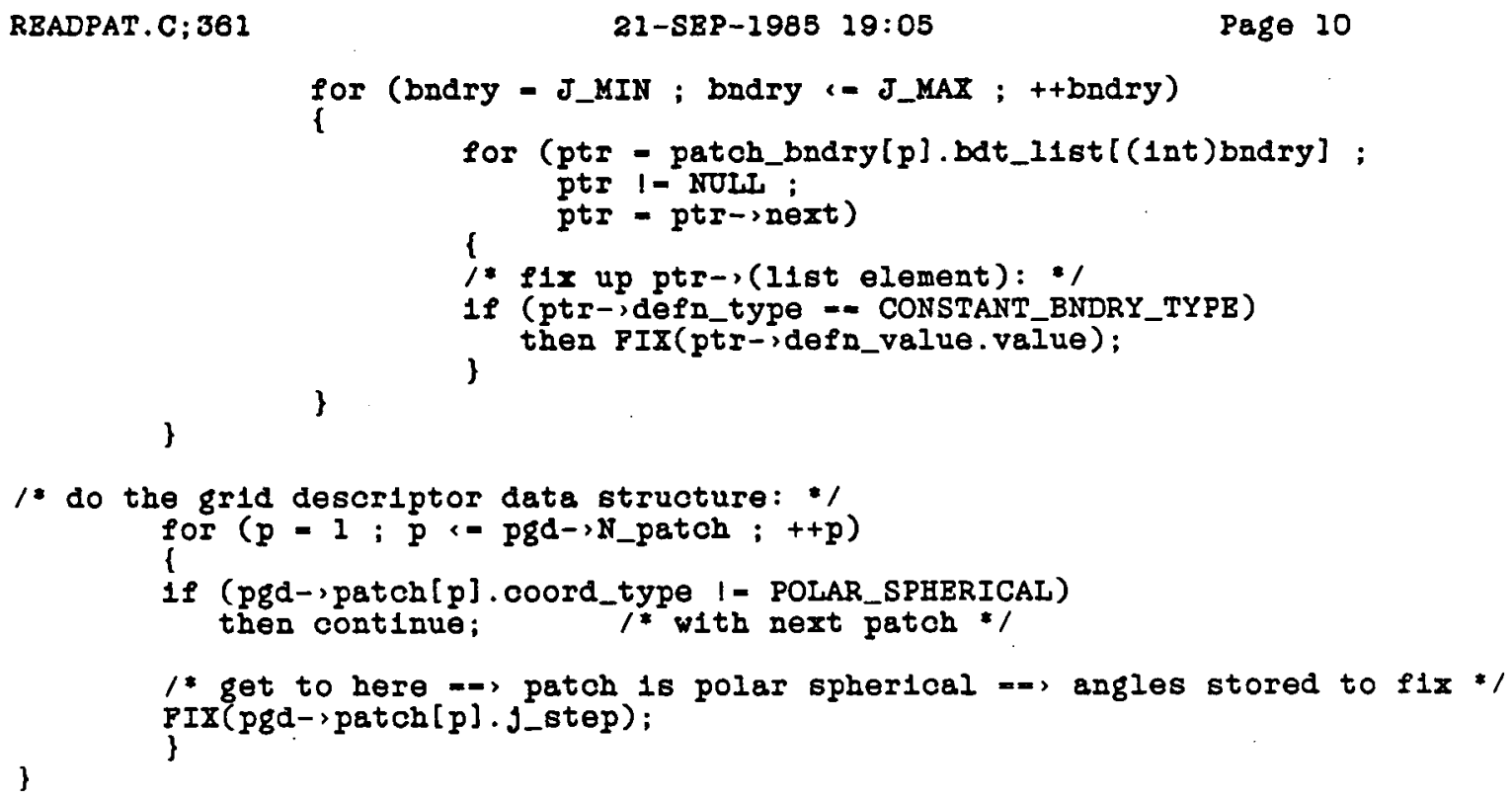


patchut1l directory

SBTBNDRY . C; 491

21-SBP-1985 19:06

Page 1

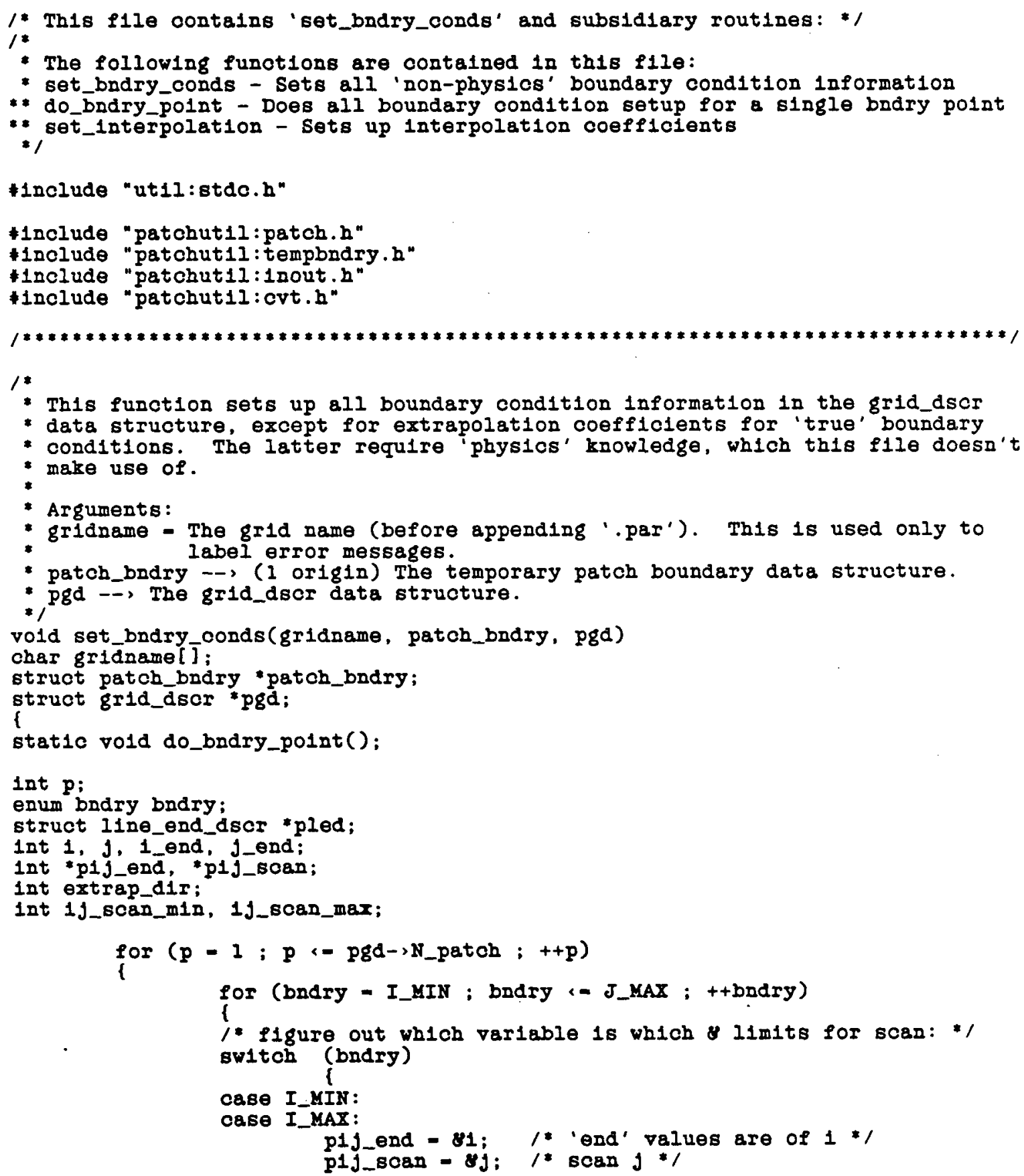




\section{patchutil directory}

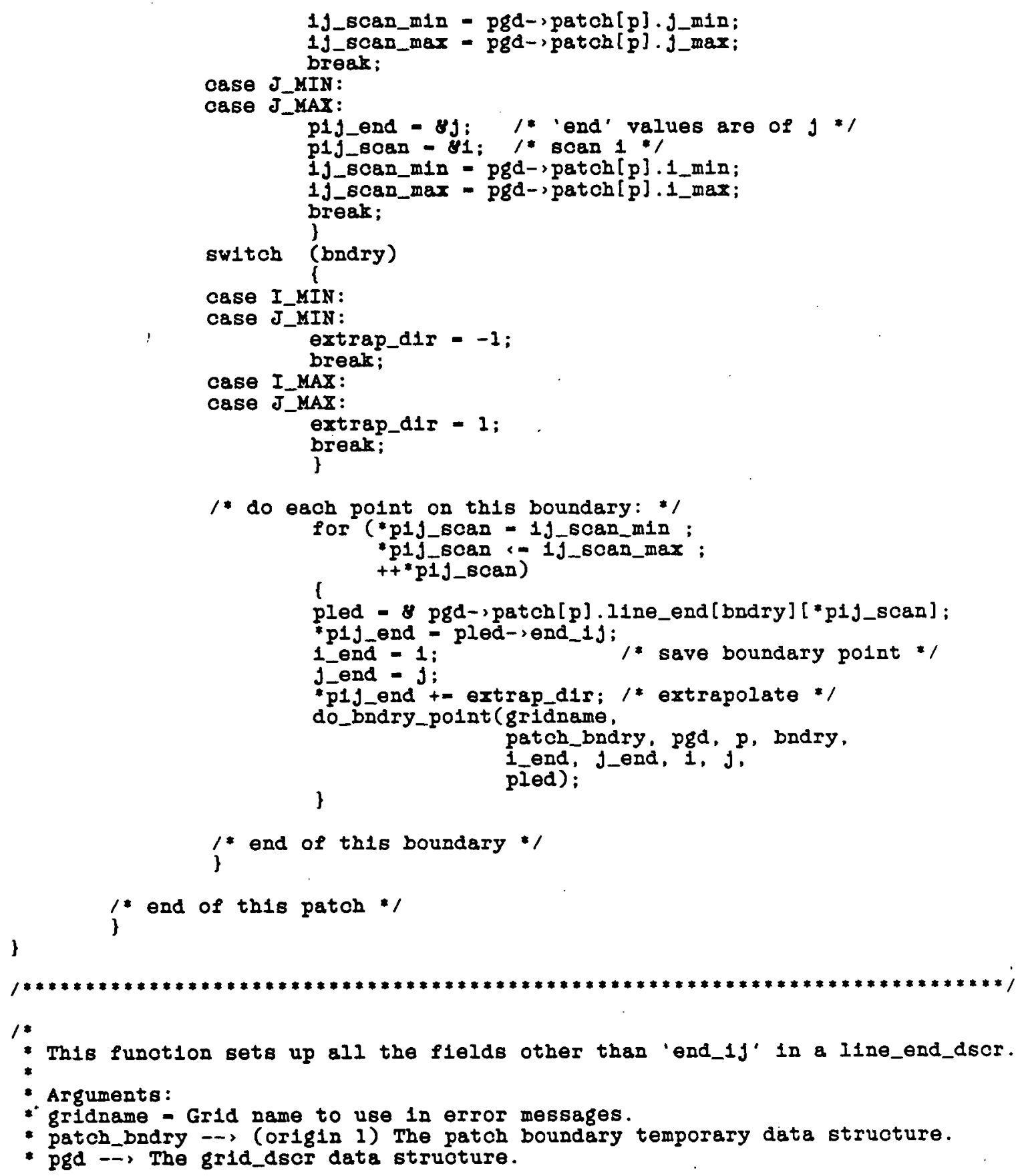


patchutil directory

* $p$ - The patch number.

* bndry - The boundery this point lies on in this patch.

* (1_end, j_end) - The 1nteger coordinates of the 'end_1f' point.

* (1_extrap.j_extrap) - The integer coordinates of the extrapolated point.

* pled - The line_end_dsor 1tself to be filled in.

stat10 vold do_bndry_polnt(gridname, patch_bndry, pgd, p, bndry, 1_end, J_end, 1_extrap. j_extrap.

char gridname []; pled)

reg1ster struct patoh_bndry patch_bndry;

reg1ster struct gr1d_dscr *pgd:

int $p$;

enum bndry bndry;

1nt 1_end, J_end, 1_extrap, J_extrap;

register struct i1ne_end_dsor "pled;

\{

vold cvt_1j_to_xy():

vo1d crt_xy_to_xy():

enum relation temp_bndry_1nout_term();

enum relation inout_xy():

int cvt_bndry_cond_type_to_code( );

char *crt_bndry_to_char( );

vold error_exit():

static vold set_bndry_trouble();

static vold set_interpolation();

register struct bndry_defn_term "ptr;

double $x_{\text {_end, }}$ Y_end;

double x_extrap. Y_extrap;

int other_patch;

double x_extrap_other, y_extrap_other;

/* Find applicable bndry_defn_term in patch boundary data structure: */ for (ptr - patch_bndry [p].bdt_ilst [bndry] ;

ptr $1=$ NOLL;

ptr = ptr->next)

\{

if ( (ptr-)defn_type $=-$ CONSTANT_BNDRY_TYPE)

88 (ptr-,bndry_cond_type =- TRUE_BNDRY_COND) then

1. 'constant-... bndry_cond-...(true)...': :

ovt_1j_to_ry ( 8 pgd->patch[p], 1_end, j_end, sx_end, yy_end);

if (temp_bndry_inout_term(patch_bndry, pqd, p, x_end, y_end, bndry.

1- INSIDE)

ptr)

then 1

Idef1ne WHATSIT ptr->bndry_cond_value.bndry_cond

pled->Plag - ovt_bndry_cond_type_to_code(WHATSIT);

19 (pled->11ag =- CVT_BNDRY_COND_TYPE_ERROR)

1* Note non-standard error codelll */ 


\section{patchutil directory}

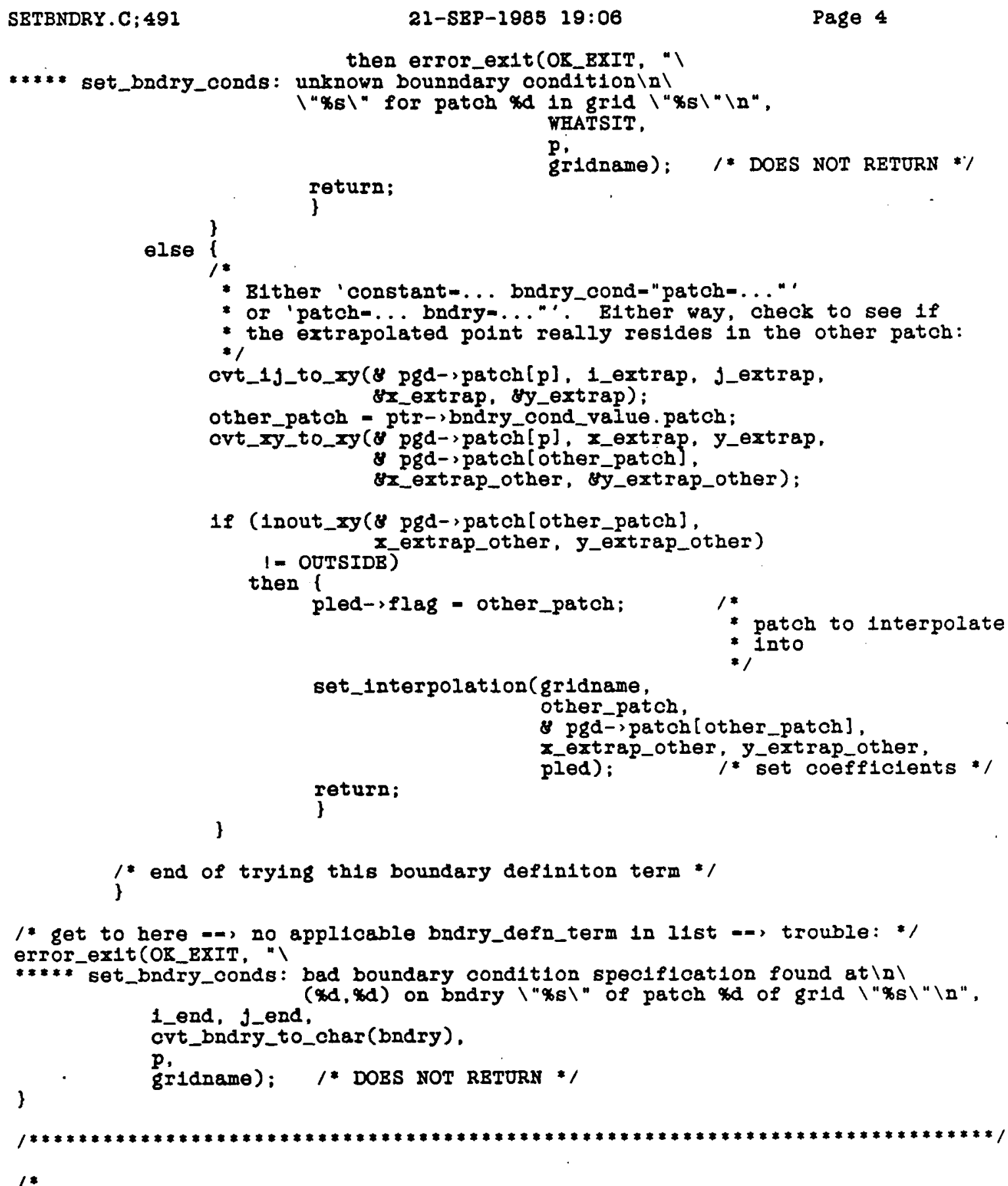


patchutil directory

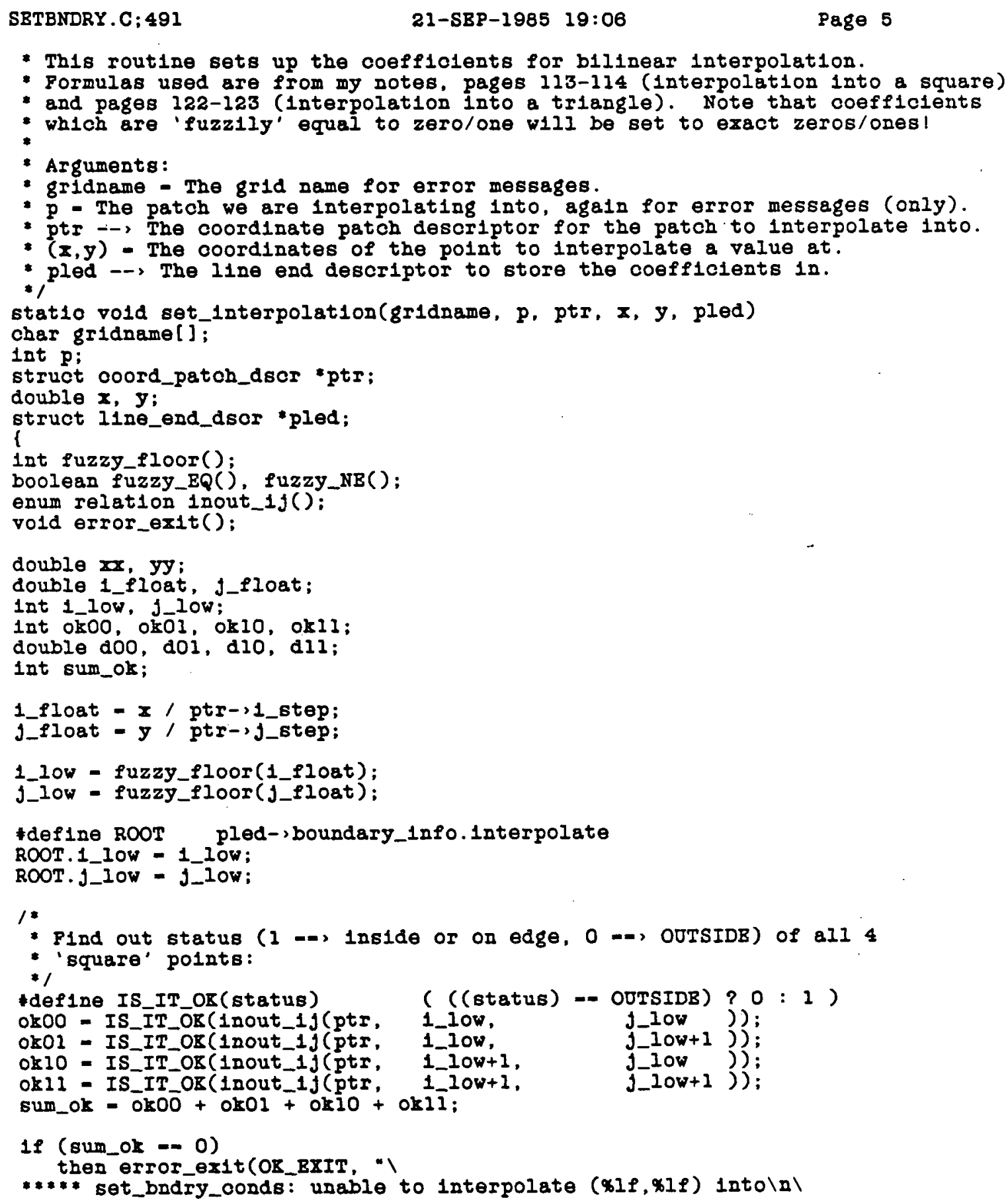

- Th1s routine sets up the coefficients for bilinear interpolation.

- Formulas used are from my notes, pages 113-114 (1nterpolation into a square)

* and pages 122-123 (1nterpolation 1nto a triangle). Note that coefficients

- whlch are 'fuzz1ly' equal to zero/one will be set to exact zeros/ones!

$*$

- Arguments:

- gridname - The grid name for error messages.

- p - The patch we are 1nterpolating into, again for error messages (only).

- ptr -- The coordinate patch descriptor for the patch to interpolate into.

- $(x, y)$ - The coordinates of the point to interpolate a value at.

* pled $\rightarrow$ The line end descriptor to store the coefficients in.

statio vold set_1nterpolation(gridname, p, ptr, x, y, pled)

char gridname[ ];

lnt $p$;

struct coord_patch_dscr "ptr;

double $x, y$;

struct line_end_dsor "pled; i

lat fuzzy_floor( );

boolean fuzzy_EQ( ), fuzzy_NB( );

enum relation lnout_1f():

vold error_exit();

double $2 x, y y$ :

double 1_float, J_float;

1nt 1_low, j_low;

int okO0, ok01, oklo, okl1;

double doo, dol, dlo, dil;

int sum_ok;

1_float $=x /$ ptr->1_step;

j_float $=g /$ ptr-sj_step;

1_low - fuzzy_floor(1_ploat);

j_low - fuzzy_floor(j_flost);

tdefine ROOT pled-sboundary_info.interpolate

ROOT.1_IOW - 1_IOW;

ROOT.j_IOW - j_low: 


\section{patchutil directory}

SETBNDRY.C; 491

- 21-SEP-1985 19:06

Page 6

$\mathbf{x}, \mathbf{y}$,

p,

gI1dname); /* DOES NOT RETURN */

xx - 1_float - 1_low:

yy - J_float - J_low;

11 (sum_ok $=-3$ )

then ?

* bilinear interpolation into a triangle: "

$1 *$

* There are 4 cases, depending on which point of the square is missing

- (to form the triangle):

$* 1$

If $(1$ okoO)

then 1

doo $=0.0$

$\mathrm{dOI}=1.0-\mathbf{x x}$

d10 $-1.0-\mathrm{yy} ;$

if (1 0kO1)

d

then

$d 00=1.0-2 x$;

$\mathrm{dOI}=0.0$;

$\mathrm{d} 10=\mathbf{x x}-\mathbf{y y}$

dII = yy:

if ( 1 okio)

then 1

doo $=1.0-y y ;$

$d 01=y y-x x$

d10 $=0.0$;

dII = xx:

If ( 1 okl 1 )

then \{

doo - 1 - xa - yy;

dOI = yy;

d10 - xx;

dl1 = 0.0;

else

* b1l1near 1nterpolation into a square: *

$\mathrm{dOO}=(0 \mathrm{k} 00) ?((1.0-\mathrm{xx}) *(1.0-\mathrm{yy})): 0.0$

d01 - (Ok01) ? ((1.0 - xx) yy) : 0.0;

d10 $-(0 k 10) ?(x:(1.0-\mathrm{yy}): 0.0$

dl1-(okl1)? (xx yy):0.0;

\}

1. Force coefflclents fuzzily equal to zero/one, to be exact zeros/ones: *I 4define PIX(dif,val) if (fuzzy_EQ(dif, val)) then dif = val;

*def1ne FULLFIX(d1j) FIX(d1j, 0.0) PIX(d1j, 1.0) 
patchutil directory

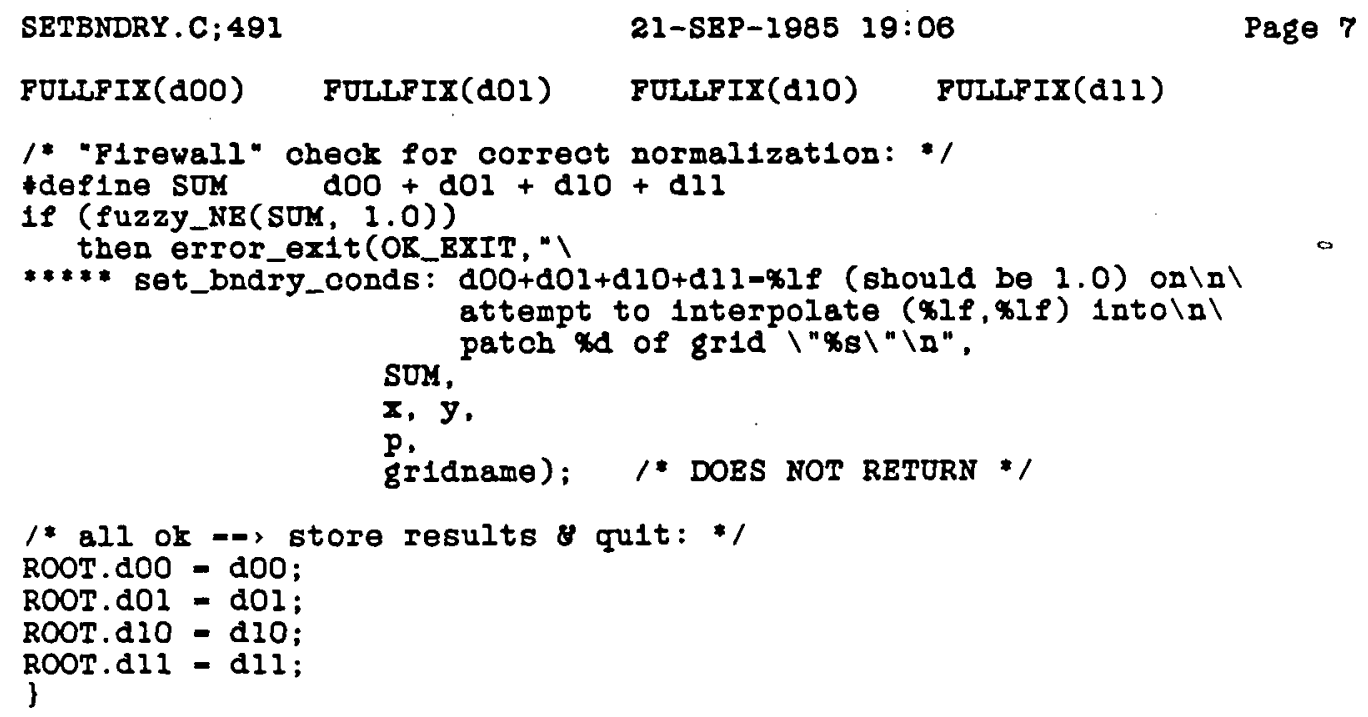


patchutil directory

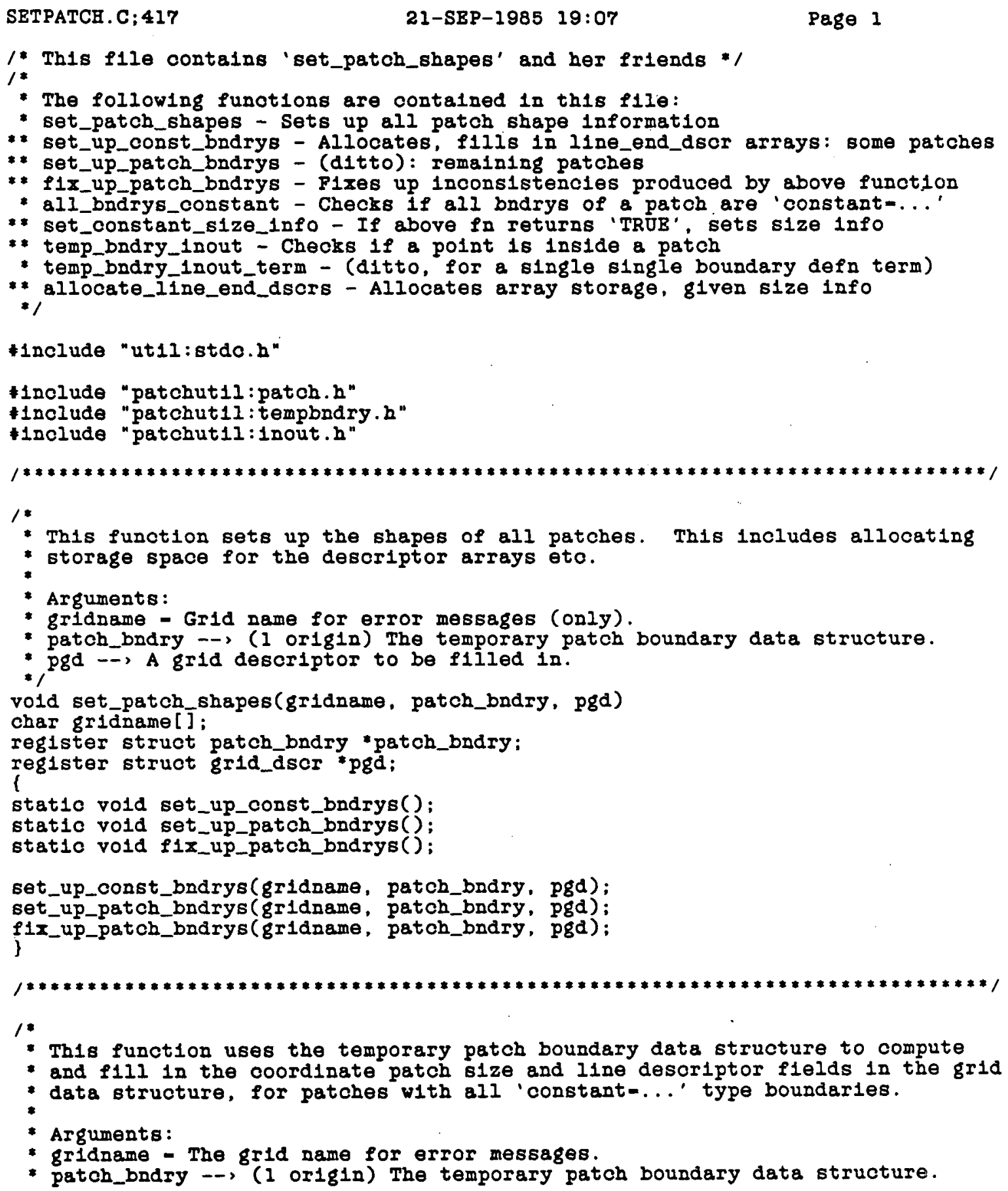


patchutil directory

SETPATCH.C; 417

21-SBP-1985 19:07

Page 2

:pgd $\rightarrow$ The grid desoriptor struoture to be fllled in.

static vold set_up_const_bndrys(gridname, patch_bndry, pgd)

char gridname[];

reg1ster struct patch_bndry *patch_bndry;

register struct grid_dsor "pgd;

boolean all_bndrys_constant():

stat1o vold set_constant_size_info()

static vold allocate_line_end_dscrs () ;

lnt $p ; 1$ current patch number */

enum bndry bndry;

int range_end;

int $1 j, 1 j \_m i n, 1 j \_m a x ;$

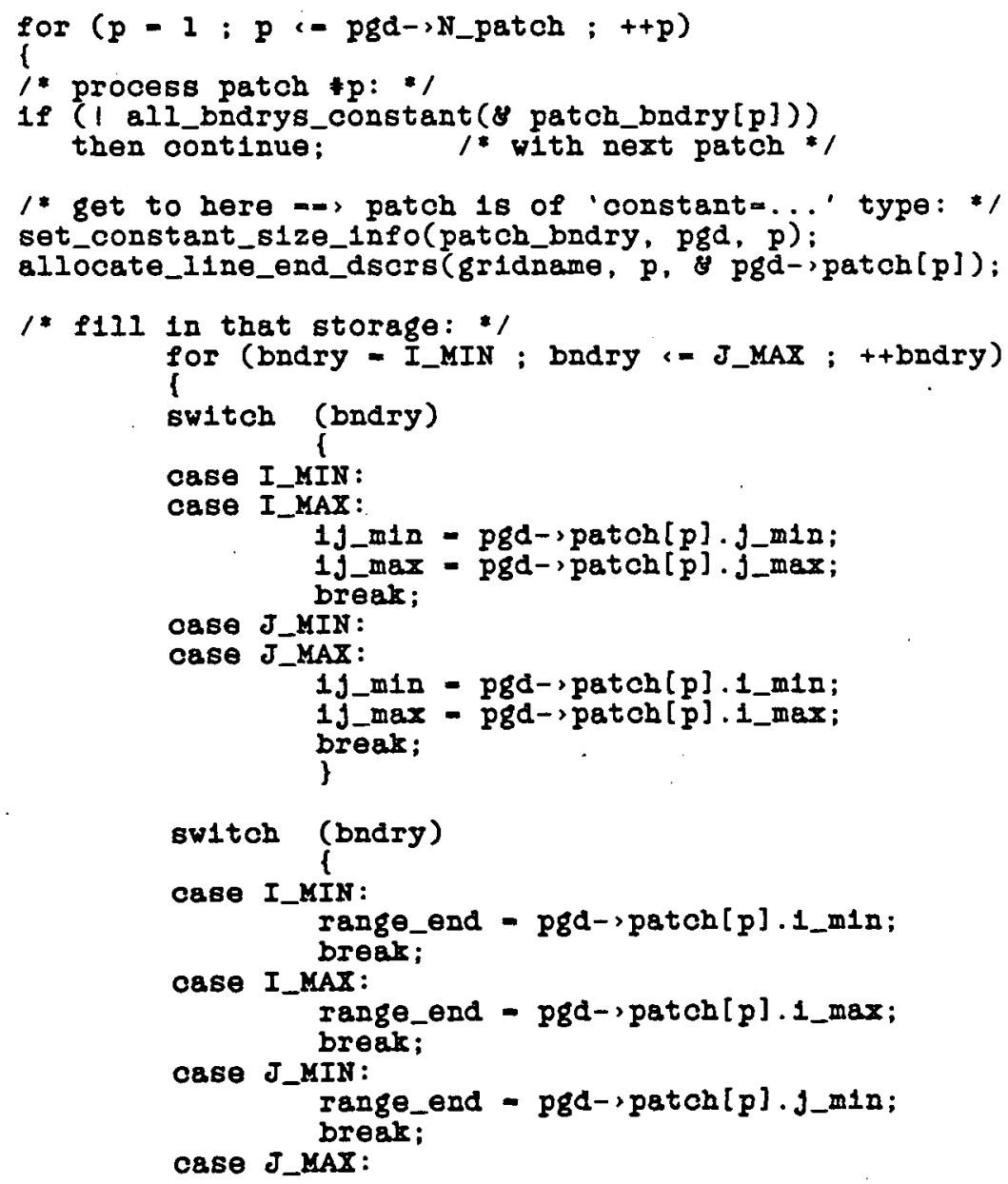


patchutil directory

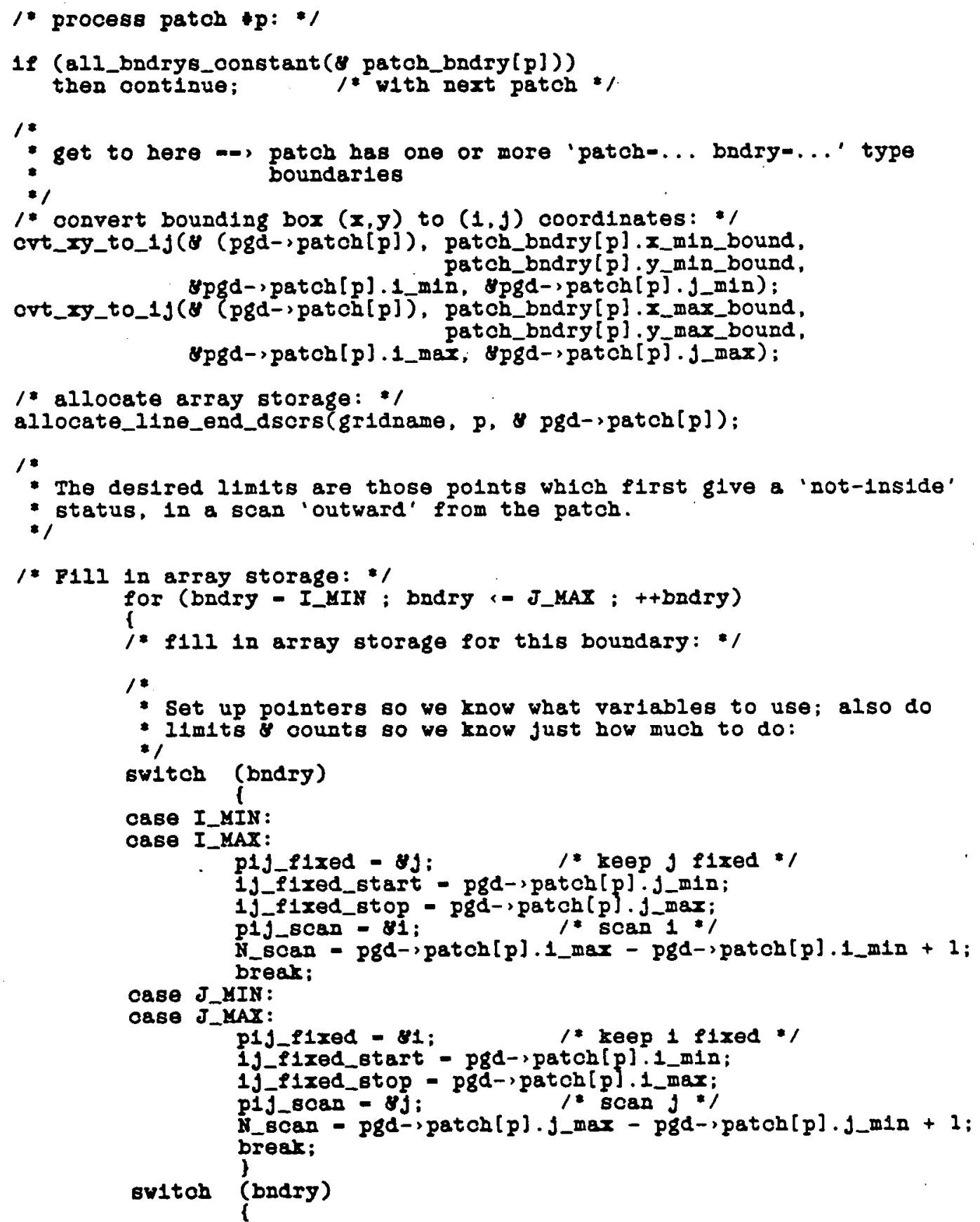


patchutil directory

Case I_MIN :

1j_scan_start - pgd-'patch $[p]$.1_max;

1j_scan_d1r - - I;

cose I_MAX:

$$
\text { break; }
$$

1j_scan_start - pgd->patch [p].1_min;

Ij_scan_d1r - 1

case J_KIN :

break;

1j_scan_start - pgd->patch[p].j_max;

1j_scon_dir - - I;

Case J_MAX: break;

1)_scan_start - pgd->patch [p].j_m1n;

ij_scan_d1r - 1;

break;

)

$f *$

- Pirst step the 'flxed' variable, which specifies the grid

* line being considered: (Note that the order in which the

* different values of this vartable are tried is unimportant)

$* 1$

for (*p1j_f1xed - $1 j_{-} f 1 x e d \_s t a r t$; * pij_fixed $-1 j$ fixed_stop ; \{ ++ plj_f1xed)

$1 *$

- Then step the 'scan' variable to find the first

* 'non-inside' value, checking against the desired

* boundary only. This is the desired linlt value.

- modulo a bit of fixing up for the Unruh gap et al.

for (*pij_scan - 1j_scan_start, scan_count=0 ; scan_count, $\mathrm{N}_{\text {scan }}$;

I pij_scen +- 1j_scan_d1r, ++scan_count)

ovt_1j_to_xy( $\mathscr{E}(\mathrm{pgd->patch}[\mathrm{p}]), 1, g, \& x, \& y)$;

if (temp_bndry_1nout(patch_bndry, pga, p, $\mathbf{x}, \mathbf{y}$, bidry.

1 - INSIDE)

intterm)

\}

then goto found;

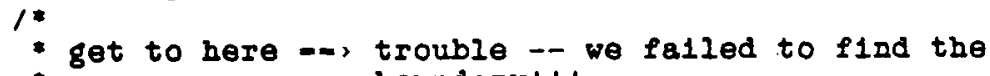


patchutil directory

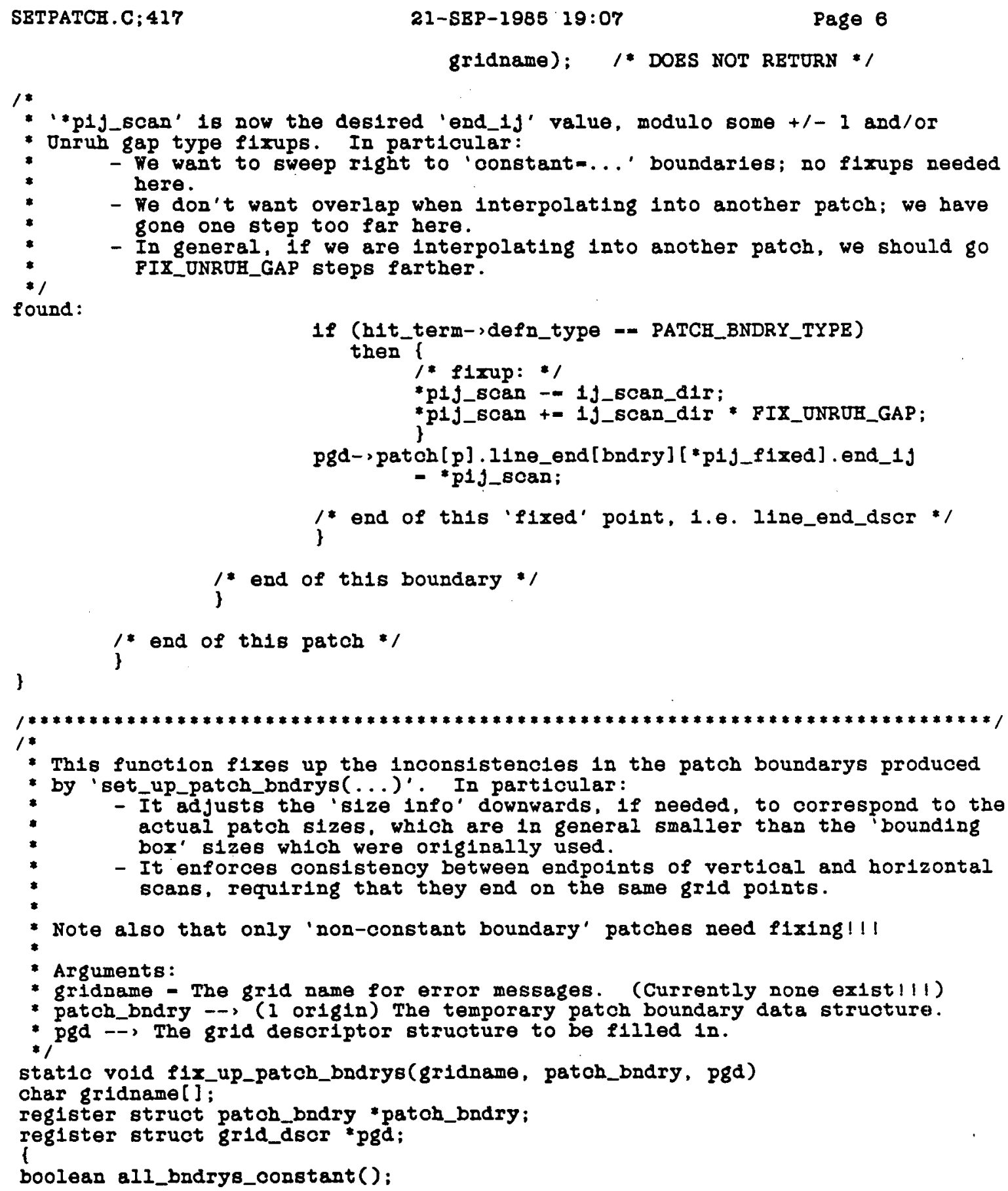




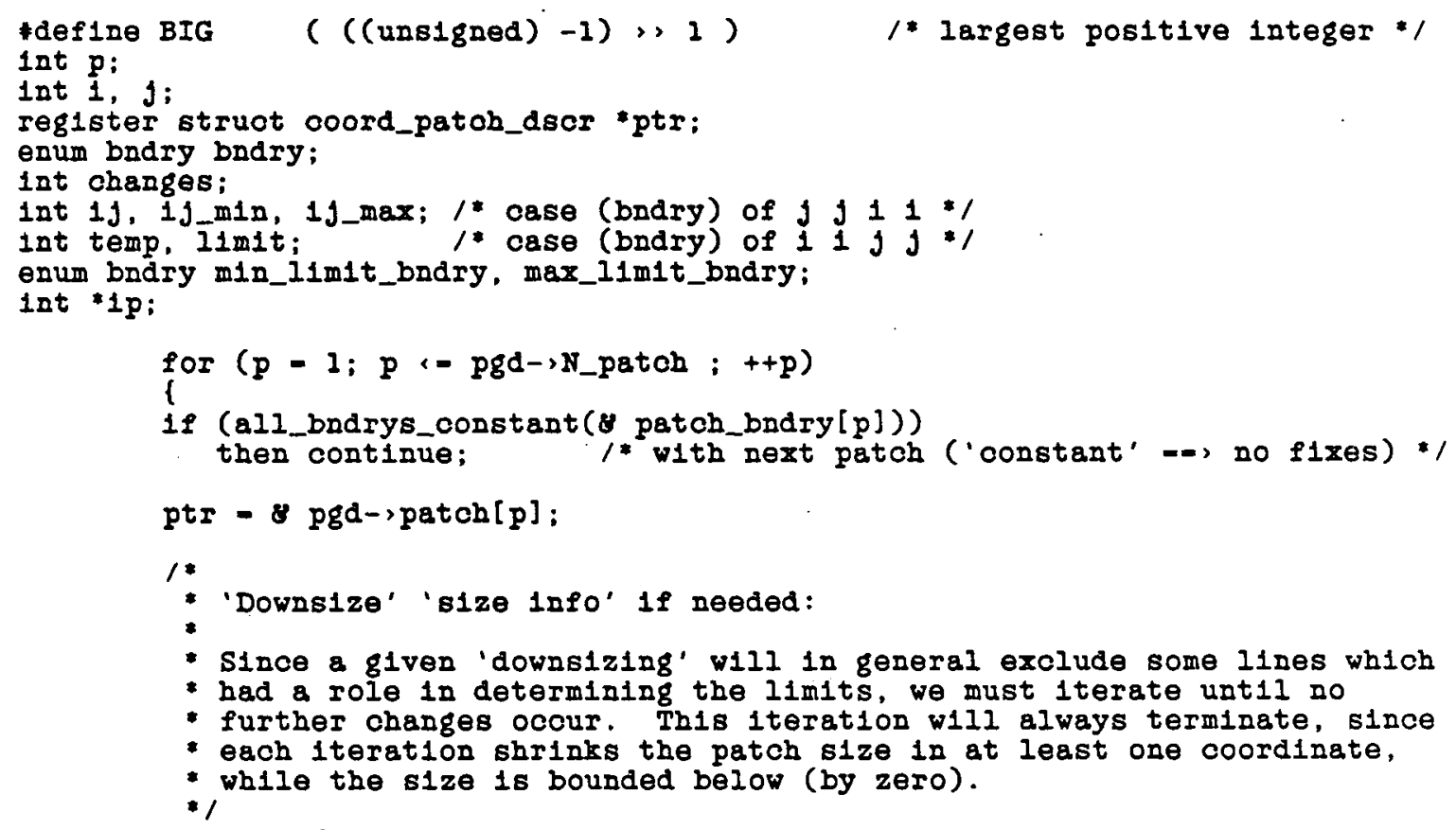




\section{patchut1l directory}

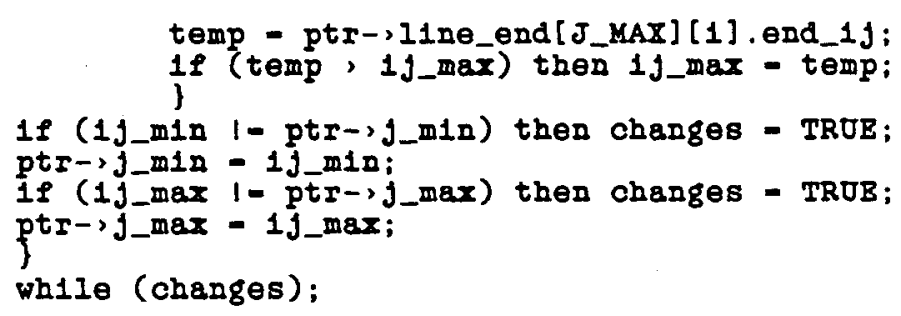


patchutil directory

SETPATCE.C;417

21-SBP-1985 19:07

Page 9

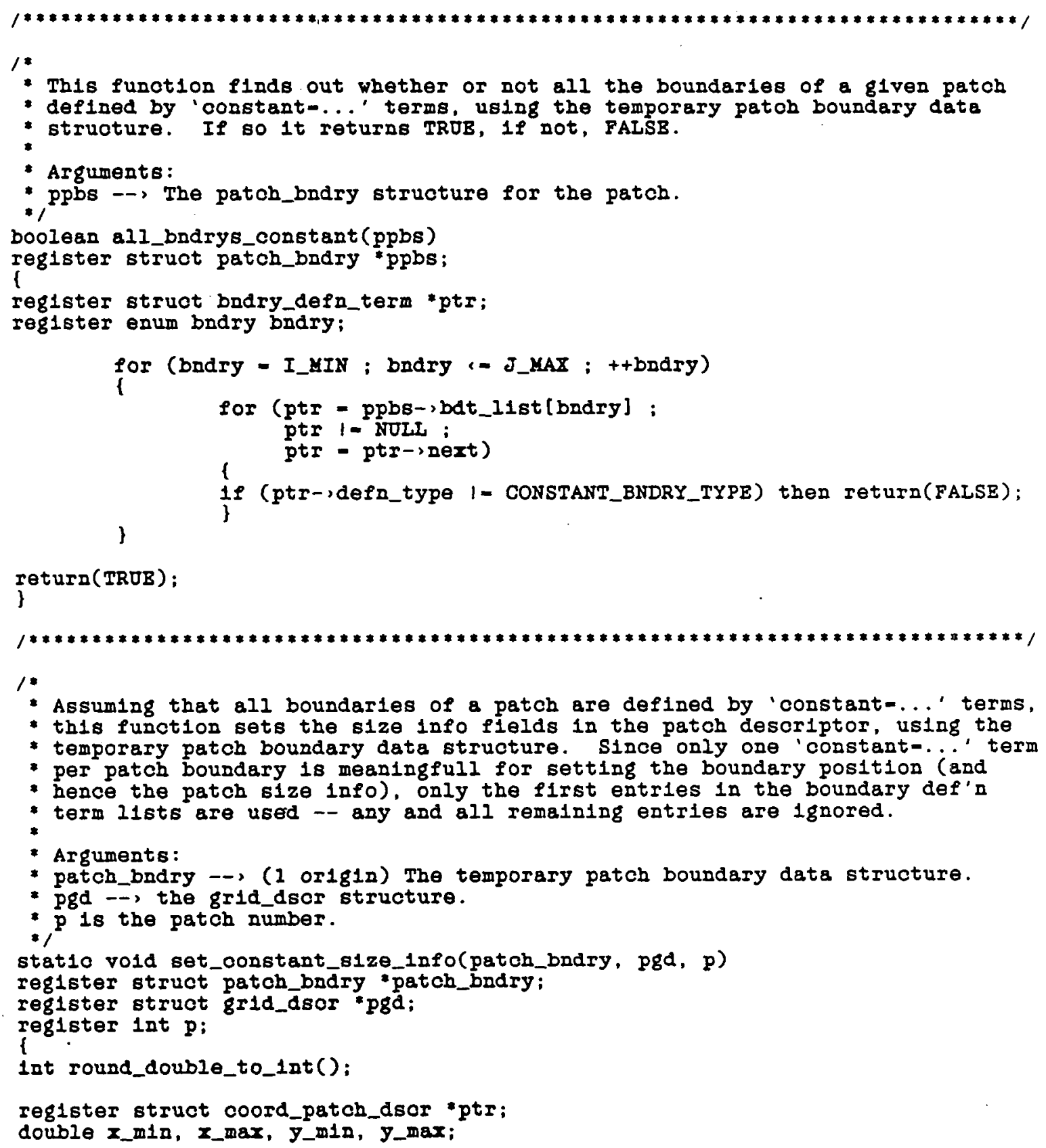




\section{patchut1l directory}

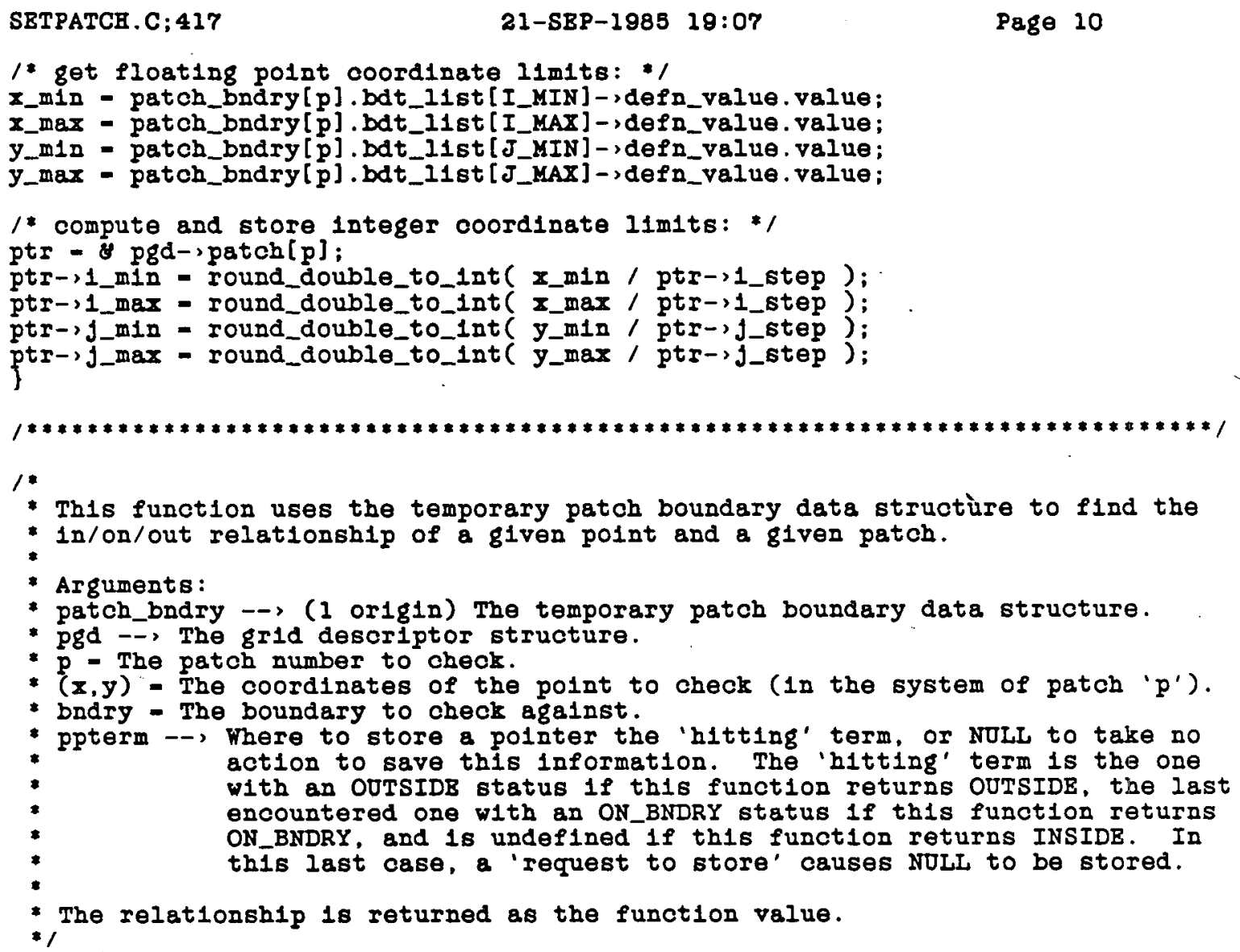




\section{patchut1l directory}

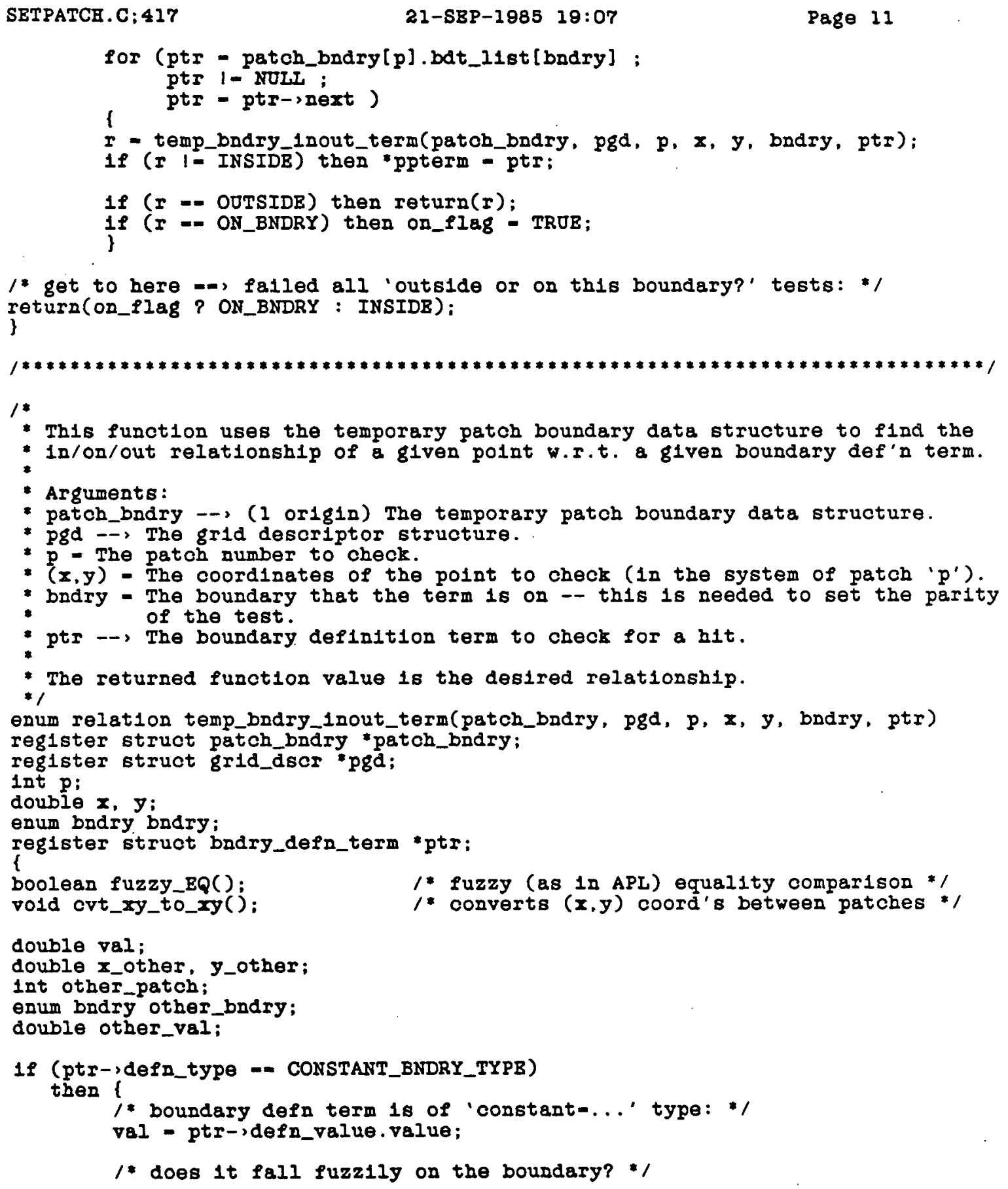


patchutil directory

switch

case I MIX:

case I_YAX:

if (fuzzy_EQ(x, val)) then return(ON_BNDRY);

case J MIN : break;

cese J_MAX:

if (fuzzy_EQ(y, val)) then return(ON_BNDRY); break;

\}

/ does it fall outside the boundary: *

switch (bndry)

case I_kIN :

$1 f(x$, val) then return(OUTSIDB) bresk;

case I_KAX

If (x, val) then return(OUTSIDB);

case J_KIN : break;

case J_MAX:

if ( $y$; val) then return(OUTSIDE); break:

$1 f(y$, val) then return(OOTSIDE)

break;

else \{

1* bndry defn term 1s of 'patch-... bndry=...' type: *I

/* get coordinates of point in other patch's coordinate system: */

other_patch - ptr->defn_value.patch_info.patch;

other_bndry = ptr->defn_value.patch_info.bndry;

other_val - patch_bndry[other_patch]

bdt_list [ other_bndry]

->defn_value.value;

cvt_xy_to_xy(y pgd-'patch[p], $x, y$,

8 pgd-ipatch[other_patch], sx_other, sy_other);

1* does 1t fall fuzzily on the (other) boundary? *

switch (other_bndry)

case I_MIN :

case I_MAX:

if (fuzzy_BQ(x_other, other_val)) then return(ON_BNDRY);

breal brege

Case J_MAX:

$1 f$ ( $f u z z y+B Q\left(y \_o t h e r\right.$, other_val)) then return(ON_BNDRY); break; \} 
patchutil directory

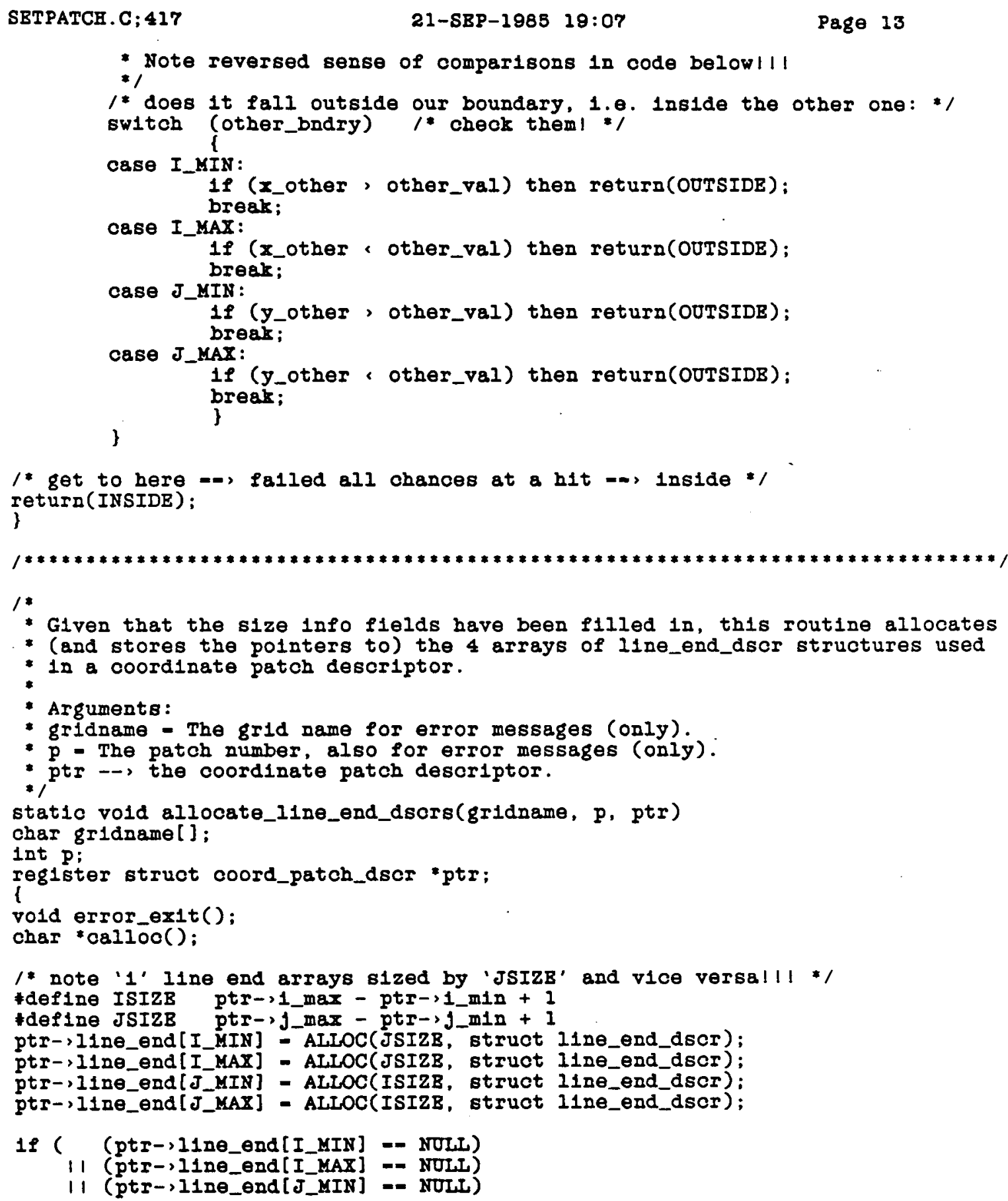


patchutil directory

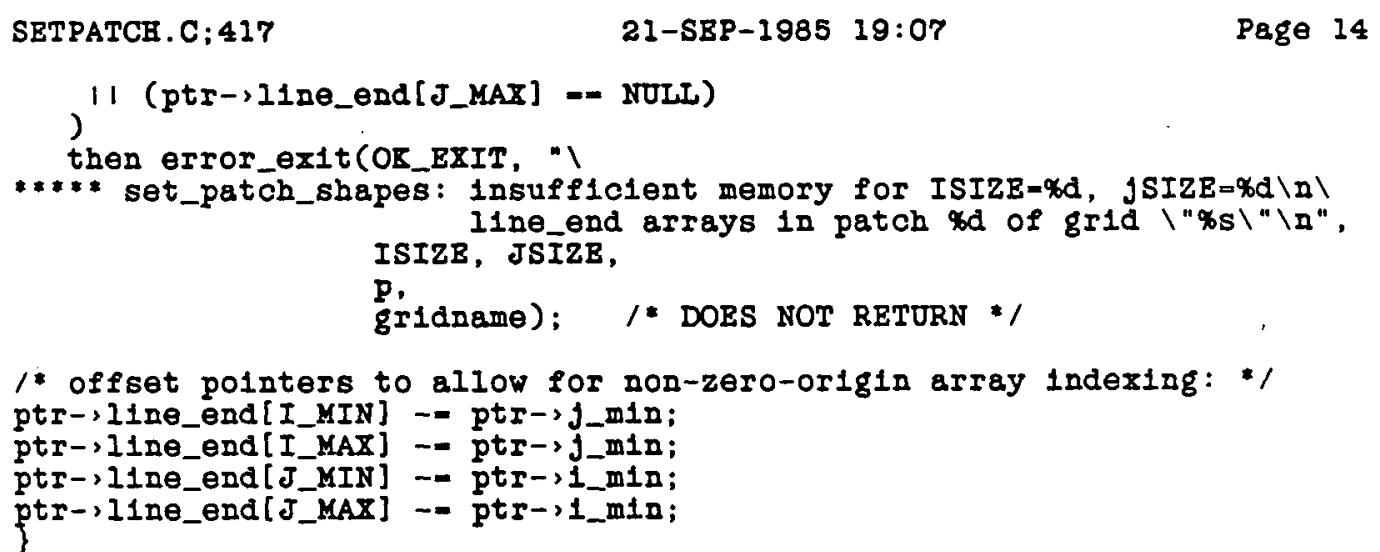




\section{ut1l directory}

BANDMAT. $\mathrm{H} ; 8$

21-SBP-1985 20:02

Page 1

1* Th1s file defines my C 'row-orlented' storage format for band matrices: */ * Ifdef COMMENT

The format is perhaps best 1llustrated by the following example - this matrix:

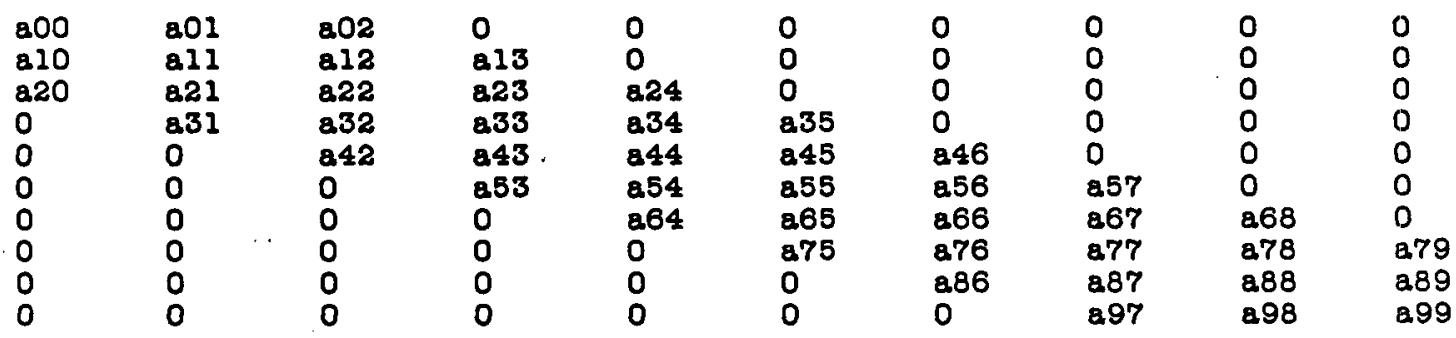

w111 be stored I1ke th1s:

\begin{tabular}{|c|c|c|c|}
\hline $\begin{array}{l}0 \\
0 \\
820 \\
831 \\
842 \\
853 \\
864 \\
875 \\
886 \\
897\end{array}$ & $\begin{array}{l}0 \\
810 \\
821 \\
a 32 \\
a 43 \\
054 \\
865 \\
876 \\
287 \\
898\end{array}$ & $\begin{array}{l}800 \\
811 \\
822 \\
833 \\
244 \\
855 \\
268 \\
877 \\
888 \\
899\end{array}$ & $\begin{array}{l}801 \\
812 \\
823 \\
234 \\
845 \\
856 \\
267 \\
878 \\
889 \\
0\end{array}$ \\
\hline
\end{tabular}

Note that my conventions are such that this matrix has 'bandwidth' 5 . tendif

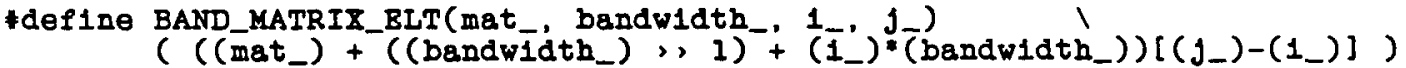




\section{util directory}

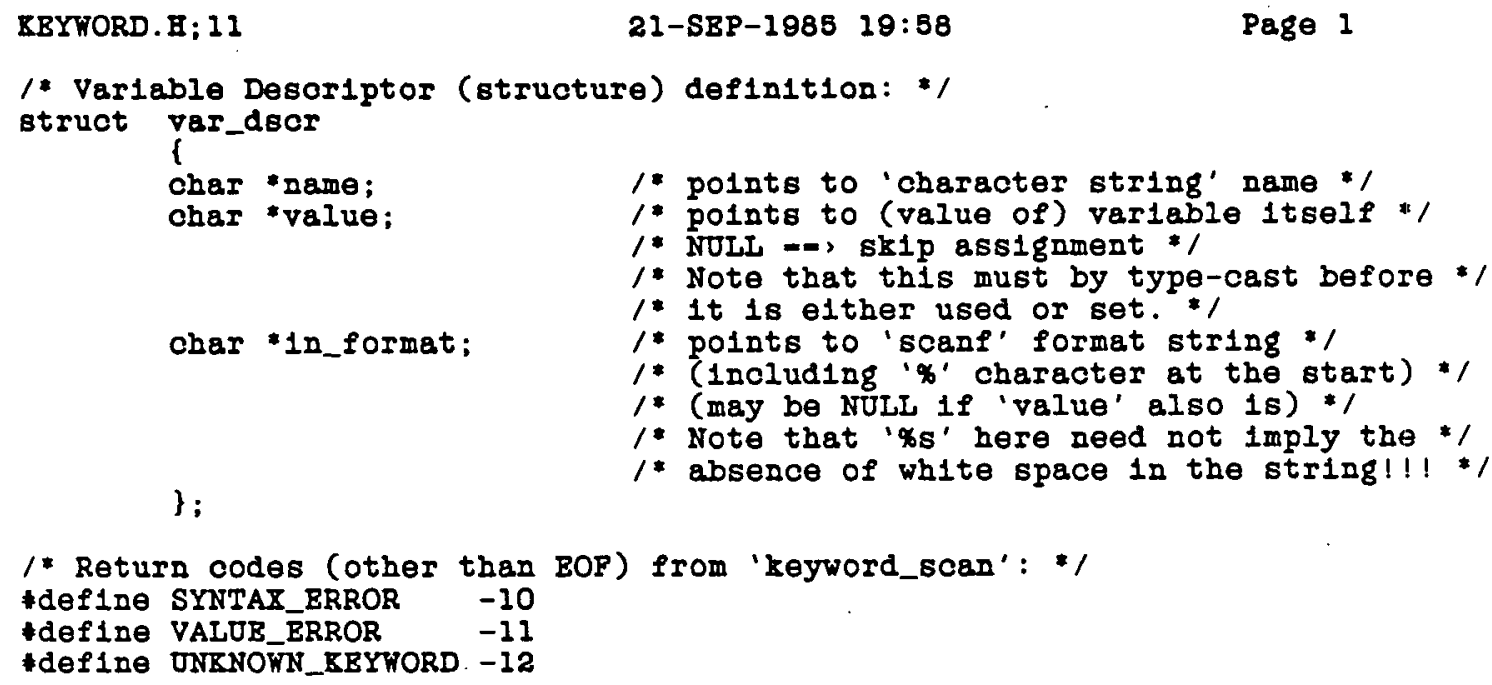




\section{util directory}

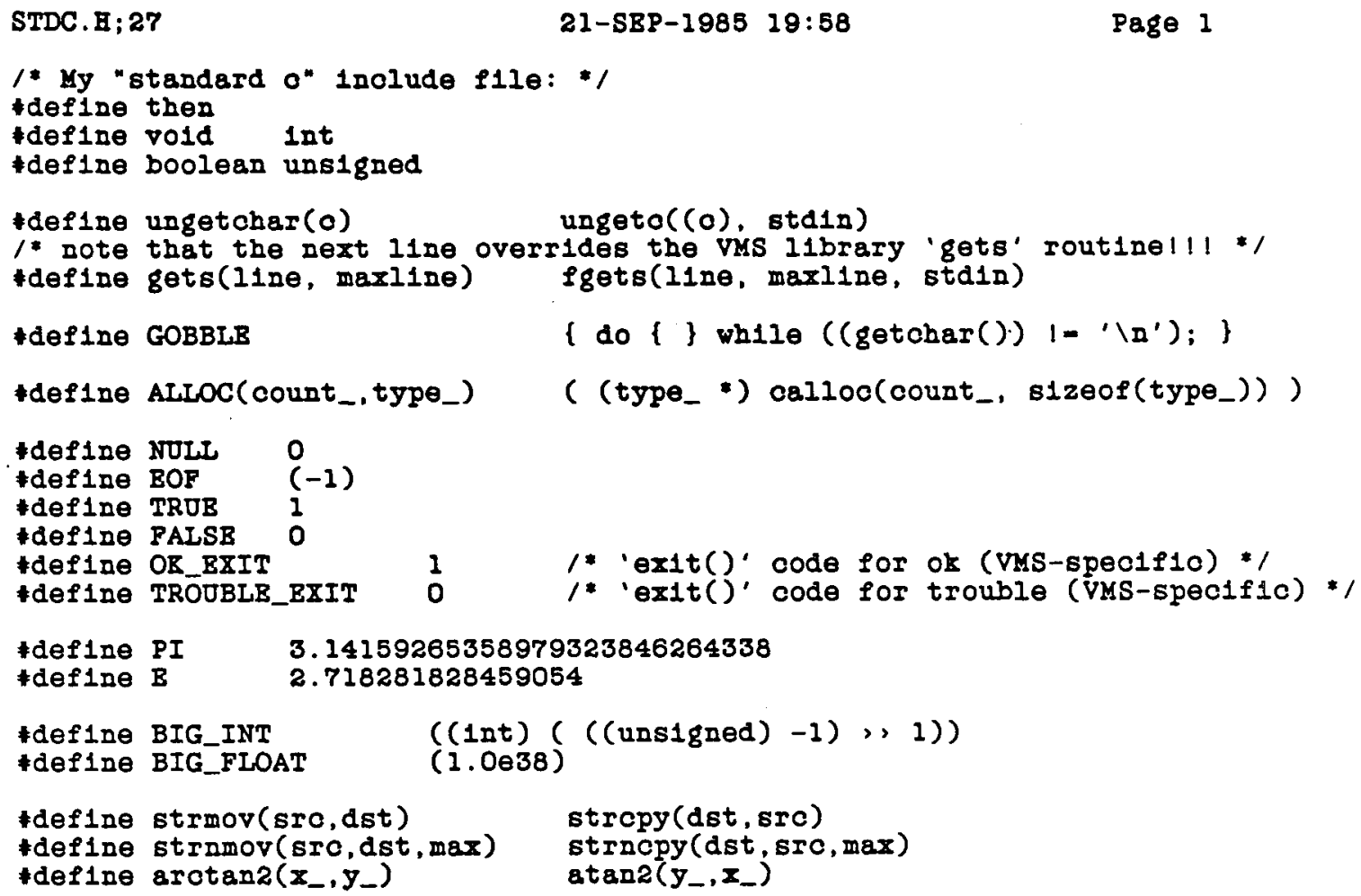




\section{ut1l directory}

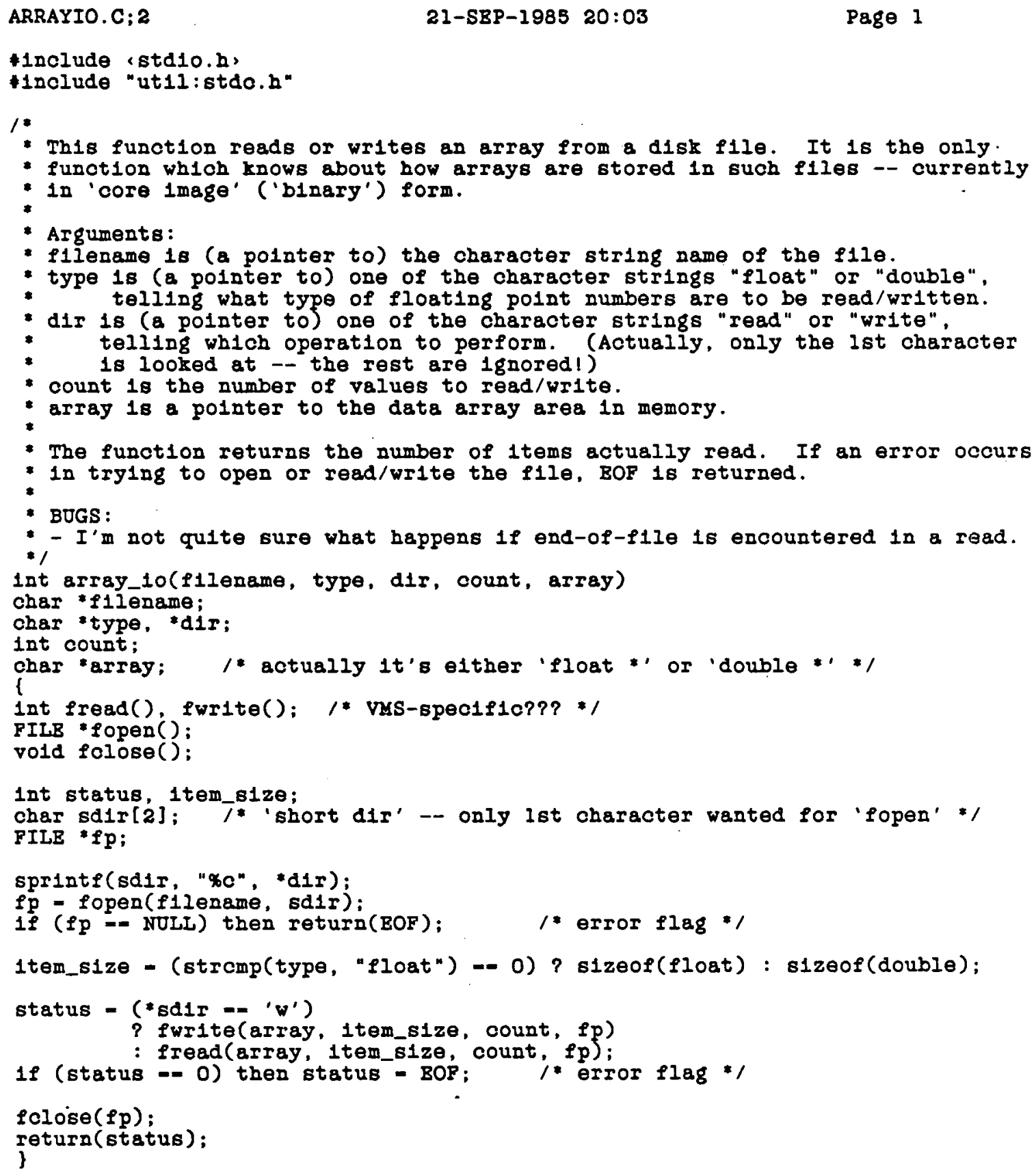




\section{util directory}

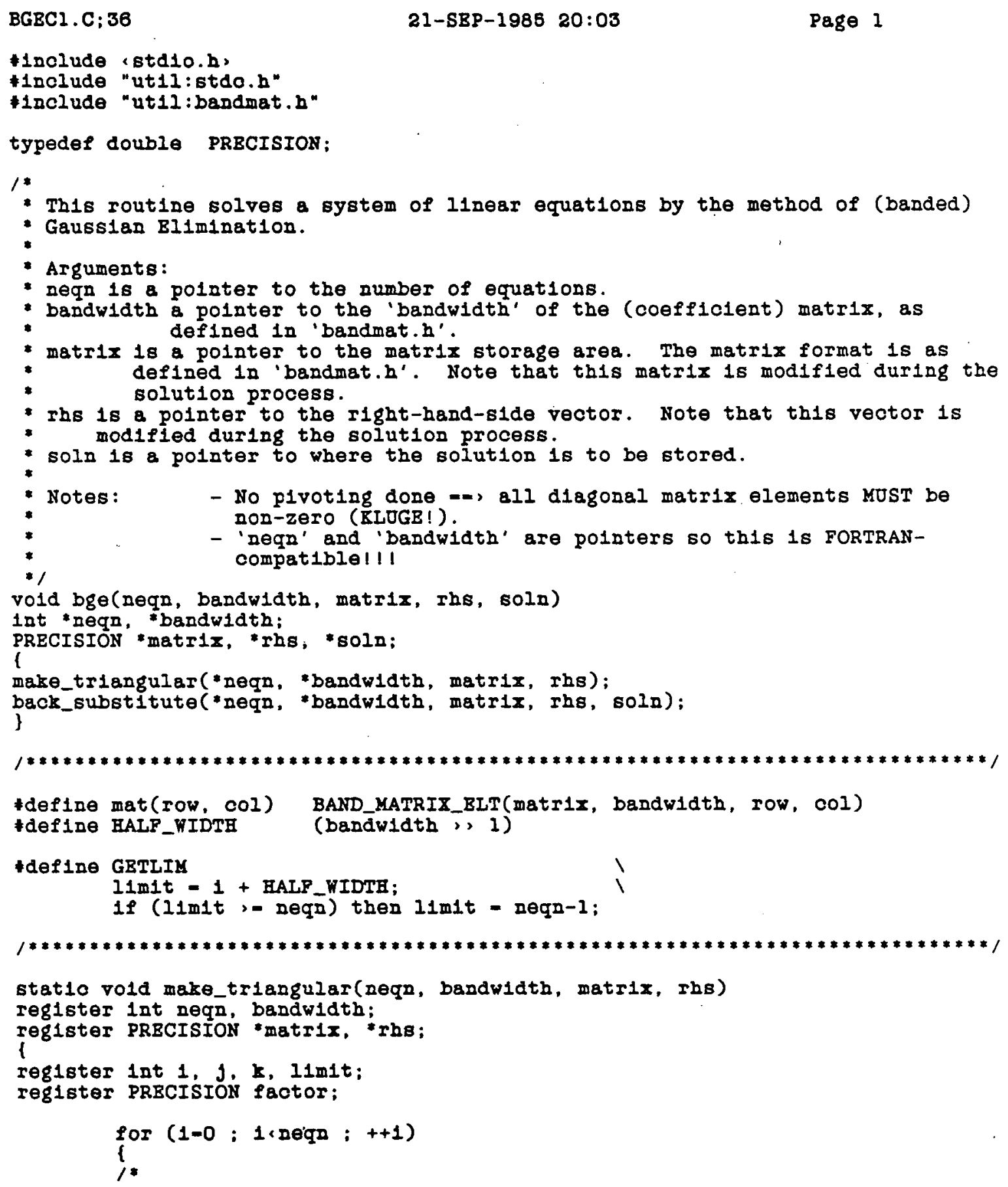




\section{util directory}

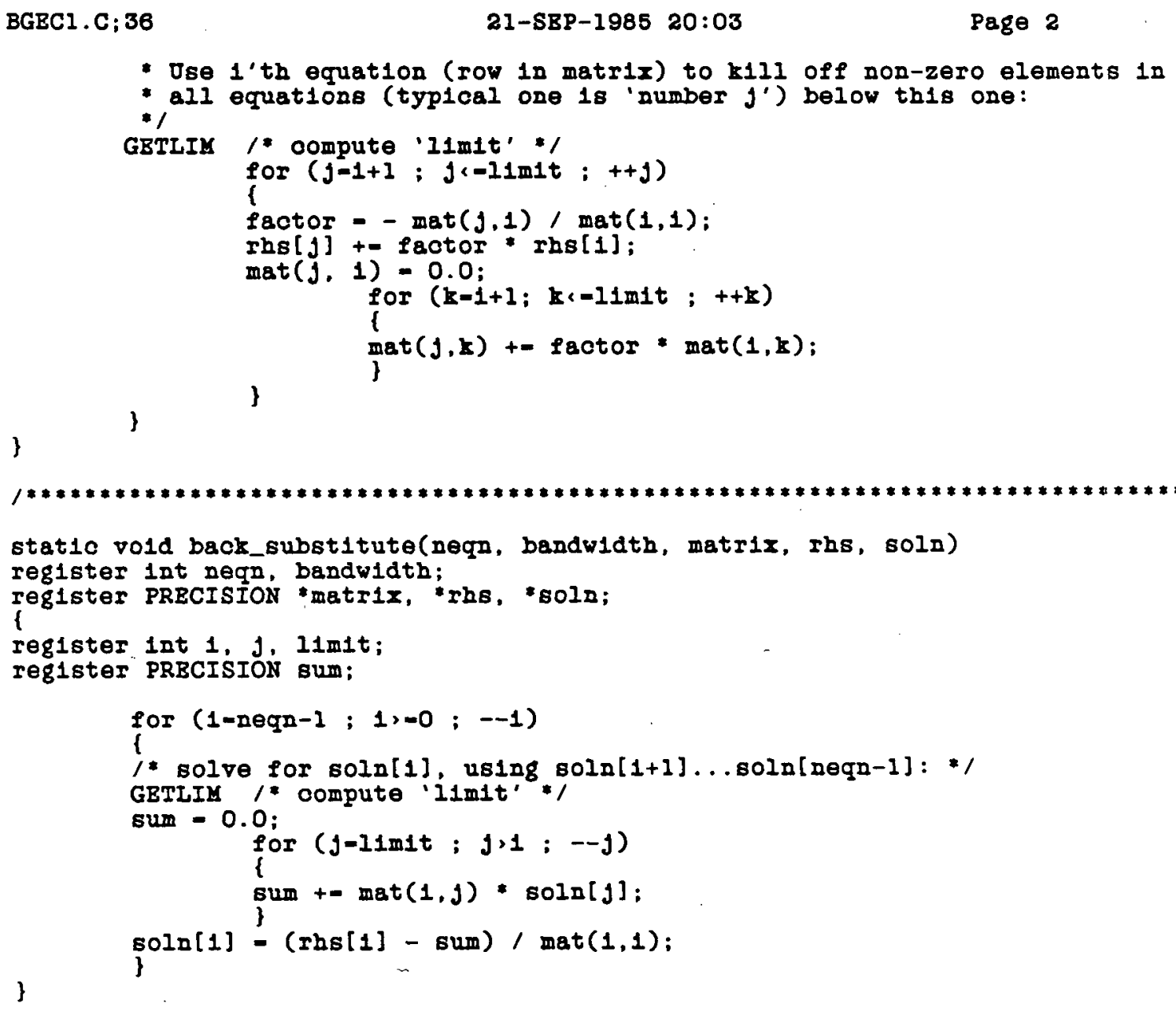




\section{ut1l directory}

CALLTRAIL. MAR; 13

t1tle call_trail

sbttl call_trall

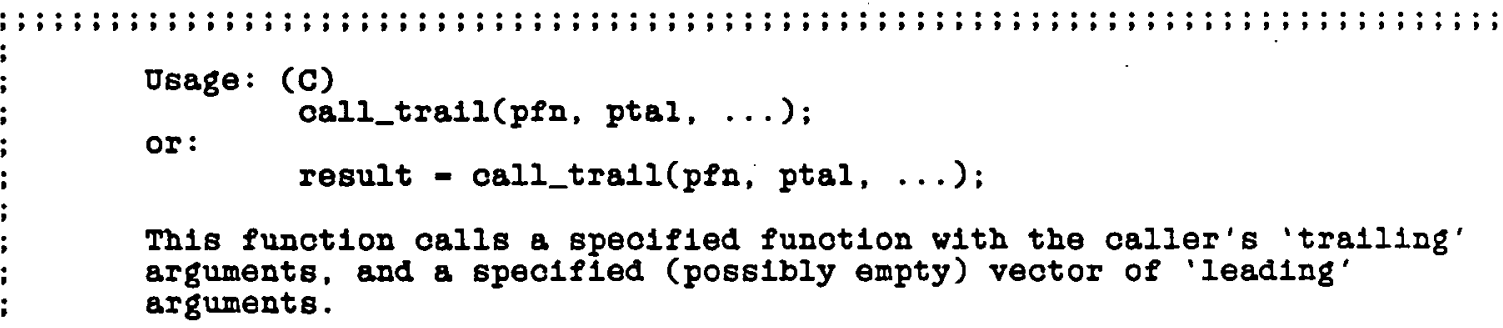

or:$$
\text { call_trall(pen, ptal, } \ldots) \text { ); }
$$$$
\text { result - call_tra1l(ppn, ptal, } \ldots) \text {; }
$$

This function calls a speolfled function with the caller's 'trailing' arguments, and a specified (possibly empty) vector of 'leading' arguments.

Arguments :

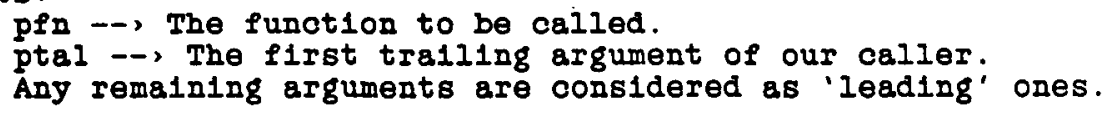

Bxample:

\#1nolude (std10.h)

foo( $111 \theta$, format)

char flle[], format [];

I

FILB " fopen(),

vold eprinte(), folose():

vold call_trall():

PILE * flleptr;

fileptr - fopen(file, "a");

call_trall(\&printf, eformat, flleptr); fclose(flleptr);

Th1s C function, when invoked with foo "flie.ext", "1-xd $\mathrm{j-xd} \backslash n ", 1, j$ )

logs a line giving the values of 1 and $j$ to the file 'file.ext'.

Example argument lists:

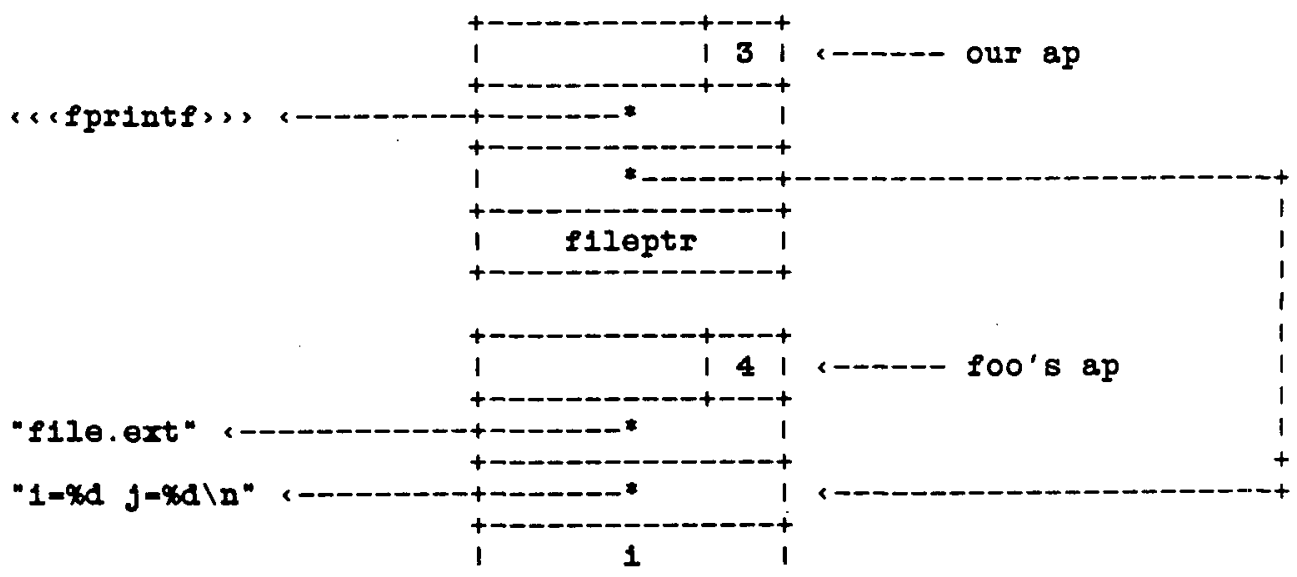




\section{ut11 directory}

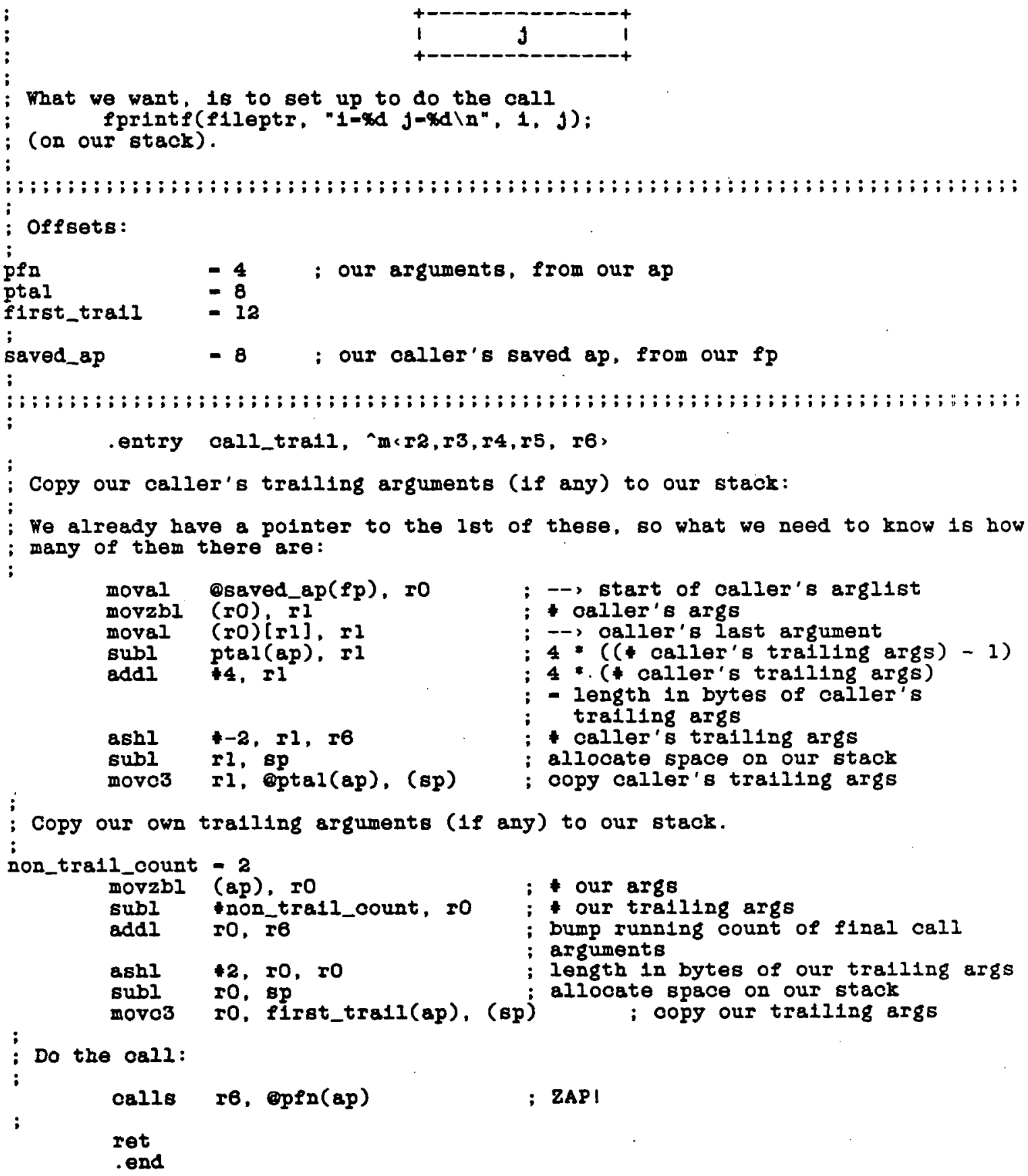




\section{ut1l directory}

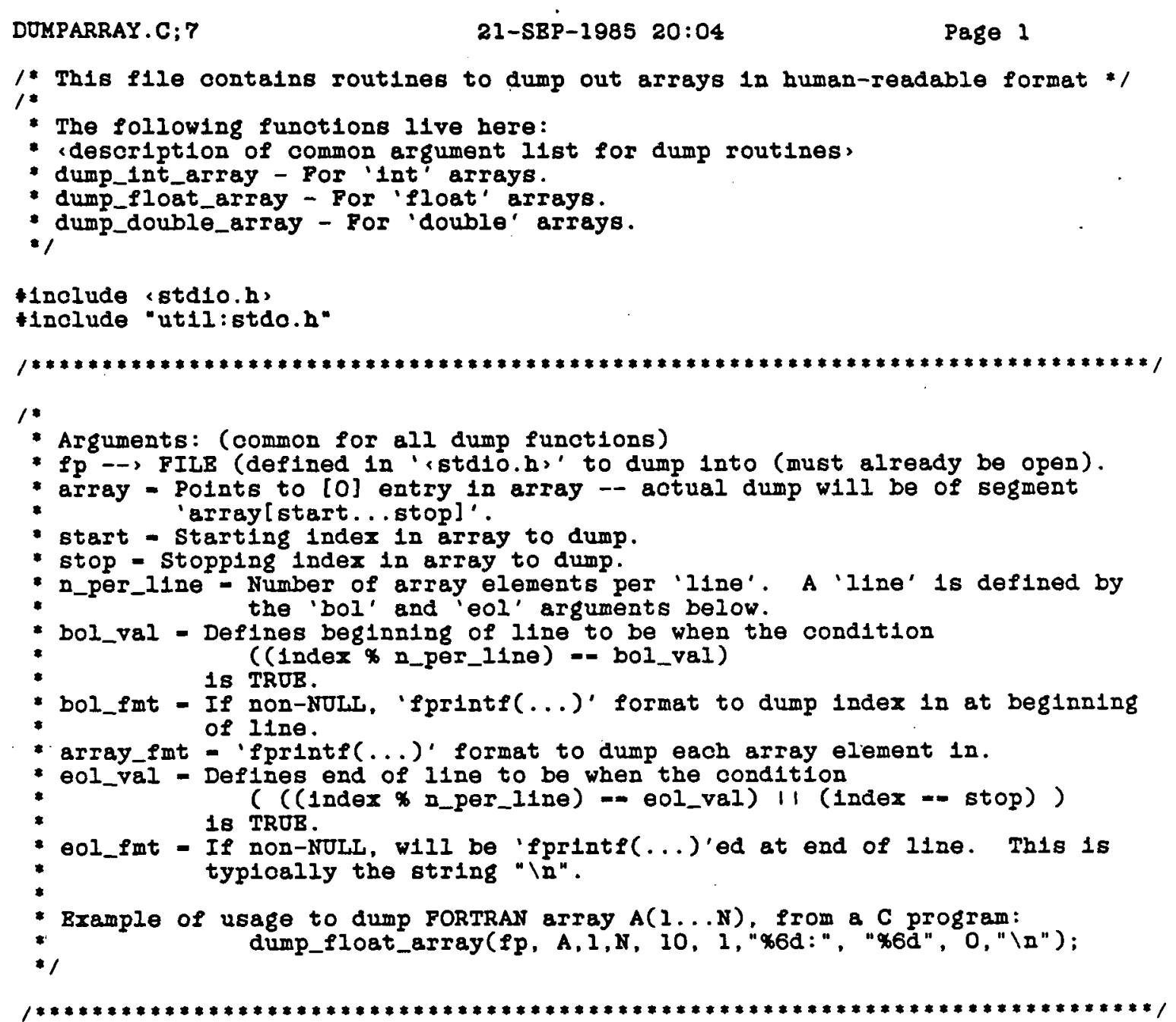

vold dump_1nt_array( $f p$. array, start, stop,
n_per_line,
bol_val, bol_fmt,
array_fmt,
eol_val, eol_fmt)

PILB * $P$;

lnt array [];

Int start, stop, n_per_11ne;

lnt bol_val;

char bol_fmt[];

char array_fmt[];

lnt $\theta 01$ ial;

char eol_fmt[]: 


\section{ut11 directory}

DULPARRAY.C; 7

\{ vold fprinte();

1nt 1;

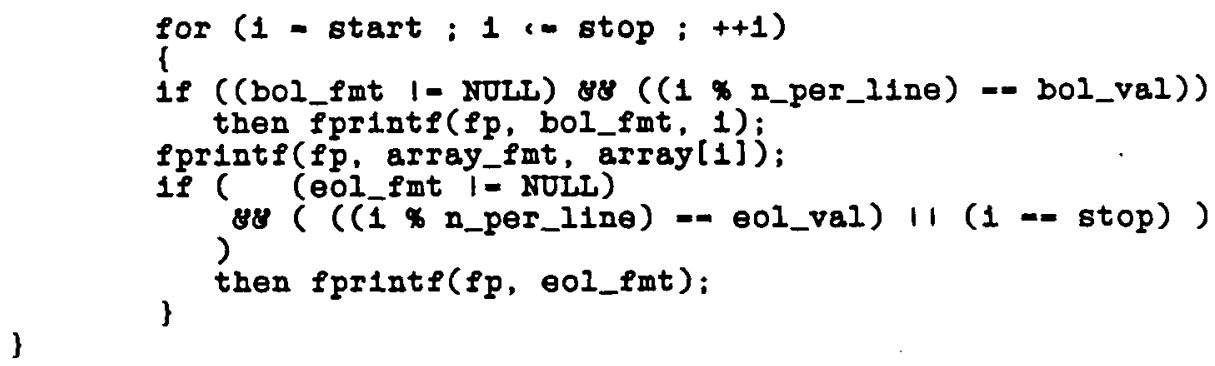

\}

Int 1;

\}

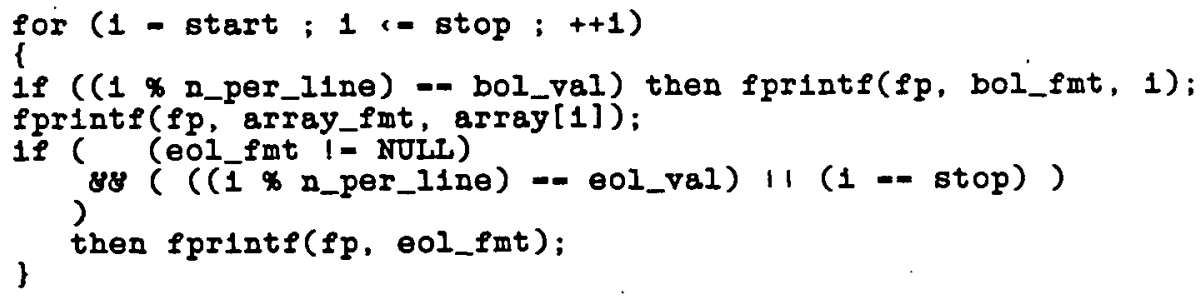


util directory

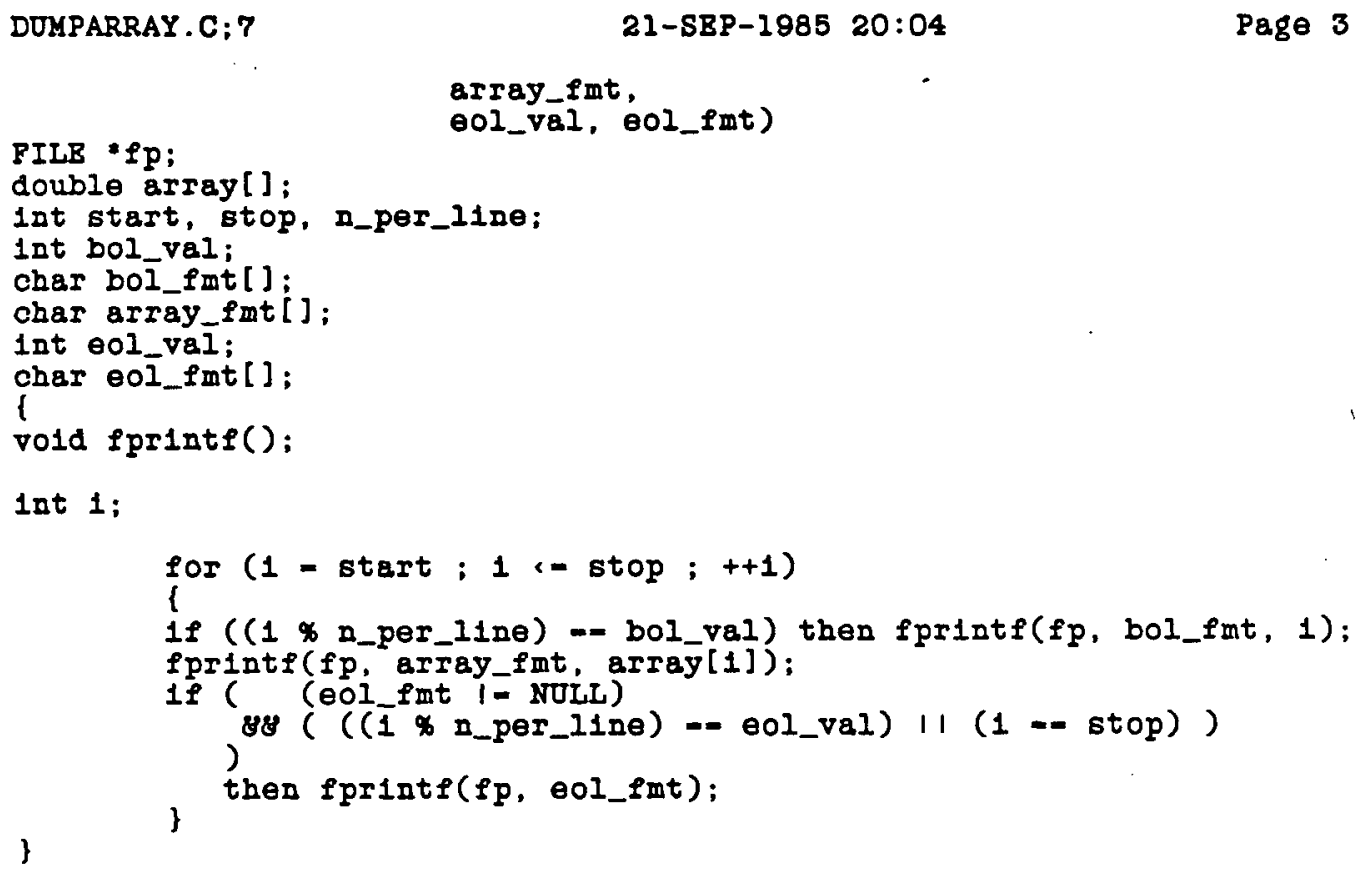


ut1l directory

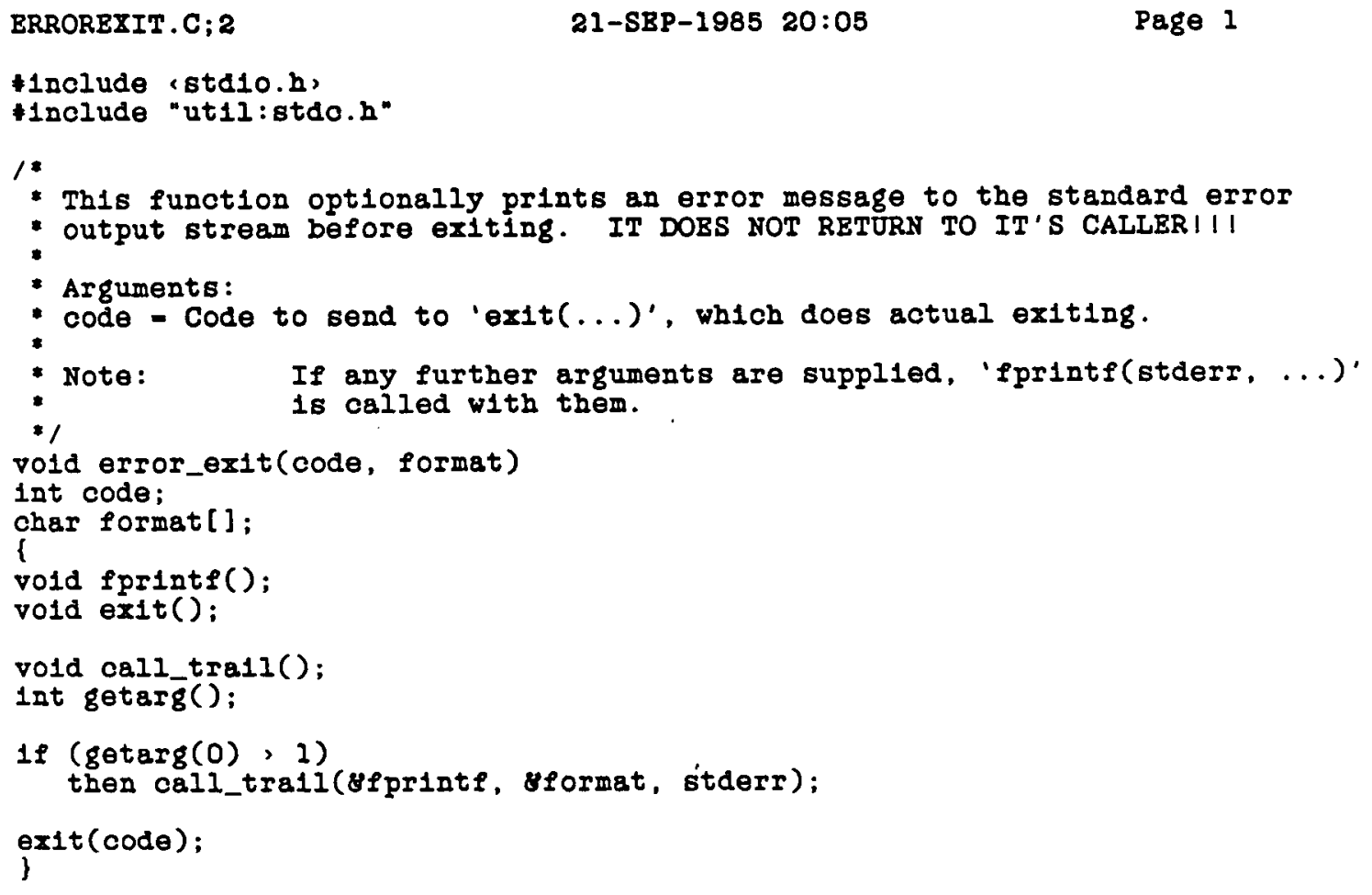




\section{util directory}

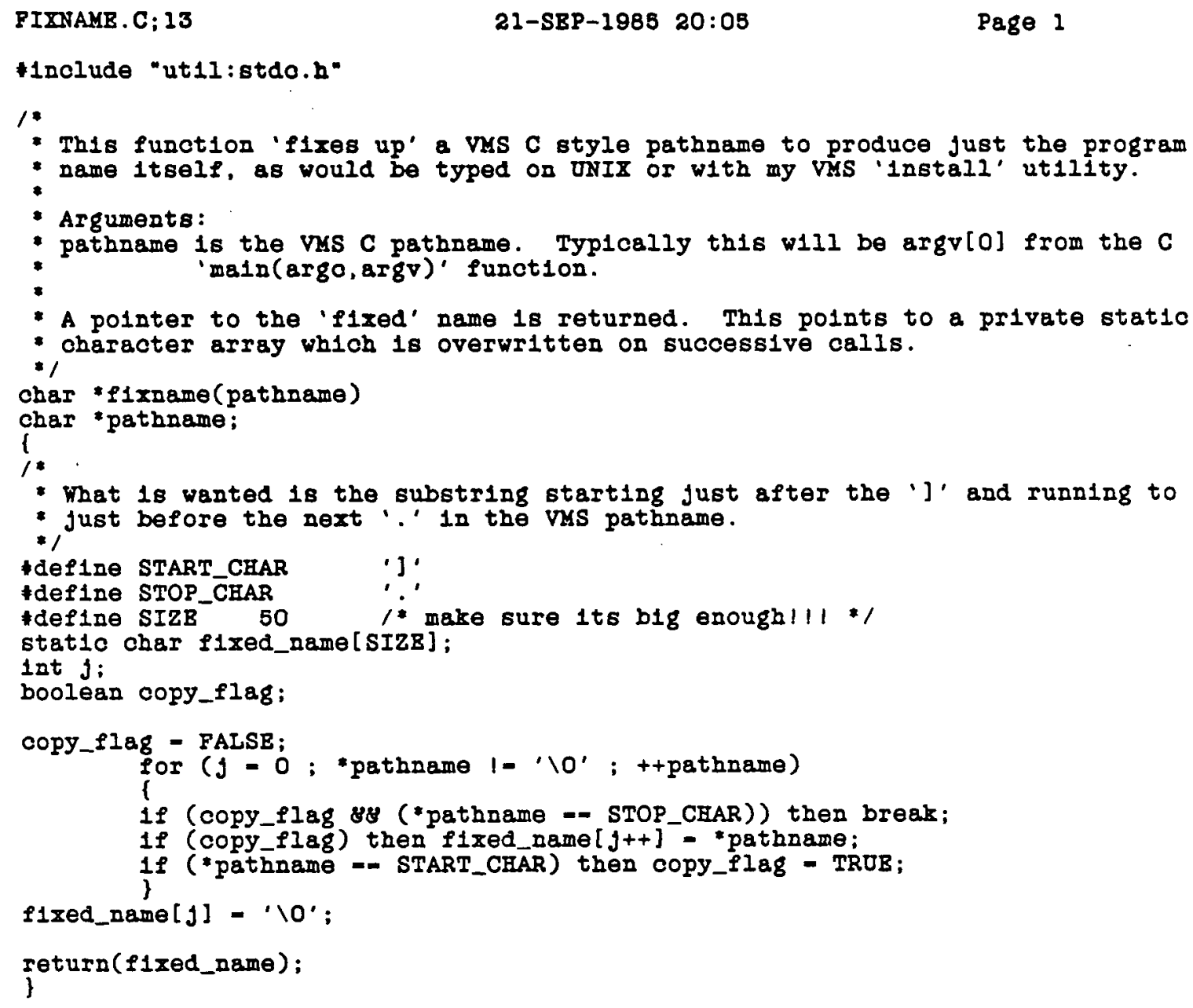




\section{util directory}

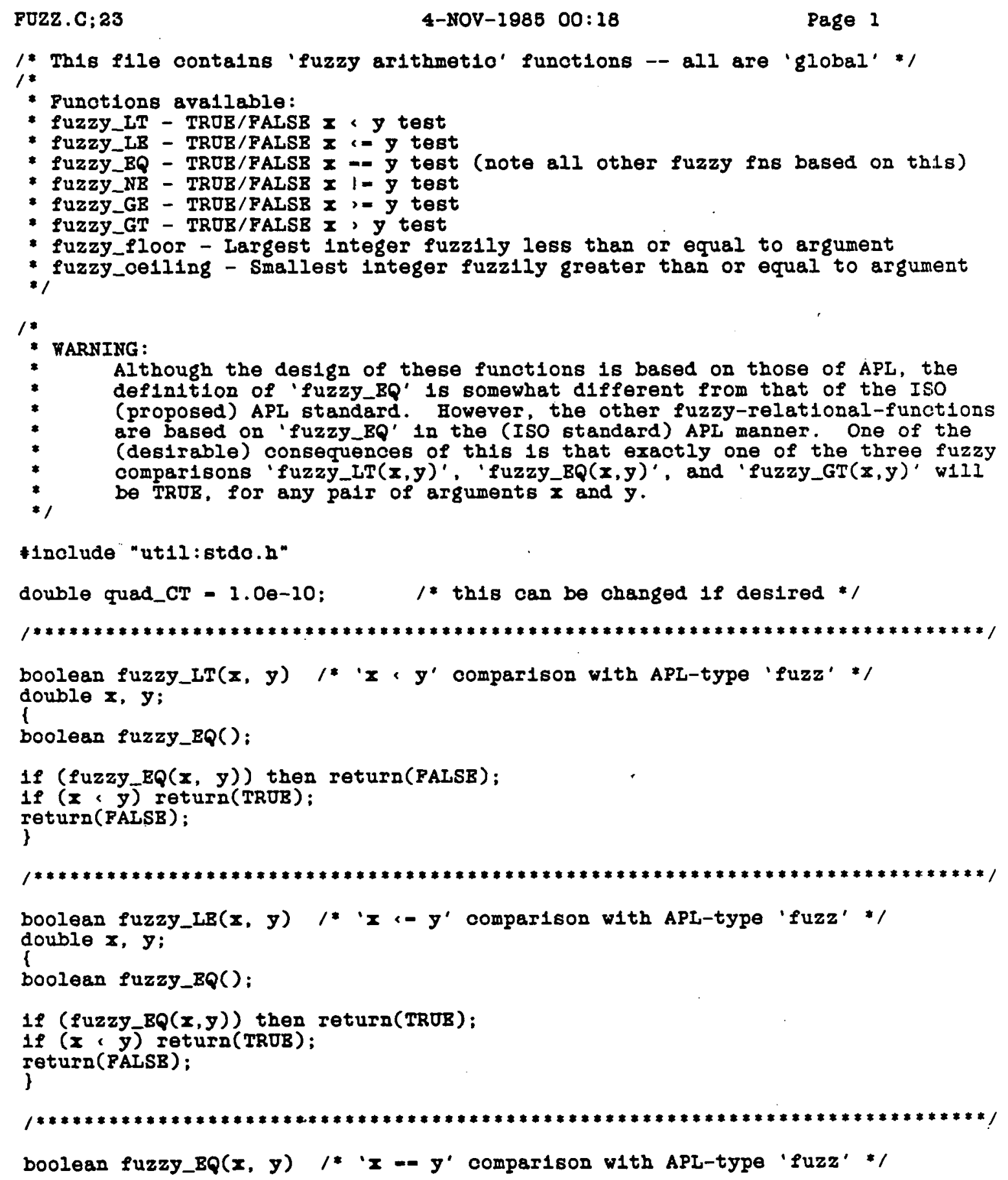




\section{ut1l directory}

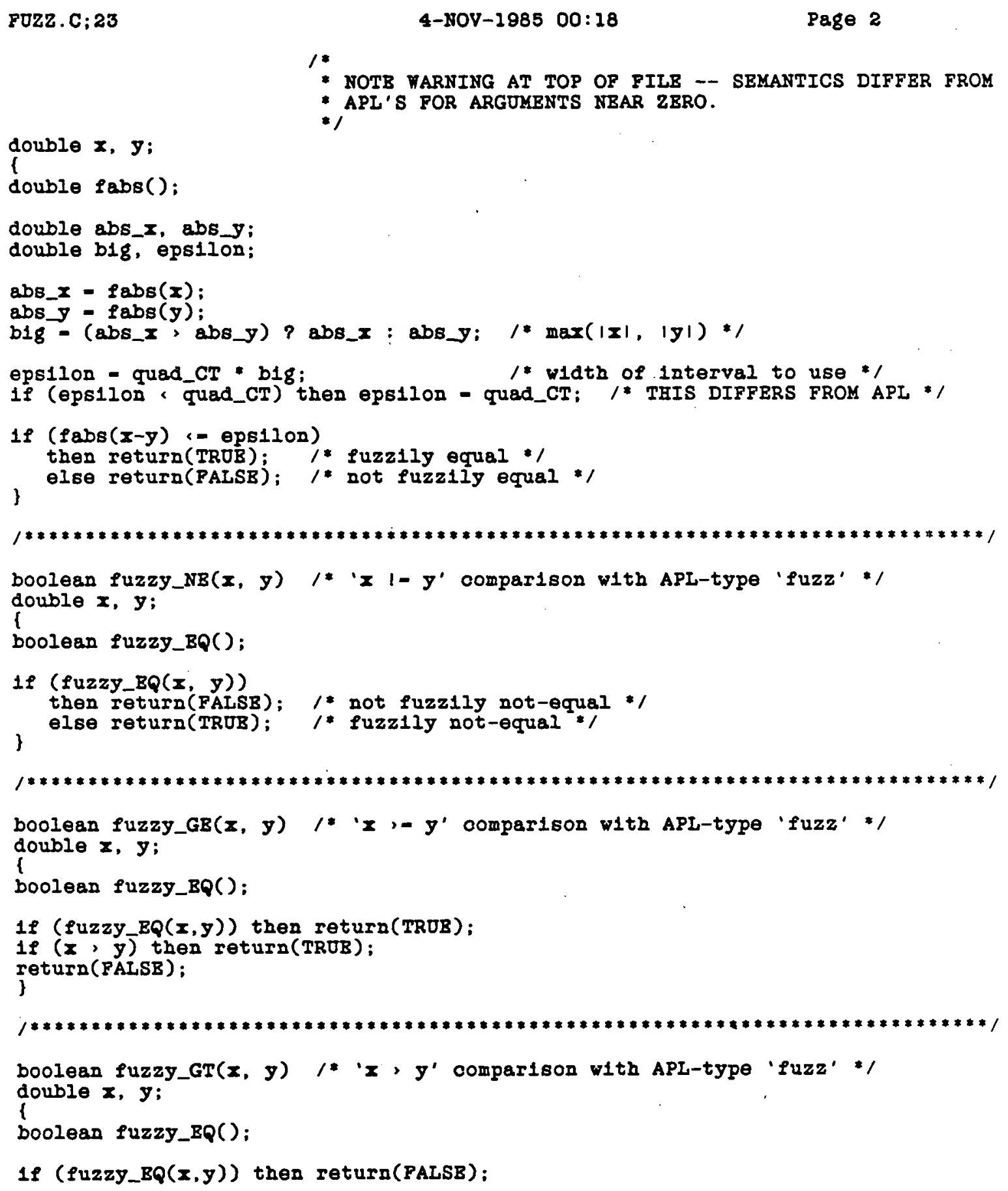


util directory

FOZZ.C:23

4-210V-1985 $00: 18$

Page 3

$11(x, y)$ then retura (TRUS):

return(FALSB);

\}

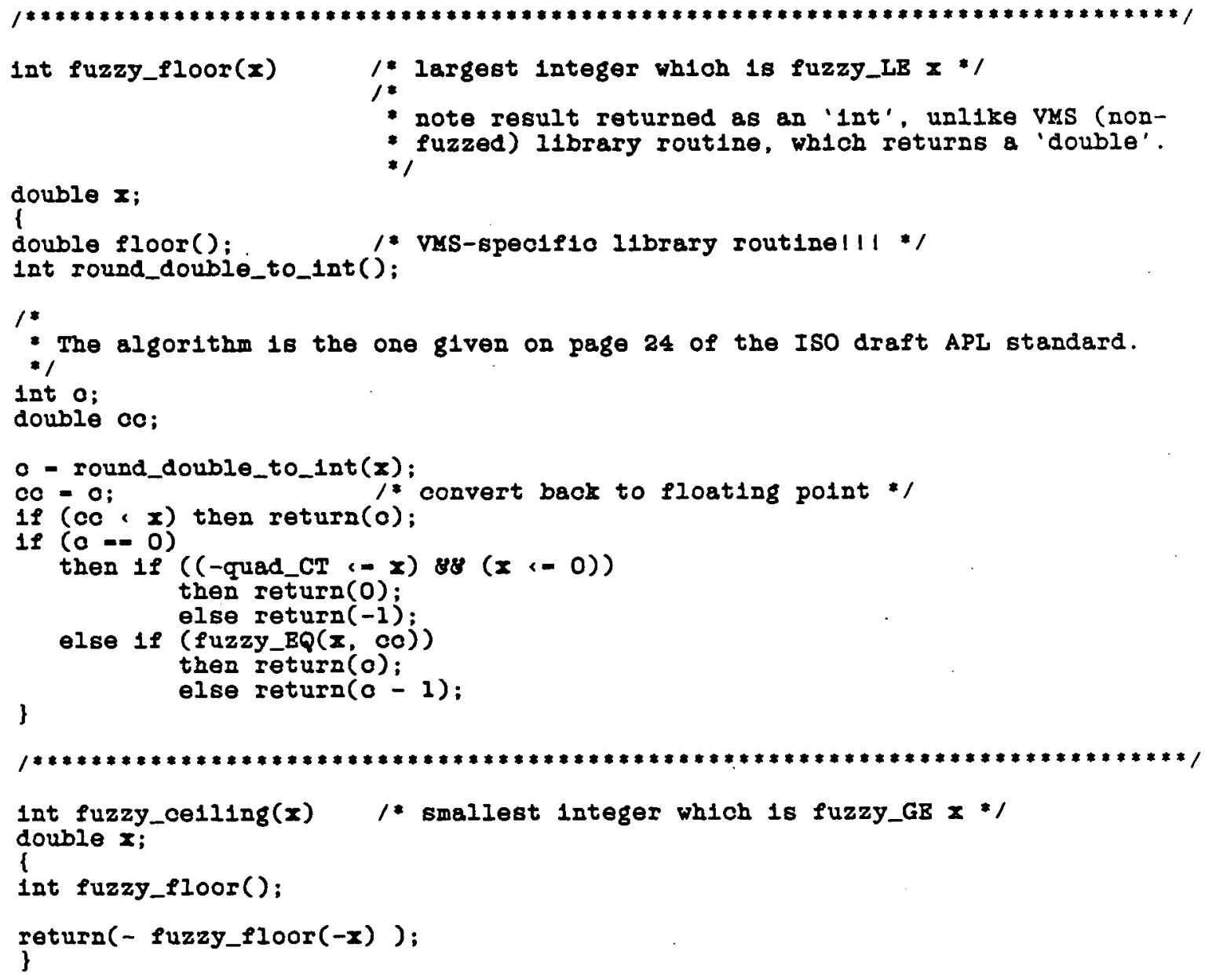




\section{ut1l directory}

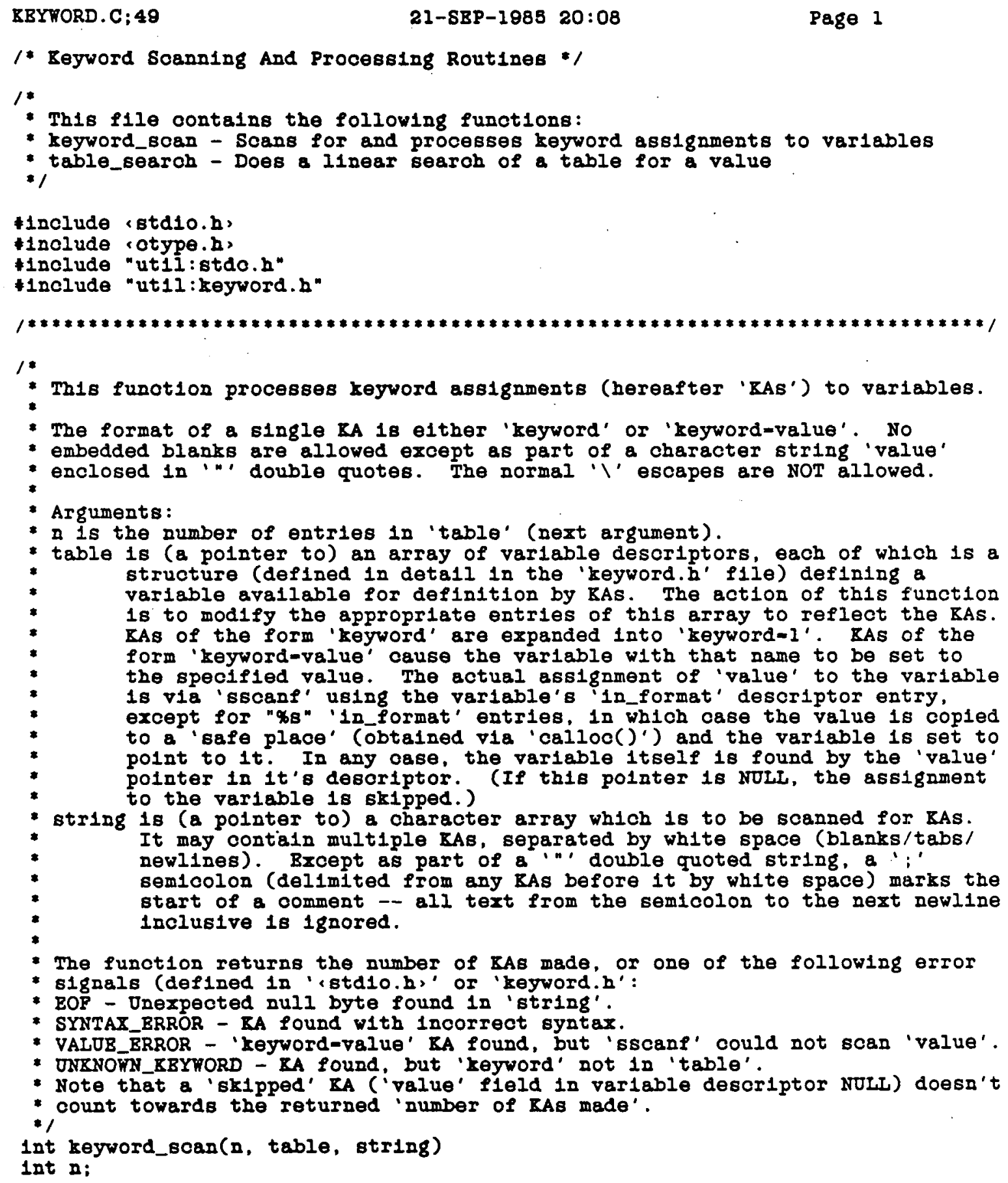




\section{ut1l directory}

BBYFORD. C; 49

21-SEP-1985 20:08

Page 2

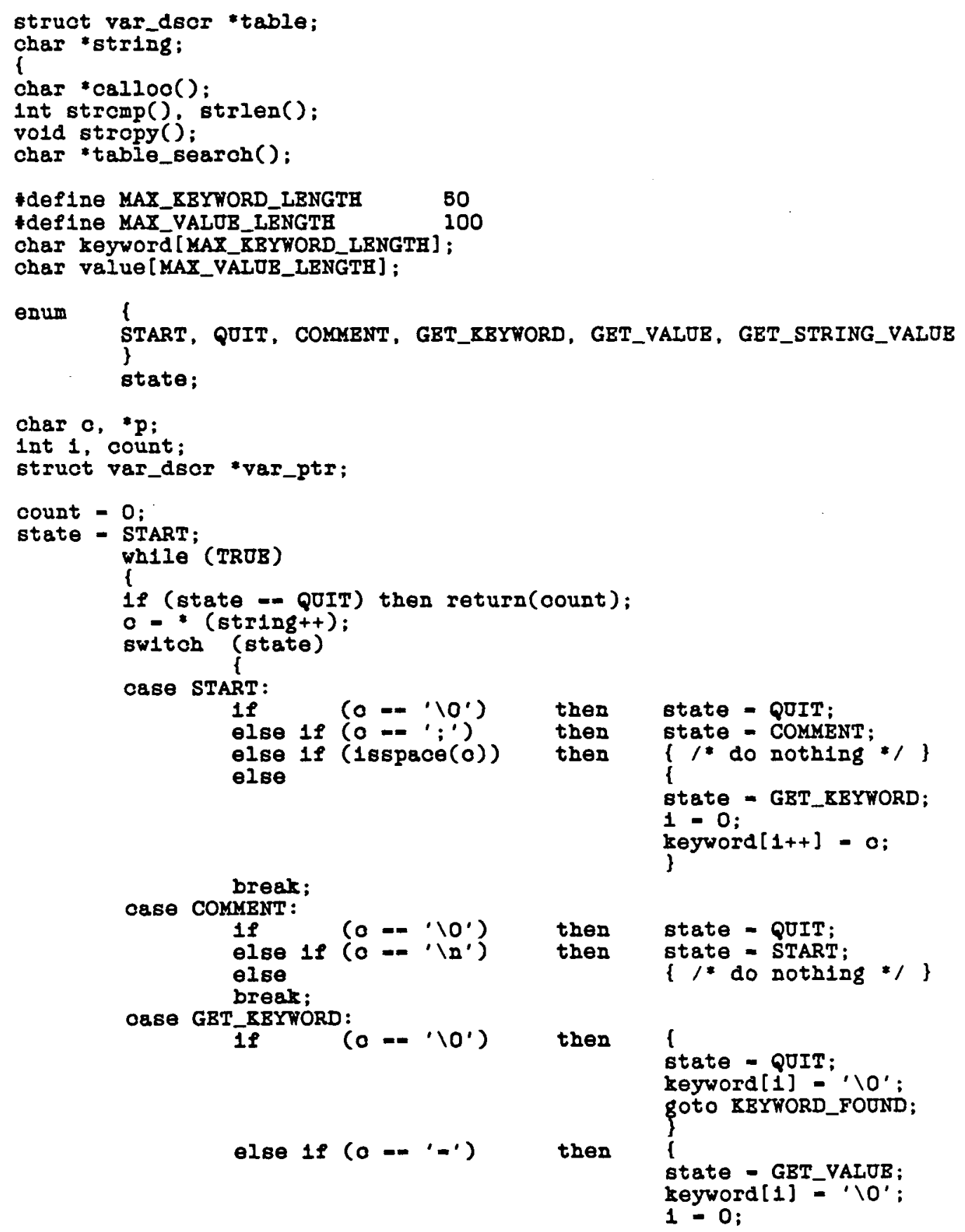




\section{util directory}

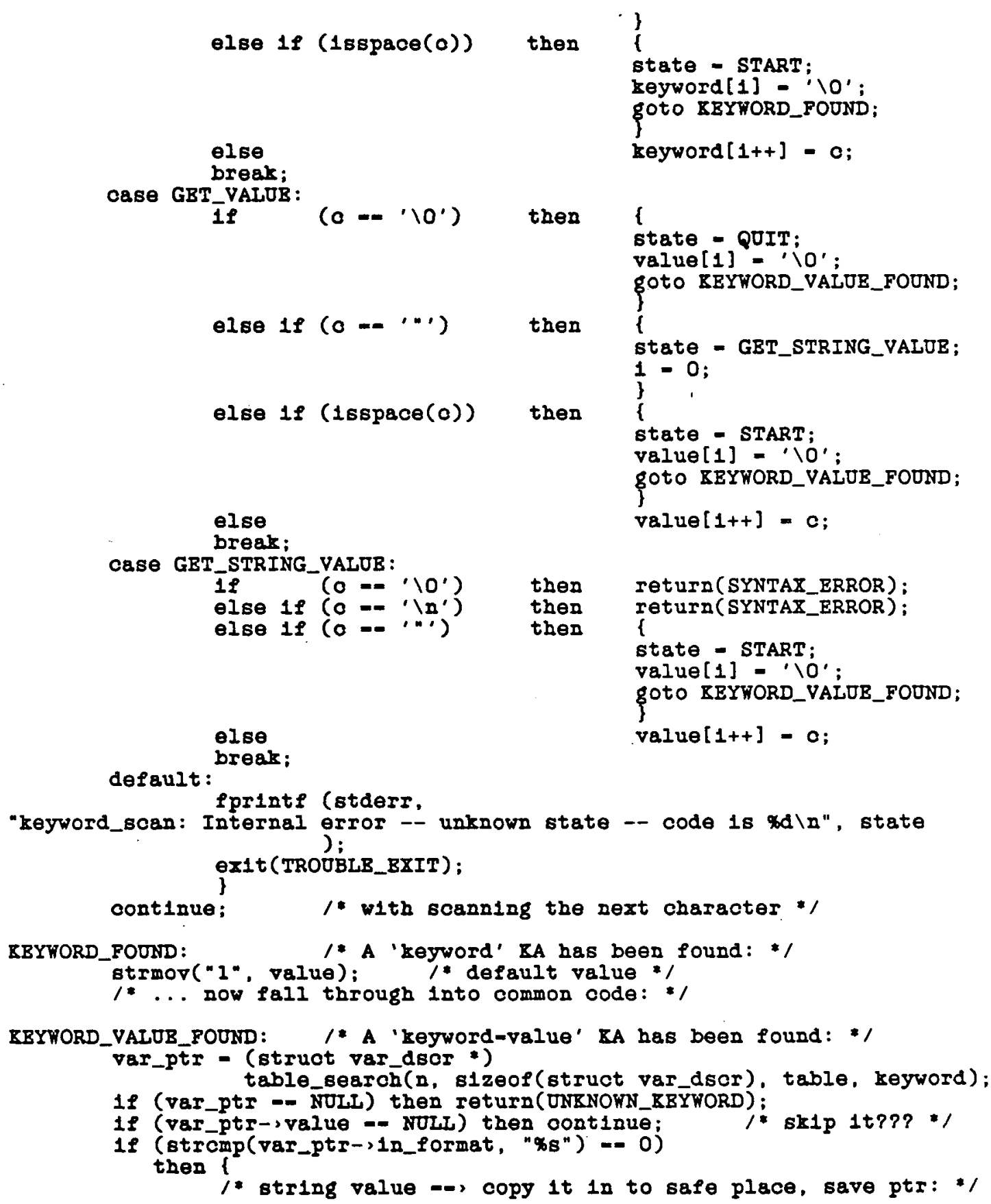




\section{ut1l directory}

BEYTORD.C; 49

$$
\text { 21-SBP-1985 20:08 }
$$

Page 4

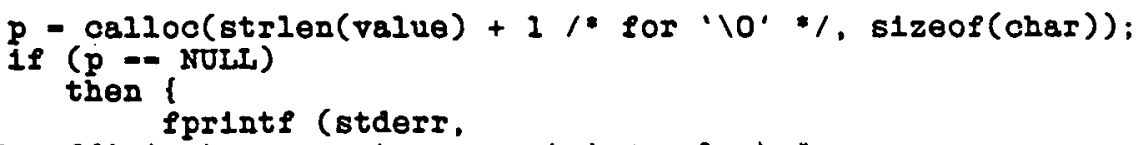

"keyword_scan: Insufficlent memory to save str1ng value

):

exit(OK_BXIT); \}

otrmov(value, p):

( (ohar *)(var_ptr-ivalue) ) - p;

else ++ count: \}

I* non-string value $=-$ let 'sscanf' store 1t: */

1 - sscanf(value, var_ptr-i1n_format, var_ptr-ivalue):

if ( 1 l- 1 ) then return(VALOB_ERROR); + +oount;

\}

\} )

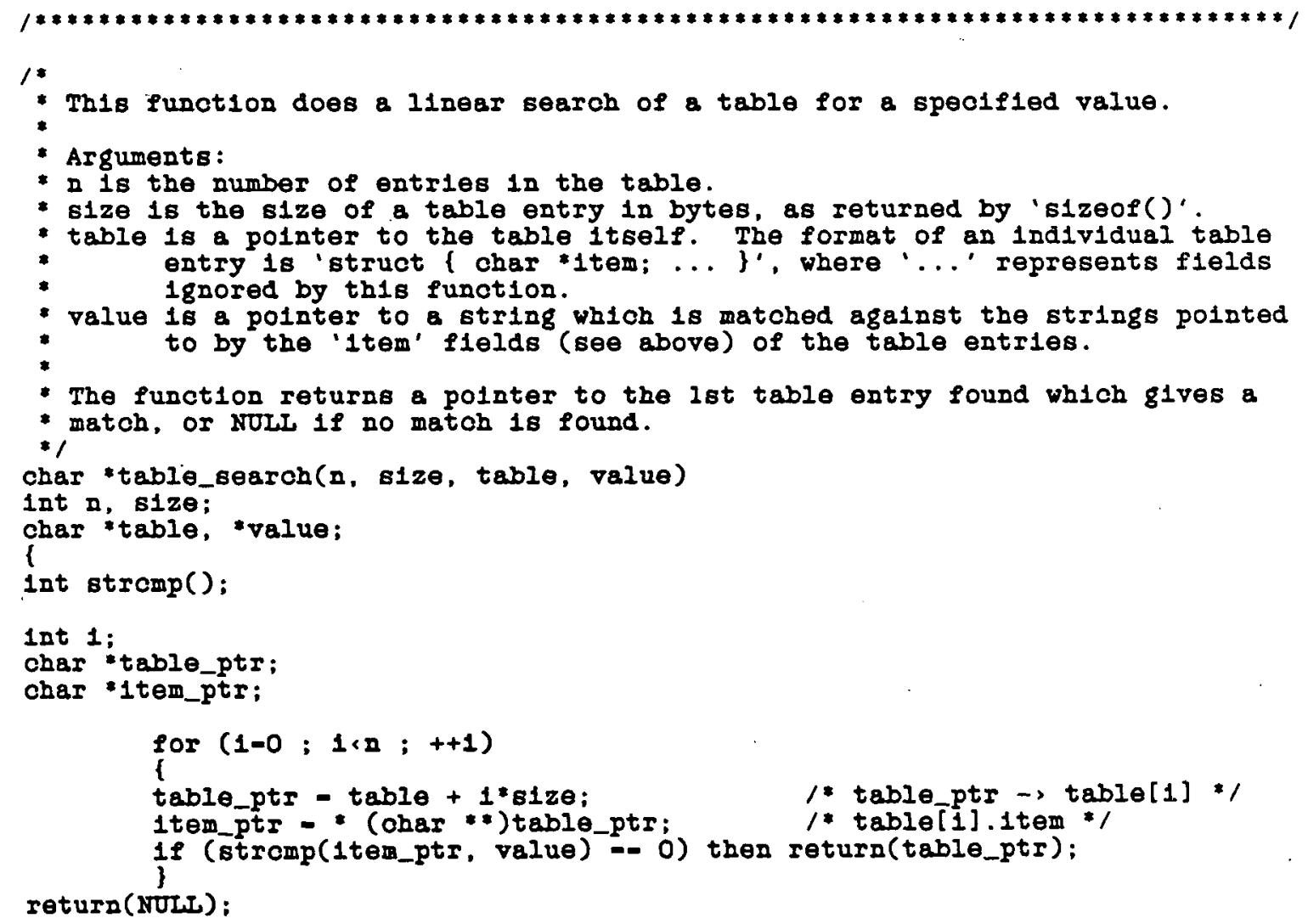


ut1l directory

BBYWORD.C;49

21-SBP-1985 20:08

Page 5

\} 
ut1l directory

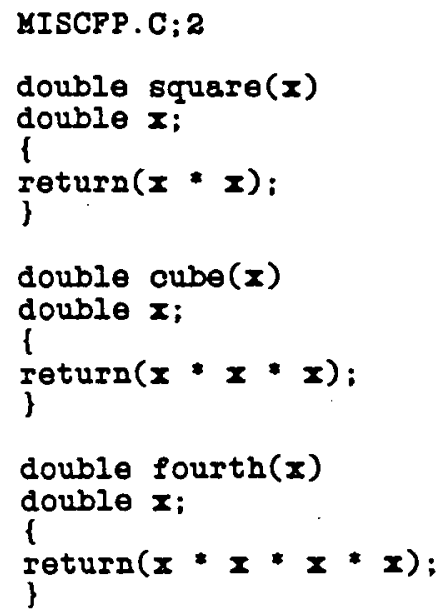


ut11 directory

ROUND.C; 1

21-SBP-1985 20:08

Page 1

\#1nclude "ut1l:stdo.h"

1nt round_double_to_1nt(d)

double d;

1

1nt 1;

1* note $C$ double-- int conversion truncates */

if (d, 0.0)

then $1=d+0.5$;

else

d - -d; $\quad /$ force positive -- negatives are system-dependent */

$1=d+0.5$

$\frac{1}{1}=-1 ;$. compensatell 1

return(1);

\} 


\section{ut1l directory}

SPLINB.C; 8

21-SBP-1985 20:08

Page 1

1: This file contains routines for using one dimensional cuble splines. "/

1

- The following functions live in this file:

" ¿definition of spline functional form.

- spline_calo - Calculates coefficlents for a cublo spline given data polnts

- spline_1nt - Integrates evenly spaced 1-dimensional data by spline pitting

$\because$

Hnclude "util:stdo.h"

1nolude "ut1l:bandmat. $h$ "

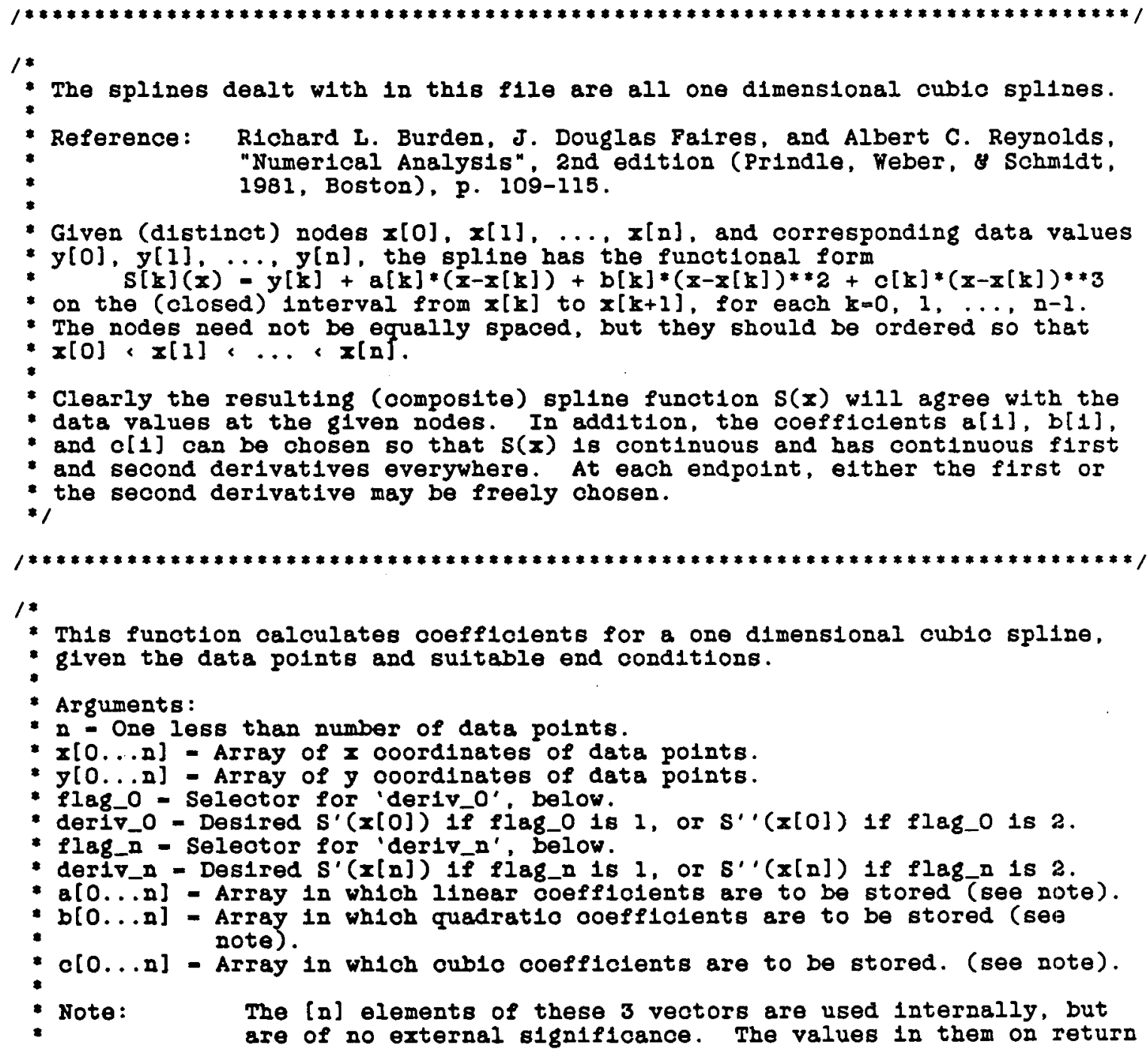




\section{ut1l directory}

SPLINB.C; 9

21-SBP-1985 20:08

Page 2

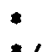

vold spline_calc(n, $x, y, 128 g$,, der1v_o, flag_n, der1v_n, a, b, c)

from this function should be 1 gnored.

int $n$;

register double $x[], y[]$;

Int flag_o;

double deriv_o;

1nt flag_n;

double deriv_n;

register double a[], b[], $a[]$; 1

char *alloo();

vold ceree();

vold error_exit():

vold bge (); 1 ; banded Gauss elimination $A * x=b$ solver *

1nt $\mathbf{k}$;

lnt neqn, bandwidth;

double h_k, h_kml ;

double (matrix) [3], shs;

$1 *$

* To flnd the coefflolents, we f1rst solve for the quadratio coefficients b[k]

- which satisfy a tridiagonal linear system of order $n+1$. We can then easiliy

* calculate the IInear and cublo coefficients from the quadratic ones.

- 1

matr1x $=\operatorname{AILOC}(3 *(\mathrm{n}+1)$, double $)$

rhs - Alloc $(n+1$, double);

$1 f($ matrix $==$ NULL) I। (rhs $==$ NULL))

then error_ex1t(OR_BXIT,

**** spline_calo: Insufficlent memory for a \%d point spline n+1); 1* DOBS NOT RETURN *

I. f1ll in interior coefflolent matrix and rhs veotor: *

for ( $k=1 ; k<-n-1 ;++k)$

(

1. formulas from the reference, p. 111-112: *

h_k $\mathbf{x}[\mathbf{k}+1]-\mathbf{x}[\mathbf{k}]$;

$\mathrm{h}$ km $1-x[k]-x[k-1] ;$

$\operatorname{mats} 1 x[k][0]=b \_k m l ;$

$\operatorname{matr1x}[k][1]=2.0 *\left(h_{-} k+h_{-} k m 1\right)$;

$\operatorname{matr1x}[k][2]-h \_k$;

$\operatorname{rhs}[k]-3.0 *\left((y[k+1]-g[k]) / h \_k-(y[k]-y[k-1]) / h \_k m l\right)$;

* 1111 in lower end point values: *!

matrix[0][0] = 0.0; $/$ not used *

$1 \rho$ ( 1 log_0 $0=1$ )

then

$f$ der1v_o 18 desired first derivative at $x[0]:$ *

h_k $-x[\bar{l}]-x[0]$;

$\operatorname{matr1x[0][1]=2.0*h+k;~}$

$\operatorname{matr} 1 x[0][2]=$ h_k;

rbs[0] $\left.=3.0 *(y[1]-y[0]) / h \_k-\operatorname{der} 1 v_{-0}\right)$; 


\section{util directory}

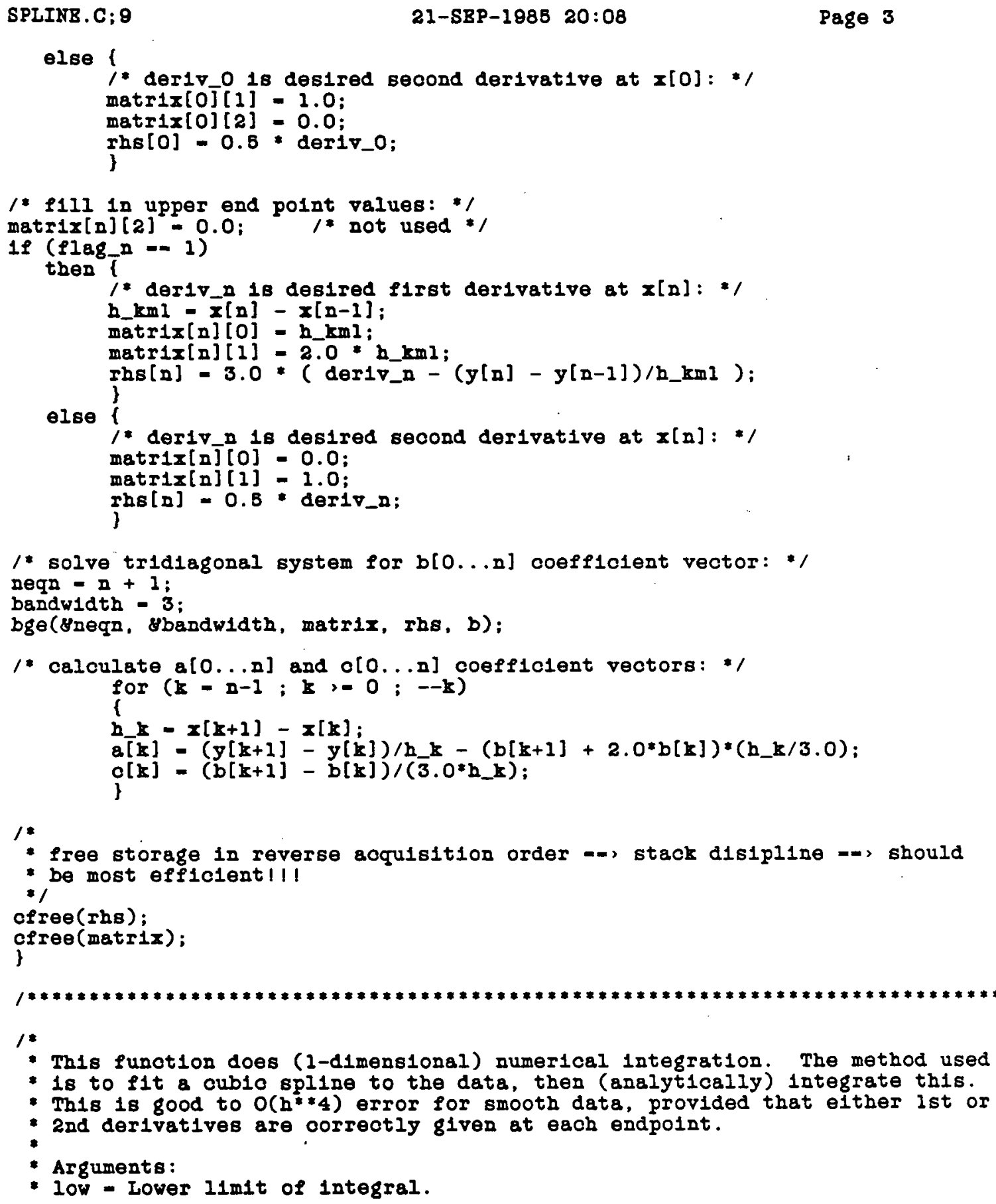

- This function does (1-dimensional) numerical 1ntegration. The method used

- Is to flt a cubio spline to the data, then (analytically) 1ntegrate this.

- Th18 18 good to $O\left(h^{*} * 4\right)$ error for smooth data, provided that e1ther ist or

- 2nd derivatives are correctly given at each endpoint.

- Arguments:

* low - Lower limit of integral. 


\section{ut11 directory}

- h1gh - Opper l1mit of Integral.

- n- Number of intervals.

- $y[0 \ldots z]$ - vector of data values to be integrated.

- flag_low - Seleotor for 'deriv_low', below.

- deriv_low - $y^{\prime}[0]$ if flag_low is 1 , or $y^{\prime \prime}[0] 1 f$ flag_low is 2.

- flag_high - Selector for 'der1v_high', below.

- deriv_high - $y^{\prime}[n]$ if flag_high is $I$, or $y^{\prime}$ '[n] if flag_high is 2.

- The estimated 1ntegral is returned as the function value. * 1

double spline_1nt(low, h1gh, n, g, flag_low, der1v_low, flag_h1gh, der1v_h1gh) double low, high;

int $\mathrm{I}$;

reglster double $\mathrm{yl}]$;

1nt flag low;

double deriv_low;

1nt flag_high ;

double dertv_high;

1

char calloo():

vold efree();

vold error_extt();

vold spline_calc();

double h, h22, h33, h44;

register double $x,=a,=b, c$;

register double sum;

register 1nt 1;

1* allocate storage: *1

$x=\operatorname{ALLOC}(n+1$, double):

a - ALIOC $(n+1$, double):

$b=\operatorname{ALLCC}(n+1$, double);

$c=\operatorname{ALLOC}(n+1$, double);

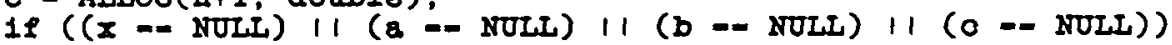

then error_exit(OR_BXIT.

"*:*** integrate: Insufflcient memory for n=od pointsin",

a); 1* DOES NOT RETURN *1

/ set up for and do spline fit: *

h - (b1gh - low) / n;

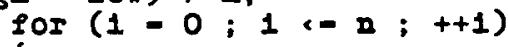




\section{util directory}

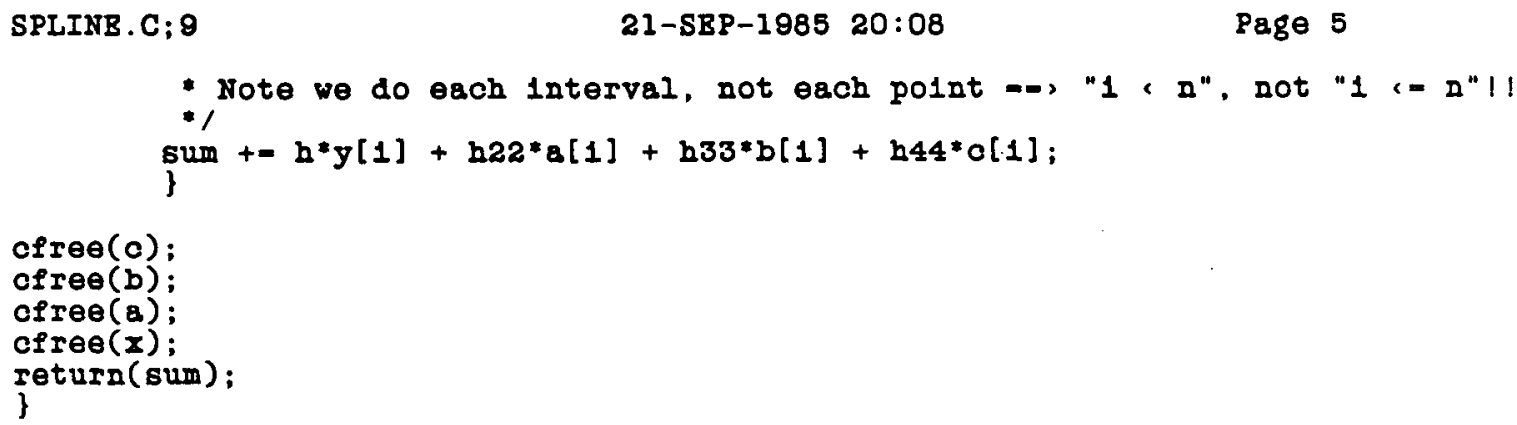




\section{ut1l directory}

TIMB.C; 20

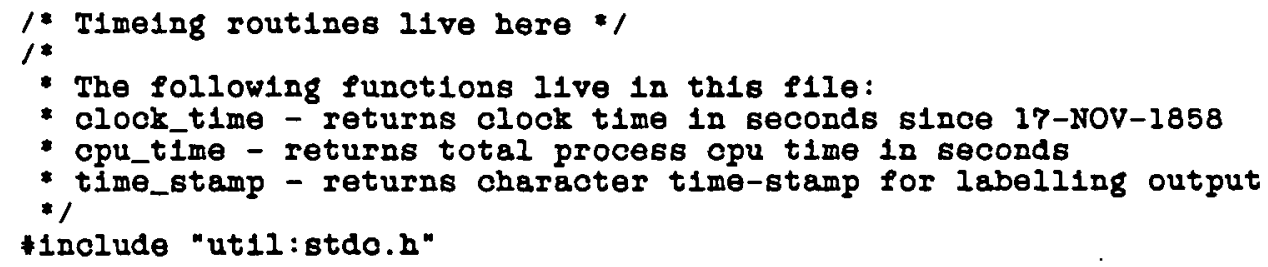

\{

tnt IIB\$STAT_TIMBR( ) ;

Int clock_code $=1$ :

short unsigned enswer [4];

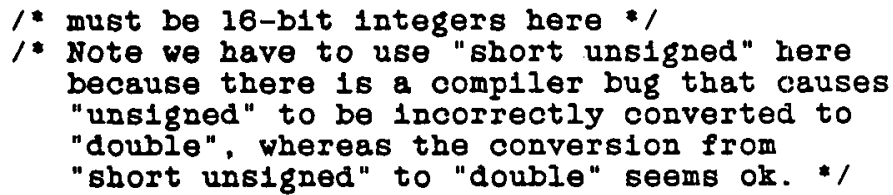

1nt 1;

double sum, weight;

1 returns "answer" in 100-nanosecond units as a 64-b1t integer: $/$ LIB\$STAT_TIMER (\&clock_code, sanswer [0]);

$\operatorname{sum}=0.0$;

welght $=1.0$;

for $(1-0 ; 1,4 ;++1)$

t

sum +- welght (double)answer[1];

we1ght $=65536.0 ; \quad / * 2$ to the $16 \%$

)

return $(100.00-9 *$ sum);

\}

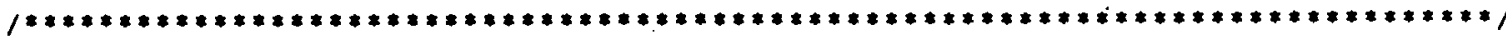

double opu_time() / Ieturns total process opu time in seconds */

(

int IIB\$STAT_TIYER();

Int opu_oode - 2; 


\section{ut11 directory}

TIKB.C; 20

21-SBP-1985 20:11

Page 2

uns1gned enswer;

1 * must be 32-b1t 1nteger here *

/ returns "answer" in 10-m1li1second units as a 32-b1t 1nteger: *

LIB\$STAT_TIMBR (80pu_code, Eanswer);

retura $(10.0 \theta-3 *((d 0 u b l \theta)$ answer $))$ :

)

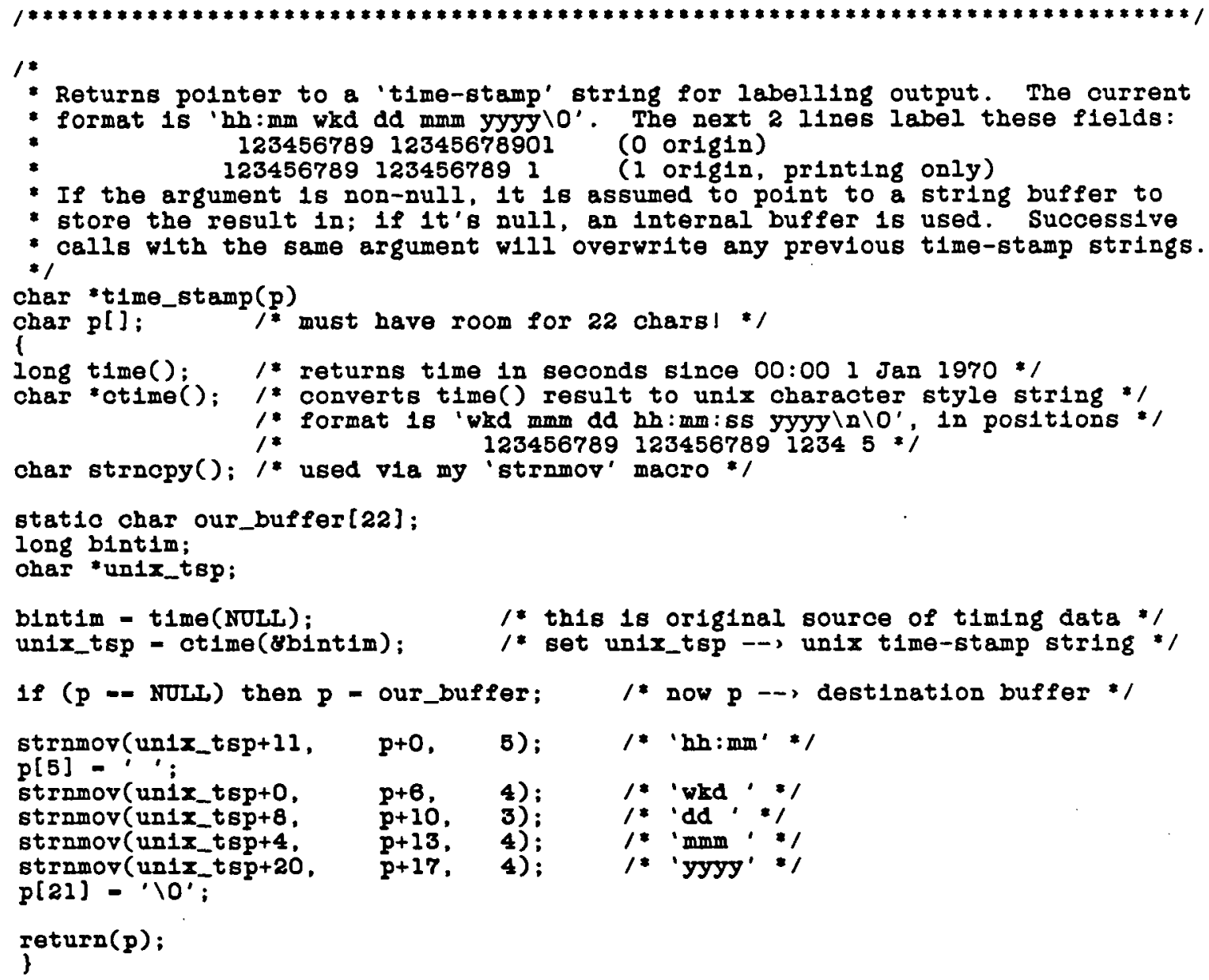


Hobson, R. F., Hannon, P., and Thornburg, J. (1981): "H1gh-Leve1 Microprograming with APL Syntax", Proc. 14th Annual Microprogramming Workshop, 131-139.

Hobson, R. F., Guda1tis, J., and Thornburg, J. (1984): "A New Machine Model for High-Level Language Interpretation", Simon Fraser University Computer Science Technical Report TR84-18.

Thornburg, J. (1985): "On Polygon Cl1pping", APL Quote Quad 15(3), 29-30; errata in press.

Thornburg, J. (1985): "An Upper Limit for the Solar Acceleration", Monthly Notices of the Royal Astronomical Society 213, 27p-28p.

Thornburg, J. (1985): "Further Notes on the Modulo Operator", Sigplan Notices 20(9), 94.

Thornburg, J. (1985): "Global Character String Search and Replace", APL Quote Quad, in press. 